DEVELOPING A MODERN RECYCLED MIX DESIGN AND AUTOMATIC

PAVEMENT MONITORING SYSTEM

A Dissertation
presented to
the Faculty of the Graduate School
at the University of Missouri-Columbia
In Partial Fulfillment
of the Requirements for the Degree
Doctor of Philosophy
Professor William G. Buttlar, Dissertation Supervisor
HAMED MAJIDIFARD
By

Columbia, Missouri

May 2021 
The undersigned, appointed by the dean of the Graduate School, have examined the dissertation entitled

\section{DEVELOPING A MODERN RECYCLED MIX DESIGN AND AUTOMATIC PAVEMENT MONITORING SYSTEM}

Presented by Hamed Majidifard

a candidate for the degree of Doctor of Philosophy in Civil Engineering and hereby certify that, in their opinion, it is worthy of acceptance.

Professor William G. Buttlar

Dr. Yaw Adu-Gyamfi

Professor Carlos Sun

Dr. Amir H. Alavi

Professor Zhihai (Henry) He 


\section{ACKNOWLEDGEMENTs}

First and foremost, I would like to express my profound gratitude and sincere appreciation to my advisor Professor William G. Buttlar who has been a continuous source of guidance, encouragement and support not only throughout the study but also throughout my $\mathrm{PhD}$ life. His suggestions and comments helped me improve the content and clarity of this dissertation. I would like to express my sincerest gratitude to my committee members for their willingness to serve on my committee and for providing me with precious suggestions and insightful comments. I am also indebted to Dr. Adu-gyamfi and Dr. Amir Alavi for their support and assistance. I am also grateful to my committee members Prof. Sun and Prof. He for their help and valuable input in this study.

I would also like to thank my fellow labmates for their stimulating discussions, friendship, and all the fun we have had in the past years. Dr. Behnam Jahangiri, Dr. Punyaslok Rath, Mrs. Loreto Urra, Mrs. Shishi Chen and Mr. Helmut Leodarta it would have been a lonely lab without you. I wish you all the best and I look forward to working with you in the future. Thanks also go to Mr. James Meister lab manager of MAPIL for his help in sample collection and technical input.

Very special thanks are to my parents, Tooba and Mansour, who love and encourage me by standing by me to overcome all difficulties in study and life. Your sacrificial love and gracious support are my drive to reach my goals in all my life. You have given me so much, and in return, so little. There is no way for me to express my utmost gratitude to you. 


\section{Table of Content}

ACKNOWLEDGEMENTS...

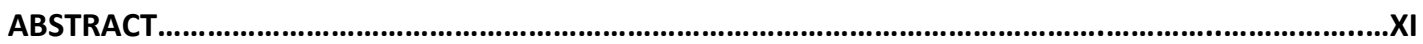

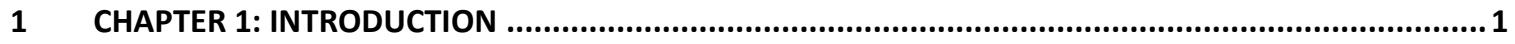

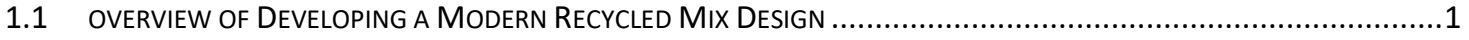

1.2 OVerview of Machine Learning-Based Prediction Models for Performance of Asphalt Mixtures ..........3

1.3 OVerview of Developing a Prediction Model for Pavement Condition of Asphalt Roads using Deep-

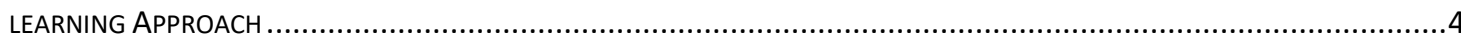

2 CHAPTER 2: DEVELOPING A MODERN RECYCLED MIX DESIGN ...........................................5

2.1 Performance Characteristics of Modern Recycled Asphalt Mixes in Missouri, Including Ground TiRe

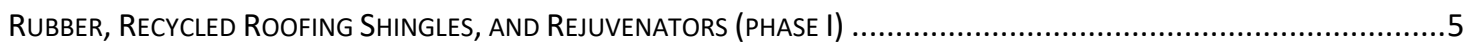

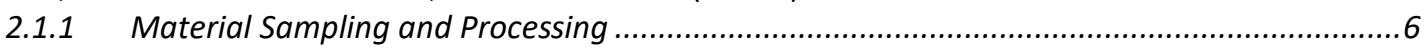

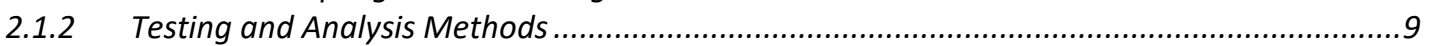

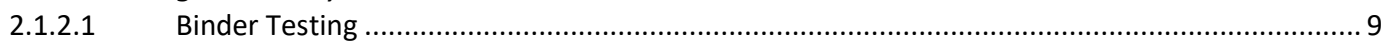

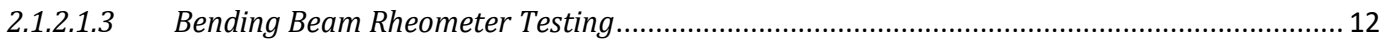

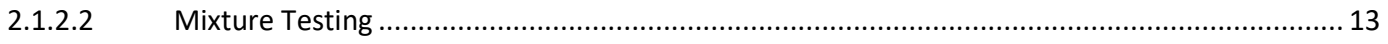

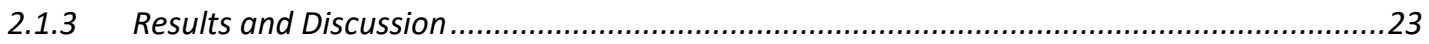

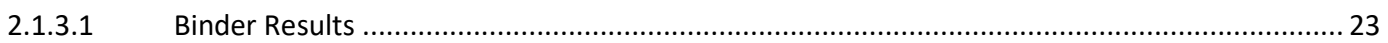

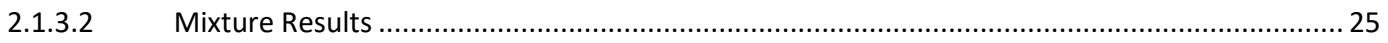

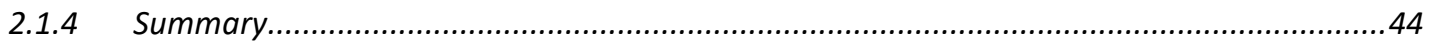

2.2 Understanding and Improving Heterogeneous, Modern Recycled Asphalt Mixes (4R-2) .................44

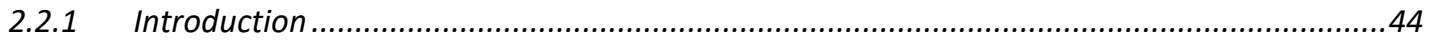

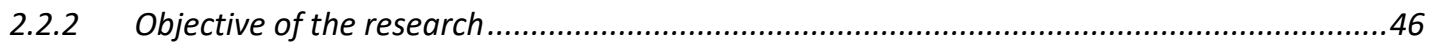

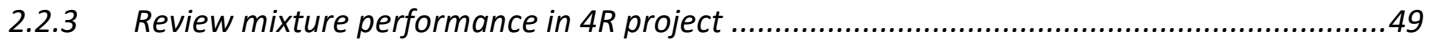

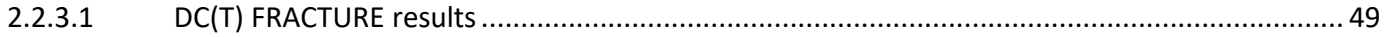

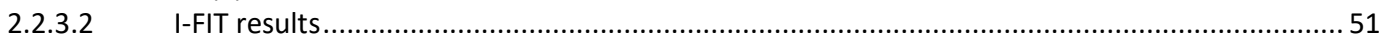

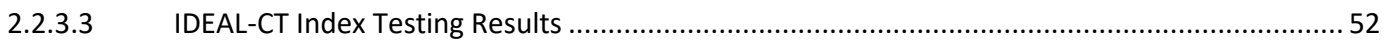

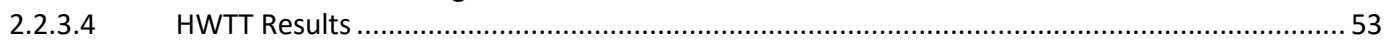

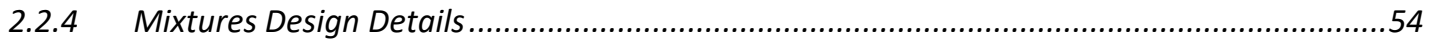

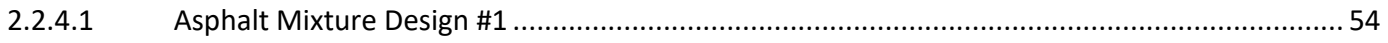

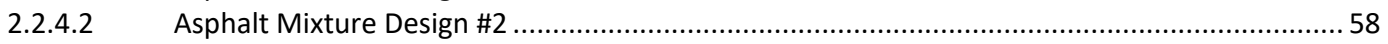

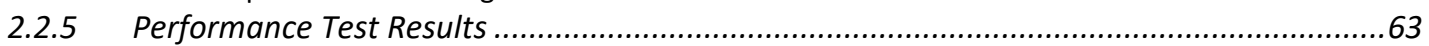

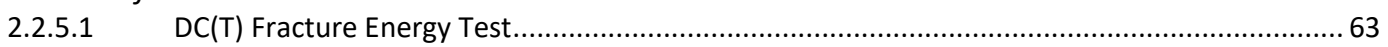

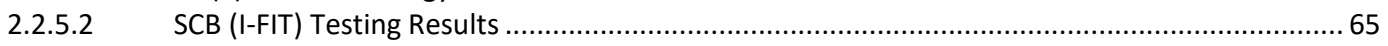

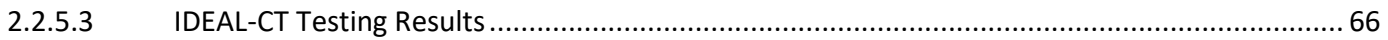

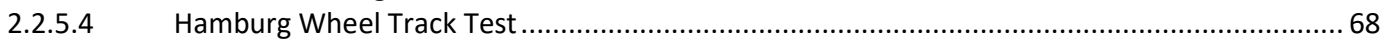

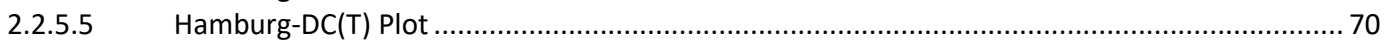

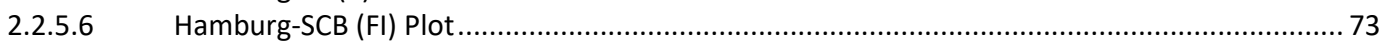

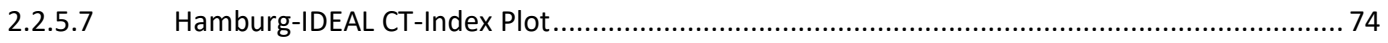

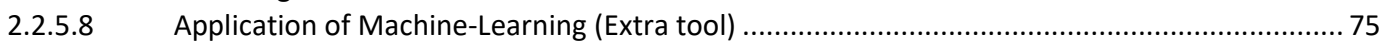

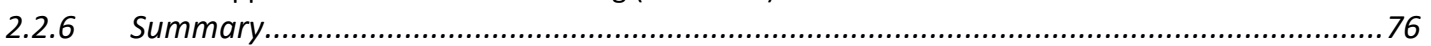

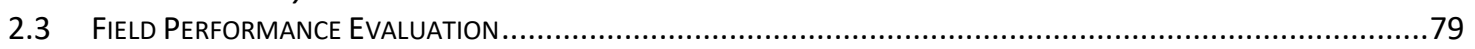

2.3.1 Field Sections Studied Investigated in this Chapter ..........................................................79

2.3.2 Relation of Field Performance to Recycling Levels and Performance Tests..........................82

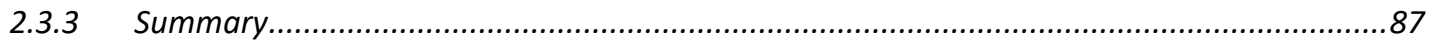


2.4 Investigating Asphalt Pavement Cracking Performance Using Different Cracking Indices.................89

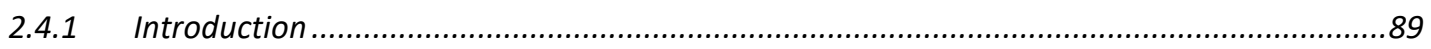

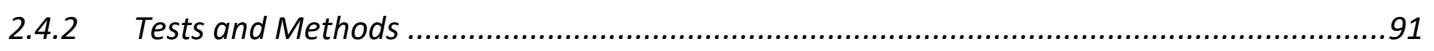

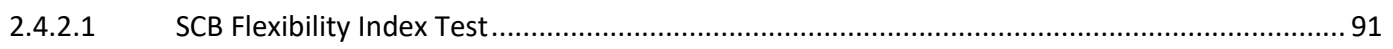

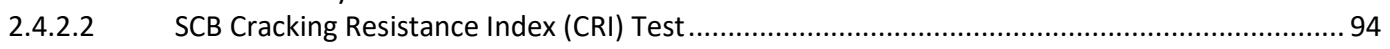

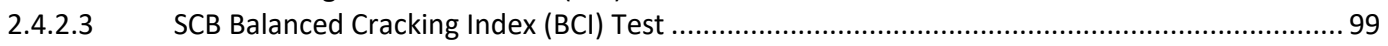

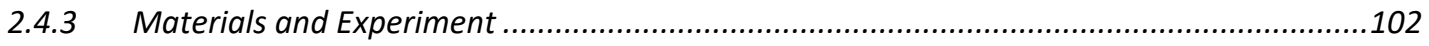

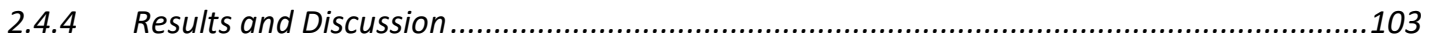

2.4.4.1 Cracking Resistance Using SCB (FI), SCB (BCI) and SCB (CRI) .......................................... 103

2.4.4.2 SCB Indices Repeatability and their Correlation........................................................... 105

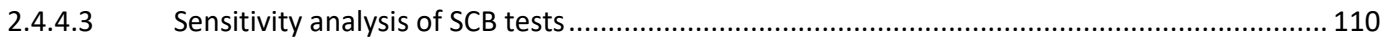

2.4.4.4 Field Performance Evaluation and Statistical Analysis ..................................................... 124

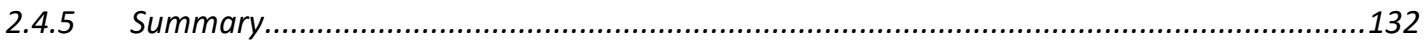

3 CHAPTER 3: MACHINE LEARNING-BASED PREDICTION MODELS FOR PERFORMANCE OF ASPHALT

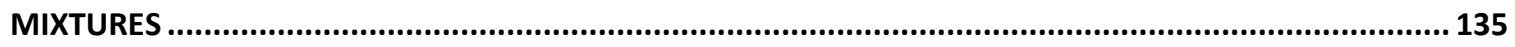

3.1 New Machine Learning-based Prediction Models for Fracture Energy of Asphalt Mixtures............135

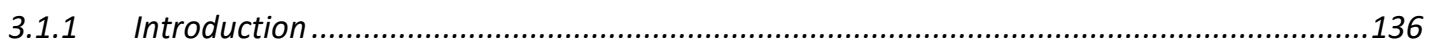

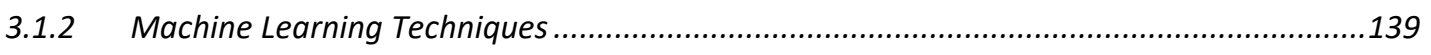

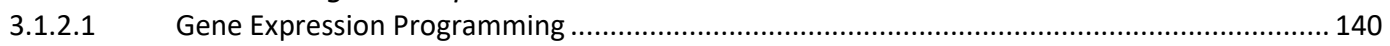

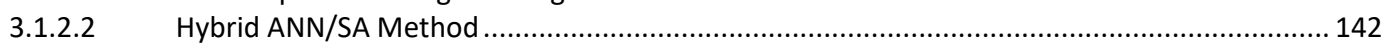

3.1.3 Modeling of Fracture Energy of Asphalt Concrete ........................................................146

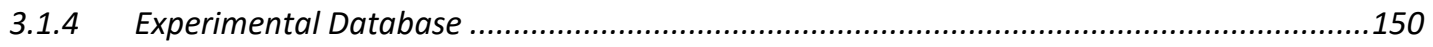

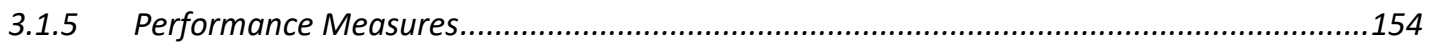

3.1.6 Model Development Using the GEP Method ...............................................................155

3.1.7 Model Development Using the Hybrid ANN/SA Method .........................................158

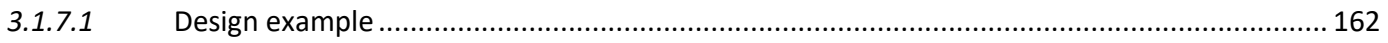

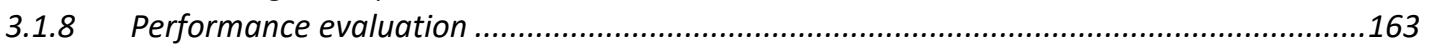

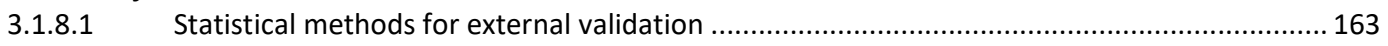

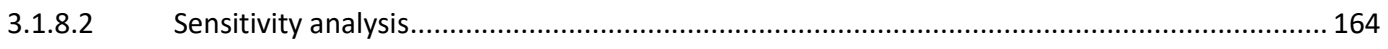

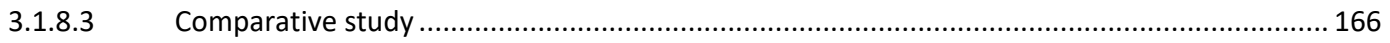

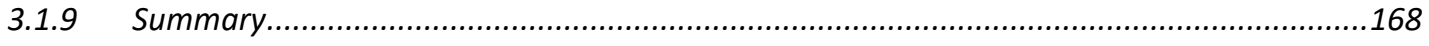

3.2 DeVeloping a Prediction Model for Rutting DePth of Asphalt Mixtures Using Gene Expression

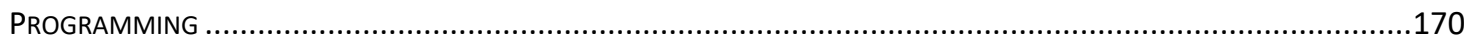

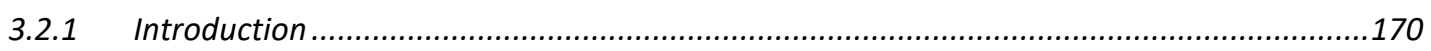

3.2.2 Genetic Expression Programming ........................................................................ 175

3.2.3 Development of HWTT Prediction Model ..................................................................176

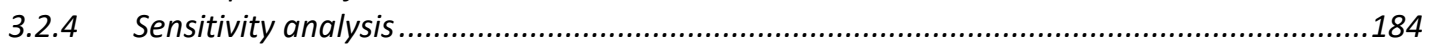

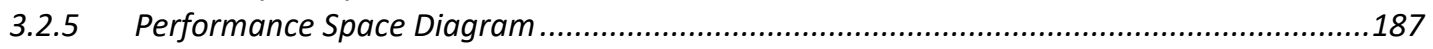

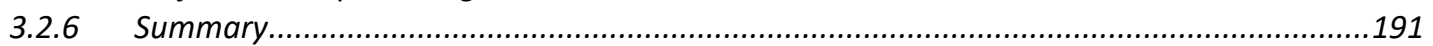

3.3 Implementing Deep Learning Convulsion Neural Network to Predict Hamburg Rutting Curve........193

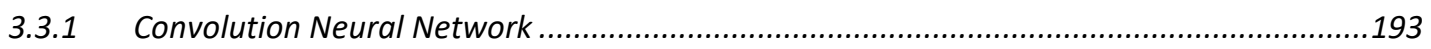

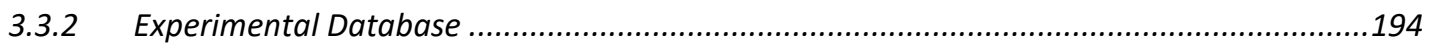

3.3.3 Development of HWTT Prediction Model .....................................................................199

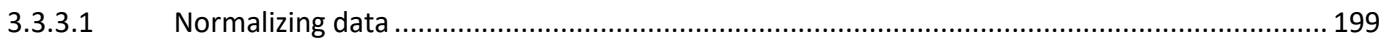

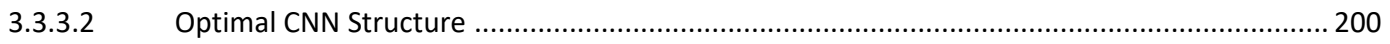

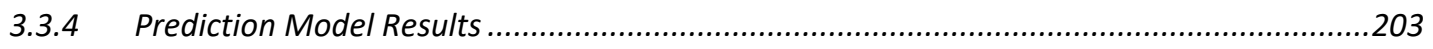

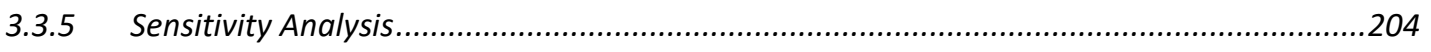

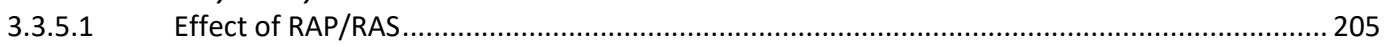

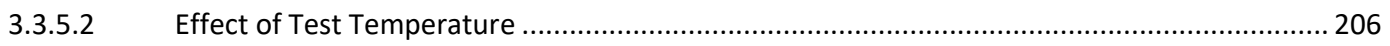

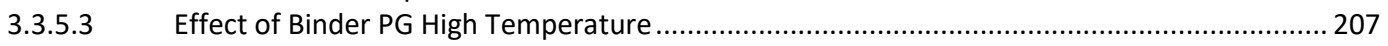

3.3.5.4 Effect of Mixture Production (Lab vs. Plant) .................................................................. 208

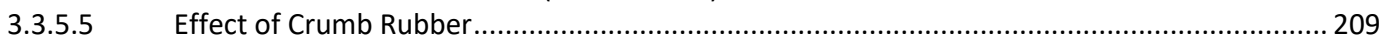

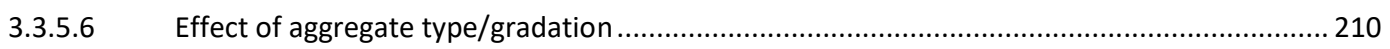

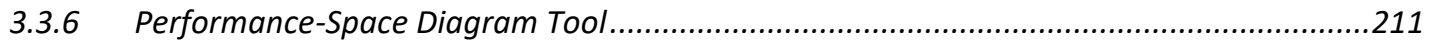


3.3.7 Comparison of rut profiles Obtained from the CNN and GEP models .............................213

3.3.8 Application of the CNN Rutting Prediction Model for Paved Mixtures ................................215

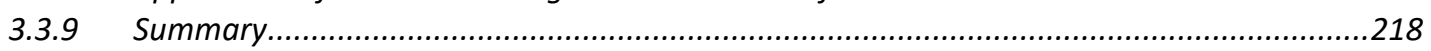

4 CHAPTER 4: DEVELOPING A PREDICTION MODEL FOR PAVEMENT CONDITION OF ASPHALT ROADS

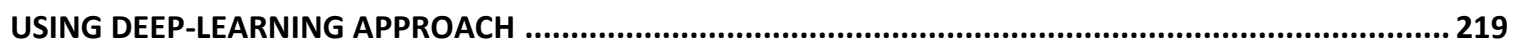

4.1 PaVement Image Dataset: A New Benchmark Dataset to Classify AND DENSIFy PaVement Distresses ..220

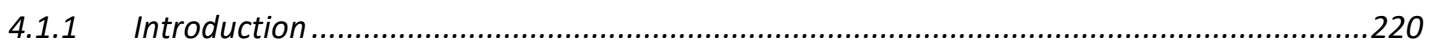

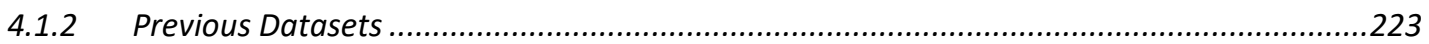

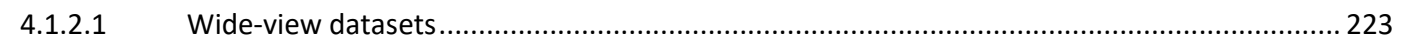

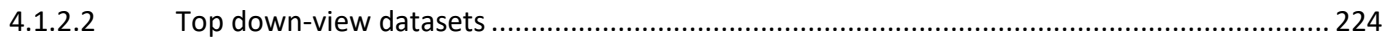

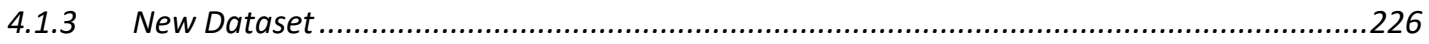

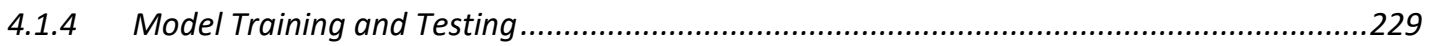

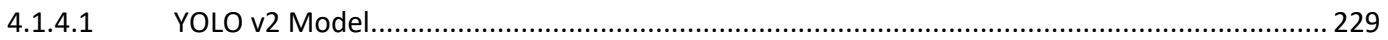

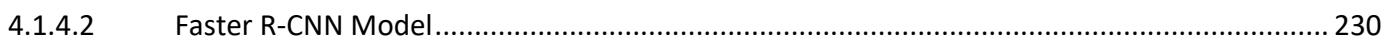

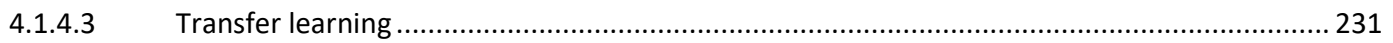

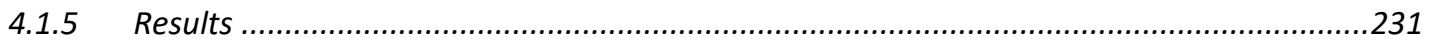

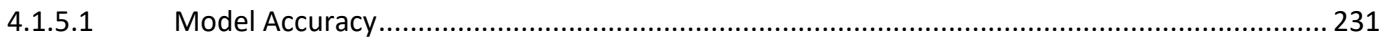

4.1.5.2 Model Performance when Using Top-Down Images......................................................... 238

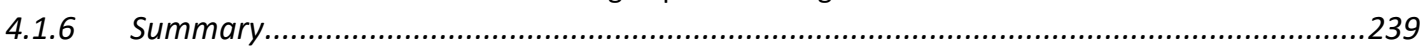

4.2 DEEP MACHINE LEARNING APPROACH TO DEVELOP A NEW ASPHALT PAVEMENT CONDITION INDEXAVEMENT CINDEX 242

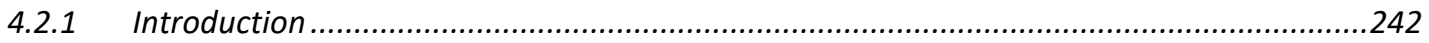

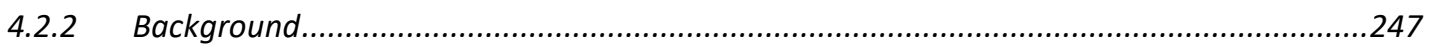

4.2.2.1 Pavement condition index development approaches....................................................... 247

4.2.2.2 Automated Crack detection, segmentation and classification approaches ........................... 248

4.2.2.3 Developing Crack Detection Model using Deep-Learning Framework (Majidifard et al., 2020) 252

4.2.3 Methodology of Developing Pavement Condition Prediction Models .............................258

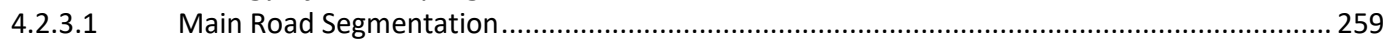

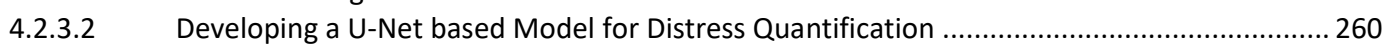

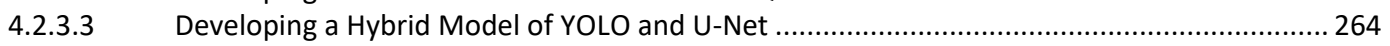

4.2.3.4 Developing Pavement Condition Prediction Models............................................................ 266

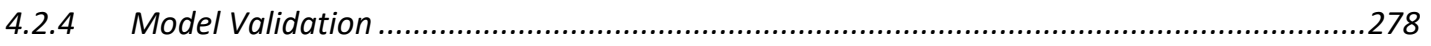

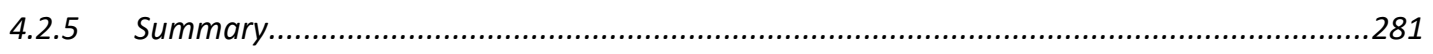

5 SUMMARY, CONCLUSIONS AND RECOMMENDATIONS FOR FUTURE WORK ............................ 285

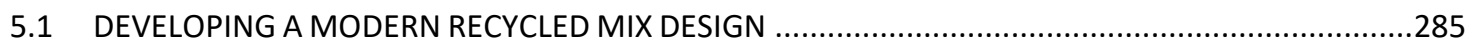

5.2 Machine Learning-based Prediction Models for PerformanCe of AsPhalt Mixtures .........................289

5.3 DeVeloping a Prediction Model for PaVement Condition of Asphalt RoAds using DeEP-LEARNing

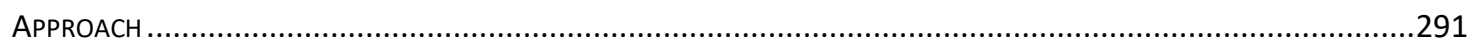

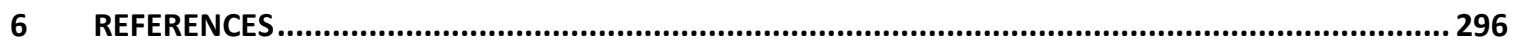

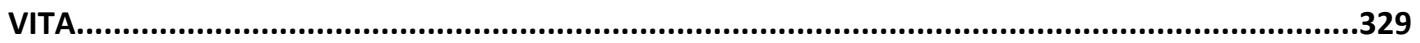




\section{LIST OF FIGURES}

Figure 2-1 Other fabrication equipment: (a) Tile saw, and; (b) Coring rig; (c) Block saw............................8

Figure 2-2 Brookfield DV3T digital rotary viscometer at MAPIL...........................................................10

Figure 2-3 Anton Paar dynamic shear rheometer at MAPIL........................................................11

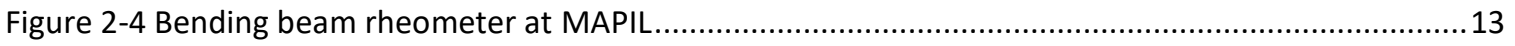

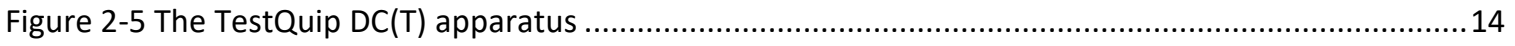

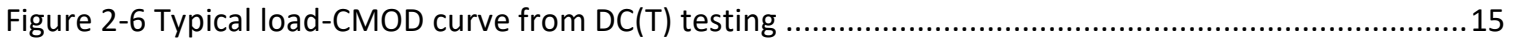

Figure 2-7 The Test Quip IDEAL-CT apparatus at MAPIL ..................................................................20

Figure 2-8 Typical load-displacement curve from Test Quip software.................................................21

Figure 2-9 Hamburg Wheel Tracking device: a) Test device b) mixtures after test ..................................23

Figure 2-10 DC(T) fracture energy test results of field cores ............................................................28

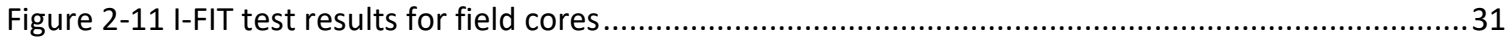

Figure 2-12 An example of very brittle behavior (US54_4-4ay tested sample) .....................................32

Figure 2-13 Comparison of DC(T) FE \& SCB FI test results of field cores .................................................3

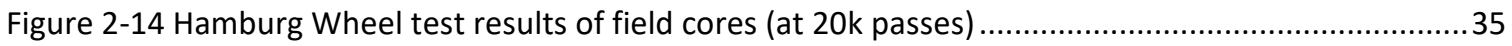

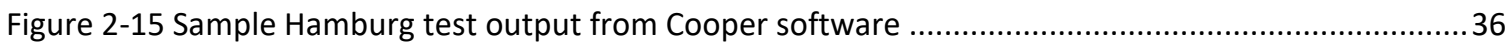

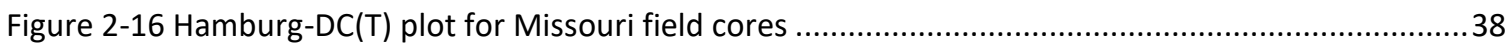

Figure 2-17 Moisture sensitivity in Hamburg Test for SPS10_1 (Sample 2) ............................................43

Figure 2-18 Hamburg-DC(T) plot for 10k Hamburg Wheel Passes ..........................................................43

Figure 2-19 Laboratory material including virgin aggregate, RAP, virgin binder, rejuvenators, additives, and

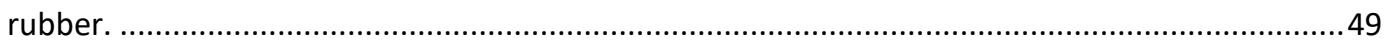

Figure 2-20 Comparing $\mathrm{DC}(\mathrm{T})$ fracture energy of field cores and plant mixes ........................................51

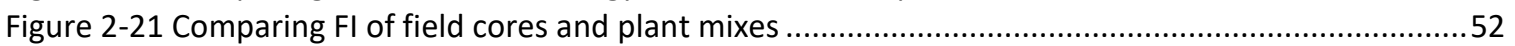

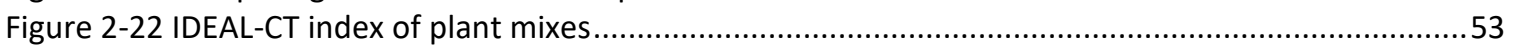

Figure 2-23 Comparing HWTT rut depth of field cores and plant mixes ................................................5

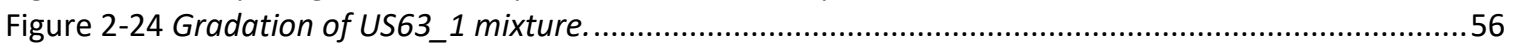

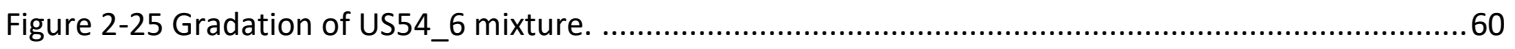

Figure 2-26 DC(T) Fracture energy test results for US63_1 mixtures...................................................64

Figure 2-27 DC(T) fracture energy test results for US54_6 mixtures. .................................................65

Figure 2-28 Flexibility Index results for US63_1 and US54_6 mixtures.................................................66

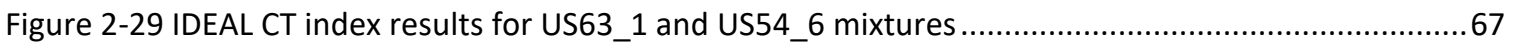

Figure 2-30 Hamburg wheel track test results for US63_1 mixtures .................................................68

Figure 2-31 Hamburg wheel track test results for US54_6 mixtures. ..................................................70

Figure 2-32 Hamburg-DC(T) performance space diagram for US63_1 mixture ........................................71

Figure 2-33 Hamburg-DC(T) performance space diagram for US54_6 mixture...................................72

Figure 2-34 Hamburg-SCB (FI) performance space diagram for US63_2 mixture....................................73

Figure 2-35 Hamburg-SCB (FI) performance space diagram for US54_6 mixture..................................73

Figure 2-36 Hamburg-IDEAL CT-Index performance space diagram for US63_2 mixture..........................74

Figure 2-37 Hamburg-IDEAL CT-Index performance space diagram for US54_6 mixture...........................75

Figure 2-38 Machine learning based prediction model for performance diagram developed by Majidifard

et al. (Majidifard, Jahangiri, Buttlar, \& Alavi, 2019); Majidifard et al., 2020)..............................76

Figure 2-39 Pavement condition for ten field sections from high deterioration rate to low deterioration

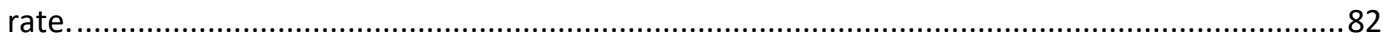

Figure 2-40 PASER rating vs. years in service for a) phase I and b) phase II.........................................84

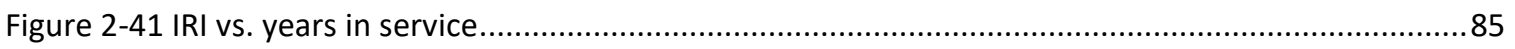

Figure 2-42 Correlation of $\mathrm{DC}(\mathrm{T}) \mathrm{FE}$, FI with deterioration rate from the field .......................................86

Figure 2-43 Correlation of DC(T) FE, FI with deterioration rate from the field (excluding the outlier) .........86

Figure 2-44 Recorded load (P) versus LLD (u) curve (AASHTO TP124-16) ............................................92

Figure 2-45 The load-displacement curve of SCB (IFIT) test for a brittle mixture...................................94

Figure 2-46 The load-displacement curve of two distinct asphalt mixtures [58]....................................96

Figure 2-47 SCB load-displacement curve for two modified mixtures..........................................98 
Figure 2-48 Illustration of the PP 75 point and the corresponding $\mathrm{L}_{75}$ in SCB test, where $\mathrm{PP} 75=75 \%$ peak

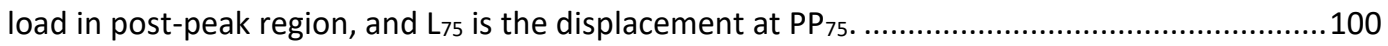

Figure 2-49 a) FI results for 52 sections, b) CRI results for 52 sections, c) BCI results for 52 sections........104

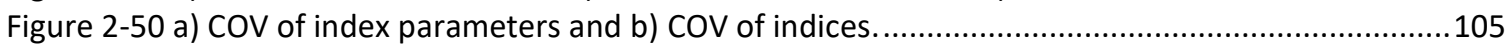

Figure 2-51 Normalized results of SCB (FI), SCB (BCI) and SCB (CRI) for 52 different mixtures. ...............106

Figure 2-52 a, b, c, d, e, f, g, h, i, j. Correlation between indices parameters.......................................109

Figure 2-53 Effect of air voids on $\mathrm{FI}, \mathrm{CRI}$, and $\mathrm{BCl}$ shown for five mixtures including SMAs, and dense-

graded mixes used in various layers of a pavement. .....................................................112

Figure 2-54 Trends of individual parameters shown for IT 1845 mix (Note: All mixtures showed similar

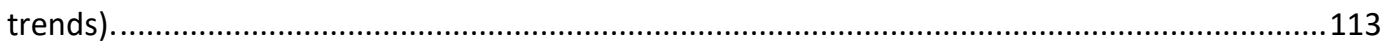

Figure 2-55 Effect of gradation and binder content on different SCB test indices ................................115

Figure 2-56 Effect of softer binder on different SCB test parameters ...............................................116

Figure 2-57 Effect of softer binder on different SCB test indices ..................................................117

Figure 2-58 Effect of crumb rubber on different SCB indices shown for three dense-grade mixtures.......119

Figure 2-59. Effect of crumb rubber on, a) fracture energy, b) slope, c) peak load, and d) L75 parameters

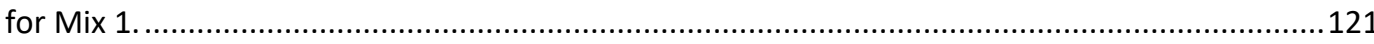

Figure 2-60. Effect of crumb rubber on, a) fracture energy, b) slope, c) peak load, and d) L75 parameters

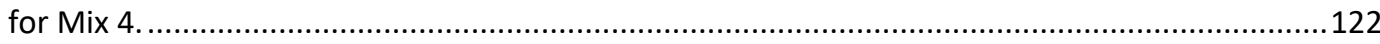

Figure 2-61 Pavement condition for ten field sections from high deterioration rate to low deterioration

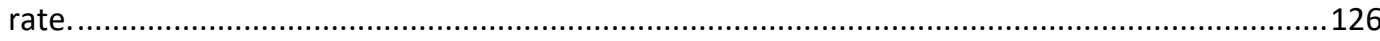

Figure 2-62 Correlation of $\mathrm{FI}, \mathrm{CRI}$, and $\mathrm{BCl}$ indices with deterioration rate for the ten sections..............127

Figure 3-1 Typical thermal cracks in a pavement segment located on U.S. Route 63 in Missouri.............137

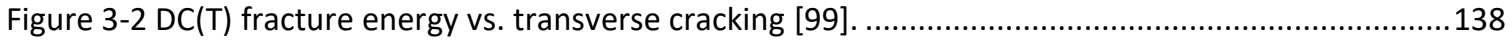

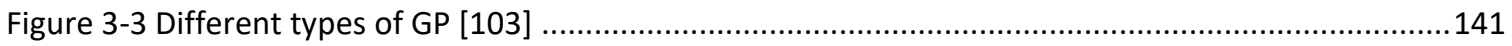

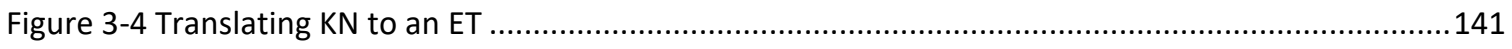

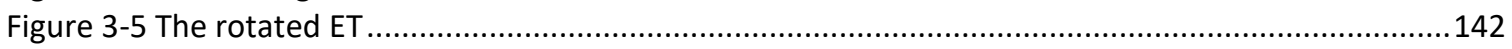

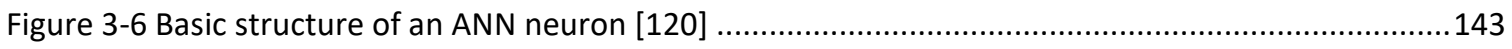

Figure 3-7 Flowchart of the ANN/SA method .................................................................................. 144

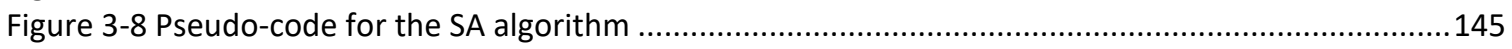

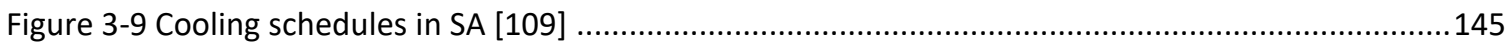

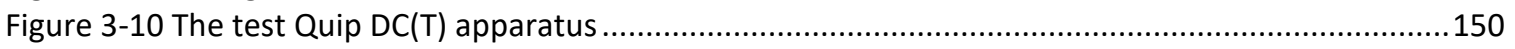

Figure 3-11 Typical Load-CMOD curve from DC(T) testing ......................................................... 151

Figure 3-12 Distribution histograms of the variables .....................................................................154

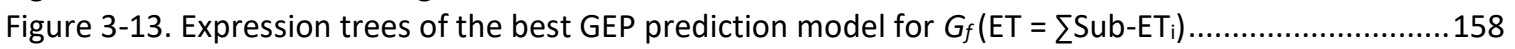

Figure 3-14 Measured against predicted $G_{f}$ using the GEP model: (a) training data, (b) testing data........158

Figure 3-15 The optimal ANN/SA architecture for predicting Gf ......................................................161

Figure 3-16 Measured against predicted $G_{f}$ using the ANN/SA model: (a) training data, (b) testing data .161

Figure 3-17 The procedure to evaluate the relative importance of inputs [144]...............................166

Figure 3-18 Sensitivity analysis of the final predictor variables in: (a) GEP analysis and (b) ANN/SA analysis

Figure 3-19 A comparison of the GEP and ANN/SA models on the entire database .............................167

Figure 3-20 (a) A Hamburg wheel tracking device, (b) Asphalt sample after 20,000 passes in the Hamburg

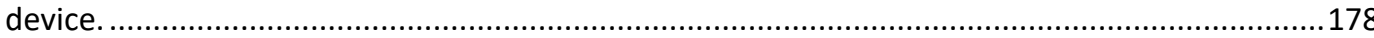

Figure 3-21 Typical rut depth curve versus number of passes in Hamburg wheel tracking test. ..............178

Figure 3-22 Performance of the GEP model: (a) learning data, (b) testing data, (c) validation data..........183

Figure 3-23 Measured versus predicted rut depth across all data points...........................................184

Figure 3-24 Variable importance in the GEP model. .................................................................. 185

Figure 3-25 Effect of a) recycling content b) binder content c) binder PG and d) test temperature on rutting depth................................................................................................. 187

Figure 3-26 Effect of using softer binder in rubber modified mixture with the application of performance space diagram, (a) measured laboratory values versus, (b) predicted values.............................189

Figure 3-27 Effect of using softer binder in recycled mixture with the application of performance space diagram, (a) measured laboratory values versus, (b) predicted values. 
Figure 3-28 Effect of RAS and binder PG grade in asphalt mixture with the application of performance space diagram, predicted (a) for 58-28 binder, (b) for 46-34 binder.

Figure 3-29 Typical rut depth curve versus number of passes in Hamburg wheel tracking test (200 data

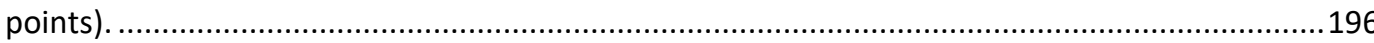

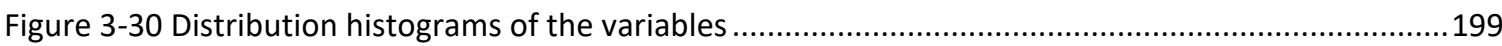

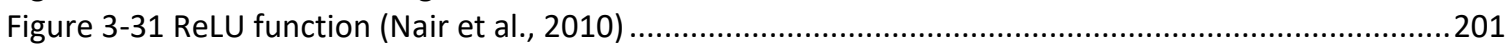

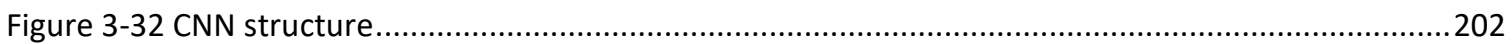

Figure 3-33 Performance of trained model on training and validation dataset versus 1000 epoch...........203

Figure 3-34 Performance of trained model on training and validation dataset versus 40 epoch...............203

Figure 3-35 Measured against predicted rut depth using the CNN model: (a) training dataset, (b) testing dataset and c) validation dataset........................................................................................204

Figure 3-36. Sensitivity analysis of the CNN and GEP models: a) Effect of RAP and RAS, b) Effect of RAS and

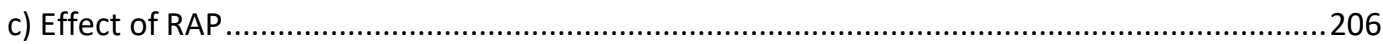

Figure 3-37. Sensitivity analysis of the CNN and GEP models: a) Effect of temperature on rut depth versus number of passes in CNN model, b) Effect of test temperature on rut depth in CNN and GEP models.

Figure 3-38. Effect of Binder PG high temperature on model prediction: (a) CNN model predictions with progressively stiffer binder, (b) Comparison of the CNN and GEP predictions with progressively stiffer binders

Figure 3-39. Effect of production (Lab vs. Plant produced) on model predictions: (a) CNN model, (b) GEP

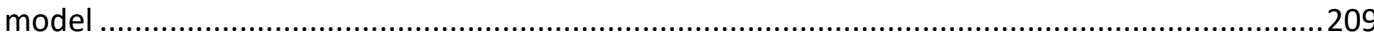

Figure 3-40. Effect of GTR\% on model predictions, (a) CNN model, (b) GEP model ................................210

Figure 3-41. The CNN model predictions for aggregate type and gradation ...........................................211

Figure 3-42. The GEP-CNN based prediction model for performance diagram developed by Majidifard et

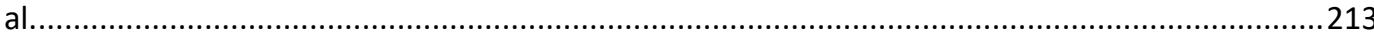

Figure 3-43. Comparison of the rut depth profiles predicted by the GEP and CNN models.....................215

Figure 3-44. Comparison of predicted and actual Hamburg rut depth using the GEP- and CNN models...217

Figure 4-1 a) Nine different distress classes considered in the PID dataset; b) sample of annotated images

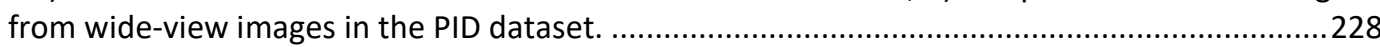

Figure 4-2 a) Number of boundary boxes for each class, b) Number of images for each class. .................229

Figure 4-3. Classification of predicted crack from validation dataset: a) true positive, b) false positive and false negative, c) false negative, d) missed annotations identified by YOLO v2 ..........................234

Figure 4-4. Confusion matrices obtained on the classification dataset using a) YOLO v2 and b) Fast R-CNN models

Figure 4-5. a) detection from YOLOv2 model b) detection from Faster R-CNN model, 1, 2, 3 and 4 represent plain top-down, plain wide-view, shadowed top-down and shadowed wide view image. UNET output, overlapped modified UNET on original image .....................................................241

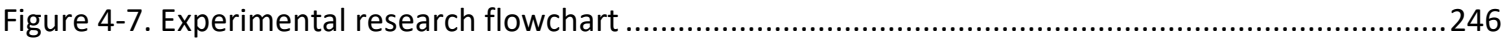

Figure 4-8. Annotated images (wide-view images) in the PID dataset [235] ............................................25

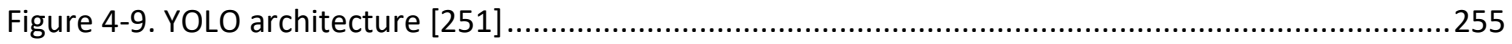

Figure 4-10. Samples of detecting pavement distresses from top-down images [235]...........................256

Figure 4-11. Example of inconsistency in PASER rating among the investigated sections..........................258

Figure 4-12. The primary PASER prediction models developed in this study ...........................................259

Figure 4-13. Automatic cropping of the main-road from shoulder via CNN ............................................260

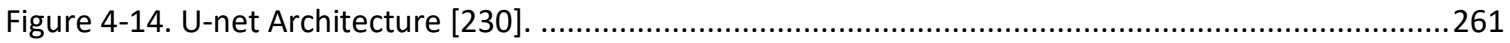

Figure 4-15 a. One example of the training data to developed the U-net model, b. hand-crafted annotations that were used to retrain the model. ..................................................................262

Figure 4-16. Examples of detecting shadows and cars as cracks a) raw image b) pre-trained model output,

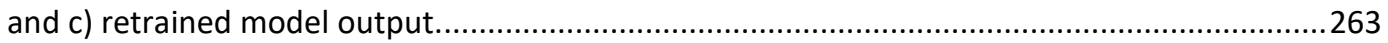

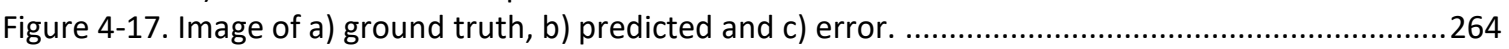

Figure 4-18. Integrated Yolo and U-Net based model and calculation of ratio of white pixels ..................265 
Figure 4-19. Distribution histograms of a) the features and b) output.

Figure 4-20. Measured versus predicted value for the entire data.

Figure 4-21. Measured against predicted PASER using the GEP model: (a) training data; (b) testing data, and; (c) validation data. ..........................................................................................2 272

Figure 4-22. Variable importance in the GEP model. ......................................................................273

Figure 4-23. Correlation of a) distress density with different threshold, b) PASER versus distress density

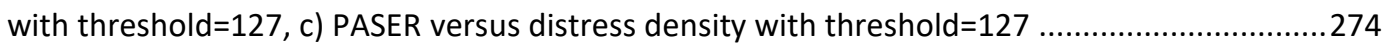

Figure 4-24. Example of problems associated with crack density U-Net based model .............................2275

Figure 4-25. Measured PASER versus predicted PASER using linear regression: (a) training data; (b) testing

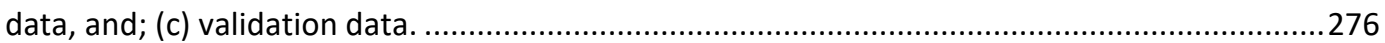

Figure 4-26. Measured PASER versus predicted PASER using Weight Method: (a) training, b) testing and (c) Validation dataset. .............................................................................................278

Figure 4-27. Pavement condition, a) I70 (good condition), b) US50 , c) MO11, d) US24, e)US71 and f) MO52 (dense-block cracks).............................................................................................................280

Figure 4-28. Changing of Predicted PASER using different models a) Hybrid model-weight based, b) Hybrid model-linear regression, c) YOLO model-GEP based and d) Measured PASER, for six sections alongside pavement profile. 


\section{LIST OF TABLES}

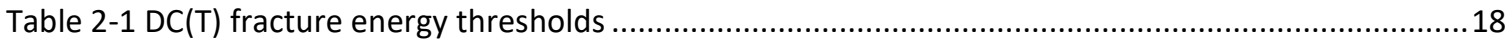

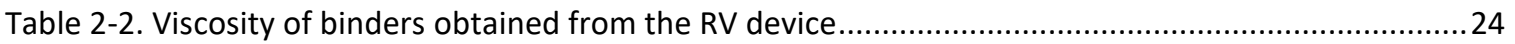

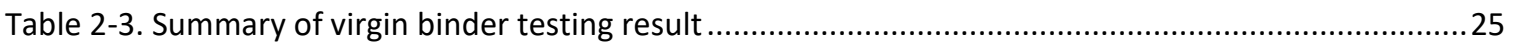

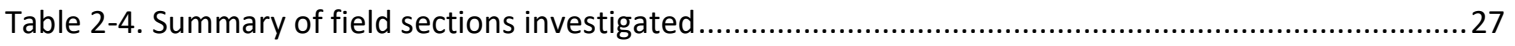

Table 2-5. Coefficient of variance (COV) of DC(T) and I-FIT measurements on field cores for Missouri

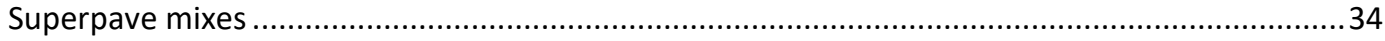

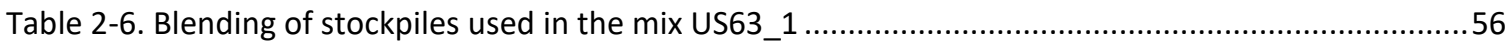

Table 2-7. Iterations of modifications applied to US63_1 mixes...............................................................57

Table 2-8. Details for US63_1_C (Control Mix) compacted to 4.0+0.5\% air voids ......................................58

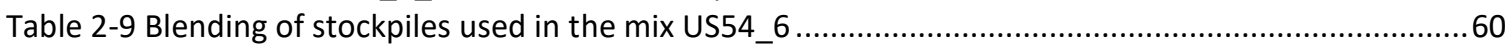

Table 2-10. Iterations of modifications applied to the US54_6 mixes .....................................................61

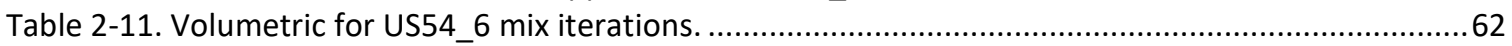

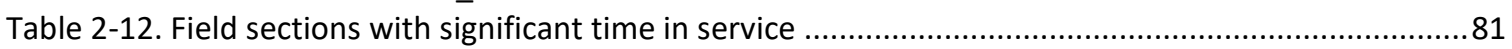

Table 2-13. Performance measures before running the performance tests at laboratory ...........................82

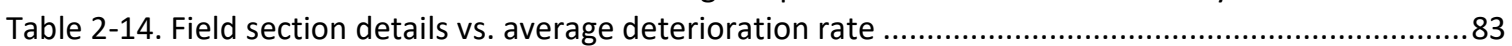

Table 2-15. Test averages and coefficient of variability for field sections ..................................................8 87

Table 2-16. Range of materials $s^{a}$ and other mixture characteristics represented in the 52 asphalt sections

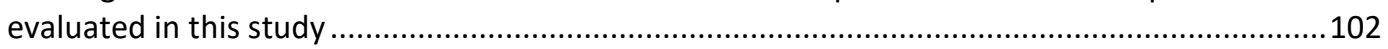

Table 2-17. Summary of mixtures properties to investigate the effect of gradation type ........................114

Table 2-18. Summary of mixtures properties to investigate the effect of softer binder .............................115

Table 2-19. Summary of mixtures properties to investigate the effect of crumb rubber ............................118

Table 2-20. Statistical analysis to investigate the performance of each index to delineate mixes with

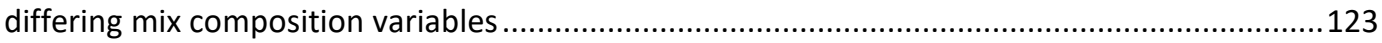

Table 2-21. Mixture properties, field performance, and SCB indices result for ten sections. ....................126

Table 2-22 Statistical analysis of performance test results. ..................................................................129

Table 3-1 A summary of the parameters affecting low-temperature cracking resistance of asphalt concrete

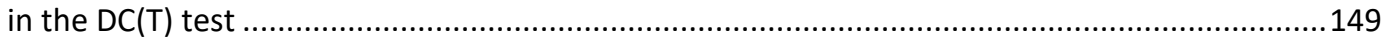

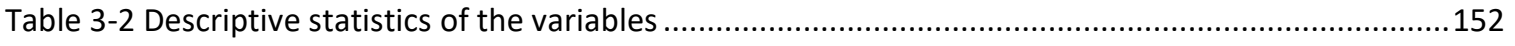

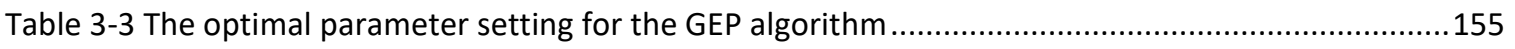

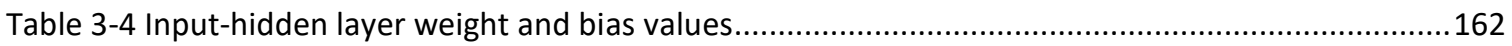

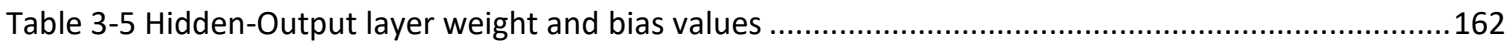

Table 3-6 Performance measures for further validation of the GEP and ANN/SA models .........................164

Table 3-7 A summary of the parameters affecting rutting resistance of asphalt concrete. .......................173

Table 3-8 Statistical parameters of the dependent and independent variables......................................180

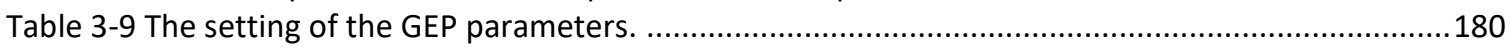

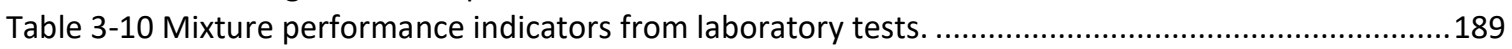

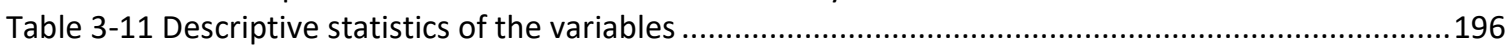

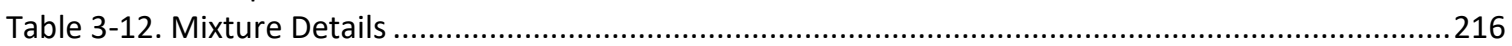

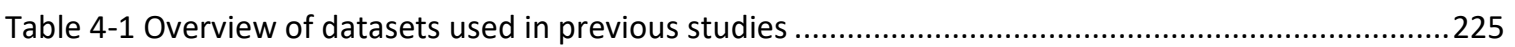

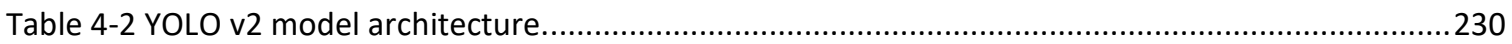

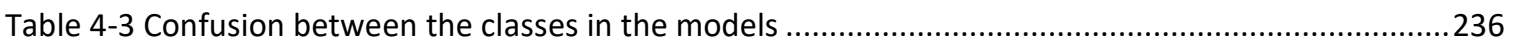

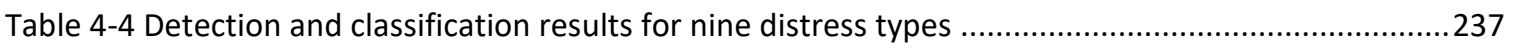

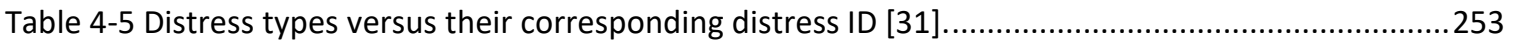

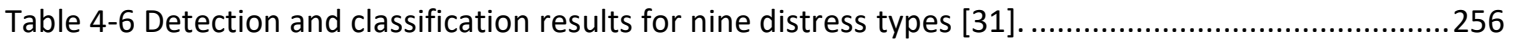

Table 4-7 Statistical parameters of the dependent and independent variables.........................................266

Table 4-8 The optimal parameter setting for the GEP algorithm..............................................................269

Table 4-9 Estimated coefficient for the fitted prediction model using linear regression from R software 276

Table 4-10 Distress weights to calculate the predicted PASER using weight method ..............................277 


\begin{abstract}
The main aim of agencies involved in the construction of asphalt roads is to improve the field performance of the asphalt mixtures. The rising use of recycled and novel materials in asphalt mixture has rendered the previous semi-empirical methods of mixture design partly incapable of accurately predicting the mixture field performance with high precision. Meeting this challenge calls for a shift towards an approach involving mixture performance tests. This project deals with investigating the performance of modern recycled asphalt mixes containing ground tire rubber, Recycled Asphalt Shingles (RAS), Recycled Asphalt Pavement (RAP) and rejuvenators. Various performance tests for various type of distresses were considered to evaluate the effect of using these components in asphalt mixtures. Combining these performance tests with prediction of field performance of mixtures should provide more robust and reliable design criteria for the modern recycled asphalt mixtures leading to better roads. To this end, the performance of eighteen different dense-graded asphalt mixtures paved in Missouri were investigated. The sections contain a wide range of reclaimed asphalt pavement (RAP) and recycled asphalt shingles (RAS), and different types of additives. The large number of sections investigated and the associated breadth of asphalt mixtures tested provided a robust data set to evaluate the range, repeatability, and relative values provided by modern mixture performance tests. As cracking is one of the most prevalent distresses in Missouri, performance tests such as the disk-shaped compact tension test (DC[T]) and Illinois flexibility index test (I-FIT) were used to evaluate the cracking potential of the sampled field cores. In addition, the Hamburg wheel tracking test (HWTT) was employed to assess rutting and stripping potential. Asphalt binder replacement $(\mathrm{ABR})$ and binder grade bumping at low temperature were found to be critical
\end{abstract}


factors in low-temperature cracking resistance as assessed by the $\mathrm{DC}(\mathrm{T})$ fracture energy test. Six sections were found to perform well in the $\mathrm{DC}(\mathrm{T})$ test, likely as a result of binder grade bumping (softer grade selection) or because of low recycling content. However, all of the sections were characterized as having brittle behavior as predicted by the I-FIT flexibility index. Service life and ABR were key factors in the I-FIT test. Finally, a performance-space diagram including DC(T) fracture energy and HWTT depth was used to identify mixtures with higher usable temperature interval (UTI mix), some of which contained significant amounts of recycled material. In the second phase of chapter 2, the poor performing mixtures were redesigned in order to improve their performance by changing the components of the mixtures including recycling content, rejuvenator type and amount, binder type, crumb rubber quantity, etc.. Finally, the optimum content of the components based on mixture performance and materials costs was determined. The testing results along with the field performance data was used to develop a specification for MoDOT to screen the mixtures and use it for quality control and quality assurance of plantproduced asphalt concrete. Field monitoring is a potential means to identify the most reliable cracking performance test. Also, a new cracking index was introduced based on SCB (I-FIT) test to improve the test reliability and correlation with field results.

In the third chapter of this study a prediction tool was developed to predict the performance of asphalt mixture at high and low temperatures. This tool is based on two different prediction models for DC(T) fracture energy and Hamburg wheel track tests. For $\mathrm{DC}(\mathrm{T})$ fracture energy model, genetic programming was used to develop the prediction model, and Convolution Neural Network (CNN) was used to train the Hamburg wheel track model on 10,000 data points. A database containing a comprehensive collection of 
Hamburg and $\mathrm{DC}(\mathrm{T})$ tests results were used to develop the machine learning-based prediction models. This tool can be used for pre-design purposes to design an asphalt mixture with balanced performance in rutting and cracking. The models were formulated in terms of typical influencing mixture properties variables such as asphalt binder hightemperature performance grade (PG), mixture type, aggregate size, aggregate gradation, asphalt content, total asphalt binder recycling content and tests parameters like temperature and number of cycles. Models accuracy were assessed through a rigorous validation process and found to be quite acceptable, despite the relatively small size of the training set. Since performing performance tests might be cost-restrictive for some users, using the proposed ML-based models can save time and expense during the material screening phase.

Pavement distress inspections are performed using sophisticated data collection vehicles and/or foot-on-ground surveys. In either approach, the process of distress detection is human-dependent, expensive, inefficient, and/or unsafe. Automated pavement distress detection via road images is still a challenging issue among pavement researchers and computer-vision community. In the forth chapter of dissertation, we extracted 7237 google street-view, manually annotated for classification (nine categories of distress classes). Afterward, the YOLO (you look only once) deep learning framework was implemented to train the model using the labeled dataset. Also, U-net based model is developed to quantify the severity of the distresses, and finally, a hybrid model is developed by integrating the YOLO and U-net model to classify the distresses and quantify their severity simultaneously. The output of the distress classification and segmentation models are used to develop a comprehensive pavement condition tool which rates each pavement image according to the type and severity of distress extracted. As a result, we 
are able to avoid over-dependence on human judgement throughout the pavement condition evaluation process. The outcome of this study could be conveniently employed to evaluate the pavement conditions during its service life and help to make valid decisions for rehabilitation of the roads at the right time. 


\section{CHAPTER 1: INTRODUCTION}

\subsection{OVERVIEW OF DEVELOPING A MODERN RECYCLED MIX DESIGN}

Asphalt concrete is the most recycled material on the planet. Yet, after several decades of increased usage, the procedures for incorporating reclaimed asphalt pavement (RAP) into asphalt mix designs is not completely performance-based. Hundreds-ofmillions of scrap tires can be found stockpiles in the United States, leading to an ample supply of potentially recyclable ground tire rubber (GTR).

Over ten million tons of tear-off roofing shingles are currently stockpiled, creating the potential for large-scale recycling of recycled asphalt shingles (RAS). Recycling these materials in asphalt pavements is a potentially sustainable solution and can often yield performance benefits if used correctly. However, a lack of scientific test results and effective tests, especially to evaluate new products and manufacturing processes, and a lack of clear quantification of costs vs. benefits impedes implementation by state transportation agencies and industry. There is a particular lack of literature and research experience with regards to newer ground tire rubber (GTR) asphalt products and their use in the Midwest. Adjustments to mix design procedures, particularly for those containing recycled asphalt shingles (RAS), also need to be studied. The role of rejuvenators in adding resiliency to recycled materials as blending components, and to the restoration of pavement surfaces, is also an open research topic. The first phase of this study involved an agency-sponsored project entitled "Performance Characteristics of Modern Recycled Asphalt Mixes in Missouri, Including Ground Tire Rubber, Recycled Roofing Shingles, and Rejuvenators". In this phase, a comprehensive lab and field investigation was carried out to evaluate the 
performance of recycled asphalt mixtures in Missouri by researchers at the University of Missouri-Columbia, in collaboration with the Missouri Department of Transportation and the Midwest Transportation Center. In this project the laboratory tests were performed on sixteen field sections, including a number of sections from the recent Long-Term Pavement Performance (LTPP), Special Pavement Sections (SPS-10) project in Osage, Beach, MO, which was constructed in 2016. Binder testing and mix performance tests were carried out on field cores and laboratory compacted specimens. Afterward the second phase of the study was defined as "Understanding and Improving Heterogeneous, Modern Recycled Asphalt Mixes". This study suggests a procedure and protocol to design high-performance mixture with the available asphalt components and materials. Various studies such as adding rejuvenators, softer binder, and crumb rubber were implemented to boost mixtures performance according to Balanced Mix Design approach. Field performance evaluation was the third phase of this chapter and 11 old field section were investigated and their field performance were compared with the laboratory test results. This helped to select the most reliable laboratory test to simulate field performance and confirmed the importance of material selection and accurate mix design strategies to meet the highest performance during pavement service life. The last phase of this chapter involved with developing a new index for semi-circular bending (SCB-Ifit) test which increased the repeatability and reliability of the test. Field performance results were used to evaluate the accuracy of the index compared to the older ones. 


\subsection{OVERVIEW OF MACHINE LEARNING-BASED PREDICTION MODELS FOR PERFORMANCE OF ASPHALT MIXTURES}

Rutting and low-temperature performance are among the most important performance tests which are critical to design a superior asphalt mixture. However, preparing asphalt samples and running the tests are expensive (each test about 1500\$) and time consuming. Therefore, developing a prediction tool based on real data using machinelearning techniques is very demanding. Various mixture and test condition parameters were considered and finally the operator can estimate the output results (rutting or lowtemperature cracking performance) by feeding the parameters into the model.

This project presents innovative machine learning methods called gene expression programming (GEP) and hybrid artificial neural network/simulated annealing (ANN/SA) to predict the fracture energy of asphalt mixture specimens. The GEP and ANN/SA models are developed using an experimental database including a number of disk-shaped compact tension $(\mathrm{DC}(\mathrm{T}))$ test results for fracture energy. The $\mathrm{DC}(\mathrm{T})$ testing has been widely used to address low temperature cracking of asphalt pavements. The fracture energy is formulated in terms of various predictor variables such as asphalt binder performance grading $(\mathrm{PG})$, asphalt content, aggregate size, aggregate gradation, reclaimed asphalt pavement (RAS) content, reclaimed asphalt shingles (RAS) content, crumb rubber content, and test temperature. A calculation procedure is presented to interpret the models and transform them into practical design equations.

In phase two of this study, a new prediction model for the rutting depth of asphalt mixtures using a machine learning (ML) technique called gene expression programming 
(GEP) and deep learning techniques. The Hamburg wheel tracking test has been widely used to evaluate the high temperature performance of asphalt paving mixtures. A database containing a comprehensive collection of Hamburg test results was used to develop a GEPbased CNN-based rutting depth models. The model was formulated in terms of typical influencing variables such as asphalt binder high temperature performance grade (PG), mixture type, aggregate size, aggregate gradation, asphalt content, and total asphalt binder recycling content. The models are recommended for pre-design purposes or as a tool to determine rut depth of asphalt mixtures when laboratory testing is not feasible. Since performing Hamburg wheel track test might be cost-restrictive for some users, using the proposed ML-based model can save time and expense during the material screening phase.

\subsection{OVERVIEW OF DEVELOPING A PREDICTION MODEL FOR PAVEMENT CONDITION OF ASPHALT ROADS USING DEEP-LEARNING APPROACH}

Pavement distress detection is the key to organize a pavement management system. Pavement distress detection intends to obtain the pavement condition which provides information to make more consistent, cost-effective, and defensible decisions related to the

preservation of a pavement network. Generally, pavement distress inspection is performed by ARAN machines traveling on the road. However, the process of distress detection is human-dependent, expensive, inefficient, and unsafe. For instance, a vehicle equipped with modern sensor and computing systems was purchased by the Ohio Department of Transportation for US\$1,179,000, with an annual operating cost US\$70,000. Therefore, a fully automatic pavement crack detection is very attractive for practitioners because of its low cost, good repeatability, and ease of use. 


\section{CHAPTER 2: DEVELOPING A MODERN RECYCLED MIX DESIGN}

This chapter is divided to four phases.

- Performance Characteristics of Modern Recycled Asphalt Mixes in Missouri, Including Ground Tire Rubber, Recycled Roofing Shingles, and Rejuvenators (abbreviated hereafter as the '4R project')

- Understanding and Improving Heterogeneous, Modern Recycled Asphalt Mixes (or the '4R-2 project')

- Field Performance Evaluation

- Investigating Asphalt Pavement Cracking Performance Using Different Cracking Indices

\subsection{PERFORMANCE CHARACTERISTICS OF MODERN RECYCLED ASPHALT MIXES IN MISSOURI, INCLUDING GROUND TIRE RUBBER, RECYCLED ROOFING SHINGLES, AND REJUVENATORS (PHASE I)}

A comprehensive lab and field investigation was carried out to evaluate the performance of recycled asphalt mixtures. Sixteen field sections were evaluated, including a number of sections from the recent Long-Term Pavement Performance (LTPP), Special Pavement Sections (SPS-10) project in Osage, Beach, MO, which was constructed in 2016. Good and poor performing sections dating back as far as 2003 construction were sampled and tested. Binder testing and mix performance tests were carried out on field cores and laboratory compacted specimens. The study focused on medium traffic volume Superpave mixes. The advanced laboratory tests were carried on mixtures having three distinct 
preparation methods, including field produced - laboratory compacted (reheated plant mix), field cores, and field sampled mixture components - laboratory prepared. In addition to a full suite of Superpave binder testing, including continuous grading and an extensive suite of asphalt mixture performance tests was carried out. This suite included Hamburg wheel track testing (submerged), disk-shaped compact tension, or DC(T) fracture testing, semi-circular bend (SCB) crack testing using the Illinois 'iFIT' procedure, the IDEAL cracking test.

A laboratory and field investigation of modern asphalt rubber products, RAP, RAS, and rejuvenators available in the Midwest was conducted. Investigations included both asphalt binder and mixture performance characterization, including materials sampled from the field projects.

\subsubsection{Material Sampling and Processing}

Based on the project proposal, a total of 18 projects were selected for sampling and testing in this study. An effort was made to encompass the following factors:

- New vs. older projects

- Good and poor performers

- Range of recycled materials and additives, i.e., RAP, RAS, GTR, Rejuvenator, Polymer

- Geographic distribution across Missouri, balanced against a concentration of a majority of projects around the center of the state to reduce sample transportation costs 
- Heavy sampling (coring plus plant and paver sampling), to enable future testing and testing of reconstituted lab mixes, vs. light sampling for economy (coring only)

For mixture testing, a majority of tests conducted in this study were performed on 150-mm diameter by $50 \mathrm{~mm}(2$ ”) thick specimens. This includes disk-shaped compact tension testing, semi-circular bend tests, and indirect tension creep and strength tests. In addition, further sample fabrication cuts and coring operations were required, depending on the test requirements. A block saw was used to cut cores and gyratory specimens (Figure 2-1), while a tile saw and small coring rig were used to meet additional fabrication requirements for mixture mechanical tests. The fabrication equipment used was obtained from Test Quip, LLC, and featured a 16" diameter block saw blade (stiffer than the more common 20" diameter blade), and purpose-built masonry (tile) saw and coring rig (Figure 2-1), which are equipped with adjustable fixtures to facilitate proper sample dimensioning. All devices were water cooled to avoid overheating of samples.
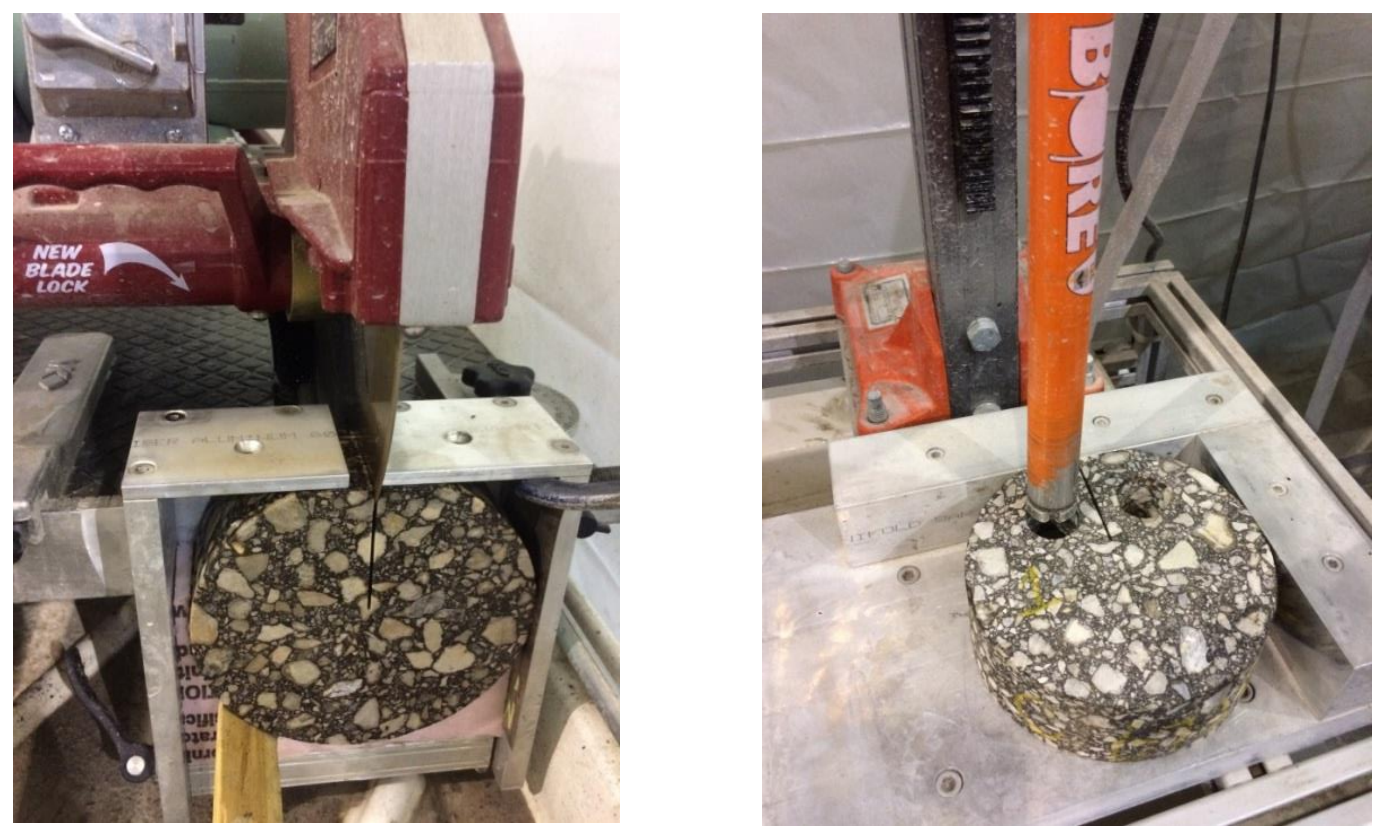


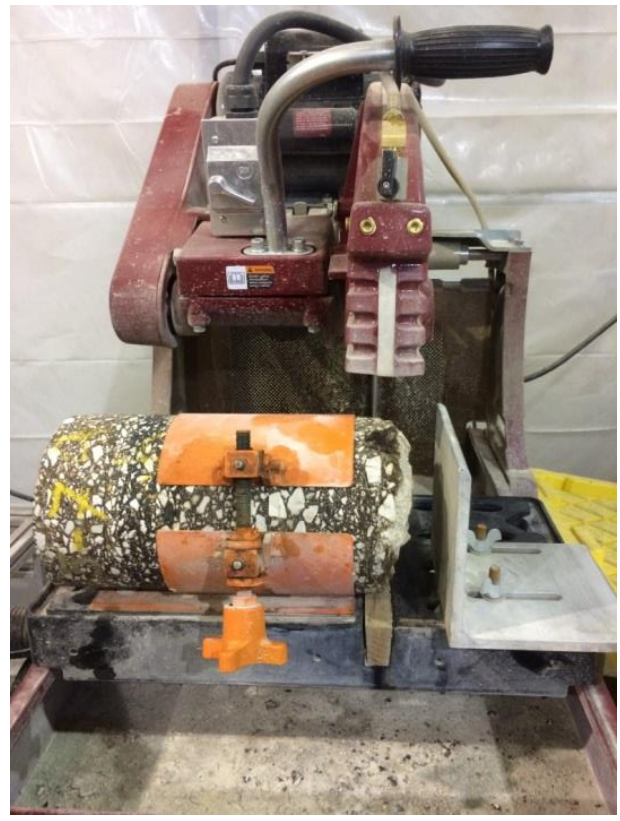

\section{Figure 2-1 Other fabrication equipment: (a) Tile saw, and; (b) Coring rig; (c) Block saw.}

The experimental design called for testing both field cores and gyratory compacted specimens.

Plant-mixed samples were brought back to the lab in 5-gallon steel pails. The plastic handles were removed and then the pails were placed in a forced draft oven to heat the asphalt mixture to a workable consistency. The heated mixture was then reduced to the gyratory sample mass following the quartering method in AASHTO R47. After splitting to sample mass, the asphalt mixture was heated to compaction temperature as set by the JMF. All samples were compacted to $7 \%$ air voids. The $7 \%$ AV was measured on the $50 \mathrm{~mm}$ slices before notching and coring for the DCT specimens, or before cutting the slice in half and notching for the IFit specimens. For the Hamburg specimens the original 
gyratory specimen (62mm in height) was used for Gmb testing prior to cutting the flat face on one side.

Lab mixed samples were created from the aggregates and the binders collected from the asphalt plants. These materials were proportioned according to the job mix formula used for paving the field sections. A bucket of an aggregate was dumped on a table and aggregate samples were reduced by the quartering method according to ASTM C702. Aggregates were dried at $110^{\circ} \mathrm{C}\left(212^{\circ} \mathrm{F}\right)$ overnight and then batched according to the JMF. Batched aggregates were then preheated with binder to the appropriate mixing temperature and mixed in a bucket style lab mixer. All lab mixes were short term aged in a forced draft oven for 2 hours according to AASHTO R30. Similar to the plant mix gyratories, $7 \%$ air voids were measured on the slices rather than targeting $7 \%$ on the full gyratory. There is generally a 0.5 to $1.0 \%$ reduction in air voids from the full gyratory to the sliced specimen. This is a technique often employed in research studies, but it is admittedly rather cumbersome. As balanced mix design and associated performance testing gain traction nationwide and in Missouri, a standard, practical method will need to be established to set void levels and void determination technique for asphalt mixture performance tests.

\subsubsection{Testing and Analysis Methods}

This chapter provides information regarding the test methods applied to materials sampled (as described before). Sample splitting and fabrication details were provided in last section. 


\subsubsection{Binder Testing}

Binder tests were focused on the suite of mechanical tests specified in the Superpave Performance Graded (PG) binder grading system, along with continuous PG grade determination.

\subsection{Rotary Viscometer Testing}

To measure the viscosity of the virgin binders sampled in this study, rotational viscometer (RV) tests were performed at two different temperatures $\left(135\right.$ and $\left.155^{\circ} \mathrm{C}\right)$. A Brookfield DV3T digital RV, Thermosel environmental chamber, and \#21spindle was used (Figure 2-2). For each sample, approximately 9 grams of binder was poured into the sample chamber and allowed to equilibrate for 20 minutes. RV testing was carried out according to ASTM D4402-15 [1]

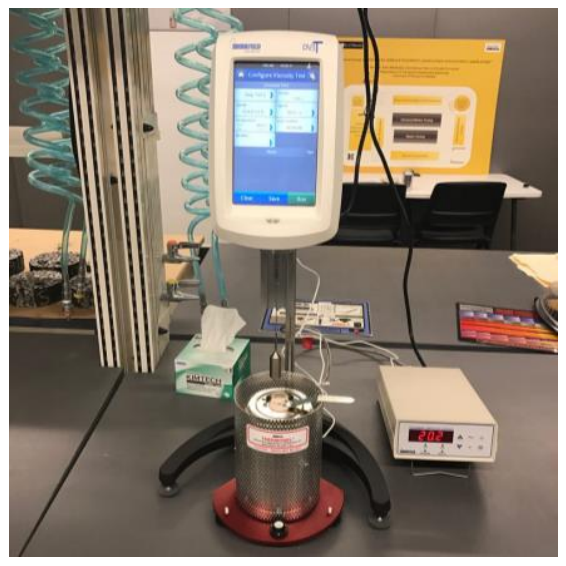

Figure 2-2 Brookfield DV3T digital rotary viscometer at MAPIL

\subsection{Dynamic Shear Rheometer Testing}

Superpave performance grading of binders requires viscoelastic characterization tests to be performed across a wide range of temperatures. To this end, one of the key devices 
required for rutting and fatigue crack mitigation is the Dynamic Shear Rheometer test (DSR), which was run on the five different neat binders sampled in this study. An Anton Paar MCR-102 SmartPave DSR, located at MAPIL was used (Figure 2-3). Dynamic shear modulus and phase angle are outputs from the DSR test, and are used to characterize the rheological properties of binders. First, testing in the high pavement summer temperature range is conducted to obtain the rutting parameter, or $\mathrm{G}^{*} / \sin \delta$. Testing is conducted across a range of Superpave PG high temperature grades, such as $58{ }^{\circ} \mathrm{C}, 64{ }^{\circ} \mathrm{C}, 70{ }^{\circ} \mathrm{C}$ and $76^{\circ} \mathrm{C}$. Samples having $25 \mathrm{~mm}$ diameter are prepared using silicone molds, then loaded in the DSR, trimmed, conditioned and tested according to ASTM D 7175-15. Testing of both tank and RTFO-aged (short-term aged) binder is then conducted. The critical high temperature grade is mathematically determined as either the temperature at which the tank binder rutting parameter reaches a threshold of $1 \mathrm{kPa}$, or the temperature at which the RTFO-aged binder rutting parameter reaches a threshold of $2.2 \mathrm{kPa}$ - whichever temperature is lower.

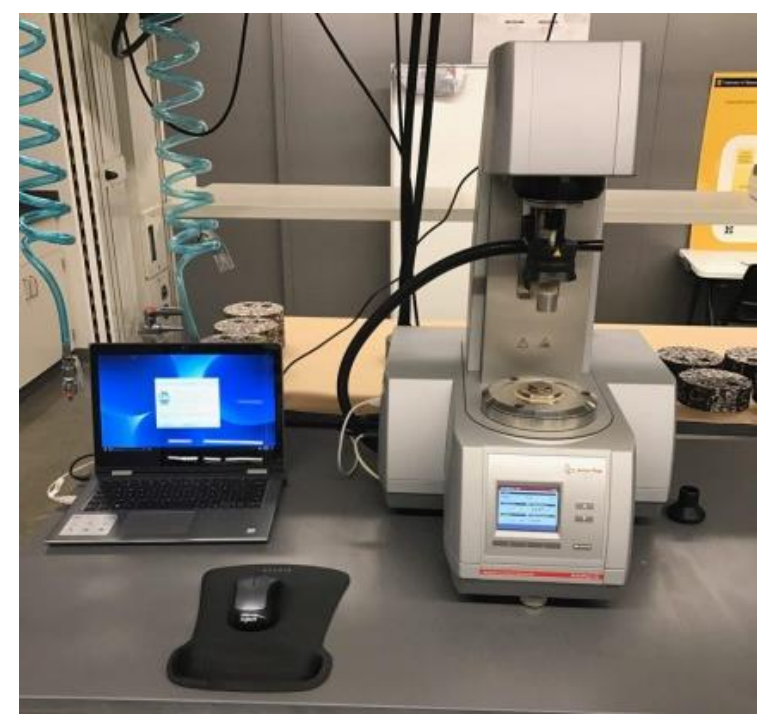

Figure 2-3 Anton Paar dynamic shear rheometer at MAPIL 
Next, testing in the intermediate temperature range on long-term aged binder is conducted to obtain the fatigue parameter, or $\mathrm{G}^{*}(\sin \delta)$. The pressure aging vessel (PAV) was used to age the binders to the long-term age condition. Testing was conducted at the Superpave intermediate temperature for the reported grade of the binder, which is generally either $22{ }^{\circ} \mathrm{C}, 25^{\circ} \mathrm{C}$ or $28^{\circ} \mathrm{C}$. Samples having $8 \mathrm{~mm}$ diameter were prepared using silicone molds, then loaded in the DSR, trimmed, conditioned and tested according to ASTM D 7175-15. The fatigue parameter was then compared against an upper threshold value of $5000 \mathrm{kPa}$.

\subsection{Bending Beam Rheometer Testing}

The Bending Beam Rheometer (BBR) test (Figure 2-4) provides a measure of low temperature stiffness and relaxation properties of asphalt binders. These parameters give an indication of an asphalt binder's ability to resist low temperature cracking. The BBR device is used to determine an asphalt binder's low temperature PG grade in accordance with AASHTO PP 42. BBR tests are conducted on PAV-aged asphalt binder samples. The continuous low temperature grade of the binder is determined as the higher of the two temperatures associated with a threshold creep stiffness of $300 \mathrm{MPa}$, or m-value of 0.3. The $\mathrm{m}$-value is automatically computed in the BBR software, and represents the absolute value of the slope of the log creep stiffness-log time curve, evaluated at a loading time of 60 seconds. For Missouri binders, the continuous low temperature grade is expected to be in the range of $-22{ }^{\circ} \mathrm{C}$ to slightly below $34{ }^{\circ} \mathrm{C}$, depending on the binder grade being tested. PGXX-22 binders are generally required for the Missouri climate; however, binders as soft as PG XX-34 are sometimes used to counterbalance the binder stiffness arising from the 
use of recycled materials, such as reclaimed asphalt pavement (RAP) or recycled asphalt shingles (RAS).

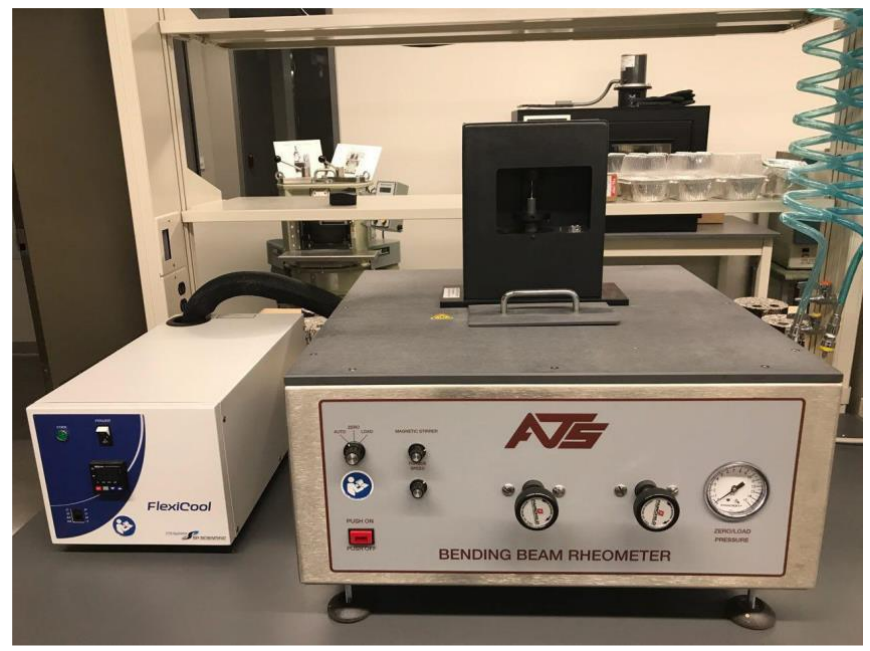

Figure 2-4 Bending beam rheometer at MAPIL

\subsubsection{Mixture Testing}

\subsection{Disk-shaped Compact Tension Testing}

The DC(T) test was developed to characterize the fracture behavior of asphalt concrete mixtures at low temperatures. The testing temperature is $10^{\circ} \mathrm{C}$ warmer than the PG low temperature grade of the mixture, per ASTM D7313-13 [2]. Thermal cracking in asphalt pavements can be considered as occurring in pure tensile opening or fracture Mode I, as the cracks propagate perpendicular to the direction of the thermal-induced stresses in the pavement, i.e., transverse to the direction of traffic. Block cracking develops in a similar manner; however, is generally confined to the upper 'crust' of the surface layer, and manifests itself as large, hexagonal or rectangular cracks. It is believed that the lowtemperature $\mathrm{DC}(\mathrm{T})$ test controls both of these two cracking modes and assists in slowing down reflective and fatigue cracking rates as well. For more direct control of reflective 
and fatigue cracking, the $\mathrm{DC}(\mathrm{T})$ is generally run across a range of temperatures. However, this was considered to be beyond the scope of the current study.

The $\mathrm{DC}(\mathrm{T})$ test procedure includes conditioning of the fabricated specimen at the selected test temperature in a temperature-controlled chamber for a minimum of two hours. After the conditioning, the specimens are suspended on loading pins in DC(T) machine, shown in Figure 2-5. A portable Test Quip DC(T) device was used, which is housed at the MAPIL lab. The test is performed at a constant Crack Mouth Opening Displacement (CMOD) rate, which is controlled by a CMOD clip-on gage mounted at the crack mouth. The CMOD rate specified in ASTM D7313-13 is $0.017 \mathrm{~mm} / \mathrm{s}(1 \mathrm{~mm} / \mathrm{min})$ [2]. To begin the testing sequence, a seating load no greater than $0.2 \mathrm{kN}$ (typically about $0.1 \mathrm{kN}$ ) is applied to 'seat' the specimen. The test is completed when a crack has propagated and the post-peak load level is reduced to $0.1 \mathrm{kN}$. The fracture energy can be obtained by measuring the area under the load-CMOD curve and dividing it by the fractured area (ligament length times thickness). A typical load-CMOD curve is shown in Figure 2-6.

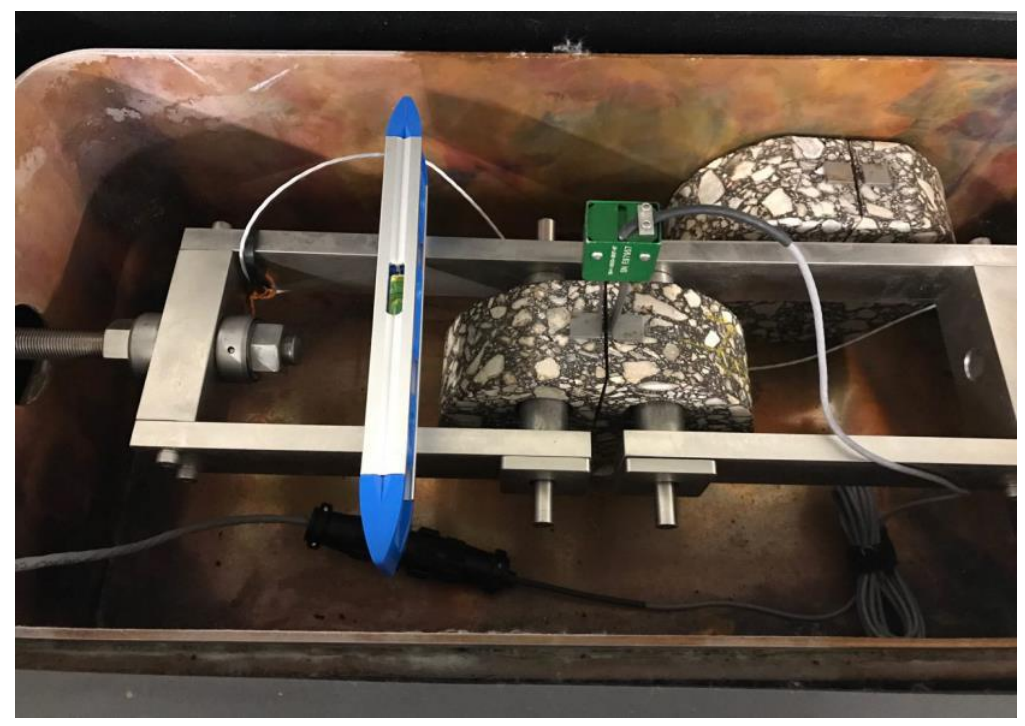


Figure 2-5 The TestQuip DC(T) apparatus

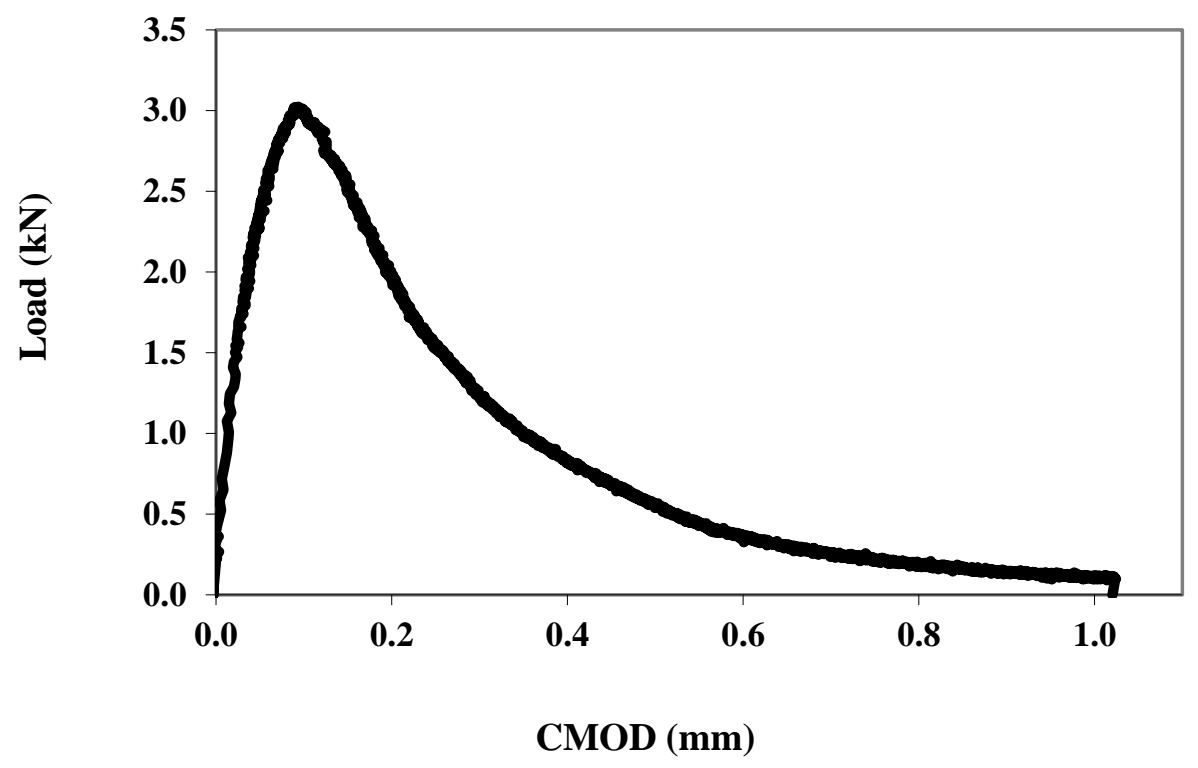

Figure 2-6 Typical load-CMOD curve from DC(T) testing

The fracture energy is computed as follows:

$G_{f}=\frac{A R E A}{B \cdot L}$

Where,

Gf $=$ Fracture energy, in $\mathrm{J} / \mathrm{m} 2$

AREA $=$ Area under Load-CMODFIT curve, until the terminal load of $0.1 \mathrm{kN}$ is reached

$\mathrm{B}=$ Specimen thickness, in $\mathrm{m}$, generally $0.050 \mathrm{~m}$ (except for field cores)

$\mathrm{L}=$ Ligament length, usually around $0.083 \mathrm{~m}$

The numerator of the equation represents the area under the Load-CMOD curve, which is the work required to create the fracture surface of size $b * a$. The area is generally computed using the quadrangle rule for numerical integration. The CMOD curve is generally the fitted CMOD, where a straight line is fit through the CMOD vs. time curve 
to enable data smoothing (ASTM D7313-07). The denominator of Eq. 2-1 represents the fractured area, i.e., B*L. Thus, fracture energy is computed as the work of fracture divided by the area fractured, which represents an average fracture energy density. Higher fracture energy values are associated with more crack resistant mixtures.

For specimen thicknesses other than $50 \mathrm{~mm}$, a size effect correction factor should be applied. This may seem counterintuitive, since after all, the fracture energy equation itself is scaled for the specimen thickness. However, that alone is not enough to fully account for effects on total fracture energy caused by variations in the sample thickness. This is because the damage zone ahead of the moving crack tip, called the fracture process zone, is sensitive to the three-dimensional stress state in that volume of material. The threedimensional stress state approaches a plane stress condition (2-dimensional stress state, as the stress normal to the face will be zero) at the edges of the specimen (flat, circular faces of the specimen - i.e., the faces cut with the chop saw), which is less confined than the stress state along the crack front in the mid-thickness of the specimen (approaching plane strain). For thinner specimens, such as field cores with thin surface lifts, a larger proportion of the fracture process zone volume is affected by proximity to the free edge, and thus, the crack tends to propagate with less applied energy in those areas. As a result, when the total energy is summed up, there will be a lower experimentally determined fracture energy for thinner specimens. In order to arrive at the fracture energy that would have been measured had the specimen possessed a $50 \mathrm{~mm}$ thickness, a correction factor (multiplier) greater than 1.0 should be applied. Previous research has led to the following correction factor: 


$$
\mathrm{CF}_{\mathrm{t}}=21.965(\mathrm{~B})^{-0.788}
$$

Where Gf from Eq. 2-1 is then multiplied by CFt to arrive at the final, thicknesscorrected Gf for instances where B is less than $50 \mathrm{~mm}$. Practically speaking, this correction should be applied when the thickness is more than $1 \mathrm{~mm}$ away from the target $50 \mathrm{~mm}$ sample thickness. For example, the correction factor for a sample thickness of $48 \mathrm{~mm}$ is 1.035. Thus, ignoring the correction factor in this instance would lead to an error in the estimated fracture energy of $3.5 \%$.

In terms of relation of $\mathrm{DC}(\mathrm{T})$ results to field performance, fracture energy thresholds to control thermal cracking with the DC(T) were first introduced in an FHWA Pooled fund study [3], as shown in

Table 2-1 For the study conducted herein, which focused on Superpave mixtures, the moderate traffic level would be appropriate. This suggests a minimum fracture energy threshold for newly conducted pavements (thus, mixture in the short-term aged condition) of $460 \mathrm{~J} / \mathrm{m} 2$. When evaluating long-term field-aged mixtures ( $>7$ years of field aging), a fracture energy threshold of $400 \mathrm{~J} / \mathrm{m} 2$ is generally used as a benchmark. This is because field aging reduces the mixture's ability resist cracking, as the binder becomes more stiff and brittle, while the aggregates generally retain their original physical properties over time 
(although some degree of freeze-thaw and fatigue damage may accumulate in the aggregate over time).

Table 2-1 DC(T) fracture energy thresholds

\begin{tabular}{cccc}
\hline & \multicolumn{3}{c}{ Project Criticality/ Traffic Level } \\
\cline { 2 - 4 } Contents & High & Moderate & Low \\
& $>$ 30M ESALs & 10-30 M ESALs & $<$ 10M ESALs \\
\hline Fracture Energy, & 690 & 460 & 400 \\
min. $\left(\mathrm{J} / \mathrm{m}^{2}\right)$ & & & Not required \\
Predicted & $<4$ & $<64$ & \\
Thermal Cracking & & & \\
Using ILLI-TC $(\mathrm{m} / \mathrm{km})$ & & & \\
\hline
\end{tabular}

\subsection{Illinois Flexibility Index Testing}

In 2016, Ozer et al. introduced the IL-SCB method for cracking resistance characterization in asphalt mixes (AASHTO TP124, 2016). The goal of the research was to develop an inexpensive, rapid test as a means to limit general pavement cracking. It was decided to simplify the test procedure by testing at room temperature, or $25^{\circ} \mathrm{C}$. It was observed that fracture energy obtained in this compact, arched bending mode at this temperature did not uniquely characterize mixture cracking properties. Rather than 
abandoning the test, the researchers observed that the post-peak slope of the loaddisplacement curve from SCB test was sensitive to the changes in the asphalt mixture specimen composition and subsequently used this to develop the Flexibility Index (FI). The FI is an empirical index parameter that is computed as the total fracture energy divided by the absolute value of the slope of the post-peak softening curve. FI is proposed to provide a means to identify brittle mixtures that are prone to premature cracking and was specifically developed to be sensitive to recycled material content.

$$
F I=\frac{G_{f}}{|m|}(0.01)
$$

Where Gf is computed in a similar manner as to the $\mathrm{DC}(\mathrm{T})$ test, and $\mathrm{m}$ represents the slope of the post-peak softening curve. There are countless ways to estimate the slope of a curve resulting from a material test, and this became a challenge for test standardization early in the development of the I-FIT. At present, to address this source of variability, the slope parameter is usually determined using a sophisticated software program available from the Illinois Center for Transportation (SCB Testquip LLC. V2.0.0rc4).

To fabricate samples, a notch is cut along the axis of symmetry of a semi-circular bend specimen to a depth of $15 \pm 1 \mathrm{~mm}$. Test specimens are then conditioned in the environmental chamber at $25 \mathrm{oC}$ for $2 \mathrm{hr} \pm 10 \mathrm{~min}$. After a contact load of $0.1 \mathrm{kN}$ is reached, the test is carried out at a rate of $50 \mathrm{~mm} / \mathrm{min}$. The test is considered to be complete when the load drops below $0.1 \mathrm{kN}$, which is identical to the $\mathrm{DC}(\mathrm{T})$ test termination definition.

\subsection{IDEAL CT-index test}

The IDEAL-CT cracking test is a recent mix performance test developed by the Texas Transportation Institute (TTI). The test is developed for routine quality control (QC) and 
quality assurance (QA). The test is still under evaluation (NCHRP IDEA 195 [5]). Additional mixes, field data, varied traffic load spectrum, and environmental conditions are required for further evaluation and validation [6]. The IDEAL-CT test was developed to characterize the potential of cracking in asphalt concrete mixes at room temperature. The test set-up is similar to the traditional indirect tensile strength test, but it is performed at $25^{\circ} \mathrm{C}$ at a constant loading rate of $50 \mathrm{~mm} / \mathrm{min}$ until failure occurs (Figure 2-7). The specimens are cylindrical with a diameter of 100 or $150 \mathrm{~mm}$ and a thickness of $38,62,75$ $\mathrm{mm}$, etc. The specimen does not require gluing, notching, drilling or additional cutting. For simplicity, in Missouri a current practice is to use a specimen with the same thickness as the Hamburg Wheel tracking test with a $7 \pm 0.5$ air voids. In this study, specimens were fabricated at both 62 and $95 \mathrm{~mm}$ thickness with a $150 \mathrm{~mm}$ diameter for comparison.

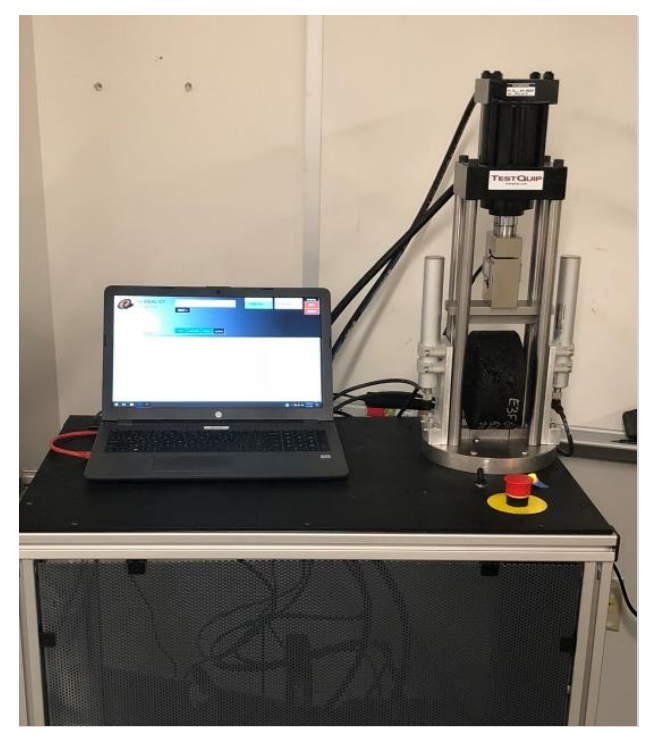

Figure 2-7 The Test Quip IDEAL-CT apparatus at MAPIL

The test procedure includes conditioning the specimens in a temperature-controlled chamber for a minimum of 2 hours at a $25{ }^{\circ} \mathrm{C}$. After conditioning, the specimens are centered between loading platens. A seating load of $0.1 \mathrm{kN}$ is applied in order to make 
appropriate contact between the loading platens and the sample. The sample is then loaded under a displacement control mode of $50 \mathrm{~mm} / \mathrm{min}$ while the loading level is measured and recorded by the device. Figure 2-8 shows a sample of the software output, i.e., the load vs. displacement curve.

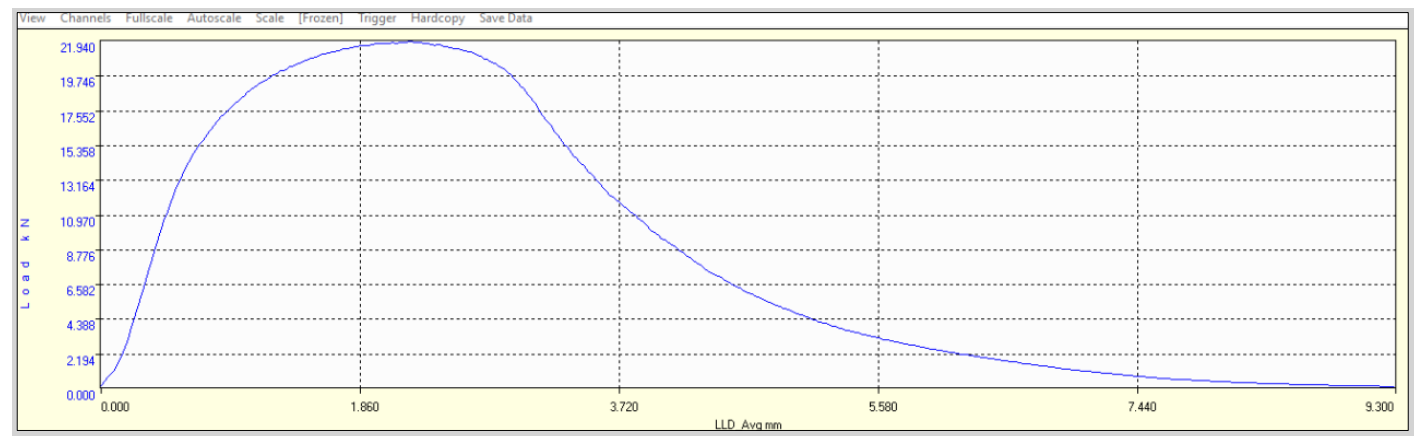

Figure 2-8 Typical load-displacement curve from Test Quip software

The cracking parameter for the IDEAL-CT is derived from the load vs. displacement curve and is inspired by the Paris' law [7] and by [8], dealing with crack propagation. The $\mathrm{CT}$ index equation for a specimen of $62 \mathrm{~mm}$ thickness is as follows:

$$
C T_{\text {index }}=\frac{G_{f}}{\left|m_{75}\right|} \times\left(\frac{l_{75}}{D}\right)
$$

where, $G_{f}=$ Fracture energy (area under the curve normalized by the area fractured) $A R E A=$ Area under the load - displacement curve, until the terminal load of $0.1 \mathrm{kN}$ is reached $m_{75}=$ Modulus parameter (absolute value of the slope at $75 \%$ of peak load) $\frac{l_{75}}{D}=$ Strain tolerance parameter (when load is reduced to $75 \%$ of peak load) $l_{75}=$ Vertical displacement when the load is reduced to $75 \%$ of peak load $D=$ Diameter of the sample 
$t=$ Specimen thickness

For samples other than $62 \mathrm{~mm}$ thickness, the following equation is used:

$$
C T_{\text {index }}=\frac{G_{f}}{\left|m_{75}\right|} \times\left(\frac{l_{75}}{D}\right) \times\left(\frac{t}{62}\right)
$$

Where the thickness is in $\mathrm{mm}$ to be consistent with the ratio $\left(\frac{t}{62}\right)$. The larger the CTindex, the better cracking resistance of the mixture; however, no widely accepted thresholds or criteria have been established.

\subsection{Hamburg Wheel Track Testing}

Permanent deformation (rutting) in an asphalt pavement is a result of consolidation and shear flow caused by traffic loading in hot weather. This results in gradual accumulation of volumetric and shear strains in the HMA layers. The measured deformation of different layers of flexible pavement revealed that the upper $100 \mathrm{~mm}$ (4in.) serves the main portion of the pavement rut depth such that the asphalt layer accumulates up to 60 percent of total permanent deformation. Lack of shear strength of the asphalt layer to resist the repeated heavy static and moving loads results in downward movement of the surface and provides the potential for upheaval and microcracks along the rut edges. In addition to the structural failure issues, safety concerns rise when the steering becomes difficult and also the surface water flows through the ruts and causes hydroplaning. Wheel load tracking (WLT) tests are the most common performance tests for measuring rutting potential of HMA mixes. The WLT methods simulate traffic by passing over standardized wheels simulating real-life traffic loads on HMA specimen at a given temperature. The two most common WLT test devices are Hamburg Wheel Tracking Test (HWTT) and the Asphalt Pavement Analyzer (APA) (formerly known as Georgia-loaded wheel tester). The 
HWTT is performed in accordance to AASHTO T324 standard (AASHTO 324, 2011). A loaded steel wheel, weighing approximately $71.7 \mathrm{~kg}$ tracks over the samples placed in a water bath at $50^{\circ} \mathrm{C}$ (Figure 2-9). The vertical deformation of the specimen is recorded along with the number of wheel passes. The test is generally stopped when either the specimen deforms by $20 \mathrm{~mm}$ or the number of passes exceeds 20,000 (Figure 2-9). A Cooper Hamburg device (Figure 2-9) was used in this study.
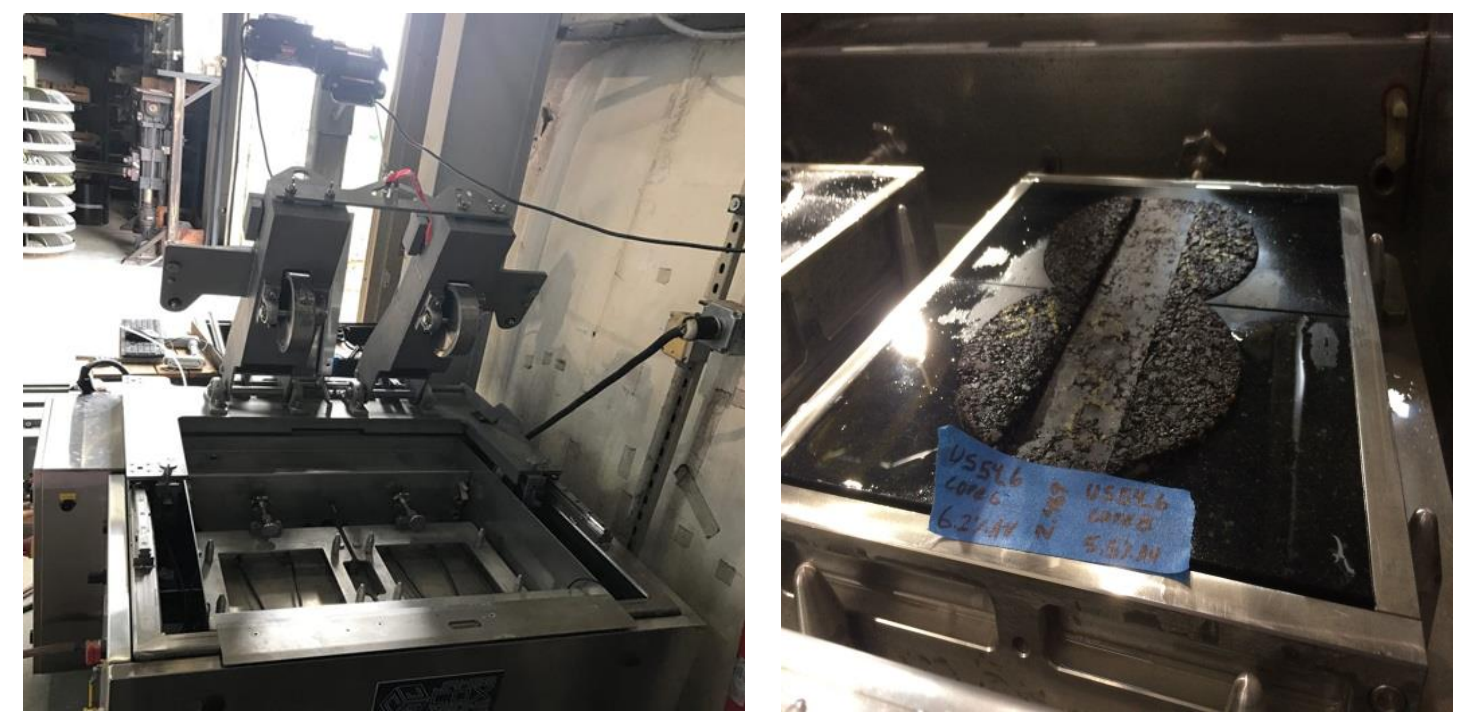

Figure 2-9 Hamburg Wheel Tracking device: a) Test device b) mixtures after test

\subsubsection{Results and Discussion}

Asphalt binder testing results including the RV, DSR, and BBR tests are discussed in this part of chapter 2. A detailed discussion regarding the test results is provided in the following sections. 


\subsubsection{Binder Results}

\subsection{Rotational Viscosity (RV) Test on Binders}

After the temperature stabilization conditioning process is complete, rotational viscosity testing begins and data is collected at one minute intervals for a total of 3 minutes. The viscosity of the tested binder in mPa.s (miliPascal times second) is averaged over these measurements and can then be used to calculate appropriate mixing and compaction temperatures for the purposes of asphalt mixture production (Table 2-2). Mixing and compaction temperatures correspond to 170 and $280 \mathrm{cP}$ viscosity levels, respectively. The viscosity at $135^{\circ} \mathrm{C}$ can also be checked against the Superpave upper limit of $3000 \mathrm{mPa} . \mathrm{s}$, which was easily met in all cases.

Table 2-2. Viscosity of binders obtained from the RV device

\begin{tabular}{cccccc}
\hline & \multicolumn{2}{c}{ Viscosity (mPa.s) } & & Mixing \\
\cline { 2 - 3 } Binder Type & $135{ }^{\circ} \mathrm{C}$ & $155^{\circ} \mathrm{C}$ & $\begin{array}{c}\text { Temperature } \\
\left({ }^{\circ} \mathbf{C}\right)\end{array}$ & $\begin{array}{c}\text { Compaction } \\
\text { Temperature }\left({ }^{\circ} \mathbf{C}\right)\end{array}$ \\
MO13-64-22H & 653.7 & 261.8 & 165.4 & 153.4 \\
US54-64-22H & 901.5 & 360.8 & 173.7 & 161.1 \\
US63-58-28 & 217.2 & 96.4 & 140.7 & 129.3 \\
US54-58-28 & 278.8 & 123.2 & 146.8 & 134.9 \\
US54-46-34 & 181.0 & 83.6 & 136.5 & 124.9 \\
\hline
\end{tabular}

\subsection{Dynamic Shear Rheometer (DSR) Test on Binders}

The results of DSR testing of virgin binders are shown in Table 2-3. The continuous high temperature $\mathrm{PG}$ is calculated based on the critical high temperature $\mathrm{PG}$, which is obtained based on RTFO and tank binder test results. 


\subsection{Bending Beam Rheometer (BBR)}

The results of the BBR test are used to evaluate the low temperature cracking resistance of the binder. Besides the low temperature PG, $\Delta$ Tc can be calculated. This parameter has been proposed to evaluate age-related cracking potential of the binder. It is defined as the numerical difference between the low continuous grade determined from the Bending Beam Rheometer (BBR) by stiffness criteria (which is the temperature at which the stiffness, $\mathrm{S}(\mathrm{t}=60 \mathrm{sec}$.$) , equals 300 \mathrm{MPa})$ and the low continuous grade temperature determined from the BBR m-value (which is the temperature at which the m-value equals 0.3). Higher $\Delta \mathbf{T c}$ values are associated with better aging and cracking resistance. According to the summary of binder results which is provided in Table 2-3, US 54-58-28 has the highest (least negative) $\Delta \mathbf{T c}$ value, while MO 13-64-22H exhibited the lowest $\Delta \mathbf{T c}$ value.

Some of the study binders were not found to meet the reported performance grade (Table 2-3), which in theory would lead to an increased pavement deterioration risk. However, the deviations were less than $2^{\circ} \mathrm{C}$ in both cases. Quite a large difference existed between the two 64-22 $\mathrm{H}$ binders evaluated in this study, where the MO13 binder had a UTI of $91.9^{\circ} \mathrm{C}$, while the US54 binder possessed a considerably better UTI value of $98.3^{\circ} \mathrm{C}$.

Table 2-3. Summary of virgin binder testing result

\begin{tabular}{ccccccc}
\hline Binder Type & \multicolumn{2}{c}{ High Temperature } & \multicolumn{2}{c}{ Low Temperature } & UTI* \\
& $\begin{array}{c}\text { Continuous } \\
\text { PGHT }\end{array}$ & PGHT & $\begin{array}{c}\text { Continuous } \\
\text { PGLT }\end{array}$ & PGLT & $\Delta$ Tc & $\left({ }^{\circ} \mathbf{C}\right)$ \\
\hline MO13-64-22H & 70.7 & 70.0 & -21.2 & -16.0 & -8.6 & 91.9 \\
US54-64-22H & 73.1 & 70.0 & -25.2 & -22.0 & -3.3 & 98.3 \\
US63-58-28 & 56.1 & 52.0 & -29.0 & -28.0 & -3.9 & 85.1
\end{tabular}




\begin{tabular}{ccccccc} 
US54-58-28 & 60.6 & 58.0 & -28.8 & -28.0 & -1.4 & 89.4 \\
US54-46-34 & 52.7 & 52.0 & -33.7 & -28.0 & -2.5 & 86.4 \\
\hline *Useful temperature interval & & &
\end{tabular}

\subsubsection{Mixture Results}

In this portion of the study, field cores and lab-produced samples obtained from the study field sections were characterized by mixture mechanical tests (mix 'performance' tests). This included the DC(T), I-Fit and Hamburg tests.

Table 2-4 provides details of the mixtures associated with each field section. Most of the sections (13 out of 18 sections) were constructed in 2016 and cored soon thereafter (within two weeks after construction), which represents the short-term aging condition. The other five sections are highlighted in different colors to easily distinguish between the recently constructed and aged sections. The oldest section investigated was US54_7, which was constructed in 2003 and did not contain any RAP and RAS. Asphalt binder replacement (ABR) by RAP and RAS, the total percentage of asphalt content by mixture mass $(\mathrm{Pb}), \mathrm{PG}$ of the virgin binder, the type and dosage of additives, and nominal maximum aggregate size (NMAS) are summarized in

Table 2-4. For convenience, the percentage of ABR, and RAP / RAS contents have been included in the section labels. As an example, MO13_1 (17-17-0) has an ABR of $17 \%$, resulting from $17 \%$ replacement by RAP and $0 \%$ by RAS. As shown, 11-out-of- 18 sections had a Superpave Performance Grade low temperature (PGLT) grade of -22, which is the low temperature binder grade currently specified currently in Missouri. 
Table 2-4. Summary of field sections investigated

\begin{tabular}{|c|c|c|c|c|c|c|c|c|}
\hline \multirow{2}{*}{ No. } & \multirow{2}{*}{$\begin{array}{l}\text { Cons. } \\
\text { Year }\end{array}$} & \multirow{2}{*}{ Section } & \multicolumn{2}{|c|}{$\mathbf{A B R}^{9} \%$} & \multirow{2}{*}{ Total $\mathbf{P}_{\mathbf{b}}{ }^{10} \%$} & \multirow{2}{*}{ Virgin Binder } & \multirow{2}{*}{ Additive } & \multirow{2}{*}{$\begin{array}{l}\text { NMAS } \\
(\mathbf{m m})\end{array}$} \\
\hline & & & \%RAP & $\%$ RAS & & & & \\
\hline 1 & 2016 & MO13_1 (17-17-0) & 17 & 0 & 5.7 & PG64-22 $\mathrm{H}^{11}$ & Type $1^{1}: 0.5 \%$ & 9.5 \\
\hline 2 & 2016 & US63_1 (35-35-0) & 35 & 0 & 5.1 & PG58-28 & Type $2^{2}: 0.5 \%+$ Type $3^{3}: 1.75 \%$ & 12.5 \\
\hline 3 & 2016 & US54_6 (31-31-0) & 31 & 0 & 5.1 & PG58-28 & Type $1: 1 \%$ & 12.5 \\
\hline 4 & 2016 & US54_1 (33-0-33) & 0 & 33 & 5.2 & PG58-28 & Type $4^{4}: 2.5 \%+$ Type $5^{5}: 3.5 \%+$ Type $1: 1.5 \%$ & 12.5 \\
\hline 5 & 2011 & US50_1 (25-25-0) & 25 & 0 & 4.5 & PG64-22 & Type $6^{6}: 1.5 \%+$ Type $7^{7}: 1 \%$ & 12.5 \\
\hline 6 & 2010 & MO52_1 (34-0-34) & 0 & 34 & 4.8 & PG64-22 & Type 6: $1.5 \%$, Type 7:0.8\% & 12.5 \\
\hline 7 & 2008 & US63_2(30-20-10) & 20 & 10 & 5.6 & PG64-22 & Type 6: $1.5 \%$ + Type $7: 0.5 \%$ & 12.5 \\
\hline 8 & 2016 & US54_2 (33-33-0) & 33 & 0 & 5.3 & PG58-28 & Type 1: $1 \%$ & 12.5 \\
\hline 9 & 2016 & US54_3 (33-18-15) & 18 & 15 & 5.2 & PG58-28 & Type 1: $1 \%$ & 12.5 \\
\hline 10 & 2016 & US54_4 (35-35-0) & 35 & 0 & 4.8 & PG64-22 H & Type 5:3\% + Type $1: 1 \%$ & 12.5 \\
\hline 11 & 2016 & US54_5 (0-0-0) & 0 & 0 & 5.4 & PG64-22 H & Type 1: $1 \%$ & 12.5 \\
\hline 12 & 2003 & US54_7 (0-0-0) & 0 & 0 & 6.2 & PG64-22 & Type $8^{8}: 0.25 \%$ & 12.5 \\
\hline 13 & 2006 & US 54_8 (9-9-0) & 9 & 0 & 5.6 & PG70-22 & Type 7: $0.5 \%$ & 12.5 \\
\hline 14 & 2016 & SPS10-1 (24-24-0) & 24 & 0 & 5.2 & PG64-22 H & Type $1: 1 \%$ & 12.5 \\
\hline 15 & 2016 & SPS10-2 (25-25-0) & 25 & 0 & 5 & PG64-22 H & Type 1:1\% & 12.5 \\
\hline 16 & 2016 & SPS10-3 (25-25-0) & 25 & 0 & 5 & PG64-22H & Type $1: 1 \%+$ Type $2: 0.5 \%$ & 9.5 \\
\hline 17 & 2016 & SPS10-6 (17-0-17) & 0 & 17 & 5.4 & PG58-28 & Type 1: $1 \%$ & 9.5 \\
\hline 18 & 2016 & SPS10-9 (46-16-30) & 16 & 30 & 5.3 & PG46-34 & Type 1: $2 \%$ & 12.5 \\
\hline
\end{tabular}

1. Type 1. Anti-stripping agent ('Morelife T280')

2. Type 2. Warm-mix additive ('Evotherm')

3. Type 3. Rejuvenator additive ('EvoFlex CA')

7. Type 7. Anti-stripping agent ('AD-here HP Plus')

4. Type 4. Anti-stripping agent ('IPC-70')

Anti-stripping agent ('LOF 65-00LS1')

5. Type 5. Warm-mix additive ('PC 2106')

9. $\mathrm{ABR}=$ Asphalt binder replacement

10. By total mass of binder, including neat and recycled

6. Type 6. Bag house fines

11. Heavy traffic designation (from MSCR test) 


\subsection{Field Core Fracture Energy Results}

This section presents $\mathrm{DC}(\mathrm{T})$ fracture energy testing results obtained from field cores. Testing of at least 3 , and usually 4 sample replicates were performed. A testing temperature of $-12{ }^{\circ} \mathrm{C}$ was used in all cases, which is 10 degrees warmer than the plan low temperature Superpave PG grade for binders specified in Missouri. Note that for convenience, the percentage of ABR, and RAP / RAS contents have been included in the section labels. As an example, MO13_1 (24-24-0) has an ABR of 24\%, resulting from 24\% replacement by RAP and $0 \%$ by RAS. Figure 2-10 presents the fracture energy results obtained for all sixteen study mixtures. The total asphalt binder replacement (ABR) is also shown on the plot, as indicated by the green symbols.

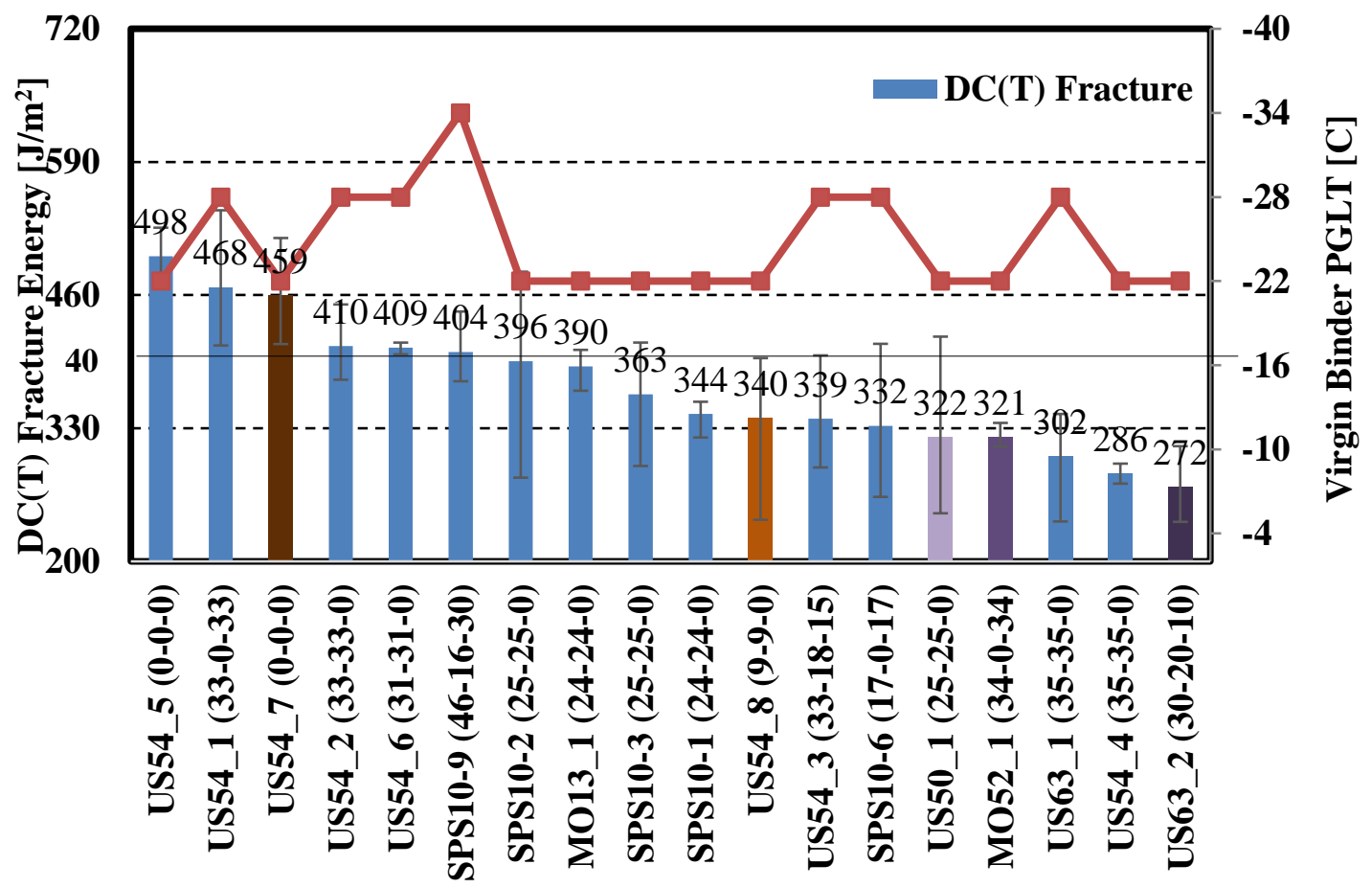

Figure 2-10 DC(T) fracture energy test results of field cores

Some general observations regarding $\mathrm{DC}(\mathrm{T})$ fracture energy from cores are: 
All aged mixtures fall below the desirable $400 \mathrm{~J} / \mathrm{m}^{2}$ minimum threshold for the longterm aging he criteria except US54_7 (14 years aging), which is a virgin mixture with 6.2\% binder content. MO 52_1 (6 years aging), US 54_8(11 years in service), US 50_1 (6 years aging) and US 63_2 (9 years aging) possessed fracture energy levels of 321, 340, 322 and $272 \mathrm{~J} / \mathrm{m}^{2}$, respectively. This indicates a sensitivity to thermal and block cracking in these sections, particularly US 63_2.

Among the highest fracture energy results obtained, a combination of virgin mixes and those containing RAP and/or RAS fell in this top grouping with fracture energy values above the recommended $460 \mathrm{~J} / \mathrm{m}^{2}$ threshold for short-term aged mixes. These particular recycled mixes appeared to benefit from the use of a softer base binder, for example:

- US54_1 had 33\% ABR by RAP with a PG 58-28 binder (468 J/m²), and;

- SPS10_9 had 46\% ABR (16\% by RAP and 30\% by RAS), along with a PG 46-34 binder $\left(467 \mathrm{~J} / \mathrm{m}^{2}\right)$.

- By comparison, US 54_5 is a virgin mix with a PG 64-22H binder $\left(497 \mathrm{~J} / \mathrm{m}^{2}\right)$.

- Only two of the newer mixes investigated (i.e., those sampled in the short-term aged condition in 2016), had noticeably low fracture energy. These were:

- US63_1, which had 35\% ABR by RAP and a single grade bump to PG 58-28 (302 $\mathrm{J} / \mathrm{m}^{2}$ ). This may indicate that a second grade bump or lower RAP level was needed in the design. Later in this chapter we will find that the aggregate or RAP source used appears to have a moisture sensitivity issue as well. 
US54_4, which also had 35\% ABR by RAP, but which used a modified, but stiff PG64-22 H binder $\left(286 \mathrm{~J} / \mathrm{m}^{2}\right)$. This may indicate that a better choice for binder would have been a PG XX-28 or even a PG XX-34, rather than investing in the polymer modified PG 64-22 H. Later in this chapter, we will see that this section had a very low Hamburg rut depth, supporting the notion for binder bumping to a softer low temperature grade.

A number of the other newer mixes would have also benefitted from minor adjustments to a softer low temperature grade, in order to reach the $460 \mathrm{~J} / \mathrm{m}^{2}$ fracture energy level.

\subsection{Field Core Flexibility Index Results}

Figure 2-11 presents the results of I-FIT testing of Missouri mixes as sampled from field cores. None of the mixtures tested possessed an FI value in excess of 8.0, and in fact all samples were found to be below an FI value of 6.0. Recall from

Table 2-4 that the 16 mixtures tested were comprised of 11 newer mixes, and 5 fieldaged mixes. Some samples had very low FI values, less than 2.0 , and in some cases, nearly zero. These specimens exhibited very brittle behavior in the I-FIT test, with snap-back type softening curves and very few data points following the peak load, indicating a very brittle failure. Analysis of data sets with very steep post-softening curves is not adequately described in the test specification, and requires analyst judgment. These mixes often possess the highest variability between test replicates, which is addressed in the following section. Considering the I-FIT testing results, some comparisons can be made as follows:

- The source of ABR was found to be very important. Considering US54_2 and 
US54_3, which have the same ABR but different combinations of RAS and RAS, US54_2 showed 400\% higher FI than US54_3.

- US54_6 and US63_1 with different additives had a difference of 50\% in FI. The significant effect of additive was also noticeable in DC(T) fracture energy.

- Comparing US54_2 and SPS10-1, one grade bump could not compensate for the presence of 9\% ABR --- the FI of SPS10-1 (24-24-0) is 320\% higher than US54_2 (33-33-0).

- Comparing to US54_3, one grade bump and 1\% more M-life additive did not help SPS10-9 to compensate for $15 \%$ additional ABR by RAS.

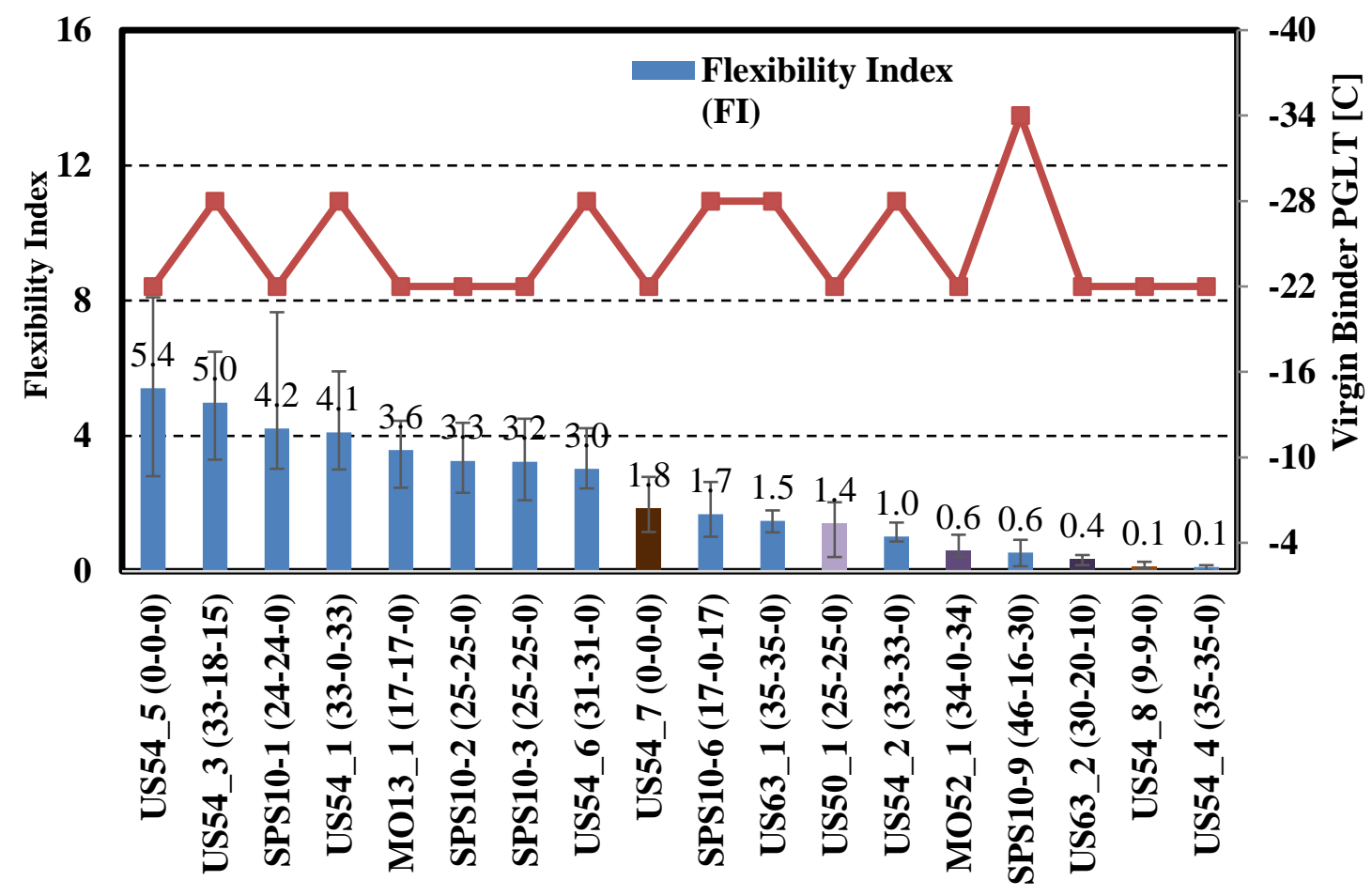

Figure 2-11 I-FIT test results for field cores

It is also worth mentioning that four replicates were tested in I-FIT and the results were averaged out and reported in Figure 2-11. However, for two sections (SPS10-9 and 
US54_4), no FI could be calculated for one of the tested replicates due to the very brittle post-peak behavior, as shown in Figure 2-12 (quadrilateral and cut-back shape of loaddisplacement plot). In this case, the slope which is used to determine the FI could not be calculated. Therefore, no FI was considered for those two replicates in the average as it was reported as "None" by software.

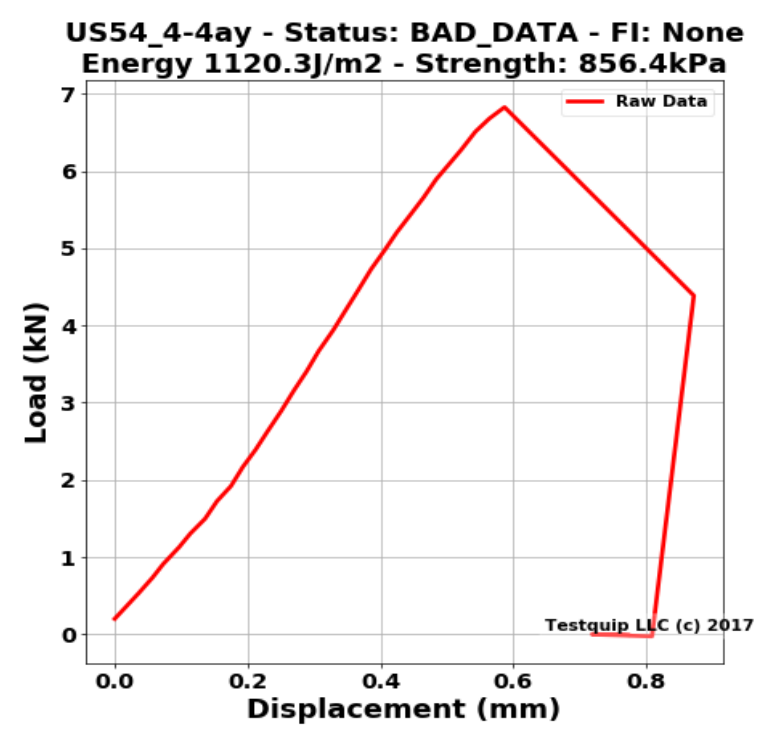

Figure 2-12 An example of very brittle behavior (US54_4-4ay tested sample)

\subsection{Comparing DC(T) and I-FIT test results}

Fracture energy and flexibility index for the various field core samples were normalized and plotted in Figure 2-13. Some similarity in trends were observed, but it is clear that the $\mathrm{DC}(\mathrm{T})$ and IFIT tests provide different cracking measures overall. Thermal cracking resistance as measured in the $\mathrm{DC}(\mathrm{T})$ for various Missouri mixtures tends to vary by about $40 \%$ for Superpave mixtures (around 0.6 to 1.0 on the normalized plot). On the other hand, the I-FIT parameter varies from nearly zero to just over 5.0, and on the normalized FI plot, varies across the entire scale from zero to 1.0. This clearly results from 
the wide array of post-peak slope behavior observed in the test. Apparently, because of the stiff specimen geometry and very fast loading rate, the fracture process in some of the stiffer specimens becomes unstable, leading to an explosive failure (very fast crack propagation) once the peak load is reached and the stored strain energy from bending begins to transfer to the crack face.

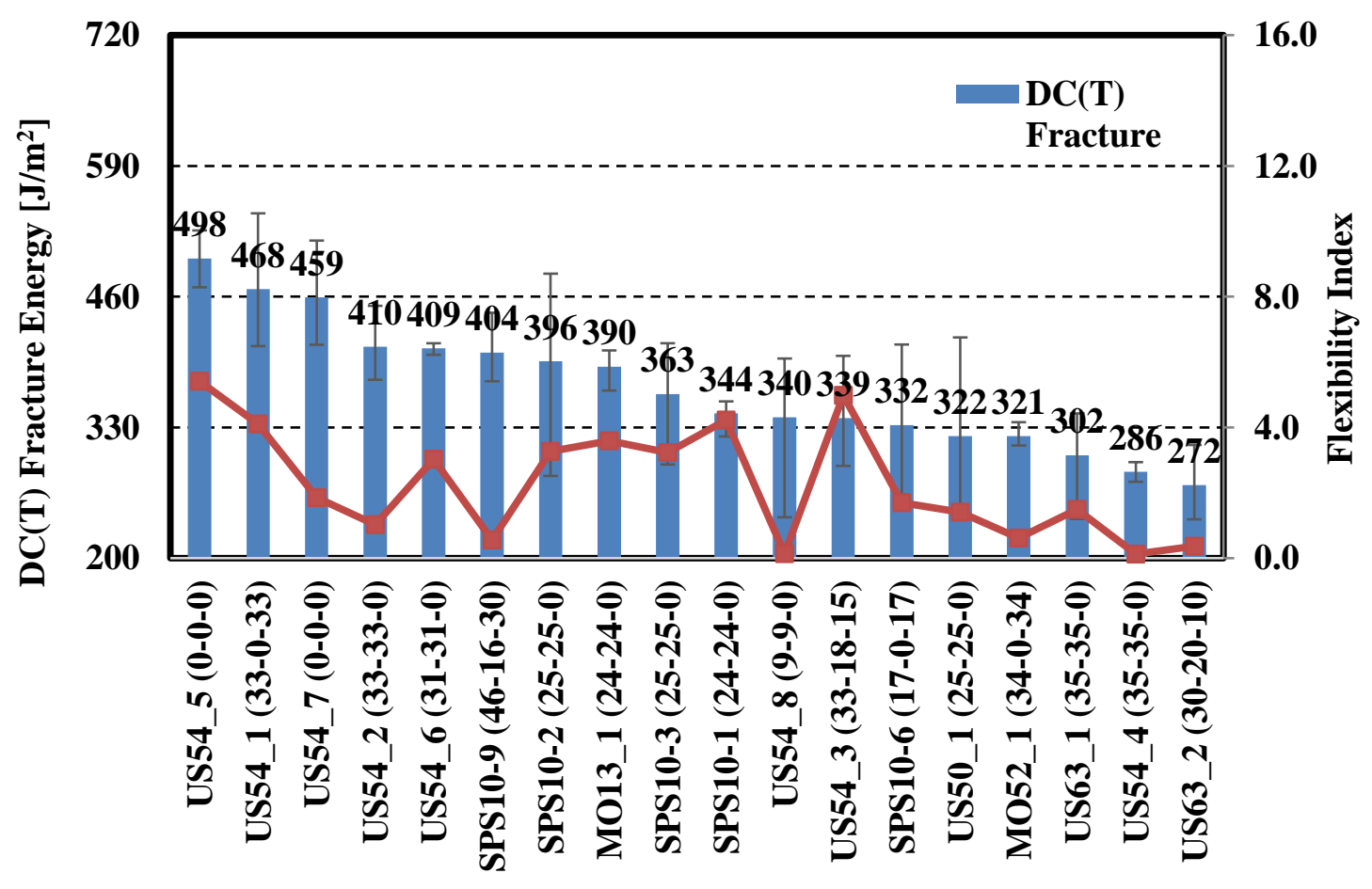

\section{Figure 2-13 Comparison of DC(T) FE \& SCB FI test results of field cores}

Table 2-5 shows the coefficient of variability (COV) of the field cores tested for both the DC(T) test and the I-FIT test. DC(T) COVs ranged from $1.4 \%$ to $27.6 \%$, with an average of $12.7 \%$. I-FIT COVs ranged from $23.3 \%$ to $80.7 \%$, with an average of $44.6 \%$. In general, for mix testing, it is desirable to keep COV values under $15-20 \%$. 
Table 2-5. Coefficient of variance (COV) of DC(T) and I-FIT measurements on field cores for Missouri Superpave mixes

\begin{tabular}{|c|c|c|c|c|c|c|c|c|}
\hline & \multicolumn{2}{|c|}{$\mathbf{D C}(\mathbf{T})$} & \multicolumn{6}{|c|}{ IFIT } \\
\hline Section & $\begin{array}{c}\text { Avg. FE } \\
\left(\mathrm{J} / \mathrm{m}^{2}\right)\end{array}$ & $\begin{array}{c}\text { COV } \\
\%\end{array}$ & $\begin{array}{c}\text { Avg. FE } \\
\left(\mathrm{J} / \mathrm{m}^{2}\right)\end{array}$ & $\begin{array}{c}\mathrm{COV} \\
\%\end{array}$ & $\begin{array}{c}\text { Avg. } \\
\text { Slope } \\
(\mathrm{kN} / \mathrm{mm})\end{array}$ & $\begin{array}{c}\mathrm{COV} \\
\%\end{array}$ & Avg. FI & $\begin{array}{c}\mathrm{COV} \\
\%\end{array}$ \\
\hline MO13_1 (17-17-0) & 390.3 & 5.4 & 1623.6 & 4.4 & 4.73 & 25.5 & 3.59 & 23.5 \\
\hline US63_1 (35-35-0) & 302.4 & 18.4 & 1092.6 & 15.0 & 7.59 & 25.1 & 1.50 & 23.3 \\
\hline US54_6 (31-31-0) & 408.8 & 1.4 & 1883.6 & 7.8 & 6.56 & 27.2 & 3.03 & 32.1 \\
\hline US54_1 (33-0-33) & 467.6 & 14.5 & 1788.4 & 15.2 & 4.53 & 17.4 & 4.11 & 32.2 \\
\hline US54_2 (33-33-0) & 410.4 & 9.1 & 1410.1 & 9.1 & 14.41 & 19.7 & 1.02 & 29.9 \\
\hline US54_3 (33-18-15) & 339.2 & 16.5 & 2227.9 & 18.8 & 4.57 & 16.4 & 4.98 & 32.2 \\
\hline US54_4 (35-35-0) & 285.8 & 3.4 & 1208.7 & 21.2 & 301.18 & 124.0 & 0.13 & 77.8 \\
\hline US54_5 (0-0-0) & 497.9 & 5.7 & 2229.1 & 14.7 & 4.62 & 33.2 & 5.41 & 43.7 \\
\hline SPS10-1 (24-24-0) & 343.9 & 5.8 & 2183.6 & 20.0 & 6.04 & 37.7 & 4.22 & 57.6 \\
\hline SPS10-2 (25-25-0) & 395.6 & 26.1 & 1864.6 & 10.2 & 5.39 & 16.7 & 3.27 & 28.7 \\
\hline SPS10-3 (25-25-0) & 363.0 & 17.2 & 2152.2 & 10.7 & 7.14 & 30.5 & 3.24 & 31.8 \\
\hline SPS10-6 (17-0-17) & 332.3 & 22.7 & 1837.5 & 13.0 & 11.97 & 32.2 & 1.69 & 41.0 \\
\hline SPS10-9 (46-16-30) & 404.4 & 8.8 & 1142.7 & 12.5 & 34.80 & 86.6 & 0.56 & 76.1 \\
\hline US50_1 (25-25-0) & 321.5 & 27.6 & 759.4 & 7.6 & 7.68 & 81.7 & 1.41 & 51.6 \\
\hline MO52_1 (34-0-34) & 321.2 & 3.8 & 546.2 & 13.1 & 10.67 & 43.3 & 0.61 & 51.4 \\
\hline US63_2 (30-20-10) & 272.4 & 13.7 & 634.7 & 3.0 & 20.14 & 49.9 & 0.36 & 35.4 \\
\hline US54_8 (9-9-0) & 340.2 & 25.5 & 1170.7 & 35.4 & 262.08 & 141.2 & 0.14 & 80.7 \\
\hline US54_7 (0-0-0) & 459.3 & 11.4 & 1221.2 & 7.4 & 7.19 & 30.1 & 1.85 & 37.6 \\
\hline Ave COV\% & & 13.2 & & 13.3 & & 46.6 & & 43.7 \\
\hline
\end{tabular}

\subsection{Hamburg Wheel Track Test Results}

Figure 2-14 presents the results of Hamburg wheel tracking tests. All mixtures rutted less than $12.5 \mathrm{~mm}$ at 20,000 passes except US 63_1, which is a section that contained a relatively high percentage of RAP. It should be noted that Superpave mixtures in Missouri 
are used on medium-volume facilities, and a rutting threshold of $12.5 \mathrm{~mm}$ at 10,000 passes is more appropriate. However, 20k pass tests were run in order to better delineate between mixes, and to identify any potential moisture sensitivity. Although RAP usually tends to lower Hamburg rut depths, sometimes moisture sensitivity will result. In these instances, Hamburg rut depths will often increase.

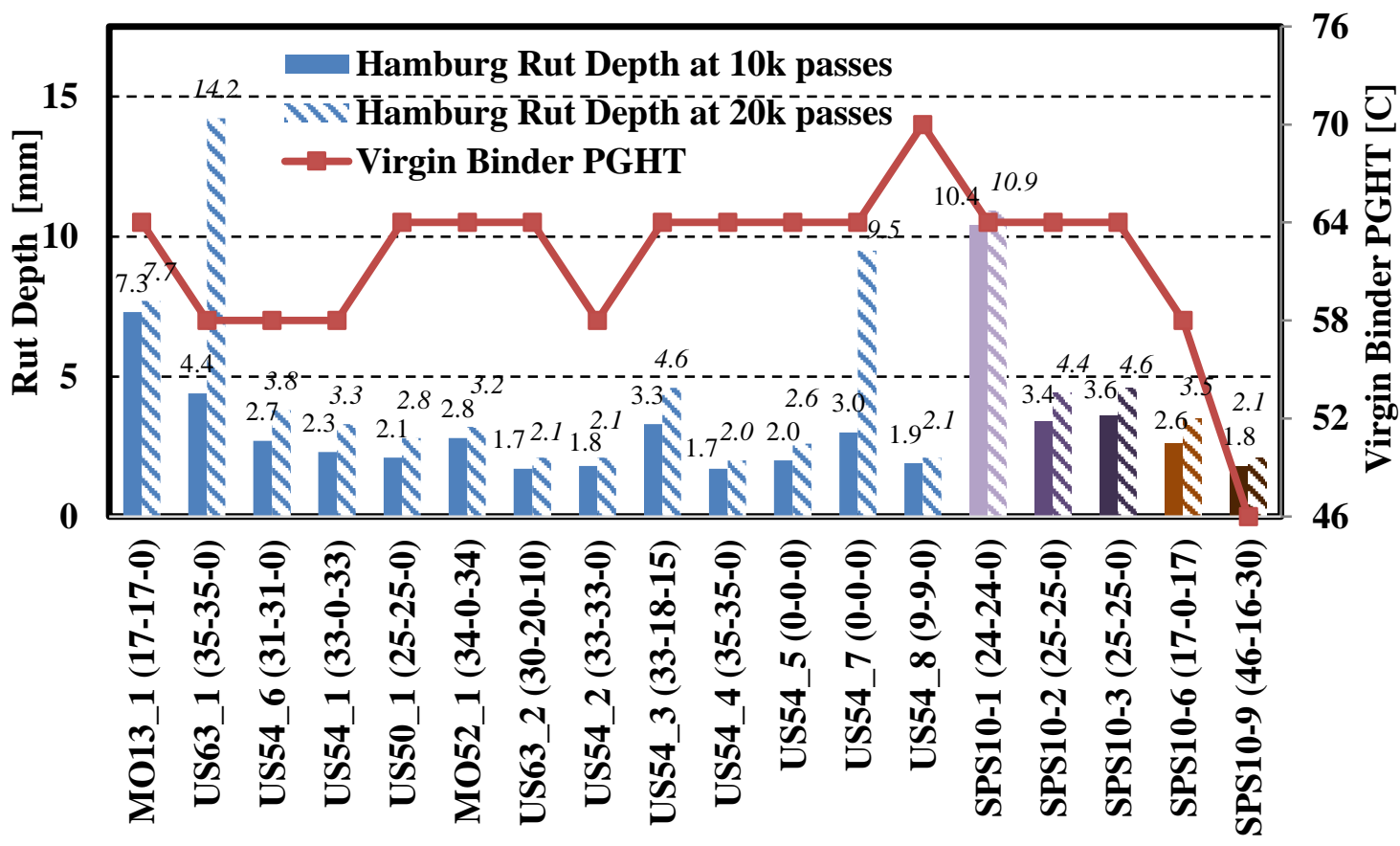

\section{Figure 2-14 Hamburg Wheel test results of field cores (at 20k passes)}

Figure 2-15 shows a sample output from the Hamburg test, where 'Sample 1' in this case is the US 63_1 field core result. The white data trace (curve) on the top plot shows the maximum rut depth plotted against the wheel pass number. The white trace on the bottom plot shows the rutting profile along the length of wheel travel. Because two cores are placed side-by-side to create a sufficiently long wheel track for testing (see Figure 2-9) it is clear from the bottom plot that although both replicates lying on the same wheel track 
had poor rutting resistance, the second specimen had a deeper rut depth than the first half. The final test result, however, represents the largest rut depth along the total wheel track.
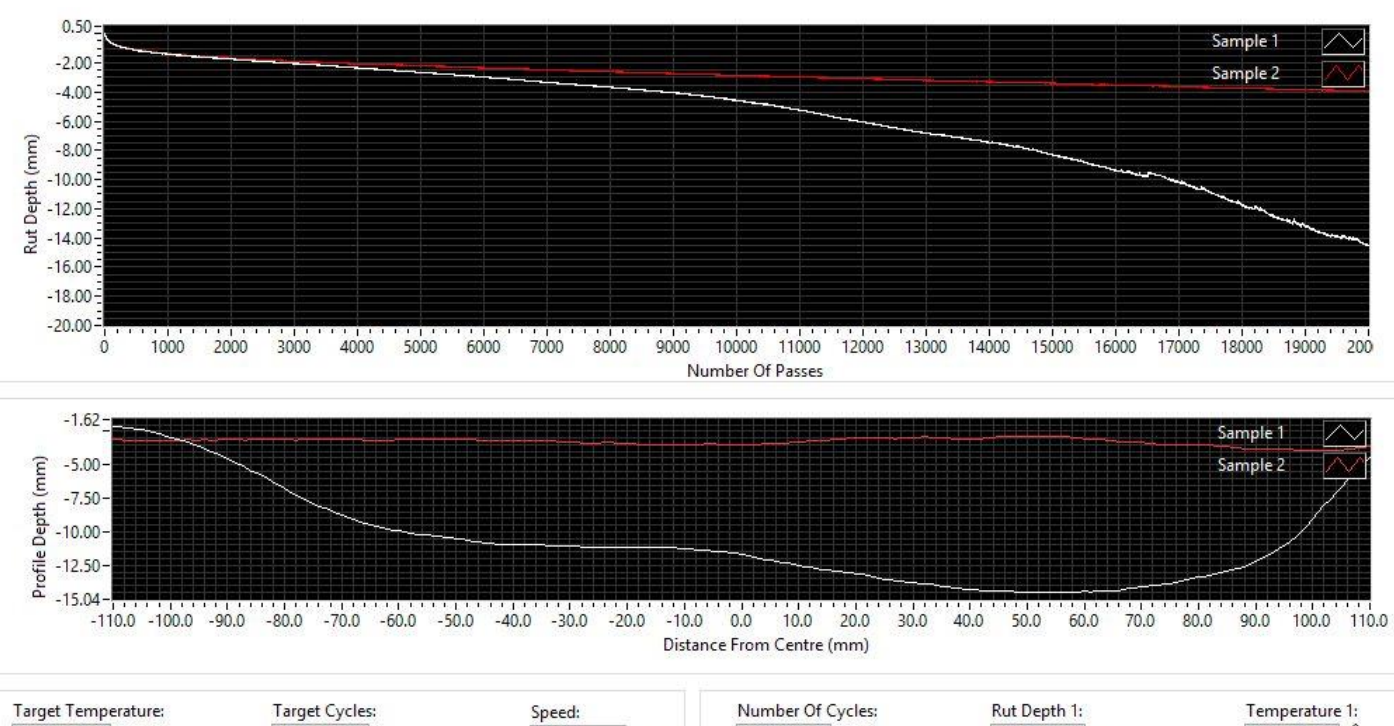
$50.0 \quad{ }^{\circ} \mathrm{C}$ Tank Temperature 50.1

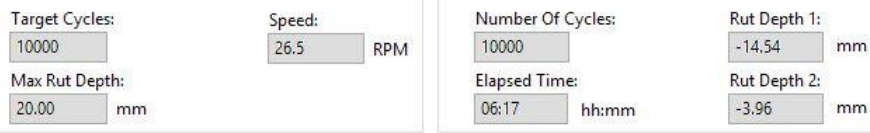

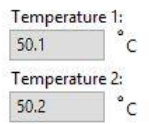

Figure 2-15 Sample Hamburg test output from Cooper software

Moreover, the white trace on the top plot shows an inflection in the curvature, starting at around 3000 passes. The presence of a 'stripping inflection point,' is viewed as an indication of moisture susceptibility in the mix. There was only one other field core set that exhibited a distinct stripping inflection point. This was the US 54_7 section (Brazito, MO). Unlike, US 63_1, this mix did not contain any recycled materials. Both mixes contained a liquid anti-strip product; however, this did not seem to mitigate moisture sensitivity in either of these mixes. The virgin aggregates in these mixes could also underlie the cause of the moisture sensitivity measured. 


\subsection{Hamburg-DC(T) Performance Space Diagram Results}

Figure 2-16 presents a useful $\mathrm{x}-\mathrm{y}$ plotting form known as the "performance space diagram,' or more specifically in this case, the Hamburg-DC(T) plot. This plot allows the simultaneous evaluation of rutting and cracking behavior. Some useful trends that can often be observed when viewing data in this form are:

The best overall performing mixtures will appear in the upper-right corner of the diagram (low rutting depth, high fracture energy). These can be considered as high 'total energy' mixtures; i.e., rut and crack (or damage) resistant. These are high toughness mixtures, and the best candidates for surfacing materials especially in demanding climates and for high traffic volumes.

Mix variables that increase net total energy in the mix and thus 'move' mixtures in the direction of the upper-right corner of the plot include:

- Higher quality binder (low temperature susceptibility, higher Useful Temperature Range, or UTI), degree of polymer modification;

- Higher quality aggregate (stronger, more angular, better bond with asphalt), and;

- The presence of crack interceptors or rut mitigators, such as fibers, rubber particles, and even RAS (but only if properly used). 


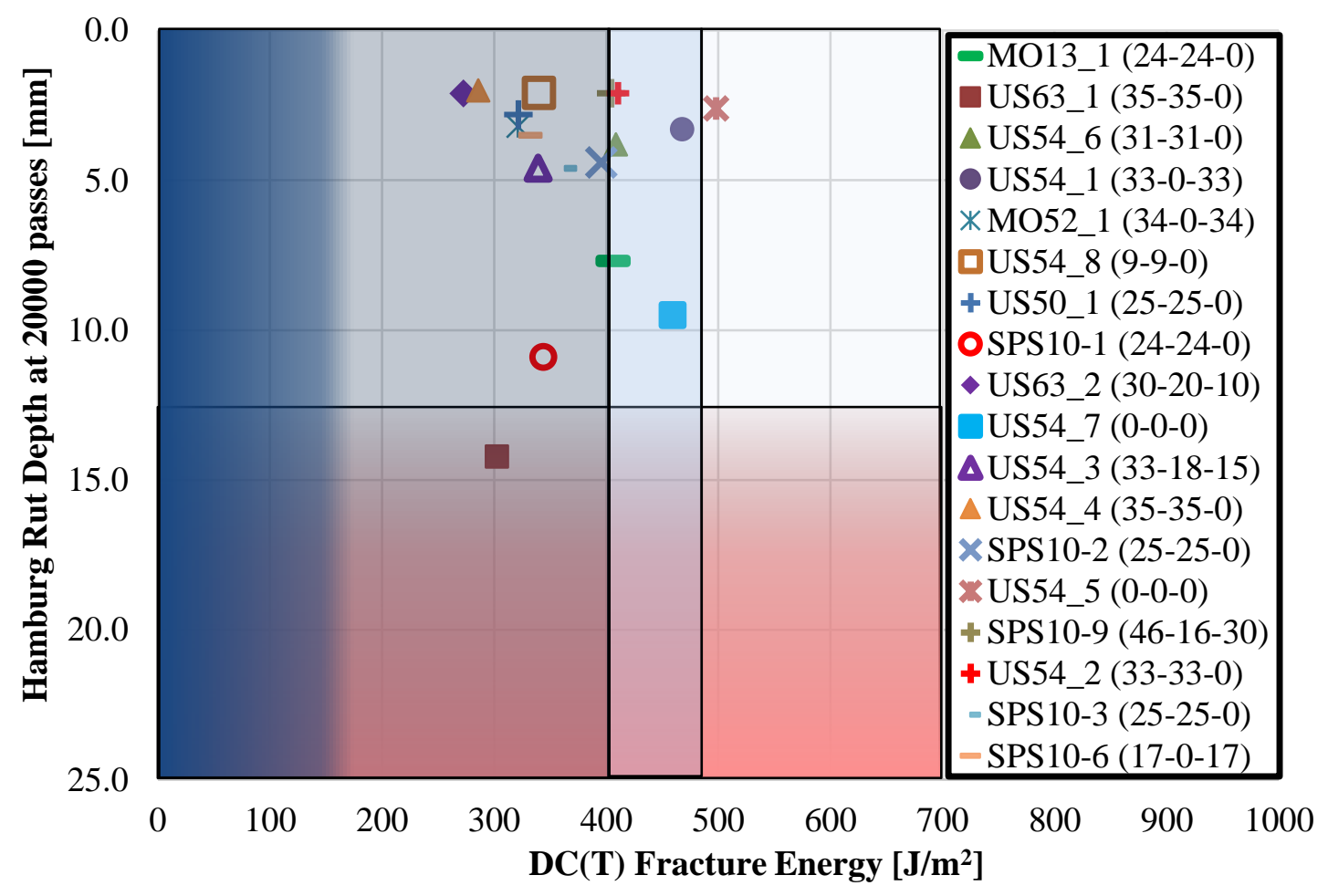

Figure 2-16 Hamburg-DC(T) plot for Missouri field cores

Other salient features of the plot include:

- Binders with different grades but similar UTI tend to move a mixture along a 'binder tradeoff axis', or roughly speaking, diagonal lines moving in the upwards-left or downwards-right directions, for stiffening and softening, respectively;

- Pure stiffening elements, such as RAP, tend to move points upwards and to the left;

- Pure softening elements, such as rejuvenators, tend to move points downwards and to the right;

- Binders with higher UTI, where the grade bump is on the high temperature grade, tend to move points mainly upwards, but also slightly to the right due to the benefits of polymer in intercepting cracks, and; 
- Binders with higher UTI, where the grade bump is on the low temperature grade, tend to move points mainly to the right, but also slightly upwards, again, due to the benefits of polymer in intercepting cracks.

- Data points that appear in the undesirable middle-to-lower-left portion of the plot are sometimes those that contain RAP and insufficient binder bumping, and possibly poor bond, where the RAP tended to cause lower $\mathrm{DC}(\mathrm{T})$ values, and the nature of the RAP-virgin material combination led to a moisture-susceptible mix with high Hamburg rut depth value.

A number of interesting findings can be extracted from the results of Missouri field cores (Figure 2-16), including:

- The best performing mix overall was US54_5, which is a virgin mix with a relatively high UTI binder (PG 64-22 H, with a UTI of 98.3). It has a gold-colored asterisk symbol, and is the furthest data point to the upper right.

The next two best performing mixtures were:

- SPS10_9: An innovative mix, containing 46\% ABR, with 16\% binder replacement from RAP, and 30\% from RAS, along with PG46-34 binder, with a relatively moderate UTI (86.4), and;

- US54_1: Comprised of 33\% ABR, all coming from RAS, and PG58-28 binder with a UTI of 89.6. 
Two poor-performing mixes in the Hamburg-DC(T) space contained RAS and exhibited stripping in the Hamburg test, including:

- US63_1 (Figure 2-17, 'Sample 2'), with 35\% RAS and PG 58-28 binder. The results suggest that the mixture could have benefitted from a second binder bump (such as PG XX-34 binder) to improve DC(T) fracture energy, along with more effective measures to improve moisture sensitivity.

- SPS10_1, with 24\% RAP and PG64-22 H binder. The Hamburg results for this mix are shown in Figure 2-17 ('Sample 2' data trace). The thin core sample had a fast rutting rate, and possible stripping inflection, followed by a complete flattening and bottoming out in the Hamburg wheel track test at around 7000 passes. The results also suggest that the mixture could have benefitted from binder bumping (softening) to improve $\mathrm{DC}(\mathrm{T})$ fracture energy, along with more effective measures to improve moisture sensitivity.

A large cloud of mixtures had similar 'total energy' on the Hamburg-DC(T) plot, but with a range of 'mix stiffness.' These mixes fell along a similar diagonal contour, spanning from the US57_7 mix (light blue square) to the US63_2 mix (purple diamond). The results suggest that these mixes have similar overall performance, with some performing better on the Hamburg relative to the $\mathrm{DC}(\mathrm{T})$ and vice-versa.

In order to further evaluate the study mixtures against performance thresholds, they were plotted using their 10,000 Hamburg wheel pass results (Figure 2-18), which is more appropriate for the moderate traffic levels experienced on the roadways sampled. The 
short-term aged mix data points should ideally fall in the medium traffic passing zone, which is the box near the upper-right which contains the gold-colored asterisk symbol (US 54_5), and two other symbols. This zone requires a minimum of $460 \mathrm{~J} / \mathrm{m}^{2}$ fracture energy and a maximum of $12.5 \mathrm{~mm}$ of rutting in the Hamburg test. The five field-aged mixtures can be judged against a slightly relaxed criteria for the $\mathrm{DC}(\mathrm{T})$, with a minimum long-term aging fracture energy threshold of $400 \mathrm{~J} / \mathrm{m}^{2}$ recommended. This adds the box just to the left of the aforementioned medium traffic zone box to the passing zone for field-aged mixes.

The 10k Hamburg-DC(T) plot led to the following conclusions:

- Three of the newly constructed mixes met both the high and low temperature medium traffic criteria. These were US 54_5 (0, 0, 0), US 54_1 $(33,0,33)$, and SPS10_9 (46, $16,20)$.

- A number of the remaining mixes appear to have the capacity (sufficient 'total energy') to meet both $\mathrm{DC}(\mathrm{T})$ and Hamburg criteria for medium-traffic volume roads, however, they will need a softer binder with similar UTI to pass the DC(T) test. This will shift the points downward, and to the right, and hopefully into the medium traffic passing zone. They could also be improved with a higher UTI binder, but that would be a more expensive option to improve the cracking performance of these mixes. For instance, you can imagine that US 54_2 (red '+' symbol) will only need a slightly softer binder or a small amount of rejuvenator to move the data point slightly down and to the right, into the medium traffic passing zone.

- One of the five field projects was in the appropriate zone for a field-aged mix. This 
was US 54_7, a virgin mix constructed in 2003, which retained and impressive 459 $\mathrm{J} / \mathrm{m}^{2}$ of fracture energy even after 14 years of aging. As expected, the aged field core exhibited a low rut depth in the Hamburg.

- None of these mixes would be suitable for use on high traffic volume facilities, unless a higher UTI binder and perhaps additional measures were taken. Referring back to the 20k Hamburg results (Figure 2-16), which would be appropriate for high-traffic volume facilities, the use of a softer binder with a similar UTI would move the points in the diagonal direction of the US54_7 mixture, and beyond (along that particular binder tradeoff axis line), which would cause a failure in the Hamburg test. Thus, the total energy of these mixes would need to be enhanced, which could be achieved by use of a higher UTI binder, stronger aggregates (or aggregate structure), and by the use of rut and/or crack mitigating materials (rubber, RAS pulp, fibers). Fortunately, high traffic volume facilities are usually comprised of stone mastic asphalt mixes (SMAs). SMAs benefit from hard rock, thick asphalt films, polymer-modified binder, and fibers, which give them an edge in meeting even stricter rutting and cracking criteria. 

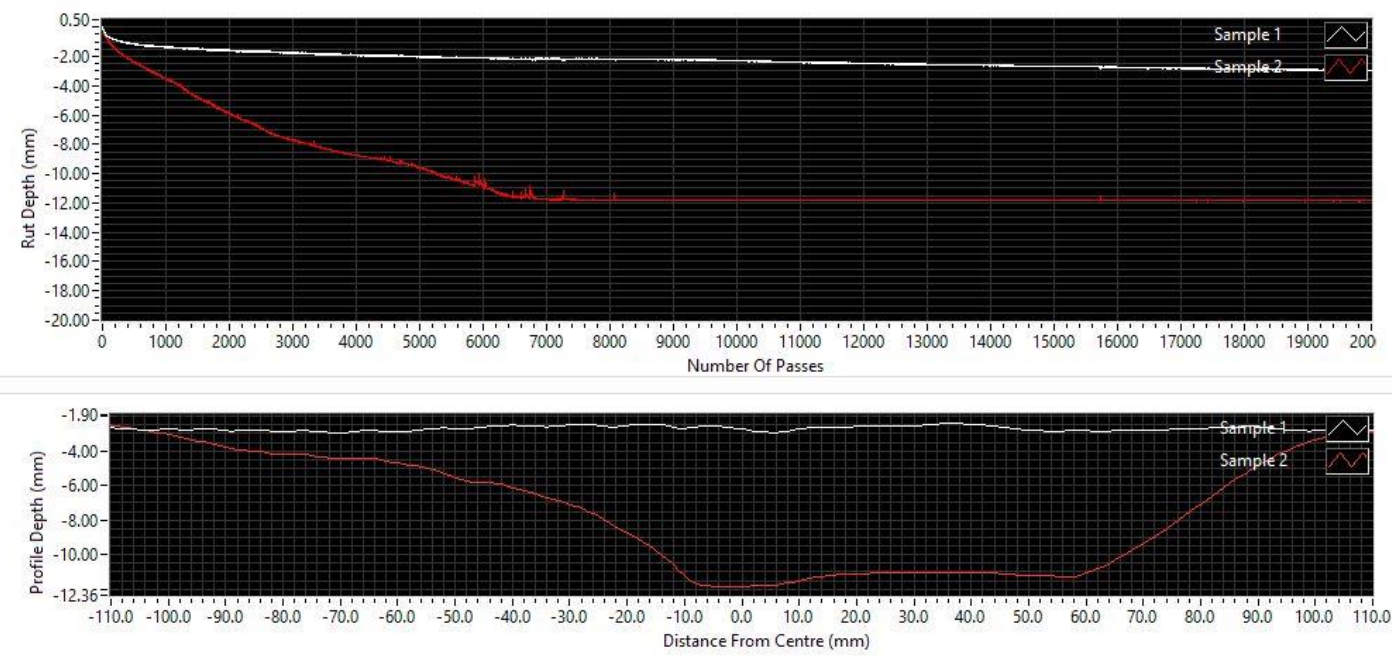

\begin{tabular}{|c|c|c|c|c|c|c|c|c|c|c|c|}
\hline \multicolumn{2}{|c|}{ Target Temperature: } & \multicolumn{2}{|c|}{ Target Cycles: } & \multicolumn{2}{|c|}{ Speed: } & \multicolumn{2}{|c|}{ Number Of Cycles: } & \multicolumn{2}{|c|}{ Rut Depth 1: } & \multicolumn{2}{|c|}{ Temperature 1: } \\
\hline 50.0 & ${ }^{\circ} \mathrm{C}$ & 10000 & & 26.5 & RPM & 10000 & & -2.97 & $\mathrm{~mm}$ & 50.0 & ${ }^{\circ} \mathrm{C}$ \\
\hline \multicolumn{2}{|c|}{ Tank Temperature } & \multicolumn{2}{|c|}{ Max Rut Depth: } & & & \multicolumn{2}{|c|}{ Elapsed Time: } & \multicolumn{2}{|c|}{ Rut Depth 2: } & \multicolumn{2}{|c|}{ Temperature 2: } \\
\hline 26.4 & ${ }^{\circ} \mathrm{C}$ & 20.00 & $\mathrm{~mm}$ & & & $06: 17$ & hh:mm & -11.86 & $\mathrm{~mm}$ & 50.1 & ${ }^{\circ} \mathrm{C}$ \\
\hline
\end{tabular}

Figure 2-17 Moisture sensitivity in Hamburg Test for SPS10_1 (Sample 2)

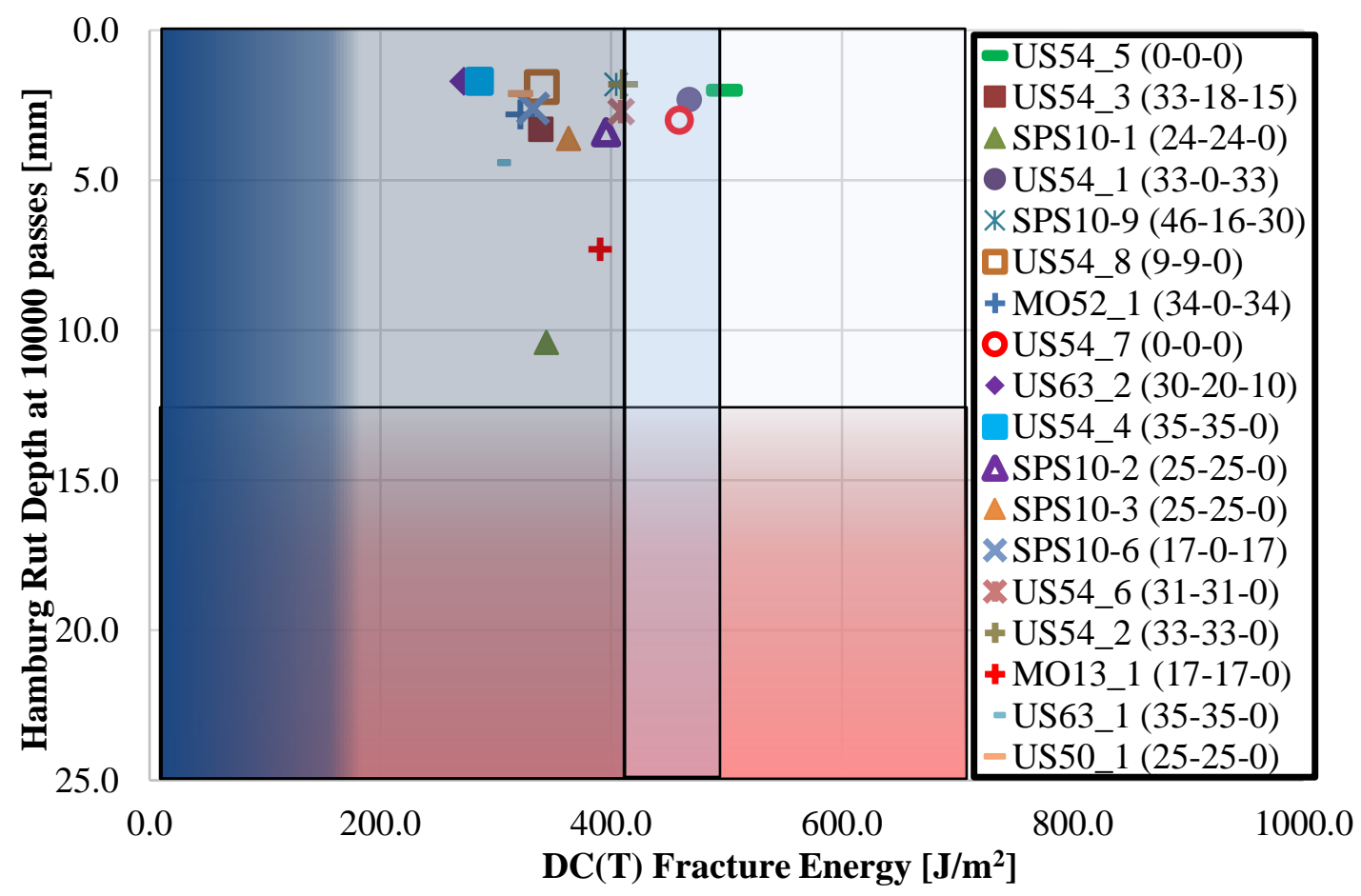

Figure 2-18 Hamburg-DC(T) plot for 10k Hamburg Wheel Passes 


\subsubsection{Summary}

Based on the findings of this part of study, the following conclusions were drawn: (1) Missouri's practices for the responsible and effective use of recycled materials is sound, and continues to improve over time - recent mix designs demonstrate more appropriate balancing between recycled material levels and virgin binder selection, resulting in better performance tests results when compared to older recycled mix designs; (2) Opportunities exist for further improving recycled mix design methods and recycling optimization in Missouri, including (a) Moving to higher ABR levels, by implementing mixture performance tests (balanced mix design); (b) Increasing the use of recycled ground tire rubber (GTR) in Missouri mixes, by using balanced mix design to certify mixes using new, more economical GTR recycling methods, and; (c) Researching the use of recycled materials in stone-mastic-asphalt (SMA) designs; (3) The Hamburg-DC(T) plot can be used to quickly and effectively design and adjust recycled mixtures to meet rutting and cracking performance requirements.

\subsection{UNDERSTANDING AND IMPROVING HETEROGENEOUS, MODERN RECYCLED ASPHALT MIXES (4R-2)}

\subsubsection{Introduction}

Balanced Mix Design (BMD) was introduced by the Federal Highway Administration (FHWA) Expert Task Group (ETG) on Mixtures and Construction in September 2015 [10]. The ETG defined BMD as "asphalt mix design using performance tests on appropriately conditioned specimens that address multiple modes of distresses taking into consideration mix aging, traffic, climate, and location within the pavement structure." In essence, it is an iterative mix design method that is based on the results 
obtained from the chosen mixture performance tests. While volumetrics are still a part of the design method, the concept of BMD is such that mixture performance tests are given a higher weight in the final mix design iteration. Further details about the BMD can be found in the NCHRP report titled "Development of a Framework for Balanced Mix Design", published in 2018 [10]. BMD performance targets for Missouri were recently published based on a research study completed in 2020 [11].

Based on the concept of BMD, various strategies can be employed by the paving agency to improve the mixture designs and produce more durable asphalt pavements. For example, Wisconsin DOT evaluated the effect of using a regressed air voids approach wherein the final binder content of a mixture is based on $3.0 \%$ air voids, which is a departure from the traditional $4.0 \%$ air void target. The results showed an increase in cracking resistance without an adverse effect on rutting resistance [12]. Multiple state agencies have also looked into similar methods such as increased Voids in Mineral Aggregates (VMA) requirements, increased in-field density, etc [4, 5].

Another popular way of enhancing mixture durability is by adjusting its baseline constituents, such as shifting to softer base binder grades or incorporating modifiers such as ground tire rubber, rejuvenators, adjusting recycled asphalt pavement (RAP) sources and usage levels, and so on. It is common knowledge that the Superpave mix design provides enhanced protection against rutting, but cracking has become an increasingly pervasive issue over the past few decades [15]. Various researchers have shown the advantage of using a softer base-binder to address the cracking behavior of modern asphalt mixtures, especially when higher recycled content is used $[5,7,8]$. Furthermore, polymer 
or rubber modification has also been used in conjunction with a softer base binder to increase the binder useable temperature interval (UTI) and to provide additional protection against rutting and cracking [9-11]. To this end, modern recycled mixtures have been successfully designed with BMD tools using a combination of softer binders, additional virgin binder, and various modifiers when relatively high recycling rates were targeted [2, 12].

In this study, the performance of asphalt mixtures in Missouri were evaluated in terms of rutting and cracking behavior, followed by the implementation of strategies to improve and to balance the performance without changing the aggregate structure $[6,13,14]$. The scope of the study was limited to leaving the aggregate blend used in the existing reference mixture unchanged while changing/modifying the virgin binder system, binder content, and by employing additives to improve and balance the overall performance. The following sections describe the objectives of the study in more detail and outlines the steps implemented for mixture improvement.

\subsubsection{Objective of the research}

This study aims to employ BMD principles and produce a better performing mix by altering its constituents while retaining the original aggregate blend. Two base mixture designs from different field sections located in Missouri were chosen for this study. The first section sampled was recently used on the southbound lanes of US Route 63 in Randolph County near Moberly, MO, and is hereby referred to as US63_1. The second section was a mainline mix placed adjacent to recently installed Long-Term Pavement 
Performance (LTPP) test sections on US route 54 in Camden and Miller Counties near Eldon, MO. This test section is hereby referred to as US54_6.

The main steps followed to pursue the objectives of this study were:

- A baseline asphalt mixture design was adopted, which followed the Superpave volumetric design principles used in Missouri.

- Keeping the aggregate gradation/system intact, various modifications were applied to the mixture designs to achieve a superior and/or more balanced mix in terms of rutting and cracking behavior.

- Multiple cracking tests were employed (DC(T), I-FIT, and IDEAL), while only a single rutting test (Hamburg was used).

- The modifications included replacing the base binder with a softer grade binder, adding a rejuvenator to the base mix, using a softer grade binder, and/or adding various percentages of recycled ground tire rubber (GTR) to the mix via the dryprocess method. The use of GTR was motivated by a recent study [24], which provided new insights regarding the toughening mechanisms resulting in properly designed GTR mixes, such as macro-scale crack pinning.

- The goal of the study was not to pursue an exhaustive, full-factorial exploration of additive dosage rates, various combinations, etc. Rather a systematic approach towards achieving a more balanced mix design in the fewest possible iterations was employed, akin to the exercise a contractor might pursue when implementing BMD for the first time.

- When dry-process GTR was used, supplemental binder was also automatically used 
following the manufacturer's recommendation. For each 5\% of GTR by weight of binder used, an additional $0.1 \%$ of supplemental virgin binder was added to the mix.

- Performance test thresholds were based on recommendations provided in [11].

- Mixes were adjusted in an attempt to balance between recommended DC(T) and HWTT cracking thresholds. The same mixture iterations were then tested in the IFIT and IDEAL cracking tests so that differences in strategies for maximizing scores in the various cracking tests could be discussed based on the results obtained.

- Although out of the scope of the current study, mixes could be re-optimized using the IFIT and/or IDEAL tests to set cracking test thresholds based on the findings of this study.

The following sections provide more details about the mixtures used in this study, followed by an introduction of the performance tests and a summary of baseline mixture characteristics.
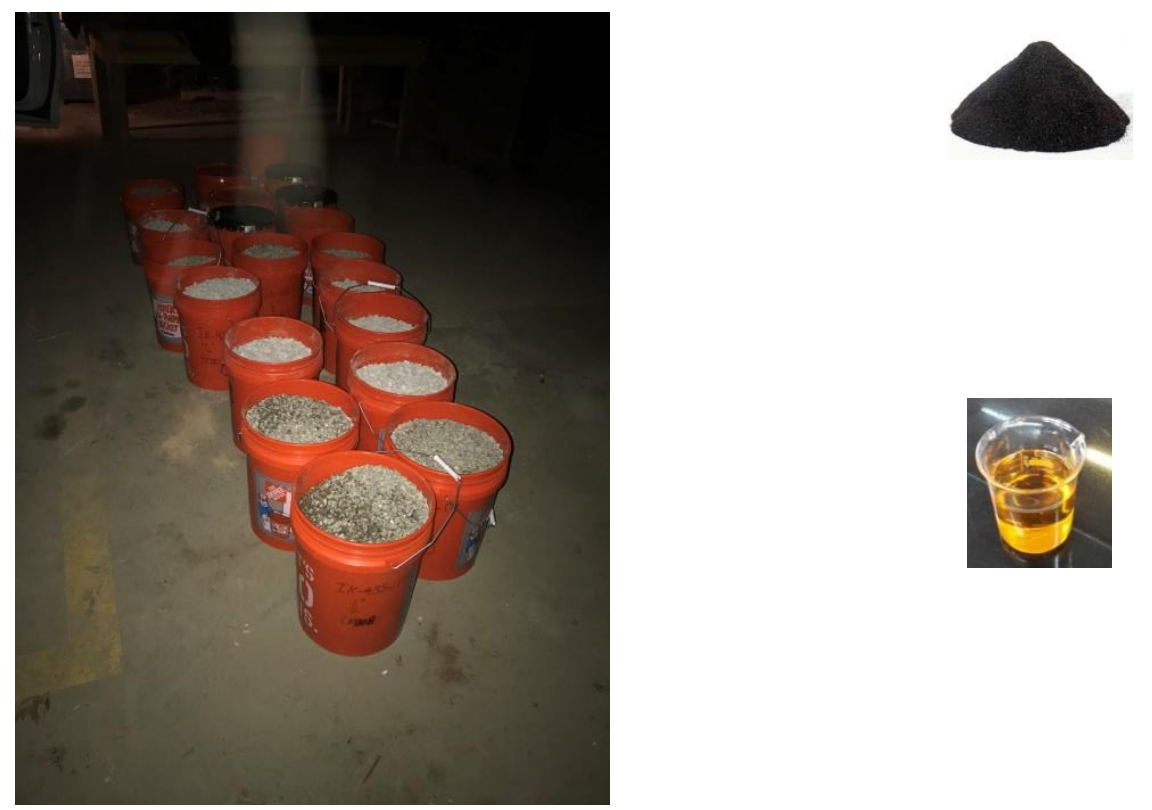


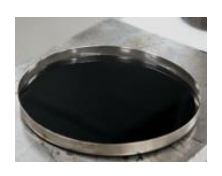

Figure 2-19 Laboratory material including virgin aggregate, RAP, virgin binder, rejuvenators, additives, and rubber.

\subsubsection{Review mixture performance in $4 R$ project}

In this part, the performance of four mixtures in $4 \mathrm{R}$ project (2.1 Section) were reviewed. Two of these mixtures were selected in this part of study, and various strategies were implemented to improve the performance of these mixtures.

\subsubsection{DC(T) FRACTURE results}

Figure 2-20 presents the $\mathrm{DC}(\mathrm{T})$ fracture energy of field cores and plant mixtures. Considering DC(T) fracture energy threshold of $460 \mathrm{~J} / \mathrm{m}^{2}$ set for medium traffic roads the plant mixes, only the field core of US54_1 could pass, albeit barely. The US63_1 section was the most susceptible section to the onset of thermal cracking; it would probably show signs of thermal cracking distress within the first few winters after construction. The US54_6 section showed much better fracture energy with comparable RAP \% to the US63 section. However, both the sections lack a grade bumping on the low temperature side to account for the stiffness imparted by the addition of RAP to the mixture. The other observations from $\mathrm{DC}(\mathrm{T})$ fracture tests are as follows.

- The MO13_1 (17-17-0) section used the stiffest virgin binder (PG64-22 H) and has the lowest amount of ABR. Also, having the NMAS of $9.5 \mathrm{~mm}$, this section was the finest mixture. Since the yielded fracture energy is close to the limit of $460 \mathrm{~J} / \mathrm{m} 2$, one grade bump on the low temperature side of the PG is believed to help this mix pass the 
criterion.

- Comparing US54_6 (31-31-0) and US54_1 (33-0-33) with the same neat binder grade, identical aggregate structure and amount of $\mathrm{ABR}$, it appears that the combination of RAS and warm WMA agent improved the DC(T) fracture energy.

- Although the US63_1 (35-35-0) has only slightly higher ABR than US54_6 (31-31-0), they used a different additive. In both cases, one grade bumping (from -22 to -28) did not suffice to compensate for the brittleness due to addition of RAP.

- Comparing the field cores with plant mix DC(T) fracture energies, it can be observed that the field cores consistently exhibited higher fracture energy values than their plant mixed counterparts. For example, the fracture energy of US63_1 and US54_6 field cores are about $50 \mathrm{~J} / \mathrm{m} 2$ higher than their corresponding plant mixtures. Although it is desirable that both the values agree with each other, field construction has a lot of variabilities in comparison to plant production conditions that can affect the final fracture energy of the laid-out asphalt mixtures. In this particular case, the plantcompacted specimens have a conservative estimation of fracture energy of the asphalt mixture. The field variability could include compaction level and the temperature at which the mix has been compacted. The observed difference between fracture energies of plant mixtures and field cores can also be attributed to the reheating that buckets of plant mixtures experienced before lab compaction. 


\begin{tabular}{|lcc|c|c|}
\cline { 2 - 5 } \multicolumn{1}{l||}{} & \multicolumn{4}{c|}{ Plant Mix- DC(T) Fracture } \\
\hline
\end{tabular}

Figure 2-20 Comparing DC(T) fracture energy of field cores and plant mixes

\subsubsection{I-FIT results}

Figure 2-21 presents the side-by-side comparison of the field core and plant mix flexibility index for four sections. None of the mixtures tested possessed an FI value in excess of 8.0, and in fact, all samples were found to be below an FI value of 5.0. These mixtures exhibited very brittle behavior in the I-FIT test, with snap-back type softening curves and very few data points following the peak load, indicating a very brittle failure. In addition, there is a significant difference in the average FI between these two types of samples. Unlike DC(T) fracture energy, the field cores and plant mixtures of the sections do not follow the same trend; as the US54_1 which had best field core performer, exhibited the poorest performance in plant mixtures. The following observations were made on analyzing the FI results.

- US54_6 and US63_1 with different additives had a difference of 50\% in FI. The significant effect of additive was also noticeable in DC(T) fracture energy. 
- The lower values of FI for PPLC mixes could be due to reheating of the mixes in laboratory before compaction, as I-FIT results are known to be sensitive to aging [25].

- The tested mixes often possess the highest variability between test replicates which is also indicated by the error bars.

\begin{tabular}{|c|c|c|c|c|c|}
\hline \multirow{4}{*}{ 厌 } & $\begin{array}{l}16.0 \\
12.0\end{array}$ & \multicolumn{3}{|c|}{$\begin{array}{l}\text { Plant Mix- FI } \\
\text { Field Core-FI } \\
\text { Neat Binder PGLT }\end{array}$} & \\
\hline & 8.0 & \multicolumn{4}{|c|}{ 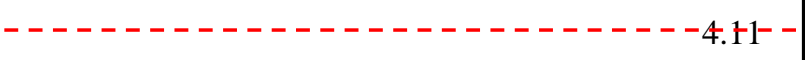 } \\
\hline & \multirow[t]{2}{*}{$\begin{array}{l}4.0 \\
0.0\end{array}$} & $\begin{array}{r}1.62^{3} .59 \\
\times 19\end{array}$ & $\begin{array}{r}1.50 \\
0.40^{-I}\end{array}$ & $0.85^{3.03}$ & 0.23 \\
\hline & & MO13_1 & US63_1 & "US54_6 & US54_1 \\
\hline \multicolumn{2}{|c|}{ Plant Mix- FI } & 1.62 & 0.40 & 0.85 & 0.23 \\
\hline \multicolumn{2}{|c|}{ Field Core-FI } & 3.59 & 1.50 & 3.03 & 4.11 \\
\hline \multicolumn{2}{|c|}{ Neat Binder PGLT } & -22 & -28 & -28 & -28 \\
\hline \multicolumn{2}{|c|}{ Total ABR } & 17 & 35 & 31 & 33 \\
\hline \multicolumn{2}{|c|}{ ABR by RAP } & 17 & 35 & 31 & 0 \\
\hline \multicolumn{2}{|c|}{ ABR by RAS } & 0 & 0 & 0 & 33 \\
\hline
\end{tabular}

\section{Figure 2-21 Comparing FI of field cores and plant mixes}

\subsubsection{IDEAL-CT Index Testing Results}

The IDEAL-CT index of the mixtures is presented in Figure 2-22. As shown, all of the indices are far below the suggested values (min CT=105 for Superpave mixes) [26]. As the other cracking tests suggested, these mixtures are very prone to cracking and do not exhibit enough capability to dissipate the input energy. While the MO13_1 with the lowest amount of ABR was the best performer with CT-index of almost 40, none of the other three mixtures could yield CT-index higher than 20. It is expected that the grade bumping results in softer post-peak behavior and leads to a higher CT-index. 


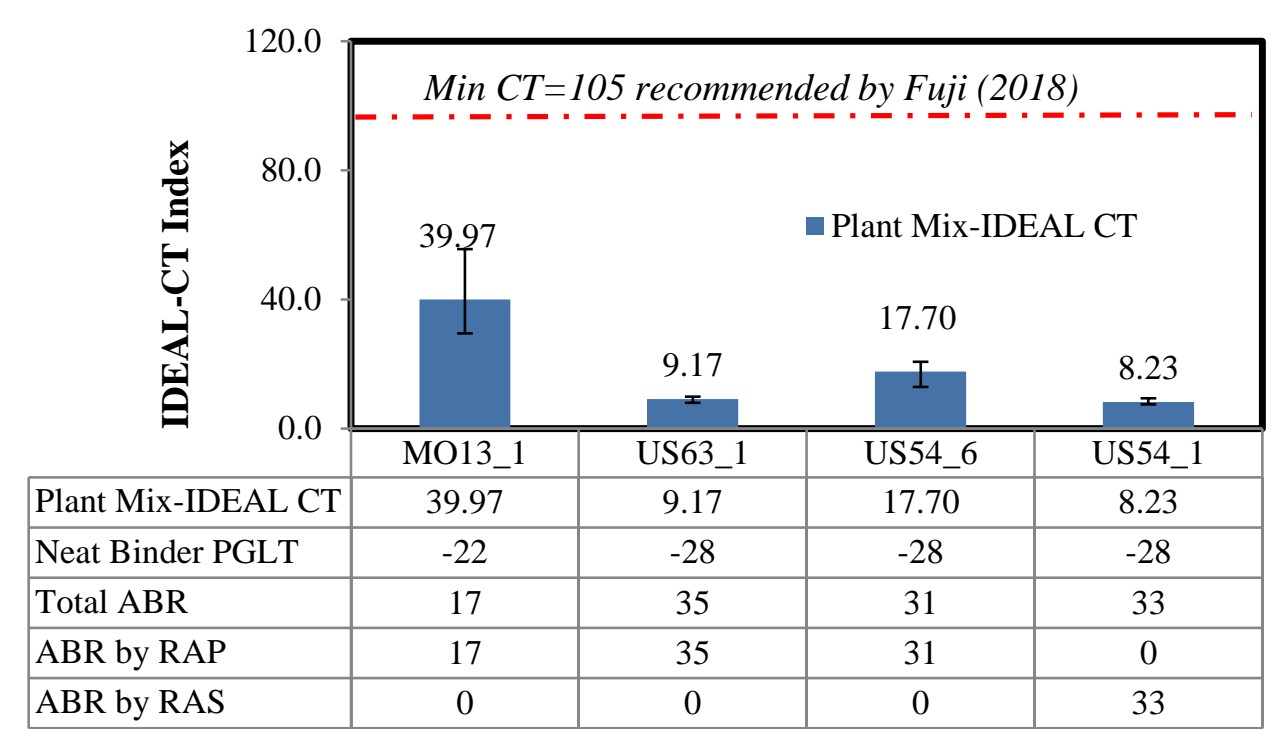

Figure 2-22 IDEAL-CT index of plant mixes

\subsubsection{HWTT Results}

Considering the rut depth of PPLC samples presented in Figure 2-23, the MO13_1 section has the worst performance in the Hamburg test. The MO13_1 section used only one stockpile of limestone and one of manufactured sand, in conjunction with the RAP stockpile in the aggregate blend. Considering the weak aggregate skeleton, the mixture did very well in rutting test. The RAP probably helped the mixture in rutting test by imparting some stiffness to it. The US63_1 and US54_6 sections showed similar rutting results, most likely due to the usage of similar types of aggregates and gradation. The US54_1 showed the best performance in rutting test. This could be due to the use of RAS in the mixture which has been known to improve the rutting performance of the mixture [27]. All the plant mixes pass the Hamburg rutting threshold of 12.5 practiced by most of the agencies [28]. The rutting results of field cores for the corresponding asphalt mixtures show a stark difference when compared to the plant mixes, except for the US54_6 sections. As mentioned in the discussion for the DC(T) fracture energy results, while it is very desirable 
for the plant mixes and field cores to have similar results, the field conditions implicitly introduce a lot of variabilities in the laid-down asphalt mixtures. The plant mixes are usually reheated before compaction and that might impart some stiffness in the mixes, resulting in low rut depths.

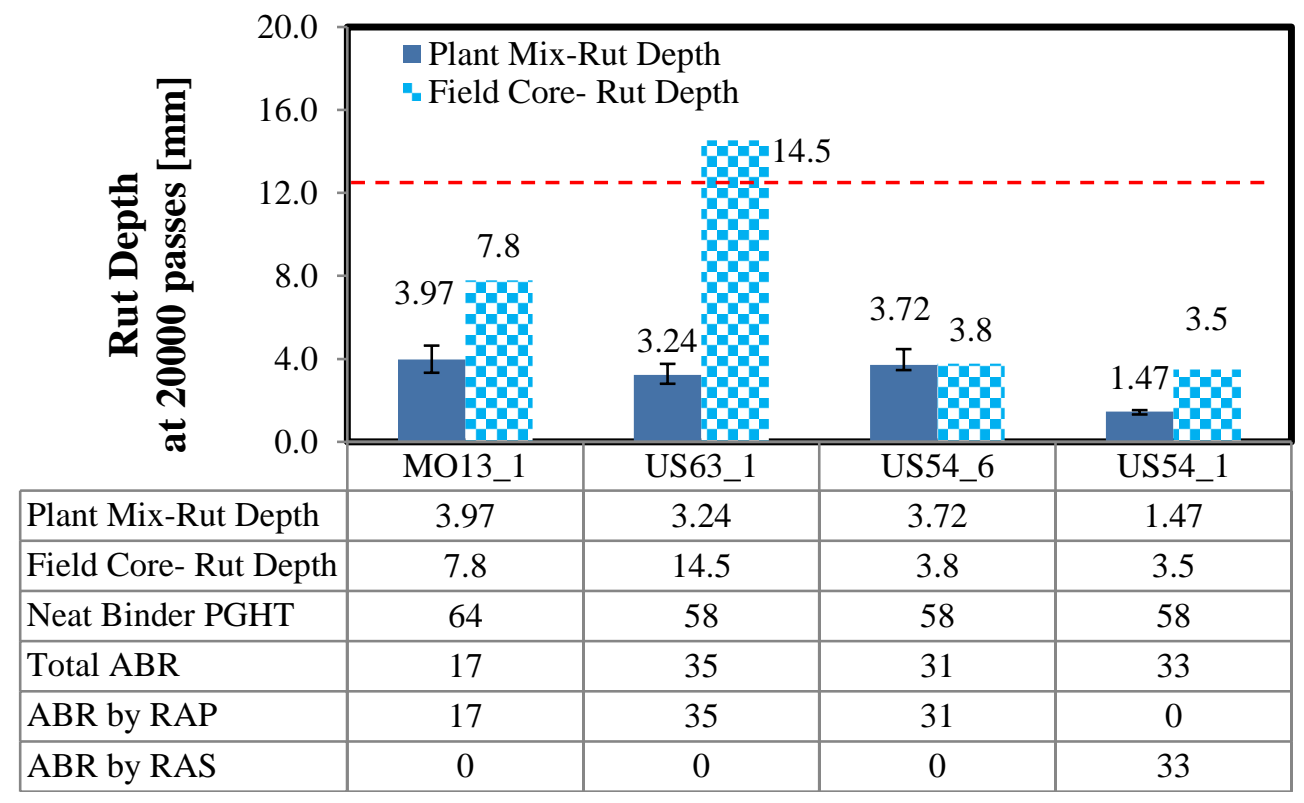

Figure 2-23 Comparing HWTT rut depth of field cores and plant mixes

\subsubsection{Mixtures Design Details}

\subsubsection{Asphalt Mixture Design \#1}

The first MoDOT mix investigated was a $12.5 \mathrm{~mm}$ NMAS dense-graded mixture, using four aggregates sources: two coarse aggregate sources collected from Burlington and Cedar Valley, MO, respectively, a manufactured sand collected from Cedar Valley, MO, and finally a RAP source with $4.5 \%$ asphalt content, creating an asphalt binder replacement (ABR) level of 33.3\% Figure 2-24 and Table 2-6. 
The mix used a base binder graded as Superpave PG58-28 as supplied from the Philips 66 refinery in Kansas City, MO. The base mix design contained Evotherm J12 ( $0.5 \%$ by weight of binder) and Evoflex CA ( $1.75 \%$ by weight of binder). The mixtures in this study were prepared using a standard bucket mixer, where aggregates and binder were heated in a forced-draft oven before being mixed. Evoflex and Evotherm additives were stirred into the binder before mixing with aggregates.

The base mixture design was subjected to the performance testing suite to establish the baseline performance of the mixture. Based on the baseline performance, various modifications were adopted, as outlined in Table 2-7. Again, a non-exhaustive approach was taken to arrive at a more balanced design in the fewest number of iterations possible, followed by a discussion of what seemed to be the most effective adjustments to move existing non-BMD mix designs to meet BMD requirements. 


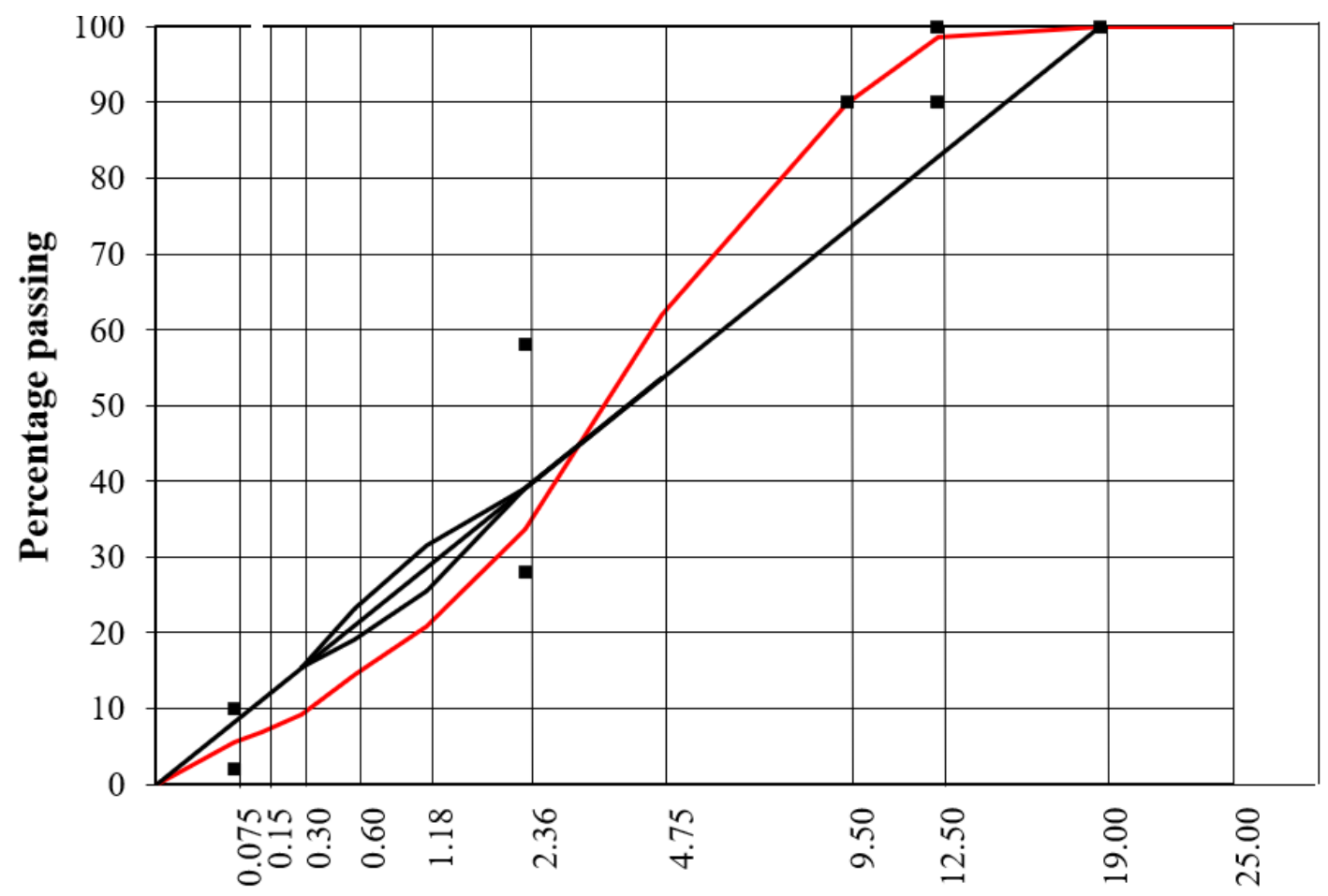

Sieve mm ( 0.45 power $)$

Figure 2-24 Gradation of US63_1 mixture.

Table 2-6. Blending of stockpiles used in the mix US63_1

\begin{tabular}{|c|c|c|c|c|c|c|}
\hline $\begin{array}{l}\text { Sieve Size } \\
\text { (No.) }\end{array}$ & $\begin{array}{l}\text { Sieve Size } \\
\quad(\mathbf{m m})\end{array}$ & $\begin{array}{l}\text { Stockpile } 1 \\
(\%)-3 / 4^{\prime \prime}\end{array}$ & $\begin{array}{c}\text { Stockpile } 2 \\
(\%)-3 / 8^{\prime \prime}\end{array}$ & $\begin{array}{c}\text { Stockpile } 3 \\
(\%) \text {-MAN } \\
\text { SAND }\end{array}$ & $\begin{array}{c}\text { Stockpile } 4 \\
\text { (\%)-RAP }\end{array}$ & $\begin{array}{c}\text { Blend } \\
(\%)\end{array}$ \\
\hline Percentage $\%$ & Percentage $\%$ & 35 & 18 & 7 & 40 & \\
\hline 2 inches & 50.00 & 100.00 & 100.00 & 100.00 & 100.00 & 100.00 \\
\hline $11 / 2$ inches & 37.50 & 100.0 & 100.0 & 100.0 & 100.0 & 100.0 \\
\hline $1 \mathrm{inch}$ & 25.00 & 100.0 & 100.0 & 100.0 & 100.0 & 100.0 \\
\hline $3 / 4$ inch & 19.00 & 100.0 & 100.0 & 100.0 & 100.0 & 100.0 \\
\hline $1 / 2$ inch & 12.50 & 96.0 & 100.0 & 100.0 & 100.0 & 98.6 \\
\hline $3 / 8$ inch & 9.50 & 81.2 & 100.0 & 100.0 & 91.1 & 89.9 \\
\hline
\end{tabular}




\begin{tabular}{ccccccc} 
No. 4 & 4.75 & 37.1 & 92.5 & 99.5 & 63.4 & 62.0 \\
No. 8 & 2.36 & 11.3 & 33.6 & 96.8 & 42.1 & 33.6 \\
No. 16 & 1.18 & 5.3 & 5.4 & 75.7 & 31.6 & 20.8 \\
No. 30 & 0.60 & 4.1 & 2.8 & 40.2 & 24.2 & 14.4 \\
No. 50 & 0.30 & 3.4 & 2.4 & 15.4 & 16.4 & 9.3 \\
No. 100 & 0.15 & 2.9 & 2.1 & 6.0 & 12.6 & 6.9 \\
No. 200 & 0.075 & 2.5 & 1.9 & 3.6 & 10.2 & 5.5 \\
\hline
\end{tabular}

Table 2-7. Iterations of modifications applied to US63_1 mixes

\begin{tabular}{ccccc}
\hline Mixture & $\begin{array}{c}\text { Virgin } \\
\text { Binder }\end{array}$ & $\begin{array}{c}\text { Crumb Rubber } \\
(\%)\end{array}$ & Additive \\
\hline & US 63_1_C (Control) & PG 58-28 & 0 & $\begin{array}{c}1.75 \% \text { Evoflex } \\
\text { (no change) }\end{array}$ \\
& US 63_1_46_R & PG 46-34 & 0 & $\begin{array}{c}1.75 \% \text { Evotherm } \\
0.5 \% \text { Evother }\end{array}$ \\
US63_1 (35-35-0) & US 63_1_46 & PG 46-34 & $0 \%$ & No \\
& US 63_1_46_E10 & PG 46-34 & $10 \%$ & No \\
& US 63_1_46_E20 & PG 46-34 & $20 \%$ & No \\
\hline
\end{tabular}

Volumetrics for all mix iterations based on this blend are shown in Table 2-8. All mixtures were compacted to 80 gyrations in the Superpave Gyratory Compactor (SGC). As part of establishing improved mixes, the traditional Superpave method of designing at 4\% air voids design was relaxed, as modern BMD principles do not typically require strict adherence to a $4 \%$ air void target. Also, for comparison purposes, only one factor was altered between experimental trials. For instance, when a softer binder was used, other factors such as binder content were kept constant. In terms of ingredient compatibility, 
none of the mixtures appeared to have a problem except in the trial labeled as US63_1_46, wherein a softer binder was used without the use of warm mix additives. The final air voids level after 80 gyrations was $5.6 \%$, which was relatively far from the reference MoDOT mix originally designed at $4 \%$ air voids (and found to possess an average $4.4 \%$ voids in the materials sampled and produced in this study). This confirmed the positive effect of warm mix additives in terms of

Table 2-8. Details for US63_1_C (Control Mix) compacted to 4.0+0.5\% air voids

\begin{tabular}{|c|c|c|c|c|c|}
\hline Mix Name & US 63_1_C & US63_1_46 & US63_1_46_R & US63_1_46_E10 & US63_1_46_E20 \\
\hline Binder Used & PG 58-28 & PG 46-34 & PG 46-34 & PG 46-34 & PG 46-34 \\
\hline Total Binder \% & 5.1 & 5.1 & 5.1 & 5.3 & 5.5 \\
\hline Virgin Binder \% & 3.4 & 3.4 & 3.4 & 3.6 & 3.8 \\
\hline $\mathrm{ABR} \%$ & 33.3 & 33.3 & 33.3 & 32.1 & 30.9 \\
\hline $\mathrm{Gmm}$ & 2.455 & 2.460 & 2.458 & 2.438 & 2.425 \\
\hline Va $\%$ at 80 Gyration & 4.4 & 5.6 & 4.4 & 4.5 & 3.9 \\
\hline $\begin{array}{c}\mathrm{Va} \% \text { for performance } \\
\text { tests }\end{array}$ & 7 & 6.9 & 6.9 & 6.8 & 6.7 \\
\hline VMA $(14<)$ & 14.1 & 16.5 & 15.4 & 16.2 & 16.2 \\
\hline VFA (65-78) & 72 & 65.8 & 71.6 & 72.2 & 75.3 \\
\hline
\end{tabular}

\subsubsection{Asphalt Mixture Design \#2}

The other mix design was, once again, a 12.5 NMAS dense-graded mixture placed as an LTPP section on US 54 in Camden County, near Eldon, MO. The mix used four stockpiles consisting of: coarse aggregates having NMAS levels of $3 / 4$ " and $1 / 2$ " (with 8 and 
7\% chert respectively), collected from Gasconade, Missouri; manufactured sand collected from the vicinity of Osage River and finally; a RAP source with $5.1 \%$ asphalt leading to an asphalt binder replacement (ABR) level of about $30 \%$. The mix gradation is shown in Figure 2-25 and Table 2-9. The mix incorporated the same base binder as the first mix (PG58-28 collected from the Philips 66 refinery in Kansas City, MO).

Table 2-10 shows the modifications applied to the baseline mix to finally achieve the required BMD test thresholds. Three variables were selected to be altered during this phase of the study- the use of a softer binder grade (PG46-34), the addition of rejuvenator (3\% Evoflex CA), and the addition of dry-process rubber (levels of 5\% and 20\% GTR by weight of virgin binder were used). For the mixes with GTR, supplemental binder was added according to the manufacturers recommendation $(0.1 \%$ supplemental virgin binder was added for the 5\% GTR mix, while $0.4 \%$ supplemental binder was used for the $20 \%$ GTR mix). An engineered crumb rubber (ECR) product was used in the current study, which has been used as a modifier in several hundreds-of-thousands of tons of SMA and dense-graded mixtures placed since 2016 on the Illinois Tollway, as described in [29]. The variables were strategically chosen after examining the baseline performance of the control (unmodified) US 54_6 mix. 


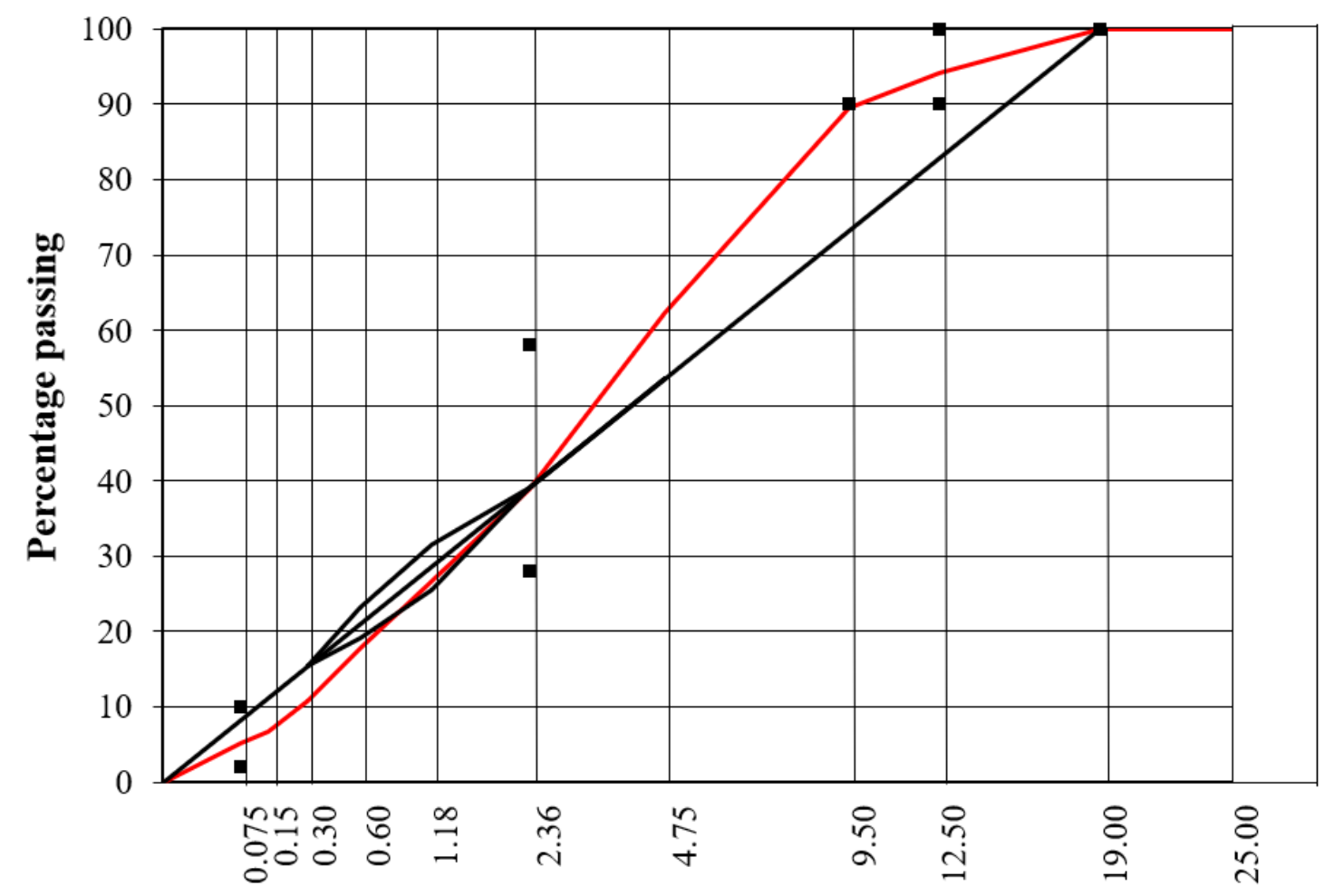

Sieve $\mathrm{mm}(0.45$ power $)$

Figure 2-25 Gradation of US54_6 mixture.

Table 2-9 Blending of stockpiles used in the mix US54_6

\begin{tabular}{|c|c|c|c|c|c|c|}
\hline $\begin{array}{l}\text { Sieve Size } \\
\text { (No.) }\end{array}$ & $\begin{array}{l}\text { Sieve Size } \\
\quad(\mathbf{m m})\end{array}$ & $\begin{array}{c}\text { Stockpile } 1 \\
(\%)-3 / 4 "\end{array}$ & $\begin{array}{c}\text { Stockpile } 2 \\
(\%)-1 / 2 "\end{array}$ & $\begin{array}{c}\text { Stockpile } 3 \\
\text { (\%)-MAN } \\
\text { SAND }\end{array}$ & $\begin{array}{c}\text { Stockpile } 4 \\
\text { (\%)-RAP }\end{array}$ & Blend $(\%)$ \\
\hline $\begin{array}{c}\text { Percentage } \\
\%\end{array}$ & -- & 14 & 29 & 25 & 32 & 100 \\
\hline 2 inches & 50.00 & 100.00 & 100.00 & 100.00 & 100.00 & 100.00 \\
\hline $11 / 2$ inches & 37.50 & 100.00 & 100.00 & 100.00 & 100.00 & 100.00 \\
\hline 1 inch & 25.00 & 100.00 & 100.00 & 100.00 & 100.00 & 100.00 \\
\hline $3 / 4$ inch & 19.00 & 100.0 & 100.00 & 100.00 & 100.00 & 100.00 \\
\hline $1 / 2$ inch & 12.50 & 58.0 & 100.00 & 100.00 & 100.00 & 94.12 \\
\hline $3 / 8$ inch & 9.50 & 28.0 & 99.9 & 100.00 & 99.00 & 89.57 \\
\hline
\end{tabular}




\begin{tabular}{ccccccc} 
No. 4 & 4.75 & 5.0 & 38.9 & 100.00 & 79.00 & 62.26 \\
No. 8 & 2.36 & 4.3 & 3.2 & 80.00 & 54.00 & 38.81 \\
No. 16 & 1.18 & 4.0 & 2.6 & 49.00 & 41.00 & 26.68 \\
No. 30 & 0.60 & 3.2 & 2.4 & 27.00 & 31.00 & 17.81 \\
No. 50 & 0.30 & 3.0 & 2.2 & 13.00 & 20.00 & 10.71 \\
No. 100 & 0.15 & 2.5 & 1.9 & 4.00 & 15.00 & 6.70 \\
No. 200 & 0.075 & 2.2 & 1.5 & 2.0 & 12.00 & 5.08 \\
\hline
\end{tabular}

Using the afore-mentioned blend as the base mixture design, a series of modifications were made to achieve an optimized mix in terms of performance, and sustainability. The strategies were in line with the concepts of the Balanced Mix Design method that dictates producing a final mix design that satisfies the chosen performance test's threshold. Table 2-10 shows the modifications applied to the base mix to achieve the required thresholds. Volumetric for the mix design iterations are shown in Table 2-11. Three variables were chosen to be changed during the course of this phase of the study- use of a softer binder grade (PG46-34), addition of rejuvenator (3\% Evoflex CA) using the method mentioned in previous sections, addition of dry-process rubber ( $5 \%$ and $20 \%$ by weight of virgin binder used). The variables were chosen on the basis of the performance test results of the control mix, as will be discussed in the following sections.

Table 2-10. Iterations of modifications applied to the US54_6 mixes

\begin{tabular}{|c|c|c|c|c|}
\hline Mixture & Type & $\begin{array}{l}\text { Softer } \\
\text { binder }\end{array}$ & Rubber & Rejuvenator \\
\hline US 54_6 (31-31-0) & US 54_6_C (control) & $\begin{array}{c}\text { PG 58-28 } \\
\text { (no change) }\end{array}$ & 0 & \\
\hline
\end{tabular}




$\begin{array}{cccc}\text { US 54_6_46 } & \text { PG 46-34 } & 0 & \text { No } \\ \text { US 54_6_46_E5 } & \text { PG 46-34 } & 5 \% & \text { No } \\ \text { US 54_6_R } & \text { PG 58-28 } & 0 & 3 \% \text { Evoflex } \\ \text { US 54_6_46_E20 } & \text { PG 46-34 } & 20 \% & \text { No }\end{array}$

The volumetric properties of the US54_6 mixture trials are shown in Table 2-11. For this mix, the iteration with 5\% dry GTR had issues with compaction and exhibited high air voids $(6.8 \%)$ at 80 gyrations. A possible reason for poor compaction could be insufficient space for rubber particles to fit in the aggregate skeleton of the dense-graded mix. It was surprising to see that the compaction issues were resolved in $20 \%$ dry GTR mix, perhaps due to the enhanced lubricity provided by the additional supplemental binder accompanying the higher amount of rubber used and the engineered chemical coating on the surface of the ECR rubber particles, which include a compaction aid according to the manufacturer

Table 2-11. Volumetric for US54_6 mix iterations.

\begin{tabular}{cccccc}
\hline Mix & US54_6_C & US 54_6_R & US 54_6_46 & US 54_6_46_E5 & US 54_6_46_E20 \\
\hline Binder Used & PG 58-28 & PG 58-28 & PG 46-34 & PG 46-34 & PG 46-34 \\
Total Binder \% & 5.1 & 5.1 & 5.1 & 5.2 & 5.5 \\
Virgin Binder \% & 3.6 & 3.6 & 3.6 & 3.7 & 4.0 \\
ABR (\%) & 29.4 & 29.4 & 29.4 & 28.8 & 27.3 \\
Gmm & 2.468 & 2.474 & 2.477 & 2.481 & 2.446 \\
Va \% & 4.6 & 4.5 & 5.2 & 6.8 & 3.8
\end{tabular}




\begin{tabular}{cccccc} 
Va \% for & 7.2 & 7.1 & 7.4 & 7.0 & 6.5 \\
performance tests & & & & & 15.3 \\
VMA (14<) & 15.2 & 15.0 & 15.5 & 16.7 & 75.3 \\
VFA (65-78) & 70.0 & 70.0 & 66.6 & 59.9 & \\
\hline
\end{tabular}

\subsubsection{Performance Test Results}

\subsubsection{DC(T) Fracture Energy Test}

Figure 2-26 shows the $\mathrm{DC}(\mathrm{T})$ fracture energy for the five iterations undertaken for the US63_1 mix. This study adopted a fixed test temperature of $-12^{\circ} \mathrm{C}$, based on the midMissouri climatic conditions which usually call for a PGXX-22 plan binder grade (where XX varies depending on design traffic and geographic location in Missouri). Results show that the control mix (no additional modifications) had very low fracture energy compared to the lowest recommend threshold for mainline mixes in Missouri - $400 \mathrm{~J} / \mathrm{m}^{2}$ for lower traffic volume roads [11], and $500 \mathrm{~J} / \mathrm{m}^{2}$ for moderate traffic volume routes. The roads investigated herein are borderline between the low/moderate traffic volume levels described in [11]. The lower fracture energy could be attributed to the absence of bumping of the virgin binder grade considering the high ABR level of the mixture. Thus, a softer binder grade was used as a first step to improve/balance performance. The softer binder grade mix, with and without rejuvenator, performed better in fracture energy but was still below the lowest threshold of $400 \mathrm{~J} / \mathrm{m}^{2}$.

For the next iteration, $10 \%$ dry-process ground tire rubber was added to the mix along with the softer binder. The modification proved to be beneficial with a fracture energy increase of about $80 \mathrm{~J} / \mathrm{m}^{2}$. For the final iteration, $20 \%$ rubber with softer binder grade led 
to a fracture energy increase of close to $150 \mathrm{~J} / \mathrm{m}^{2}$ as compared to the control mixture, pushing it just beyond the $400 \mathrm{~J} / \mathrm{m}^{2}$ threshold for low traffic routes in Missouri.

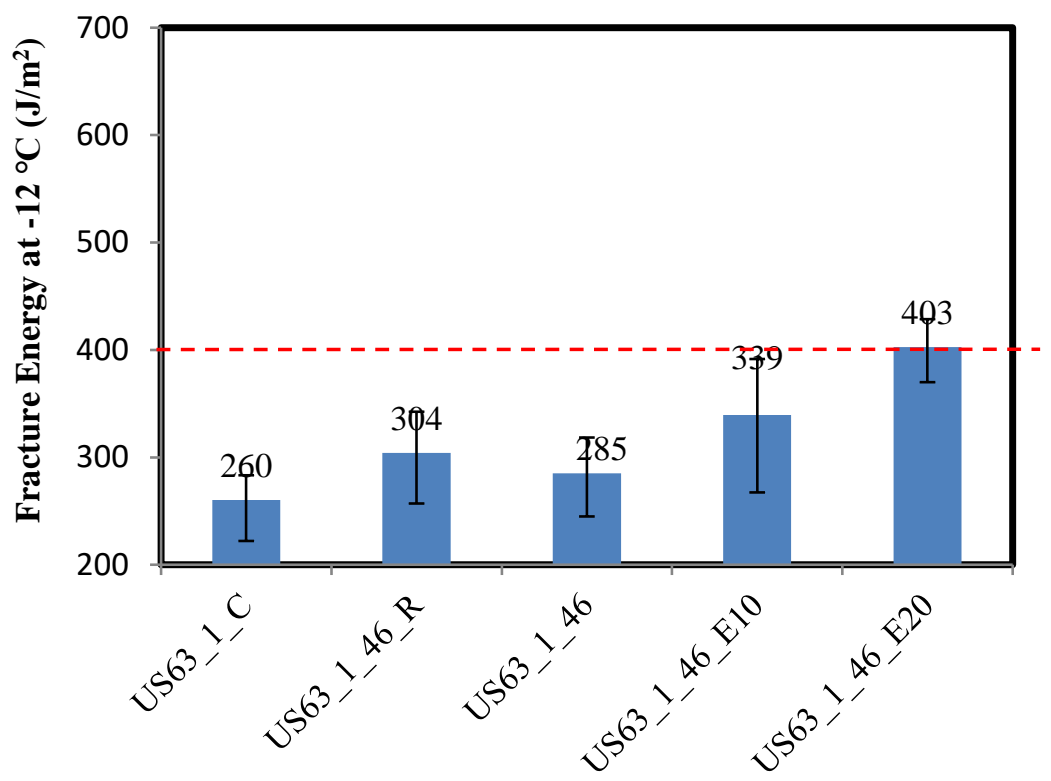

Figure 2-26 DC(T) Fracture energy test results for US63_1 mixtures.

Figure 2-27 shows the fracture energy results obtained from $\mathrm{DC}(\mathrm{T})$ testing at $-12{ }^{\circ} \mathrm{C}$ for the US54_6 mix iterations. The control mix, which had 29.4\% ABR, resulted in average fracture energy of $378 \mathrm{~J} / \mathrm{m}^{2}$, failing to cross the lowest threshold of $400 \mathrm{~J} / \mathrm{m}^{2}$. Adding $3 \%$ Evoflex CA to the mix (US54_6_58_R) did not appreciably alter the fracture energy in this mix. This somewhat unexpected result might be due to the specific chemical interactions between the binder, RAP, and rejuvenator in this mix - resulting in slightly lower fracture energy as compared to the control mix. Next, a softer binder system (PG46-34) was incorporated into the mixture, which led to an improvement in fracture energy. Compared to the control mix, the $\mathrm{DC}(\mathrm{T})$ fracture energy increased by $53 \mathrm{~J} / \mathrm{m}^{2}$. This placed the second trials between the low and moderate traffic level fracture energy thresholds of 400 and 500 $\mathrm{J} / \mathrm{m}^{2}$, respectively. Thus, additional trials were carried out to strive towards further 
improvements. While retaining the softer virgin binder grade, dry-process GTR was added to the mixture, at levels of $5 \%$ and $20 \%$ by weight of the base binder, respectively, in iterations 4 and 5. The incorporation of 5\% rubber by weight of binder (US54_6_46_E5) increased mixture fracture energy to $483 \mathrm{~J} / \mathrm{m}^{2}$. In the final iteration, using $20 \% \mathrm{GTR}$, a significant boost in fracture energy to a level $567 \mathrm{~J} / \mathrm{m}^{2}$ was observed, which finally surpassed the fracture energy threshold of $500 \mathrm{~J} / \mathrm{m}^{2}$ recommended for medium traffic level road facilities in Missouri.

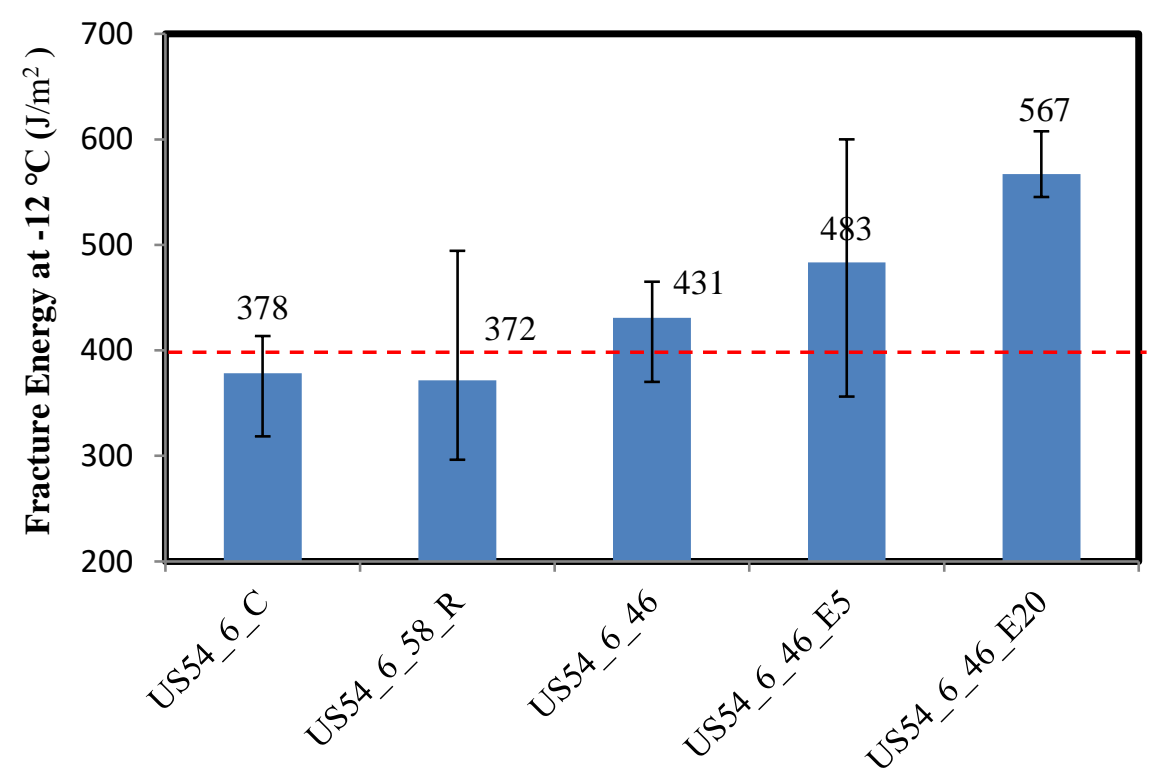

Figure 2-27 DC(T) fracture energy test results for US54_6 mixtures.

\subsubsection{SCB (I-FIT) Testing Results}

The results of SCB (I-FIT) tests performed on the five mixture iterations for the two study mix types are shown in Figure 2-28. Using a softer binder improved the FI value significantly in the US54_6 mixture, while it did not significantly affect the US63_1 mixture. In both mixtures, the rejuvenator did not help to improve the cracking performance as gauged by the FI. When crumb rubber was added to the mixture with a 
softer binder, the FI score decreased significantly. This was in contrast to $\mathrm{DC}(\mathrm{T})$ fracture results, where the addition of dry-process GTR and supplementary binder increased the fracture energy quite significantly in some cases.
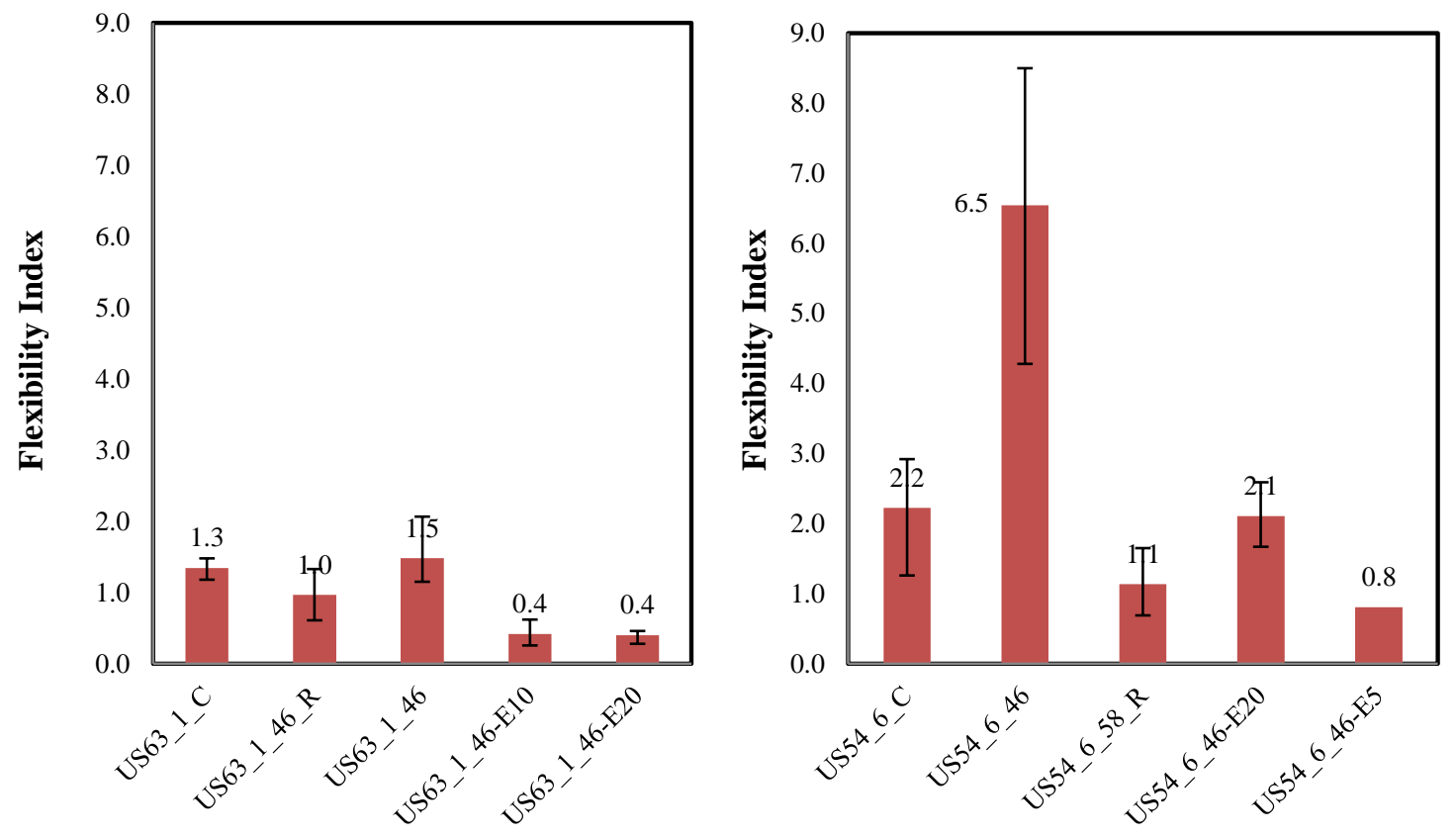

Figure 2-28 Flexibility Index results for US63_1 and US54_6 mixtures

\subsubsection{IDEAL-CT Testing Results}

Figure 2-29 represents IDEAL CT results for the tested mixtures. The use of a softer binder and rejuvenator in the US63 mixture did not improve the IDEAL CT index. The consistency of this result between all 3 cracking tests appears to suggest the presence of a relatively weak aggregate structure in this mixture. However, the softer binder plus rejuvenator had a positive effect on the CT index for the US54 mixture. However, the use of crumb rubber along with supplemental binder significantly reduced the IDEAL CT values measured for both mixtures, consistent with the trend observed in the SCB (I-FIT) 
results. The disagreement of IDEAL CT and FI with DC(T) FE test for the rubber modified mixtures is stark and certainly deserves more investigation in future studies. The intermediate cracking test results are somewhat counter-intuitive as field results suggest that rubber modification provides cracking resistance benefits to asphalt mixtures in the field, even under extreme environments and loading conditions [16, 17]. For now, it can be reasonably concluded that the different test modes, test temperatures and loading rates used in the three cracking tests underlie the differences observed in cracking index trends observed with the mix alterations investigated herein.
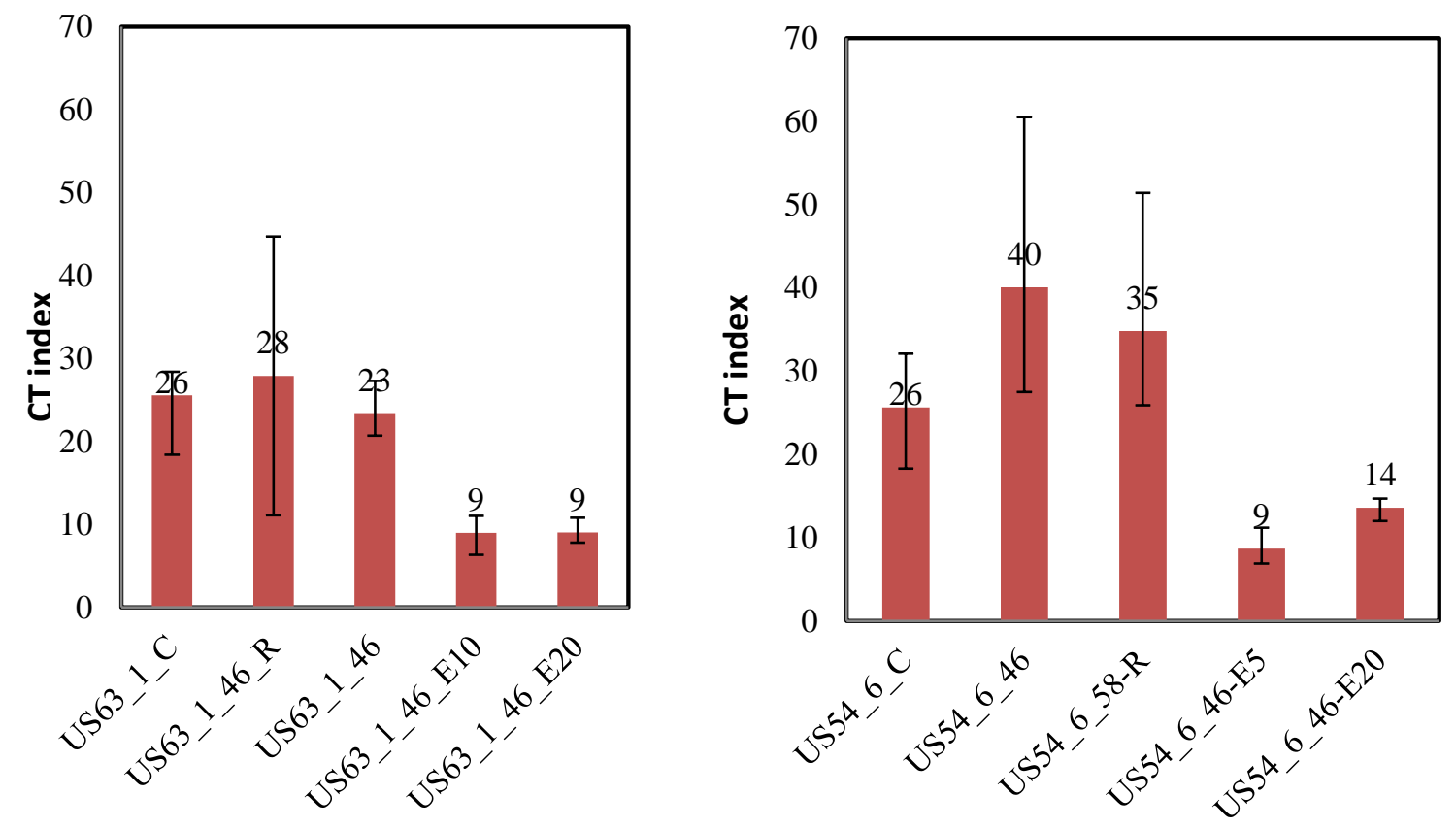

Figure 2-29 IDEAL CT index results for US63_1 and US54_6 mixtures 


\subsubsection{Hamburg Wheel Track Test}

The rutting resistance of the baseline control mixtures was excellent, providing a large factor of safety and motivation for the mixture iterations described in the preceding sections (aimed at 'softening' the mix and other strategies to increase crack resistance). The results of the mixture iterations are shown in Figure 2-30. Replacing the base binder with the softer binder grade increased the max rut depths measured, as expected, but well within recommended tolerances [11]. Notably, the addition of rubber not only increased the fracture energy but also helped in increasing the rut resistance (GTR mixes possessed the lowest HWTT rut depths).

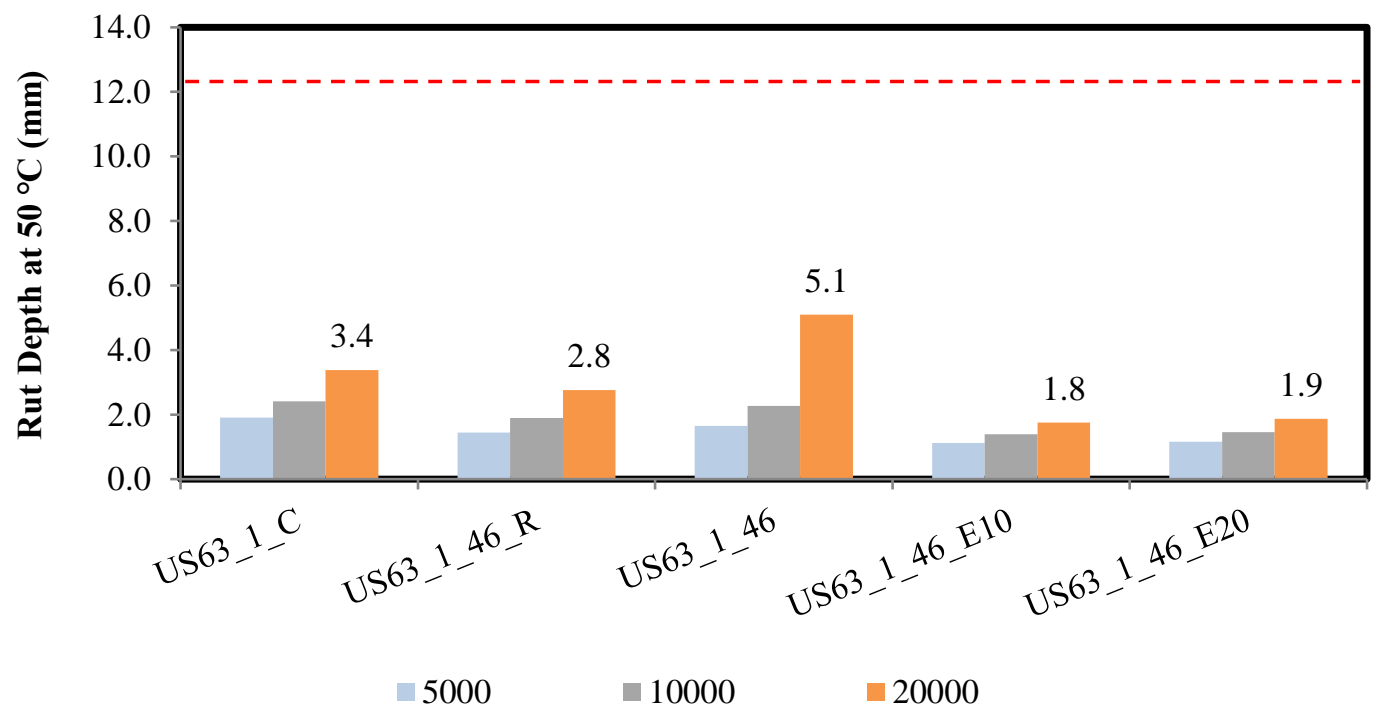

Figure 2-30 Hamburg wheel track test results for US63_1 mixtures

Figure 2-31 shows the HWTT results for the US54_6 mix trials. As shown in this figure, all of the trials could easily meet the maximum rut depth criterion $(12.5 \mathrm{~mm})$, with minimal rutting potential even at 20,000 passes. Once again, replacing the base binder with a softer grade binder slightly increased the permanent deformation compared to the control 
mix, as expected. The addition of ground tire rubber also produced the best performing mixtures in terms of rutting resistance, as evidenced by the last two iterations of the mixture. However, unlike DC(T) fracture energy, which benefitted from the higher GTR level of $20 \%$, in terms of rutting resistance, the 5\% GTR mixes slightly outperformed the $20 \%$ GTR mixes. However, since all of the GTR test results were below $2 \mathrm{~mm}$ of rutting after 20,000 passes, all can be considered as very low rut potential mix designs.

Although outside of the scope of the current study, the results, especially those for the GTR mixes, suggest that an additional factor of safety against rutting exists in the investigated mixtures. However, in locations such as Missouri, it is not currently practical to obtain binders softer than the PG 46-34 grade investigated in this study. Thus, as modern, recycled asphalt mixtures continue to evolve in the evolving circular economy, asphalt technologists must address the trends towards stiffer materials present in RAP stockpiles, less ductile base binder materials, higher presence of fines in recycled materials, and the challenge of incorporating waste plastic as another candidate recycling ingredient. In other words, how can we make sustainable mix design - sustainable? 


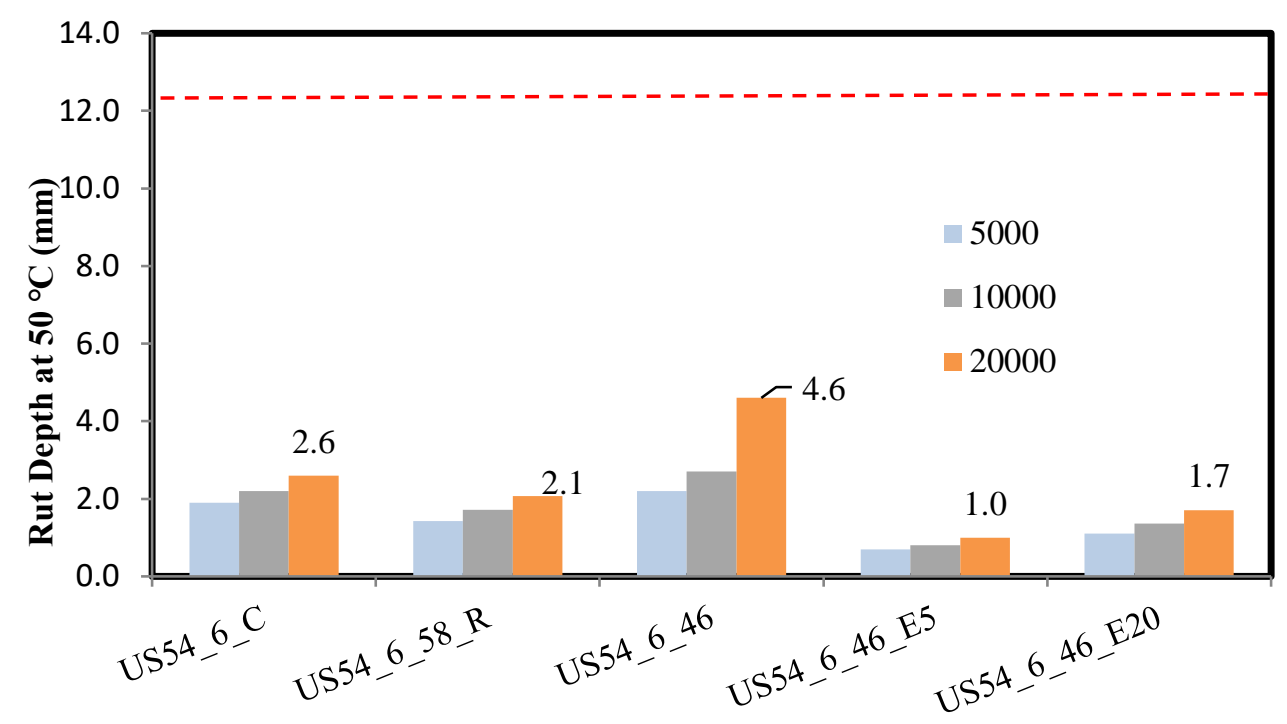

Figure 2-31 Hamburg wheel track test results for US54_6 mixtures.

\subsubsection{Hamburg-DC(T) Plot}

As mentioned in the previous section, plotting in Hamburg-DC(T) space allows simultaneous comparison of the overall performance of the mix. Results from the US63_1 mixtures are shown in Figure 2-32. As expected, all the mixes fall in the red section of the plot, indicating inadequate thermal cracking performance for the requirements of this project. While the employed strategies did push the mix in the right direction (shown with green arrow), none of the modifications were enough to push it over the set thermal cracking threshold. 


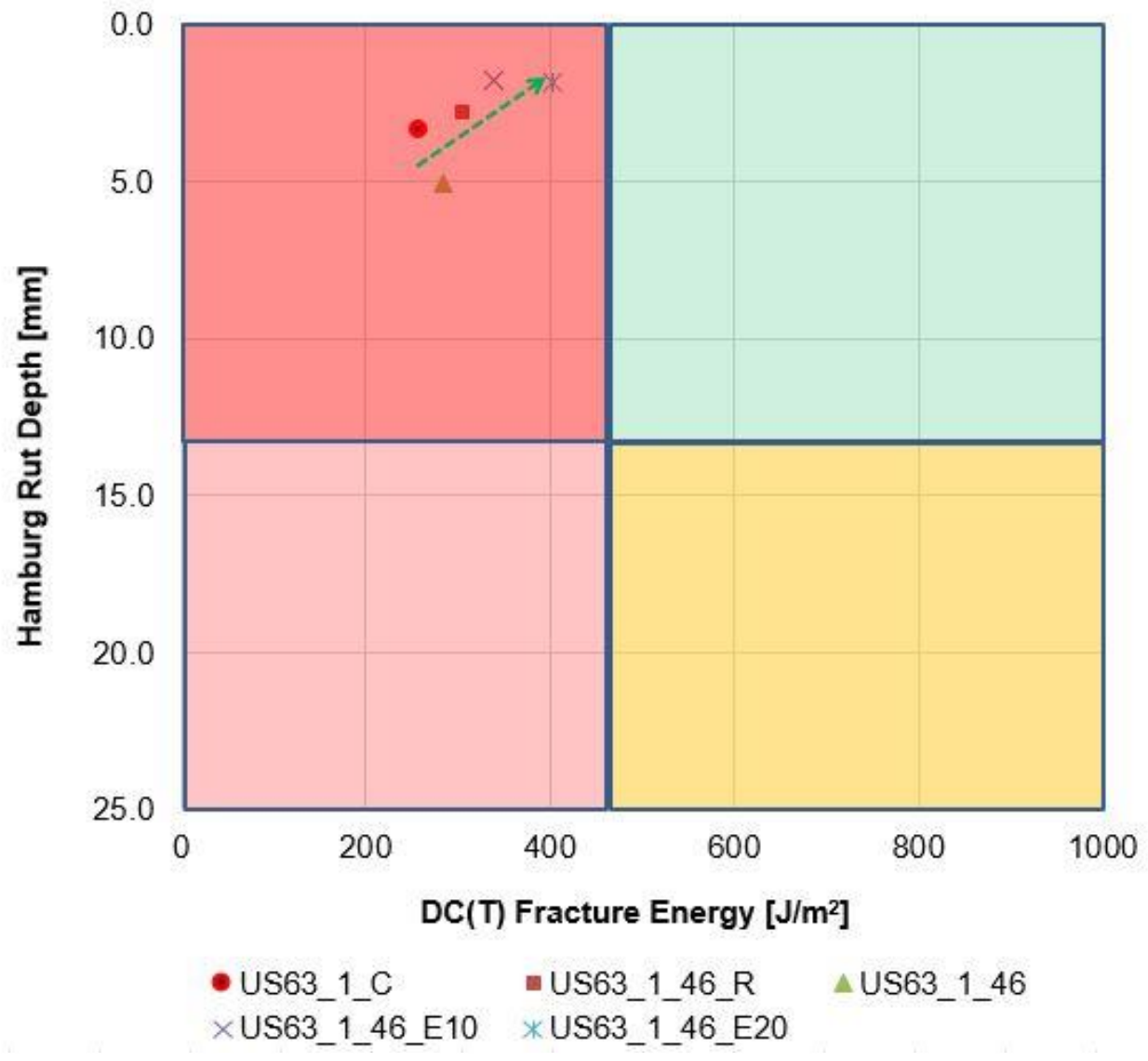

Figure 2-32 Hamburg-DC(T) performance space diagram for US63_1 mixture

Although the addition of rubber helped to improve the mix in terms of thermal cracking and rutting, the mixture was still very prone to thermal cracking with the fracture energy barely crossing the lowest threshold of $400 \mathrm{~J} / \mathrm{m}^{2}$. This could be due to the poor quality of aggregates used in this mix. Thus, another local mix design was adopted to be modified following BMD principles. This failed mix iteration, however, showed the immense potential of ground tire rubber in resisting thermal cracking and improving rut resistance of the asphalt mixture.

Figure 2-33 shows the DC(T) and Hamburg results for US54_6 mixture iterations. As seen from the plot, the addition of rejuvenator and then replacing the base binder with 
softer binder pushed the mixture towards better fracture energy but sacrificed rut resistance (shown with blue arrow). However, the addition of rubber in conjunction with softer grade binder replenished the rut resistance of the mix and added thermal cracking resistance, allowing the mix to cross the set threshold (shown with green arrow).

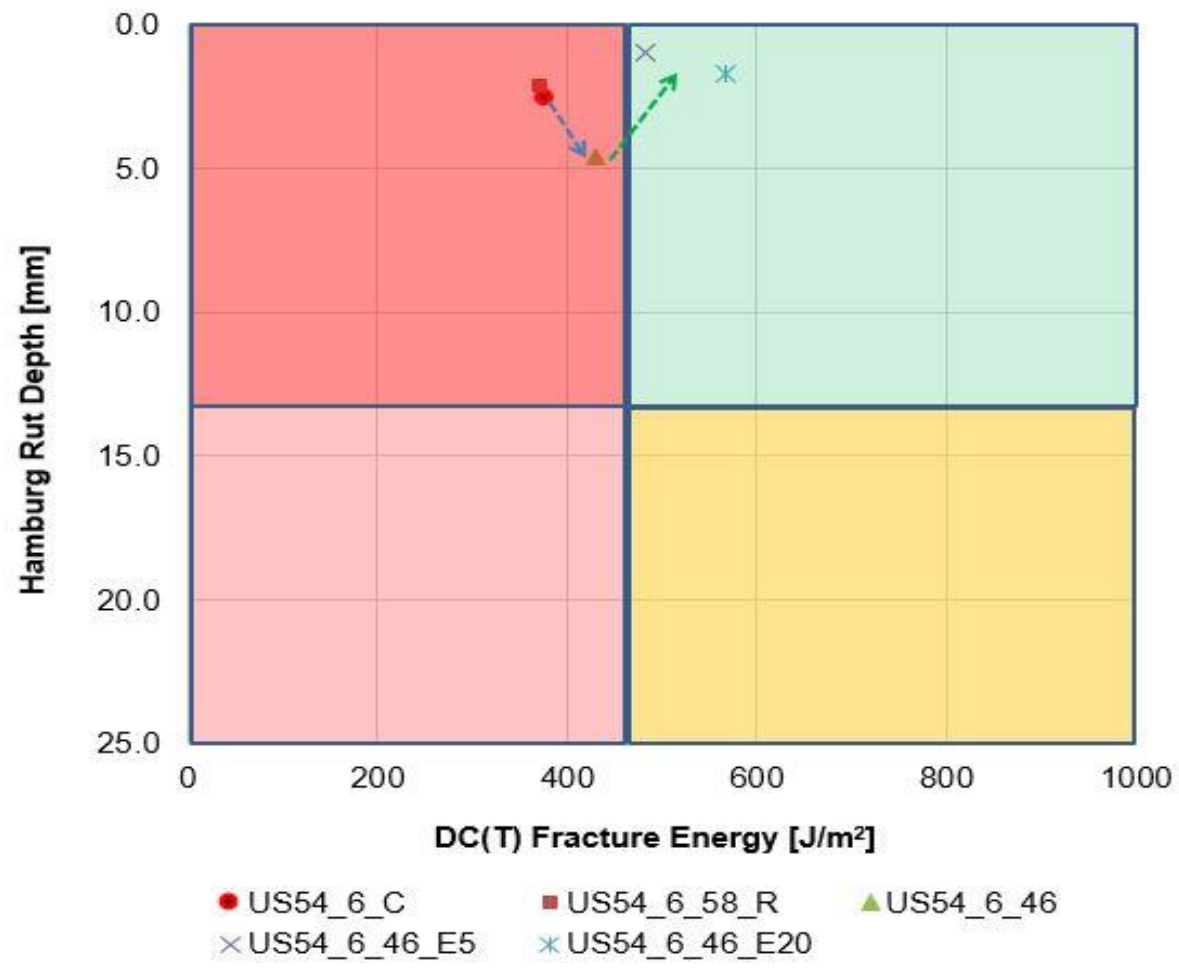

Figure 2-33 Hamburg-DC(T) performance space diagram for US54_6 mixture.

In summary, as shown in the previous sections, the $1^{\text {st }}$ mix iteration, US63_1, could not pass the thermal cracking threshold adopted for this study. Thus, a $2^{\text {nd }}$ mix iteration, US54_6, was tried, and various modification strategies were tried in line with the concepts of BMD. Finally, by using softer grade binder and rubber in conjunction, the thermal and rutting thresholds were achieved. The following section estimates the material cost of producing the US54_6 mix iterations. 


\subsubsection{Hamburg-SCB (FI) Plot}

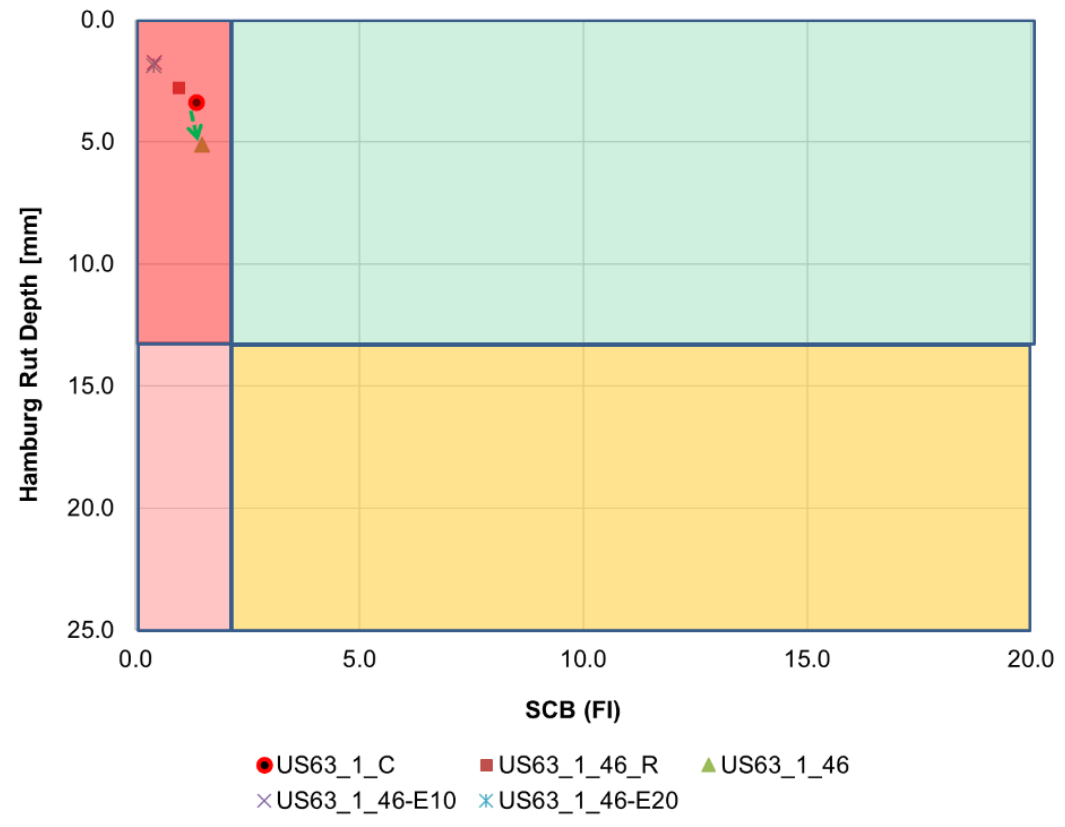

Figure 2-34 Hamburg-SCB (FI) performance space diagram for US63_2 mixture.

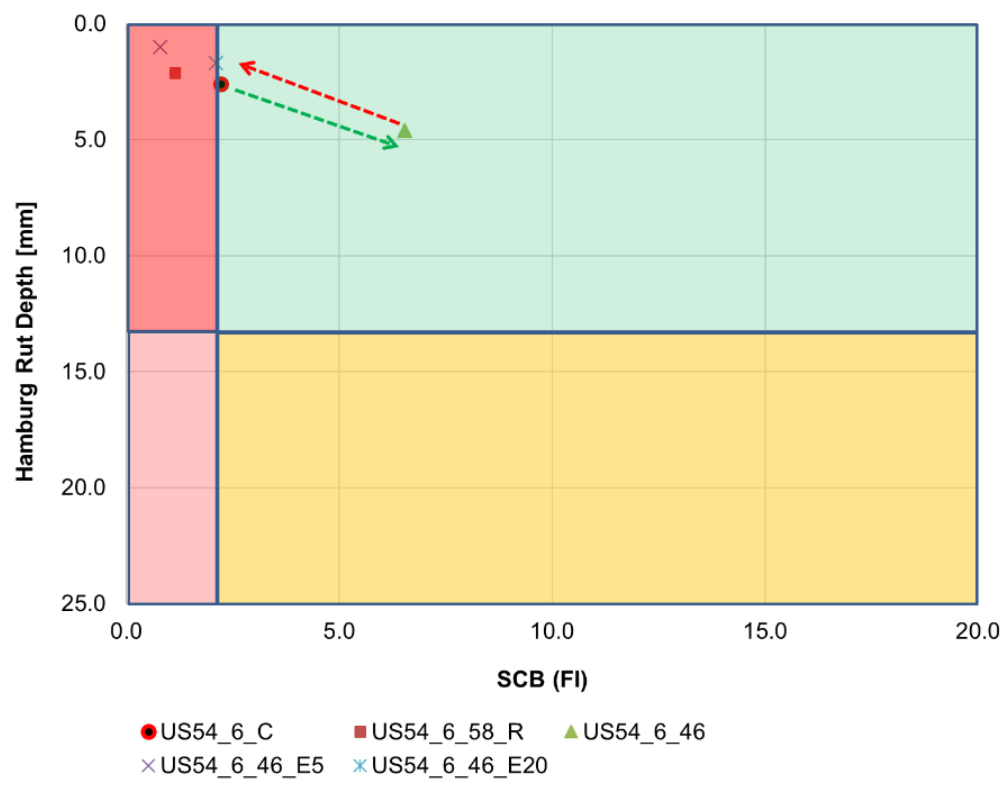

Figure 2-35 Hamburg-SCB (FI) performance space diagram for US54_6 mixture. 


\subsubsection{Hamburg-IDEAL CT-Index Plot}

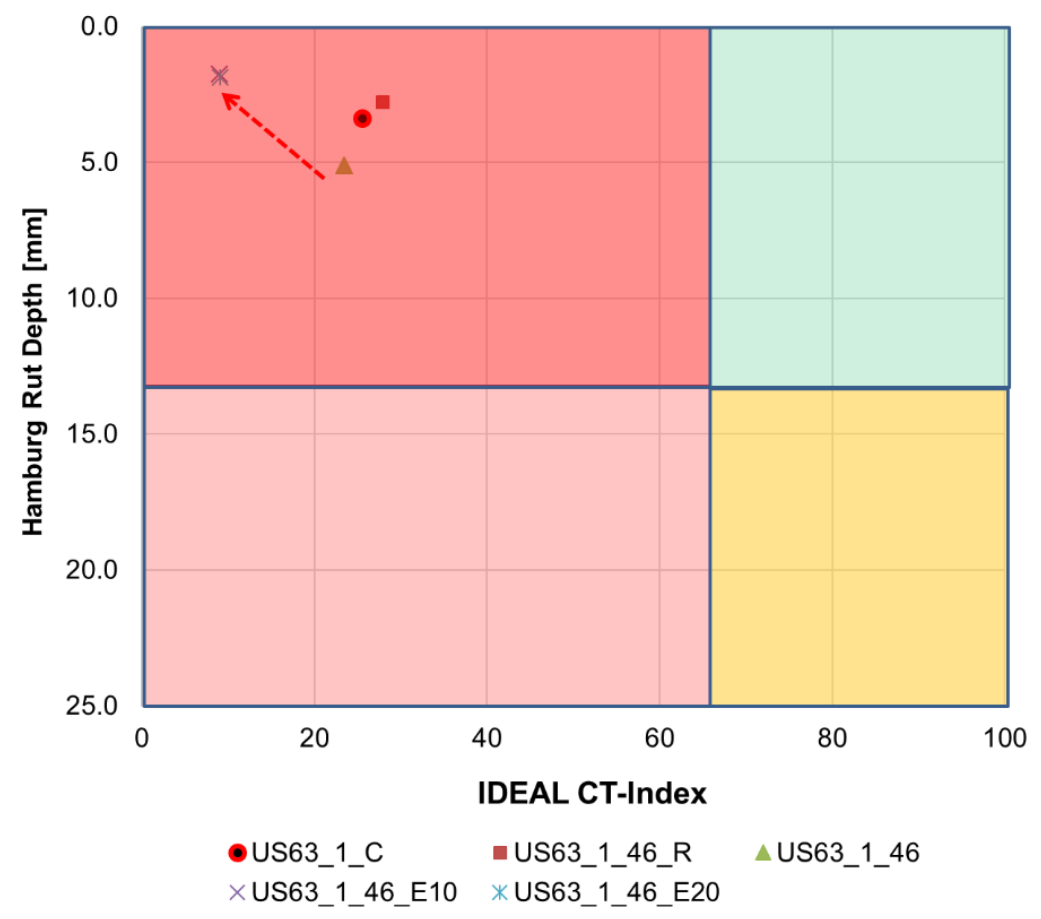

Figure 2-36 Hamburg-IDEAL CT-Index performance space diagram for US63_2 mixture. 


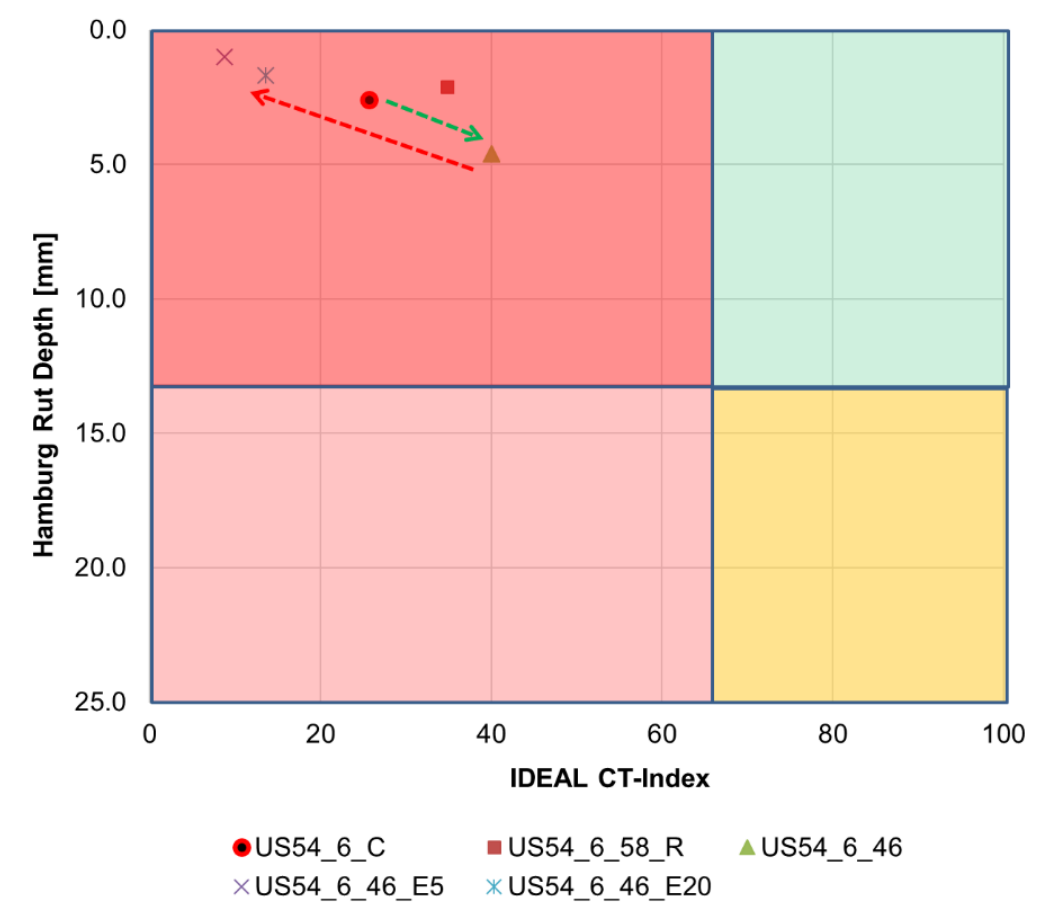

Figure 2-37 Hamburg-IDEAL CT-Index performance space diagram for US54_6 mixture.

\subsubsection{Application of Machine-Learning (Extra tool)}

Majidifard et al. developed a prediction tool to predict the performance of asphalt mixture at high and low temperatures (Majidifard, Jahangiri, Buttlar, \& Alavi, 2019; Majidifard et al., 2020). This tool is based on two different prediction models for DC(T) fracture energy and Hamburg wheel track tests. For DC(T) fracture energy model, genetic programming was used to develop the prediction model, and Convolution Neural Network (CNN) was used to train the Hamburg wheel track model on 10,000 data points. A database containing a comprehensive collection of Hamburg and $\mathrm{DC}(\mathrm{T})$ tests results were used to develop the machine learning-based prediction models. This tool can be used for pre-design purposes to design an asphalt mixture with balanced performance in rutting and cracking. The models were formulated in terms of typical influencing mixture properties variables such as asphalt binder high-temperature performance grade (PG), mixture type, aggregate 
size, aggregate gradation, asphalt content, total asphalt binder recycling content and tests parameters like temperature and number of cycles (Figure 2-38). Models accuracy were assessed through a rigorous validation process and found to be quite acceptable, despite the relatively small size of the training set. Since performing performance tests might be cost-restrictive for some users, using the proposed ML-based models can save time and expense during the material screening phase. This software can be used in this protocol when testing is not feasible. Figure 2-38 shows the schematic view of the software. After filling the inputs on the left and clicking "Run" button, the point will appear on the Hamburg-DC(T) performance diagram, and the rutting curve versus cycles will be available.

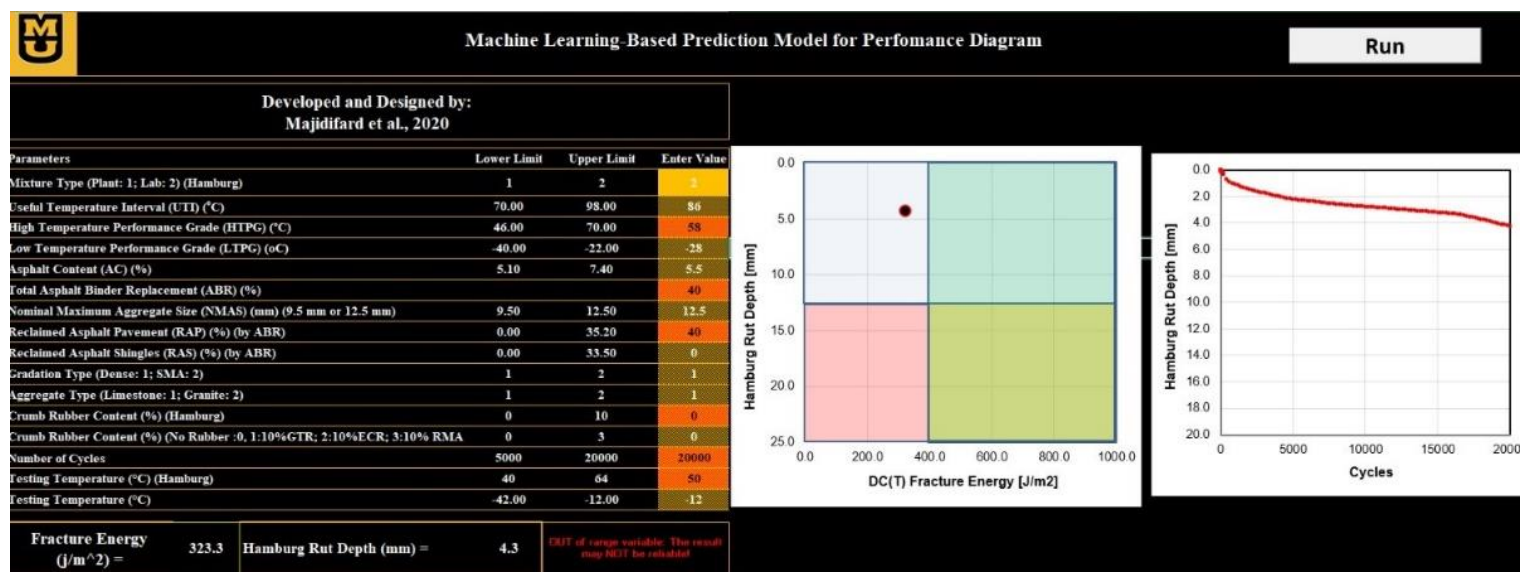

Figure 2-38 Machine learning based prediction model for performance diagram developed by Majidifard et al. (Majidifard, Jahangiri, Buttlar, \& Alavi, 2019); Majidifard et al., 2020).

\subsubsection{Summary}

In this study, an attempt was made to shed light on effective strategies to iterate existing mix designs into more 'balanced mix designs' for modern, heterogeneous recycled mixtures in the Midwest. Different strategies were employed, such as the use of a softer 
virgin binder, the addition of a rejuvenator, and the employment of $5 \%$ to $20 \%$ of dryprocess, engineered crumb rubber by weight of total binder. Two dense-graded asphalt mixtures designed and placed on Missouri roadways prior to the use of BMD were adopted as the baseline mix designs. These mixes were subjected to a suite of cracking and rutting mixture performance tests to establish baseline performance, followed by four mix design iterations per mix (for a total of 10 investigated mixtures). The DC(T), I-FIT, IDEAL-CT, and Hamburg wheel tracking tests were used in the performance testing suite.

The strategies implemented to balance the performance of the mixtures led to the following observations:

- Replacing the base binder with a softer virgin binder grade:

This strategy was generally effective in both the US63_1 and US54_1 mixtures. According to performance test results, it was concluded that the US63 aggregate structure can be considered as generally weak. Therefore, the use of a softer binder was not as effective as compared to the US54_1 section, where all three cracking tests showed a positive effect when a softer based binder was used. The softer base binder led to higher HWTT rut depths, but still far from the maximum threshold of $12.5 \mathrm{~mm}$ even for the highest traffic designs entailing 20,000 wheel track passes.

- Adding a rejuvenator:

In this study, the addition of a rejuvenator was not as effective as the other strategies investigated. However, the use of a rejuvenator served the purpose of helping achieve desirable volumetric properties in the mixtures, likely by increasing the lubricity/workability of the mixtures. According to the results, when the rejuvenator was eliminated from the mixture, it did not meet the volumetric properties ( $4 \%$ voids at Ndesign). This suggests the value of using a rejuvenator when designing highRAP mixtures.

- Adding ground tire rubber: 
Engineered crumb rubber was introduced via a dry-process in an attempt to further improve the cracking and rutting performance of the mixtures. The results showed that the $\mathrm{DC}(\mathrm{T})$ fracture energy test disagrees with the results obtained from the SCB (I-FIT) and IDEAL CT-index when GTR was introduced into the study mixtures. The use of rubber up to $20 \%$ increased the fracture energy of mixtures significantly (almost doubled), while the FI and CT-index scores were significantly reduced. This is somewhat counter-intuitive as field results suggest that rubber modification provides cracking resistance benefits to asphalt mixtures in the field, even under extreme environments and loading conditions. In terms of rutting, the addition of rubber increased the rutting resistance of the mixtures.

Comparing BMD optimization as a whole, the use of a softer binder was the most effective strategy to optimize SCB(I-FIT) and IDEAL CT cracking test scores, while the incorporation of rubber along with a softer base binder and supplemental binder was the most effective method to maximize $\mathrm{DC}(\mathrm{T})$ fracture energy test results. In all cases, modern recycled mixtures appear to have a significant factor of safety against rutting, which suggests the increasing importance of softer virgin binder grades and effective rejuvenators and the importance of accessing these materials without greatly increasing asphalt mixture costs. The movement towards increased sustainability in asphalt mixtures will require continued balancing of increased recycled material usage, mixture durability, and mixture economics. Major sacrifices in one or more of these three categories will not truly lead to long-term, sustainable solutions. Finally, this study highlights the significant challenges confronting the industry with respect to the need for even softer base binder supplies and cost- and performance-effective rejuvenators, with broad compatibility in the brave new world of modern, heterogeneous recycled asphalt mixes. 


\subsection{FIELD PERFORMANCE EVALUATION}

\subsubsection{Field Sections Studied Investigated in this Chapter}

Field monitoring is a potential means to identify the most reliable cracking performance test (Faheem, Hosseini, Titi, \& Schwandt, 2018; Majidifard, Adu-Gyamfi, \& Buttlar, 2020; Majidifard, Jin, Adu-Gyamfi, \& Buttlar, 2020). This part of the study evaluates the field performance of ten sections located in Missouri. Field performance data for the 11 oldest sections was collected from MoDOT online PASER rating system, including ARAN video logs, which were used to delineate thermal cracks from block cracks. A series of pavement condition images are shown in Figure 2-39 for each section investigated in this study. Pavement Surface Evaluation and Rating (PASER) were extracted from MoDOT portal for all the sections. To exclude the dependency of pavement condition on aging, a deterioration rate was computed based on the average decline in PASER rating over the service life of the overlay. Table 2-12 summarizes the details of the sections, along with their PASER ratings and performance test results obtained from the extracted field cores for respective sections. As can be seen from the table, the field sections investigated in this study cover a wide range of recycling in the mixtures and also include sections age 5- 16 years on the field. These sections are summarized in Table 2-12. 

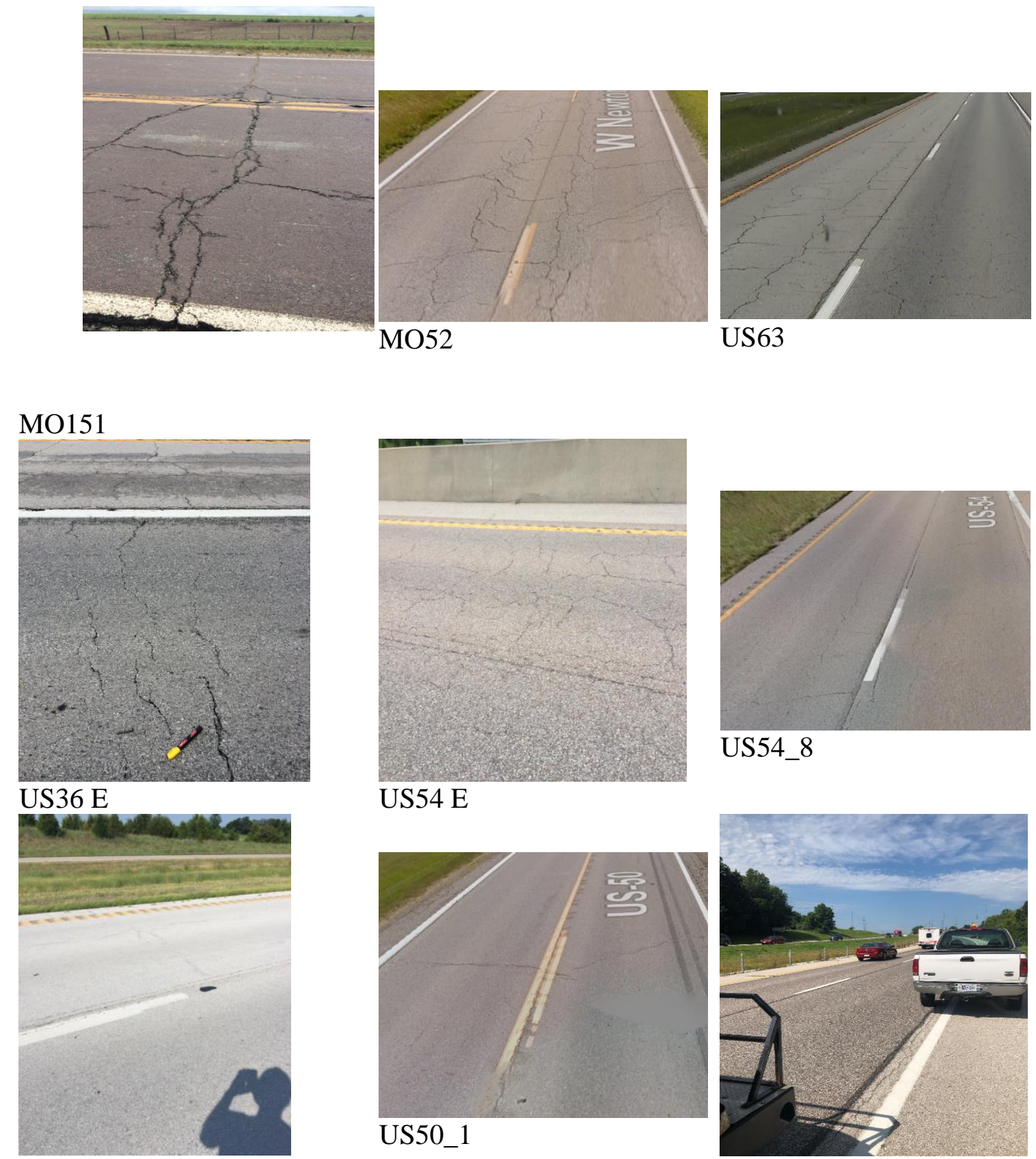

US61
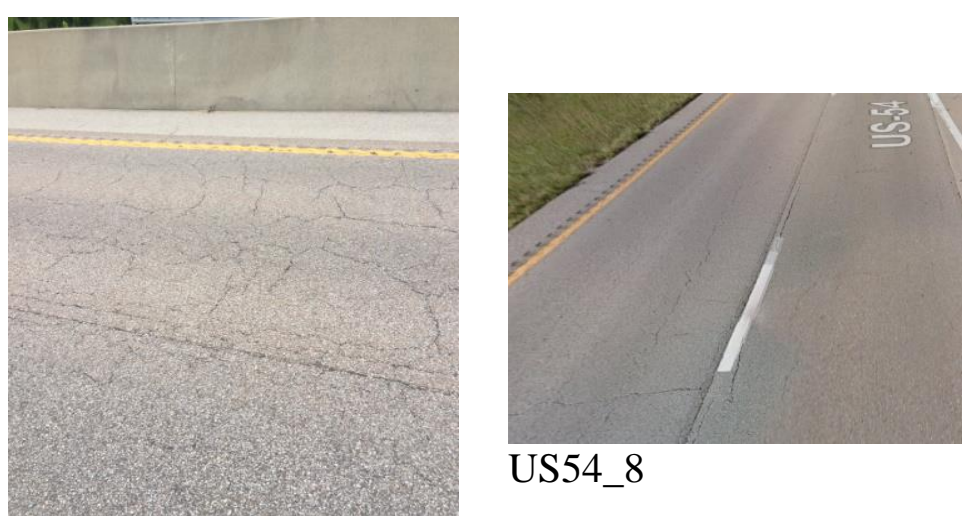

US54_8

US54 E

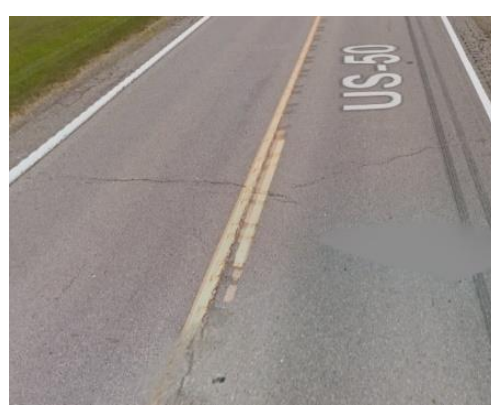

US50_1

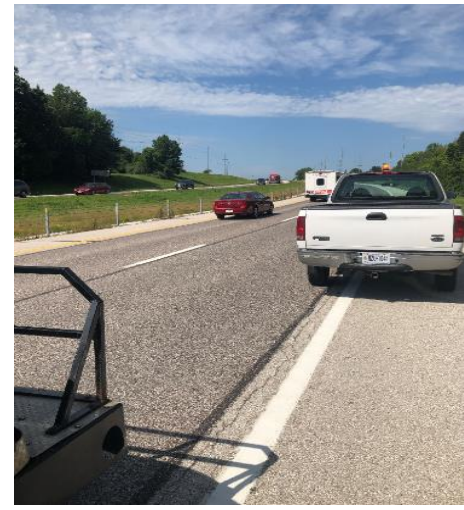

MO94

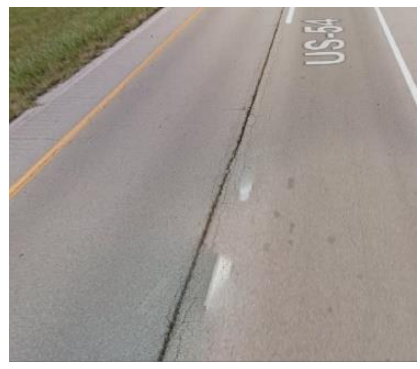

US54_7

Figure 2-39 Pavement condition for ten field sections from high deterioration rate to low deterioration rate. 
Table 2-13 shows the latest field performance measures available for these sections.

Table 2-12. Field sections with significant time in service

\begin{tabular}{cccccccc}
\hline \multirow{5}{*}{ Shase I } & Section \# & $\begin{array}{c}\text { Constr. } \\
\text { Year }\end{array}$ & $\begin{array}{c}\text { Virgin } \\
\text { Binder } \\
\text { Grade }\end{array}$ & $\begin{array}{c}\text { Asphalt } \\
\text { Content } \\
(\boldsymbol{\%})\end{array}$ & $\begin{array}{c}\text { ABR } \\
(\boldsymbol{\%})\end{array}$ & $\begin{array}{c}\text { ABR by } \\
\text { RAP } \\
(\boldsymbol{\%})\end{array}$ & $\begin{array}{c}\text { ABR by } \\
\text { RAS } \\
(\boldsymbol{\%})\end{array}$ \\
\hline \multirow{5}{*}{ MO52_1 } & 2010 & PG64-22 & 4.8 & 33.5 & 0 & 33.5 \\
& US 54_8 & 2006 & PG70-22 & 5.6 & 8.6 & 8.6 & 0 \\
& US50_1 & 2011 & PG64-22 & 5.0 & 24.6 & 24.6 & 0 \\
& US63_2 & 2008 & PG64-22 & 5.6 & 29.9 & 19.9 & 10 \\
& US54_7 & 2003 & PG64-22 & 6.2 & 0 & 0 & 0 \\
\hline \multirow{5}{*}{ Phase II } & MO 151 & 2010 & PG64-22 & 4.7 & 30.6 & 15.9 & 14.7 \\
& US 36 E & 2011 & PG64-22 & 5.1 & 24.7 & 24.7 & 0 \\
& US 54 E & 2010 & PG70-22 & 5.7 & 11.8 & 11.8 & 0 \\
& MO 94 & 2005 & PG64-22 & 5.6 & 0 & 0 & 0 \\
& MO 6 W & 2015 & PG58-28 & 5.9 & 29.6 & 29.6 & 0 \\
& US 61 N & 2013 & PG64-22H & 5.3 & 29.6 & 29.6 & 0 \\
\hline
\end{tabular}
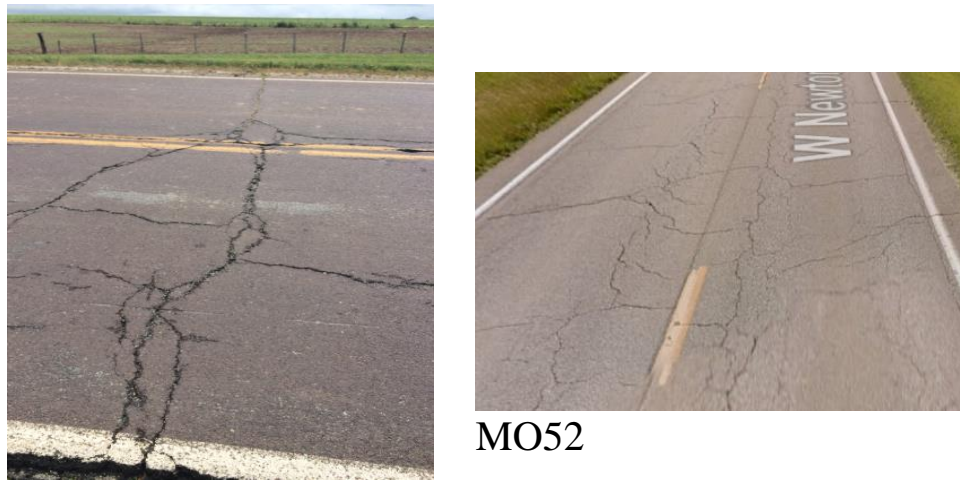

MO52
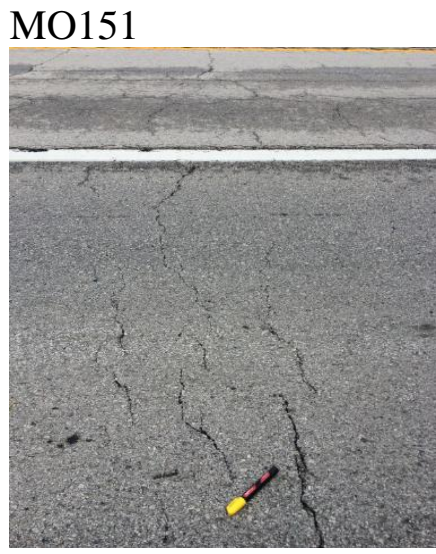

US36 E

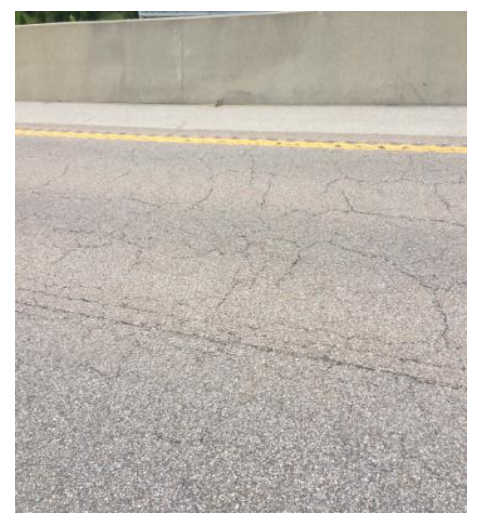

US54 E

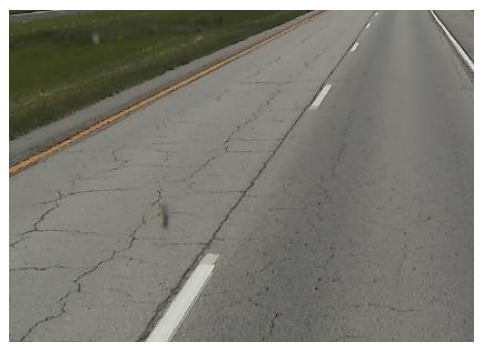

US63

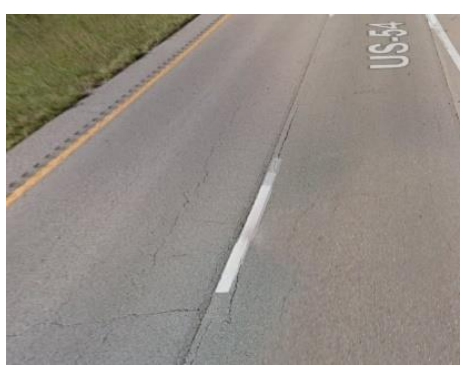

US54_8 


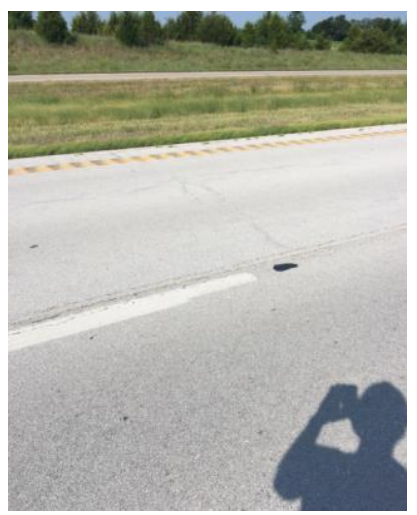

US61

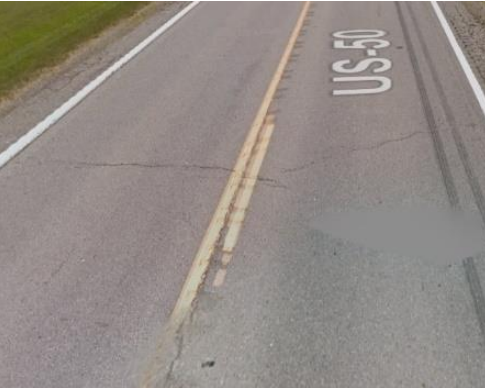

US50_1

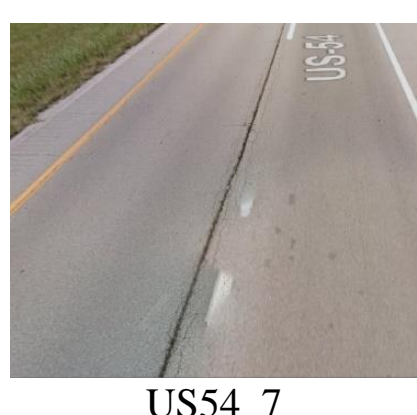

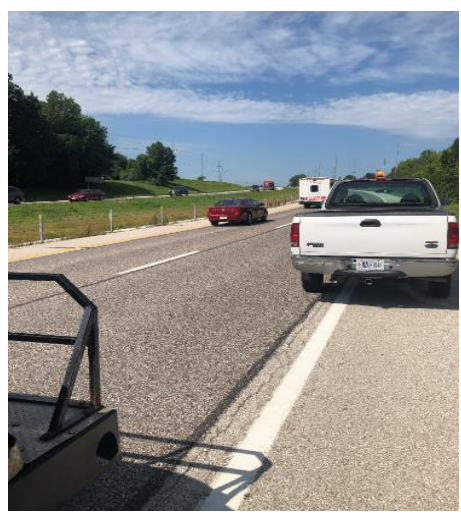

MO94

US54_7

Figure 2-39 Pavement condition for ten field sections from high deterioration rate to low deterioration rate.

Table 2-13. Performance measures before running the performance tests at laboratory

\begin{tabular}{cccccc}
\hline Section \# & $\begin{array}{c}\text { Constr. } \\
\text { Year }\end{array}$ & $\begin{array}{c}\text { IRI } \\
(\mathbf{i n} / \mathbf{m i})\end{array}$ & $\begin{array}{c}\text { PASER } \\
\text { Rating }\end{array}$ & $\begin{array}{c}\text { ARAN } \\
\text { Rut } \\
\text { Depth } \\
(\mathbf{m m})\end{array}$ & Visual Observations \\
\hline MO52_1 & 2010 & 91 & 4.0 & 7.1 & Mainly joint reflective cracking \\
US 54_8 & 2006 & 64 & 5.5 & 2.0 & Block cracking developing \\
US50_1 & 2011 & 61 & 6.5 & 2.2 & Very little distress \\
US63_2 & 2008 & 78 & 4.5 & 1.5 & Dense block \& thermal cracking \\
US54_7 & 2003 & 53 & 7.5 & 3.0 & Very little distress after 14 years \\
MO 151 & 2010 & & 6.4 & 4.7 & Dense block \& thermal cracking \\
US 36 E & 2011 & & 5 & 2.5 & Dense block \& thermal cracking \\
US 54 E & 2010 & & 5 & 2.5 & Block cracking developing \\
MO 94 & 2005 & & 7.8 & 0.9 & Very little distress after 14 years \\
MO 6 W & 2015 & & 8.7 & 2.5 & Very little distress \\
US 61 N & 2013 & & 8 & 2.5 & Very little distress \\
\hline
\end{tabular}

\subsubsection{Relation of Field Performance to Recycling Levels and Performance}




\section{Tests}

Table 2-14 summarizes the 11 field sections with respect to overlay date, PASER rate, Deterioration rate, ARAN rut depth, DC(T) FE, FI and HWWT test results. A deterioration rate was computed based on the average decline in PASER rating over the service life of the overlay. Clearly, the US 54_7 section was the best performer, with a very low deterioration rate (still very little cracking after 15 years in service). This section contained no recycling, and also possessed a relatively high binder content for a 12.5 NMAS mix (6.2\%). The worst 2 performers were MO52_1 and US63_2, which had the highest recycling rates (34 and 30\% ABR, respectively), but without any bumping/softening of the virgin binder grade.

Table 2-14. Field section details vs. average deterioration rate

\begin{tabular}{ccccccccl}
\hline & Route & $\begin{array}{c}\text { Overlay } \\
\text { Year }\end{array}$ & $\begin{array}{c}\text { PASER } \\
(\mathbf{2 0 1 9})\end{array}$ & $\begin{array}{c}\text { Det } \\
\text { rate* }\end{array}$ & $\begin{array}{c}\text { DC(T) } \\
\text { FE }\end{array}$ & $\begin{array}{c}\text { FI } \\
\text { (SCB) }\end{array}$ & $\begin{array}{c}\text { HWTT Rut } \\
(\mathbf{m m}) \\
\text { @ 10,000 }\end{array}$ & $\begin{array}{l}\text { ARAN } \\
\text { Rut } \\
(\mathbf{m m})\end{array}$ \\
\hline \multirow{6}{*}{ Phase I } & MO52_1 & 2010 & 4 & 0.83 & 321.2 & 0.6 & 2.8 & 7.1 \\
& US54_8 & 2006 & 5.5 & 0.45 & 340.2 & 0.1 & 1.9 & 2 \\
& US50_1 & 2011 & 6.5 & 0.35 & 321.5 & 1.4 & 2.1 & 2.2 \\
& US63_2 & 2008 & 4.5 & 0.63 & 272.4 & 0.4 & 1.7 & 1.5 \\
& US54_7 & 2003 & 7.5 & 0.14 & 459.3 & 1.8 & 3 & 3 \\
\hline \multirow{3}{*}{ Phase II } & UO151 & 2014 & 4 & 0.90 & 179.5 & 0.3 & 17.5 & 4.7 \\
& US36 E & 2011 & 5 & 0.50 & 226 & 0.2 & 1.6 & 2.5 \\
& MO94 E & 2010 & 5 & 0.50 & 229 & 0.6 & 2.3 & 2.5 \\
& MO6 & 2005 & 7.8 & 0.16 & 348.7 & 0.8 & 2.8 & 0.9 \\
& US61 & 2016 & 8.6 & 0.43 & 262 & 5.4 & 3.9 & 2.5 \\
\hline
\end{tabular}

*Average deterioration rate in terms of change in PASER rating per year. 


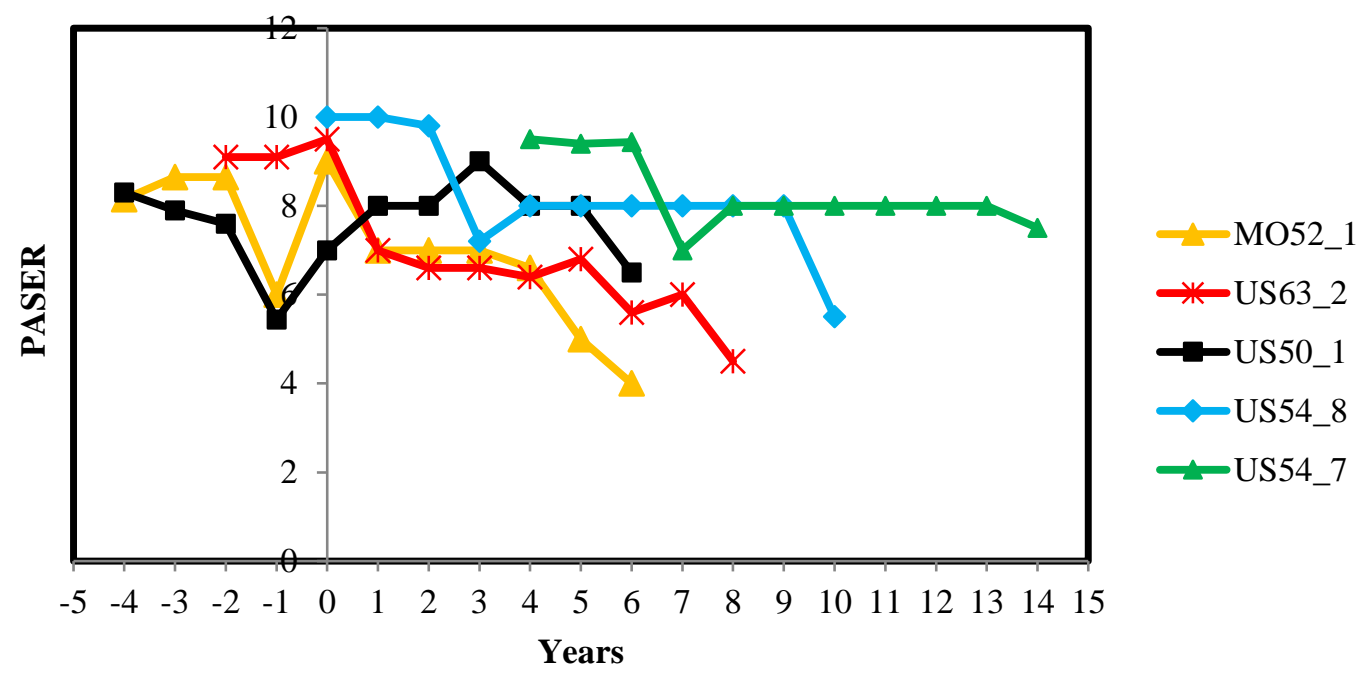

a)

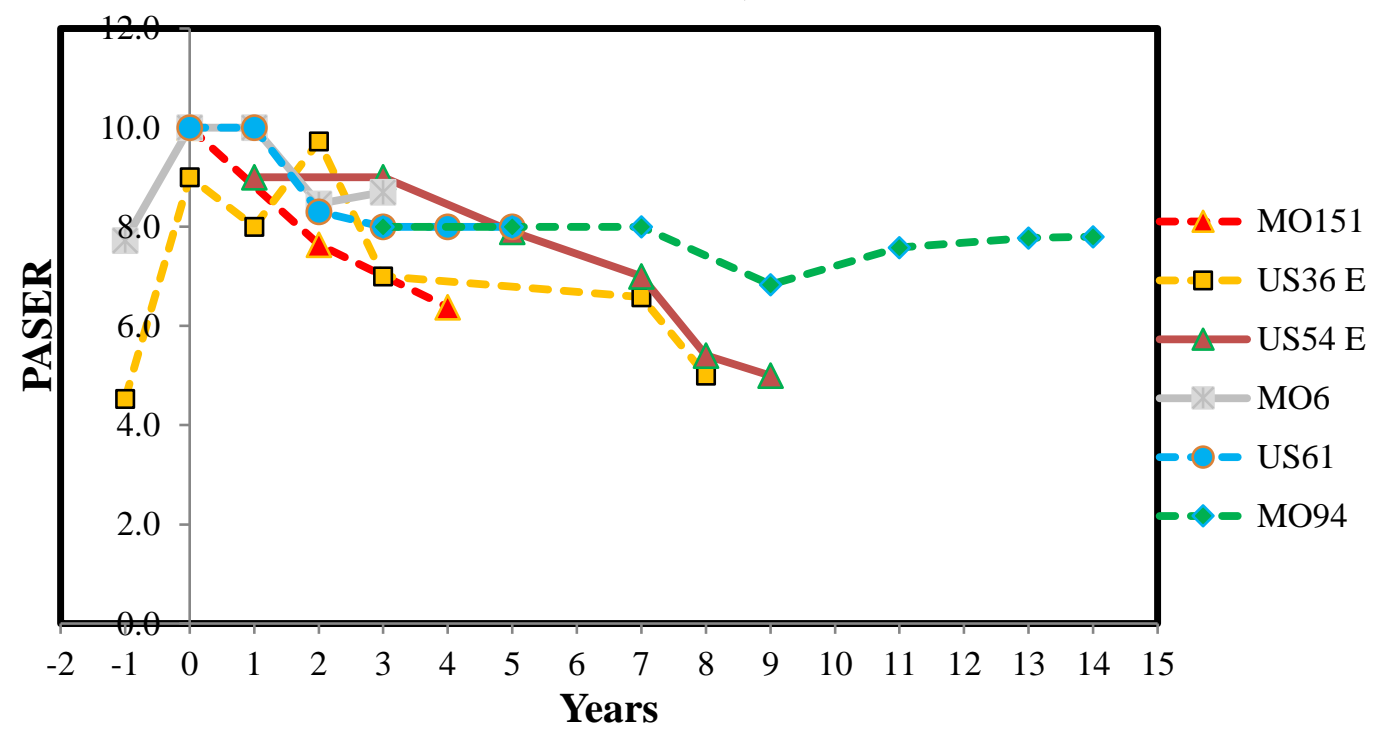

b)

Figure 2-40 PASER rating vs. years in service for a) phase I and b) phase II 


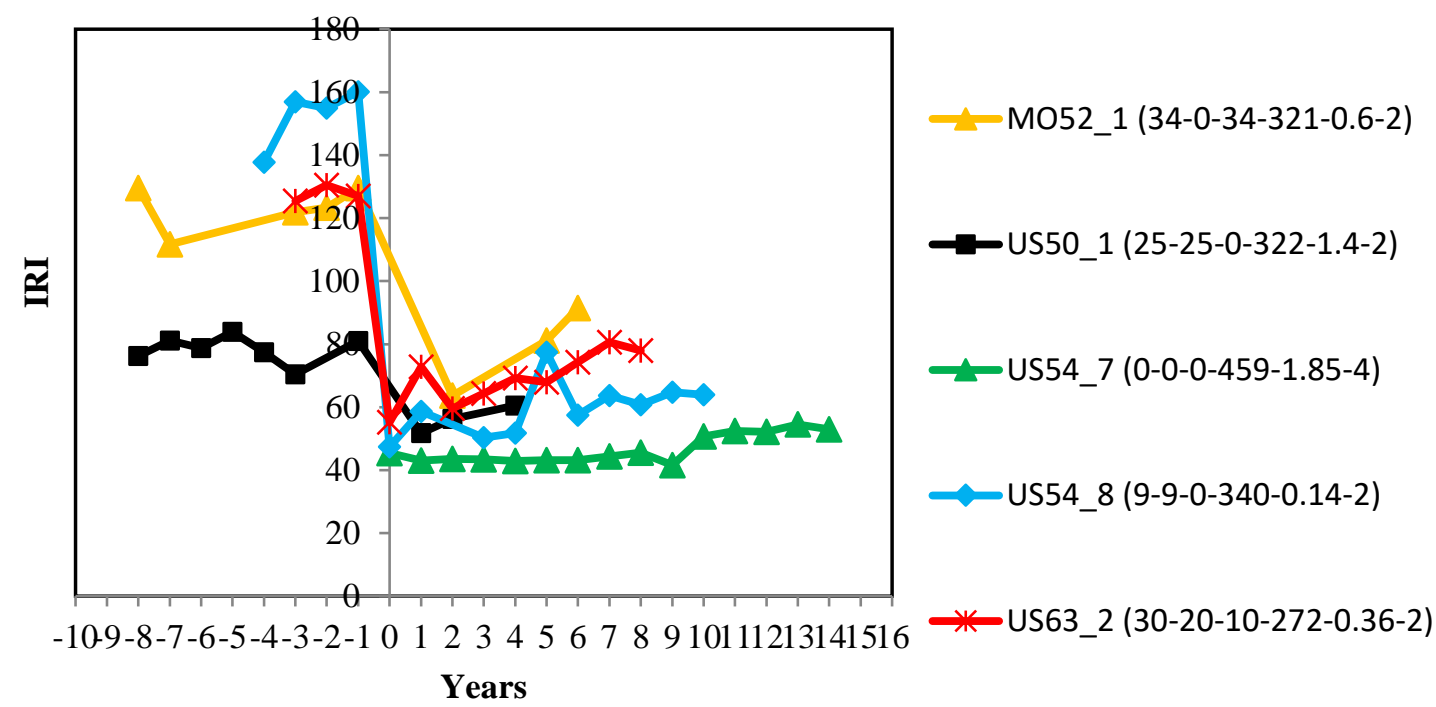

Figure 2-41 IRI vs. years in service

The time of construction was taken as year zero for each section, and thus the condition of the pre-existing pavement is represented by 'negative' year labels on the plots. Although fluctuations in the PASER and IRI data create some ambiguity, the overall deterioration rate and overlay performance can be reasonably assessed. These plots further demonstrate the relatively good performance of US54_7 and MO 94, and relatively poor performance of MO52_1, US63_2 and MO 151. Identical rankings in terms of IRI and PASER deterioration rates are noted.

Figure 2-42 provides a summary of field performance vs. lab cracking performance tests. The values of DC(T) FE and FI for these 11 sections were plotted versus deterioration rate. According the calculated $\mathrm{R}^{2}$, the $\mathrm{DC}(\mathrm{T}) \mathrm{FE}$ value are more correlated with the field data than FI ones. As seen in the figure in the both figures, one point can be considered as the outlier. Therefore, the outlier point was removed from both figures and the data replotted again. The $\mathrm{R}^{2}$ for the $\mathrm{DC}(\mathrm{T}) \mathrm{FE}$ curve increased to 0.7 while the FI figure only delivered 0.23 as the $\mathrm{R}^{2}$ (Figure 2-42). 

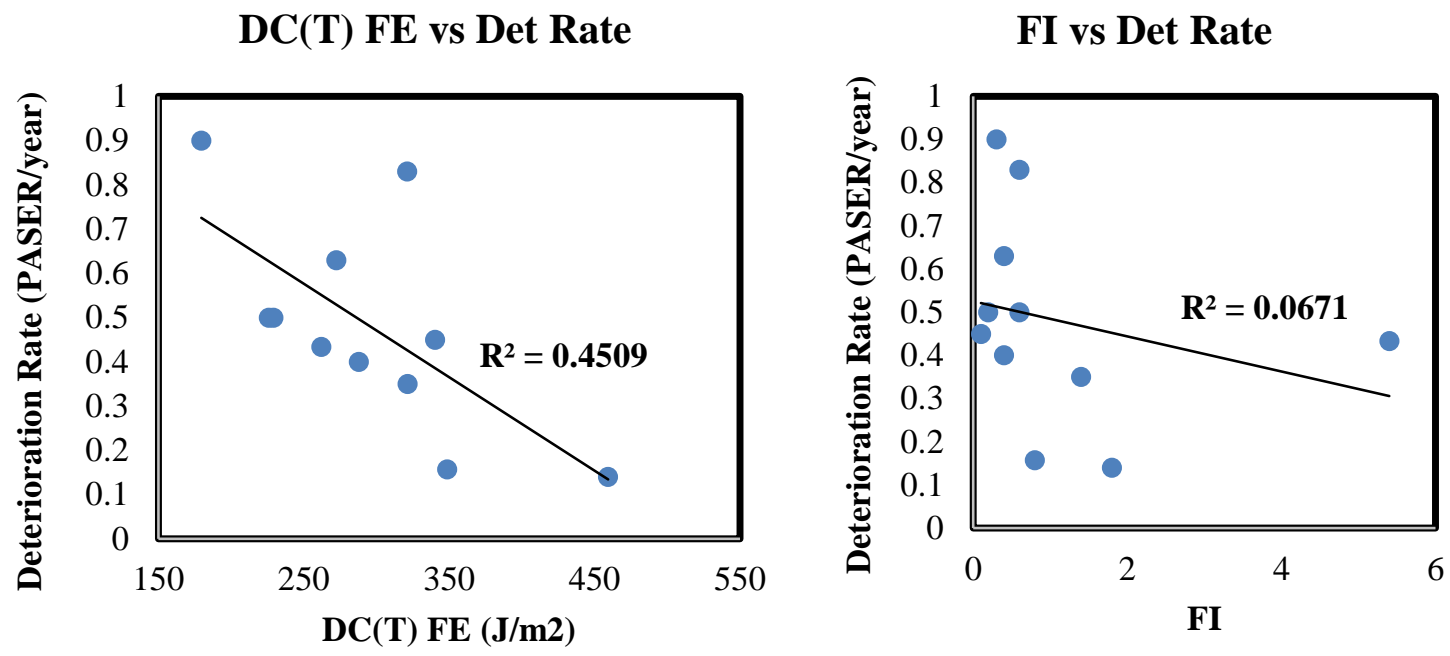

Figure 2-42 Correlation of DC(T) FE, FI with deterioration rate from the field

DC(T) FE vs Det Rate

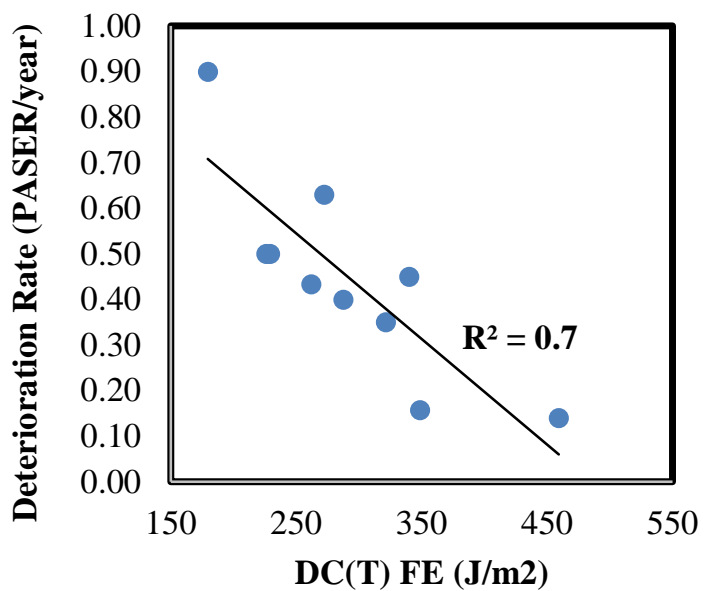

FI vs Det Rate

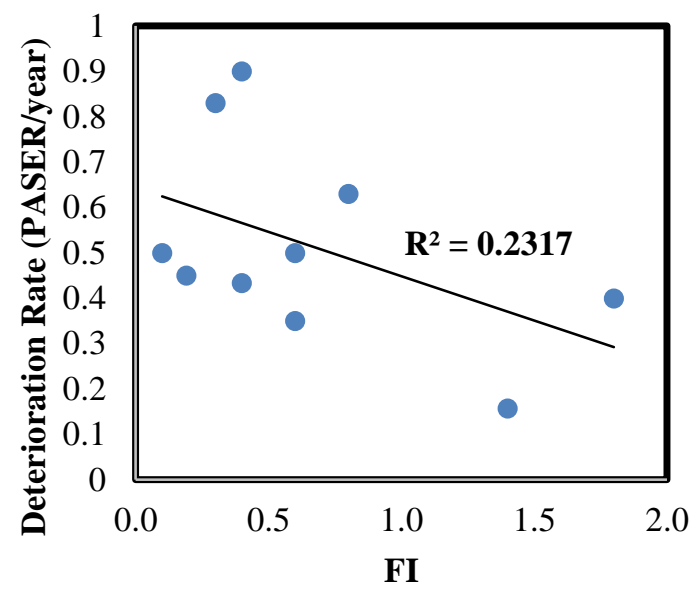

Figure 2-43 Correlation of DC(T) FE, FI with deterioration rate from the field (excluding the outlier)

Table 2-15 lists the Coefficient of Variation (COV) for the two performance tests for the field sections tested. The average DC(T) COV for the field sections was $19.5 \%$, slightly higher than the overall COV for the 18 mixtures investigated (Table 2-5), but still within the recommended maximum COV range of $20 \%$. On the other hand, the I-FIT test had an 
average COV of $52.2 \%$ for the field section evaluations. Higher COV values make it more difficult to draw inferences regarding statistical differences between means of sample populations to be compared (for instance, between testing parties), and create more uncertainly when comparing mean values against specification requirements.

Table 2-15. Test averages and coefficient of variability for field sections

\begin{tabular}{ccccc}
\hline Section \# & $\begin{array}{c}\text { DC(T) FE } \\
(\mathbf{J} / \mathbf{m} 2)\end{array}$ & $\mathbf{C O V}(\boldsymbol{\%})(\mathbf{D C}(\mathbf{T}))$ & FI & COV(\%) (FI) \\
\hline MO 52_1 & 321 & 3.8 & 0.6 & 51.4 \\
US 54_8 & 340 & 25.5 & 0.14 & 80.7 \\
US 50_1 & 322 & 27.6 & 1.4 & 51.6 \\
US 63_2 & 272 & 13.7 & 0.36 & 35.4 \\
US 54_7 & 459 & 11.4 & 1.85 & 37.6 \\
MO151 & 179.5 & 44 & 0.3 & 80.2 \\
US36 E & 226 & 7.3 & 0.2 & 21.1 \\
US54 E & 229 & 32 & 0.6 & 33.8 \\
MO94 & 348.7 & 13.8 & 0.8 & 91.9 \\
MO6 & 262.3 & 9.4 & 5.4 & 61.5 \\
US61 & 287.7 & 25.8 & 0.4 & 29.2 \\
Average & N/A & 19.5 & N/A & 52.2 \\
\hline
\end{tabular}

Clearly, more tests sections are needed to further evaluate performance tests and specification limits needed to establish a balanced mix design approach for recycled materials. That notwithstanding, the results obtained herein show the strong possibility of arriving at a specification in the near future that will allow contractor innovation in designing modern, heterogeneous recycled mixtures.

\subsubsection{Summary}

A very good performing field section was identified and tested in this study (US54_7 and MO94). This sections has performed very well after 15 years in service, and still has low rutting $(3 \mathrm{~mm})$ and a high PASER rating (7/10). The correlation of deterioration rate 
of the sections with FI and $\mathrm{DC}(\mathrm{T})$ values, confirmed that, $\mathrm{DC}(\mathrm{T})$ test can be a more reliable test than SCB (IFIT) to evaluate the cracking performance. Also DC(T) test results were more repeatable $(\mathrm{COV} \%=19.5)$ than FI $(\mathrm{COV}=52 \%)$ and it can proves the higher reliability of the $\mathrm{DC}(\mathrm{T})$ test compared to SCB IFIT test. Another finding of this part of the research is that the $\mathrm{SCB}$ (IFIT) test is highly depend on aging of the section. The recommended values and thresholds should be adjusted based on the section old. 


\subsection{INVESTIGATING ASPHALT PAVEMENT CRACKING PERFORMANCE USING DIFFERENT CRACKING INDICES}

\subsubsection{Introduction}

Service-life improvement of asphalt pavements requires the availability of welldesigned standardized testing procedures. In the United States, Superpave volumetric mix design and balanced mix design methods have continued to evolve. While the former cannot adequately predict the field performance of asphalt mixtures, the latter provides the opportunity to more closely control mixture field performance. The advantage of the balanced mix design approach is its ability to predict asphalt mix performance characteristics such as fatigue cracking, thermal cracking, rutting, and moisture damage [27], [29], [31], [34]-[45].

Traffic-induced cracking (often referred to as fatigue cracking), which is commonly controlled through testing in the intermediate temperature range, is one of the principal distresses in asphalt pavements [46]-[49]. Fatigue cracking performance is usually evaluated through one of the following two approaches: the first involves the application of cyclic loading on asphaltic specimens and measuring the number of cycles to a defined failure threshold. Several tests have been developed based on this approach, including Push-Pull Fatigue, Beam Fatigue, and Texas Overlay tests [50]. The second approach introduces fracture-type testing and the development of simple cracking indices as indicators of fatigue cracking resistance. Fracture energy $\left(G_{\mathrm{f}}\right)$ is a material property and is simply defined as the energy required to generate a unit fracture surface area in the material [51]. The Illinois Semi-Circular Bending (IL-SCB) test, Louisiana SCB, and IDEAL 
Cracking Test Index (CT) tests have been proposed, in some cases involving empirical combinations of parameters derived from a load-displacement type curve [6], [52], [53].

Traditional fatigue and fracture tests are generally quite complex and computationally expensive [54], [55]. In some cases, complex specimen geometries are involved [56], and some systems require complex modeling schemes [35], [44], [45], [57]. A recent trend, akin to turning the clock back towards simpler tests with very simplified analysis schemes, has gained popularity in the United States. Various simplified indices such as the IDEAL Cracking Test Index (CT), the Flexibility Index (FI), and the Cracking Resistance Index (CRI), have been proposed in recent years. These indices are derived from simplified, or pseudo-fracture tests conducted in the intermediate temperature range (room temperature, or $25^{\circ} \mathrm{C}$ ), as described elsewhere [6], [50], [52], [58].

The accuracy of these simplified indices in predicting asphalt cracking performance, and the repeatability of some of the methods have been vigorously debated in recent years [27], [50], [58]. It appears that there is room to improve the repeatability of these modern, simple cracking tests and to improve their links to performance, while maintaining their practitioner-friendly, simplified attributes in regards to testing and analysis.

This part of research begins by briefly reviewing two of the popular simple cracking test indices (FI and CRI), followed by introducing a newly-developed index based on the IL-SCB test, which has been termed herein as the Balanced Cracking Index (BCI). The cracking performance of 52 asphalt mixtures is compared with the existing and proposed cracking indices. The sensitivity of the various cracking test parameters investigated to 
various mix ingredients and modifiers is investigated. A detailed statistical analysis is then presented. Correlation to field cracking performance is then conducted for ten recent field studies as a means to evaluate the efficacy of the various cracking tests and associated cracking indices.

\subsubsection{Tests and Methods}

The IL-SCB test at $25{ }^{\circ} \mathrm{C}$ was performed to evaluate the cracking performance of asphalt mixtures. For the IL-SCB, several cracking indices were computed, including a newly-proposed BCI parameter,

\subsubsection{SCB Flexibility Index Test}

The Illinois Semi-Circular Bending (IL-SCB) test is based on 3-pt bending of a semi-circular shaped specimen to evaluate cracking resistance of an asphalt [59]-[61]. In 2016, Ozer et al. described a practitioner-friendly SCB test as a means to control pavement cracking in a general sense [4], [61]. The operating temperature for the test was set at room temperature or $25^{\circ} \mathrm{C}$. However, since fracture energy at $25^{\circ} \mathrm{C}$ did not appear to capture mixture cracking properties, a post-peak load slope parameter was additionally considered, which led to a new crack test parameter called the Flexibility Index (FI). The FI is an empirical index defined as the total fracture energy divided by the absolute value of the slope at the inflection point of the portion of the load-displacement curve accruing after the peak load, as shown in Figure 2-44, and by Equation 2-6 and 2-7. Please note that the displacement is measured in terms of Load Line Displacement (LLD) in IL-SCB test. 


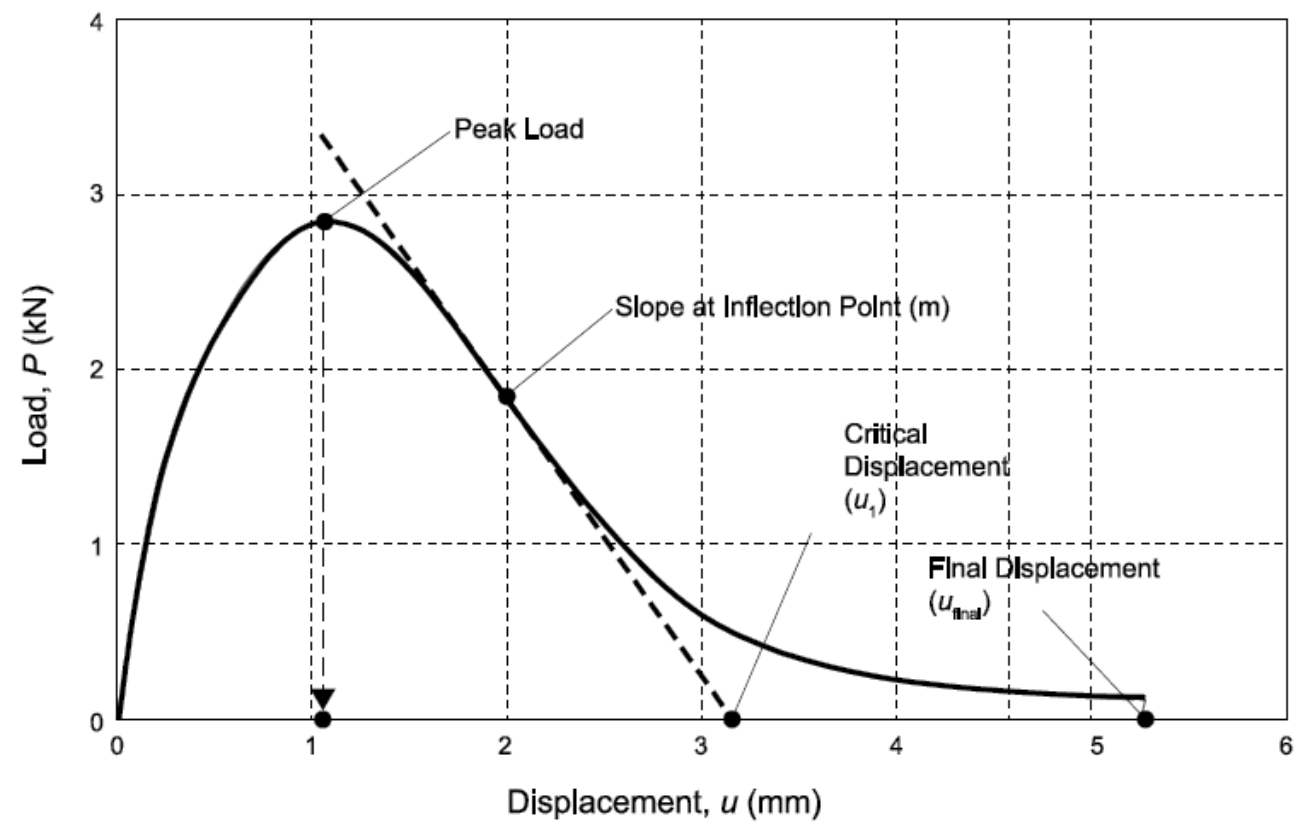

Figure 2-44 Recorded load (P) versus LLD (u) curve (AASHTO TP124-16).

$$
G_{f}=\frac{W_{f}}{\text { Area lig }_{\text {lig }}} \times 10^{6}
$$

Where,

$$
\begin{aligned}
& G f=\text { fracture energy }\left(\mathrm{J} / \mathrm{m}^{2}\right) \\
& W f=\text { work of fracture }(\mathrm{J}) \\
& P=\text { load }(\mathrm{kN}) \\
& u=\text { load line displacement }(\mathrm{mm}) \\
& \text { Arealig = ligament area }\left(\mathrm{mm}^{2}\right) \\
& \text { Arealig = ligament length } \times t \text { and } \\
& t=\text { specimen thickness }(\mathrm{mm}) \\
& F I=\frac{G_{f}}{|m|}(0.01)
\end{aligned}
$$

Where,

$$
m=\text { absolute value slope of the curve at inflation point }
$$


Although the FI was shown to distinctively rank asphalt mixtures by Ozer et al. [61], further research with wider datasets revealed that the index is not without drawbacks. First, the FI test has been shown to have issues in characterizing brittle mixtures, especially aged field cores. In these brittle materials, the post-peak load-LLD curve is affected by the release of significant stored elastic energy, a fast moving crack, and sometimes snap-back fracture behavior [56]. This phenomenon has been addressed using more sophisticated, closed-loop control systems [56], [62], allowing the post-peak behavior of brittle materials to be more accurately characterized. However, in the simple tests investigated herein, simple load-line displacement machine control is used, which tends to lower device cost and operational complexity. The erratic curve that results will have very few data points (perhaps only one or two), and is therefore poorly characterized, making it difficult to determine an " $m "$ value and subsequently the FI index [50], [58] (Figure 2-45).In general, the calculation of the post-peak slope value in the calculation of the FI involves complex curve-fitting techniques, as described in Ozer et al. (2016). It is difficult to accurately d etermine the inflection point, which is needed to compute the post-peak slope parameter, at least from the standpoint of test standardization. For example, when irregular load-LLD curves are recorded, errors and discrepancies can arise by introducing ad-hoc fitting techniques in an attempt to smooth, or to extrapolate, load-LLD curves.

Finally, the FI parameter has been shown to have a high Coefficient of Variation $(\mathrm{COV})$, which brings into question whether the parameter has sufficient repeatability to enable reliable asphalt cracking performance evaluation [27], [40], [58], [63]. A wellknown statistical principle exists, which pertains to the use of replicate measurements to estimate the mean of a population with inherent material and measurement variability [64]. 
This principle states that the required number of testing replicates needed to arrive at a given level of reliability will vary with the square of the standard deviation of the test. Because COV is linearly related to standard deviation (it is simply the standard deviation divided by the sample mean), then a test method having 1.5 times the COV of another test would require 2.25 times more testing replicates to arrive at a similar level of parameter estimation reliability. Recently Kaseer et al. introduced an alternative cracking index parameter from I-FIT test data, aimed at reducing COV, called the Cracking Resistance Index (CRI). Details are provided in the following section.

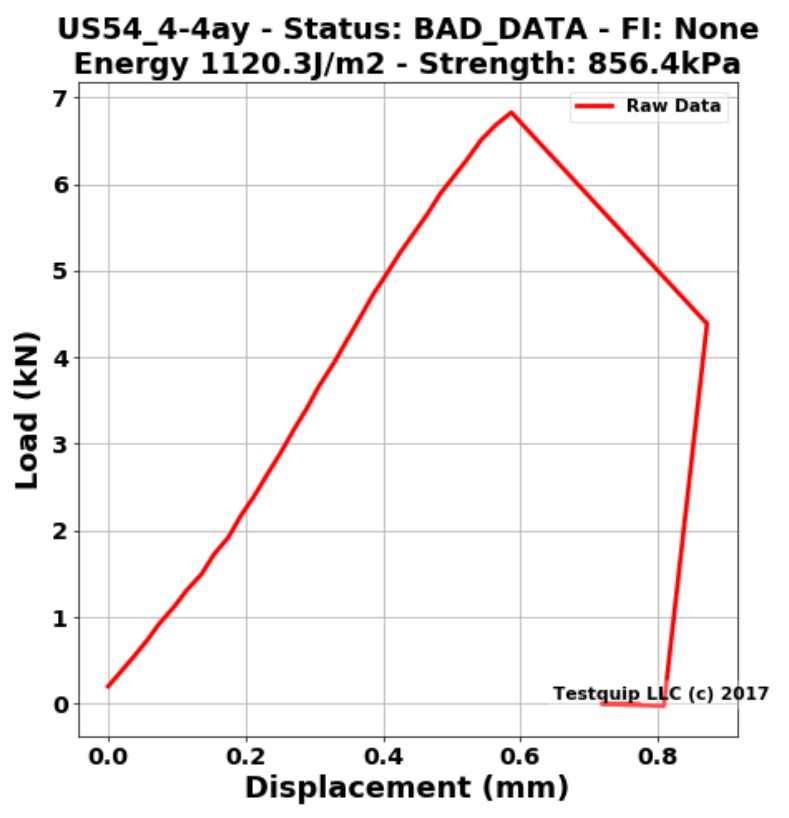

Figure 2-45 The load-displacement curve of SCB (IFIT) test for a brittle mixture.

\subsubsection{SCB Cracking Resistance Index (CRI) Test}

The CRI was proposed as an alternative to the FI parameter from IL-SCB test. The advantages of the CRI is its simpler analysis and higher repeatability than the FI [58]. It is expressed as the ratio of total fracture energy and the peak load, as shown in Equation 2-8, 


$$
C R I=\frac{G_{f}}{P_{\max }}
$$

where,

$G_{f}$ is the total fracture energy $\left(\mathrm{J} / \mathrm{m}^{2}\right)$

$P_{\text {max }}$ is the peak load $(\mathrm{kN})$

As suggested by researchers during the development of the FI, the fracture energy for tests conducted at an intermediate testing temperature may not be able to distinguish between mixtures. Figure 2-46 shows the fracture energy of a virgin and a high-recycled mix to illustrate this point. However, the CRI of the virgin mixture was found to be $50 \%$ higher than that of a recycled mixture (possessing the larger $\mathrm{P}_{\max }$ ), as shown in Figure 2-46. Based on the simple ratio used in this index, the CRI accounts for both fracture energy and mixture strength in the ranking of mixtures. However, while fracture energy boosts the CRI score, peak load decreases the score. This is because higher peak load indirectly relates to a high post-peak softening curve slope, as discussed in the paragraphs that follow. 


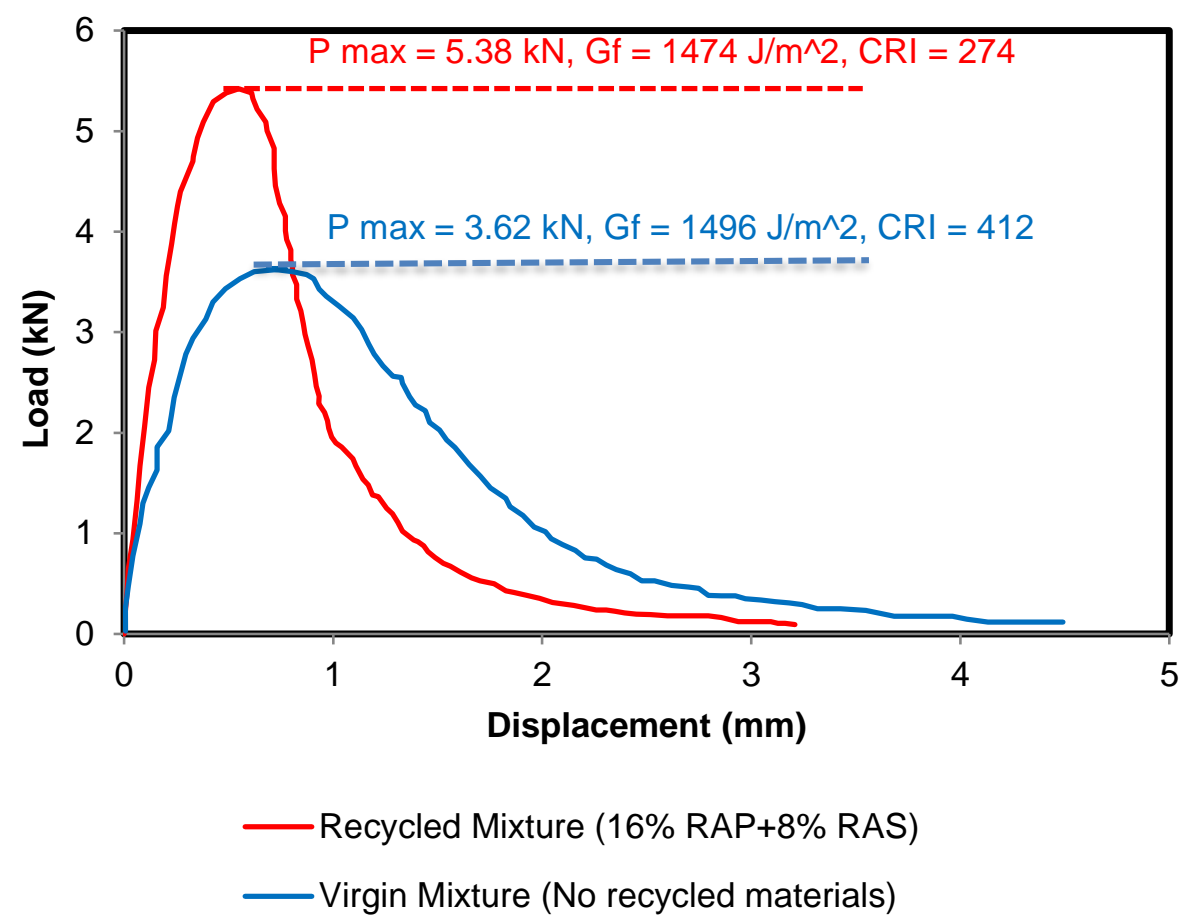

Figure 2-46 The load-displacement curve of two distinct asphalt mixtures [58].

The CRI, as compared to the FI, improves on repeatability, provides a simpler analysis approach for analyzing load-LLD data, and is not as punitive in assessing stiff mixtures [58]. It should be noted that while the FI test directly uses post-peak slope as one of the input parameters, the CRI is indirectly affected by slope. For instance, if both the fracture energy $\left(\mathrm{G}_{\mathrm{f}}\right)$ and peak load $(\mathrm{P})$ are high, the fracture energy benefit is reduced in the resulting CRI parameter. If one considers an over-simplified model of a Load-CMOD curve as being roughly triangular in shape, having both high $\mathrm{G}_{\mathrm{f}}$ and high $\mathrm{P}$ creates a tall, triangular Load-CMOD curve with a steep post-peak slope. These mixes are subsequently characterized as being brittle; however, the effect of slope is not punitive as directly including it in the calculation (as is the case with the FI index). 
It can be argued that the CRI has its own distinct limitations. First, the correlation between the CRI and field performance data has not been adequately documented in the literature. In addition, the CRI has the potential to confound the dependence of fracture energy on $\mathrm{P}_{\max }$, which could lead to misleading conclusions for some polymer-modified mixtures with both high fracture energies and high peak loads. Stated simply, with an increase in $P_{\max }$, the fracture energy increases, and consequently, both the numerator and the denominator in Equation 2-8 increase. Figure 2-47 illustrates the inherent difficulty in the CRI and FI indices in terms of their ability to properly characterize the inherent cracking resistance shown to exist in polymer-modified mixtures. These mixes are characterized as being relatively poor in cracking resistance (brittle) despite their extraordinary strength and high fracture energy.

To delve deeper into this phenomenon, consider the mixture labeled as 1823 , which has an SBS-polymer-modified binder (PG 64-34), a 4.75 mm NMAS aggregate structure, a 38.3\% ABR recycling content (24.1\% ABR from RAS and 14.2\% ABR from RAP) and a total binder content of $7.94 \%$. Then, compare mixture 1823 to mixture 1834 , which involves an unmodified binder (PG 58-28), a 9.5 mm NMAS, 20\% ABR from RAP), and a total binder content of $6.0 \%$. 


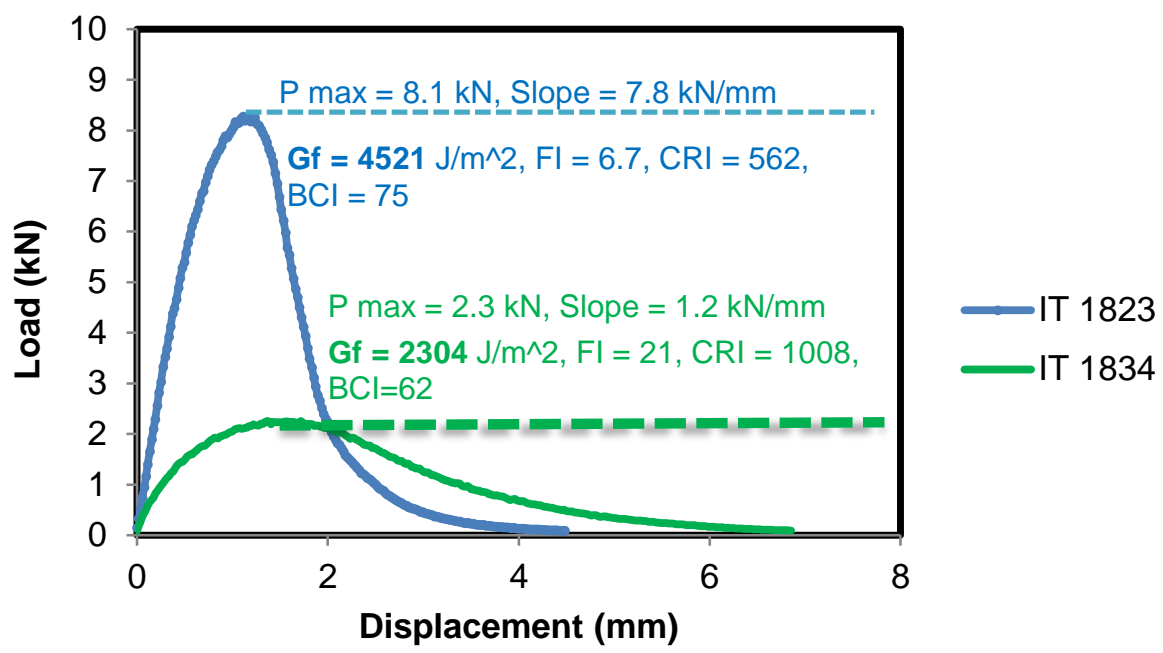

Figure 2-47 SCB load-displacement curve for two modified mixtures.

Comparing fracture energy, FI, and CRI of the mixes, mix 1834 has half the fracture energy, but three times the FI, and twice the CRI as compared to mix 1823. This is due to the fact that mix 1823 has a higher post-peak slope, resulting in a lower FI, and higher peak load, resulting in a lower CRI, when compared to mix 1834. While the high values of FI and CRI for mix 1834 suggest high cracking resistance, the small peak load brings into question the tensile loading resistance of this mixture. Thus, while it appears that the CRI and FI parameters allow for clear ranking of brittle mixtures when fracture energies are almost equal, these indices create ambiguity in cases where fracture energy values differ significantly between the two mixes being compared. The FI and CRI indices, in essence, penalize mixes with high peak load, which often includes mixes with modifiers/additives. However, often modified mixtures have shown improvements in the field cracking performance of asphalt mixtures [65]-[67] [68]-[70]. Therefore, cracking indices that penalize mixes for high tensile load carrying capacity at intermediate temperatures may 
not produce desirable results for mixes that are both strong (high peak load) and tough (high fracture energy, high strain tolerance).

While the CRI parameter appears to be a step forward in characterizing brittle mixes as compared to the FI, there is still a need to identify a simple-yet-robust post-peak parameter in order to distinguish between a wide array of unmodified and modified mixtures, and able to characterize more brittle, field-aged asphalt mixtures (which are necessary to calibrate tests to long-term field cracking performance).

To this end, an alternative SCB cracking index determined from the load-LLD curve, herein referred to as the Balanced Cracking Index (BCI), is introduced in this study. The following sections describe the development of the BCI, demonstrate its potential advantages in ranking asphalt mixtures, and compares it to the FI and CRI parameters, and to field performance

\subsubsection{SCB Balanced Cracking Index (BCI) Test}

Taking into account mixture fracture energy in conjunction with a post-peak softening curve parameter from the IL-SCB test, a new index, termed the Balanced Cracking Index (BCI), is proposed in this study. The BCI was defined as the product of: a) fracture energy of the mix computed as described in previous sections $\left(\mathrm{G}_{\mathrm{f}}\right)$; $\left.\mathrm{b}\right)$ the load-line displacement corresponding to the time when $75 \%$ of the peak load in the post-peak region $\left(\mathrm{PP}_{75}\right)$ is reached (L75), and; c) a scaling factor set to 0.01 (which is also used in the FI parameter). The computation of $\mathrm{BCI}$ is described in Equation 2-9: 
Balanced Cracking Index $(B C I)==0.01 \times G_{f} \times L_{75}$

where,

$$
\begin{aligned}
& G_{f}=\text { fracture energy }\left(\mathrm{J} / \mathrm{m}^{2}\right) \\
& L_{75}=\text { Displacement corresponding to } \mathrm{PP}_{75}(\mathrm{~mm})
\end{aligned}
$$

Figure 2-48 illustrates the procedure to calculate the BCI for a typical sample:

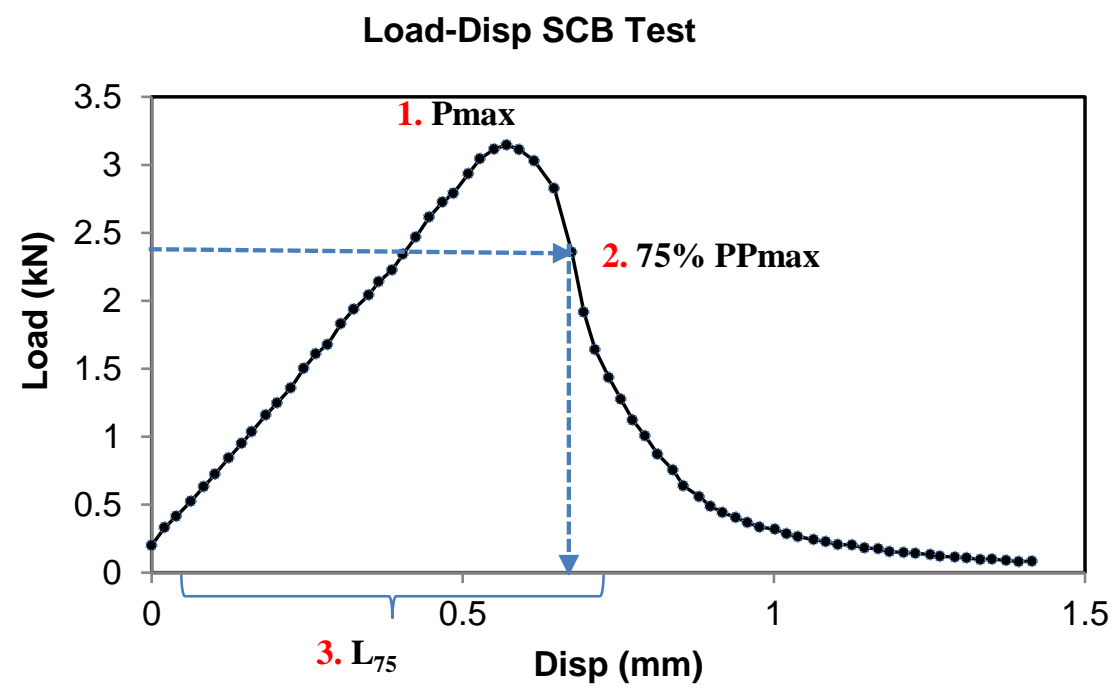

Figure 2-48 Illustration of the $\mathrm{PP}_{75}$ point and the corresponding $\mathrm{L}_{75}$ in SCB test, where PP75 $=75 \%$ peak load in post-peak region, and $L_{75}$ is the displacement at PP75.

The use of the LLD at $75 \%$ peak load in the post-peak region was first proposed in the IDEAL-CT test, reported by Zhou et al. where a $62 \mathrm{~mm}$ gyratory is tested in an Indirect Tension Test (IDT) setup [6]. The authors reasoned that, in most mixes, the inflection point of the post-peak softening curve occurs approximately when $75 \%$ of the peak load is reached. Unlike the FI slope parameter, and identical to the technique used in the IDEALCT test, determining the LLD at PP75 is a simple task that can be achieved without the 
need for sophisticated data fitting techniques or software. The L75 parameter provides a measure of the mixtures ability to tolerate strain after the peak load is reached. According to [71], the transition from fracture process zone development to stable crack propagation occurs shortly after the peak load is reached in tensile fracture tests of asphalt concrete. Mixes with high L75 values possess considerable strain tolerance, in this case, the ability to withstand bending strain and, therefore to undergo high crack mouth opening displacement without allowing significant crack propagation. These mixes are therefore expected to withstand straining caused by thermal cycling and traffic loading, especially in overlays placed over cracked pavements.

The BCI (Equation (2-9)) is proportional to both the fracture energy of the mix and the L75 parameter, giving equal weight to both factors. As a preliminary observation, applying the $\mathrm{BCI}$ analysis to the mixtures used in Figure 2-47 shows that the $\mathrm{BCI}$ for mix 1823 is higher than that of the 1834 mix. The 1823 mixture carries a $\mathrm{BCI}$ of 75 , whereas the 1834 mixture possesses a BCI score of 62 . Given the fact that this mixture possessed exceptional fracture energy along with reasonable strain tolerance, the relative scoring provided by the BCI parameters appears to be reasonable in this case. Subsequent sections show a detailed analysis of the characterization of 52 asphalt mixtures in terms of FI, CRI, and BCI. Next, a rigorous sensitivity analysis of factors affecting each index is presented. Field cracking results from ten sections located in Missouri are then correlated with the existing and new cracking indices and compared. 


\subsubsection{Materials and Experiment}

A variety of testing specimens fabricated from different asphalt mixtures, including field cores (labeled herein as 'Field') placed between the years of 2003 to 2018, plantproduced/laboratory-compacted ('Plant') mixtures, and laboratory-produced laboratorycompacted ('Lab') mixtures were tested in the SCB at $25^{\circ} \mathrm{C}$ and characterized using the FI, CRI, and BCI indices. The test performed on four SCB samples for each mixture and none of the test result were removed for calculating the average index. The aggregate and binder were mixed together in the bucket at mixing temperature and kept in the oven for 2 hours at compaction temperature according to AASHTO R35. Mixing and compaction temperature were different based on the total binder performance grade. For plant mixtures, loose mixture kept in the oven till reaching the compaction temperature, and afterward, all samples were compacted to have $7 \pm 0.5 \%$ air void. Details about the mixtures investigated are provided in Table 2-16, with additional details provided elsewhere [27], [29], [40].

Table 2-16. Range of materials $\mathrm{s}^{\mathrm{a}}$ and other mixture characteristics represented in the 52 asphalt sections evaluated in this study

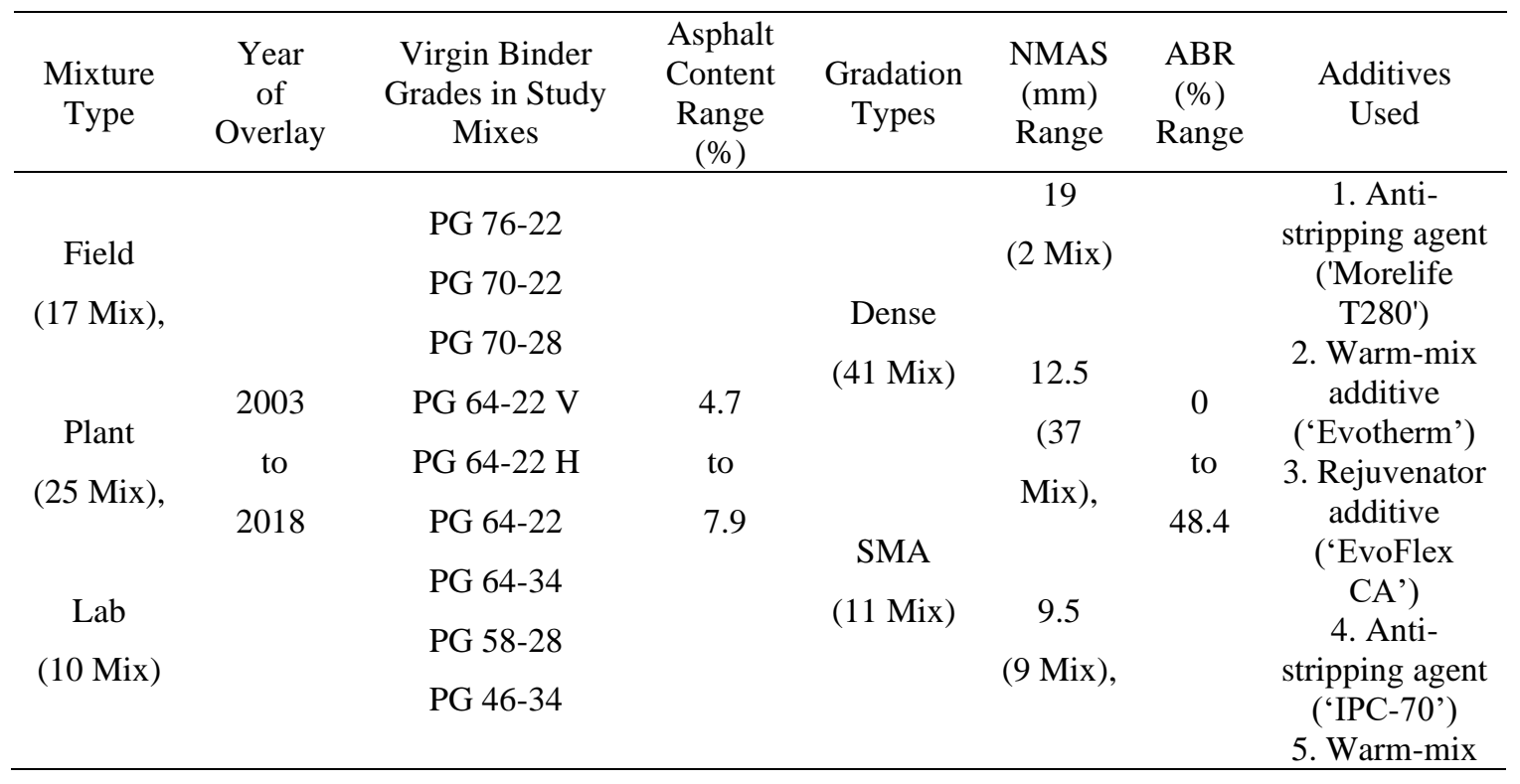




$\begin{array}{cc}4.75 & \text { additive ('PC } \\ \text { (4 Mix) } & 2106 \text { ') } \\ & \text { 6. Crumb } \\ & \text { rubber (dry } \\ & \text { process and } \\ & \text { terminal } \\ & \text { blend) } \\ & \text { 7. Steel Fiber }\end{array}$

${ }^{a}$ For convenience, property ranges are given. Details are provided in [27], [29], [40].

\subsubsection{Results and Discussion}

\subsubsection{Cracking Resistance Using SCB (FI), SCB (BCI) and SCB (CRI)}

All 52 mixtures were tested in the IL-SCB test at $25^{\circ} \mathrm{C}$. Then, the FI, CRI, and BCI values were computed from the load-LLD curves.. Figure 2-49 first ranks the mixtures according to decreasing FI (Figure 2-49a), followed by CRI and BCI for all mixes to demonstrate the degree of agreement and disagreement noted between the three indices.

$\mathrm{SCB}(\mathrm{FI})$

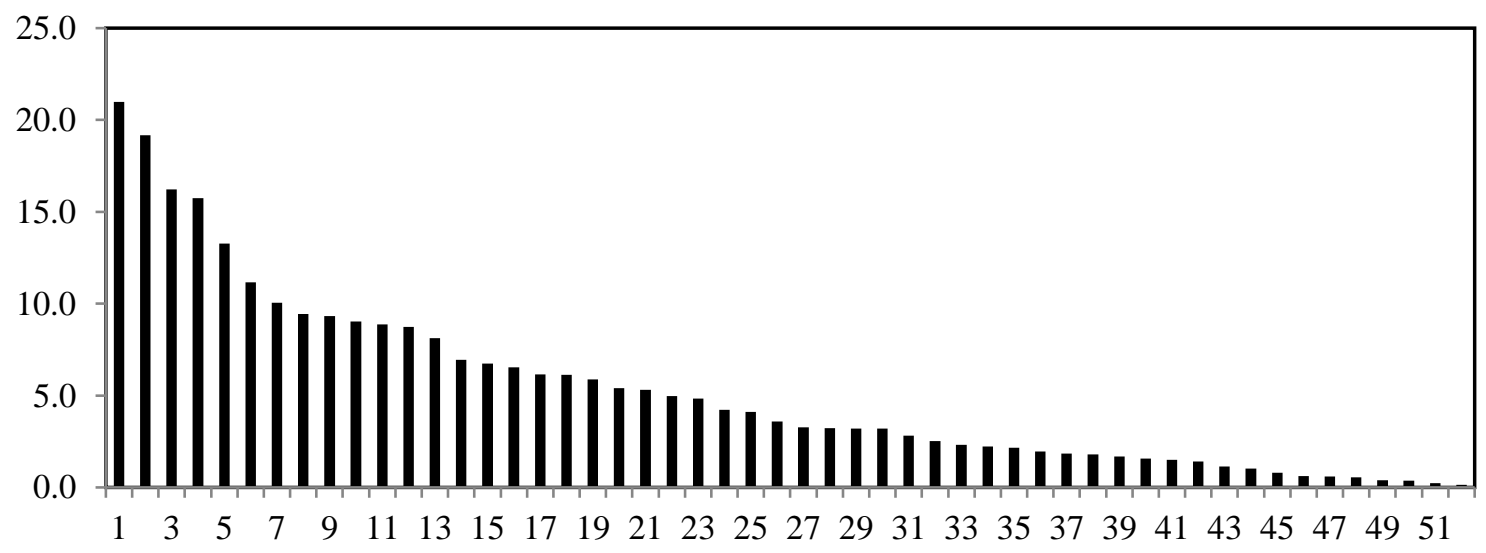

a) 
$\mathrm{SCB}(\mathrm{CRI})$

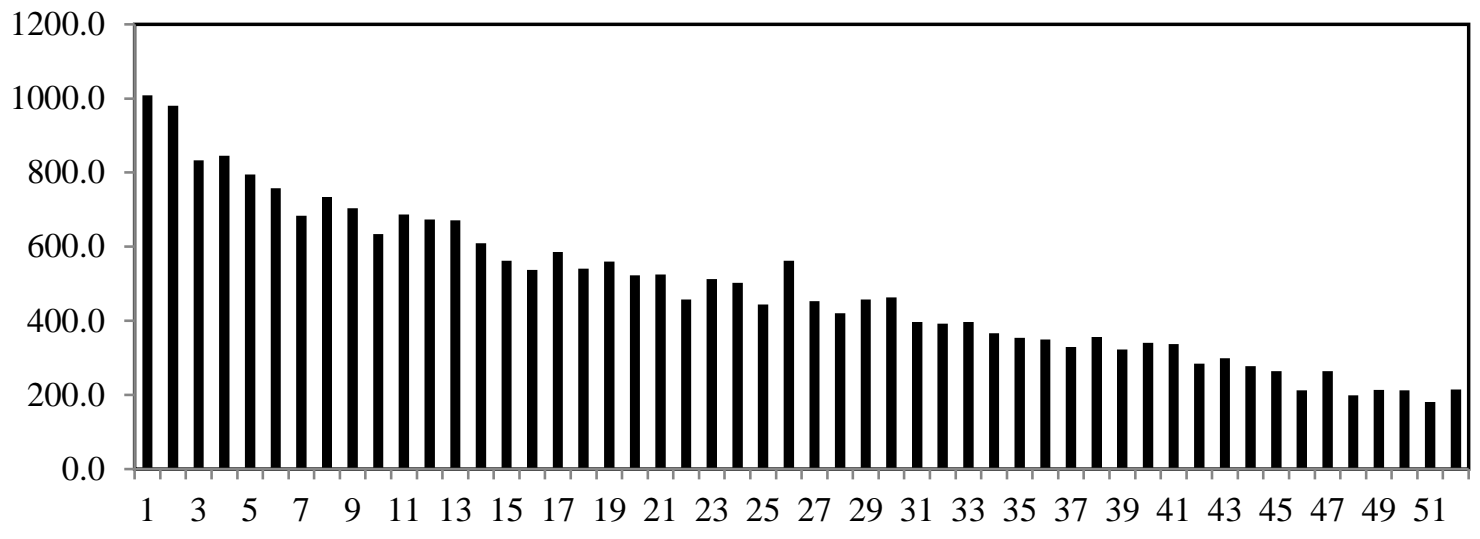

b)

SCB (BCI)

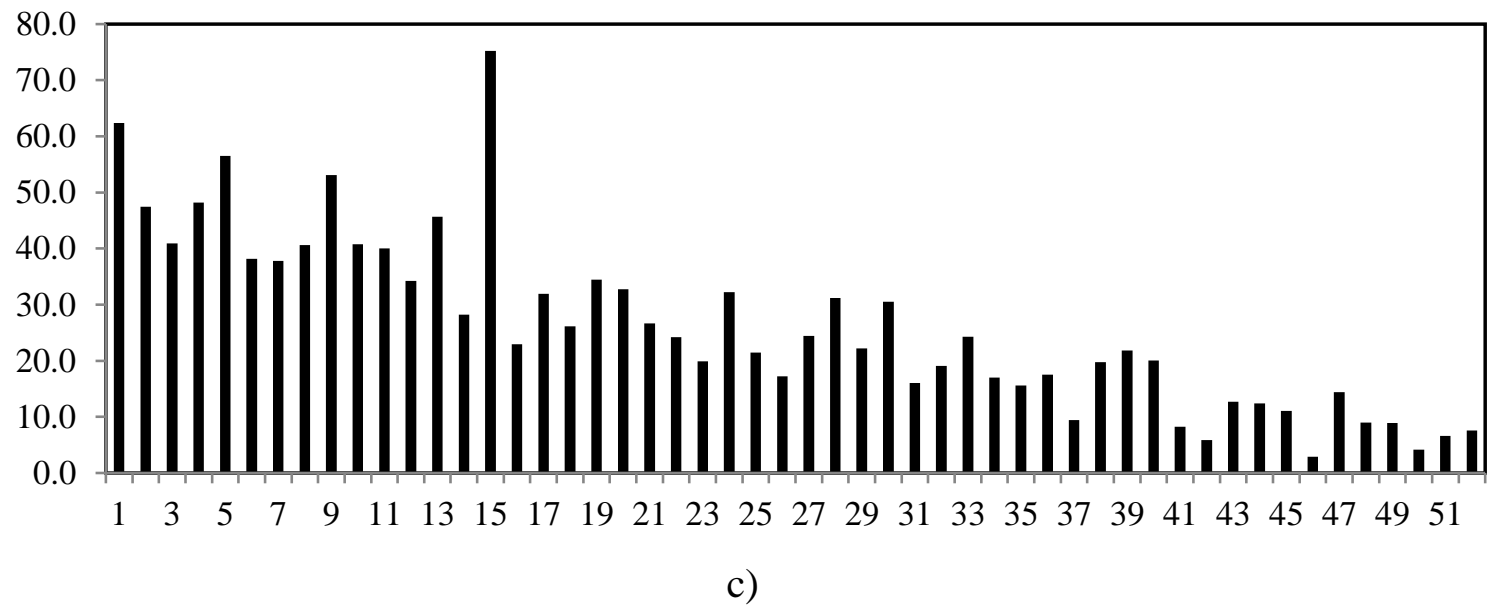

Figure 2-49 a) FI results for 52 sections, b) CRI results for 52 sections, c) BCI results for 52 sections.

It is observed from Figure 2-49 that except for a few mixtures, the three SCB indices follow a similar overall trend. However, the FI and CRI indices follow each other much more closely in comparison to the BCI index. This is not a surprising observation considering the similar formulation of FI and CRI, where both give modest credit for higher fracture energy while applying a fairly severe penalty for higher post-peak slope or peak load, respectively. The BCI index showed a reasonable departure from the trend, as it not 
only credits the mix for possessing a higher fracture energy, but also for possessing a higher post-peak displacement value (L75).

\subsubsection{SCB Indices Repeatability and their Correlation}

Figure 2-50 shows the Coefficient of Variability (COV) computed for the three investigated cracking indices. As shown in Figure 2-50b, the FI and CRI have the largest and the smallest COV values, $34.6 \%$, and $11.4 \%$, respectively (Figure 2-50b). This follows the results shown in Figure 2-50a where the slope, used in the calculation of FI, has high variability, and $\mathrm{P}_{\max }$, used in computing CRI, has low variability. The high variability introduced by the slope parameter has also been reported in the literature [27], [52], [63]. The new BCI parameter was found to have a COV of $17.5 \%$, somewhat higher than the CRI but considerably lower than the FI. Both parameters used in the computation of the BCI parameter possessed COV values of $10.3 \%$ or below, which is reasonable considering the inherent variability in fracture testing of granular, heterogeneous, quasi-brittle infrastructure materials.

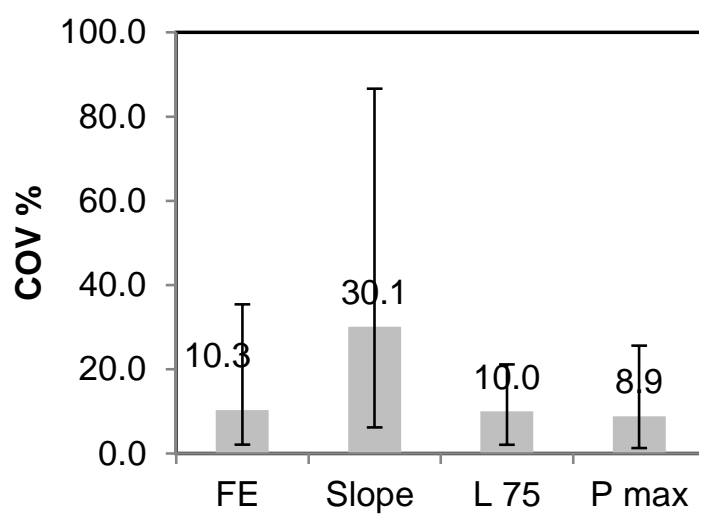

a)

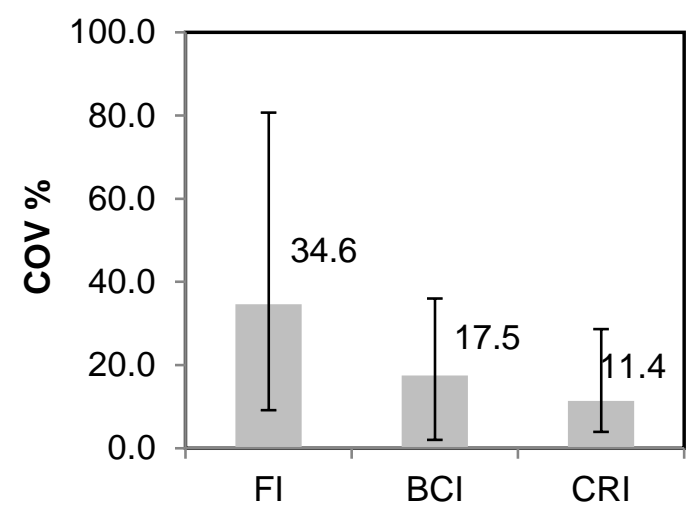

b)

Figure 2-50 a) COV of index parameters and b) COV of indices. 
In order to plot the indices on an equivalent scale, the indices values were normalized. The normalized values were calculated by dividing each value to the index's maximum value among 52 mixtures. Figure 2-51 shows the normalized values of the investigated SCB indices, indicating similar trends. However, because the FI term approaches zero in a number of cases, the very strong influence of the slope parameter in the denominator of the index is clear. To statistically evaluate correlations between cracking test indices and parameters from the IL-SCB test, regression analysis was performed as presented in Figure 2-52. Each plot provides the fitted model equation (either linear, quadratic, exponential) and the coefficient of determination $\left(\mathrm{R}^{2}\right)$ as a quantitative estimation of the correlation between the two values examined for any given plot.

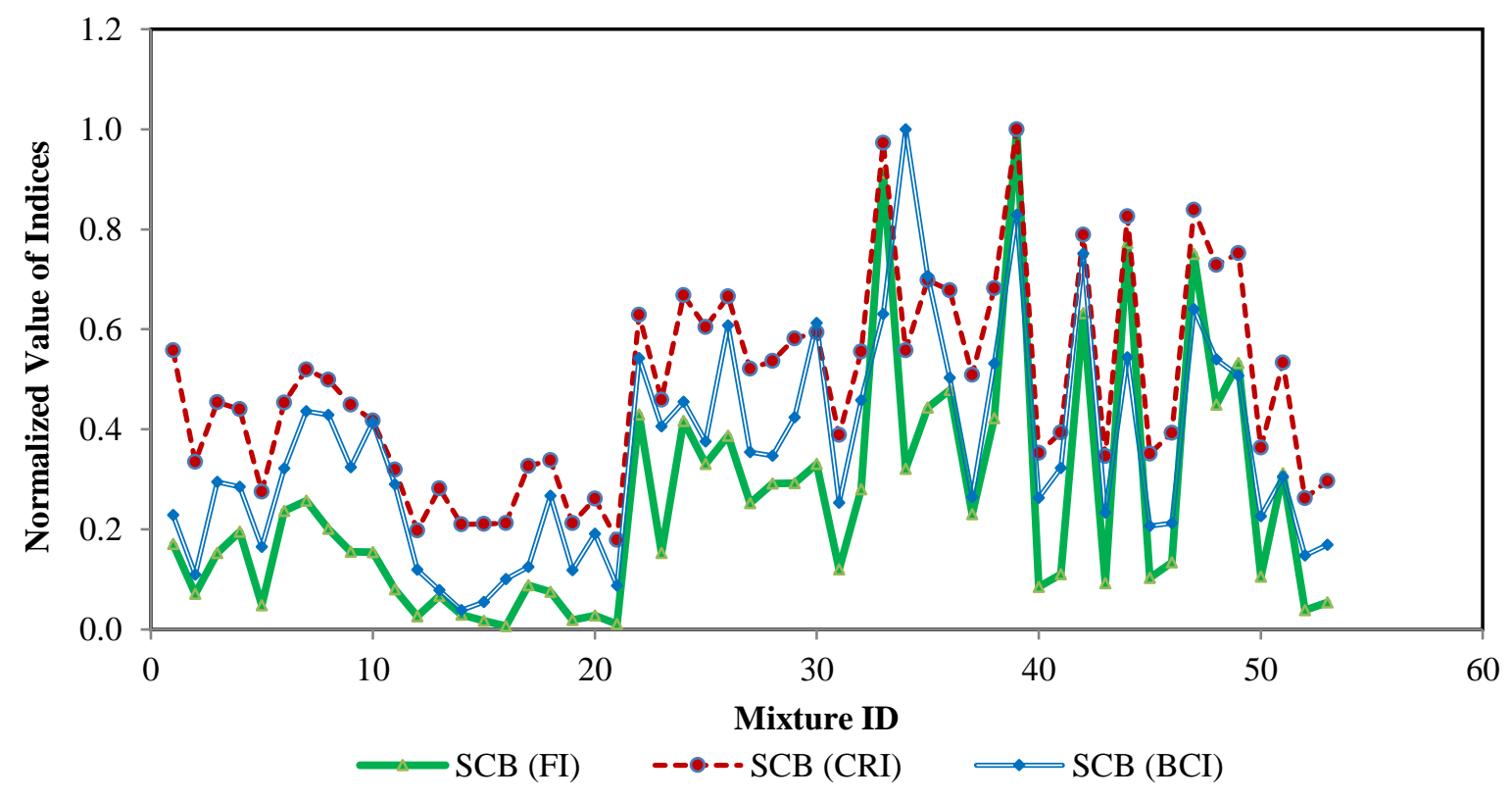

Figure 2-51 Normalized results of SCB (FI), SCB (BCI) and SCB (CRI) for 52 different mixtures.

Given an abundance of parameters used in this study and for different indices, it was of interest for the authors to look into the possible correlations in the parameters. To 
examine correlations between parameters, regression analysis was performed on the indices and the individual parameters and are presented in a series of graphs in Figure 2-52.

Figure 2-52a, b, and c show correlation between the different indices. Figure 2-52 d and e show the correlation between the different parameters used to compute the indices. Figure 2-52 $\mathrm{f}$ and g compare the correlation between the post-peak parameters for FI, i.e. " $\mathrm{m}$ ", and for BCI, i.e. L75. Figure 2-52 h, i, and j show correlation between the fracture energy to the index values. Each graph shows the type of fitting and the coefficient of determination $\left(\mathrm{R}^{2}\right)$ as a quantitative estimation of the correlation between two values.

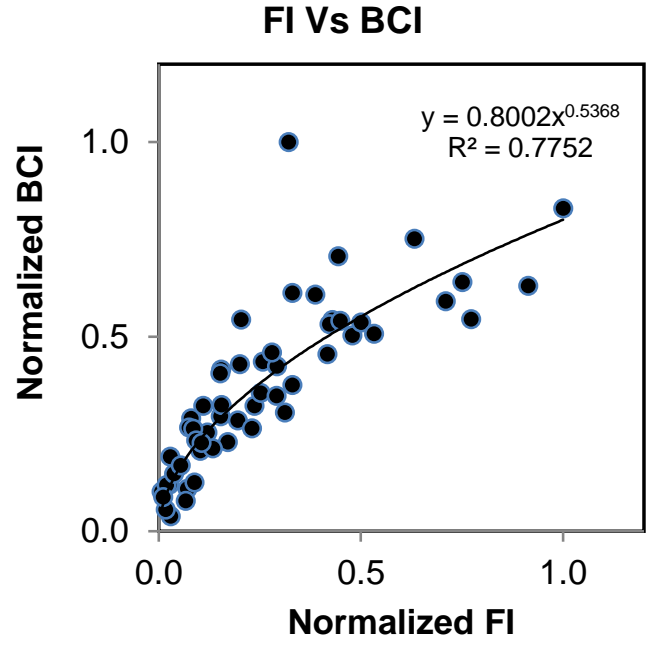

a)

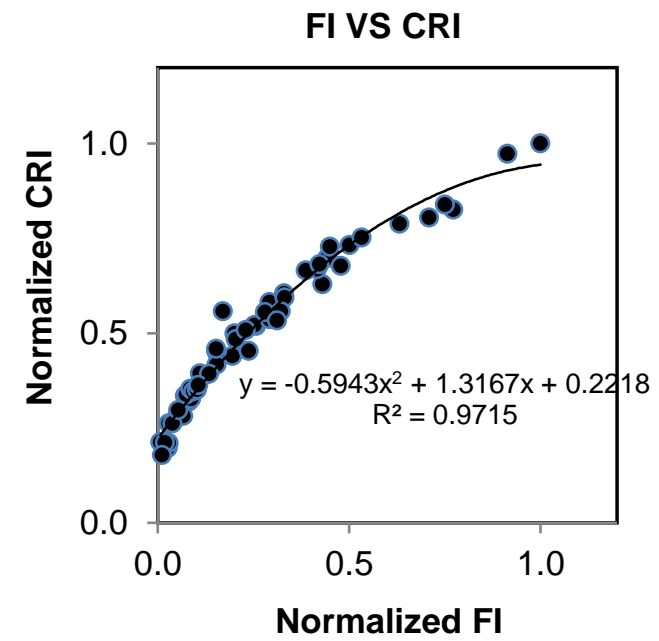

b) 
BCI VS CRI

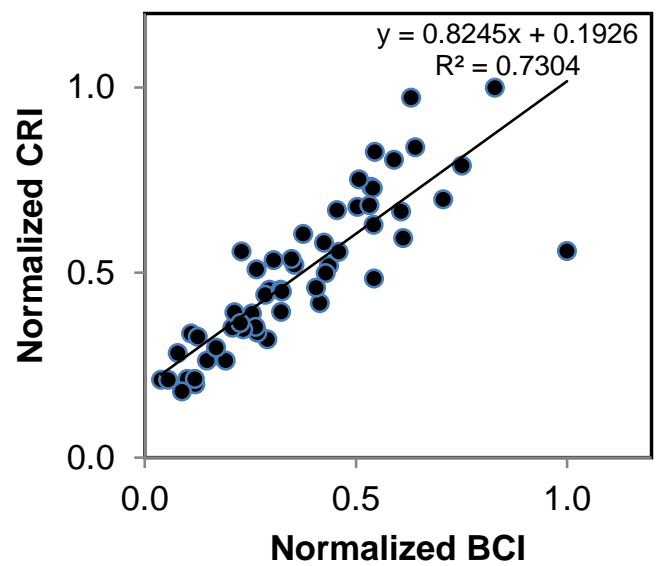

c)

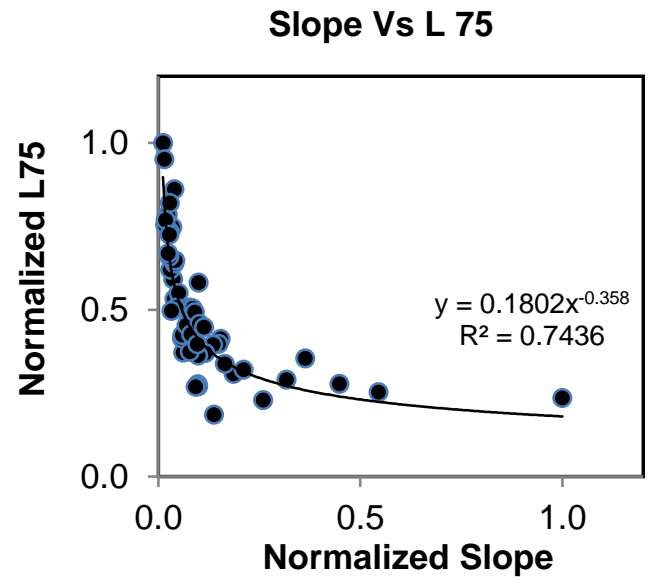

e)

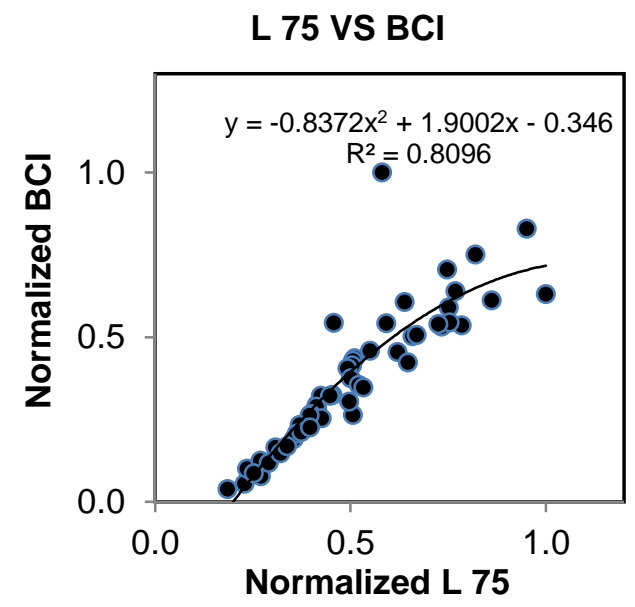

g)

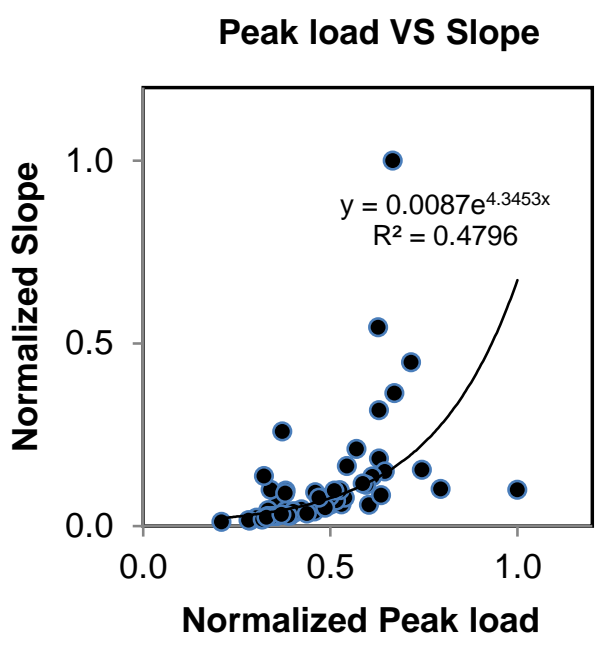

d)

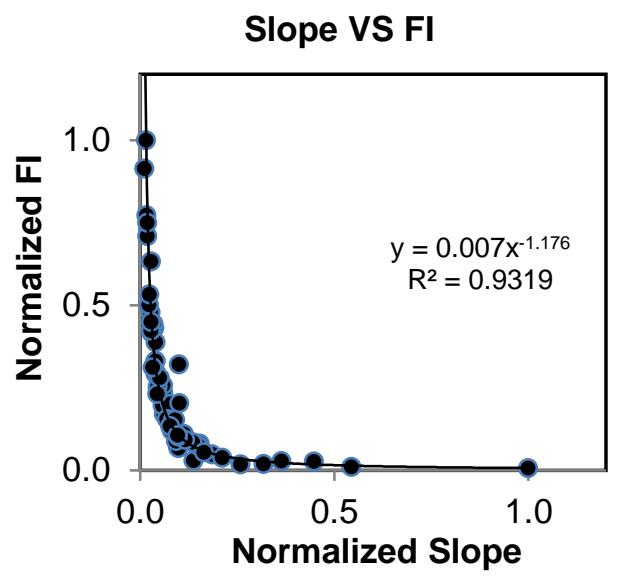

f)

FE Vs FI

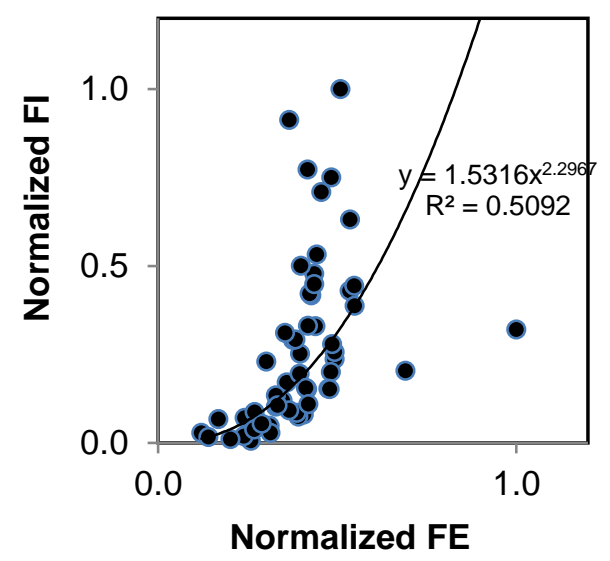

h) 
FE Vs BCl

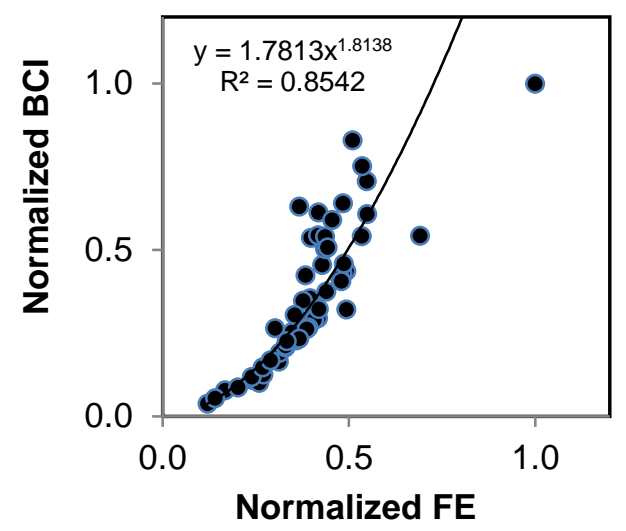

i)
FE VS CRI

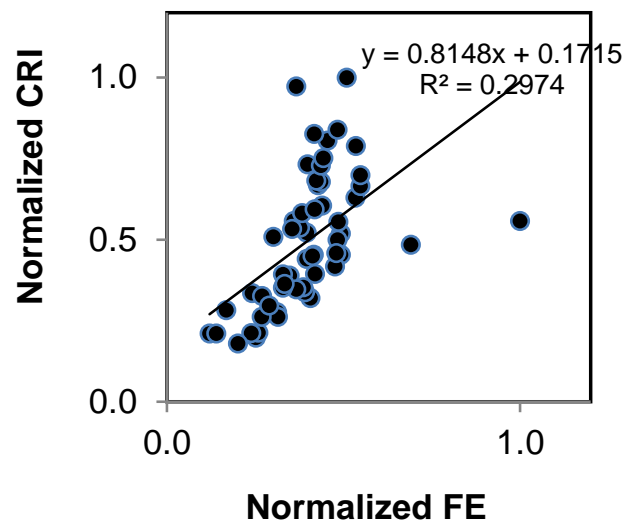

j)

Figure 2-52 a, b, c, d, e, f, g, h, i, j. Correlation between indices parameters.

The correlations shown in Figure 2-52 led to the following observations:

- The correlation between the FI and CRI is quite strong, indicating strong similarity between these two indices (Figure 2-52b), while the correlation between BCI and the other two indices is lower (Figure 2-52a and Figure 2-52c). This reinforces the notion that the BCI parameter presents a significant departure from the FI index.

- The FI index is almost perfectly correlated to slope, as shown in Figure 2-52f. Unfortunately, the large COV associated with the slope parameter in turn significantly raises the COV associated with the FI. The L75 parameter is more repeatable than the slope (Figure 2-52a).

- Neither the FI nor the CRI index is strongly correlated to fracture energy, as each parameter seems to be overwhelmed by the influence of slope, either directly (FI uses slope as a parameter) or indirectly (load in the denominator of the CRI equation indirectly places a penalty on post-peak softening curve slope).

- The BCI index on the other hand is correlated to both L75 and fracture energy, 
allowing each of these parameters to weigh into the overall composite cracking score provided, i.e., without either parameter overwhelming the index.

The poor correlation of FI to fracture energy and the strong correlation of FI to the slope parameter observed in the data investigated herein is in agreement with other recently reported data [72].

\subsubsection{Sensitivity analysis of SCB tests}

In this section, a sensitivity analysis is carried out on the effects of air voids, gradation, binder content, binder PG, and additives like crumb rubber on each of the SCBtype index.

\subsection{Air void effect}

Research studies on prevailing index-type cracking tests, such as IFIT and IDEALCT, have shown an increase in the index parameter with an increase of air voids [58], [73][76]. The increase is usually associated with a decrease of post-peak slope, and peak load in the load-LLD curve obtained from tests. This study tested five different mixtures to obtain the three SCB indices and examine the trends.

Figure 2-53 shows the trends from FI, CRI, and BCI for five mixtures, including dense-graded, and stone matrix asphalt, used in different layers of the pavement, along with a shoulder mix. These mixes were part of a larger study that included comprehensive testing of mixtures laid on Illinois Tollway routes with high traffic. All the mixes and indices reveal the aforementioned trend with regards to air void level vs. cracking score. 
Mix ID: IT 1828

N50, 4.75 mm Dense Grade Binder Course, PG46-34 + 10\% GTR (dry process), ABR* by RAP = 35.3, by RAS $=9.2$
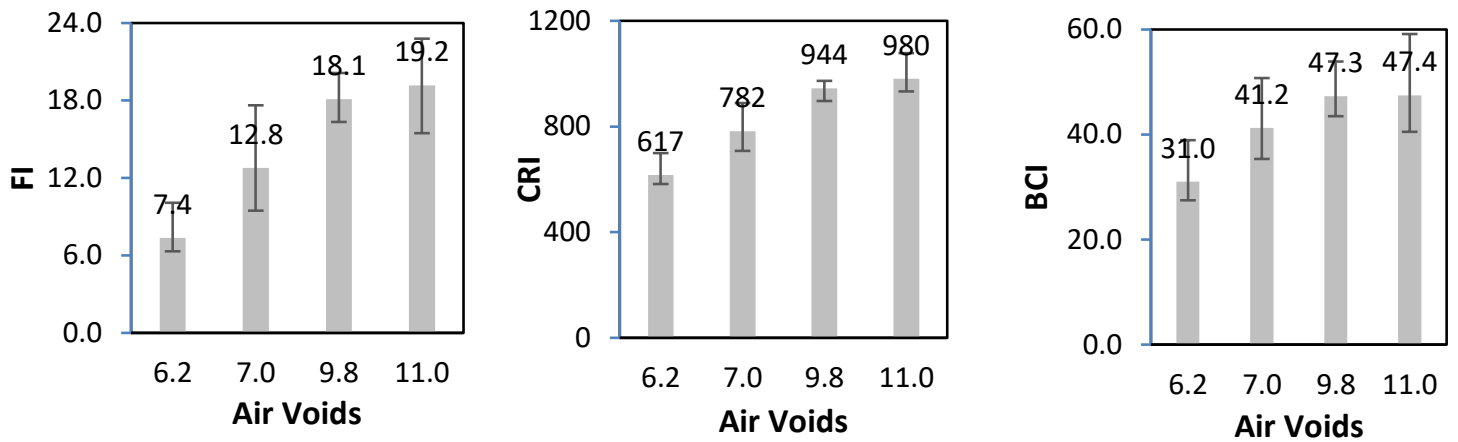

Mix ID: IT 1829

N50, 4.75 mm Dense Grade Binder Course, PG58-28 + 12\% GTR (wet process), ABR* by RAP = 17.8, by RAS $=9.3$
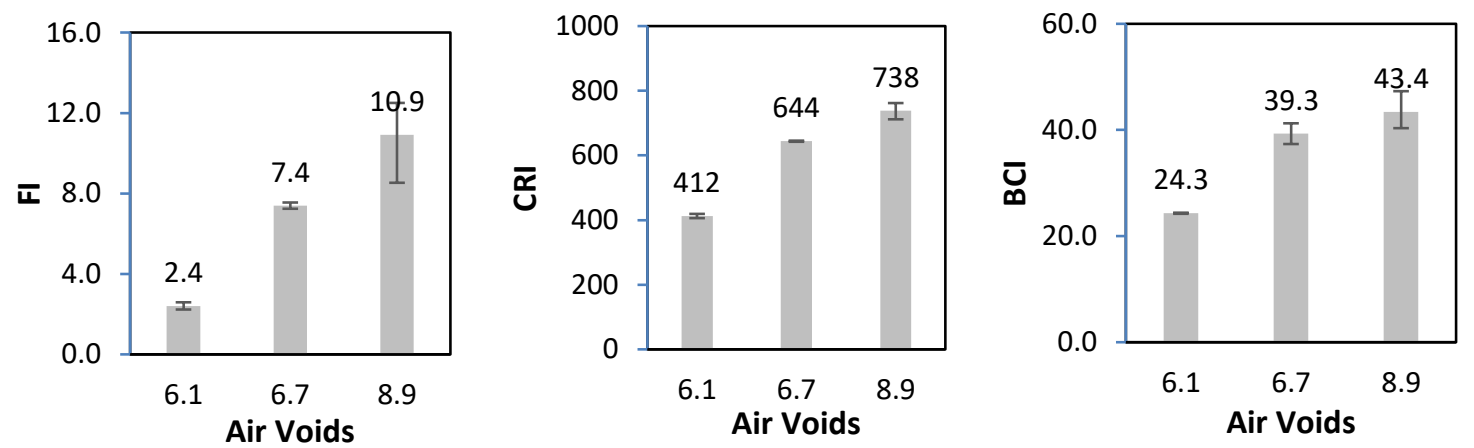

Mix ID: IT 1824

N80, 12.5 mm SMA Friction Surface Course, PG 64-34 (SBS modified), ABR by RAP = 20.4, by RAS $=16.7$
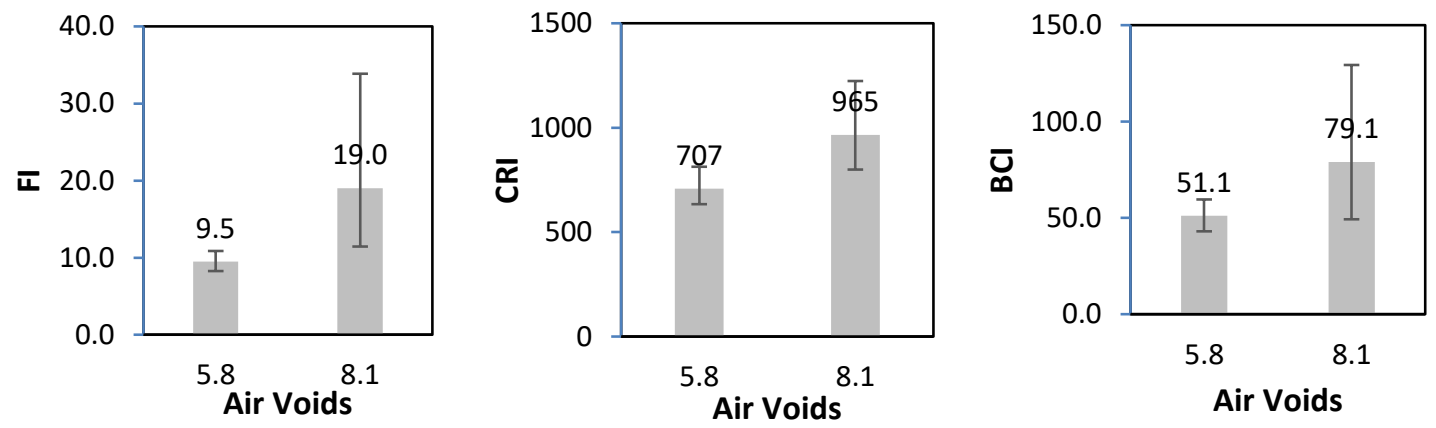

Mix ID: IT 1845

N80, 12.5 mm SMA** Friction Surface Course, PG 46-34 + 10.5\% GTR (Terminal Blend), ABR by $\mathrm{RAP}=23.9$, by RAS $=15.4$ 

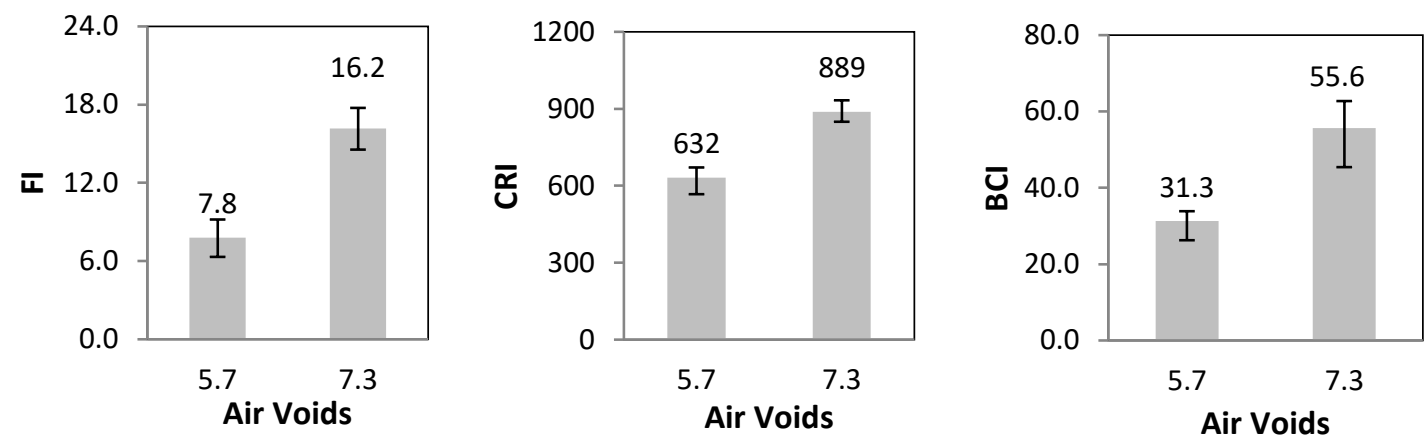

Mix ID: IT 1818

N70, 9.5 mm Dense Grade Shoulder Mix, PG 64-22, ABR by RAP $=20.4$
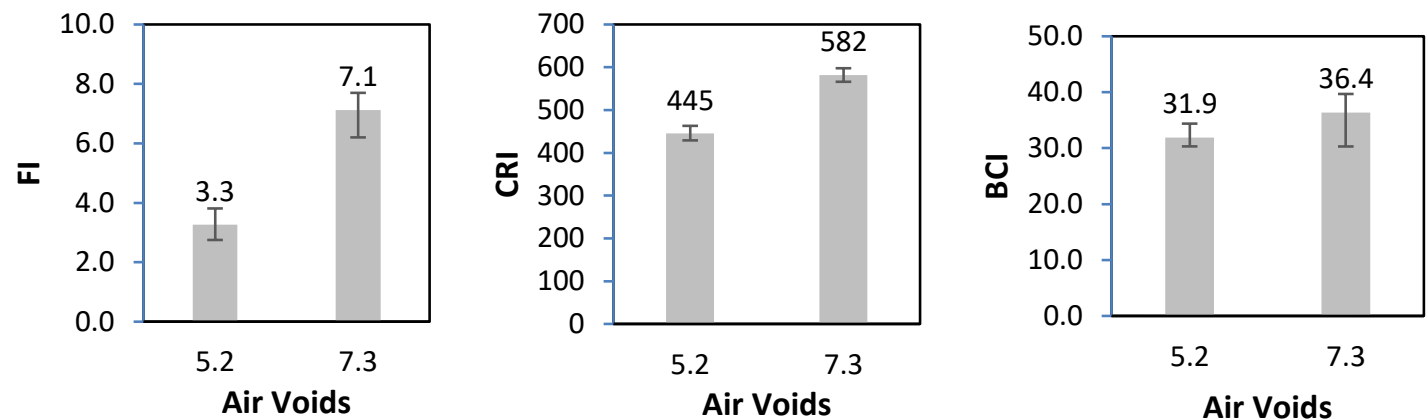

$* \mathrm{ABR}=$ Asphalt Binder Replacement (Ratio of Recycled binder to total binder)

**SMA = Stone Matrix Asphalt

Figure 2-53 Effect of air voids on FI, CRI, and BCI shown for five mixtures including SMAs, and dense-graded mixes used in various layers of a pavement.

Figure 2-54 examines air voids effects on indices for one of the mixes, IT 1845, in detail with regards to the effects of various parameters used in the indices. The other four mixtures showed very similar trends. With an increase in air voids, the peak load and the post-peak slope (used in the denominator of FI and CRI respectively) decrease, while the fracture energy and L75 parameter (used in numerators) increase. As a result, cracking scores increase with an increase in air voids. In reality, higher air voids generally lead to higher pavement deterioration rates [35], [77]. It follows from this observation that the SCB-based indices and associated specification thresholds must be approached with care. While correction factors for air void levels have been proposed [75], they appear to be mix- 
dependent. Therefore mix-specific calibration is recommended in order to properly account for varying air void levels.
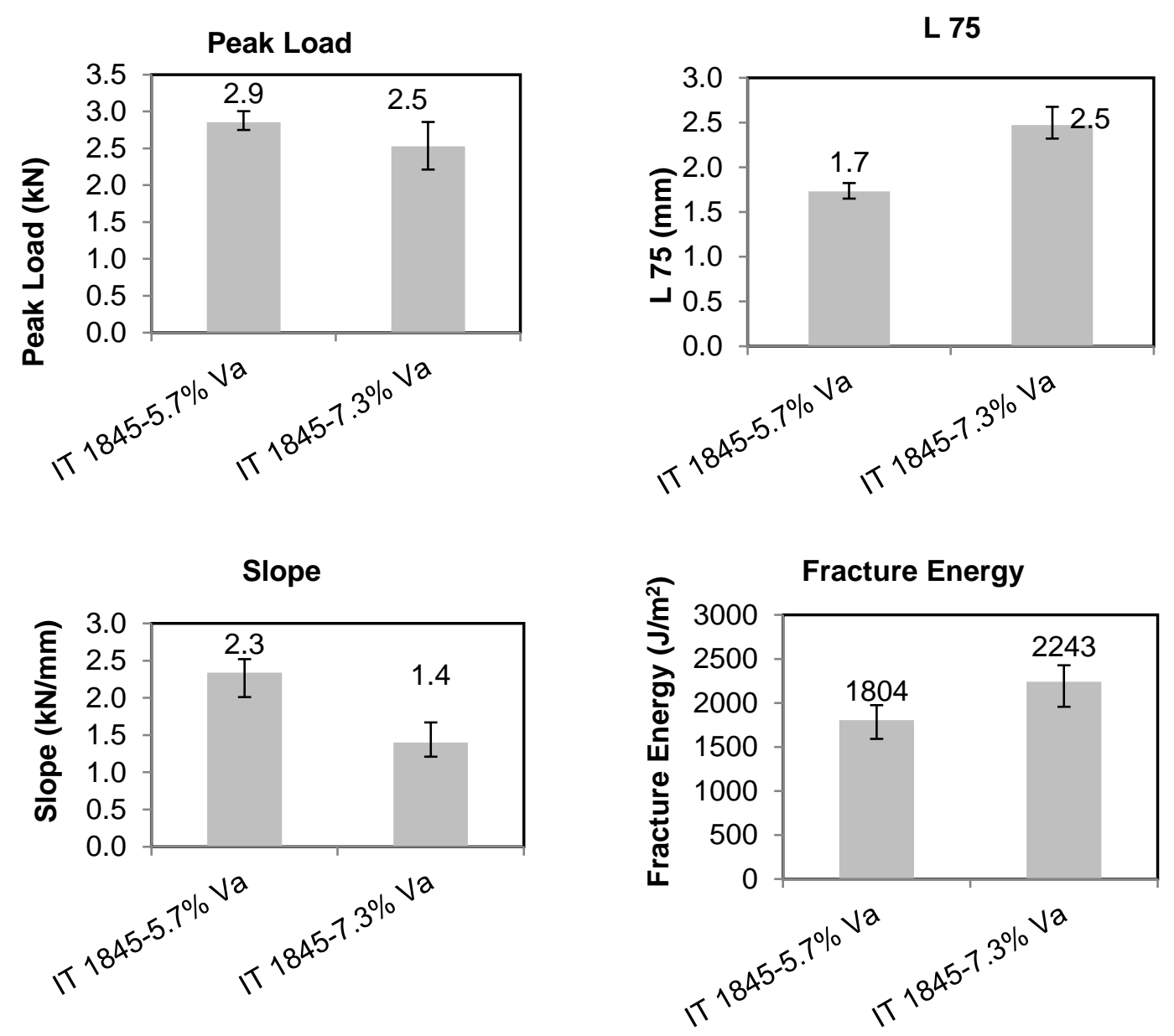

\section{Figure 2-54 Trends of individual parameters shown for IT 1845 mix (Note: All mixtures showed similar trends).}

\subsection{Gradation and binder content effect}

Aggregate type and gradation are among the most important factors affecting the performance of asphalt mixtures, as aggregates comprise around $95 \%$ of mixture weight and over $80 \%$ of mixture volume in most cases. Two mixtures were evaluated in this regard, one dense-graded and one stone matrix asphalt mix, with equal recycling contents and binder types. The binder contents of the two mixtures differed significantly (5.9\% vs. 
7.8\%). An SMA with a lower asphalt content than the dense-grade mix was intentionally selected to illustrate a point. The reverse is often the case, where SMA mixes generally have higher asphalt content than dense-graded mixes due to their higher voids in the mineral aggregate (VMA) requirements, with all other factors being equal. However, the juxtaposition in the relative levels of optimized binder content in this case is due to the fact that the SMA had a higher NMAS (lower surface area) and higher compaction level as compared to the $4.75 \mathrm{~mm}$ dense-graded mix (Table 2-17).

Table 2-17. Summary of mixtures properties to investigate the effect of gradation type

\begin{tabular}{cccccccccc}
\hline $\begin{array}{c}\text { Mixture } \\
\text { Type }\end{array}$ & Binder & $\begin{array}{c}\text { AC } \\
(\%)\end{array}$ & Gradation & $\begin{array}{c}\text { NMAS } \\
(\mathrm{mm})\end{array}$ & $\begin{array}{c}\text { ABR } \\
(\%)\end{array}$ & $\begin{array}{c}\text { RAP } \\
(\%)\end{array}$ & $\begin{array}{c}\text { RAS } \\
(\%)\end{array}$ & Additive & $\begin{array}{c}\text { VMA } \\
(\%)\end{array}$ \\
\hline $\begin{array}{c}\text { IT 1835 } \\
(41-25-16)\end{array}$ & $\begin{array}{c}\text { PG } \\
\text { PG-34 }\end{array}$ & 5.9 & SMA $^{\mathrm{a}}$ & 12.5 & 41 & 25 & 16 & $10 \% \mathrm{GTR}$ & 16.2 \\
$\begin{array}{c}\text { IT 1828 } \\
(45-35-9)\end{array}$ & $\begin{array}{c}\text { PG } \\
46-34\end{array}$ & 7.8 & Dense & 4.75 & 45 & 35 & 9 & $10 \% \mathrm{GTR}$ & 18.6 \\
\hline
\end{tabular}

Figure 2-55 shows that the cracking scores for the dense graded mixture is much higher than those of the SMA mixture. This finding runs contrary to the widely-accepted proposition that SMAs perform better with regards to cracking resistance, due to their strict aggregate quality requirements and VMA [78]. The reason for the higher cracking index of dense-grade mixture in this case could be due to higher binder content. SCB-type tests rewards higher flexibility, which has a direct correlation with binder content in the asphalt mixture, but does not consider the strength/quality of the aggregates. This finding brings into question the ability of SCB-type fracture tests and paraments in evaluating the relative performance of SMA vs. dense-graded mixtures. 
FI

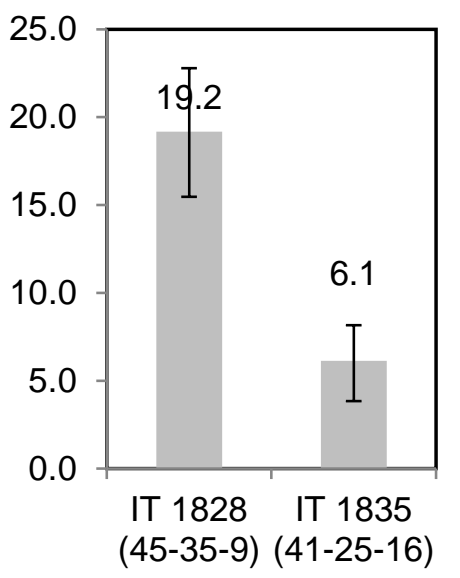

CRI

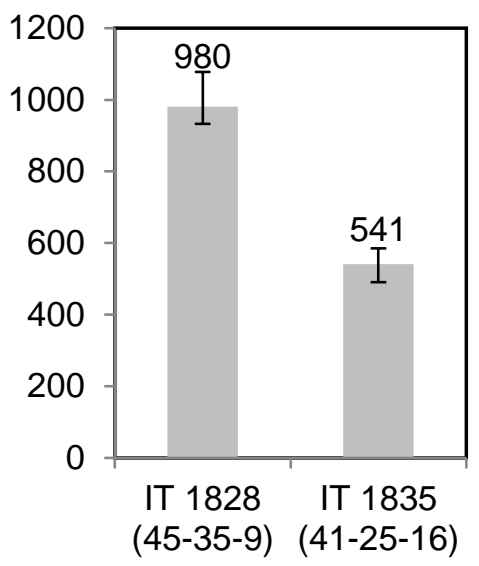

$\mathrm{BCl}$

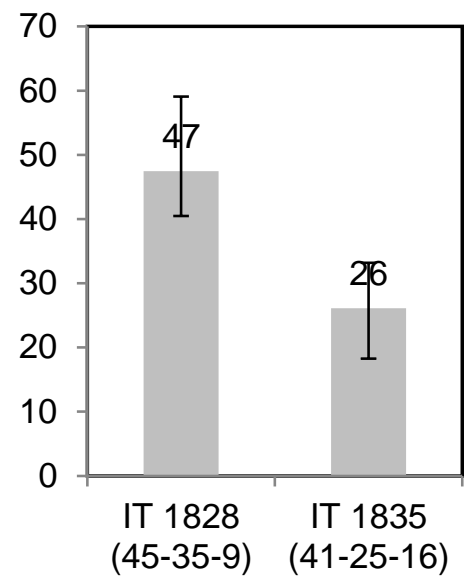

Figure 2-55 Effect of gradation and binder content on different SCB test indices

\subsection{Softer binder effect}

Next, the effect of binder type on cracking performance was evaluated using the three SCB indices investigated herein for two selected study mixtures (Table 2-18).

Table 2-18. Summary of mixtures properties to investigate the effect of softer binder

\begin{tabular}{ccccccccc}
\hline Mixture Type & +-Binder & $\begin{array}{c}\text { AC } \\
(\%)\end{array}$ & Gradation & $\begin{array}{c}\text { NMAS } \\
(\mathrm{mm})\end{array}$ & $\begin{array}{c}\text { ABR } \\
(\%)\end{array}$ & $\begin{array}{c}\text { RAP } \\
(\%)\end{array}$ & $\begin{array}{c}\text { RAS } \\
(\%)\end{array}$ & Additive \\
\hline $\begin{array}{c}\text { US54_6_C } \\
(31-31-0) \\
\begin{array}{c}\text { US54_6_46 } \\
(31-31-0)\end{array}\end{array}$ & PG 58-28 & 5.1 & Dense & 12.5 & 31 & 0 & 0 & 0 \\
\hline
\end{tabular}

The use of PG46-34 binder as a softer alternative to PG58-28 binder in the US54_6 mixture raised the fracture energy as well as the $\mathrm{L}_{75}$ and decreased the $\mathrm{P}_{\max }$ and post-peak slope (Figure 2-56). Consequently, and as expected, all SCB indices increased as a result of the use of a softer binder (Figure 2-56). 

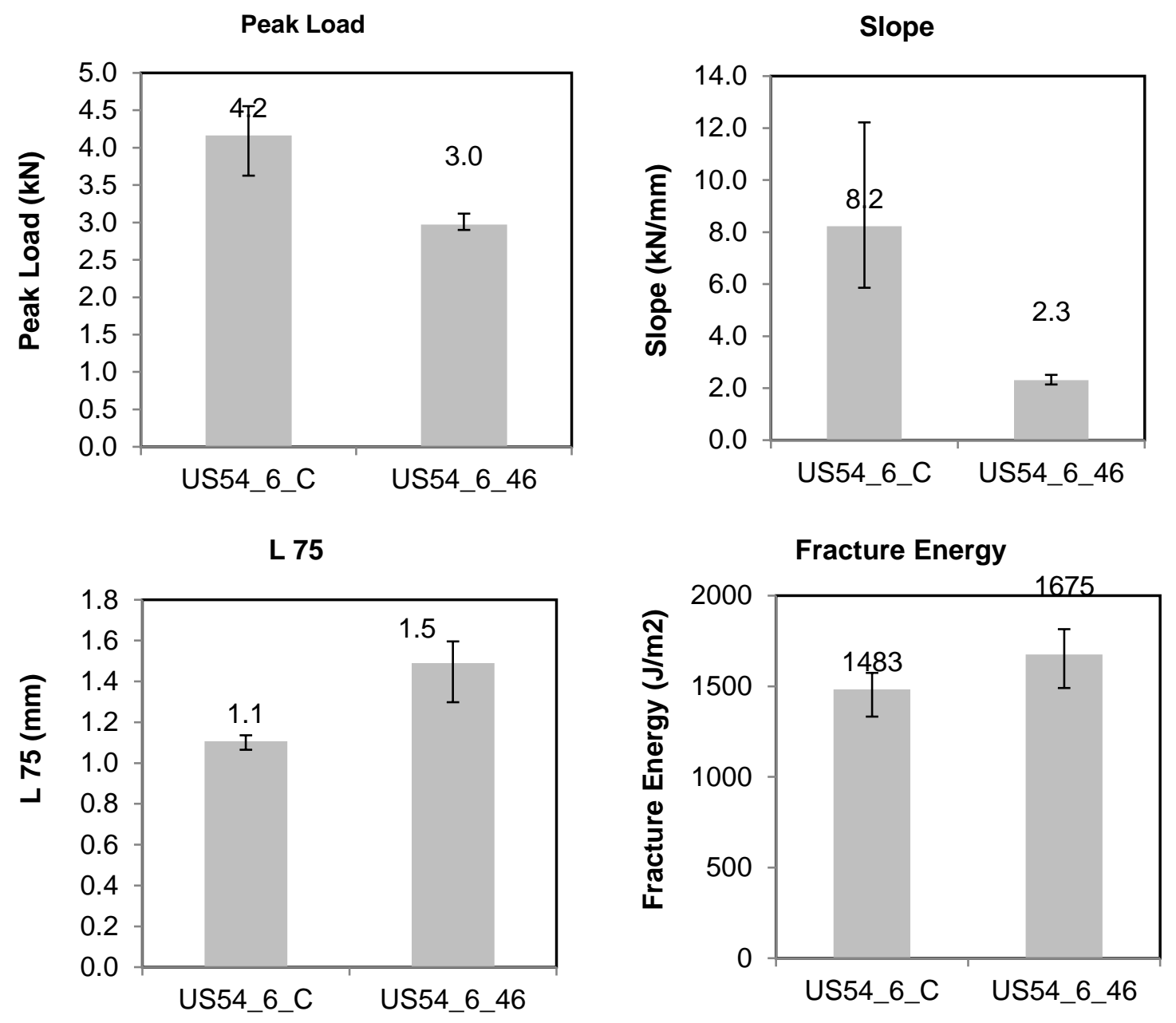

Figure 2-56 Effect of softer binder on different SCB test parameters
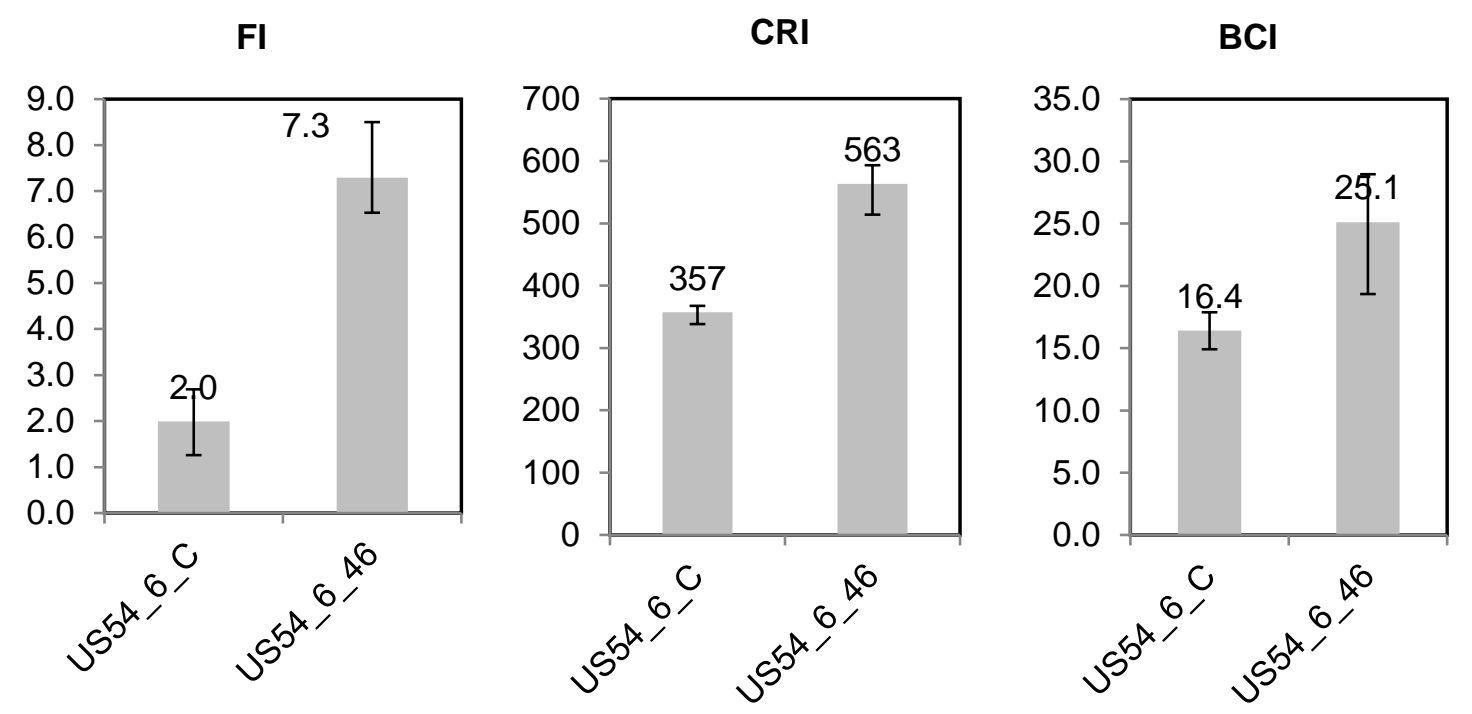


\section{Figure 2-57 Effect of softer binder on different SCB test indices}

\subsection{Crumb rubber effect}

Ground tire rubber (GTR) has been used in modifying asphalt pavements in the United States since the late 1960s. While the early use of GTR in asphalt mixtures in the 1990s were largely unsuccessful [79], [80], advancements in the GTR technology, has renewed the interest of paving agencies in its use [29], [40], [81]-[85], [86]-[88]. In this study, a comparison of the indices in ranking asphalt mixture modified with rubber in dry process technology, a popular way of incorporating rubber in asphalt mixtures is presented. It should be noted that modification with rubber in itself contains a number of important variables, such as rubber particle size, type of grinding process used to manufacture the recycled crumb rubber (ambient, cryogenic), the incorporation of other chemicals or inclusion of other modifiers, etc. Additionally, there is no universal consensus on a laboratory preparation method for rubber-modified mixes. This has resulted in differing experimental trends reported in the literature [76], [89]. In this study, a dry-process modification approach was used with dense-graded mixture types. The size of the rubber particles were passing the \#30 mesh, i.e., 400-500 microns in diameter. Lab preparation of the mixtures was performed in a standard bucket mixer, and special steps were taken to allow the lab-produced mixture to more closely resemble a plant-produced dry-process mixture. To this end, the GTR was pre-blended with binder prior to bucket mixing by shear mixing at 3500 rotations per minute for 30 minutes at $170^{\circ} \mathrm{C}$. The mixture samples were then short-term aged for 2 hours at $170^{\circ} \mathrm{C}$ and then compacted using a Superpave Gyratory Compactor (SGC). After compaction, a 20 pound weight $(\sim 9.1 \mathrm{~kg})$ was placed on the top of the compacted gyratory still held inside the mold to prevent the potential swelling due 
to rebounding of the rubber particles. This step was very important to maintain uniform air voids across all specimens tested. Table 2-19 provides a summary of mixtures properties used in the study of the effect of crumb rubber.

Table 2-19. Summary of mixtures properties to investigate the effect of crumb rubber

\begin{tabular}{|c|c|c|c|c|c|c|}
\hline Mixture ID & Binder & $\begin{array}{c}\text { AC } \\
\text { \% }\end{array}$ & Gradation & $\begin{array}{c}\text { N } \\
\text { design }\end{array}$ & $\begin{array}{c}\text { NMAS } \\
(\mathbf{m m})\end{array}$ & \%GTR \\
\hline Mix1_Unmodified & PG 58-28 & 4.5 & Dense & 80 & 12.5 & 0 \\
\hline Mix1_10GTR & PG 58-28 & 4.7 & Dense & 80 & 12.5 & 10 \\
\hline Mix2_Unmodified & PG 64-22 & 4.7 & Dense & 35 & 12.5 & 0 \\
\hline Mix2_12GTR & PG 64-22 & 4.7 & Dense & 35 & 12.5 & 12 \\
\hline Mix3_Polymer & PG 70-28 & 4.7 & Dense & 65 & 12.5 & 0 \\
\hline Mix3_P+5GTR & PG 70-28 & 4.8 & Dense & 65 & 12.5 & 5 \\
\hline Mix4_Unmodified & PG 64-22 & 5.2 & Dense & 80 & 12.5 & 0 \\
\hline Mix4_10GTR & PG 64-22 & 5.4 & Dense & 80 & 12.5 & 10 \\
\hline
\end{tabular}

As shown in Figure 2-58, all three SCB indices predicted that the GTR, plantproduced mixture was less crack-resistant at the intermediate temperature as compared to the control mix. This is somewhat counter-intuitive as field results suggest rubber modification provides cracking resistance benefits to asphalt mixtures place in the field, even under extreme environments and loading conditions [29], [40], [81]-[85].

a) $\operatorname{Mix} 1$
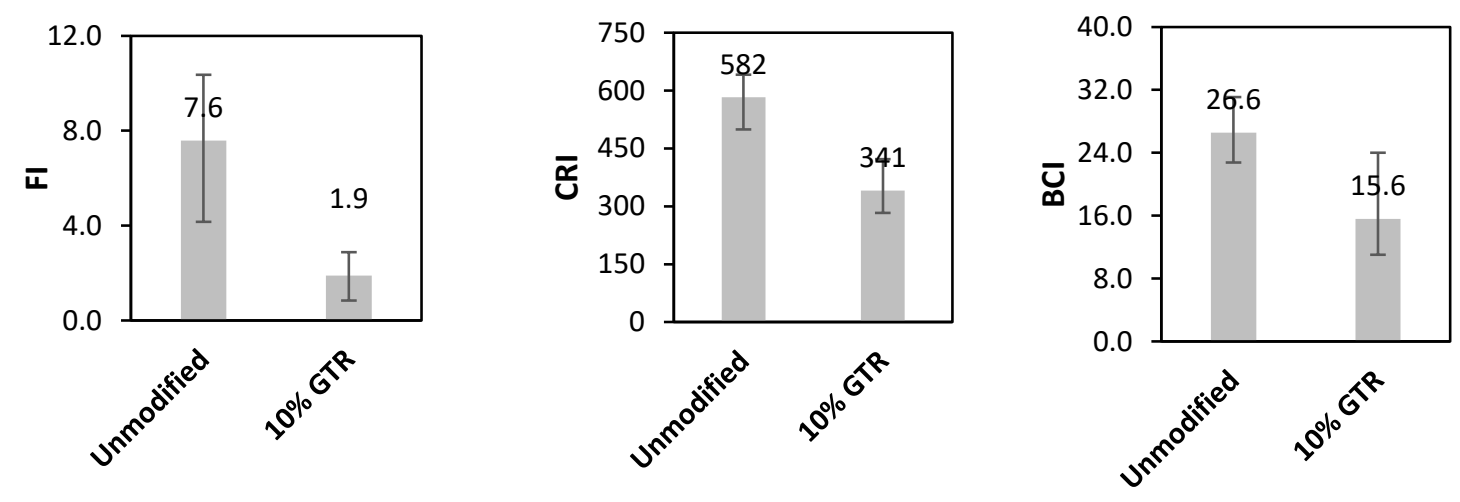
b) Mix 2
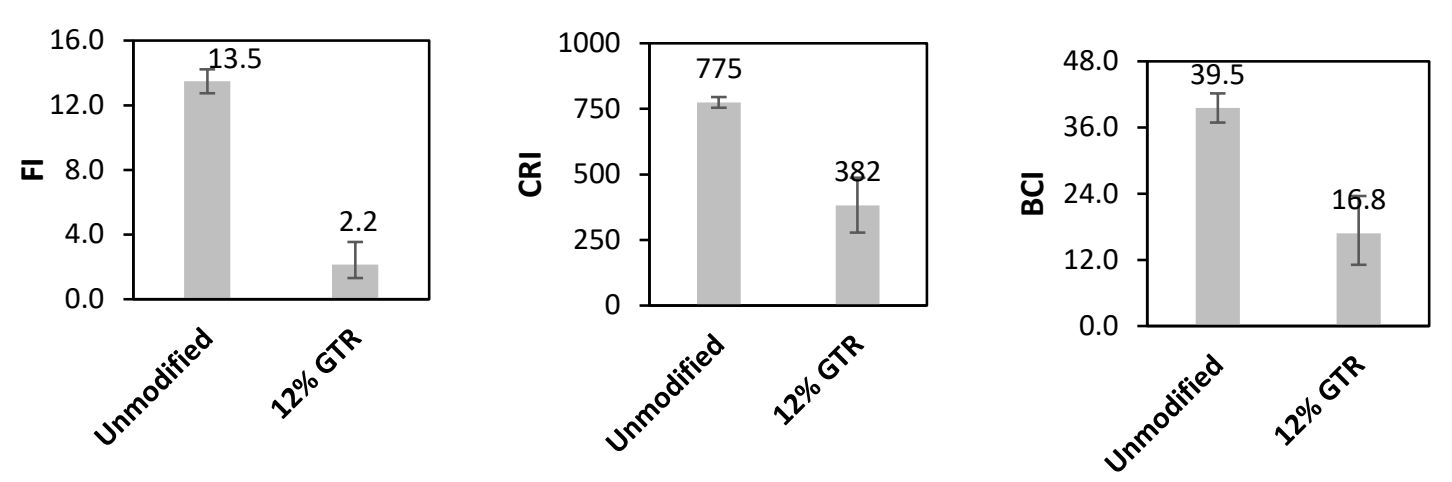

c) Mix 3
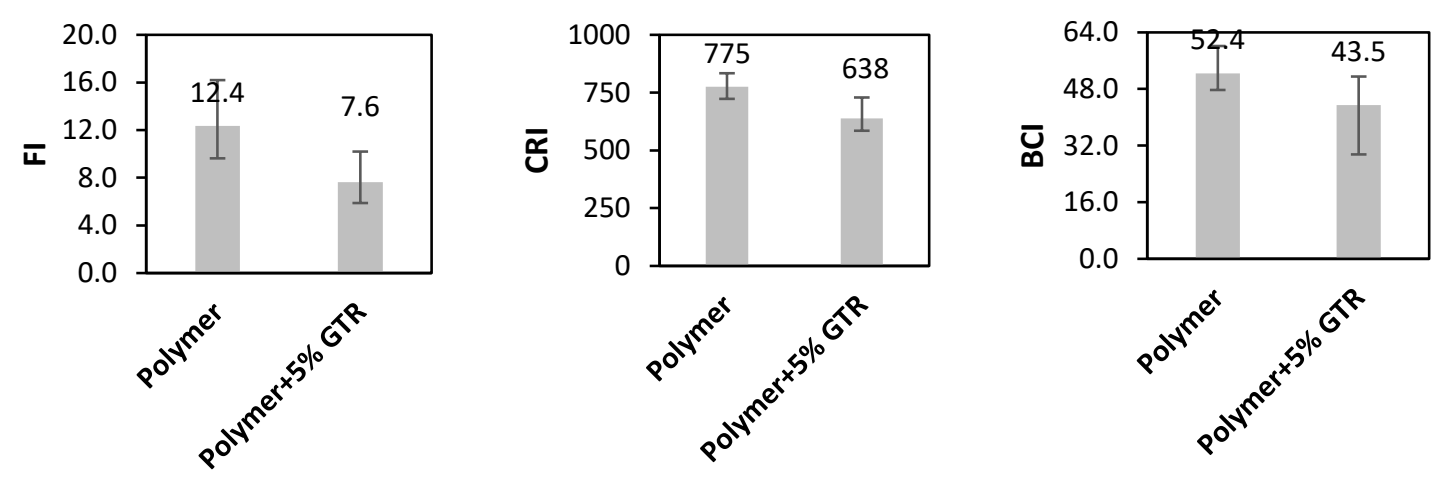

Figure 2-58 Effect of crumb rubber on different SCB indices shown for three densegrade mixtures.

Upon closer examination of the load-displacement curves obtained from SCB test, it was observed that the rubber-modified mixtures had a higher peak load, and much steeper post-peak slope as compared to the unmodified mixtures, as measured by the SCB test at the standard conditions of $25^{\circ} \mathrm{C}$ test temperature and $50 \mathrm{~mm} / \mathrm{min}$ displacement rate. The higher peak load signifies the higher strength of the rubber mixtures, which in turn resulted in a higher accumulation of stored strain energy (as the specimen bends before breaking). The stored strain energy is rapidly released following the onset of crack propagation, leading to steeper post-peak slopes (higher "m" parameter in the FI test, lower L75). This results in lower FI and CRI values. 
Figure 2-59 and Figure 2-60 show the trends of individual parameters used in measuring the cracking indices for Mixes 1 and 4, respectively. Figure 2-59 demonstrates that the fracture energy of the GTR mix is lower as measured by the SCB test at $25^{\circ} \mathrm{C}$ and $50 \mathrm{~mm} / \mathrm{min}$, and the post-peak slope is higher as compared to the unmodified mix, leading to a much lower FI. On the other hand, as shown in Figure 2-60, the fracture energy of Mix 4 with GTR modification is higher than the unmodified mix, but the high slope value nullifies any credit that the higher fracture energy attributes to this mix, ultimately resulting in a lower FI score. In this case, the BCI parameter credits the mixture for a higher fracture energy, as seen by the relatively close proximity in the BCI values for Mix 4 with and without GTR additive (Figure 2-58). That notwithstanding, none of the three SCB-based indices were able to fully account for the excellent cracking resistance generally observed in the field when GTR is used as a recycled modifier.

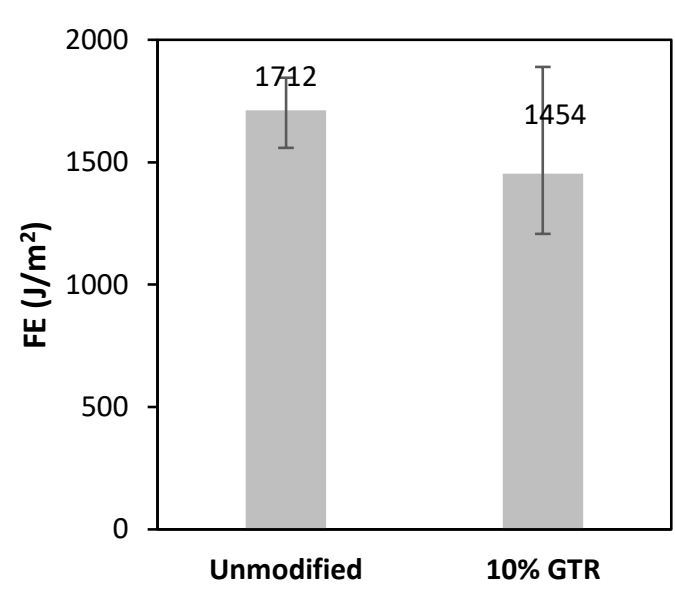

(a)

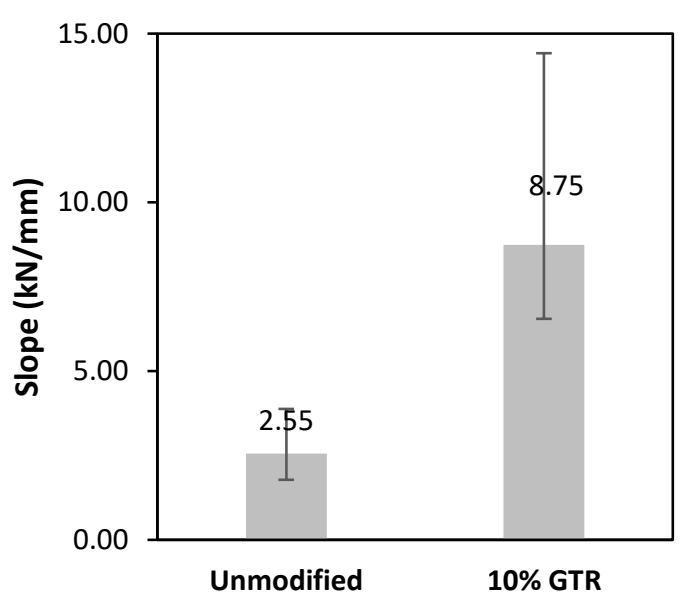

(b) 


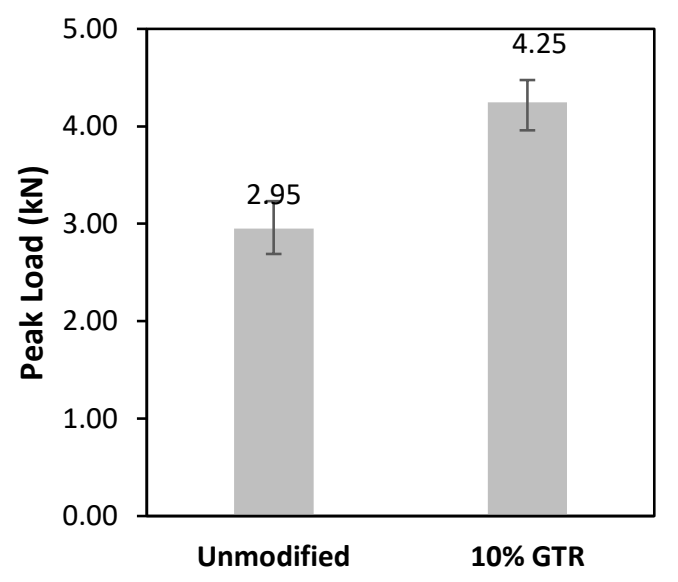

(c)

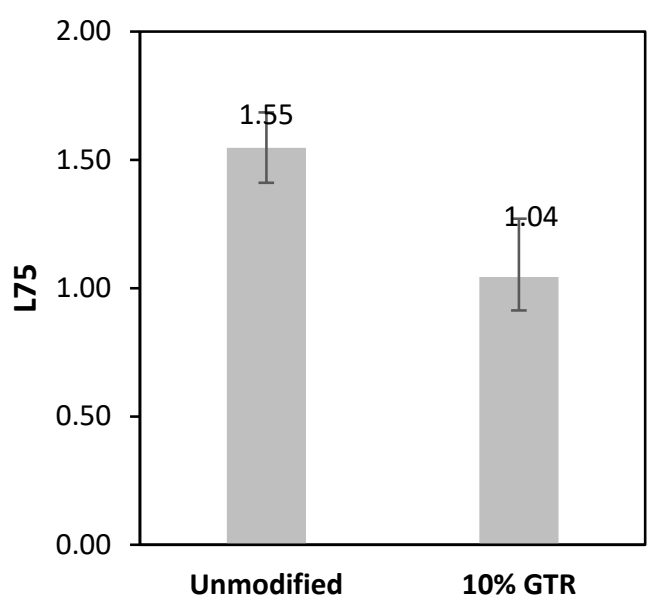

(d)

Figure 2-59. Effect of crumb rubber on, a) fracture energy, b) slope, c) peak load, and d) L75 parameters for Mix 1.

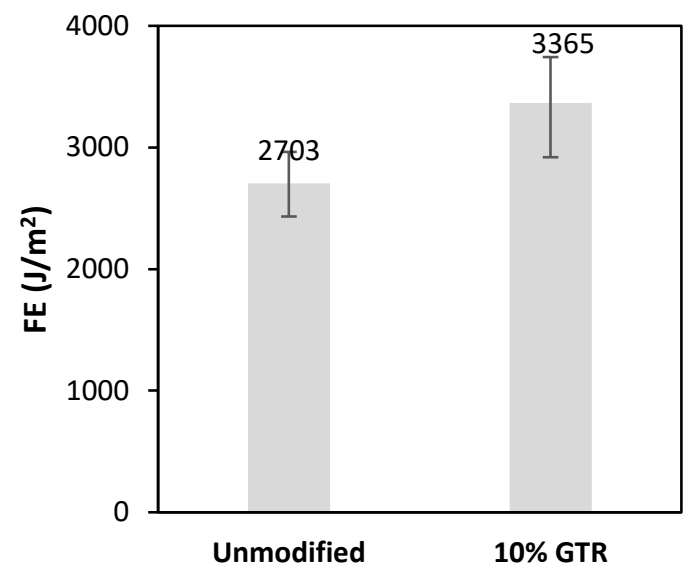

(a)

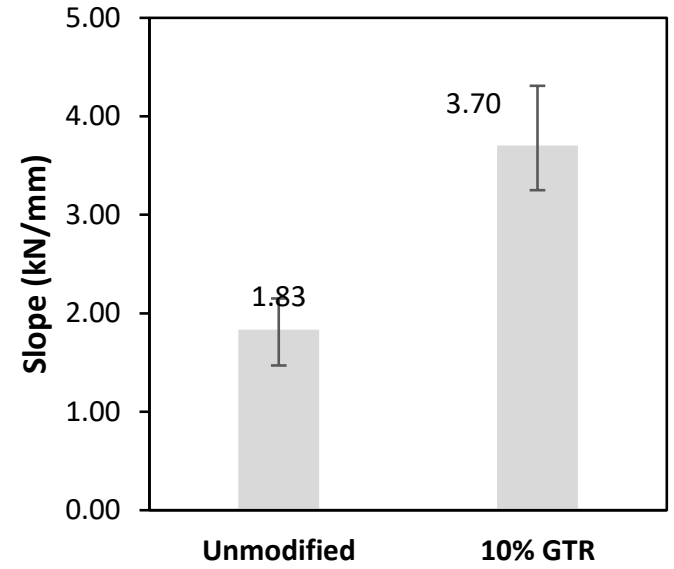

(b) 


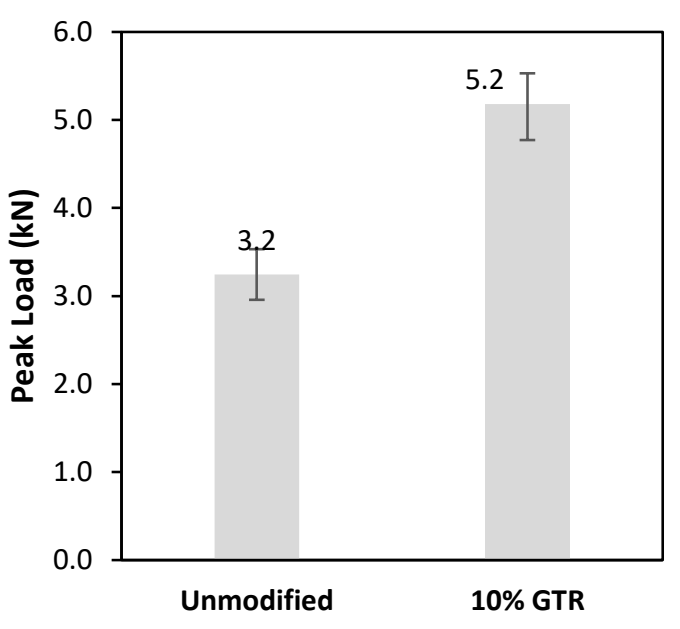

(c)

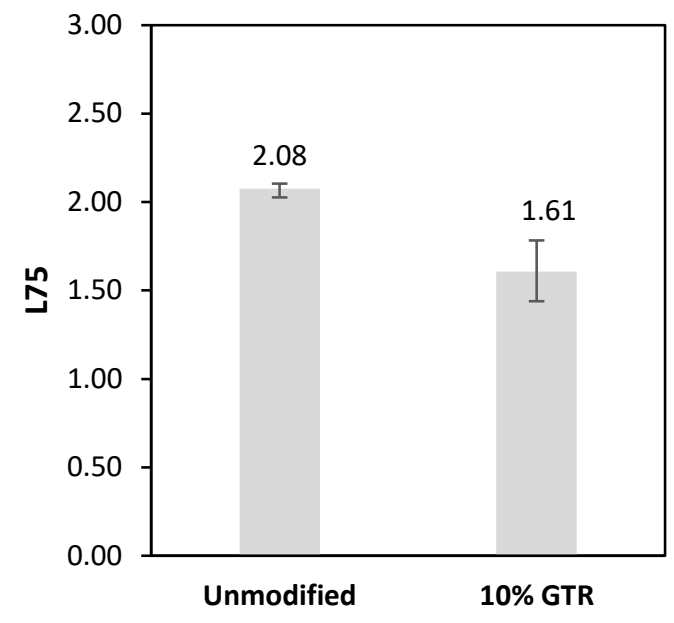

(d)

Figure 2-60. Effect of crumb rubber on, a) fracture energy, b) slope, c) peak load, and d) L75 parameters for Mix 4.

\subsection{Statistical Analysis of Mix Design Variables}

In this study, a statistical analysis is performed to check if different indices are able to detect the effect of different mix design parameters on the cracking performance. To this end, we conducted multi-way analysis of variance (ANOVA), also known as multi-factor ANOVA tests with replication. Minitab 2017 software was employed to run the statistical analysis Table 5 presents a statistical analysis of the investigated mixtures in terms of each performance test conducted. The F-value and P-value results obtained from the ANOVA test are included in the tabulated results. A significance level of 0.05 was used, as described earlier. The 0.05 level of confidence is commonly used in statistical analysis which indicates that there is a 5\% risk of concluding that an effect exists when there are no actual effects existing. P-values lower than 0.05 reject the null hypothesis and provide statistical evidence that the selected parameter is significant. Total Degrees of Freedom (total $\mathrm{DF}=$ total number of replicates -1 ) for all of the mix variables and indices is 7 as four replicates for each of the two compared mixtures are used to check if the index is able to 
detect the effect of variables. Yielding $p$-values lower than 0.05 indicates that all the indices are able to distinguish the compared mixtures. Therefore, the introduced BCI parameter is capable to capture the effect of different mix variables such as air void, aggregate gradation, binder type, and rubber modification.

The analysis revealed that all of the three indices are significantly affected by the mixture design variable studied. The order of the SCB indices from smallest-to largest with respect to their p-values is FI, CRI, and BCI. Specifically, a p-value is the probability of obtaining an effect at least as extreme as the one in the sample data, assuming that the null hypothesis is true. For example, the p-value for the $\mathrm{BCI}$ index corresponding to air void effect is 0.014 . This means that there is a $1.4 \%$ chance of computing an insignificant difference between samples due to random sampling. The FI and CRI parameters are more sensitive than BCI with respect to the effects of mixture variables on cracking scores due to their lower p-values. However, the combination of a reasonably small p-value (less than 0.05) in conjunction with a lower COV rendered the BCI parameter as the best in distinguishing between mixtures in terms of their cracking potential.

Table 2-20. Statistical analysis to investigate the performance of each index to delineate mixes with differing mix composition variables

\begin{tabular}{cccc}
\hline $\begin{array}{c}\text { Mixture Variable } \\
\text { Investigated }\end{array}$ & Index & F-value & P-value \\
\cline { 3 - 4 } & FI & 45.3 & 0.003 \\
$\begin{array}{c}\text { Effect of air voids: } \\
\begin{array}{c}\text { Comparing 5.7 and 7.3\% air } \\
\text { voids for 1845 mix }\end{array}\end{array}$ & CRI & 39.93 & 0.003 \\
$\begin{array}{c}\text { Effect of mix gradation and } \\
\text { binder content: }\end{array}$ & BCI & 17.62 & 0.014 \\
$\begin{array}{c}\text { Comparing 1835 with 1828 mix } \\
\text { Fin }\end{array}$ & CRI & 47.27 & 0.000 \\
& & 126.5 & 0.000
\end{tabular}




\begin{tabular}{cccc} 
& BCI & 16.28 & 0.007 \\
& FI & 51.98 & 0.002 \\
$\begin{array}{c}\text { Effect of the softer binder: } \\
\begin{array}{c}\text { Comparing US54_6_C with } \\
\text { US54_6_46 mix }\end{array}\end{array}$ & CRI & 60.48 & 0.001 \\
& BCI & 8.1 & 0.047 \\
$\begin{array}{c}\text { Effect of 10\% GTR addition: } \\
\text { Comparing an unmodified vs. a } \\
\text { 10\%GTR modified mix }\end{array}$ & FI & 12.2 & 0.013 \\
& BCI & 32.6 & 0.001 \\
\hline
\end{tabular}

\subsubsection{Field Performance Evaluation and Statistical Analysis}

Any new cracking performance test must ultimately prove its ability to correlate to, and better yet, to effectively control field cracking performance [32]-[34][90], [91]. In this portion of the study, the cracking behavior of ten roadway sections located in Missouri used to compare field cracking behavior with the cracking indices obtained after testing field cores. Representative pavement condition images are shown in Figure 2-61for each section investigated. The Pavement Surface Evaluation and Rating (PASER) system [92] provides a numerical rating of pavement serviceability, rating from 10 (best) to 0 (worst). PASER results for the ten field sections were extracted from the MoDOT data portal. To minimize the effect of differing pavement service life across projects, a deterioration rate was computed based on the average decline in the PASER rating over the service life of the overlay. Sections aged between 5-16 years in the field were studied. Table 2-21 summarizes pertinent details of the sections, along with their PASER ratings and SCB Index values (FI, CRI, BCI) obtained from the extracted field cores for the respective 
sections. The field sections investigated cover a wide range of recycling levels, binder types, and asphalt contents.

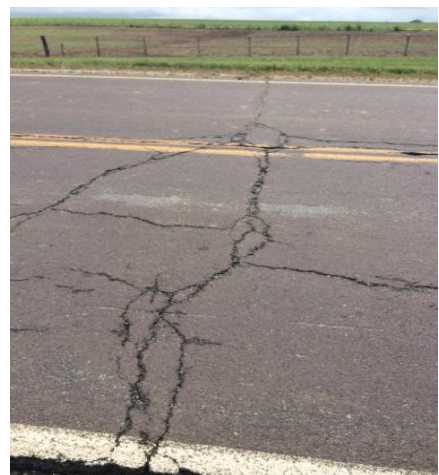

MO151
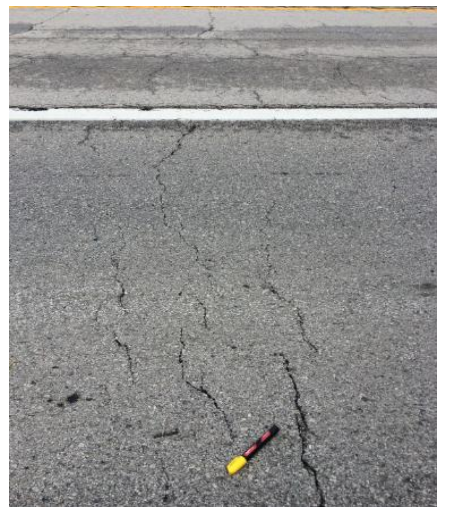

US36 E

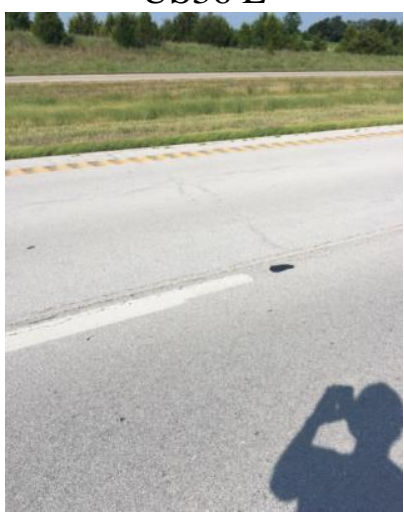

US61

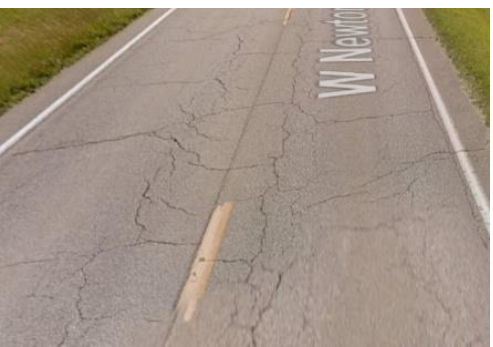

MO52

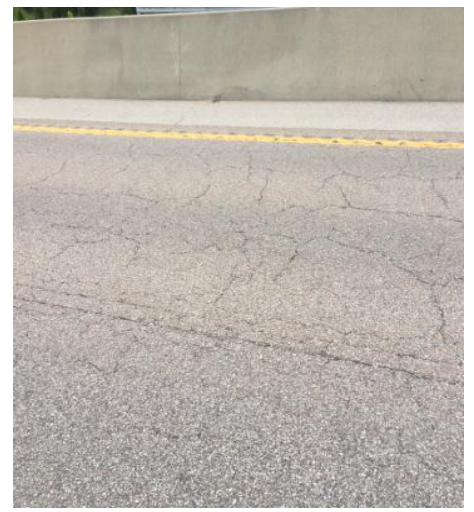

US54 E

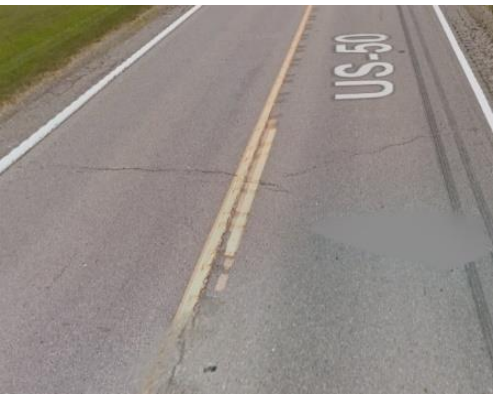

US50_1

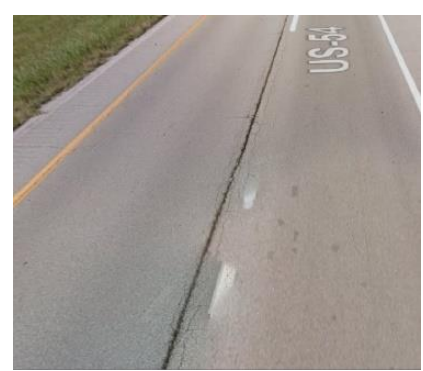

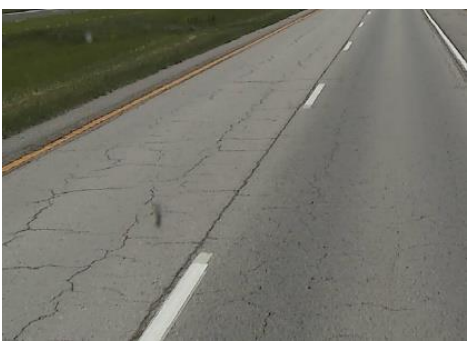

US63

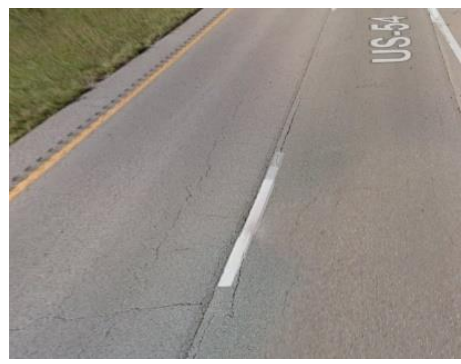

US54_8

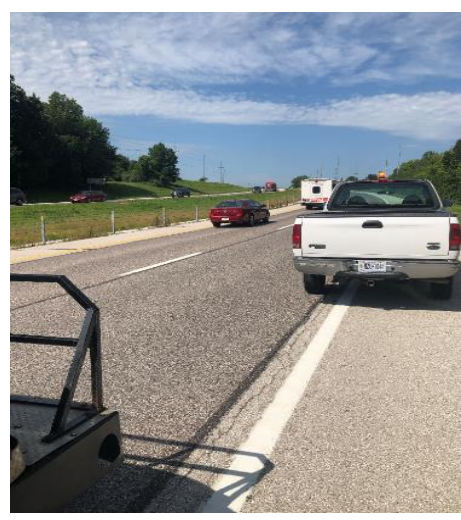

MO94 
US54_7

Figure 2-61 Pavement condition for ten field sections from high deterioration rate to low deterioration rate.

Table 2-21. Mixture properties, field performance, and SCB indices result for ten sections.

\begin{tabular}{|c|c|c|c|c|c|c|c|c|c|c|}
\hline Section & $\begin{array}{c}\text { Overlay } \\
\text { Year }\end{array}$ & $\begin{array}{c}\text { Virgin } \\
\text { Binder } \\
\text { grade }\end{array}$ & $\begin{array}{c}\text { Total } \\
\text { Asphalt } \\
\text { Content } \\
(\%) \\
\end{array}$ & $\begin{array}{c}\text { NMAS } \\
(\mathbf{m m})\end{array}$ & $\begin{array}{c}\text { ABR } \\
(\%)\end{array}$ & $\begin{array}{c}\text { PASER } \\
\text { (year=field } \\
\text { coring) }\end{array}$ & $\begin{array}{c}\text { Deterioration } \\
\text { Rate } \\
\text { (Det } \\
\text { rating/yr.)* }\end{array}$ & $\begin{array}{r}\text { SCB } \\
(\text { FI) }\end{array}$ & $\begin{array}{c}\text { SCB } \\
(\text { CRI })\end{array}$ & $\begin{array}{r}\text { SCB } \\
\text { (BCI) }\end{array}$ \\
\hline MO151 & 2014 & PG64-22 & 4.7 & 12.5 & 30.6 & 4 & 0.9 & 0.3 & 271.1 & 2.4 \\
\hline MO52_1 & 2010 & PG64-22 & 4.8 & 12.5 & 33.5 & 4 & 0.8 & 0.6 & 212.0 & 2.9 \\
\hline US63_2 & 2008 & PG64-22 & 5.6 & 12.5 & 29.9 & 5 & 0.6 & 0.4 & 213.0 & 4.1 \\
\hline US36 E & 2011 & PG64-22 & 5.1 & 12.5 & 24.7 & 5 & 0.5 & 0.2 & 161.2 & 3.7 \\
\hline US54 E & 2010 & PG70-22 & 5.7 & 12.5 & 11.8 & 5 & 0.5 & 0.6 & 231.4 & 6.6 \\
\hline US54_8 & 2006 & PG70-22 & 5.6 & 12.5 & 8.6 & 6 & 0.5 & 0.1 & 214.0 & 7.6 \\
\hline US61 & 2013 & PG64-22H & 5.3 & 9.5 & 29.6 & 8 & 0.4 & 0.4 & 237.8 & 4.2 \\
\hline US50_1 & 2011 & PG64-22 & 5 & 12.5 & 24.6 & 7 & 0.4 & 1.4 & 285.0 & 5.9 \\
\hline MO94 & 2005 & PG64-22 & 5.6 & 12.5 & 0 & 8 & 0.2 & 1.5 & 312.2 & 5.8 \\
\hline US54_7 & 2003 & PG64-22 & 6.2 & 12.5 & 0 & 8 & 0.1 & 1.8 & 329.0 & 9.4 \\
\hline
\end{tabular}

It is clear from Figure 2-61 and Table 2-21, that the US 54_7 section was the best performer, with a very low deterioration rate (very little cracking after 15 years in service). This section contained no recycling, and also possessed a relatively high binder content for a 12.5 NMAS mix (6.2\%). The worst performer was the MO151 section, which had the highest recycling (30.6\% ABR), but without any softening of the selected virgin binder grade.

To compare the three SCB indices, the FI, CRI, and BCI values for the sections were plotted versus the corresponding deterioration rate. Figure 2-62 shows the graphs along with the correlation coefficient, $\mathrm{R}^{2}$, and the average COV obtained from testing replicates. According to the calculated $\mathrm{R}^{2}$, BCI, FI, and CRI have the highest-to-lowest correlation with field performance data, respectively. Furthermore, the CRI, BCI, and FI were found 
to have the lowest-to-highest $\mathrm{COV} \%$, respectively. In this analysis, the COV parameter describes the repeatability of the test samples.
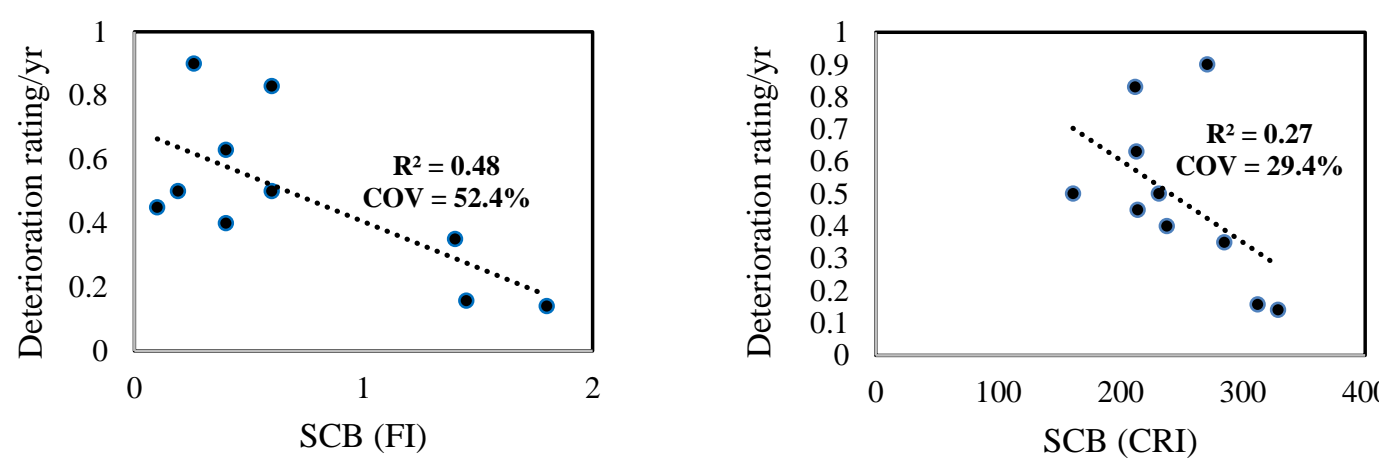
a) $\mathrm{SCB}(\mathrm{FI})$ vs det rate
b) SCB (CRI) vs det rate

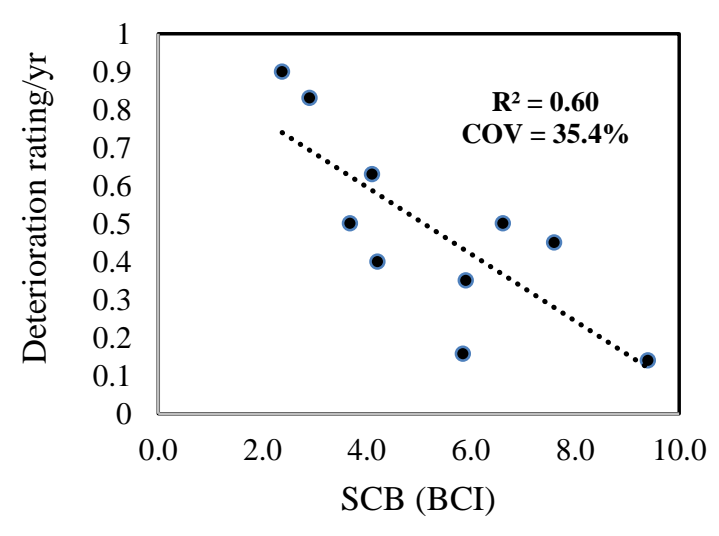

c) $\mathrm{SCB}(\mathrm{BCI})$ vs det rate

Figure 2-62 Correlation of FI, CRI, and BCI indices with deterioration rate for the ten sections.

A one-way analysis of variance (ANOVA) was carried out, along with the Fisher method, to assess the ability of the three cracking indices studied to distinguish between the ten sets of field cores tested. A significance level of 0.05 (95\% reliability) was selected to examine the null hypothesis. Because the p-values for the FI and BCI indices are less 
different than 0.05 (Table 2-22), the mean values of the cracking index for ten sections are significantly. The P-value for the CRI index was found to be 0.069 , which is higher than the threshold of 0.05 , and indicates that the index is not able to differentiate between the sections. Table 2-22 shows the grouping results obtained from running a statistical analysis, called "Fisher" test. This test can be performed using simple software tools such as MINITAB. In order to run this statistical test, we had ten mixture types used to pave ten sections across the state of Missouri. These sections have different job mix formulas (JMFs) and also performed differently in the lab and in-situ. Using the laboratory testing results on SCB samples, three index parameters including FI, CRI, and BCI were calculated for four replicates. Based on the COV and the average of the resultant indices, the group is assigned to the sections. The more distinctive means and lower COVs will results in a higher number of groups. As shown, using FI led to two distinctive groups (A and B) while the CRI parameter yielded only one group, meaning that there is no difference between the sections in terms of the cracking performance in the lab. On the other hand, the introduced BCI parameter categorized the sections in five different groups. The higher number of groups for $\mathrm{BCI}$ indicates that the mean of the BCl's for each specific mix is more significantly different from the other mixtures and the repeatability of this parameter for different replicates of the same mix is higher. It should be noted that there are sections that have shared groupings such as MO94 with A, B, C, and D labels. This happens due to the similarities observed between the means of the BCI and normally happens when the number of studied sections is high. Yet, the grouping results say that the cracking of the US54_8 (group A), is significantly better than US61 (Group C, D, and E), or MO52_1 and 
MO151 (both in Group E) are statistically different from MO94, US50_1, US54 E, and US54_8.

Table 2-22 Statistical analysis of performance test results.

\begin{tabular}{|c|c|c|c|c|c|c|c|c|c|c|c|c|}
\hline \multirow[t]{2}{*}{ Index } & \multicolumn{5}{|c|}{ ANOVA test results } & \multicolumn{7}{|c|}{ Fisher test grouping results } \\
\hline & Factor & $\begin{array}{l}\text { Adj } \\
\text { SS }\end{array}$ & $\begin{array}{l}\text { Adj } \\
\text { MS }\end{array}$ & F-value & P-value & $\begin{array}{c}\text { Mixture } \\
\text { type }\end{array}$ & Mean & Group & & & & \\
\hline \multirow{10}{*}{$\begin{array}{l}\text { SCB } \\
\text { (FI) }\end{array}$} & $\begin{array}{c}\text { Mixture } \\
\text { type }\end{array}$ & 12.1 & 1.3 & 9.8 & 0.000 & US54_7 & 1.8 & A & & & & \\
\hline & & & & & & MO94 & 1.5 & $\mathrm{~A}$ & & & & \\
\hline & & & & & & US50_1 & 1.4 & $\mathrm{~A}$ & & & & \\
\hline & & & & & & US54 E & 0.6 & & B & & & \\
\hline & & & & & & MO52_1 & 0.6 & & B & & & \\
\hline & & & & & & US61 & 0.4 & & B & & & \\
\hline & & & & & & US63_2 & 0.4 & & B & & & \\
\hline & & & & & & MO151 & 0.3 & & B & & & \\
\hline & & & & & & US36 E & 0.2 & & B & & & \\
\hline & & & & & & US54_8 & 0.1 & & B & & & \\
\hline \multirow{10}{*}{$\begin{array}{l}\text { SCB } \\
\text { (CRI) }\end{array}$} & $\begin{array}{c}\text { Mixture } \\
\text { type }\end{array}$ & 86836 & 9648 & 2.08 & 0.069 & US54_7 & 329 & A & & & & \\
\hline & & & & & & MO94 & 312 & $\mathrm{~A}$ & & & & \\
\hline & & & & & & US50_1 & 285 & $\mathrm{~A}$ & & & & \\
\hline & & & & & & MO151 & 271 & A & & & & \\
\hline & & & & & & US61 & 238 & A & & & & \\
\hline & & & & & & US54 & 231 & A & & & & \\
\hline & & & & & & US54_8 & 214 & A & & & & \\
\hline & & & & & & US63_2 & 213 & A & & & & \\
\hline & & & & & & MO52_1 & 212 & A & & & & \\
\hline & & & & & & US36 & 161 & $\mathrm{~A}$ & & & & \\
\hline \multirow{10}{*}{$\begin{array}{l}\text { SCB } \\
\text { (BCI) }\end{array}$} & $\begin{array}{c}\text { Mixture } \\
\text { type }\end{array}$ & 90.84 & 10.09 & 7.36 & 0.000 & US54_8 & 7.6 & A & & & & \\
\hline & & & & & & US54 E & 6.6 & A & B & & & \\
\hline & & & & & & US50_1 & 5.9 & A & B & $\mathrm{C}$ & & \\
\hline & & & & & & MO94 & 5.8 & A & B & $\mathrm{C}$ & $\mathrm{D}$ & \\
\hline & & & & & & US61 & 4.2 & & & $\mathrm{C}$ & $\mathrm{D}$ & $\mathrm{E}$ \\
\hline & & & & & & US54_7 & 4.1 & & & & $\mathrm{D}$ & $\mathrm{E}$ \\
\hline & & & & & & US63_2 & 4.1 & & & & $\mathrm{D}$ & $\mathrm{E}$ \\
\hline & & & & & & US36 E & 4.0 & & & & $\mathrm{D}$ & $\mathrm{E}$ \\
\hline & & & & & & MO52_1 & 2.9 & & & & & $\mathrm{E}$ \\
\hline & & & & & & MO151 & 2.4 & & & & & $\mathrm{E}$ \\
\hline
\end{tabular}


The following observations were made from the results shown in Figure 2-62 and A one-way analysis of variance (ANOVA) was carried out, along with the Fisher method, to assess the ability of the three cracking indices studied to distinguish between the ten sets of field cores tested. A significance level of 0.05 (95\% reliability) was selected to examine the null hypothesis. Because the p-values for the FI and BCI indices are less different than 0.05 (Table 2-22), the mean values of the cracking index for ten sections are significantly. The P-value for the CRI index was found to be 0.069 , which is higher than the threshold of 0.05 , and indicates that the index is not able to differentiate between the sections. Table 2-22 shows the grouping results obtained from running a statistical analysis, called "Fisher" test. This test can be performed using simple software tools such as MINITAB. In order to run this statistical test, we had ten mixture types used to pave ten sections across the state of Missouri. These sections have different job mix formulas (JMFs) and also performed differently in the lab and in-situ. Using the laboratory testing results on SCB samples, three index parameters including FI, CRI, and BCI were calculated for four replicates. Based on the $\mathrm{COV}$ and the average of the resultant indices, the group is assigned to the sections. The more distinctive means and lower COVs will results in a higher number of groups. As shown, using FI led to two distinctive groups (A and B) while the CRI parameter yielded only one group, meaning that there is no difference between the sections in terms of the cracking performance in the lab. On the other hand, the introduced BCI parameter categorized the sections in five different groups. The higher number of groups for BCI indicates that the mean of the BCI's for each specific mix is more significantly different from the other mixtures and the repeatability of this parameter for different replicates of the same mix is higher. It should be noted that there are sections that have shared groupings 
such as MO94 with A, B, C, and D labels. This happens due to the similarities observed between the means of the BCI and normally happens when the number of studied sections is high. Yet, the grouping results say that the cracking of the US54_8 (group A), is significantly better than US61 (Group C, D, and E), or MO52_1 and MO151 (both in Group E) are statistically different from MO94, US50_1, US54 E, and US54_8.

Table 2-22:

- The FI index does not have a strong correlation with field performance results $(\mathrm{R} 2=0.48)$, and the lowest repeatability among the SCB indices studied $(\mathrm{COV}=$ 52.4\%). The statistical analysis showed that the FI could categorize the ten sections into only two separate groups. The FI index identified the most crack-resistant mixture but was unable to correctly rank the most cracking-prone mixture according to PASER deterioration rate (Table 2-21).

- The BCI exhibited the best correlation with field performance (R2=0.60) and had better test repeatability $(\mathrm{COV}=35.4 \%)$ than the FI index $(\mathrm{COV}=52.4 \%)$. The $\mathrm{BCI}$ index also by far categorized the ten mixtures into the most distinct groups (five unique groups of means were statistically identified). The BCI parameter also successfully identified the best and worst field sections in terms of index ranking as compared to PASER deterioration rate (Table 2-21).

- Although CRI showed the best repeatability among the indices (COV $=29.4 \%$ ), it showed the poorest correlation with field performance $(\mathrm{R} 2=0.27)$. Furthermore, statistical analysis showed that this index was not able to distinguish between the mixtures in statistically-relevant manner, as it categorized all sections into one group. 
Like the FI index, the CRI ranked the best performing section correctly according to PASER deterioration rate, but did not correctly rank the section with the highest PASER deterioration rate.

\subsubsection{Summary}

A comprehensive evaluation of three cracking indices derived from the semi-circular bend (SCB) asphalt cracking test conducted at an intermediate test temperature was performed. Among the three indices evaluated, a new index parameter termed BCI was introduced. The BCI can be simply computed from SCB test data by multiplying fracture energy by the post-peak displacement value that occurs when the testing load falls to $75 \%$ of the peak load. The resulting quantity is then multiplied by a scaling factor of 0.01 to arrive at the BCI. The three cracking indices were analyzed and statistically compared using a robust data set containing 52 asphalt mixtures characterized in the lab, along with ten field sections. The field sections afforded a comparison to real-world pavement cracking performance data.

The key findings of this study are now summarized. The lab and field data, along with the statistical analysis performed, indicated that:

- The average COV of the FI, BCI, and CRI indices was 34.6\%, $17.5 \%$, and $11.4 \%$, respectively, for the 52 laboratory compacted asphalt mixtures tested in this study.

- The existing FI and CRI indices were strongly correlated with one another $(\mathrm{R} 2=0.97)$, while the BCI index was only moderately correlated with the other two $(\mathrm{R} 2=0.77$, and $\mathrm{R} 2=0.73$ ). This result indicates that the BCI parameter produces a different cracking 
assessment as compared to the other two tests.

- The FI index was shown to have a strong correlation with post-peak slope, but a poor correlation to the fracture energy measured on the mixtures studied. The overwhelming dependency of the FI on the slope parameter clearly leads to having the poorest repeatability of the parameters investigated, due to the high variance measured for slope across testing replicates. In contrast, the correlation of BCI with its individual input parameters, i.e., fracture energy and post-peak displacement at 75\% peak load (L75) is high $(\mathrm{R} 2=0.85)$.

- The BCI index disagrees with trends produced by both the FI and CRI indices when a polymer-modified mixture is compared to a mixture with a softer, neat binder. Both the FI and CRI produced low cracking scores due to the overwhelming influence of the slope parameter (effectively negating the high fracture energy result). On the other hand, the BCI index, which is equally affected by fracture energy and mixture ductility, produced a more rational cracking score for the polymer modified mixture.

- All three SCB cracking indices showed similar limitations in some instances. For example, all three indices are shown to increase as air voids increase, which is counterintuitive. In addition, none-of-the-three indices were found to be sensitive to aggregate type or gradation.

- Ten field sections with different materials and service lives (that is, service life (age) of the most recent asphaltic overlay) were studied to characterize the capability of each SCB index to predict relative cracking performance. Neither the FI nor the CRI index produced a strong correlation to field performance in the Missouri sections studied $(\mathrm{R} 2=0.48$, and 0.27 , respectively). 
- A statistical analysis of data showed that the FI could classify the ten field sections into only two distinct groups, and the CRI classified all of them into a single group. Additionally, although FI and CRI both identified the most crack-resistant mixture, neither was able to correctly rank the most brittle mixture.

- On the other hand, the newly introduced BCI index showed a high correlation to field performance $(\mathrm{R} 2=0.60)$. Additionally, it categorized the ten sections into five distinct groups. This appears to have been a result of a favorable combination of (1) numerical differences between population means, and; (2) the presence of a relatively low COV for individual group means.

In conclusion, the BCI parameter proposed in this study was compared with other existing cracking indices and showed promise in its ability to distinguish between asphalt mixtures and to rank them according to their field cracking potential. The BCI exhibits a strong correlation with its individual input parameters (fracture energy and post-peak displacement at $75 \%$ peak load) and, more importantly, whereas the FI and CRI indices were shown to be dwarfed by the effect of the slope and peak load parameters, respectively. Further investigation of the new BCI index is recommended, including a broader array of field sections, component materials, and aging levels. 


\section{CHAPTER 3: MACHINE LEARNING-BASED PREDICTION MODELS FOR PERFORMANCE OF ASPHALT MIXTURES}

In this part of the study a prediction tool was developed to predict the performance of asphalt mixture at high and low temperatures. This tool is based on two different prediction models for DC(T) fracture energy and Hamburg wheel track tests. For DC(T) fracture energy model, genetic programming was used to develop the prediction model, and Convolution Neural Network $(\mathrm{CNN})$ and genetic programming were used to train the Hamburg wheel track models. A database containing a comprehensive collection of Hamburg and DC(T) tests results were used to develop the machine learning-based prediction models. This tool can be used for pre-design purposes to design an asphalt mixture with balanced performance in rutting and cracking. The models were formulated in terms of typical influencing mixture properties variables such as asphalt binder hightemperature performance grade (PG), mixture type, aggregate size, aggregate gradation, asphalt content, total asphalt binder recycling content and tests parameters like temperature and number of cycles. Models accuracy were assessed through a rigorous validation process and found to be quite acceptable, despite the relatively small size of the training set. Since performing performance tests might be cost-restrictive for some users, using the proposed ML-based models can save time and expense during the material screening phase. By integrating Phase I and Phase II of the project, we developed a prediction tool to predict the performance of asphalt mixture at high and low temperature. This tool can be used for pre-design purposes to design a asphalt mixture with balanced performance in rutting and cracking. Therefore, this tool will save time and money for such design procedures.

\subsection{NEW MACHINE LEARNING-BASED PREDICTION MODELS}




\section{FOR FRACTURE ENERGY OF ASPHALT MIXTURES}

\subsubsection{Introduction}

Cracking is one of the principal distresses in asphalt pavements. This phenomenon can be classified into two main categories: load-associated and non-load-associated cracking. Fatigue cracking belongs to the first category. It occurs when tensile stresses due to repetitive traffic loading exceed the tensile strength of the material. As a result of excessive tensile stress, micro-cracks form, grow, and incorporate into macro-cracks leading to aggregate cracking and pavement structural failure [42]. Thermal cracking or low-temperature cracking is a major non-load-associated asphalt pavement distress in cold climates [93]. As the temperature drops, thermal stresses develop due to the differential contraction of the binder and aggregate in the asphalt mastic. In addition, the pavement tends to develop longitudinal tensile strain due to restrained contraction caused by decreasing temperatures [94], [95]. Continuous construction (infrequent transverse joints) and bonding to underlying layers creates the aforementioned restraint. If the thermal stresses exceed the tensile strength of the asphalt mixture, transversely-oriented, thermal cracks can form (see Figure 3-1). Reflective cracking is also a common distress in asphalt pavement surfaces, driven by stress concentrations resulting from the placement of a continuous asphalt overlay on top of a discontinuous pavement (such as jointed concrete pavement, or deteriorated, cracked pavement). 


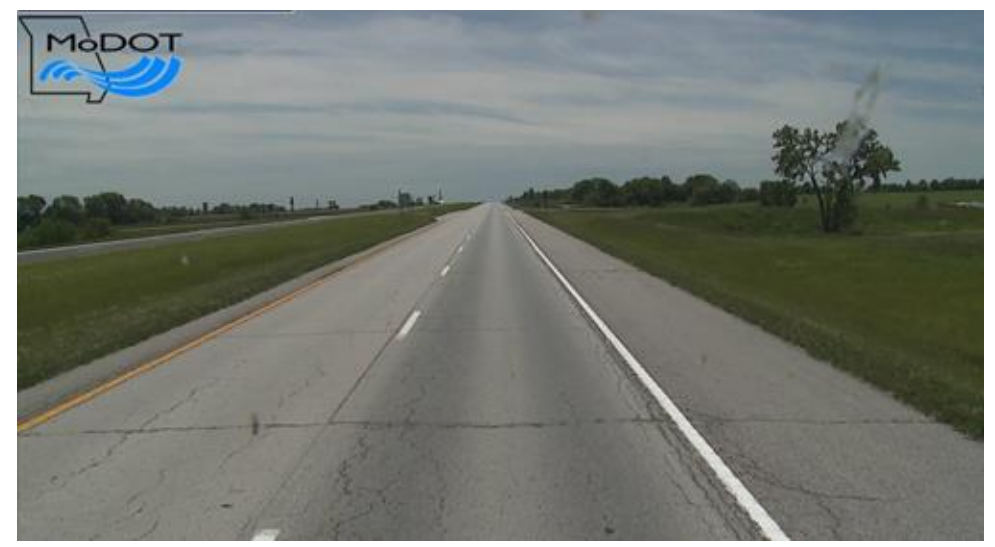

\section{Figure 3-1 Typical thermal cracks in a pavement segment located on U.S. Route 63 in Missouri.}

The most widely-used tests to evaluate the thermal cracking resistance of asphalt mixtures are thermal stress restrained specimen test (TSRST), indirect tensile creep and strength test (IDT), semi-circular bending (SCB) test, and disc-shaped compact tension (DC(T)) test. Two main features of the $\mathrm{DC}(\mathrm{T})$ test is that it allows for testing cylindrical cores obtained from the field or compacted Superpave Gyratory Compacter (SGC) specimens, and its large fracture surface area [62]. Thermal cracking in asphalt pavements commonly occurs in absolute tensile opening or fracture Mode I. In other words, the cracks grow perpendicular to the direction of the thermal-induced stresses in the pavement, i.e., transverse to the direction of traffic [62]. It is believed that the low-temperature $\mathrm{DC}(\mathrm{T})$ test controls not only the first mode of cracking but also accounts for the second mode partially [96]. Several studies have suggested advantages of the DC(T) over other testing procedures in controlling thermal cracking and correlation with field performance [3], [97]-[99]. Fracture energy of asphalt mixture is the most important output of the $\mathrm{DC}(\mathrm{T})$ test. The performance-based specifications utilize fracture energy as performance parameter because it correlates well with the field cracking performance [100]. This parameter measures the amount of work required to fracture an asphalt core along a fabricated notch 
in the specimen (ASTM D7313-13). Figure 3-2 shows the correlation between observed transverse cracking in asphalt mixtures and their corresponding $\mathrm{DC}(\mathrm{T})$ fracture energies based on the data from states of Illinois, Minnesota, Missouri, and Wisconsin [99]. As seen in this figure, fracture energy is strongly correlated with low-temperature cracking.

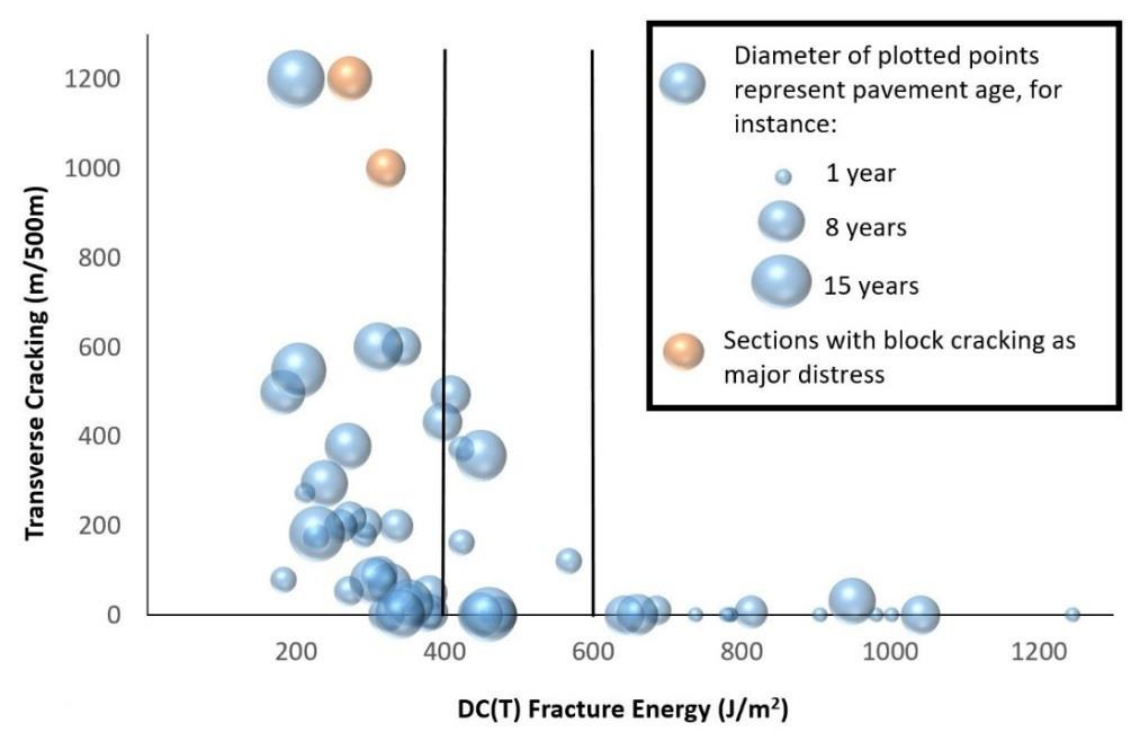

Figure 3-2 DC(T) fracture energy vs. transverse cracking [99].

However, asphalt mixture performance testing is sometimes cost-prohibitive and/or component materials may not be available or known during the pavement thickness design process. Therefore, developing a relationship between the fracture energy measured using the DC(T) test and known or estimated parameters from the asphalt mixture specimen leads to considerable savings in construction cost and time. While there are some prediction models for fracture in bituminous materials [101], [102], none of them deals with correlating the asphalt fracture energy from performance tests with component mixture properties. 
Based on this motivation, the goal of this study was to develop comprehensive models for predicting the fracture energy of asphalt mixture specimens. To this aim, robust machine learning methods termed gene expression programming (GEP) and hybrid artificial neural network/simulated annealing (ANN/SA) are used to build the prediction models. The machine learning algorithms are calibrated using a set of $\mathrm{DC}(\mathrm{T})$ fracture energy data for asphalt mixtures. A key feature of the proposed models is that they include simultaneous effects of various testing, binder and aggregate-related parameters, along with modern asphalt ingredients such as recycled materials, rejuvenators, rubber and their interactions. The derived design equations can be considered as variable tools for routine pre-design practice via spreadsheet programming or hand calculations. The models are further evaluated using some recommended criteria and are benchmarked against one other.

\subsubsection{Machine Learning Techniques}

In general, a challenging issue for modeling the fracture energy is to find the most suitable factors and simulation techniques. Traditional regression analysis is one of the basic techniques for this purpose. However, structure of a regression model should be defined in advance, which is not always an optimal solution [103]. To deal with this issue, researchers are now widely using machine learning (ML) techniques as powerful empirical regression approaches [104]. ML is a subdivision of artificial intelligence (AI) inspired by biological learning processes. The ML algorithms can learn the underlying behavior of a system from a set of training data without a prior knowledge about the nature of the relationships between the data [105]. ML is a collection of various algorithms such as ANN, support vector machines (SVM), etc. These techniques have proven useful for many 
civil engineering applications including prediction of asphalt concrete properties [106][108]

Despite the reliable performance of these techniques, they are considered as blackbox tools. That is, they are usually unable to generate practical prediction equations [103]. In addition, the ML techniques and more specifically ANNs are prone to being stuck in local minima [109]. This issue can be tackled by integrating the ANN training process with powerful optimization algorithms such as simulated annealing (SA) [109]-[113]. While a hybrid ANN and SA (ANN/SA) method has a remarkably better prediction performance than standard ANN [109], there are not any studies in the area of its application to the characterization of the bituminous material. In this study, the so-called ANN/SA-based black-box system is opened, and the developed model is converted it into a practical design equation. In addition, with the advent of genetic programming (GP) [114], the limitation of the black-box methods has been tackled. This is because the GP-based methods can generate precise non-linear mathematical models for an investigated system. The current research is also aimed at deploying one of the robust branches of classical GP called GEP to develop highly nonlinear models for the prediction of fracture energy of asphalt mixtures.

\subsubsection{Gene Expression Programming}

Invented by Koza (1992), GP is an extension to genetic algorithm (GA) that can automatically generate mathematical models following the Darwinian evolutionary theory. While the GA solutions are a sequence of numbers, GP creates programs that usually have a tree-shape structure [103]. Three major types of GP are shown in Figure 3-3. 


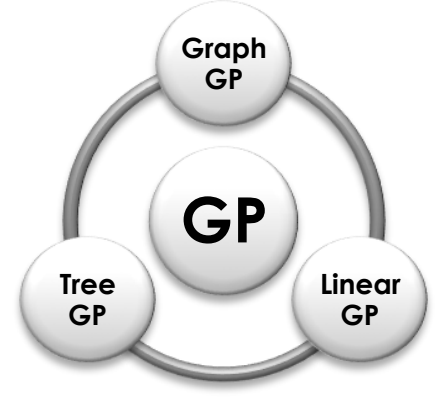

\section{Figure 3-3 Different types of GP [103]}

GEP is a linear GP method proposed by [115].GEP uses strings with a fixed length of characters to define solutions. The evolved solutions are expressed as Expression Trees (ETs). GEP can evolve complex programs composed of several subprograms [116]. Genes in GEP are a combination of functions or terminal sets [103], [117]. An example of genes expressed in Karva notation (KN) is as follows:

+.×.cos.a. -. +. +.× b.a.c. $-4 . b . a$

This KN can be transformed into a tree-shape ET as shown in Figure 3-4.

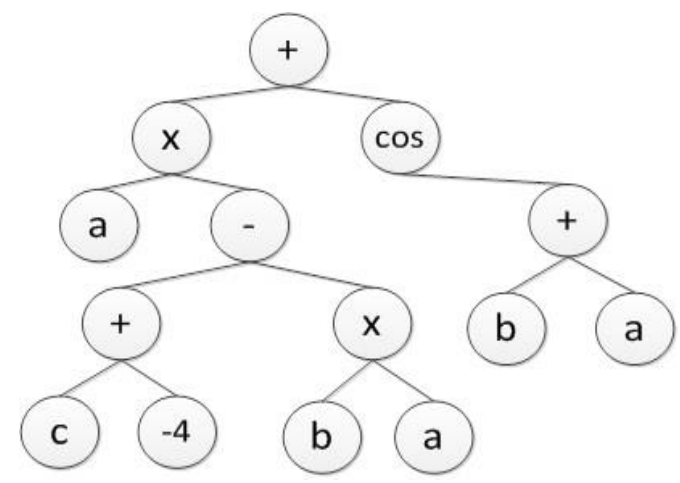

Figure 3-4 Translating KN to an ET

The ET given in Figure 3-4 can be expressed in a functional form as: 


$$
a((c-4)-(b \times a))+\cos (b+a)
$$

The main steps that GEP follows to obtain a solution can be found in [115]. GEP uses various genetic operators such as crossover, mutation and rotation [118]ra. In order to reshape the ETs, the rotation operator plays a key role by rotating two parts of a program [103], [115]. For instance, Eq. (3) shows how the first five elements of the original gene are rotated to create a new program:

$$
+.+. \times \text { b.a.c. }-4 \text {.b.a.+.×.cos.a. }-
$$

The above equation can be transformed to $(b+a)+(c \times-4)$, as visualized in Figure 3-5. More details about classical GP and GEP can be found in [103], [115].

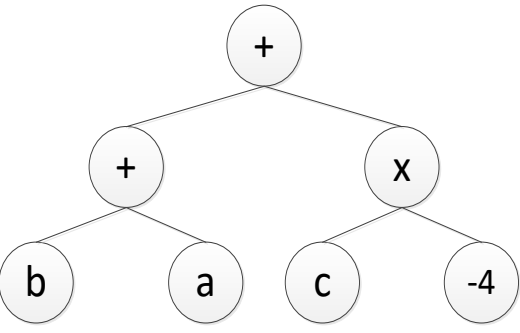

\section{Figure 3-5 The rotated ET}

\subsubsection{Hybrid ANN/SA Method}

ANNs are among the most widely used branches of ML. Figure 3-6 shows the basic structure an ANN neuron. In this neuron, a weight coefficient is applied to the inputs and a summation function sums the weighted inputs and bias. The output is then calculated by a transfer function [109], [119], [120]. 


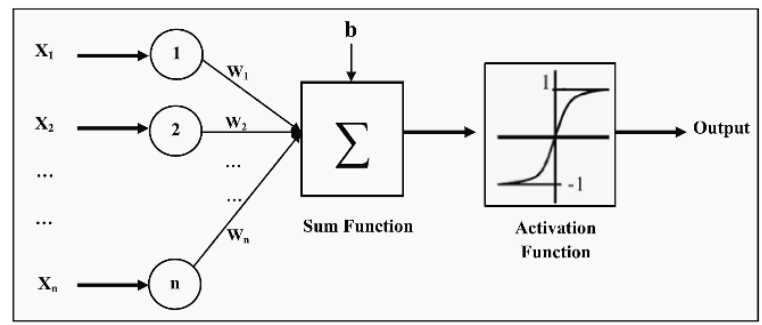

Figure 3-6 Basic structure of an ANN neuron [120]

One of the concerns during the ANN training process is to find optimal initial weight values [109].This can be done using robust optimization algorithm such as SA [113]. More details about the basic SA method can be found in [121], [122]. A flowchart describing the ANN/SA method is presented in Figure 3-7. Boltzmann distribution is used in the SA method to accept the solution improving the performance. The SA-based optimization process is shown in Figure 3-8. The parameters involved in the algorithm are configuration of energy $(E(x)), k$ is Boltzmann's constant $(k)$, and temperature $(T)$ to define the probability $\left(P_{a}\right)$ of any actual state of $x$ [121], [123]. Two cooling schedules in SA are presented in Figure 3-9. More details about these schedules and the ANN/SA training process is given in [109], [121], [123]. 


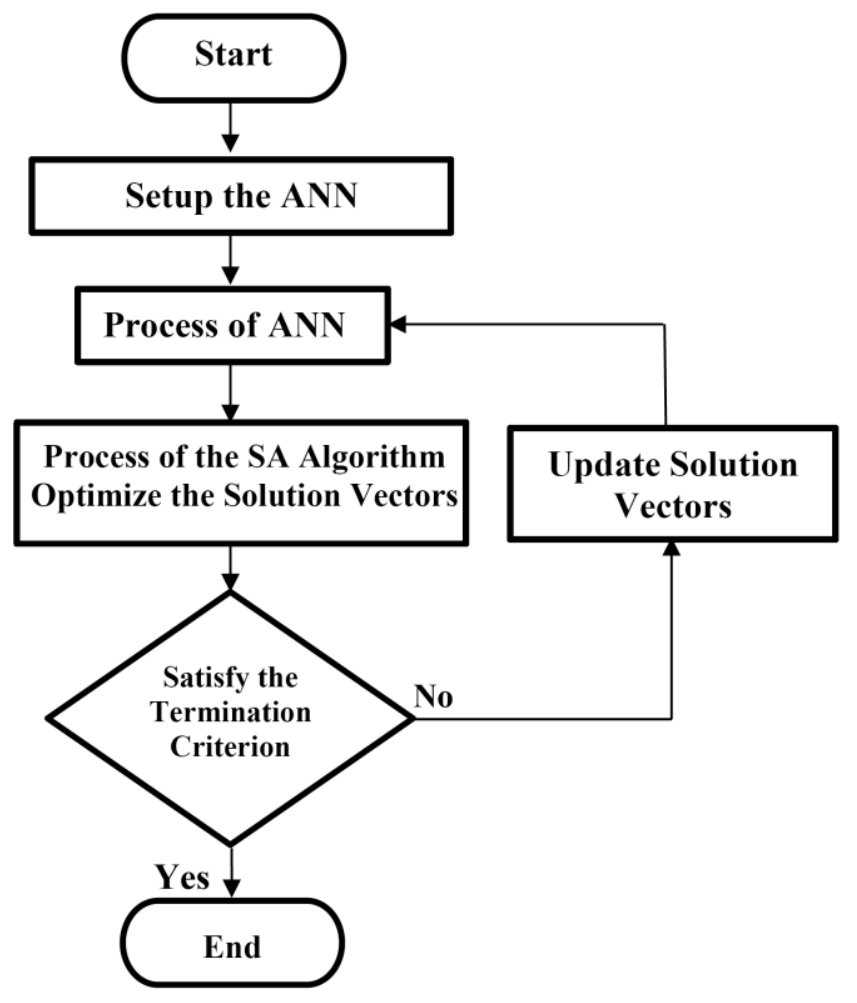

Figure 3-7 Flowchart of the ANN/SA method 


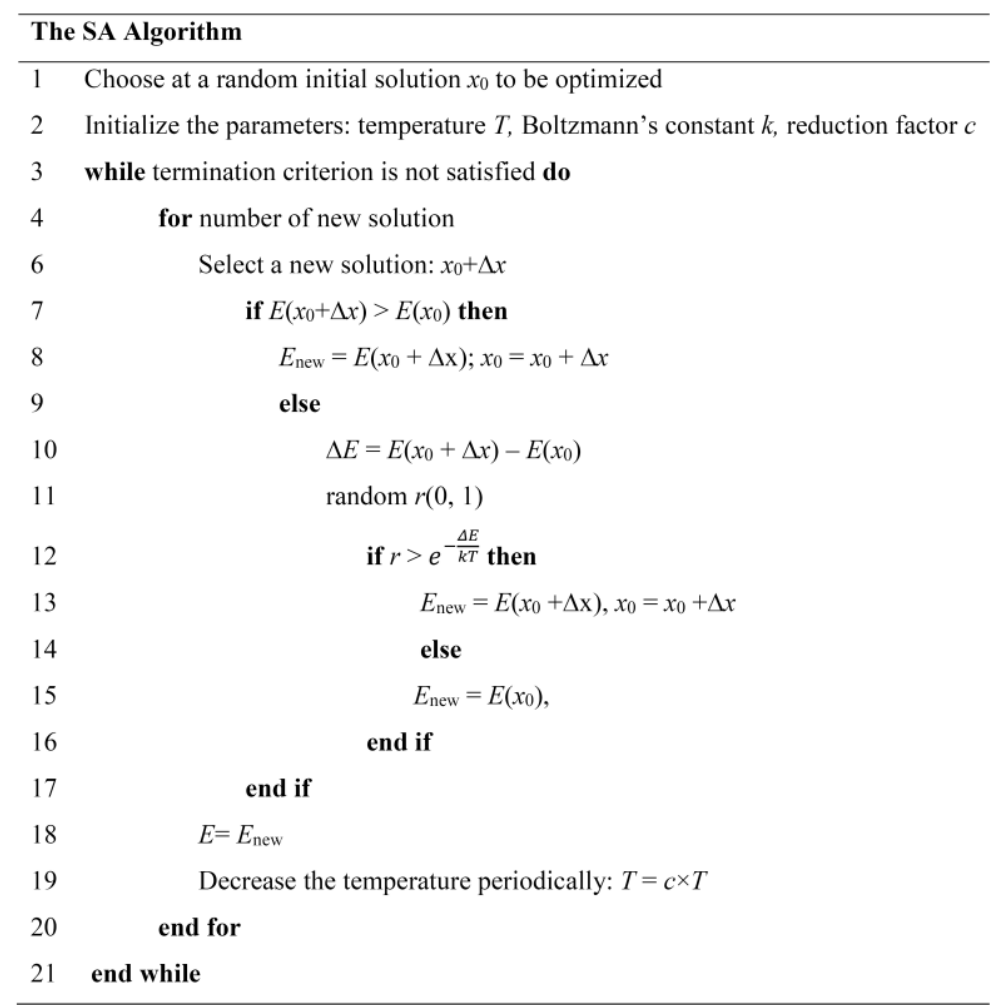

Figure 3-8 Pseudo-code for the SA algorithm

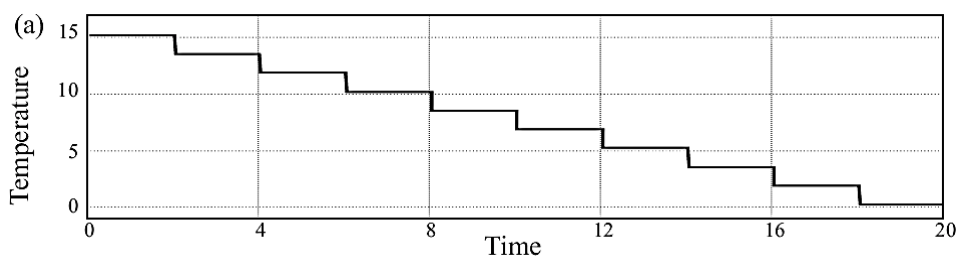

(a) Linear cooling

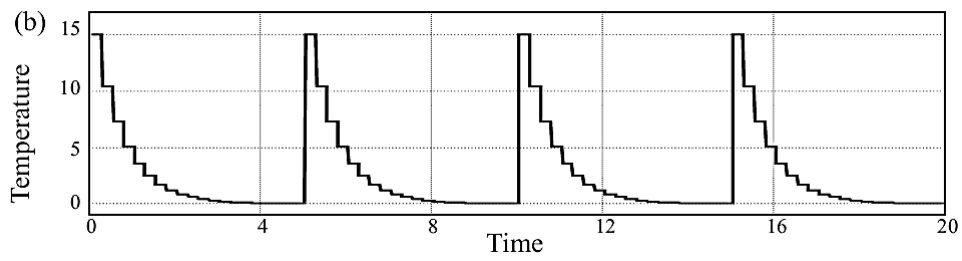

(b) Temperature cycling

Figure 3-9 Cooling schedules in SA [109] 


\subsubsection{Modeling of Fracture Energy of Asphalt Concrete}

As discussed before, none of the previous studies have taken into account the combined effect of testing, binder, aggregate, and recycling parameters for the prediction of the asphalt concrete fracture energy $\left(G_{f}\right)$. This is unfortunate, since the influence of these parameters on $G_{f}$ is now fairly well-understood [96]. In this research, the parameters which were believed to affect the low temperature cracking resistance of asphalt concrete in $\mathrm{DC}(\mathrm{T})$ test were extracted from relevant literature and are categorized in three groups: testing temperature, aggregate-related parameters, and binder-related parameters.

DC(T) testing temperature: According to the ASTM specification (ASTM D7313), the $\mathrm{DC}(\mathrm{T})$ test is performed at 10 degrees warmer than the low temperature performance grade (LTPG) of the binder. As the sample temperature is reduced, the asphalt binder becomes more brittle and its fracture resistance decreases [3], [62]. SCB and DC(T) testing temperatures are defined based on the performance grade (PG) of the binder [124]. Statistical analysis showed that testing temperature is a significant parameter to explain the variations of the SCB and $\mathrm{DC}(\mathrm{T}) G_{f}[124]$.

Aggregate-related parameters: The aggregates form the skeleton of asphalt pavements. Depending on the required performance, dense-graded, gap-graded, and opengraded gradation types are used in asphalt production. Nominal maximum size (NMAS) provides an appropriate means to compare the size range of each gradation type. In addition to the gradation, aggregate type and chemical mineralogy affect the binder-aggregate interaction and the low temperature cracking performance of asphalt mixture. Buttlar et al. (2017) observed that mixtures with crushed gravel aggregates outperformed the mixtures 
with dolomitic limestone in terms of the $\mathrm{DC}(\mathrm{T}) G_{f}$ and Hamburg rut depth. Benefiting from more durable aggregates and higher asphalt content, stone matrix asphalt (SMA) exhibited superior fracture resistance comparing to conventional dense-graded hot mix asphalt (HMA) [96]. Also, the mean $\mathrm{DC}(\mathrm{T})$ and $\mathrm{SCB} G_{f}$ of granite mixtures was measured to be higher than limestone mixtures [124], [125]

Binder-related parameters: Asphalt binder plays and important role in low temperature behavior of asphalt mixture. If properly chosen, the binder can provide the flexibility and ductility needed by asphalt concrete to relax the induced thermal stress. Therefore, a soft binder outperforms a stiff binder in terms of low temperature cracking [96]. In spite of this fact, the soft binder may lead to rutting distress at high climates. As a result, a binder with a higher range of useful temperature interval (UTI) is desirable to withstand the cracking and rutting at low and high temperatures, respectively. In addition to the neat binder gradation, application of recycled materials such as reclaimed asphalt pavement (RAP) and reclaimed asphalt shingles (RAS), and also additives (e.g. rejuvenator agents, warm mix, styrene-butadiene-styrene (SBS), and acids) affect the binder gradations and properties [126], [127].

- Recycled materials (RAP and RAS)

Behnia et al. (2011) performed DC(T) on asphalt mixtures containing 0, 10, 20, 30, 40 and $50 \%$ Asphalt binder replacement (ABR) by RAP. Results showed that addition of RAP consistently decreased the $G_{f}$ of asphalt samples containing PG58-28 binder. However, mixtures with PG64-22 binder were less sensitive to addition of RAP [16]. Moreover, researchers reported that the mixtures with RAS had lower DC(T) $G_{f}$ and higher DC(T) peak loads than the control mixture [128]. 
- Rubber modification

To address the environmental concerns about crumb tire rubber and to improve the performance of asphalt concrete, ground tire rubber (GTR) and engineered crumb rubber (ECR) have attracted considerable attention. An improvement in low temperature performance of asphalt mixture is reported after using these additives in indirect tension (IDT) and asphalt concrete cracking device (ACCD) tests. Also, incorporating ground tire rubber with PG64-28 and PG64-34 led to an improvement in the $\mathrm{DC}(\mathrm{T}) G_{f}$ of SMA mixtures at both -12 and $-18^{\circ} \mathrm{C}[96]$.

- Additives

Polyphosphoric acid (PPA)+SBS and SBS modified mixtures showed higher fracture resistance in DC(T) test than PPA and PPA+Elvaloy modified mixtures [129]. Also, PPA helped mixtures with PG58-28 binder yield higher $G_{f}$ than those with PG64-28 [96]. The DC(T) test on both the laboratory compacted and field collected samples showed that the crumb rubber warm mix asphalt (CR-WMA) with Evotherm and the CR-HMA exhibited a comparable low temperature performance [130].

Table 3-1 presents a summary of the parameters included in various studies. On the basis of this literature review, we have developed new models using the GEP and ANN/SA approaches correlating $G_{f}$ with the influencing variables as follows:

$\mathrm{G}_{\mathrm{f}}=\mathrm{f}(\mathrm{UTI}, \mathrm{LTPG}, \mathrm{AC}, \mathrm{NMAS}, \mathrm{RAP}, \mathrm{RAS}, \mathrm{G}, \mathrm{AT}, \mathrm{CRC}, \mathrm{T})$

(4)

Where,

$U T I\left({ }^{\circ} \mathrm{C}\right)$ : Useful temperature interval (i.e., for PG 64-22 binder, UTI $=64-(-22)=86^{\circ} \mathrm{C}$ )

$\operatorname{LTPG}\left({ }^{\circ} \mathrm{C}\right)$ : Low temperature performance grade (for PG 64-22 binder, this is $-22^{\circ} \mathrm{C}$ )

$A C(\%)$ : Asphalt content

NMAS (mm): Nominal maximum aggregate size. In this study, NMAS can take $9.5 \mathrm{~mm}$ or $12.5 \mathrm{~mm}$.

$R A P(\%)$ : Reclaimed asphalt pavement

RAS (\%): Reclaimed asphalt shingles 
G: Gradation type (Dense: 1; SMA: 2; Gap-Graded: 3)

AT: Aggregate type (Limestone: 1; Granite: 2; Gravel: 3)

CRC (\%): Crumb rubber content (No additive: $0 ; 12 \%$ GTR $\quad: 1 ; 10 \%$ ECR: $2 ; 10 \%$

Evoflex: 3)

$T\left({ }^{\circ} \mathrm{C}\right)$ : Testing temperature

Table 3-1 A summary of the parameters affecting low-temperature cracking resistance of asphalt concrete in the DC(T) test

\begin{tabular}{|c|c|}
\hline Authors & Variables Investigated \\
\hline \multirow{2}{*}[124]{} & Testing temperature \\
\hline & Aggregate source \\
\hline \multirow{3}{*}[96]{} & Aggregate type \\
\hline & Aggregate gradation \\
\hline & Binder PG grade \\
\hline [126] & Recycled materials (RAP) \\
\hline \multirow{3}{*}[127]{} & Additive \\
\hline & Binder PG grade \\
\hline & Polymer and rubber modification \\
\hline$[16]$ & \multirow{2}{*}{ Recycled materials (RAP) } \\
\hline$[126]$ & \\
\hline$[128]$ & Recycled materials (RAS) \\
\hline$[127]$ & Rubber modification \\
\hline$[18]$ & Rubber modification \\
\hline [129] & Additive \\
\hline [96] & Additive \\
\hline \multirow{2}{*}[130]{} & Additive \\
\hline & Rubber \\
\hline
\end{tabular}




\subsubsection{Experimental Database}

The models are developed using 51 test results obtained from previous studies [131][133] and 45 test results from the current study. The DC(T) tests were performed according to ASTM D7313-07. The testing procedure included conditioning of the fabricated specimen at the selected test temperature in a temperature-controlled chamber for a minimum of two hours. The $\mathrm{DC}(\mathrm{T})$ test temperature is generally $10{ }^{\circ} \mathrm{C}$ higher than the $\mathrm{PG}$ low-temperature grade of the binder used in the asphalt mixture. After the conditioning, the specimens were suspended on loading pins in DC(T) machine, shown in Figure 3-10. The specimens were pulled with steel loading dowels through drilled holes, forcing a crack to propagate outward from the notch. The test was carried out at a constant Crack Mouth Opening Displacement $(\mathrm{CMOD})$ rate of $1 \mathrm{~mm} / \mathrm{min}(0.017 \mathrm{~mm} / \mathrm{s})$. The test was stopped when the post-peak loading reaches a nominal level of $0.1 \mathrm{kN}$. A typical load-CMOD curve is shown in Figure 3-11.

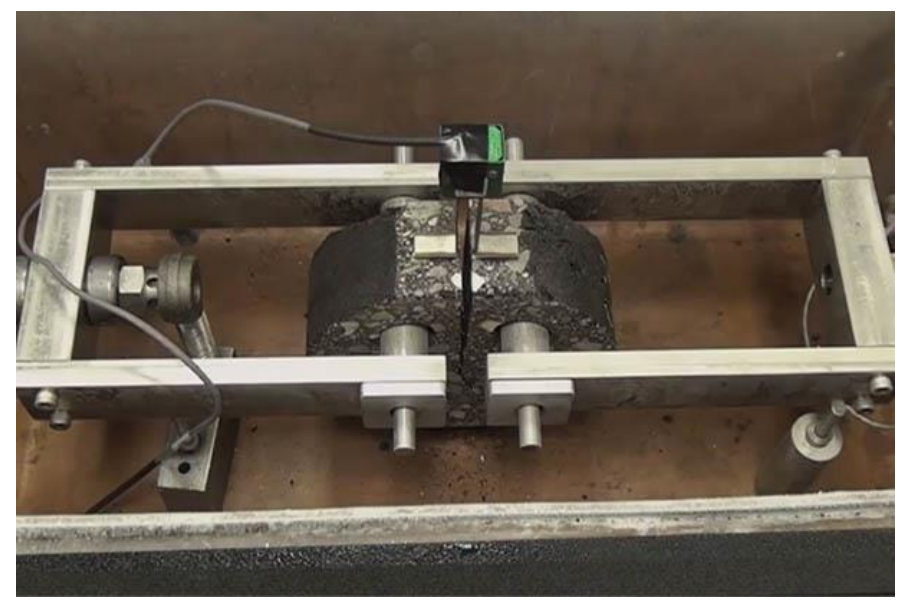

Figure 3-10 The test Quip DC(T) apparatus 


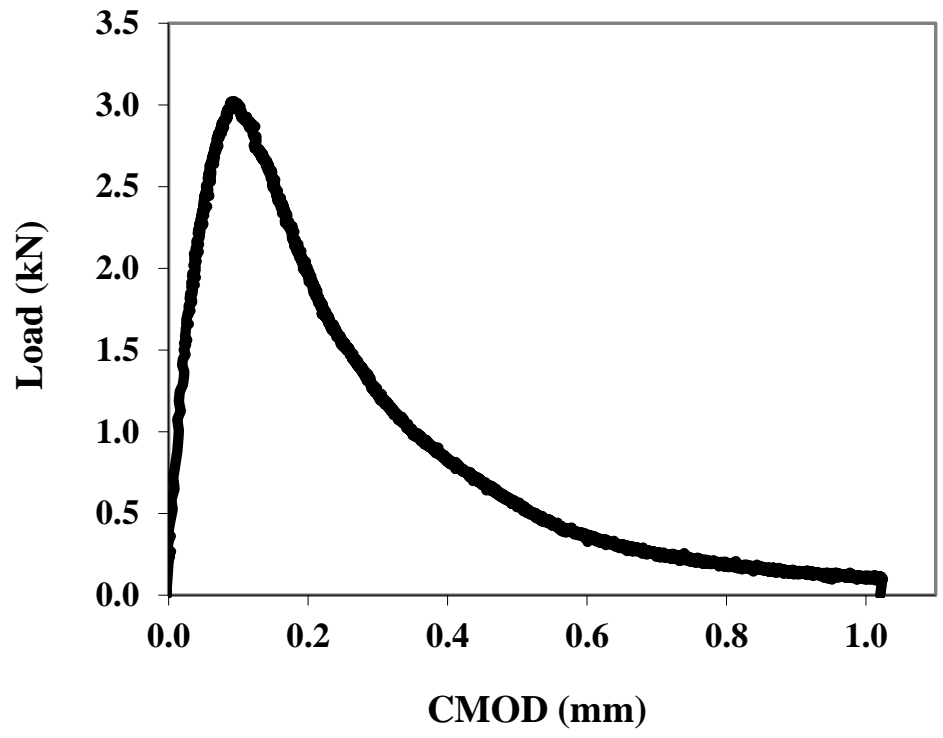

Figure 3-11 Typical Load-CMOD curve from DC(T) testing

$G_{f}$ can be obtained by measuring the area under the load-CMOD curve and dividing it by the fractured area (ligament length times thickness) as follows:

$$
G_{f}=\frac{A R E A}{B \cdot L}
$$

Where,

$G_{f}=$ Fracture energy, in $\mathrm{J} / \mathrm{m}^{2}$

AREA $=$ Area under Load-CMOD ${ }_{\text {FIT }}$ curve, until the terminal load of $0.1 \mathrm{kN}$ is reached

$\mathrm{B}=$ Specimen thickness, in $\mathrm{m}$, generally $0.050 \mathrm{~m}$ (except for field cores)

$\mathrm{L}=$ Ligament length, usually around $0.083 \mathrm{~m}$

The numerator of the equation represents the area under the Load-CMOD curve, which is the work required to create the fracture surface. The area is generally computed using the quadrangle rule for numerical integration. The CMOD curve is generally the 
fitted CMOD, where a straight line is fit through the CMOD vs. time curve to enable data smoothing (ASTM D7313-07).

Table 3-2 presents the descriptive statistics of the variables used in this study. To deal with overfitting, three random divisions were considered for the data: learning $(\sim 70 \%)$, validation $(\sim 10 \%)$ and testing $(\sim 20 \%)$ [134]-[137]. The learning and validation subsets were used to calibrate and evaluate the models, respectively. Since they were both involved in selecting the final models, they were labeled as training data in the subsequent analyses. To visualize the distribution of the samples, the data are presented by frequency histograms (see Figure 3-12). As observed from Figure 3-12, the distributions of the predictor variables are not uniform. Arguably, better model performance is expected for cases within the ranges given in Table 3-2 and where the densities of the variables are higher as shown in Figure 3-12.

Table 3-2 Descriptive statistics of the variables

\begin{tabular}{cccccccccccc}
\hline Parameter & $\begin{array}{c}U T I \\
\left({ }^{\circ} \mathrm{C}\right)\end{array}$ & $\begin{array}{c}L T P G \\
\left({ }^{\circ} \mathrm{C}\right)\end{array}$ & $\begin{array}{c}A C \\
(\%)\end{array}$ & $\begin{array}{c}\text { NMAS } \\
(\mathrm{mm})\end{array}$ & $\begin{array}{c}R A P \\
(\%)\end{array}$ & $\begin{array}{c}R A S \\
(\%)\end{array}$ & $G$ & $A T$ & $\begin{array}{c}C R C \\
(\%)\end{array}$ & $\begin{array}{c}T \\
\left({ }^{\circ} \mathrm{C}\right)\end{array}$ & $\begin{array}{c}G_{f} \\
\left(\mathrm{j} / \mathrm{m}^{2}\right)\end{array}$ \\
\hline Mean & 88.35 & -29.37 & 6.14 & - & 5.41 & 8.81 & - & - & - & -17.37 & 710.64 \\
Median & 86.00 & -28.00 & 6.00 & - & 0.00 & 0.00 & - & - & - & -12.00 & 658.00 \\
Mode & 86.00 & -28.00 & 6.00 & - & 0.00 & 0.00 & - & - & - & -12.00 & 590.00 \\
Kurtosis & -0.43 & -0.68 & 0.27 & - & 3.70 & -0.90 & - & - & - & 2.66 & 10.83 \\
Skewness & -0.09 & -0.27 & -0.03 & - & 1.89 & 0.93 & - & - & - & -1.75 & 2.78 \\
Range & 28.00 & 18.00 & 2.30 & - & 35.20 & 33.50 & - & - & - & 30.00 & 2434.70 \\
Minimum & 70.00 & -40.00 & 5.10 & 9.50 & 0.00 & 0.00 & 1 & 1 & 0 & -42.00 & 283.00 \\
Maximum & 98.00 & -22.00 & 7.40 & 12.50 & 35.20 & 33.50 & 3 & 3 & 3 & -12.00 & 2717.70 \\
\hline
\end{tabular}



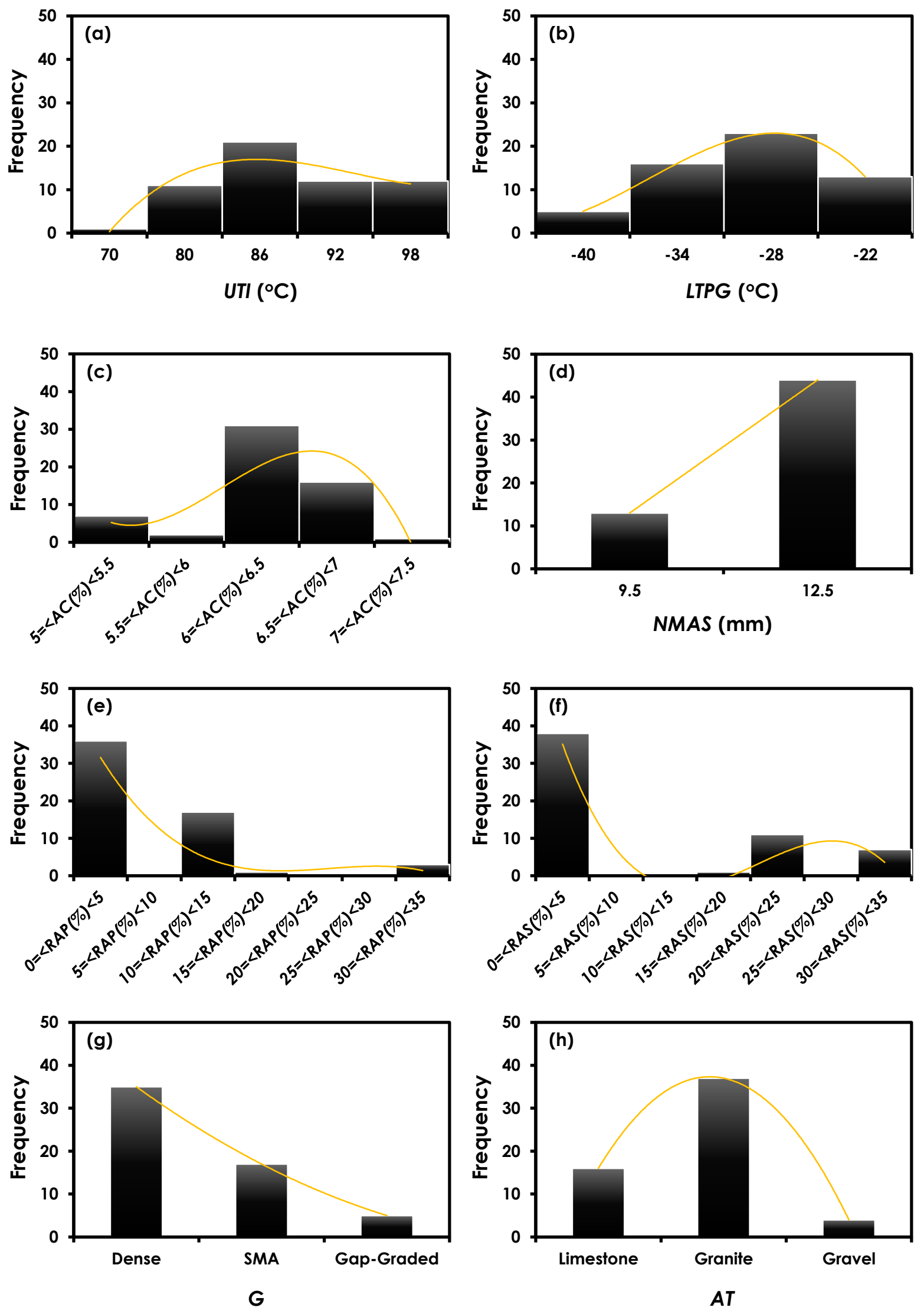

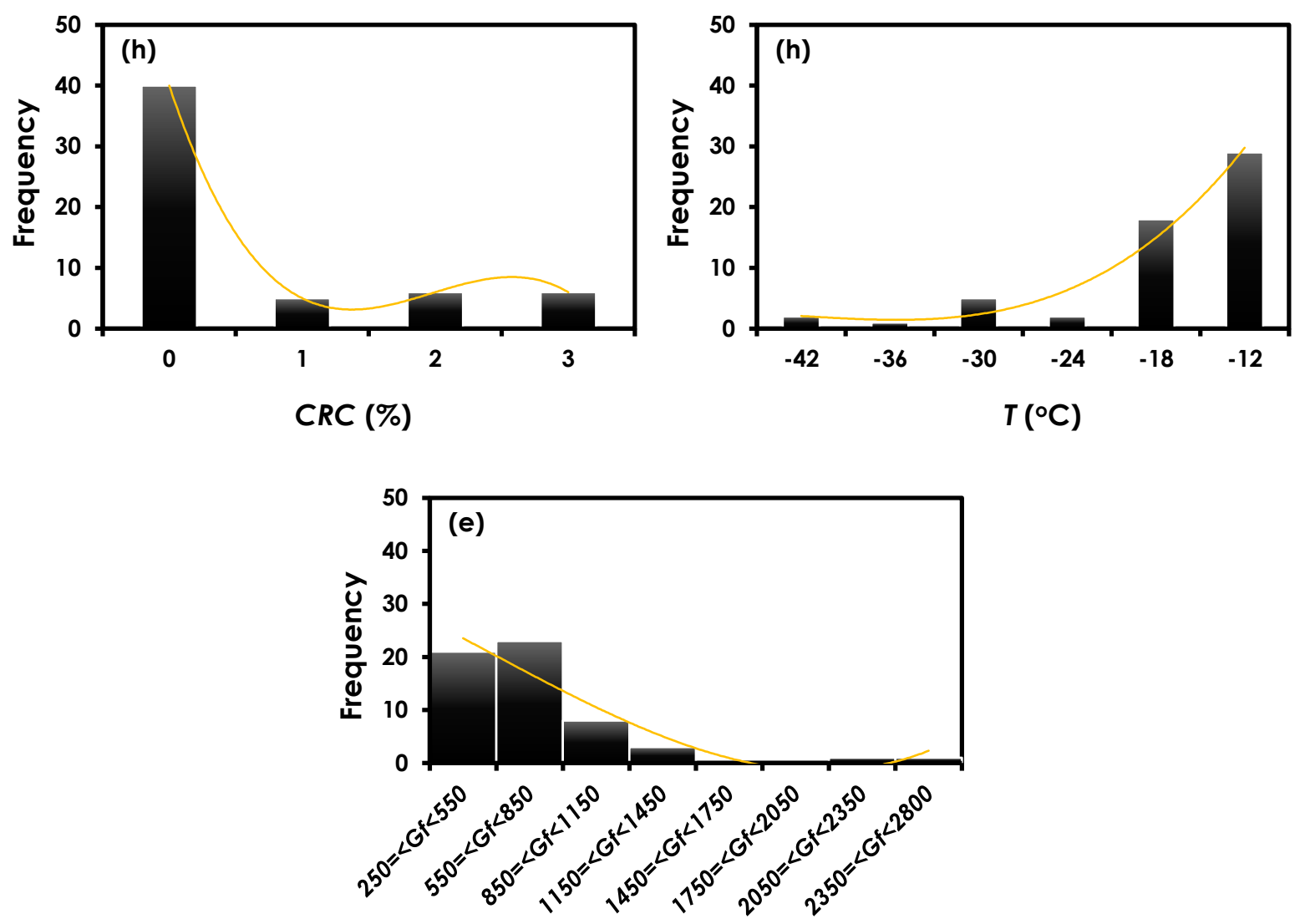

Figure 3-12 Distribution histograms of the variables

\subsubsection{Performance Measures}

Determination coefficient $\left(\mathrm{R}^{2}\right)$, root mean squared error (RMSE) and mean absolute error (MAE) were used as the performance measures:

$$
\begin{aligned}
& R^{2}=\frac{\left(\sum_{i=1}^{n}\left(O_{i}-\overline{O_{l}}\right)\left(t_{i}-\overline{t_{l}}\right)\right)^{2}}{\sum_{1=1}^{n}\left(O_{i}-\overline{O_{l}}\right)^{2} \sum_{i=1}^{n}\left(t_{i}-\bar{t}_{l}\right)^{2}} \\
& R M S E=\sqrt{\frac{\sum_{i=1}^{n}\left(O_{i}-t_{i}\right)^{2}}{n}} \\
& M A E=\frac{\sum_{i=1}^{n}\left|O_{i}-t_{i}\right|}{n}
\end{aligned}
$$


where,

$O_{i}$ : Measured output

$t_{i}$ : Predicted output

$\overline{O_{i}}$ : Average of measured outputs

$\overline{t_{i}}$ : Average of predicted outputs

$n$ : Number of samples

The best GEP models were selected based on a multi-objective strategy that involved simplicity of the models and their least prediction error during the learning and validation stages [137].

\subsubsection{Model Development Using the GEP Method}

After a series of preliminary runs and observing the GEP algorithm performance, UTI, LTPG, AC, NMAS, RAP, RAS, G, AT, CRC, and $T$ were determined to be the most influencing parameters. Then, the GEP method was used to arrive at the best mathematical models. Several runs were carried out to determine the optimized GEP parameters. There are five main parts for setting GEP parameters including: general setting, complexity increase, genetic operators, numerical constant, and fitness function. In general part, number of chromosomes affects the time of the simulation run. By increasing the number of chromosomes, the runs take longer. The head size shows the complexity of each term in the evolved model [103]. Table 3-3 shows a set of parameters used during the GEP simulations.

Table 3-3 The optimal parameter setting for the GEP algorithm 


\begin{tabular}{lll}
\hline & \multicolumn{1}{c}{ Parameter } & \multicolumn{1}{c}{ Settings } \\
\hline General & & \\
& Chromosomes & $20,30,50$ \\
& Genes & $3,4,5$ \\
& Head size & $8,10,15$ \\
& Linking function & Addition \\
& Function set & $+,-, \times, /, \sqrt{ }, \sqrt{ }, \mathrm{Ln}, \mathrm{Log}$, power, exp \\
\hline Complexity increase & & \\
& Generations without change & 2000 \\
& Number of tries & 3 \\
& Max. complexity & 5 \\
\hline Genetic operators & & \\
& Mutation rate & $0.00138,0.044$ \\
& Inversion rate & 0.00546 \\
\hline Numerical constants & & \\
& Data type & Floating-point \\
& Lower bound & -10 \\
& Upper bound & +10 \\
\hline
\end{tabular}

The overall number of runs for developing the optimal models was about 54 (number of combinations of the algorithm parameters) $\times 3$ (number of replications) $=162$. The optimal GEP-based prediction model for $G_{f}$ is as follows:

$$
\begin{aligned}
G_{f}\left(\frac{j}{m^{2}}\right)=( & (((N M A S-9) A T-R A P)(5.36+T-L T P G))-L T P G) \\
& +A T\left(G^{4}-1.7+U T I\right)+\frac{N M A S-U T I}{((T+N M A S) A T)-A T+6.45}-R A S \\
& +R A P+\frac{L T P G}{A T}+L T P G^{2}+3.49 T+A C \times R A P \\
& +T \sqrt{C R C^{3}-10 T-R A P \times A C-6.461+U T I}
\end{aligned}
$$

Where, UTI, LTPG, AC, NMAS, RAP, RAS, G, AT, CRC, and T denote useful temperature interval, low temperature performance grade, percentage of asphalt content, nominal maximum aggregate size, percentage of reclaimed asphalt pavement, percentage of reclaimed asphalt shingles, gradation type, aggregate type, crumb rubber content, and testing temperature, respectively. The model is a complicated combination of parameters and operators to predict $G_{f}$. The expression trees (ETs) of the obtained model is given in 
Figure 3-14. In this figure, d0, .., d9 represent UTI, LTPG, AC, NMAS, RAP, RAS, G, AT, $C R C$, and $T$, respectively. Parameters $\mathrm{c}_{0}$ and $\mathrm{c}_{1}$ in the ETs are numerical constants, $\mathrm{n}$ in $\mathrm{Xn}$ denotes power. This model is comprised of five individual sub-models connected using addition operation. Each of these sub-models include a part of the information provided by the final model (Ferreira, 2001). Figure 3-15 presents the predictions provided by this model. The high density of the points around the ideal 45-degree angle line clearly indicates the good performance of the model.
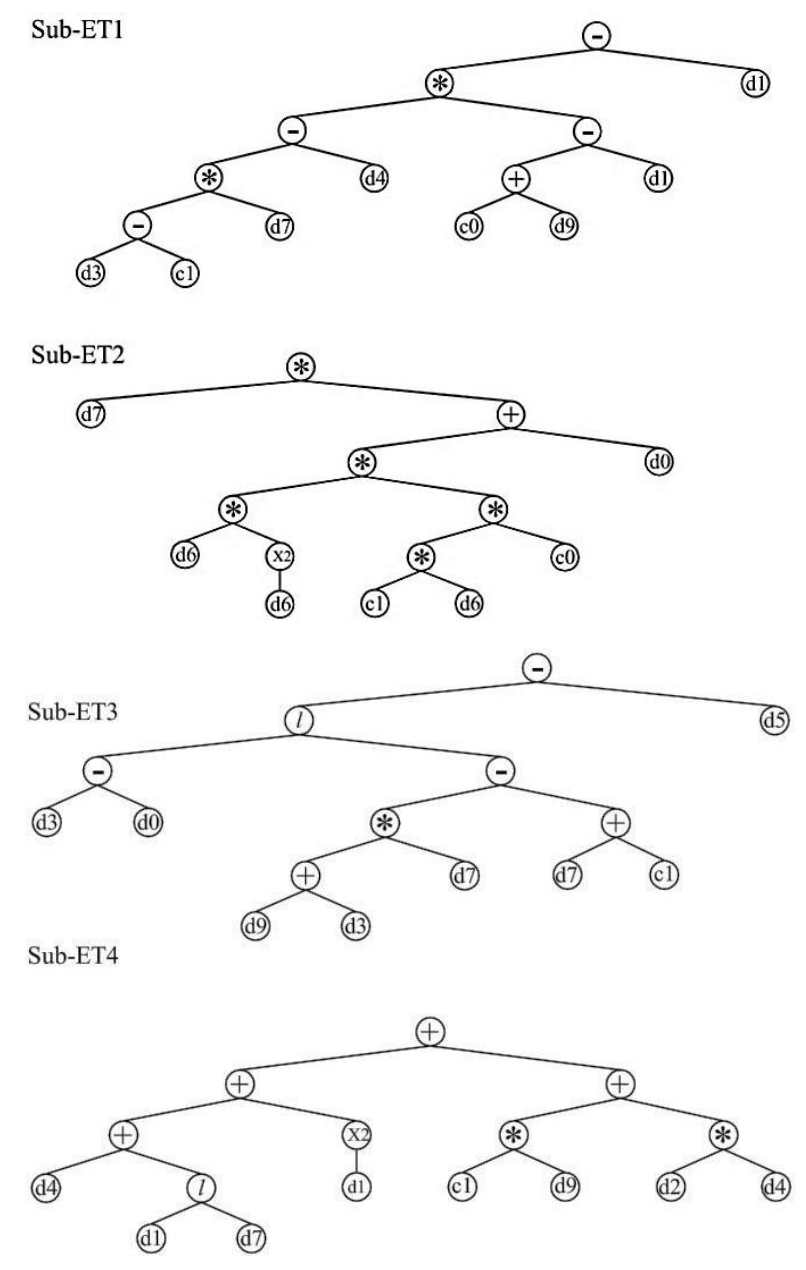

$\begin{array}{ll}\text { (a) } \mathrm{ET}_{1} \text { and } \mathrm{ET}_{2} & \text { (b) } \mathrm{ET}_{3} \text { and } \mathrm{ET}_{4}\end{array}$ 


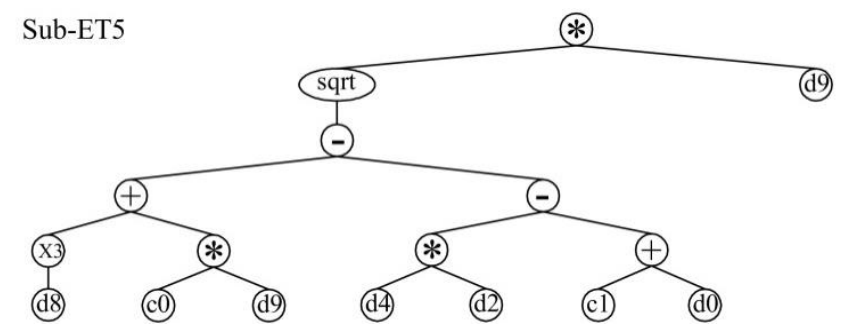

(c) $\mathrm{ET}_{5}$

Figure 3-13. Expression trees of the best GEP prediction model for $\boldsymbol{G}_{f}\left(\mathbf{E T}=\sum \mathrm{Sub}\right.$ $\left.\mathbf{E T}_{\mathbf{i}}\right)$
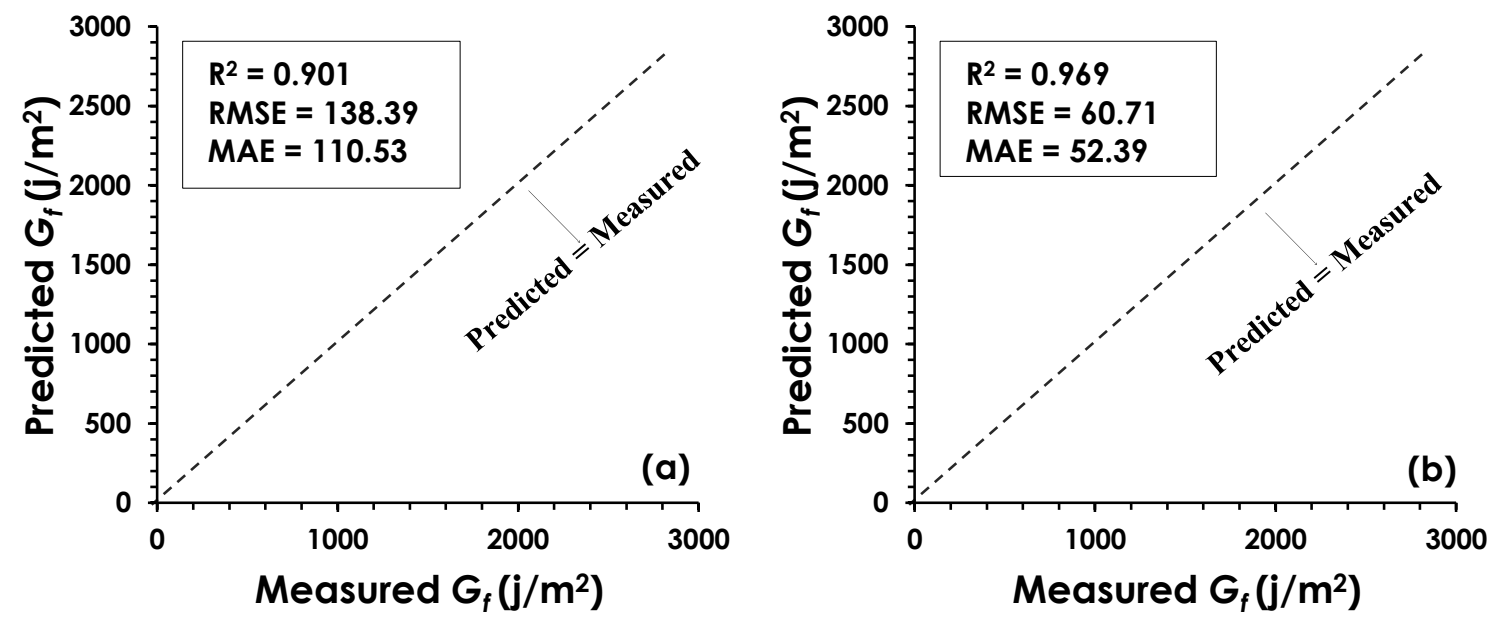

Figure 3-14 Measured against predicted $G_{f}$ using the GEP model: (a) training data, (b) testing data

\subsubsection{Model Development Using the Hybrid ANN/SA Method}

The ANN/SA models formulate $G_{f}$ in terms of UTI, LTPG, AC, NMAS, RAP, RAS,

$G, A T, C R C$, and $T$. For the ANN simulation, the data was normalized between 0.05 and 0.95 using the following method [138]:

$$
V_{n}=A \times V+B
$$

where,

$$
A=\frac{L-U}{V_{\max }-V_{\min }}
$$




$$
B=L-A \times V_{\max }
$$

$V_{\max }$ and $V_{\min }$ are the maximum and minimum of the variable $V$ in the database, respectively. In this study, $L$ and $U$ were taken 0.05 and 0.95 , respectively. The normalized data were then fed into the ANN/SA algorithm. The algorithm was implemented several times to find the best solution. The ANN/SA parameter setting was based on some previously suggested values [109], [113] and after a trial-and-error approach. The best ANN/SA simulation results were obtained with these parameter values: $k=1500$; initial temperature $=15$; final temperature $=0.015$. The number of temperature cycles were set to 10, 20 and 50. Also, 10, 15 or 20 iterations were considered for the number of iterations. Several networks with different settings for hidden layers, hidden nodes, epochs, and activation functions were trained. Conjugate-Gradient and Levenberg-Marquardt algorithms were implemented for the training of the network. The best results were obtained by the Conjugate-Gradient method. Also, the transfer function between the input and hidden layer was log-sigmoid of form $1 /\left(1+\mathrm{e}^{-\mathrm{x}}\right)$. The same function was used for the hidden layer and output layer.

In order to transform the ANN/SA model into a practical equation, the weights and biases were frozen after the networks were well trained [109]. Thereafter, the optimal model was converted to a functional form using the following equation [109], [139].

$$
h=f_{\text {нО }}\left(\operatorname{bias}_{h}+\sum_{k=1}^{h} V_{k} f_{\text {IH }}\left(\operatorname{bias}_{h k}+\sum_{i=1}^{m} w_{i k} x_{i}\right)\right)
$$


where $h$, bias, $V, w, x$, and $f$ denote output, bias, weight connection, input, and transfer function, respectively. Also, $h, I H, H O, k, i$ represent the hidden layer, input-hidden layer, hidden-ouput layer, number of neurons, and number of input parameter, respectively. The best ANN/SA prediction model for $G_{f}$ was constructed with all of the 10 input parameters (UTI, LTPG, AC, NMAS, RAP, RAS, G, AT, CRC, and T) and $11(\mathrm{~m}=11)$ nodes (see Figure 3-15). After de-normalizing the output, the ANN/SA-based formulations of $G_{f}$ is as follows:

$$
G_{f}\left(j / m^{2}\right)=-2705.22\left(\frac{1}{1+e^{-\left(A+\text { Bias }_{h}\right)}}\right)+2852.97
$$

where,

$$
A=\sum_{k=1}^{11} \frac{V_{k}}{1+e^{-F}}
$$

$$
\begin{aligned}
& F=U T I_{n} W_{1 k}+L T P G_{n} W_{2 k}+A C_{n} W_{3 k}+N M A S_{n} W_{4 k}+R A P_{n} W_{5 k}+R A S_{n} W_{6 k}+ \\
& G_{n} W_{7 k}+A T_{n} W_{8 k}+C R C_{n} W_{9 k}+T_{n} W_{10 k}+\text { Bias }_{k}
\end{aligned}
$$

in which, $U T I_{n}, L T P G_{n}, A C_{n}, N M A S_{n}, R A P_{n}, \quad R A S_{n}, G_{n}, A T_{n}, C R C_{n}, \quad$ and $\quad T_{n}$ represent the inputs variables normalized using Eq. (11). The weight and bias values of the optimal ANN/SA model are given in Table 3-4 and Table 3-5 8,000 epochs were considered for the network training. Figure 3-16 presents the predicted against the measured $G_{f}$ values. As seen in this figure, the points are densely distributed around the idea fit line which indicates that the prediction is well-performed. 

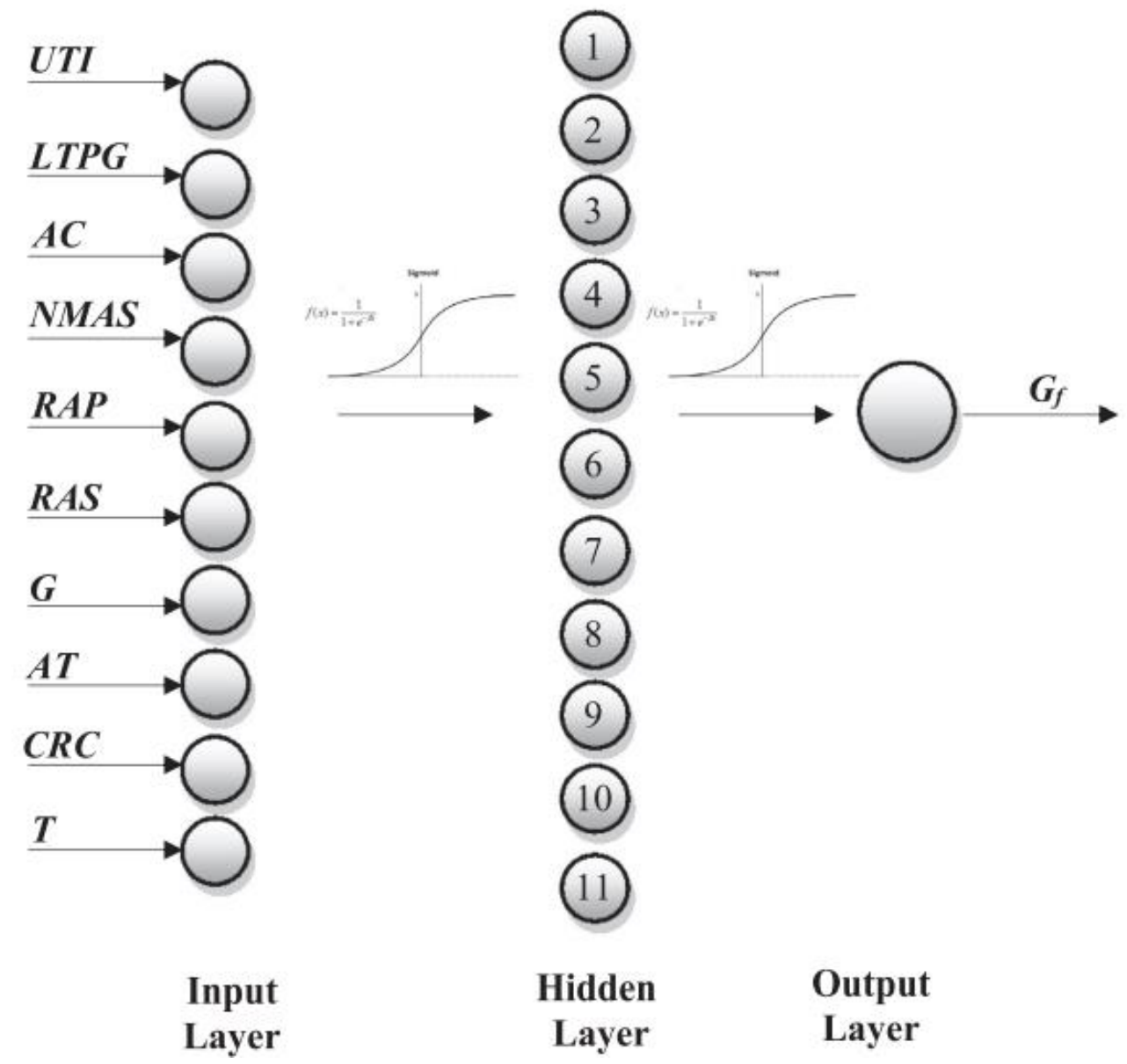

Figure 3-15 The optimal ANN/SA architecture for predicting Gf
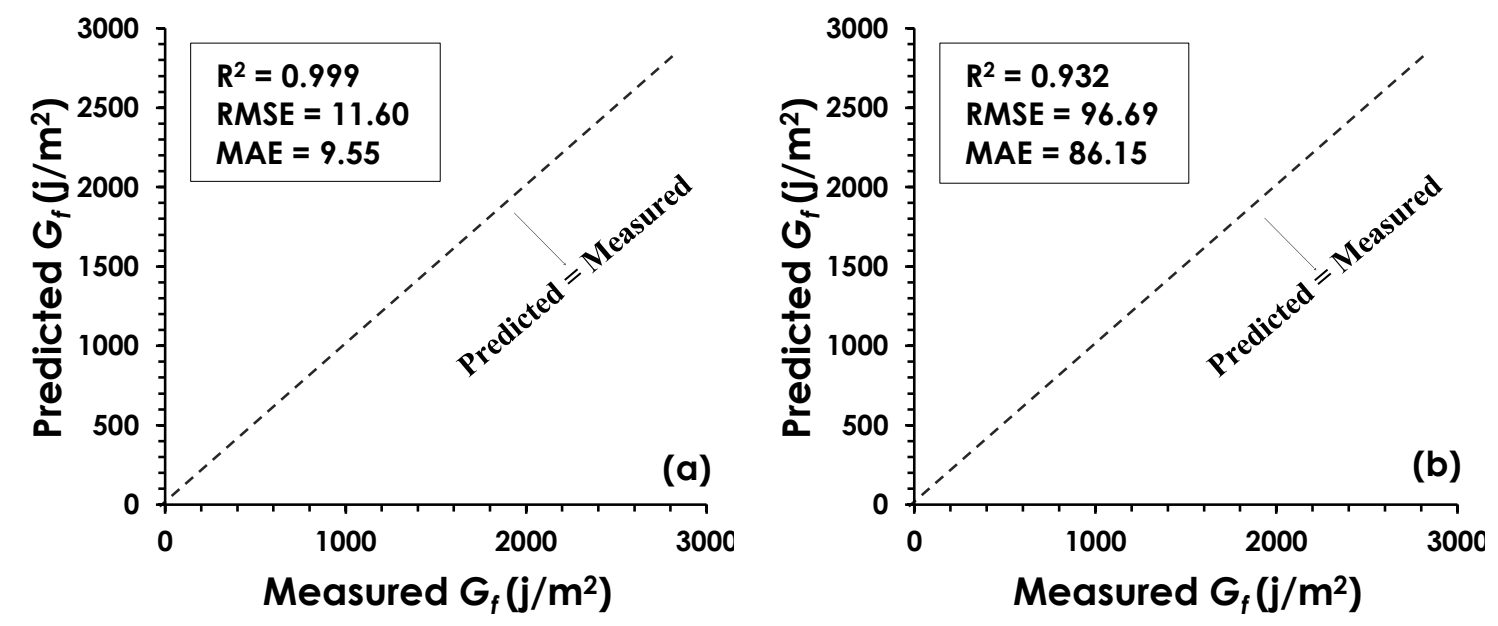

Figure 3-16 Measured against predicted $G_{f}$ using the ANN/SA model: (a) training data, (b) testing data 
Table 3-4 Input-hidden layer weight and bias values

\begin{tabular}{|c|c|c|c|c|c|c|c|c|c|c|c|}
\hline \multirow[b]{2}{*}{$\begin{array}{c}\text { Weight } \\
\text { S }\end{array}$} & \multicolumn{9}{|c|}{$K$} & \multirow[b]{2}{*}{10} & \multirow[b]{2}{*}{11} \\
\hline & 1 & 2 & 3 & 4 & 5 & 6 & 7 & 8 & 9 & & \\
\hline$W_{l k}$ & 2.080 & 2.433 & $2 . \overline{212}$ & $\begin{array}{c}- \\
2.367\end{array}$ & 2.501 & 3.292 & 2.783 & 0.072 & -0.856 & $\begin{array}{c}- \\
2.108\end{array}$ & $\begin{array}{c}3.64 \\
8\end{array}$ \\
\hline$W_{2 k}$ & $\begin{array}{c}- \\
6.347\end{array}$ & 2.589 & 7.572 & 4.444 & 2.218 & 1.010 & -1.156 & $\begin{array}{c}- \\
0.746\end{array}$ & 0.025 & 0.722 & $\begin{array}{c}3.29 \\
0\end{array}$ \\
\hline$W_{3 k}$ & $9 . \overline{186}$ & -8.646 & $2 . \overline{-}$ & 3.982 & 3.947 & $1 . \overline{383}$ & 2.968 & $\begin{array}{c}- \\
6.359\end{array}$ & 2.101 & $\begin{array}{c}- \\
0.897\end{array}$ & $\begin{array}{c}- \\
1.45 \\
8\end{array}$ \\
\hline$W_{4 k}$ & 1.552 & 4.077 & 5.969 & 4.724 & $\begin{array}{c}- \\
4.697\end{array}$ & 2.913 & 3.362 & $\begin{array}{c}- \\
1.464\end{array}$ & 2.480 & 2.120 & $\begin{array}{c}5.40 \\
4\end{array}$ \\
\hline$W_{5 k}$ & $\begin{array}{c}- \\
2.453\end{array}$ & -2.202 & 1.278 & 0.836 & 0.999 & 4. & -0.533 & 2.276 & -1.258 & 2.856 & $\begin{array}{c}-\overline{19} \\
6\end{array}$ \\
\hline$W_{6 k}$ & 1.694 & 3.175 & $\begin{array}{c}- \\
2.713\end{array}$ & $\begin{array}{c}- \\
0.825\end{array}$ & 2.747 & $6 . \overline{244}$ & -2.981 & 3.682 & -1.960 & $\begin{array}{c}- \\
2.543\end{array}$ & $\begin{array}{c}1.34 \\
5\end{array}$ \\
\hline$W_{7 k}$ & 0.243 & 3.847 & $\begin{array}{c}- \\
1.600\end{array}$ & $\begin{array}{c}- \\
1.391\end{array}$ & $4 . \overline{705}$ & 1.433 & 1.045 & 1.030 & 2.154 & $\begin{array}{c}- \\
3.012\end{array}$ & $\begin{array}{c}- \\
0.44 \\
0\end{array}$ \\
\hline$W_{8 k}$ & 7.694 & -2.359 & $\begin{array}{c}- \\
0.479\end{array}$ & $\begin{array}{c}- \\
5.490\end{array}$ & 9.166 & $0 . \overline{9} 9$ & 2.789 & 6.021 & 1.449 & $\begin{array}{c}- \\
3.242\end{array}$ & $\begin{array}{c}1.35 \\
0\end{array}$ \\
\hline$W_{9 k}$ & $\begin{array}{c}- \\
0.718\end{array}$ & 5.610 & 4.090 & 1.653 & 0.862 & $4 . \overline{424}$ & 3.739 & 1.196 & -8.570 & $\begin{array}{c}- \\
0.276\end{array}$ & $\begin{array}{c}0.33 \\
2\end{array}$ \\
\hline$W_{l o k}$ & 1.793 & $\begin{array}{c}- \\
10.472\end{array}$ & $\begin{array}{c}- \\
2.509\end{array}$ & 4.828 & 5.322 & 0.550 & -1.087 & 5.262 & 1.105 & 3.746 & $\begin{array}{c}4.01 \\
2\end{array}$ \\
\hline Bias $_{k}$ & $\begin{array}{c}- \\
1.789\end{array}$ & -5.773 & 3.986 & 0.791 & $\begin{array}{c}- \\
1.905\end{array}$ & 0.183 & 1.181 & $\begin{array}{c}- \\
3.953\end{array}$ & 5.056 & $\begin{array}{c}- \\
4.795\end{array}$ & $\begin{array}{c}0.25 \\
2\end{array}$ \\
\hline
\end{tabular}

Table 3-5 Hidden-Output layer weight and bias values

\begin{tabular}{|c|c|c|c|c|c|c|c|c|c|c|c|c|}
\hline \multirow[b]{2}{*}{ Weights } & \multicolumn{11}{|c|}{$K$} & \multirow[b]{2}{*}{ Biash $_{h}$} \\
\hline & 1 & 2 & 3 & 4 & 5 & 6 & 7 & 8 & 9 & 10 & 11 & \\
\hline$V_{k}$ & 6.964 & -9.361 & -2.696 & 2.694 & -5.021 & 1.550 & 5.092 & 3.908 & 3.591 & -14.99 & -5.08 & 4.576 \\
\hline
\end{tabular}

\subsubsection{Design example}

The ANN/SA structure interpretation procedure was shown using the following illustrative example for one of the testing data:

$U T I=98^{\circ} \mathrm{C} ; L T P G=-40^{\circ} \mathrm{C} ; A C=6.5 \% ; N A M S=12.5 \mathrm{~mm} ; R A P=0 \% ; R A S=0 \% ; G=$ 1 (Dense); $A T=2$ (Granite); $C R C=0 \% ; T=-42{ }^{\circ} \mathrm{C} . G_{f}$ is required. Here are the calculation steps: 
1: Normalization of the input parameters and calculating $U T I_{n}, L T P G_{n}, A C_{n}, N M A S_{n}$, $R A P_{n}, R A S_{n}, G_{n}, A T_{n}, C R C_{n}$, and $T_{n}$ based on Eqs. (10)- (12).

For UTI: Minimum and maximum are 70 and $98^{\circ} \mathrm{C}$, respectively. Hence:

$$
\begin{array}{r}
a=\frac{(0.05-0.95)}{(98-70)}=-0.032, b=0.05-(-0.032) \times 98=3.186 \\
U T I_{n}=\overbrace{-0.032}^{a} \times \overbrace{98}^{U T I}+\overbrace{3.186}^{b}=0.05
\end{array}
$$

Similarly,

$L T P G_{n}, A C_{n}, N M A S_{n}, R A P_{n}, R A S_{n}, G_{n}, A T_{n}, C R C_{n}$, and $T_{n}$ are equal to 0.95, 0.40, $0.05,0.95,0.95,0.95,0.50,0.95$, and 0.95 .

2: Calculation of the hidden layer. Referring to Eq. (16), $F_{1}, \ldots, F_{11}$ are obtained as:

$F_{1}=-6.954, F_{2}=-7.684, F_{3}=1.093, F_{4}=5.632, F_{5}=9.589, F_{6}=-12.211, F_{7}=3.152$, $F_{8}=-2.825, F_{9}=-1.370, F_{10}=-10.785$, and $F_{11}=4.991$.

3: Predicting $G_{f}$. The log-sigmoid function $\left(f=1 /\left(1+\mathrm{e}^{-\mathrm{x}}\right)\right)$ was used to calculate A: $\mathrm{A}=6.964 f\left(\mathrm{~F}_{1}\right)-9.361 f\left(\mathrm{~F}_{2}\right)+2.696 f\left(\mathrm{~F}_{3}\right)+2.694 f\left(\mathrm{~F}_{4}\right)-5.021 f\left(\mathrm{~F}_{5}\right)+1.550 f\left(\mathrm{~F}_{6}\right)+$ $5.092 f\left(\mathrm{~F}_{7}\right)+3.908 f\left(\mathrm{~F}_{8}\right)+3.591 f\left(\mathrm{~F}_{9}\right)-14.999 f\left(\mathrm{~F}_{10}\right)-5.085 f\left(\mathrm{~F}_{11}\right)=0.027$

Using Eq. (14), $G_{f}$ is equal to:

$$
G_{f}=-2705.22\left(\frac{1}{1+e^{-(0.027+4.576)}}\right)+2852.97=874.03 \mathrm{j} / \mathrm{m}^{2}
$$

The measured $G_{f}$ for this case was $790.2 \mathrm{j} / \mathrm{m}^{2}$ which is $11 \%$ lower than the predicted $G_{f}$.

\subsubsection{Performance evaluation}

\subsubsection{Statistical methods for external validation}

On the basis of a rational hypothesis, Smith (1986) argues that for correlation coefficient $(|\mathrm{R}|)$ higher than 0.8 , a strong correlation exists between the predicted and measured values [140]. In all conditions, the error values (e.g. RMSE, MAE) should be at 
the minimum [117]. Based on the results shown in Figure 3-14 and Figure 3-16, the proposed GEP and ANN/SA models have a satisfactory performance on the training and testing data sets. Besides, Table 3-6 shows some criteria suggested by [141], [142] for the external validation of the models. More details about these performance measures can be found in [103]. As observed from Table 3-6, the developed models satisfy all of the requisite conditions. The validation phase ensures that the proposed models are strongly suitable and applicable.

\section{Table 3-6 Performance measures for further validation of the GEP and ANN/SA} models

\begin{tabular}{ccccc}
\hline Item & Formula & Condition & GEP & ANN/SA \\
\hline 1 & $R$ & $0.8<R$ & 0.984 & 0.965 \\
2 & $k=\frac{\sum_{i=1}^{n}\left(h_{i} \times t_{i}\right)}{h_{i}{ }^{2}}$ & $0.85<k<1.15$ & 0.981 & 1.093 \\
3 & $k^{\prime}=\frac{\sum_{i=1}^{n}\left(h_{i} \times t_{i}\right)}{t_{i}{ }^{2}}$ & $0.85<k^{\prime}<1.15$ & 1.011 & 0.904 \\
4 & $m=\frac{R^{2}-R_{o}{ }^{2}}{R^{2}}$ & $|m|<0.1$ & -0.030 & 0.000 \\
5 & $n=\frac{R^{2}-R_{o^{\prime}}{ }^{2}}{R^{2}}$ & $|n|<0.1$ & -0.032 & -0.012 \\
6 & $R_{m}=R^{2} \times\left(1-\sqrt{\left|R^{2}-R_{o}{ }^{2}\right|}\right)$ & $0.5<R_{m}$ & 0.804 & 0.918 \\
7 & $R_{o}{ }^{2}=1-\frac{\sum_{i=1}^{n}\left(t_{i}-h_{i}{ }^{o}\right)^{2}}{\sum_{i=1}^{n}\left(t_{i}-\bar{t}_{l}\right)^{2}}, h_{i}{ }^{o}=k \times t_{i}$ & Should be close to 1 & 0.997 & 0.932 \\
8 & $R_{o^{\prime}}{ }^{2}=1-\frac{\sum_{i=1}^{n}\left(h_{i}-t_{i}{ }^{o}\right)^{2}}{\sum_{i=1}^{n}\left(h_{i}-\bar{h}_{l}\right)^{2}}, t_{i}{ }^{o}=k^{\prime} \times h_{i}$ & Should be close to 1 & 0.999 & 0.943 \\
\hline
\end{tabular}

\subsubsection{Sensitivity analysis}

As discusses in Section 3.3, an extensive simulation study was carried out to find the most relevant parameters and accordingly developing the best predictive models. The optimal models were built using 10 input parameters (UTI, LTPG, AC, NMAS, RAP, RAS, 
$G, A T, C R C$, and $T$ ). However, a second phase of sensitivity analysis was further performed to distinguish the parameters with higher contributions in the final models. To this aim, the frequency values [143] of the input parameters were obtained for the GEP models. In case a design variable has appeared in $100 \%$ of the best thirty GEP programs, its frequency value will be equal to 1 [143]. For the ANN/SA analysis, Garson's algorithm [144] was utilized to find the relative importance of the predictor variables based on the weights of the ANN/SA model (Figure 3-17). Figure 3-18 shows the sensitivity analysis results for the GEP and ANN/SA models. For both GEP and ANN/SA, $G_{f}$ is more sensitive to $A C$ and $A T$. Also, the influence of $L T P G$ and $T$ is high in the GEP analysis. As seen in Figure 3-18, the effect of $U T I, R A P$ and $R A S$ on $G_{f}$ is less than the other parameters. These are expected outcomes because at low temperature, the fracture phenomenon significantly relies on the aggregate type. In this case, cracks propagate through the aggregates and try to break them up and grow. Asphalt binder is the weak component in asphalt mixtures. A soft binder has a better performance compared to a stiff binder in terms of low temperature cracking [27]. Lower LTPG will provide higher ductility and flexibility at low temperature, while higher LTPG leads to brittle behavior of binder [36]. In addition, the fracture energy is dissipated significantly near the glass transition temperature [55]. Binders with lower LTPG have lower glass transition temperature. The significantly high effect of temperature on $G_{f}$ is also reported in different studies [124]. 


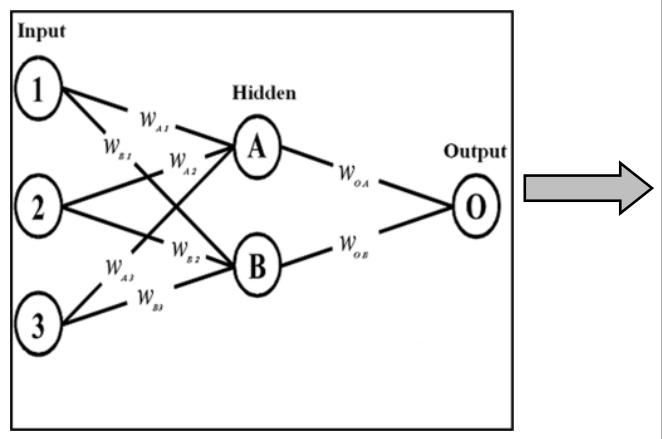

Contribution of each input neuron to the output via

each hidden neuron calculated as the product of the

input-hidden and hidden-output connections

e.g., $C_{A I}=W_{A I} \times W_{O A}$

Relative contribution of each neuron to the outgoing signal of each hidden neuron

e.g., $r_{A 1}=\left|C_{A 1}\right| /\left(\left|C_{A 1}+C_{A 2}+C_{A 3}\right|\right)$

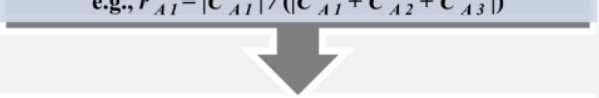

Sum of the input neuron contributions

e.g., $S_{r}=r_{A 1}+r_{B 1}$

Relative Importance (RI) of each input variable

e.g., $R I_{1}=S_{1} /\left(S_{1}+S_{2}+S_{3}\right)$

Figure 3-17 The procedure to evaluate the relative importance of inputs [144].
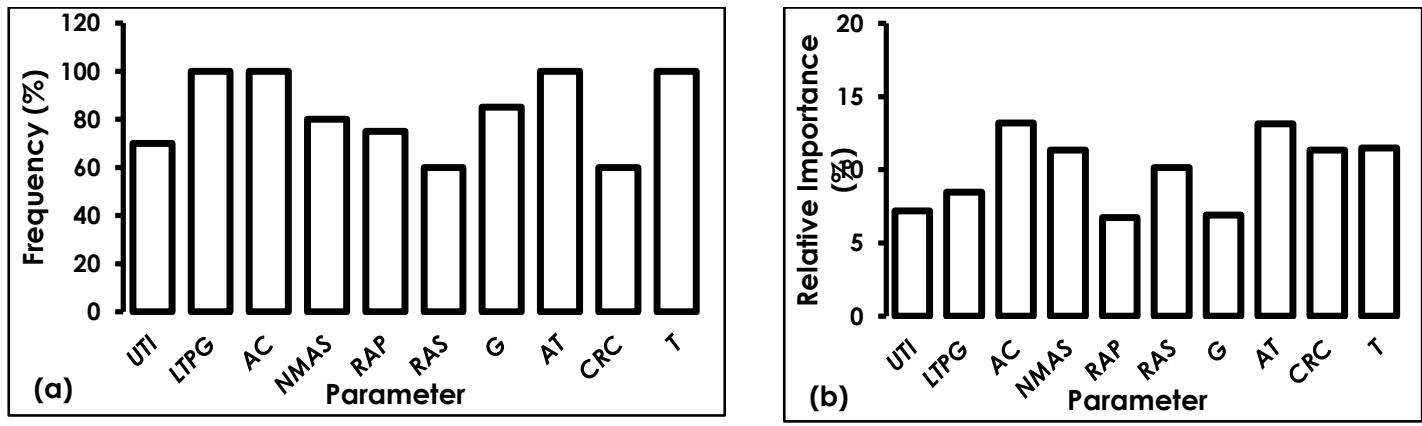

Figure 3-18 Sensitivity analysis of the final predictor variables in: (a) GEP analysis and (b) ANN/SA analysis

\subsubsection{Comparative study}

Since there were not any existing models for the $G_{f}$ of asphalt pavements, the GEP and ANN/SA were benchmarked against each other. Referring to Figure 3-14 and Figure 3-16, the ANN/SA model has a notably better performance than the GEP model on the training data. This is while GEP has a superior performance to ANN/SA on the testing data. This means that the GEP model has a better generalization compared to the ANN/SA 
model. In addition, Figure 3-19 visualizes histogram plots of measured/predicted $G_{f}$ values along with the standard deviation (STD) and average (Mean) metrics for the entire database. Evidently, the models with higher distribution around Measured $G_{f} /$ Predicted $G_{f}$ $=1$ provide better predictions. As seen in Figure 3-19b, the density of the data corresponding to ANN/SA are higher around 1 compared to the GEP model. On the other hand, GEP provides a better Mean value than the ANN/SA model. It should be noted that a major advantage of GEP over ANN-based and other classical modeling approaches lies in its capability to derive explicit relationships without assuming prior forms of the existing relationships. In addition, the final modes developed using GEP are obtained after controlling millions of nonlinear models, which is not feasible via other nonlinear regression approaches. While this study has opened the black-box on the ANN/SA model, the derived prediction equation is much more complicated than the GEP model. It must be noted better performance might be achievable by tweaking the parameters in the ANN model.
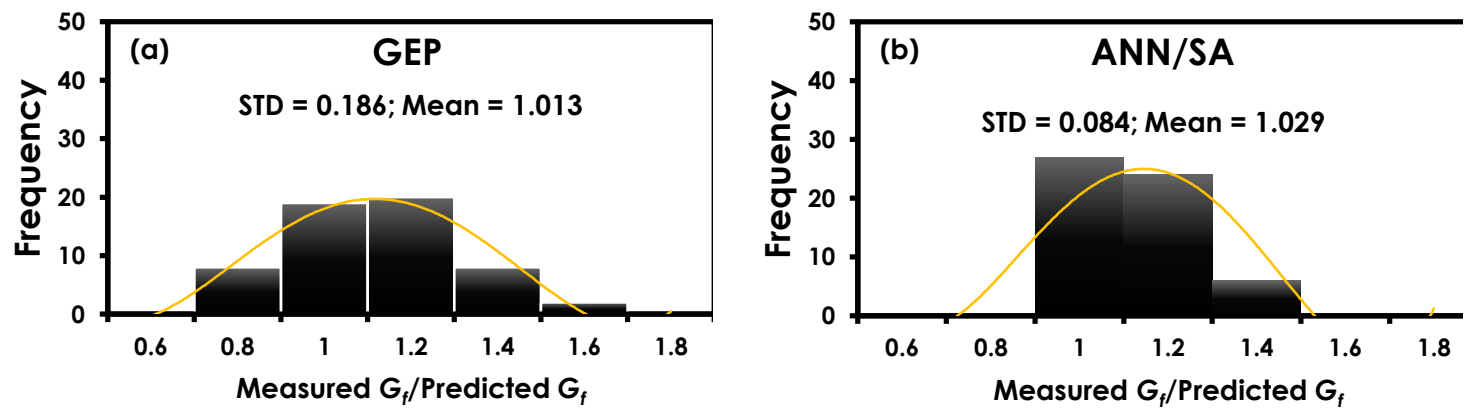

Figure 3-19 A comparison of the GEP and ANN/SA models on the entire database 


\subsubsection{Summary}

In this study, two machine learning methods called GEP and ANN/SA were used to predict the $G_{f}$ of asphalt mixtures. Two models were developed using a well-established experimental database. The proposed models include the simultaneous effect of various test, binder and aggregate-related parameters, as well as modern asphalt ingredients such as recycled materials, rejuvenators, and rubber. The models provide reliable predictions of $G_{f}$. The GEP model produces better outcomes than the ANN/SA model for the testing data. The beauty of the GEP analysis is that it can model the $G_{f}$ behavior without any need to predefine the model's functional structure. Besides, a calculation procedure was proposed to transform the optimal ANN/SA model into a functional representation. The GEP model seems to be more practical than the ANN/SA model because of its better generalization and simpler functional structure. A limitation of the derived models is that they were calibrated using a fairly small set of DC(T) test results. This limitation can be tackled by updating the models with new trained weights when more data become available. The results of the sensitivity analysis indicate that $A C, A T, L T P G$ and $T$ are more effective to explain the variations of $G_{f}$ compared with the other predictor variables. Since the proposed models are based on the data alone, they are mostly suitable for pre-design purposes. In any case, the range of the mixture properties should fall within the range considered for the development of the models. It should be noted that performing DC(T) test might be costprohibitive for some users. Thus, a trial method to design a mixture with acceptable thermal cracking performance may be expensive. Arguably, using the proposed empirical models for the estimation of the $\mathrm{DC}(\mathrm{T})$ test results will save time and money for such design procedures. 
Further research can focus on including DC(T) test results for mixtures with much wider range properties to obtain more reliable predictions. Moreover, an ML model can be developed to characterize the combined effect of asphalt additives on both rutting and thermal cracking performance. The importance of such model is that adding an additive to asphalt pavement usually improves merely one of these two performance indexes. In addition, a relationship can be developed between the density of the thermal and block cracks in pavement segments and the corresponding $\mathrm{DC}(\mathrm{T})$ fracture energy. 


\subsection{DEVELOPING A PREDICTION MODEL FOR RUTTING DEPTH OF ASPHALT MIXTURES USING GENE EXPRESSION PROGRAMMING}

\subsubsection{Introduction}

Rutting is one of the most critical types of pavement distress, which decreases road safety and ride quality [145]. This load-related distress usually occurs in the top $100 \mathrm{~mm}$ of an asphalt pavement, and involves both consolidation and shear flow type deformations [146]. The rutting resistance of asphalt mixtures depends on component materials and mix design, along with environmental and traffic effects [45], [108], [147]-[149]. The variety of climates and mix design parameters for each specific pavement section makes it difficult to obtain reliable rut resistance estimations as an alternative to tedious and expensive lab testing. Modern rut test devices provide a more realistic evaluation as compared to traditional tests such as the Marshall stability and flow procedure. The Asphalt Mixture Performance Tester (AMPT), Asphalt Pavement Analyzer (APA), and Hamburg Wheel Tracking Test (HWTT) are the most popular rutting tests used in the United States. The HWTT is designed to provide a rutting characterization by simulating traffic effect through loading by a steel wheel, at a high temperature, usually $50{ }^{\circ} \mathrm{C}$, and usually by testing in a temperature-controlled water bath (AASHTO 324, 2011).

This HWTT has been employed by many researchers to characterize the rutting potential of asphalt mixtures [18], [150]-[156]. Sel et al. (2014) used the TxDOT Hamburg test database including 840 data points to perform a statistical analysis towards test result estimation. The test results were obtained from Hamburg test at two different temperatures including $50{ }^{\circ} \mathrm{C}$ and $40{ }^{\circ} \mathrm{C}$. It was shown that 90 percent of the mixtures tested at $40{ }^{\circ} \mathrm{C}$ 
pass a $12.5 \mathrm{~mm}$ rut depth screening criteria, while 65 percent of the mixtures accumulate less than $12.5 \mathrm{~mm}$ of rut depth at $50{ }^{\circ} \mathrm{C}$ [157]. Given the fact that the commonly used binder type in Wisconsin is either PG58-28 or PG58-34, Swiertz et al., (2017) investigated the appropriate testing temperature for the Hamburg wheel track test. Tests were carried out at 40, 45 and $50{ }^{\circ} \mathrm{C}$. Using three aggregate sources, two binder types (PG58-28 and PG 5834) in modified and neat conditions resulted in nine different mixture combinations. Regardless of the mixture combination, most mixes could not pass the criteria at $50{ }^{\circ} \mathrm{C}$, while they passed 45 and $40{ }^{\circ} \mathrm{C}[158] \mathrm{w}$.

In another study, Walubita et al., (2016) found that the average temperature and max temperature measured form the surface of randomly selected, in-service highways in Texas were 58.3 and $63.1{ }^{\circ} \mathrm{C}$, respectively. Therefore, frequent rutting failures observed in Texas were attributed to the lower Hamburg testing temperature used as compared to actual pavement temperatures in that region. To address this issue, Hamburg testing at 50, 60 and $70{ }^{\circ} \mathrm{C}$ was conducted. The experimental results showed that at the $60{ }^{\circ} \mathrm{C}$ testing temperature, three-out-of-six mixtures exceeded a $12.5 \mathrm{~mm}$ rutting criteria for PG64-XX binder prior to 1000 wheel passes. In addition, all mixes rapidly failed at $70{ }^{\circ} \mathrm{C}$, and conversely, all mixes passed the criteria when tested at $50{ }^{\circ} \mathrm{C}[151]$.

Xiao et al., (2007) studied various asphalt samples using two rubber types and four rubber contents [159]. The authors investigated the effect of recycling content, rubber content, and air void. The results showed that the higher rubber dosage results in lower rut depth under the HWTT. Also, the addition of reclaimed asphalt pavement (RAP) was found to lower permanent deformation in the HWTT [159]. Vahidi et al., (2013) experimented 
with 10 and $15 \%$ ground tire rubber (GTR) and treated ground tire rubber (TGTR) along with PG58-28 asphalt binder [160] . A 40\% RAP content was also u[161]sed, resulting in high recycled content mixes. Compared with the control mix, which had 40\% RAP and no rubber, the rutting resistance was significantly improved in the GTR mixes. The lower measured rut depth was attributed to the stiffened binder resulting from absorption of volatiles in the binder by the rubber particles [160]. Shirini and Imaninasab tested porous asphalt dense graded mixtures with $0,5,10$, and $20 \%$ of crumb rubber [162]. High temperature performance was considerably improved through the use of GTR. An $8.5 \mathrm{~mm}$ Hamburg rut depth originally measured in the control mix was reduced to $1.9 \mathrm{~mm}$ after incorporation of $20 \%$ rubber by weight of the binder [162].

Mahmoud et al., (2010) evaluated four different aggregate types including a hard limestone, soft limestone, sandstone, and gravel [161]. In addition to the aggregate type, the authors studied various aggregate blends such as dense graded, open graded, and gap graded mixtures [161]. The Hamburg testing results showed that Hamburg testing is able to properly capture the effect of aggregate type, with softer aggregates leading to higher rut depths in the HWTT [161]. Also, the dense-graded mixtures recorded higher deformation under Hamburg wheel as compared to the gap- and open-graded asphalt mixtures investigated. Grebenschikov and Prozzi (2004) compared the mechanisticempirical pavement design guide (MEPDG) outputs with the Hamburg testing results [163]. To this end, the rutting performance of mixtures fabricated with four binder contents and three dense graded mixture types and the same limestone type aggregates were studied. One of the aggregate structures was coarse graded (Type $\mathrm{C}$ based on TxDOT) and another one was fine graded (Type D based on TxDOT) [163]. These two aggregate gradations 
were used as representatives of the lower and higher limits of dense graded gradation. These two border-line gradations were then compared to the target gradation. Although the Hamburg testing results showed a significant difference in terms of high temperature performance between the mixtures, the MEPDG was not able to distinguish between them [163].

Different RAP percentages have been used by Zhao et al., (2013) to make HMA and WMA mixtures in a recent rutting study [164]. The rutting behavior of the mixtures were then evaluated through Hamburg wheel track and asphalt pavement analyzer (APA) tests. The experimental results indicated that the WMA mixture without any recycled materials incurred higher rut depths as compared with HMA [164]. Also, the rut depth of the WMA mixtures consistently decreased as the RAP content increased [164]. The beneficial effect of recycled materials on high temperature performance has also been reported by other researchers (W. G. Buttlar et al., 2019; W. Buttlar, Rath, et al., 2018; Jahangiri et al., 2019; Majidifard, Tabatabaee, et al., 2019; Faramarzi, Lee, Kim, \& Kwon, 2018; Mirhosseini, Kavussi, Tahami, \& Dessouky, 2018; Notani et al., 2019; Ozer, Al-Qadi, Kanaan, \& Lippert, 2013; Zhang, Simate, Hu, Souliman, \& Walubita, 2017). Ozer et al., (2013) applied 2.5, 5, and $7.5 \%$ recycled asphalt shingles (RAS) by weight of the mix and observed a significant drop in rut depth in the Hamburg test as the recycled material content increased [166]. Also, the stiffer virgin binder grade was found to lead to a lower plastic deformation as expected [166]. Table 3-7 summarizes recent parametric studies conducted with the HWTT along with key findings.

Table 3-7 A summary of the parameters affecting rutting resistance of asphalt 
concrete.

\begin{tabular}{|c|c|c|c|}
\hline Authors & $\begin{array}{c}\text { Year } \\
\text { Published }\end{array}$ & $\begin{array}{l}\text { Variables } \\
\text { Investigated }\end{array}$ & Key Findings \\
\hline$[157]$ & 2014 & Testing Temp. & $\begin{array}{l}\text { Increasing the testing temp. from } 40 \text { to } 50^{\circ} \mathrm{C} \text { led to } \\
\text { rutting failure of many mixtures in Texas. }\end{array}$ \\
\hline$[158]$ & 2017 & Testing Temp. & Mixes in Wisconsin could not withstand $50^{\circ} \mathrm{C}$. \\
\hline [151] & 2016 & Testing Temp. & Suggesting testing temp. of $60^{\circ} \mathrm{C}$ in Texas. \\
\hline [159] & 2007 & Rubber & $\begin{array}{c}\text { Both rubber content and rubber particle size effect } \\
\text { permanent deformation }\end{array}$ \\
\hline$[160]$ & 2013 & Rubber & $\begin{array}{c}\text { Absorbption of volatiles in the binder by crumb rubber } \\
\text { leads to lower rut depth }\end{array}$ \\
\hline$[161]$ & 2011 & Aggregates & $\begin{array}{c}\text { Hamburg test captures differences in aggregate type and } \\
\text { gradation }\end{array}$ \\
\hline [163] & 2004 & $\begin{array}{l}\text { Aggregate, } \\
\text { Binder Content }\end{array}$ & $\begin{array}{c}\text { Coarse aggregate and lower asphalt content result in } \\
\text { lower rut depth }\end{array}$ \\
\hline [27] & 2019 & $\begin{array}{l}\text { RAP, RAS, } \\
\text { Binder Type }\end{array}$ & $\begin{array}{l}\text { Stiff binders along with recycled materials generally } \\
\text { result in low rut depths for Missouri surface mixtures }\end{array}$ \\
\hline [164] & 2013 & WMA and RAP & $\begin{array}{l}\text { WMA showed higher rut depth as compared to HMA, } \\
\text { RAP lowers permanent deformation }\end{array}$ \\
\hline$[166]$ & 2013 & RAS & Higher RAS content led to lower rut depths \\
\hline
\end{tabular}

Given the rapidly increasing use of the Hamburg test in balanced mix design across the United States, developing a model that can predict the rutting depth obtained from the HWTT as a function of asphalt mixture properties provides a time-and-cost-saving options for the purposes of initial design and material screening Given this motivation, this study involved the development of a new model for predicting the rutting performance of asphalt mixture specimens as characterized with the HWTT. A gene expression programming (GEP) method was used to create the resulting analytical expression, which can be easily programmed into a spreadsheet or cloud-based service. In a recent study, the authors 
mapped an extensive fracture energy database using GEP [31] with very reasonable accuracy. For a detailed review of the GEP modeling technique and its application in mapping asphalt lab testing data, the reader is encouraged to consult the aforementioned reference.

\subsubsection{Genetic Expression Programming}

GEP is currently regarded as the most advanced form of traditional genetic programming (GP) techniques, which can be used to generate nonlinear prediction models in a highly automated fashion. These techniques have been extensively deployed to help solve a myriad of complicated engineering problems (Alavi \& Gandomi, 2011a; Fathi, Mazari, Saghafi, Hosseini, \& Kumar, 2019; Rashidi, Saghafi, \& Takhtfiroozeh, 2018; Jiao et al., 2019; Nejad, Motekhases, Zakeri, \& Mehrabi, 2015). The traditional GP creates computational models by imitating the biological evolution of living organisms. It provides a tree-like form of solutions, which represents the closed-form solution of the optimization problem [114], [115]. The primary objective of GP is to generate a population of programs that correlate inputs with outputs for each data point. The initial random functions are then calibrated to obtain fitting functions that can accurately estimate the output via an administered trial-and-error methodology.

The GP algorithm has several key advantages over other machine learning (ML) techniques [105], including artificial neural networks and adaptive neuro-fuzzy inference systems. The first benefit is that GP is not a black box and outputs are in the form of simple, sometimes lengthy, mathematical expressions. The other advantage is that GPbased models benefit from their ability to obtain precise relationships without considering 
former patterns of existing relationships. On the other hand, the primary advantage of GEP over the traditional GP method is that it can compile several sub-programs to create a single complex program. Moreover, the GEP algorithm can be implemented significantly faster than GP by evolving binary bit patterns. More details regarding ML, GP and GEP methods can be found in [103], [114], [115]. In the current study, GEP is deployed for developing the HWTT rutting prediction models.

\subsubsection{Development of HWTT Prediction Model}

On the basis of the literature review as summarized in Table 3-7, this study presents

a new ML-based model which predicts the HWTT rut depth from the following variables:

$R u t(m m)=$ $f($ Mix type, UTI, HTPG $, A C, N M A S, A B R, R A P, R A S, G, A T, C R C, T$, Pass)

where, (3-17)

Mix type: Plant or lab-compacted mixture (Plant (loos asphalt mixture collected from plant and compacted in the lab) $=1$, Lab (produced from virgin materials in Lab) = 2) UTI $\left({ }^{\circ} \mathrm{C}\right)$ : Useful temperature interval (i.e., for PG 64-22 binder, UTI = 64-(-22) = $\left.86^{\circ} \mathrm{C}\right)$

$H T P G\left({ }^{\circ} \mathrm{C}\right)$ : High temperature performance grade (for PG 64-22 binder, this is $64^{\circ} \mathrm{C}$ ) $A C(\%):$ Asphalt content

NMAS (mm): Nominal maximum aggregate size. In this study, NMAS can take $4.75 \mathrm{~mm}, 9.5 \mathrm{~mm}, 12.5 \mathrm{~mm}$ or $19 \mathrm{~mm}$.

$A B R(\%)$ : Asphalt Binder Replacement (ratio of recycled binder to the total binder) $R A P(\%):$ Reclaimed asphalt pavement, \% by ABR

$R A S(\%)$ : Reclaimed asphalt shingles, \% by ABR

$G$ : Gradation type (Dense: 1; SMA: 2)

AT: Aggregate Type (Limestone: 1; Granite: 2)

$C R C$ (\%): Crumb rubber content (rubber percentage in virgin binder) 


\section{$T\left({ }^{\circ} \mathrm{C}\right)$ : Testing temperature}

Pass: Number of wheel passes

The models were developed using 96 HWTT results obtained from various tests conducted on materials from pavements in the Midwest United States, following AASHTO-T324. For each test result, the test was performed on three samples, and the average was reported as the test results. Therefore 288 samples were tested to collect 96 test results. As shown in Figure 3-20, the 71.7 kg loaded steel wheel runs over the samples placed in a water bath at $50{ }^{\circ} \mathrm{C}$ (Figure 3-20). The vertical deformation of the specimen is recorded along with the number of wheel passes. The test ends when either the specimen deforms more than $20 \mathrm{~mm}$ or the number of passes exceeds 20,000 (Figure 3-21). Please note that this study also looked at different test temperatures for the Hamburg wheel test. Various DOTs and agencies are adopting Hamburg test temperatures according to the prevailing climatic conditions instead of the standardized recommended value of $50^{\circ} \mathrm{C}$, and therein lies the merit of this prediction algorithm that can take test temperature as a variable in its predictions.

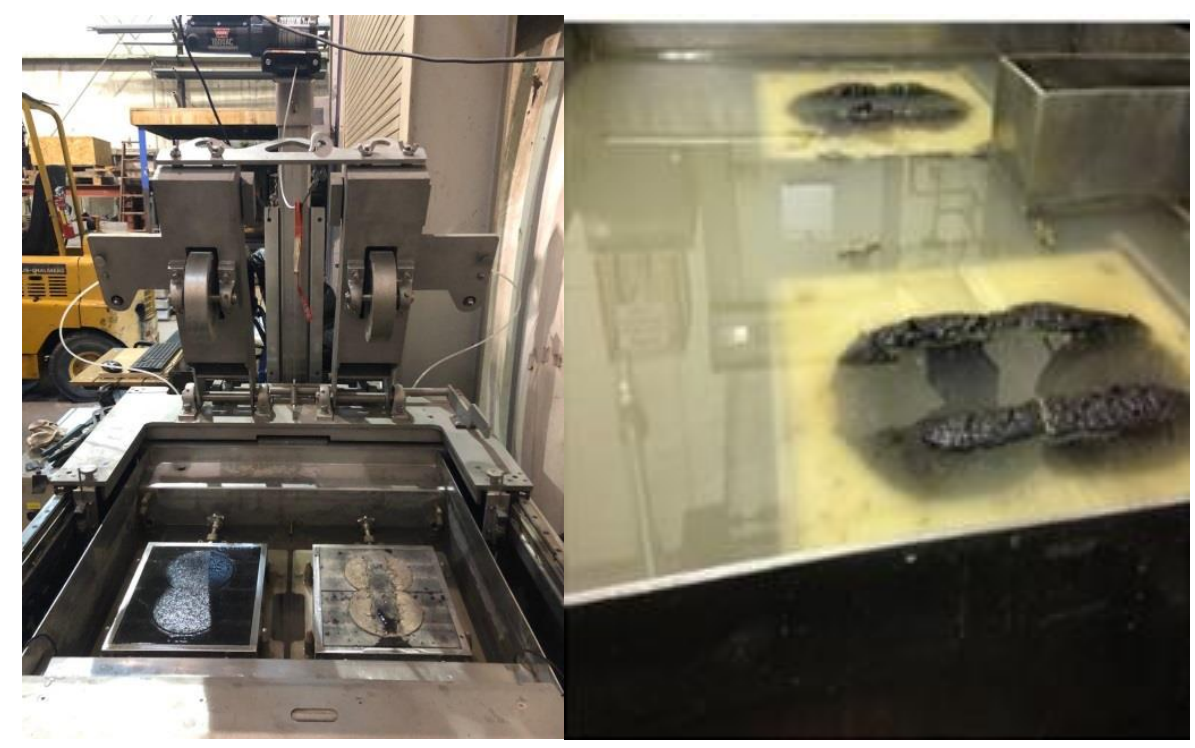


Figure 3-20 (a) A Hamburg wheel tracking device, (b) Asphalt sample after 20,000 passes in the Hamburg device.

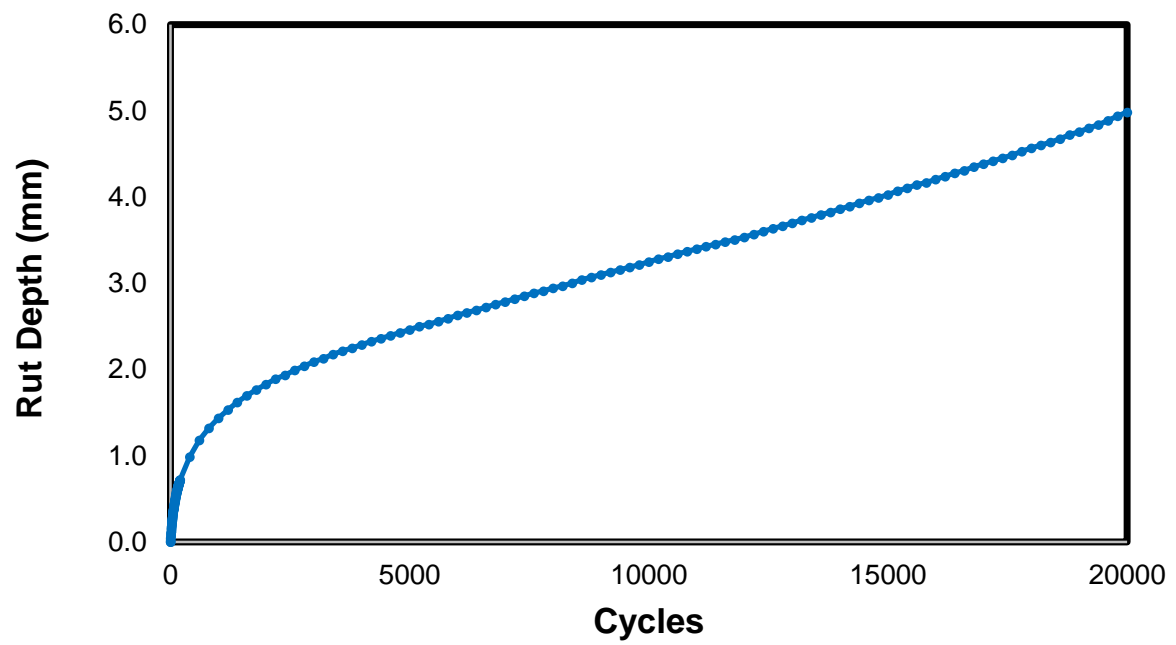

Figure 3-21 Typical rut depth curve versus number of passes in Hamburg wheel tracking test.

The range of the parameters used is summarized in Table 3-8. To avoid overfitting which the main concern in machine learning problems, the database was divided into three subsets for model evaluation purposes, namely:

- Learning: the sample of data used to fit the model ( 70\%).

- Testing: the sample of data used to provide an unbiased evaluation of a model fit on the training dataset while tuning model parameters $(\sim 15 \%)$.

- Validation: the sample of data used to provide an unbiased evaluation of a final model fit on the training dataset $(\sim 15 \%)$. 
Model calibration and preliminary evaluation was done using the learning and validation sets, respectively. The accuracy of the models was assessed using the following indexes:

- R: Correlation coefficient

- RMSE Root mean squared error

- MAE: Mean absolute error

Determination coefficient (R), root mean squared error (RMSE) and mean absolute error (MAE) were used as the performance measures:

$$
\begin{aligned}
& R=\sqrt{\frac{\left(\sum_{i=1}^{n}\left(o_{i}-\overline{o_{l}}\right)\left(t_{i}-\overline{t_{l}}\right)\right)^{2}}{\sum_{1=1}^{n}\left(O_{i}-\overline{O_{l}}\right)^{2} \sum_{i=1}^{n}\left(t_{i}-\bar{t}_{l}\right)^{2}}} \\
& R M S E=\sqrt{\frac{\sum_{i=1}^{n}\left(o_{i}-t_{i}\right)^{2}}{n}} \\
& M A E=\frac{\sum_{i=1}^{n}\left|o_{i}-t_{i}\right|}{n}
\end{aligned}
$$

where,

$O_{i}$ : Measured output

$t_{i}$ : Predicted output

$\overline{O_{i}}$ : Average of measured outputs

$\overline{t_{i}}$ : Average of predicted outputs

$n$ : Number of samples 
Table 3-8 Statistical parameters of the dependent and independent variables.

\begin{tabular}{lllllllllllllll}
\hline & $\begin{array}{l}\text { Mix } \\
\text { type }\end{array}$ & $\begin{array}{l}\text { UTI } \\
\left({ }^{\circ} \mathrm{C}\right)\end{array}$ & $\begin{array}{l}\text { HTPG } \\
\left({ }^{\circ} \mathrm{C}\right)\end{array}$ & $\begin{array}{l}\text { AC } \\
(\%)\end{array}$ & $\begin{array}{l}\text { ABR } \\
(\%)\end{array}$ & $\begin{array}{l}\text { NMAS } \\
(\mathrm{mm})\end{array}$ & $\begin{array}{l}\text { RAP } \\
(\%)\end{array}$ & $\begin{array}{l}\text { RAS } \\
(\%)\end{array}$ & G & AT & $\begin{array}{l}\text { CRC } \\
(\%)\end{array}$ & $\begin{array}{l}\text { T } \\
\left({ }^{\circ} \mathrm{C}\right)\end{array}$ & Passes & $\begin{array}{l}\mathrm{R} \\
(\mathrm{mm})\end{array}$ \\
\hline Mean & 1.1 & 87.8 & 58.9 & 5.9 & 28.8 & 11.3 & 20.9 & 8.0 & 1.2 & 1.2 & 2.3 & 52.2 & 13131 & 3.8 \\
Median & 1.0 & 86.0 & 58.0 & 5.7 & 32.5 & 12.5 & 20.4 & 0.0 & 1.0 & 1.0 & 0.0 & 50 & 10000 & 2.7 \\
Range & 1.0 & 18.0 & 24.0 & 2.8 & 48.4 & 14.3 & 35.3 & 33.0 & 1.0 & 1.0 & 10.0 & 24 & 15000 & 19.2 \\
Max & 2.0 & 98.0 & 70.0 & 7.9 & 48.4 & 19.0 & 35.3 & 33.0 & 2.0 & 2.0 & 10.0 & 64 & 20000 & 19.7 \\
Min & 1.0 & 80.0 & 46.0 & 5.1 & 0.0 & 4.8 & 0.0 & 0.0 & 1.0 & 1.0 & 0.0 & 40 & 5000 & 0.6 \\
\hline
\end{tabular}

The GEP algorithm was run multiple times to explore optimal parameter settings (Table 3-9). Different values were set for model parameters such as number of chromosomes, genes, head size, and mutation rate. In general part, number of chromosomes affects the time of the simulation run. By increasing the number of chromosomes, the runs take longer. The head size shows the complexity of each term in the evolved model [103]. Three replicate model runs were performed for each setting, which resulted in total of 162 runs.

Table 3-9 The setting of the GEP parameters.

\begin{tabular}{lll}
\hline & Parameter & Setting \\
\hline & Chromosomes & $20,30,50$ \\
& Genes & $3,4,5$ \\
& Head size & $8,10,15$ \\
General & Linking function & Addition \\
& & $+,-, \times, /, \sqrt{ }, 3 \sqrt{ }$, \\
& Function set & ln, Log, Power, \\
& & exp, Min, Min 3, \\
& Generation without change & 2000 \\
\hline \multirow{3}{*}{ Complexity increase } & Number of tries & 3 \\
& Max. complexity & 5 \\
\hline \multirow{2}{*}{ Genetic operator } & Mutation rate & $0.00138,0.044$ \\
& Inversion rate & 0.00546 \\
\hline \multirow{3}{*}{ Numerical constants } & Data type & Floating-point \\
& Lower bound & -10 \\
& Upper bound & 10 \\
\hline
\end{tabular}


The best performing GEP prediction model for rut depth prediction was found to be in the following form:

$R u t(m m)=Y_{1} \times Y_{2} \times Y_{3} \times Y_{4} \times Y_{5} \times Y_{6}$

where,

$$
\begin{aligned}
& Y_{1}= \\
& \left(\operatorname { s i n } \left(\left(\operatorname{gepMin} 4\left(G 1 C 3,\left((G 1 C 2-d(12))^{\wedge}(1 / 3)\right),\left(d(10)^{\wedge} 2\right),(G 1 C 9 / d(9))\right)\right.\right.\right. \\
& \left.+((d(10) * G 1 C 3) / \tan (d(1)))))^{\wedge} 2\right) \\
& Y_{2}= \\
& \text { gepMin } 4((((((G 2 C 7+d(12)+G 2 C 0+G 2 C 1) / 4.0)+d(11))+(G 2 C 8 \\
& +G 2 C 1)) / 2.0),(\cos (d(12))+(d(8)+d(9))), d(6),((d(4)-d(10)) \\
& +(1.0-G 2 C 9)))
\end{aligned}
$$

$$
Y_{3}=
$$

$$
\begin{gathered}
((((((d(7) * G 3 C 4)+(d(12) * d(7))+(d(12)+d(8))+(d(8) * d(7))) / 4.0) \\
\left.\left.+(d(7) * d(4)))+\left(\left(G 3 C 9^{\wedge} 2\right) * \cos (d(5))\right)\right)\right)^{\wedge}(1 / 3)
\end{gathered}
$$

$$
Y_{4}=
$$

$$
\begin{aligned}
& (1.0 /((\max (d(5),(d(5) *(d(8) * d(4))))-((1.0-(d(12) * \mathrm{G} 4 \mathrm{C} 9))-((d(3) \\
& -\mathrm{G} 4 \mathrm{C} 1) * \mathrm{~d}(2))))))
\end{aligned}
$$

$$
Y_{5}=
$$$$
\max (((d(13)+(\tan (((d(5)+d(4)+d(8)+G 5 C 7) / 4.0)) *(d(5)
$$$$
* d(4)))) / 2.0),\left(\left((G 5 C 0 * d(1))^{\wedge} 2\right) *(((d(10)+d(9)+d(6)\right.
$$$$
\left.\left.\left.+d(3))(4.0)^{\wedge} 2\right)\right)\right)
$$

$Y_{6}=$ 


$$
\begin{gathered}
\left(1.0 /\left(\text { gepMin3 } \left(\left(\left(\left(\left(\left(\left(d(5)^{\wedge}(1 / 3)\right)+(G 6 C 0+G 6 C 7)\right) / 2.0\right)+(d(11)-G 6 C 8)\right)\right.\right.\right.\right.\right. \\
+\cos ((d(12) * d(1)))) / 2.0), d(12), d(1))))
\end{gathered}
$$

where, gepMin3(x, y, z) and gepMin4(a, b, c, d), respectively, denote $\min (\min (x, y), z)$ and $\min (\min (\min (a, b), c), d)$. Also, the constants and variables included in the model are as follows:

$\mathrm{G} 1 \mathrm{C} 3=5.04 ; \mathrm{G} 1 \mathrm{C} 9=-3.70 ; \mathrm{G} 1 \mathrm{C} 2=-0.76 ; \mathrm{G} 2 \mathrm{C} 8=-3.01 ; \mathrm{G} 2 \mathrm{C} 1=-3.79 ; \mathrm{G} 2 \mathrm{C} 9=3.59$; $\mathrm{G} 2 \mathrm{C} 7=-4.81 ; \mathrm{G} 2 \mathrm{C} 0=-0.80 ; \mathrm{G} 3 \mathrm{C} 9=18.67 ; \mathrm{G} 3 \mathrm{C} 4=-17.87 ; \mathrm{G} 4 \mathrm{C} 9=-33.55 ; \mathrm{G} 4 \mathrm{C} 1=$ 2.73; G5C0 = 2.68; G5C7 = -7.16; G6C8 = -3.94; G6C0 = -1.36; G6C7 = -5.96;

$\mathrm{d}(1)=$ Mix type, $\mathrm{d}(2)=\mathrm{UTI}, \mathrm{d}(3)=$ High PG, $\mathrm{d}(4)=$ Asphalt Content, $\mathrm{d}(5)=$ ABR (\%), $\mathrm{d}(6)$ $=$ NMAS, $\mathrm{d}(7)=\operatorname{RAP}(\%), \mathrm{d}(8)=\operatorname{RAS}(\%), \mathrm{d}(9)=$ Gradation, $\mathrm{d}(10)=$ Agg Type, $\mathrm{d}(11)=$ Crumb Rubber $(\%), \mathrm{d}(12)=$ Test Temperature $(\mathrm{C}),. \mathrm{d}(13)=$ Number of Passes

Figure 3-22 exhibits the performance of the GEP-based ML models trained with the aforementioned HWTT dataset. A compassion of measured versus predicted Rut values is shown in Figure 3-23 for the entire data. Despite the relatively small data set used (from a typical ML training perspective), satisfactory performance of the GEP model was attained.

The best GEP models were selected based on a multi-objective strategy that involved simplicity of the models and their least prediction error during the learning and validation stages [137]. 

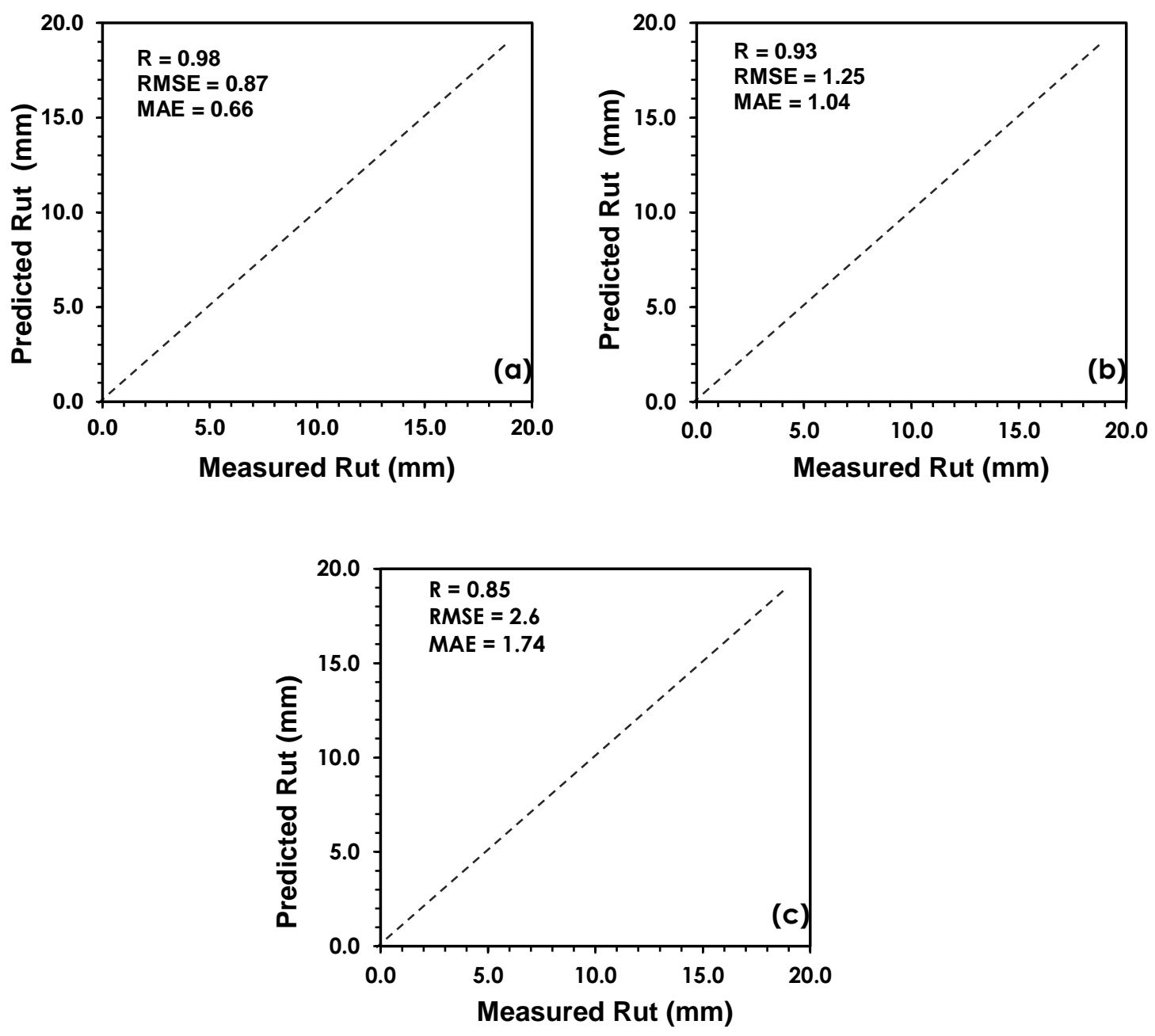

Figure 3-22 Performance of the GEP model: (a) learning data, (b) testing data, (c) validation data. 


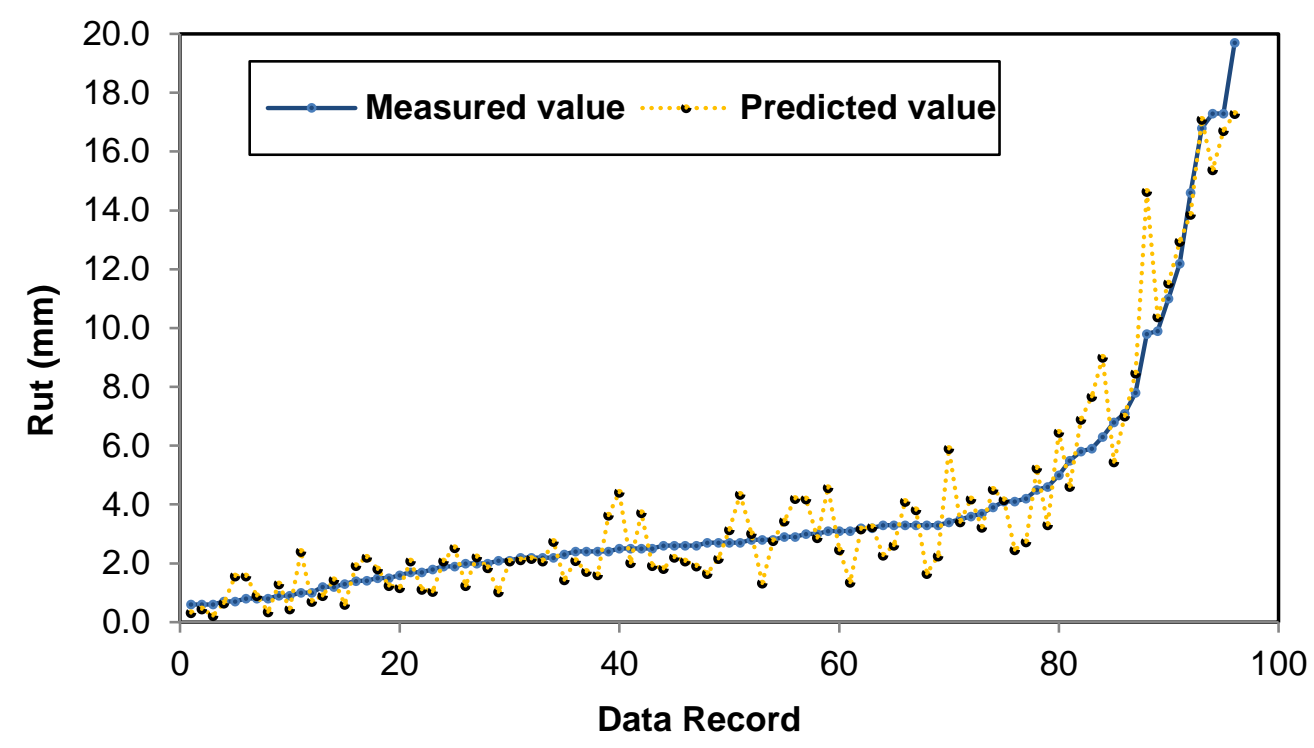

Figure 3-23 Measured versus predicted rut depth across all data points

\subsubsection{Sensitivity analysis}

A sensitivity analysis was carried out to explore the relative importance of variables in the GEP model. The results are presented in Figure 3-24. The major observations were:

- Mix type, $\mathrm{ABR} \%$, and test temperature had the highest influence on the rut depth.

- NMAS, gradation, and UTI had the lowest influence on rut depth. However, it must be noted that these variables are not independent. For example, change of NMAS and gradation will be led to change in binder content of the mixture and consequently rutting will be affected. 


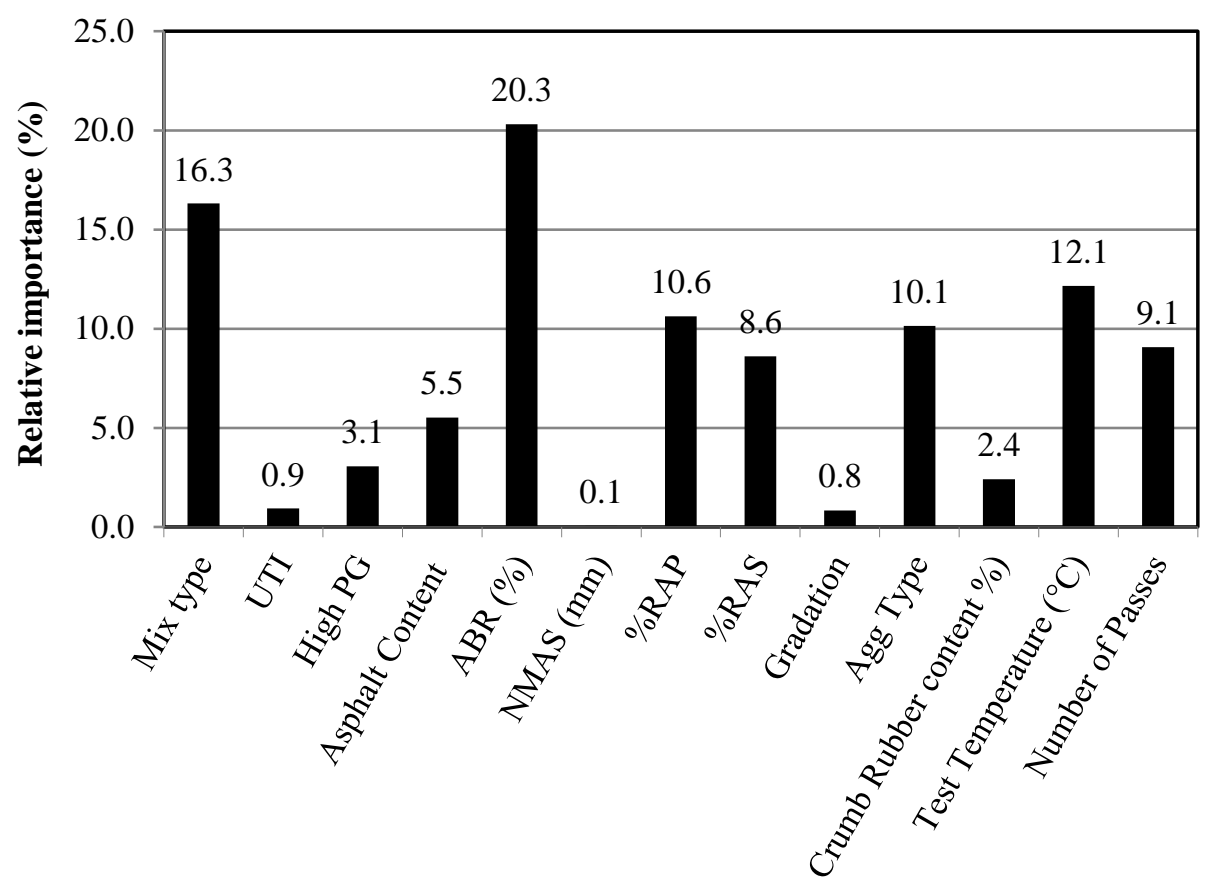

Figure 3-24 Variable importance in the GEP model.

The predictive machine-learning model presented in this study has been subsequently used to predict HWTT results for several plant- and lab-compacted mixtures across the Midwest. In this part of the study, sensitivity of the recycled material (RAP and RAS), asphalt content, binder grade type, and test temperature are evaluated. To investigate the effect of each factor, the factor of interest was incrementally changed while all others variables were held constant (Figure 3-25). The results in Figure 3-25a, b and c are predicted. Based on these results, the following expected results were obtained:

- The model is capable of capturing differences in recycling content, where increased ABR clearly reduces rutting potential.

- As binder content increases, rutting depth increases.

- The high temperature Performance Grade in the virgin binder is one of the important 
factors in determining rut depth (Figure 3-25c).

- Referring to Figure 3-25d, the model is capable of predicting a realistic trend of rutting with the number of wheel passes, and can produce a very realistic family of curves as a function test temperature. As expected, higher test temperature results in higher rutting.
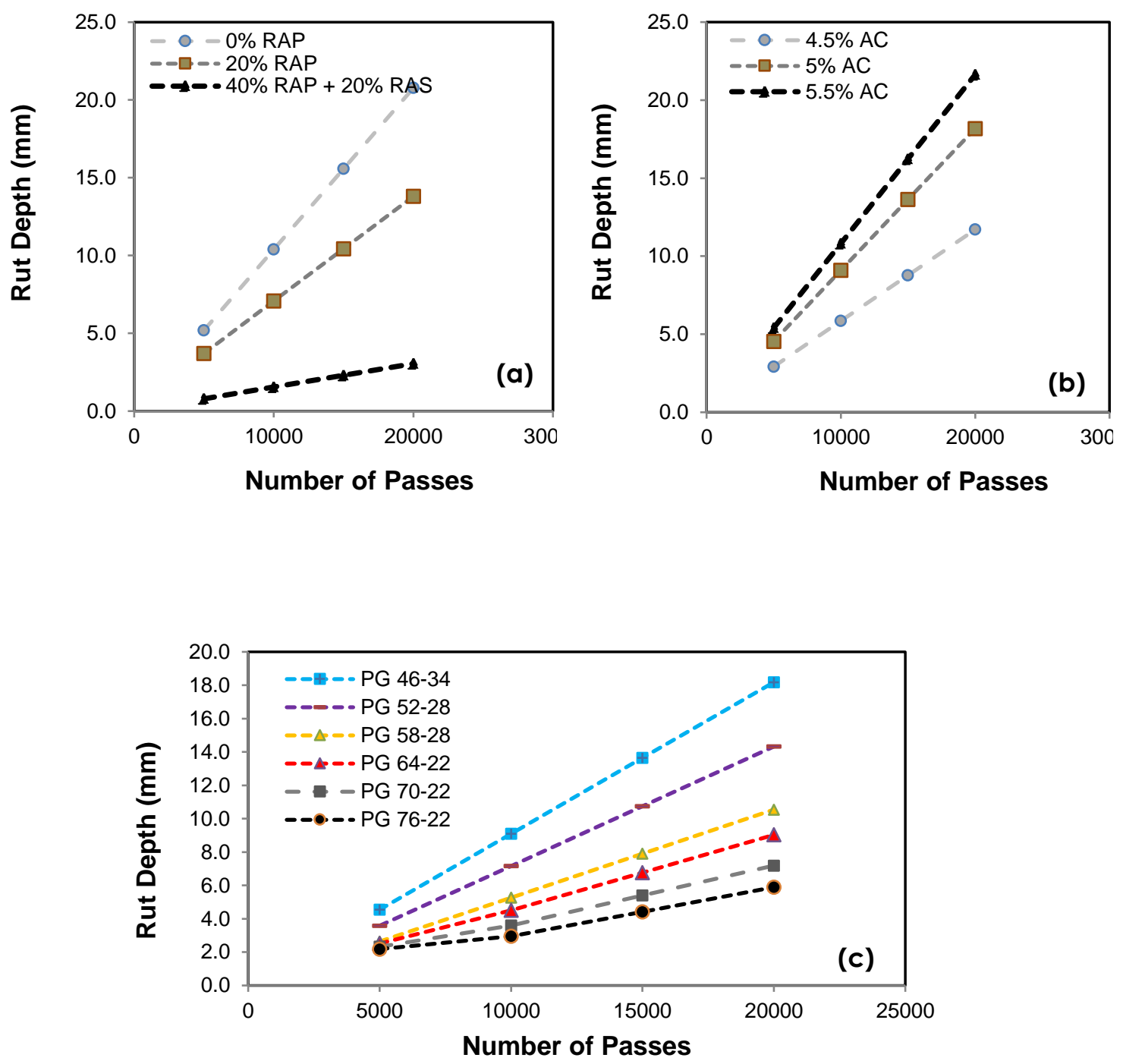


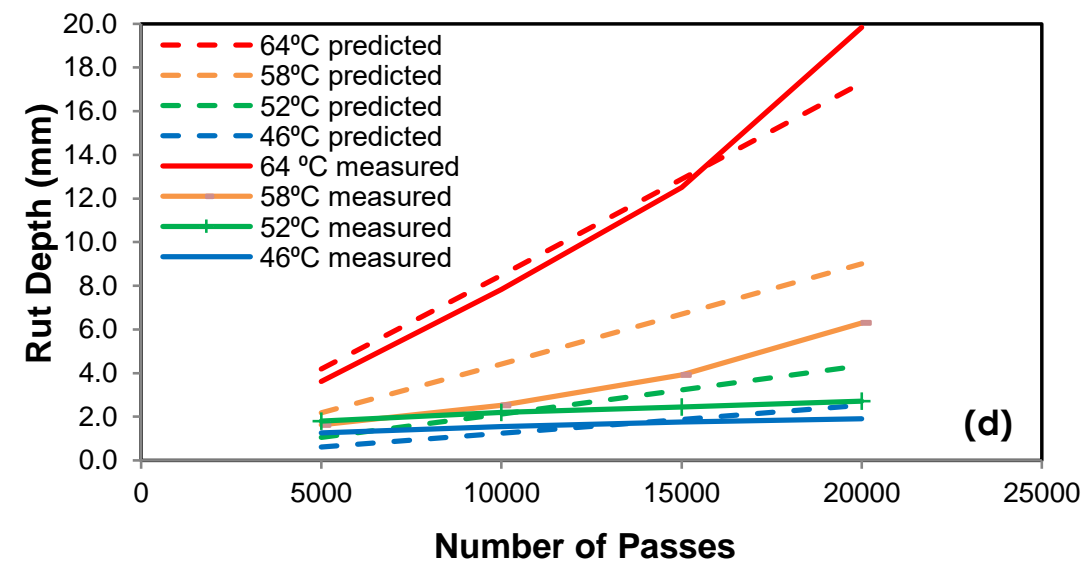

Figure 3-25 Effect of a) recycling content b) binder content c) binder PG and d) test temperature on rutting depth.

\subsubsection{Performance Space Diagram}

Buttlar et al., (2016) introduced a Performance-Space diagram concept for use in balanced mix design and evaluation [96]. In this technique, low temperature fracture energy is plotted on an X-Y style plot with HWTT rut depth (Figure 3-26). Similar approaches involving interaction-type plots have been implemented by other researchers in recent years [44], [171]-[173]. The advantage of using such a graphical approach is that it allows a holistic view of the mixture performance on the high- and low-temperature spectrum, and gives an insight into the changes in performance of the mixture with different mixture variables. For example, [96] and [18] showed, in separate studies, the possibility of using higher recycle material in asphalt mixtures if softer base binder is used by studying the relative position of the mixtures on the Performance-Space diagram ( W. G. Buttlar et al., 2017; Rath et al., 2019).

In this section, the proposed Hamburg rut depth model along with the $\mathrm{DC}(\mathrm{T})$ fracture energy prediction model by Majidifard et al. (2018) were simultaneously used to verify the 
trends exhibited by asphalt mixtures on a performance-space diagram based on varied mixture constituents [31]. Mixtures with different percentages of recycled materials (RAP/RAS, GTR), and binder types were used, and trends obtained from two the ML predictive models were compared to laboratory-measured results (shown in Table 3-10).

Figure 3-27 shows the effect of using softer base binder with various rubber additives, shown in a performance-space diagram. Rubber-modification has been shown to bump up the high temperature grade of the asphalt binder, and in this case, using a softer base binder has a similar effect as with the RAP-modified mixtures. Using a softer binder leads to a higher fracture energy while lowering rut resistance in the asphalt mixtures. The ML models were able to predict the general trend of the effect of using a softer base binder with rubber modification in asphalt mixtures.

Figure 3-27 shows the effect of using softer binder in conjunction with RAP. The use of RAP stiffens the asphalt mixture, adversely affecting low-temperature cracking resistance while improving rutting [16], [175], [176]. Addition of softer binder helps the mixture regain some of its thermal cracking resistance while sacrificing its rutting resistance. Once again, the figures show that the model is able to predict this general trend of the effect of using softer base binder with RAP.

Figure 3-28 shows the effect of RAS on asphalt mixture high and low temperature performance properties. It should be noted both figures represent the predicted values generated by the model The mixture plotted in 8 was once again used, where the RAP was reduced to $20 \%$ (from $31.8 \%$ ) and then the RAS content was varied from $5 \%$ to $15 \%$. This process was repeated with a softer base binder (PG 58-28). Clearly, adding RAS results in 
the stiffening of the mixture, resulting in turn to lowered fracture energies and increased rutting resistance in the aspahlt mixture. This trend follows the general trend reported in various studies involving RAS-modified mixtures [18].

Table 3-10 Mixture performance indicators from laboratory tests.

\begin{tabular}{|c|c|c|c|}
\hline Mix & $\begin{array}{c}\text { DC(T) Fracture } \\
\text { Energy }\left(\mathbf{J} / \mathbf{m}^{2}\right)\end{array}$ & COV $(\boldsymbol{\%})$ & $\begin{array}{c}\text { Hamburg Rut Depth } \\
(\mathbf{m m})\end{array}$ \\
\hline $58-28+10 \%$ TB-GTR* & 570 & 22.8 & 3.39 \\
\hline $46-34+10 \%$ TB-GTR & 754 & 16.3 & 6.60 \\
\hline $58-28+10 \%$ dry-GTR** & 547 & 9.0 & 3.12 \\
\hline $46-34+10 \%$ dry-GTR & 635 & 2.8 & 2.9 \\
\hline $64-22+45 \% \mathrm{RAP}^{+}$ & 286 & 8.7 & 3.7 \\
\hline $58-28+45 \% \mathrm{RAP}^{+}$ & 348 & 18.1 & 10.2 \\
\hline $46-34+45 \% \mathrm{RAP}^{+}$ & 377 & 1.4 & \\
\hline
\end{tabular}

*TB-GTR - Terminal Blend GTR. **dry-GTR - Dry process GTR. Refer to [18] for more details on the mixture.

${ }^{+}$Refer to [96]b

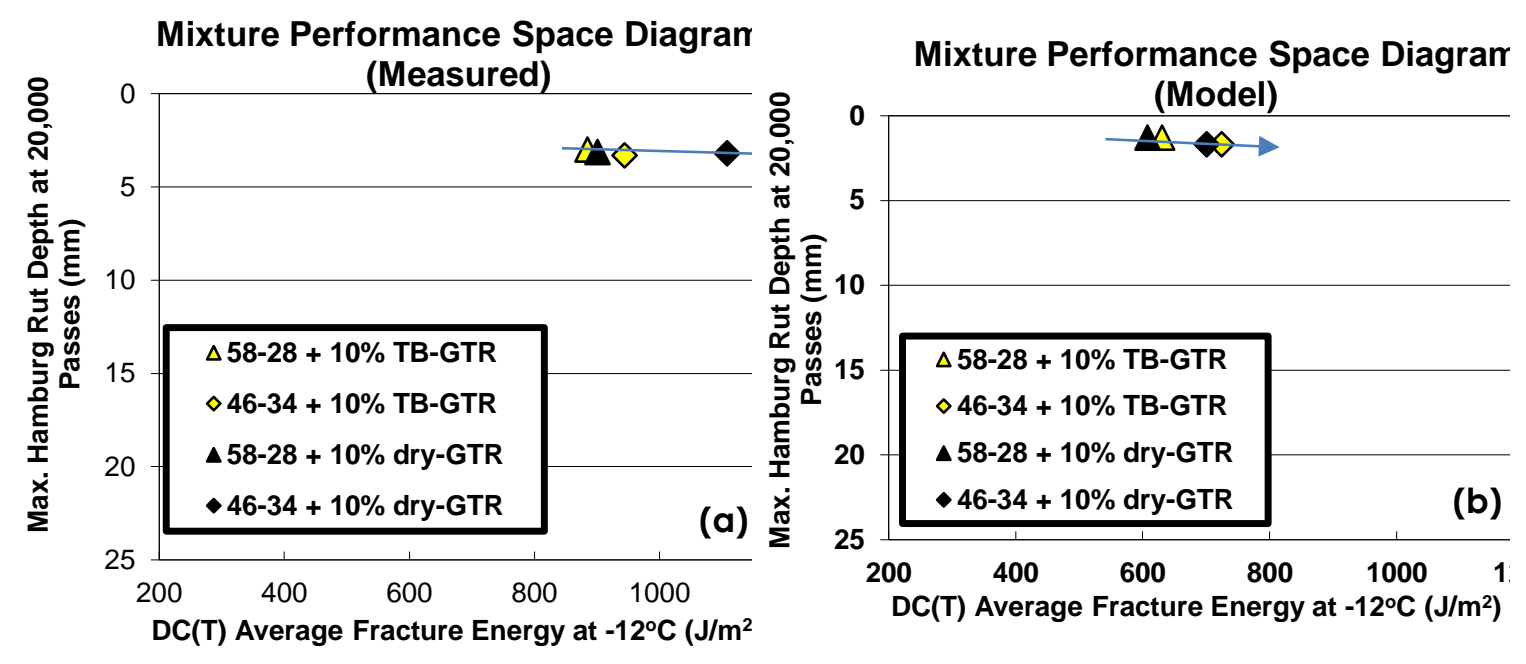

Figure 3-26 Effect of using softer binder in rubber modified mixture with the 
application of performance space diagram, (a) measured laboratory values versus, (b) predicted values.

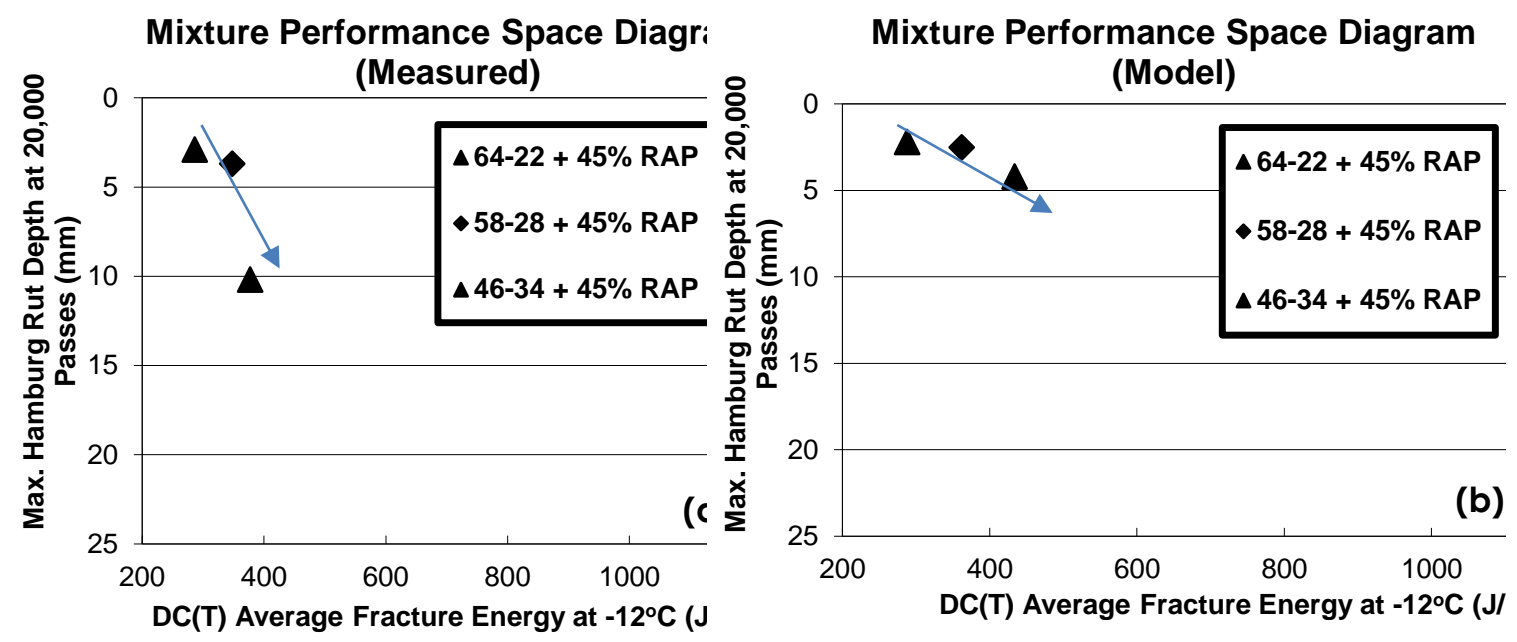

Figure 3-27 Effect of using softer binder in recycled mixture with the application of performance space diagram, (a) measured laboratory values versus, (b) predicted values.
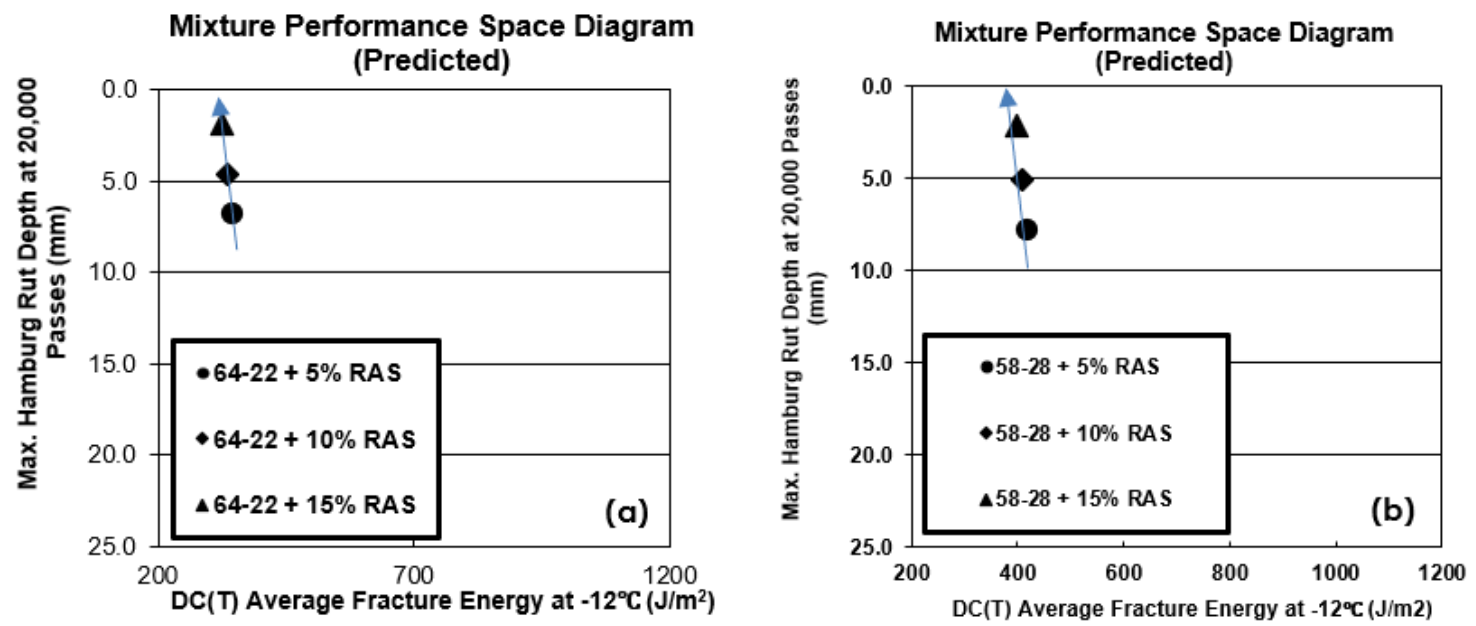

Figure 3-28 Effect of RAS and binder PG grade in asphalt mixture with the application of performance space diagram, predicted (a) for 58-28 binder, (b) for 46-34 binder. 
Although the model is able to predict the general trends with good accuracy, there is still a need to increase predictive accuracy for mixtures outside of the training dataset. Due to the relatively small training data set used, the model is not yet able to accurately distinguish between mixtures with different aggregates types. Similarly, the training data mostly consisted of mixtures that had both RAP and RAS, which currently limits the model's ability to accurately predict the performance of mixtures contain only RAS.

\subsubsection{Summary}

In this part of study, a new model was developed to predict the rutting depth of asphalt mixtures using a GEP-based modeling approach. Mix type, ABR \%, PG high temperature grade, and test temperature were found to be more highly related to HWTT rut depth as compared to other variables. The effect of asphalt additives such as ground tire rubber was characterized by the model with respect to both rutting, and by employing a recently developed related model, thermal cracking. By having both high and low temperature prediction models, screening of materials for balanced mix design can be greatly facilitated. The proposed models can and should be updated with more data in the future. Since performing Hamburg wheel track test might be cost-restrictive for some users, using the proposed ML-based model can save time and expense during the material screening phase. Further research will focus on including Hamburg wheel track test results for mixtures with a much wider range in properties, and will include a larger span of aggregate types and will include more HWTT results conducted across a range of test temperature, to assist agencies in evaluating proper HWTT test temperatures to use for the purpose of balanced mix design in various climates across the US and beyond. Use of the modeling 
technique to predict the presence and magnitude of the stripping inflection point will also be pursued. 


\subsection{IMPLEMENTING DEEP LEARNING CONVULSION NEURAL NETWORK TO PREDICT HAMBURG RUTTING CURVE}

Recently, there has been a growing trend withing the pavement community to deploy advanced machine learning and deep learning methods for the characterization of asphalt pavements. In this arena, Majidifard et al. used genetic expression programming (GEP) to develop prediction models for both fracture energy of asphalt at low temperature and rutting performance at high temperature. This study proposes a new deep learning approach to formulate the rutting depth obtained from the HWTT as a function of asphalt mixture properties. Considering the increasing use of the HWTT in a balanced mix design across the United States, such models can provide time-and-cost-saving options for the purposes of an initial design and material screening. A subset of deep learning called convolution neural network $(\mathrm{CNN})$ method is used to create an analytical model that can be easily programmed into a spreadsheet or cloud-based services. Sensitivity and parametric analyses are performed to validate the reliability of the model.

\subsubsection{Convolution Neural Network}

CNNs is a branch of deep learning motivated by physiological processes between neurons that mimic visual cortex systems [177]. A CNN structure is created by an input, multiple hidden layers, and an output layer. The hidden layers typically include a series of convolutional layers that convolve with multiplication or other dot products. Each hidden layer is followed by an activation function, which is commonly taken as Rectified linear units (RELU) layer. Hidden layers are consisting of neurons with learnable weights

and biases. Each neuron takes various inputs, exerts a weighted sum across them, transfers 
it within an activation function, and returns an output. A loss function at the end of the structure can minimize the error and re-assign new weights to the neurons. Multilayer perceptrons are regulated to form $\mathrm{CNN}$. In multilayer perceptrons structure, the layers are fully connected, which means that each neuron in one layer is connected to all neurons in the next layer [178]-[181].

The application of $\mathrm{CNN}$ in the pavement arena generally involves either classification or regression approaches. Most classification applications using the CNN model involve detecting and classifying pavement distresses [33], [34], [182]. For instance, Zhang et al. [183] established CrackNet software using CNN to detect patches [183]. Also, Majidifard et al. [34] developed YOLO v2 and Faster R-CNN frameworks to classify pavement distress using a comprehensive database. Also, Majidifard et al. [33] developed an automatic pavement distress detection tool to classify and quantify the severity of the distresses via hybrid YOLO and U-Net models [33]. Jiang et al. [184] used CNN to classify asphalt mixtures components (aggregate, mastic, and air void) from X-ray images [184]. There are limited studies with focus on using $\mathrm{CNN}$ for regression problems. Gong et al. [185] have recently used a deep neural network to predict pavement rutting for the mechanistic-empirical pavement design guide [185]. The current study aims at harnessing the power of CNNs for developing the HWTT rutting prediction models.

\subsubsection{Experimental Database}

On the basis of the literature reviewed, this study presents a new CNN-based prediction model for the HWTT rut depth vs. wheel pass as follows:

Rut depth $(\mathrm{mm})=$ 
f(Mix type, UTI, HTPG $A C, N M A S, A B R, R A P, R A S, G, A T, C R C, T, P a s s$ )

where,

Mix type: Plant or lab-compacted mixture (Plant (loos asphalt mixture collected from plant and compacted in the lab) $=1, \mathrm{Lab}$ (produced from virgin materials in lab) $=2$ )

$U T I\left({ }^{\circ} \mathrm{C}\right)$ : Useful temperature interval (i.e., for PG 64-22 binder, UTI $=64-(-22)=86^{\circ} \mathrm{C}$ )

$H T P G\left({ }^{\circ} \mathrm{C}\right)$ : High temperature performance grade (for PG 64-22 binder, this is $64^{\circ} \mathrm{C}$ )

$A C(\%)$ : Asphalt content

NMAS (mm): Nominal maximum aggregate size. In this study, NMAS can take $4.75 \mathrm{~mm}$, 9.5mm, $12.5 \mathrm{~mm}$ or $19 \mathrm{~mm}$.

$A B R(\%)$ : Asphalt Binder Replacement (ratio of the recycled binder to the total binder)

$R A P(\%):$ Reclaimed asphalt pavement, \% by $\mathrm{ABR}$

RAS (\%): Reclaimed asphalt shingles, \% by ABR

$G$ : Gradation type (Dense: 1; SMA: 2)

AT: Aggregate Type (Limestone: 1; Granite: 2)

$C R C(\%)$ : Crumb rubber content (rubber percentage in virgin binder)

$T\left({ }^{\circ} \mathrm{C}\right)$ : Testing temperature

Pass: Number of wheel passes

The models were developed using 10,000 data point from HWTT results from various tests conducted on fifty materials from pavements in the Midwest United States, following AASHTO-T324. For each test, three samples were tested and the average was reported as the test results. Therefore, 150 samples were tested to collect 10,000 test results. Each curve contained 200 data points (Figure 3-29). Moreover, this study evaluated different test temperatures for the Hamburg wheel test. Various DOTs and agencies are adopting Hamburg test temperatures according to the prevailing climatic conditions instead of the standardized recommended value of $50^{\circ} \mathrm{C}$. Therefore, the proposed model can be considered a practical tool for design purposes by DOTs as it incorporates the temperature as a variable in its predictions. 


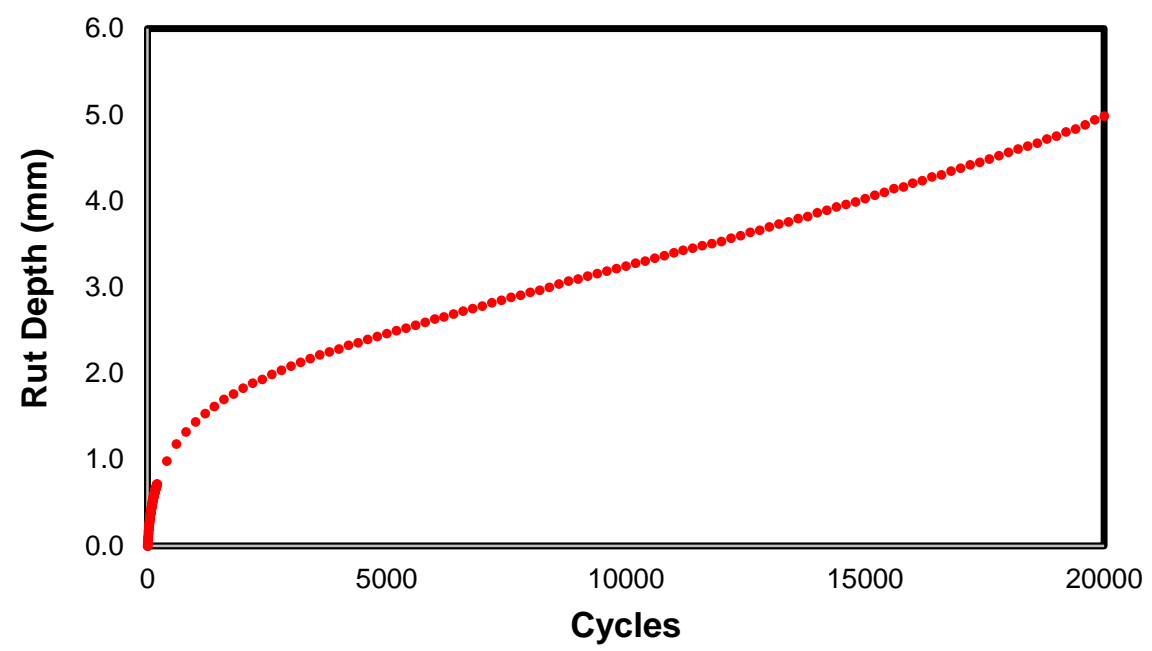

Figure 3-29 Typical rut depth curve versus number of passes in Hamburg wheel tracking test (200 data points).

The ranges of the parameters used to develop the prediction model are summarized in Table $3-11$.

Table 3-11 Descriptive statistics of the variables

\begin{tabular}{ccccccc}
\hline & Mean & Median & Mode & Max & Min & Range \\
\hline Mix type (Plant=1, Lab=2) & 1.4 & 1 & 1 & 2 & 1 & 1 \\
UTI & 86.4 & 86 & 86 & 98 & 80 & 18 \\
High PG & 56.8 & 58 & 58 & 70 & 46 & 24 \\
Asphalt Content (AC) (\%) & 5.7 & 5.4 & 5.1 & 7.9 & 5.1 & 2.8 \\
ABR (\%) & 31.2 & 32.5 & 35.2 & 48.4 & 17 & 31.4 \\
NMAS (mm) & 11.8 & 12.5 & 12.5 & 19 & 4.8 & 14.3 \\
RAP (\%) & 24.6 & 24 & 35.2 & 35.3 & 0 & 35.3 \\
RAS (\%) & 6.6 & 0 & 0 & 33 & 0 & 33 \\
Gradation (Dense=1, SMA=2) & 1.2 & 1 & 1 & 2 & 1 & 1 \\
Agg Type (limestone=1, Granite=2) & 1.2 & 1 & 1 & 2 & 1 & 1 \\
Crumb Rubber Content (CRC) & 3.7 & 0 & 0 & 20 & 0 & 20 \\
Temp ( $\left.{ }^{\circ} \mathbf{C}\right)$ & 51.6 & 50 & 50 & 64 & 40 & 24 \\
Number of Passes & 5046.3 & 198 & 0 & 20000 & 0 & 20000 \\
Rut Depth (mm) & 1.6 & 0.8 & 0 & 19.8 & 0 & 19.8 \\
\hline
\end{tabular}


Frequency histograms present the variability of the parameters (see Figure 3-30). As seen in the figure, the distribution of the variables is not uniform.
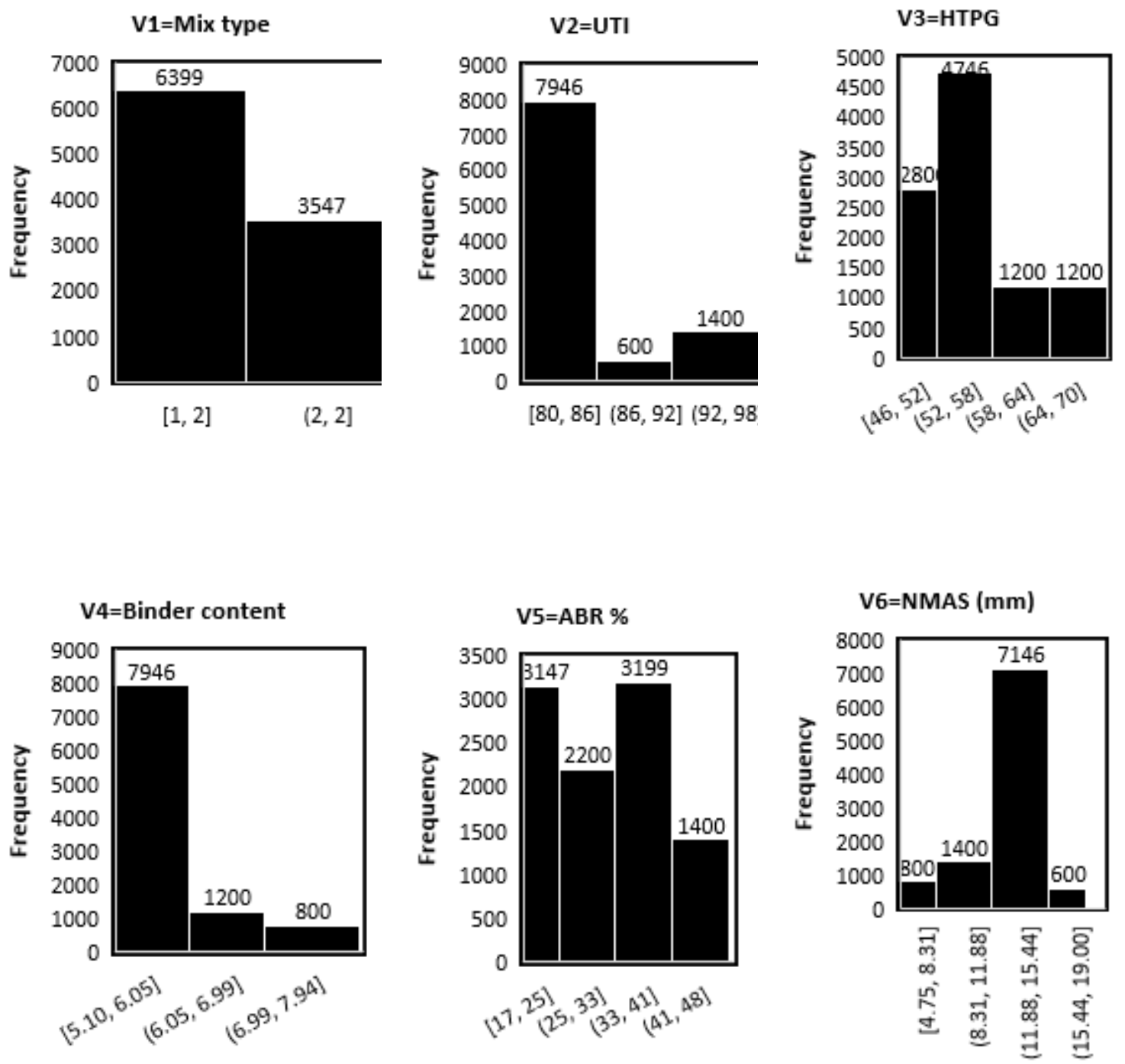

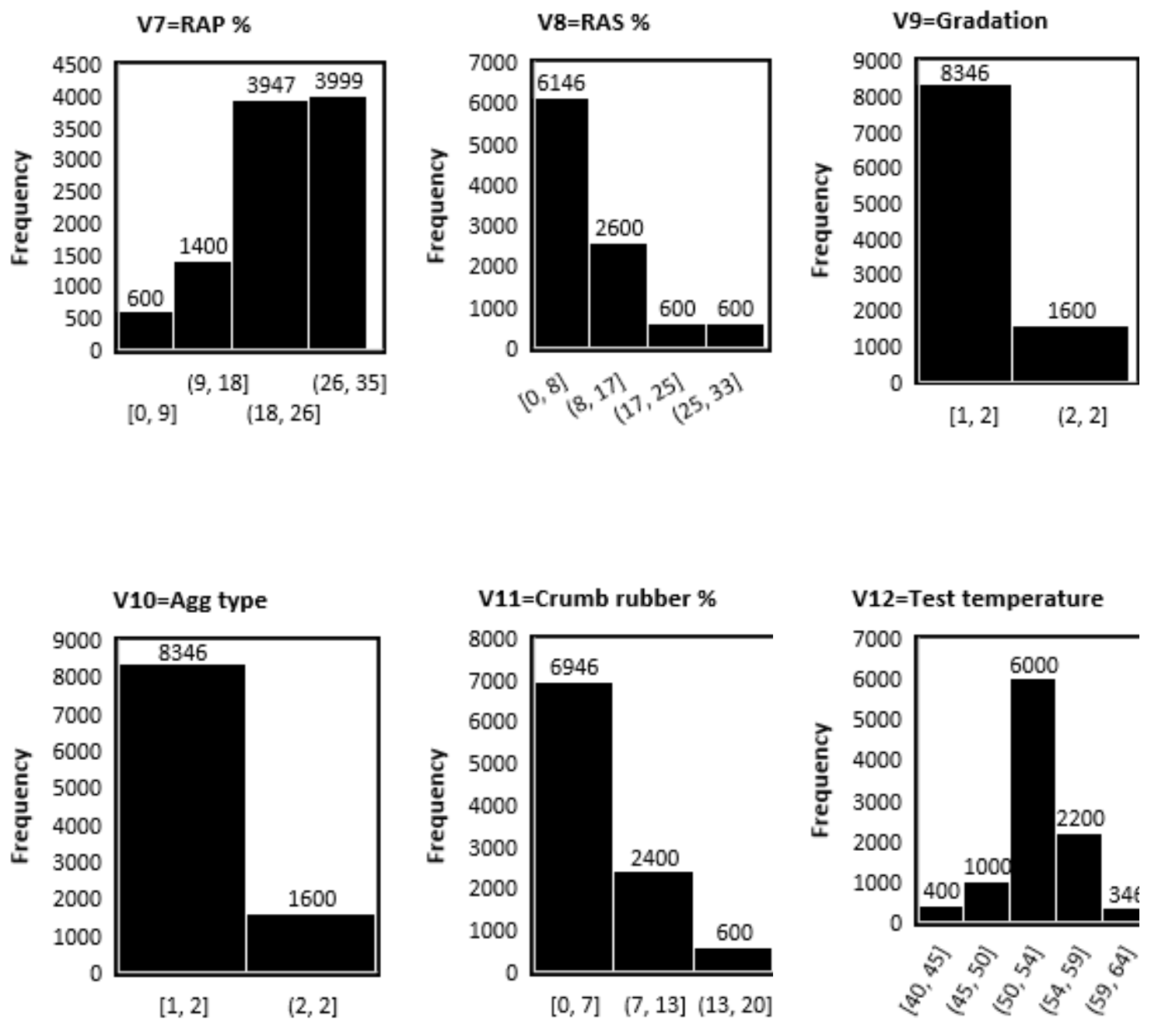

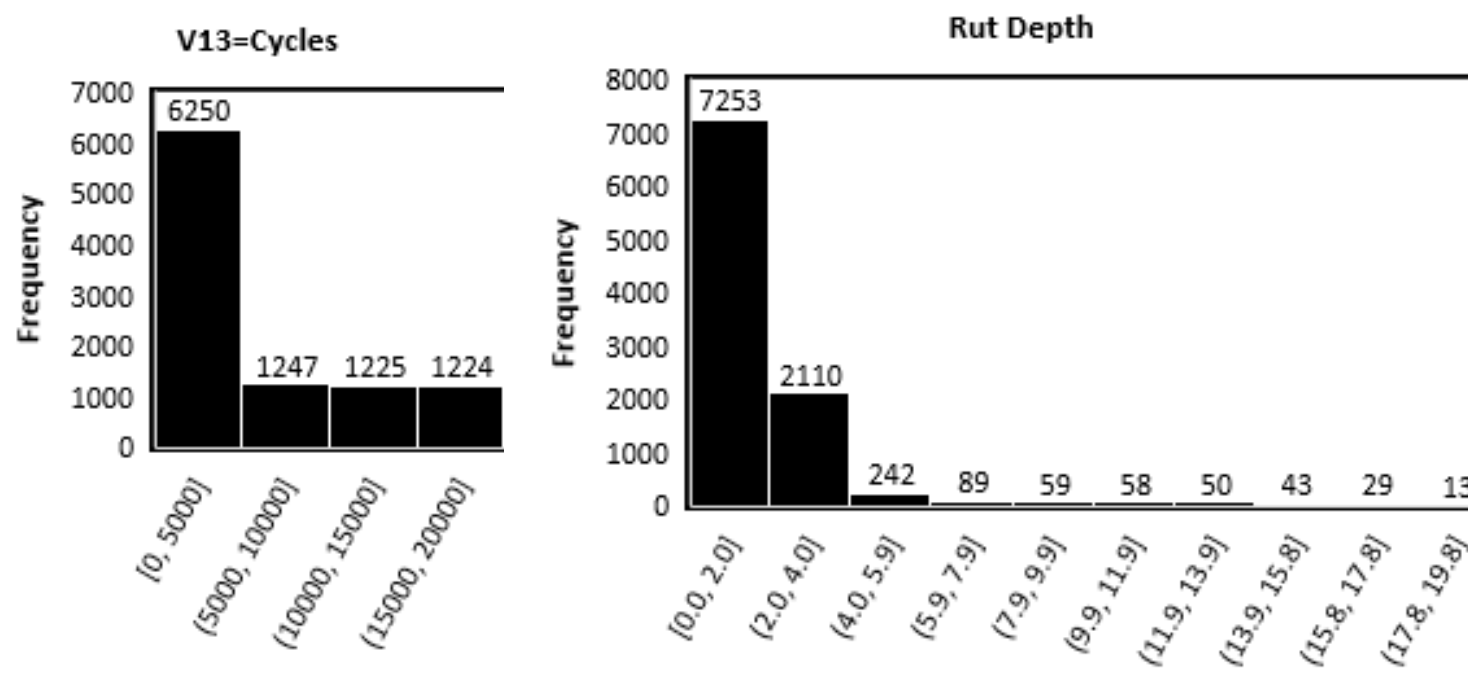

Figure 3-30 Distribution histograms of the variables

To avoid overfitting, the database was divided into three subsets:

- Learning: sample of data used to fit the model $(\sim 70 \%)$.

- Testing: sample of data used to provide an unbiased evaluation of a model fit on the training dataset while tuning model parameters $(\sim 15 \%)$.

- Validation: sample of data used to provide an unbiased evaluation of the final model on unseen dataset $(\sim 15 \%)$.

\subsubsection{Development of HWTT Prediction Model}

\subsubsection{Normalizing data}

Features normalization is the first step for training the dataset, which makes the training faster and more accurate. Therefore, both the training and testing dataset were normalized, as normalization should be applied to any data used to train or test the model. 


\subsubsection{Optimal CNN Structure}

\subsection{Convolutional layer}

The principal benefit of CNN comparing to neural networks is the use of parameter distribution in the convolutional layer. The convolutional layer utilizes multiple small filters to produce outputs instead of connecting each neuron set in the next layers [186].

\subsection{Activation units}

Activation function calculates the weighted sum and adds bias with it to determine if a neuron should be activated or not [179]. A neural network without an activation function is basically just a linear regression model. The activation function apply the non-linear conversion to the input, initiating the capability to learn and produce more complex tasks. In neural networks, the weights and biases of the neurons are updated according to the output error. Activation functions make the back-propagation feasible since the gradients are supplied along with the error to renew the weights and biases. Rectifier Linear Unit (ReLU) is the most popular and efficient activation function recently [179], [187] (Figure 3-31).

$$
f(x)=\max (0, x)
$$




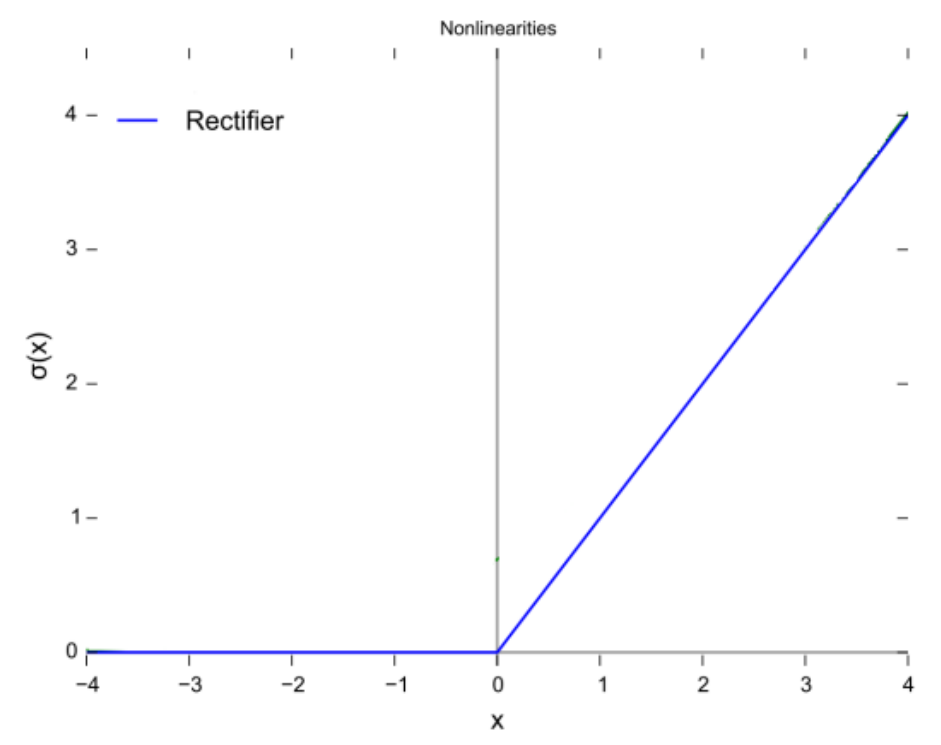

Figure 3-31 ReLU function (Nair et al., 2010)

ReLU is employed broadly in most deep learning models and greatly enhances model performance in most instances. Since the function derivation is " 1 " for positive values, it flags the errors to be back-propagated efficiently to avoid losing gradient. Also, the cost of computation reduces, and the model converges more accurately due to the simplicity of the function.

\subsection{Optimizing function}

The optimization technique is generally used in deep neural network training to minimize the loss function, precisely. The optimization function herein applied is the Root Mean Square Propagation (RMSProp) [188]. The default learning rate (0.001) suggested by the RMSprop was employed as an adaptive learning rate algorithm. 


\subsection{Regularization of overfitting}

Regularization procedures are explicitly designed to prevent overfitting and consequently enhance generalization. Some regularization technique examples include: early stopping, weight decay through L2 or L1 parameter regularization, dropout, and dataset increment [30], [44], [45]. In this study, we used an early stopping callback that examines training condition for each epoch. If a considered number of epochs lapses without showing improvement, then the algorithm stops the training.

In this study, the proposed model consisted of a sequential model with two densely connected hidden layers and an output layer that delivered unique output values (Figure 3-32).

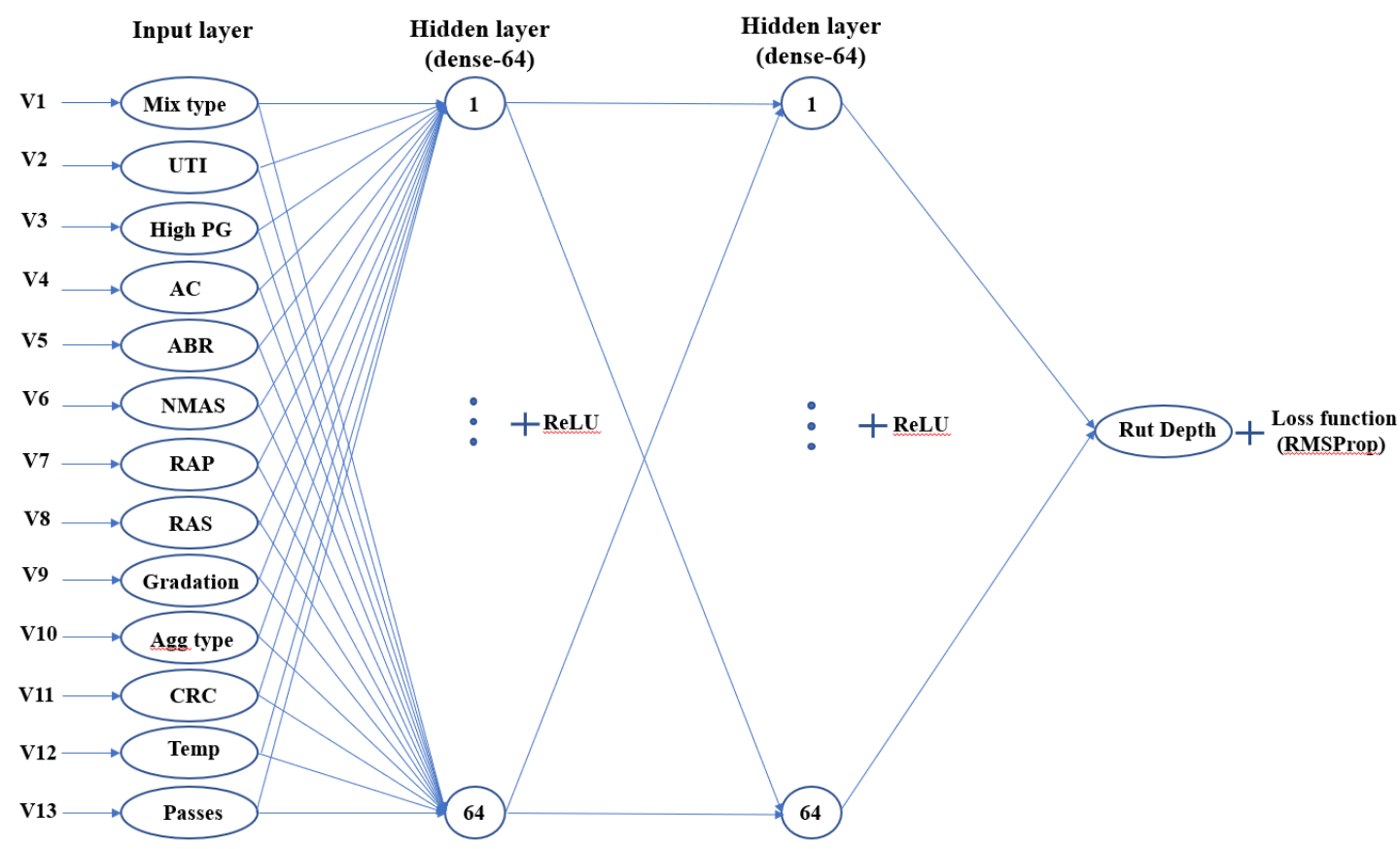

Figure 3-32 CNN structure 


\subsubsection{Prediction Model Results}

The framework was developed via Tensorflow and Keras libraries in python. A batch size of 10 was selected. The model was trained for 1000 epochs. The results for training and testing datasets were monitored (Figure 3-33). $\mathrm{R}^{2}$ calculated for both the training and testing dataset are shown in Figure 3-33 .
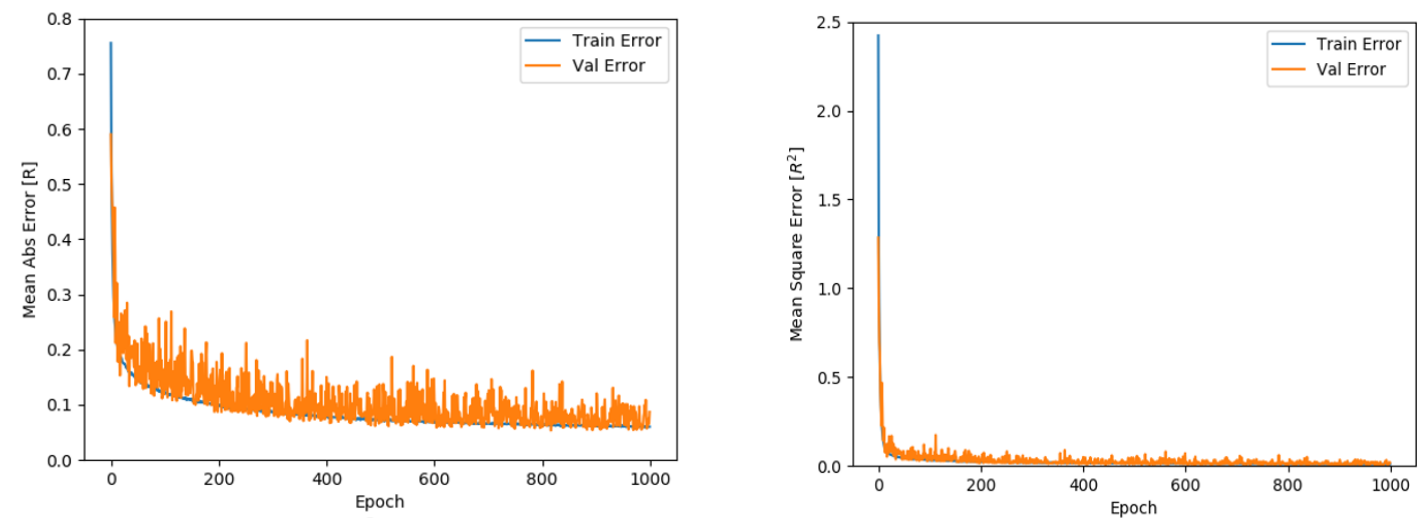

Figure 3-33 Performance of trained model on training and validation dataset versus 1000 epoch.

As mentioned before, early stopping is a beneficial technique to avoid overfitting.

Figure 3-34 shows insignificant improvement in the error after about 40 epochs.
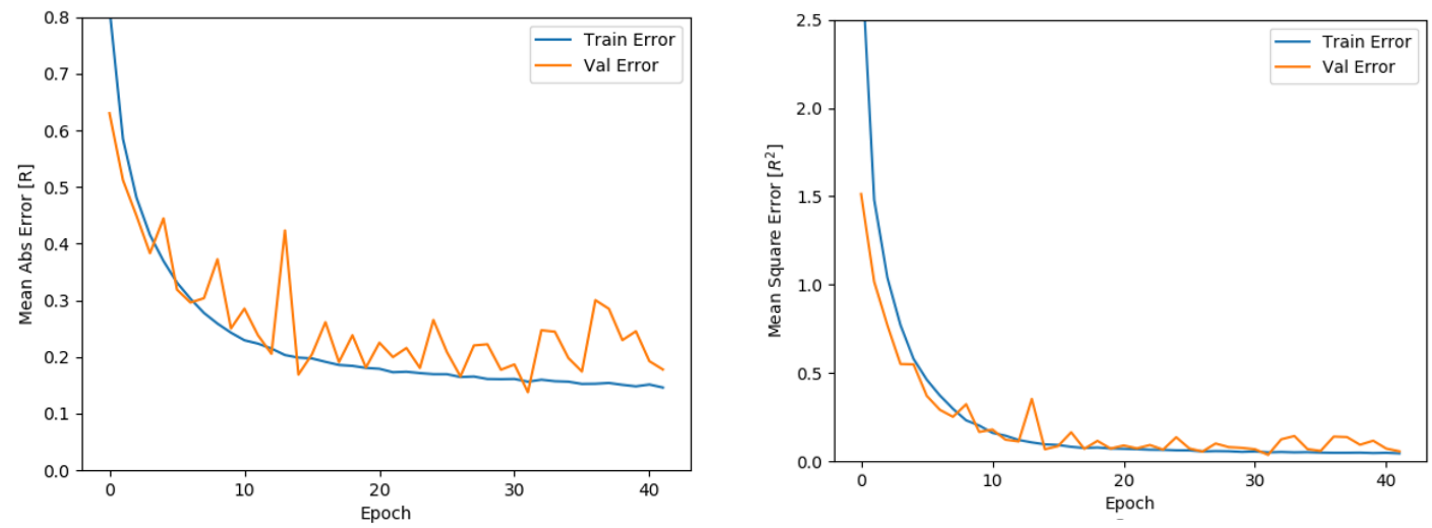

Figure 3-34 Performance of trained model on training and validation dataset versus 40 epoch. 
Figure 3-35 shows the performance indices of the optimal CNN model on the training, validation, and testing dataset, respectively. As seen, the proposed CNN model can predict the rut depth accurately.

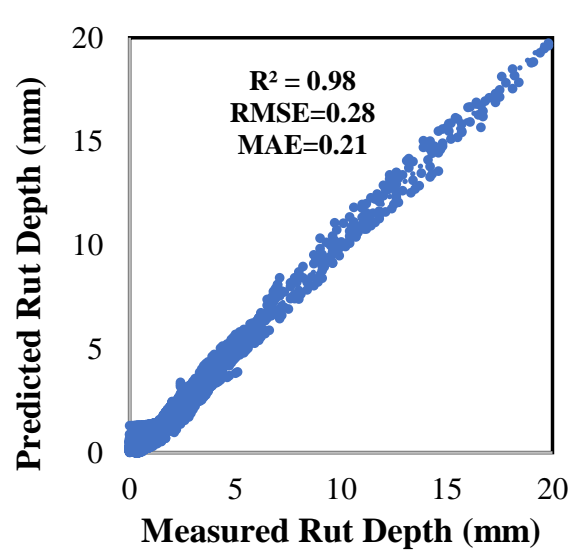

a) Training dataset

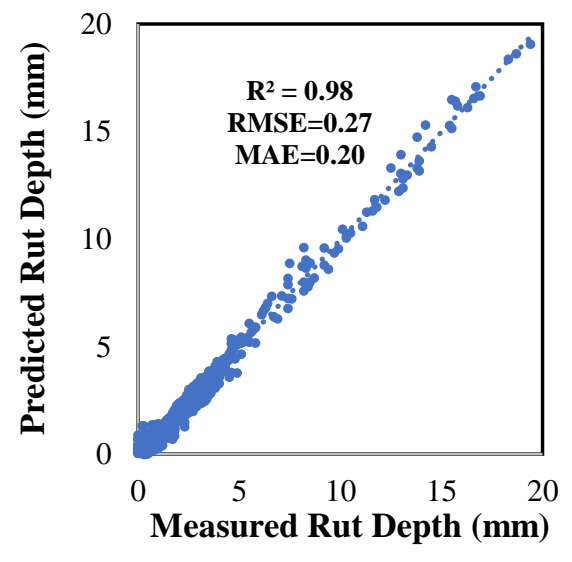

b) Testing dataset

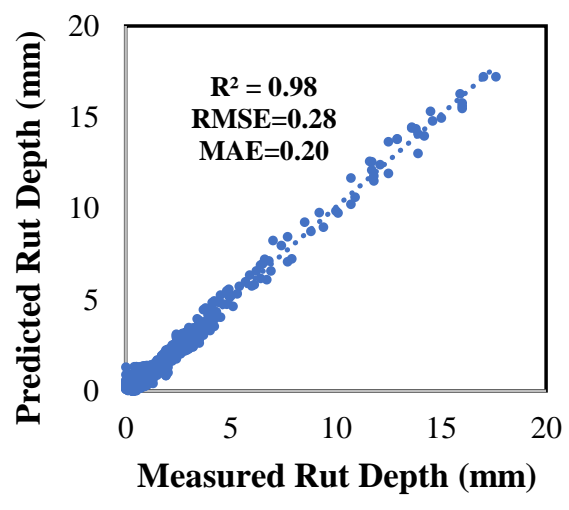

c) Validation dataset

Figure 3-35 Measured against predicted rut depth using the CNN model: (a) training dataset, (b) testing dataset and c) validation dataset.

\subsubsection{Sensitivity Analysis}

The effectiveness of the predictive models can be evaluated by inspecting model sensitivity to known influencing factors. The CNN model presented in this study has been 
used to predict HWTT results for several plant- and lab-compacted mixtures across the Midwest in the United States. For further validation, sensitivity of the proposed model to the variations of the recycled material (RAP and RAS), asphalt content, binder grade type, crumb rubber content, aggregate type, gradation, and test temperature were evaluated. To investigate the effect of each factor, the factor of interest was incrementally changed while all other variables were held constant (Figure 3-36 to Figure 3-41). In order to provide an insight into the performance of the $\mathrm{CNN}$ model, the predictions made by a previously developed GEP model [38] was included in the analysis.

\subsubsection{Effect of RAP/RAS}

Figure 3-36 shows the effect of recycled content on machine learning model predictions. The following conclusions can be drawn:

- The model is capable of capturing differences in recycling content, where increased ABR clearly reduces rutting potential. Figure 3-36 a shows the effects of increasing recycled content in a base mixture. It shows an increment amount of RAP and ARS provide higher rut resistance.

- Figure 3-36 b \& c highlights the improvements of the CNN model over the GEP model by comparing the rut depth values for different passes for a mix with different levels of RAP and RAS. While the GEP model is "unsteady," the CNN model provides an expected and a steady trend of the mixture rutting performance with an increase in RAP/RAS. 

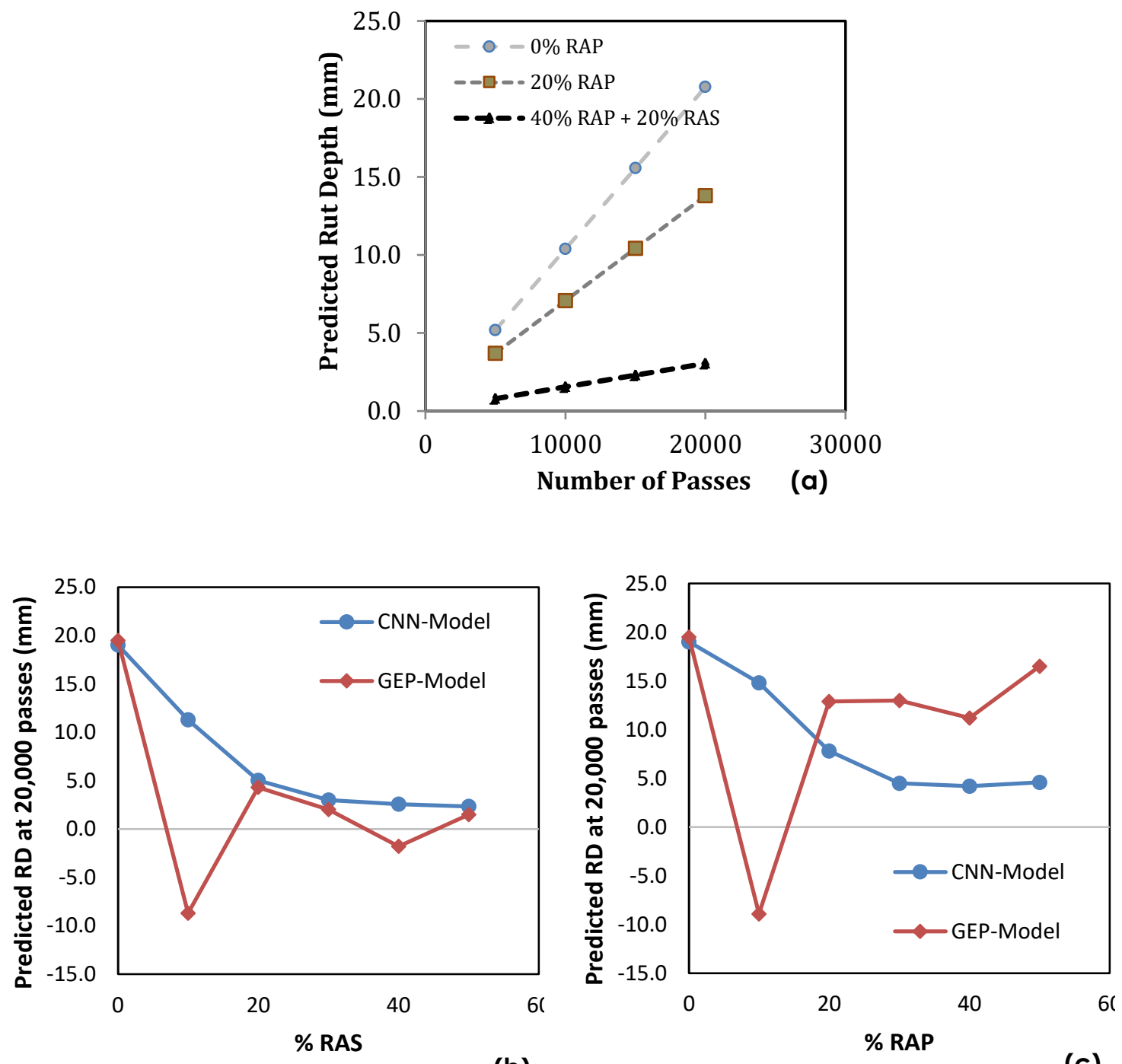

(b)

(c)

Figure 3-36. Sensitivity analysis of the CNN and GEP models: a) Effect of RAP and RAS, b) Effect of RAS and c) Effect of RAP

\subsubsection{Effect of Test Temperature}

Figure 3-37 shows model sensitivity to test temperature. The following observations were made:

- Figure 3-37a shows predicted rut depth for a base mixture at various passes and test temperatures. The observed trend was expected as rut depth values increased at warmer testing temperatures. 
- Figure 3-37b shows the sensitivity comparison of GEP and CNN models with respect to testing temperatures. While GEP model was able to exhibit the expected trend, the negative rut depth prediction at 40 and $46^{\circ} \mathrm{C}$ were unexpected results (produced by the GEP model).
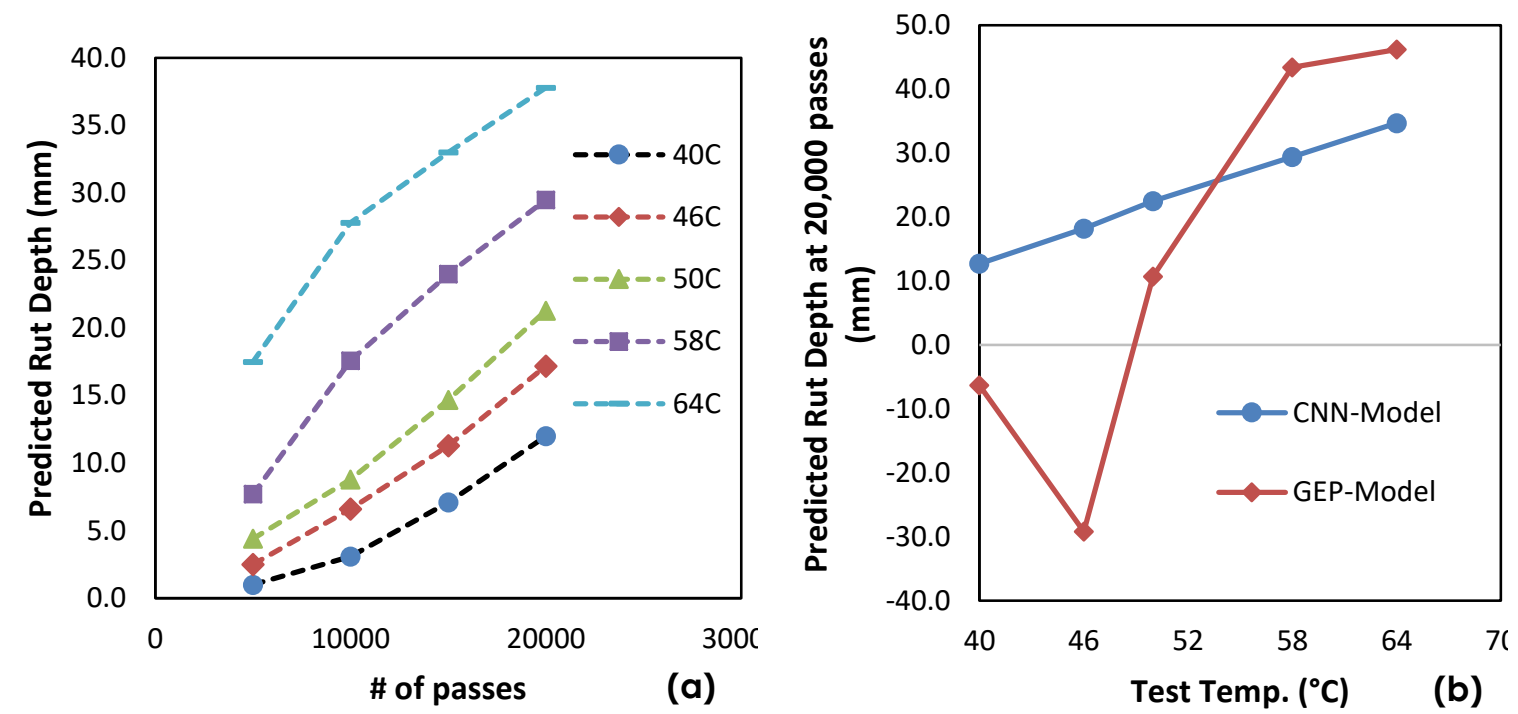

Figure 3-37. Sensitivity analysis of the CNN and GEP models: a) Effect of temperature on rut depth versus number of passes in CNN model, b) Effect of test temperature on rut depth in CNN and GEP models

\subsubsection{Effect of Binder PG High Temperature}

Figure 3-38 shows CNN model sensitivity to binder PG high temperature grade.

- Figure 3-38a shows the rut depth prediction with binders that are progressively stiffer. As expected, the model predicts a lower rut depth with stiffer binder grade.

- Figure 3-38b shows the comparison of GEP and CNN model- both the models are able to exhibit satisfactory trends for this input parameter. 

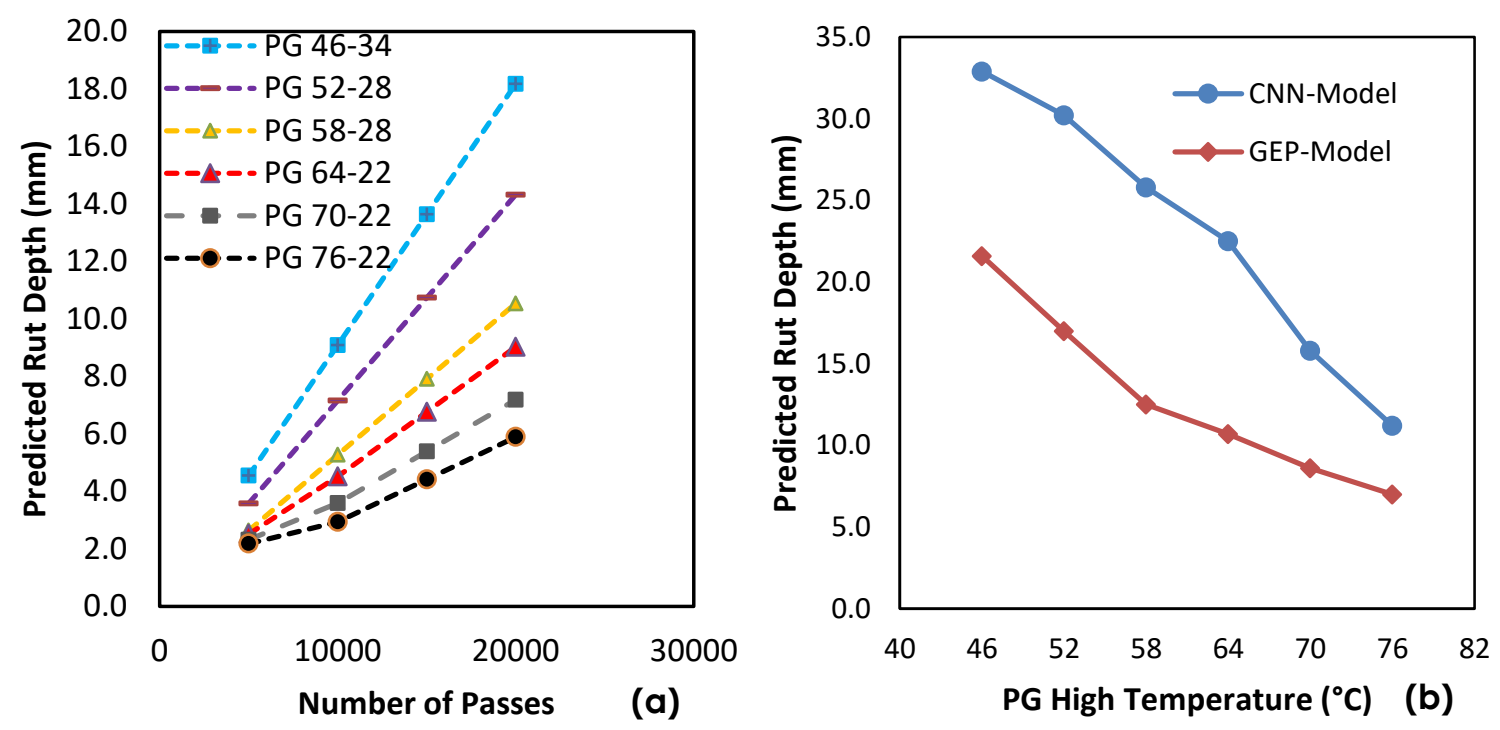

Figure 3-38. Effect of Binder PG high temperature on model prediction: (a) CNN model predictions with progressively stiffer binder, (b) Comparison of the CNN and GEP predictions with progressively stiffer binders

\subsubsection{Effect of Mixture Production (Lab vs. Plant)}

The current model also has the ability to distinguish between lab and plant produced mixtures, as shown in Figure 3-39a. It should be noted that the difference between the rutting performance of the plant- and the lab-produced mixture is very subtle in the case shown here, but this could differ with other mixtures. For the purposes of this study, it was deemed enough to show that the model is sensitive to mixture production differences.

Figure 3-39b shows GEP model sensitivity to plant- and lab-produced mixtures. While the model is able to differentiate between the mixtures, the differences are glaringly large and unexplainable. For example, the GEP model predicts $4 \mathrm{~mm}$ rut depth across all passes for the lab mix. 

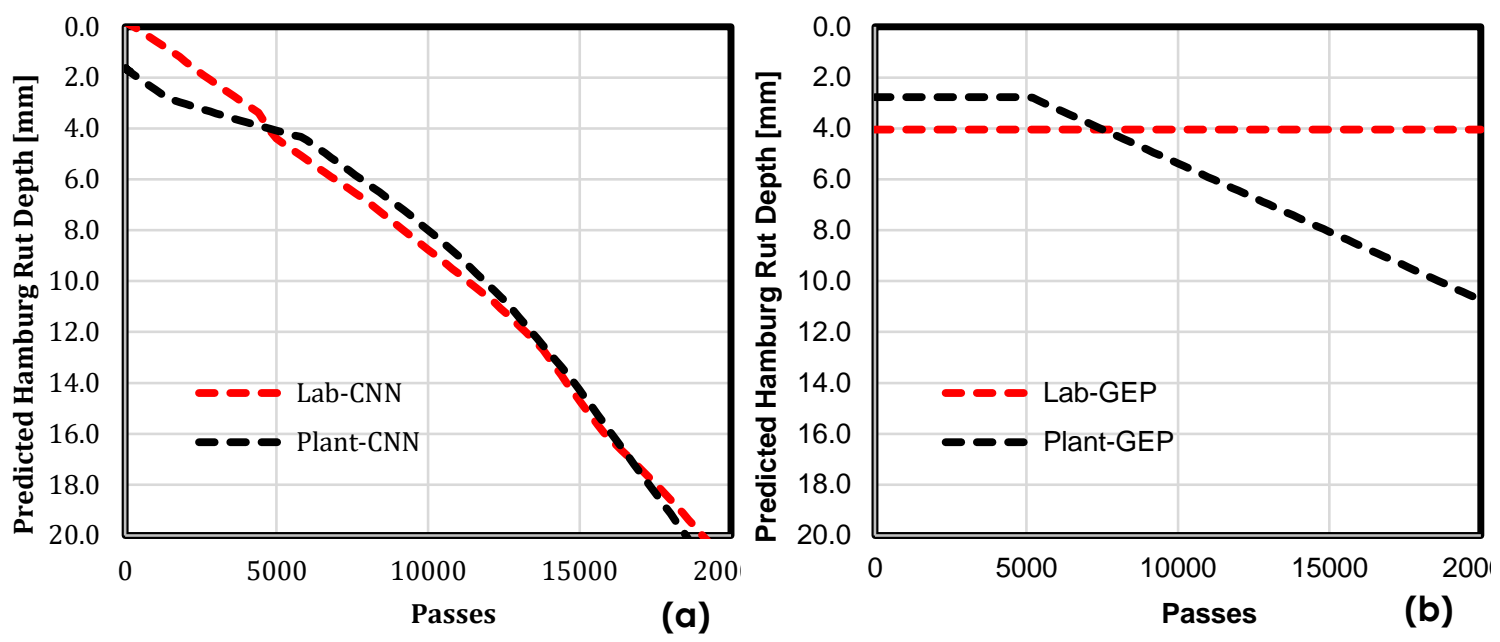

Figure 3-39. Effect of production (Lab vs. Plant produced) on model predictions: (a) CNN model, (b) GEP model

\subsubsection{Effect of Crumb Rubber}

Figure 3-40a and $\mathrm{b}$ show the effect of GTR in asphalt mixture rutting performance. As observed in Figure 3-40a, the model predicts an improvement in rutting performance of mixture with the addition of GTR, as expected. On the other hand, the GEP model fails to differentiate between any increments in GTR\%, as shown in Figure 3-40b. It must be noted that the previous dataset did not have enough crumb rubber modified mixture. 

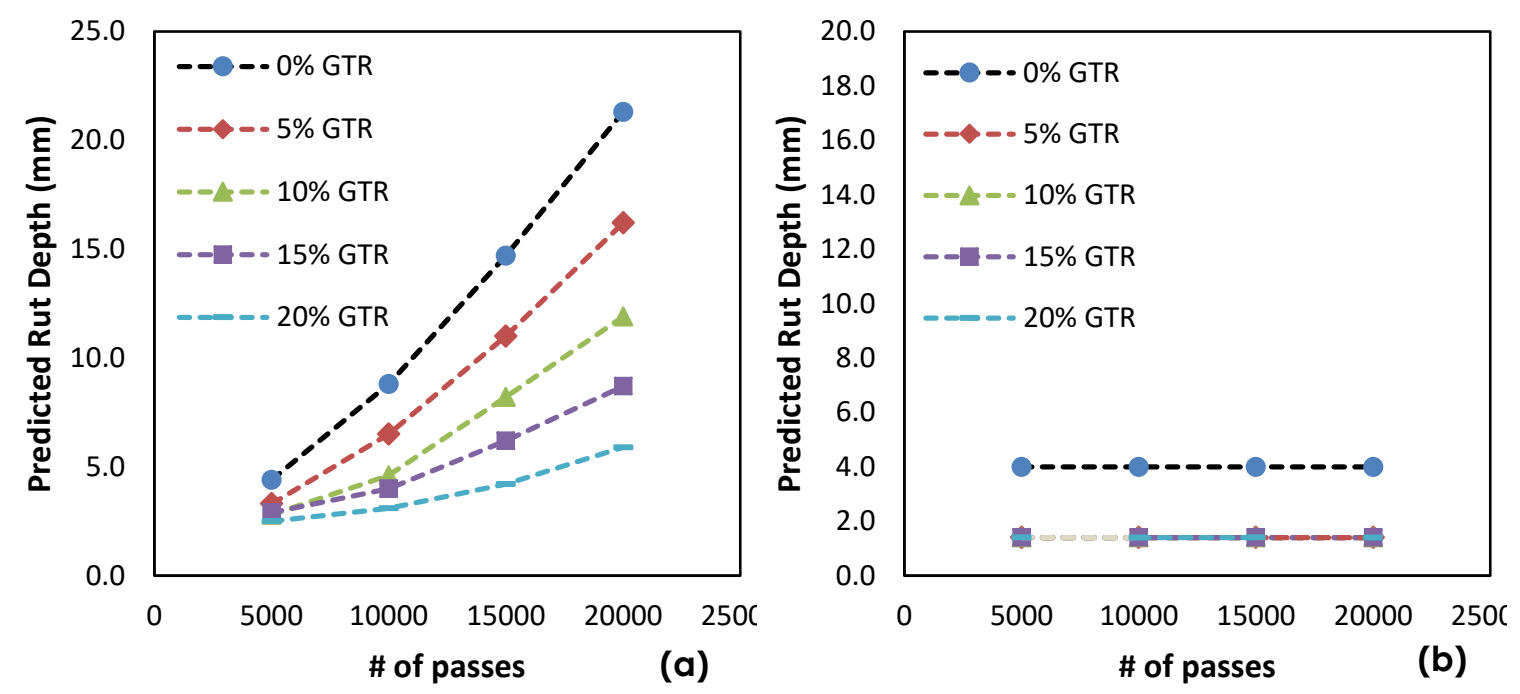

Figure 3-40. Effect of GTR\% on model predictions, (a) CNN model, (b) GEP model

\subsubsection{Effect of aggregate type/gradation}

Currently, the CNN model has been trained using only two types of aggregatelimestone and granite, and two types of gradations- dense-graded and stone matrix (mastic) asphalt (SMA). Furthermore, due to the limitation of available data, the dense-graded mixes could only be assigned to have limestone as an aggregate, while the SMAs were assigned granite as aggregate. Keeping these limitations in mind, Figure 3-41 shows the trends exhibited by the CNN model prediction for the two aggregate types and gradation with respect to changing mixture constituents. As seen in both cases, the trends are similar. The base mixture with 64-22 binder (6.6\% total asphalt content) and 0\% RAP shows high rutting potential. Addition of $45 \%$ RAP to the next mix iteration (with $6.2 \%$ total binder content) results in better rutting resistance. Further, switching the PG64-22 with to a softer base binder, PG 58-28 and subsequently PG46-34, causes progressively higher rutting in the mix. 


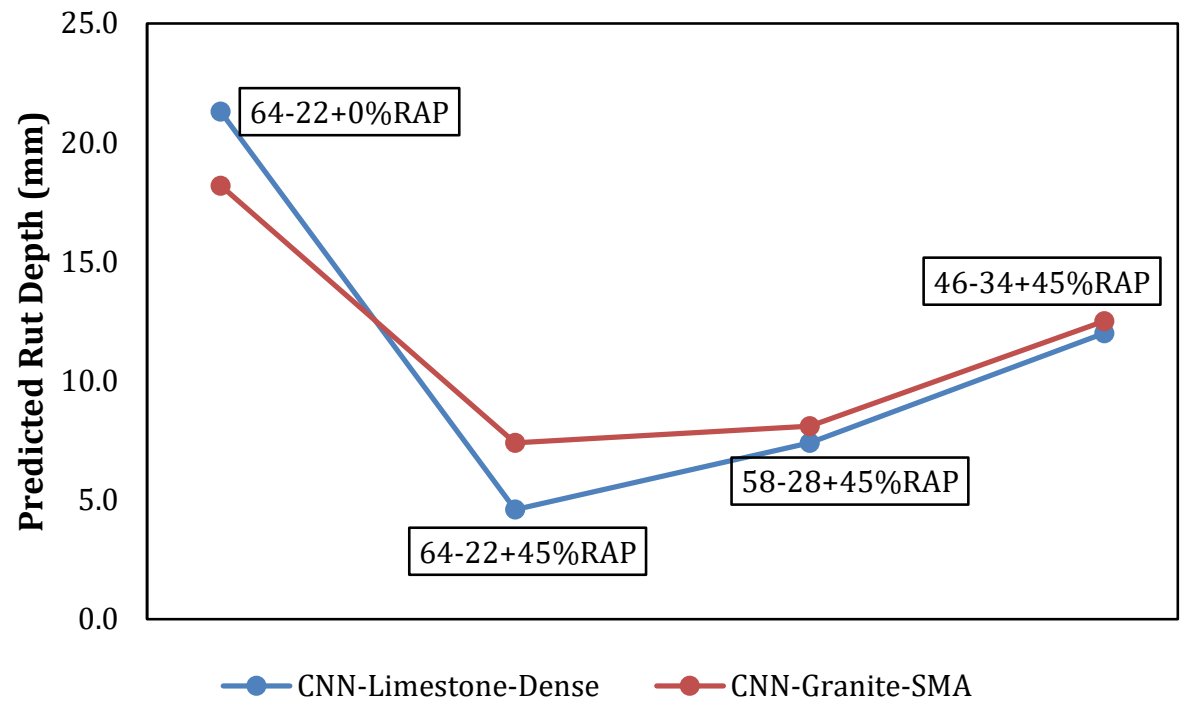

Figure 3-41. The CNN model predictions for aggregate type and gradation

\subsubsection{Performance-Space Diagram Tool}

Majidifard et al. [31], [38] implemented the GEP-based models to predict the lowand high-temperature performance in terms of $\mathrm{DC}(\mathrm{T})$ fracture energy and Hamburg rut depth, respectively. The authors used a concept called Performance-Space diagram (PSD), introduced by Buttlar et al. [96], to show the efficiency of the tool in performance prediction. PSD allows a holistic view of a mixture performance, which helps in implementing a balanced mix design approach. For example, Rath et al. [81] used PSD to show the possibility of using higher recycling in asphalt mixtures if the introduced stiffness due to recycled material was "balanced" with use of a softer base binder. A database containing a comprehensive collection of Hamburg and DC(T) tests results were used to develop these machine learning-based prediction models. The models were formulated in terms of typical influencing mixture properties variables such as asphalt binder high- 
temperature performance grade (PG), mixture type, aggregate size, aggregate gradation, asphalt content, total asphalt binder recycling content and tests parameters like temperature and number of cycles (Figure 3-42). The model's accuracy was assessed through a rigorous validation process and found to be quite acceptable, despite the relatively small size of the training set. Figure 3-42 shows the schematic view of the software. After filling the inputs on the left and clicking "Run" button, the tool will populate the Hamburg-DC(T) PSD, representing the mixture's $\mathrm{DC}(\mathrm{T})$ fracture energy at specified test temperature, and rut depth at 20,000 passes (10,000 cycles) at specified test temperature.

The prediction models combined with the PSD can be used in pre-design stages of an asphalt mixture to tweak mixture designs for suitable performance in terms of cracking and rutting, helping reduce the overall cost of mixture testing in design phase. For example, data in Figure 3-41 shows that the rut depth was improved with the addition of RAP to the PG64-22 binder. However, the addition of RAP increases the risk of thermal cracking. This can be remedied by switching to a softer base binder, but that would result in an increase in the rutting susceptibility. Thus, an iterative process can be applied according to the performance limits of the prevailing agency to design an optimized or 'balanced' mix in terms of cracking and rutting performance. These time-consuming iterative steps in real world can be simulated in this tool, and a balanced mixture can be made with minimum efforts and much lower expense. 


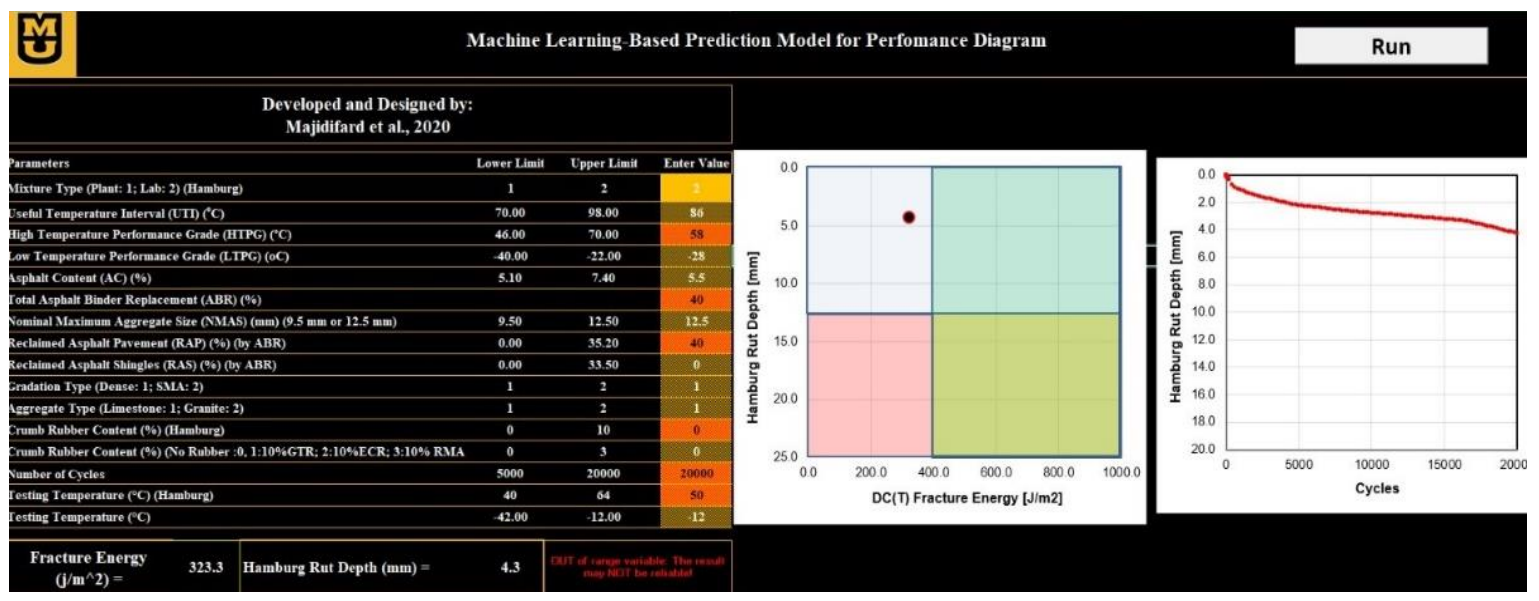

Figure 3-42. The GEP-CNN based prediction model for performance diagram developed by Majidifard et al.

\subsubsection{Comparison of rut profiles Obtained from the CNN and GEP models}

This section investigates the trends seen in the predicted rut depth values as obtained by CNN and GEP models to highlight the substantial improvements provided by the CNN model over the GEP model. Selected rut depth profiles from the investigated mixtures were plotted as predicted using the GEP- and CNN-based models (Figure 3-43). The following conclusions were then drawn:

a. The rut depth curve prediction in the GEP model was based on linear interpolation between the values predicted at discrete passes (four data points from each curve), such as 5000, 10000, 15000, and 20000 passes. This results in a rather linear behavior of the predicted rut depth profile by the GEP model. The CNN model, however, was based on data from 200 data points during 20,000 passes, allowing the model to improve its accuracy in profile prediction (Figure 3-43).

b. Due to the use of rut depth values at discrete passes, the accuracy of rut depth prediction at an intermediate number of wheel-pass, say 7500 passes, is lower than the average accuracy of the model. Using all points through 20,000 passes in the 
$\mathrm{CNN}$ model enables the user to accurately predict rut depth at any number of passes. This feature makes the model highly versatile and applicable to various cases where the number of passes is adjusted according to the requirements of the project (climates and traffic).

c. It was observed that certain asphalt mixes with recycled materials, especially roofing shingles, resulted in negative rut depth with the old GEP model. Figure 3-43 (c) and (d) show such a trend when presence of recycled roofing shingles in the mixtures lead to a negative constant rut depth profile in the GEP model. This was due to the unavailability of a broad range of data points available for recycled mixtures. The CNN model provides an improved prediction capability for recycled mixtures, as shown in Figure 3-43. It is noted by the authors that higher accuracy can be aimed in future iterations of this tool with a larger training dataset for recycled mixtures. 


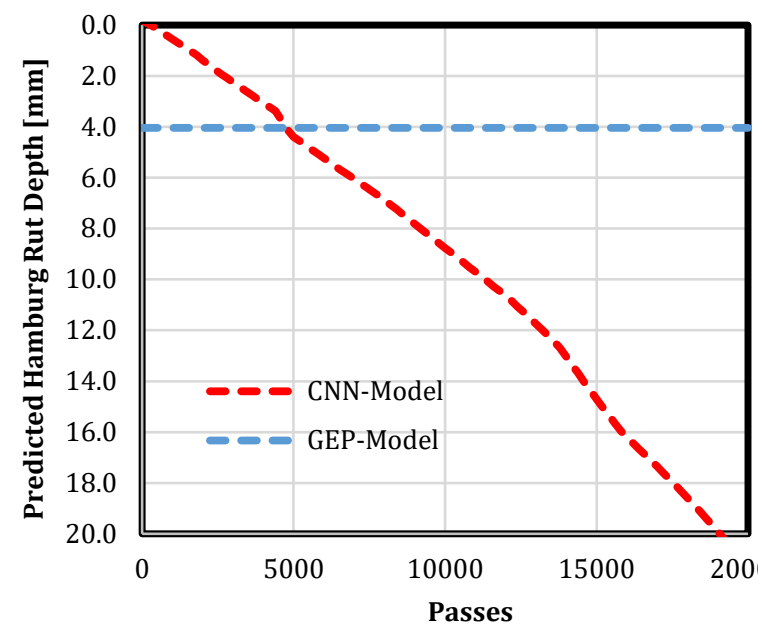

a) Mix- PG64-22, 0\% RAP/RAS, 6.6\% total binder content, limestone aggregate

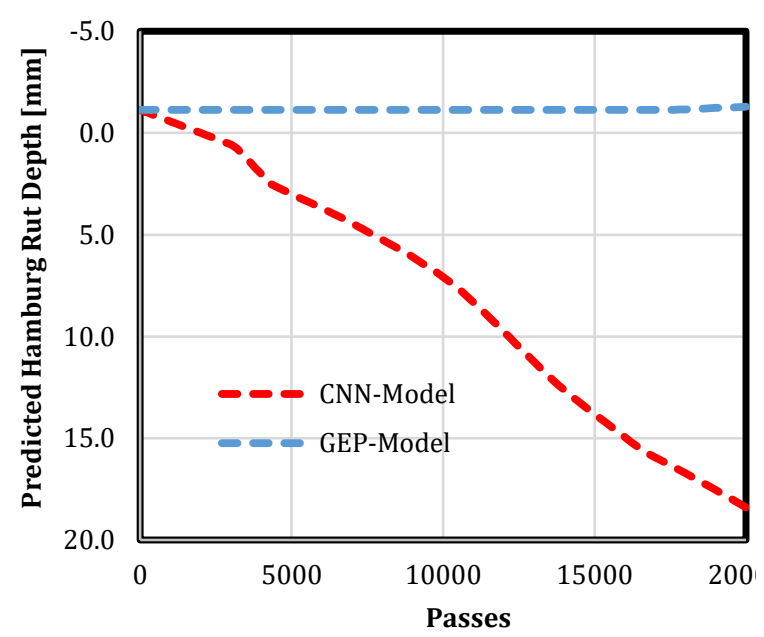

c) Mix- PG58-28, 0\% RAP, 2.5\% RAS, $6.2 \%$ total binder content, limestone aggregate

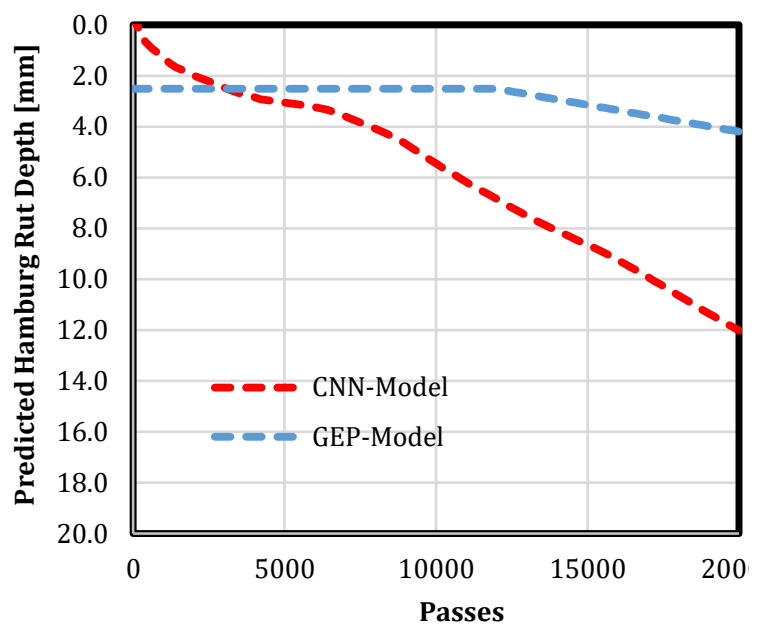

b) Mix- PG46-34, 45\% RAP, 0\% RAS, $6.2 \%$ total binder content, limestone aggregate

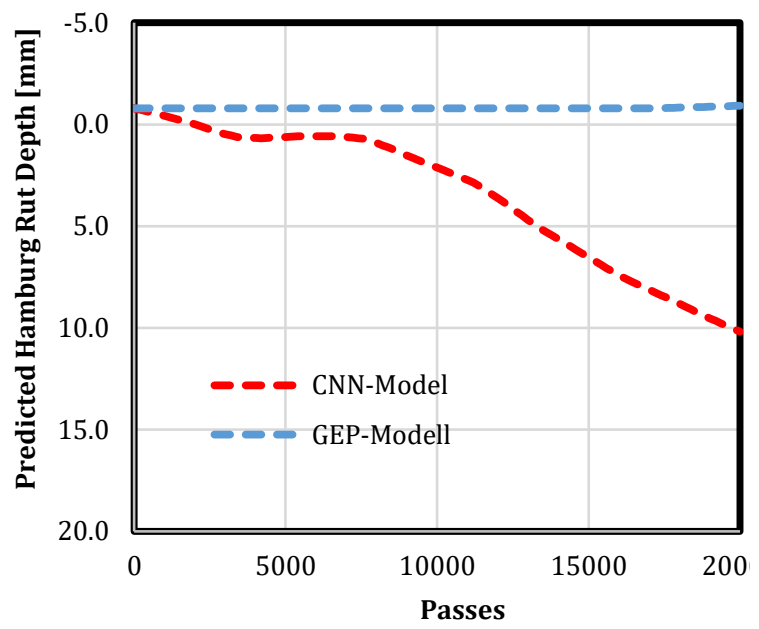

d) Mix- PG58-28, 0\% RAP, 5.0\% RAS, $6.2 \%$ total binder content, limestone aggregate

Figure 3-43. Comparison of the rut depth profiles predicted by the GEP and CNN models.

\subsubsection{Application of the CNN Rutting Prediction Model for Paved Mixtures}

Full Hamburg rutting curves of PPLC mixtures from the states of Missouri and Illinois were plotted to compare measured vs. predicted rut depth profiles. Details about the mixtures can be found elsewhere [27], [29], [132], but brief details of the mixtures are 
shown in Table 3-12.

Figure 3-44shows the rut depth profile measured in the Hamburg device compared to the predicted rut depth by the CNN and GEP models. As can be observed, the CNN model was able to increase the accuracy of the prediction of rut depth substantially in comparison to the GEP model. The CNN model was accurately able to predict the entire rut depth profile at different temperatures.

Table 3-12. Mixture Details

\begin{tabular}{cccccc}
\hline Mix ID & $\begin{array}{c}\text { Binder PG } \\
\text { used (\%) }\end{array}$ & NMAS (mm) & $\begin{array}{c}\text { ABR\% by } \\
\text { RAP }\end{array}$ & $\begin{array}{c}\text { ABR\% by } \\
\text { RAS }\end{array}$ & $\begin{array}{c}\text { Crumb } \\
\text { Rubber \% }\end{array}$ \\
\hline MO13_1 & $70-22(5.7)$ & 9.5 & 17 & 0 & 0 \\
US54_1 & $58-28(5.2)$ & 12.5 & 0 & 33 & 0 \\
1807 & $46-34(6.2)$ & 19 & 34.4 & 14.0 & 0 \\
1818 & $64-22(5.7)$ & 9.5 & 20.4 & 0 & 0 \\
1829 & $70-28(7.9)$ & 4.75 & 17.8 & 9.3 & 10 \\
1835 & $46-34(5.9)$ & 12.5 & 25.0 & 16.1 & 10 \\
\hline
\end{tabular}

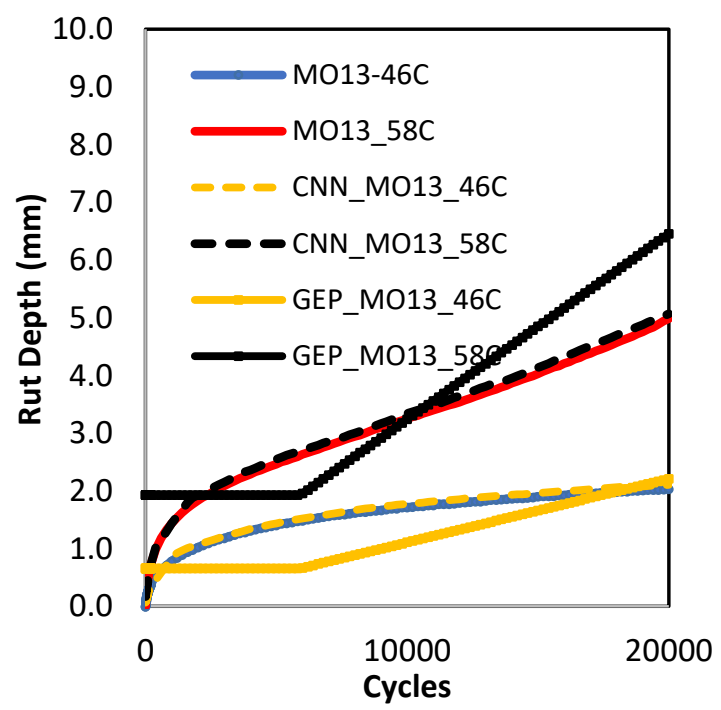

a)

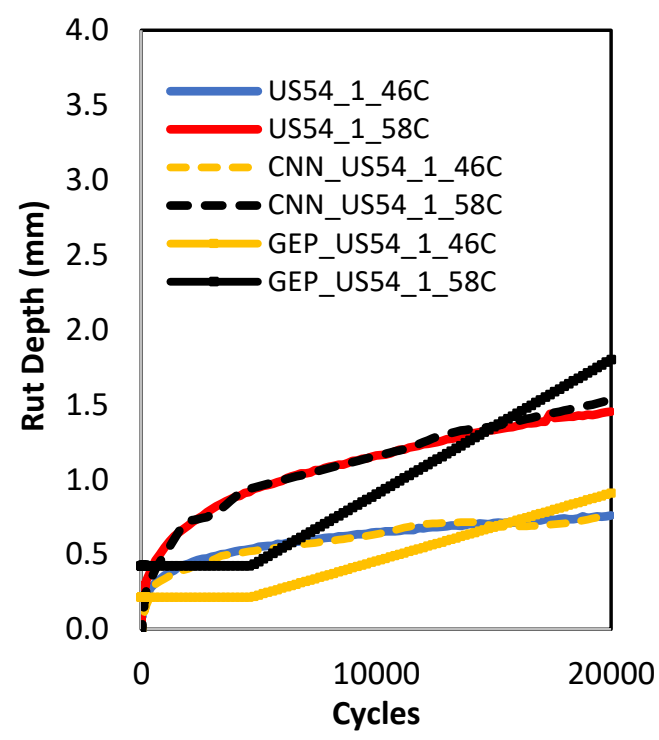

b) 


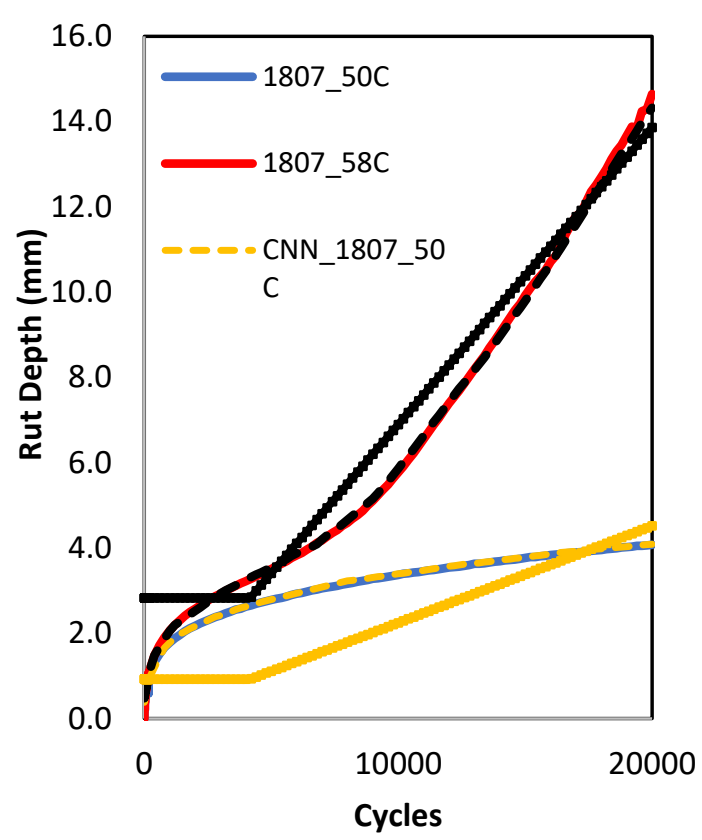

(c)

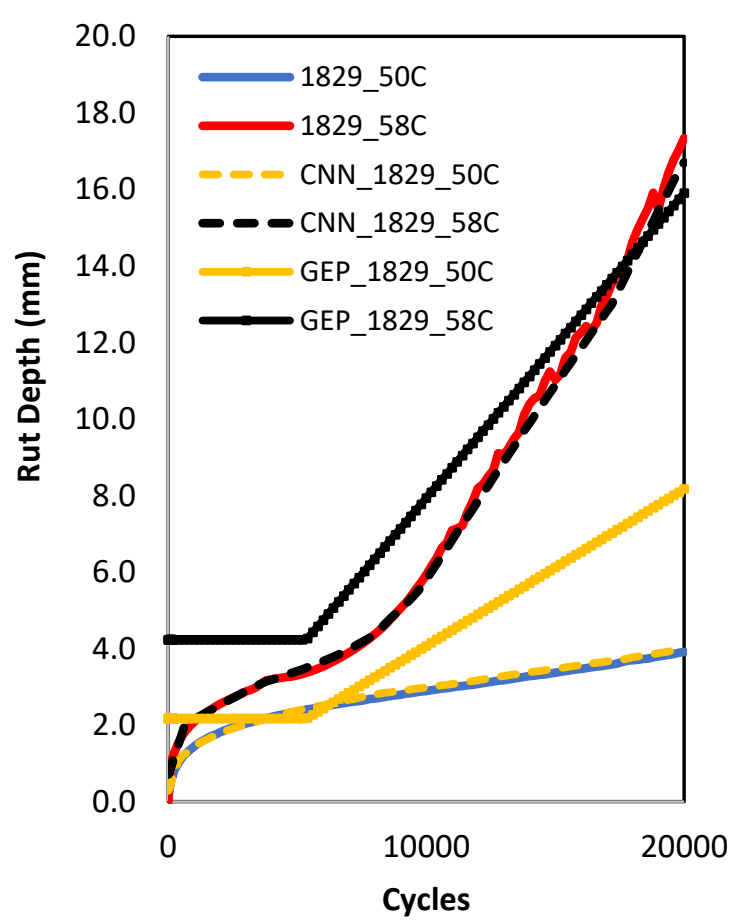

(e)

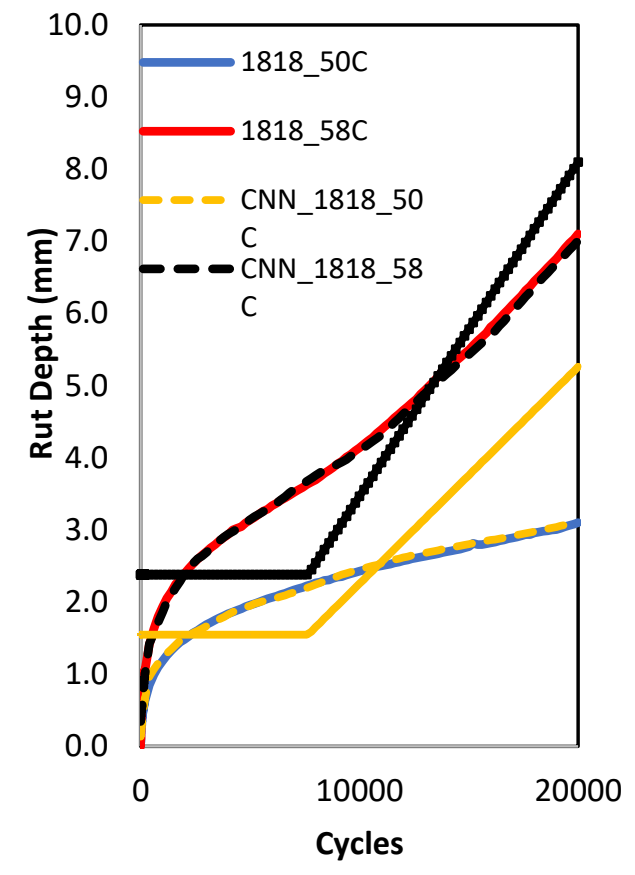

(d)

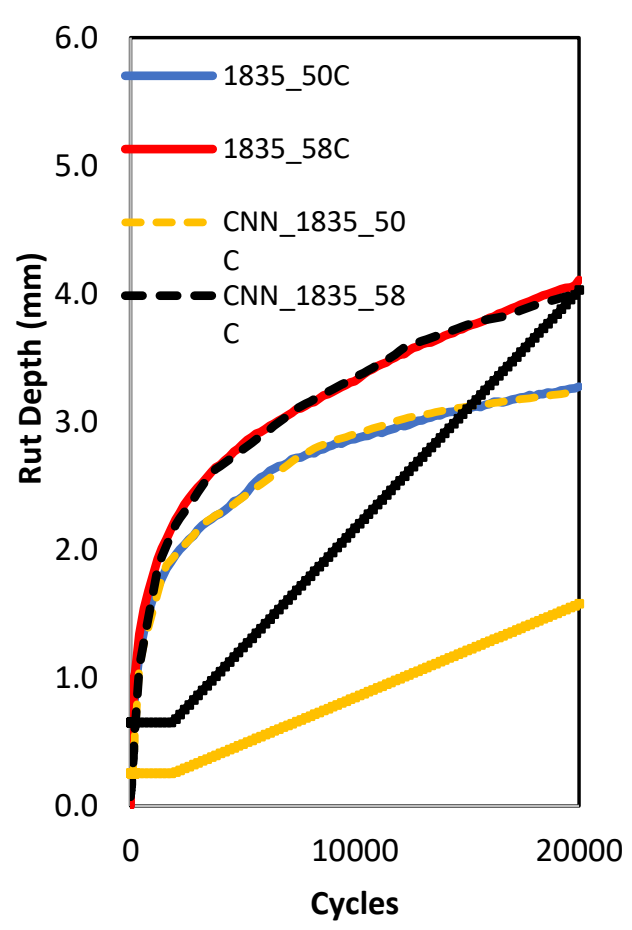

(f)

Figure 3-44. Comparison of predicted and actual Hamburg rut depth using the GEP- and CNN models 


\subsubsection{Summary}

In this part of study, a new model was developed to predict the rutting depth of asphalt mixtures using a deep learning framework, namely CNN. The dataset contained 10,000 data points collected from 50 mixture test results. Thirteen mixture parameters and testing variables were considered to develop the model. The results confirmed the acceptable performance of CNN algorithm. The results of sensitivity analysis showed that the proposed model is highly sensitive to RAP, RAS, high PG, crumb rubber content, test temperature, and numbers of cycles. The proposed model predicts the full rutting curve of asphalt mixtures across 20,000 passes at various test temperatures in the HWTT. Furthermore, a CNN-based prediction model for performance space diagram outputs is presented as a tool to predict both low- and high-temperature mixture performance in terms of DC(T) fracture energy and Hamburg rut depth. The prediction models can be used in pre-design stages of an asphalt mixture to tweak mixture designs for suitable performance in terms of cracking and rutting, helping reduce the overall cost of mixture testing in the design phase. A comparative study has been performed between a GEP-based prediction model and the new CNN model. The results clearly demonstrate that the CNN model performance is superior to the GEP model. The proposed model can be updated with additional data in the future to provide more accurate predictions over a wider range of mixture component materials, such as aggregate mineralogy, shape and gradation. More HWTT results with a wider range of test temperatures will also be incorporated into future model development to assist agencies in evaluating proper HWTT test temperatures for the purpose of balanced mix design in various climates. 


\section{CHAPTER 4: DEVELOPING A PREDICTION MODEL FOR PAVEMENT CONDITION OF ASPHALT ROADS USING DEEP- LEARNING APPROACH}

Pavement distress inspections are performed using sophisticated data collection vehicles and/or foot-on-ground surveys. In either approach, the process of distress detection is human-dependent, expensive, inefficient, and/or unsafe. Automated pavement distress detection via road images is still a challenging issue among pavement researchers and computer-vision community. In this study, we extracted 7237 google street-view, manually annotated for classification (nine categories of distress classes). Afterward, YOLO (you look only once) deep learning framework was implemented to train the model using the labeled dataset. Also, U-net based model is developed to quantify the severity of the distresses, and finally, a hybrid model is developed by integrating the YOLO and Unet model to classify the distresses and quantify their severity simultaneously. The output of the distress classification and segmentation models are used to develop a comprehensive pavement condition tool which rates each pavement image according to the type and severity of distress extracted. As a result, we are able to avoid over-dependence on human judgement throughout the pavement condition evaluation process. The outcome of this study could be conveniently employed to evaluate the pavement conditions during its service life and help to make valid decisions for rehabilitation of the roads at the right time. This project consists of two phases. In the first phase, deep learning based models developed to classify different distresses. In the second phase U-net based model was implemented to quantify the severity of distresses and a pavement condition index was developed to rank the sections based on the detected values in the first phase. 


\subsection{PAVEMENT IMAGE DATASET: A NEW BENCHMARK DATASET TO CLASSIFY AND DENSIFY PAVEMENT DISTRESSES}

\subsubsection{Introduction}

Recently, road infrastructure in the United States received a ' $D$ ' grade [189] according to the American Society of Civil Engineers (ASCE) Infrastructure Report Card, 2017. This is mainly driven by increasing budget constraints, which have created a culture of delayed maintenance and underinvestment in the renewal of transportation infrastructure systems. Strategic rehabilitation and maintenance of road surfaces require accurate data regarding overall road condition, and individual distress type, extent and severity [190]. Over the past two decades, improvement in sensor and camera technology has led to significant progress in automating pavement distress monitoring [191]-[193]. Typically, however, the sophisticated hardware and software involved results in high initial and operating expenses. For instance, a vehicle equipped with modern sensor and computing systems was purchased by the Ohio Department of Transportation for US\$1,179,000, with an annual operating cost US\$70,000 [194]. Furthermore, the final pavement condition assessment provided by these systems can be highly operator dependent [195].

Recent progress in image processing techniques and machine learning methods have motivated researchers to utilize these approaches to develop predictive models [90], [168], [196] towards well-timed repair and maintenance activities [190], [197]. Recent advances in deep learning has led to significant improvement of machine learning models in areas such as smart cities, self-driving cars, healthcare, agriculture, retailing, and finance. Similar strategies could be implemented in pavement distress monitoring. However, deep learning 
models rely on a large database of ground truth data, which is usually not available. Recent advances in vision-based, automated pavement crack detection techniques include: intensity-thresholding [198]-[201], match filtering [202], edge detection [203], seed-based approach [204], wavelet transforms [205]-[208], texture-analysis, and machine learning [209]-[212]. An automatic procedure for crack detection known as CrackTree was reported by Zou et al. [213].

Although manipulation of machine learning methods for automated pavement distress detection is no longer a new technique, application of deep learning methods is still an area of active research [183], [214]-[217]. Deep convolution neural networks (DCNNs) are defined as deep architecture with many hidden layers that enable them to acquire numerous abstraction levels [180], [218]-[220]. Zhang et al. developed a crack detection model using raw image patches via the CNN-based software CrackNet [216]. In 2019, Zhang et al. implemented the Recurrent Neural Network (RNN) technique to create CrackNet-R, which is more efficient than CrackNet in detecting small cracks and in removing noise [183]

In order to develop a robust distress detection model, critical pavement distresses must be detected. Several factors such as traffic, climate, structural layering, layer age and condition will affect the pavement deterioration rate. Once characterized, road administrators can use the information to develop strategies to repair the pavement based on the type, extent and severity of the distresses identified. Previous studies made progress towards this goal, but falling short in one area or another. For instance, [183] concentrated on determining the presence of damage, but did not specifically identify individual types 
of distress. Zalama et al. [221]categorized the types of distress horizontally and vertically, while Akarsu et al. [222] classified distresses into three categories - horizontal, vertical, and alligator. Finally, other investigations led to detection of blurry road markings [223], while other focused on classifying cracks, including sealed cracks [224].

The robustness of machine learning models is heavily dependent on the quality of data used for training them. Understanding the significance of labeled datasets for developing a robust pavement condition tool, in this study we introduce the so-called 'Pavement Image Dataset,' or (PID). The initial study utilized 7,237 images extracted from 22 different pavement sections, including both interstate and US highways. Images were extracted using the Google Application Programming Interface (API) in street-view using a code developed in Python. Initially, each image was hand-annotated by drawing a bounding box around each identified pavement distress. The dataset was evaluated using two classical deep learning frameworks, namely You Look Only Once (YOLO v2) and Faster Region Convolution Neural Network (Faster R-CNN). The following summarize the primary contributions of this study:

- Introduction of a new dataset that enables simultaneous classification and density quantification of pavement distresses using varied camera views (top-down and wideview). Wide-view images were used for classification, while top-down images were used for quantification of crack density.

- Annotation of 7,237 images (wide-view images) with nine different distress types that were deemed to be critical for assessing pavement condition. These include a number of cracking modes, including reflective, transverse, block, longitudinal, alligator, 
sealed transverse, sealed longitudinal, and lane longitudinal cracking, along with potholes.

- Implementation of two classical deep learning frameworks: YOLO-v2 and Faster RCNN, and training of the models using the aforementioned dataset.

- In the following section, we review previous datasets and introduce our proposed dataset.

\subsubsection{Previous Datasets}

Several benchmarked datasets (private and public) have been developed in previous studies in the training of machine learning models [182]. The camera views typically used can be grouped into two categories: wide-view and top-down view. The main difference between the dataset provided in this study and previous datasets is that the current study captures data from both camera-views, which was found to be useful for distress classification and density determination. In the following sections, we review previous datasets based on either wide-view or top-down view datasets.

\subsubsection{Wide-view datasets}

Wide-view datasets capture a large area of the pavement and are therefore useful for pavement distress classification. Street-view image databases normally involve a high number of images with 'non pavement' views showing sidewalks, cars, buildings, etc. In a recent study, deep learning was employed to find and remove such objects based on a database of 9,712 wide-view images obtained via a mobile mapping system [225]. In another study, Maeda et al. used an end-to-end deep learning framework for pavement distress classification based on wide-view road images captured with a smartphone 
mounted on a vehicle dashboard. Images were divided into eight output classes (five types of cracks, rutting-bump-pothole-separation, crosswalk blur, and white line blur) [215]. Zhang et al. employed a sampling approach to create one million triple-channel (RGB) 99 $\times 99$-pixel image patches based on $500(3264 \times 2448$ pixels $)$ pavement images gathered by smartphone. In this study, 640,000, 160,000, and 200,000 patches were used for training, cross-validation, and testing, respectively [216].

\subsubsection{Top down-view datasets}

Top-down images provide more accurate view of distresses compared to wide-view. However, these type of images generally required more sophisticated camera and mounting equipment as compared to wide view images. In addition, pavement distress classification based on top-down views can be challenging as they may not capture the entire view of the distress. The German Asphalt Pavement distress (GAPs) dataset introduced by Eisenbach et al. was evidently the first open source pavement distress image dataset appropriate for high-performance DCNNs training. The study involved 1,969 grayscale pavement images (1,418 for training, 500 for testing, and 51 for validation) with different distresses such as cracks (alligator, sealed/filled longitudinal/transverse), patches, open joints, potholes, and bleeding [226]. In another study Gopalakrishnan et al. used a dataset containing over 1,000 pavement images provided from the Long-Term Pavement Performance (LTPP) database of the Federal Highway Administration (FHWA), which contained a combination of PCCsurfaced and AC-surfaced pavement images [227]. Zhang et al. used rotation data augmentation and image resizing methods to generate a large block dataset from 800 images. The idea of the research focused on classifying cracked, sealed and non-cracked blocks [224]. 
New image capturing technologies have recently been implemented to characterize pavement condition. Zhang et al. used an effective DCNN for pixel-perfect crack detection on three-dimensional asphalt pavement surfaces. The dataset included 1,800, 3D asphalt surface images for training, and another 200 images for testing the system [183]. Tong et al. utilized Ground Penetrating Radar (GPR) pavement images for automated identification, measurement, and detection of concealed cracks. The dataset contains 6,832 GPR images with different damage types such as subgrade settlement, hidden cracks, roadbed cavities, and non-damaged areas. GPR is a powerful technique for evaluating pavement integrity in a non-destructive manner, and can characterize subsurface pavement defects, such as hidden cracks [228].

Table 4-1 represents a summary of the datasets utilized in previous studies. Most of the studies relied on 2D images, while the Zhang et al. study utilized 3D asphalt pavement surface images. Public datasets have clearly aided in the development of open-source deep learning methods in pavement evaluation. This has also facilitated comparisons between models, for instance, in terms of their detection accuracy.

Table 4-1 Overview of datasets used in previous studies

\begin{tabular}{cccccc}
\hline Availability & Reference & Dataset & $\begin{array}{c}\text { Number } \\
\text { of images }\end{array}$ & $\begin{array}{c}\text { Number } \\
\text { of } \\
\text { classes }\end{array}$ & $\begin{array}{c}\text { Angle of } \\
\text { camera }\end{array}$ \\
\hline \multirow{2}{*}{ Private } & {$[225]$} & Street view images & 9,712 & 1 & $\begin{array}{c}\text { wide } \\
\text { view }\end{array}$ \\
& {$[216]$} & Smartphone images & 500 & 1 & $\begin{array}{c}\text { wide } \\
\text { view }\end{array}$ \\
Public & {$[228]$} & $\begin{array}{c}\text { GPR images } \\
\text { 3D asphalt surface } \\
\text { images }\end{array}$ & 6,832 & 3 & top-down \\
& {$[183]$} & $\begin{array}{c}\text { Local (private) } \\
\text { German asphalt } \\
\text { pavement distress } \\
\text { (GAPs) }\end{array}$ & 1,900 & 1 & top-down \\
& {$[224]$} & $\begin{array}{c}\text { Smartphone street } \\
{[226]}\end{array}$ & 9,053 & 6 & top-down \\
& {$[215]$} & & 6 & top-down \\
& & wide
\end{tabular}


All this notwithstanding, none of the aforementioned studies utilized a comprehensive dataset containing all pavement distress types from sections with highly varied condition. Furthermore, the studies did not attempt classification and distress density characterization in a simultaneous fashion. In the current study, we introduce a dataset with both wide-view and top-down view images to classify and determine distress density, respectively. Furthermore, we introduce a newly developed python-based software program, which was used to rapidly extract images in bulk within the Google API.

\subsubsection{New Dataset}

The current dataset consists of 7,237 images obtained from 22 different pavement sections in the United States collected by utilizing our new python-based code. The Google API enables the extraction of pavement images automatically by specifying GPS coordinates along with camera and image parameters. For each considered section, start and endpoints were selected on the road, and interpolated 'snapping points' were determined in 15 meter intervals. Two different images were collected at each coordinate point. Images with a pitch angle of $-70^{\circ}$ and $-90^{\circ}$ were chosen for distress classification and density determination, respectively. The wide view image (at a $-70^{\circ}$ pitch) was found to be useful for distress classification. The top-down view image $\left(-90^{\circ}\right.$ pitch) led to more accurate distress quantification. Image size was $640 \times 640$ pixels for all images in the dataset. Afterwards, the wide-view images were hand annotated to characterize nine different distresses. Of the total 7,237 wide-view images, 5,789 images were used for 
training and 1,448 images were used for testing. represents provides examples of the nine different distress types that were targeted (Figure 4-1).

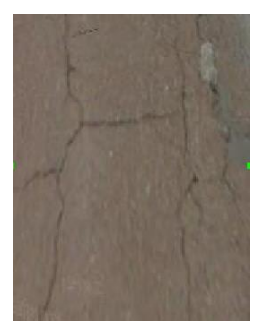

Block (D2)

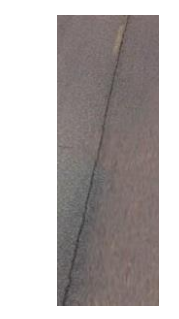

Lane longitudinal

(D6)

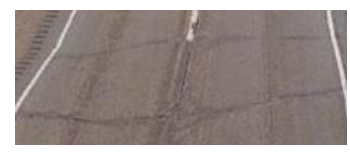

Reflective (D0)

a)

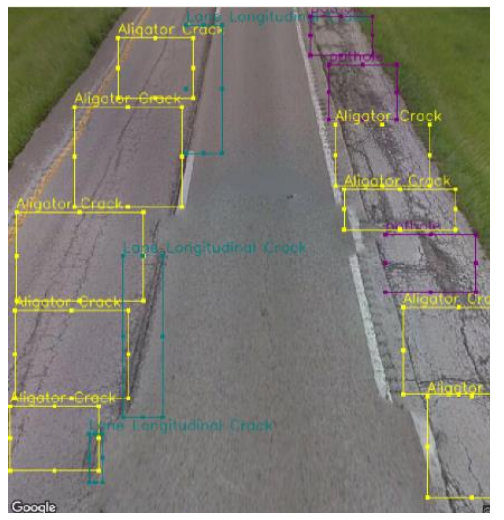

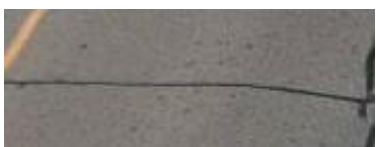

Sealed reflective (D7)

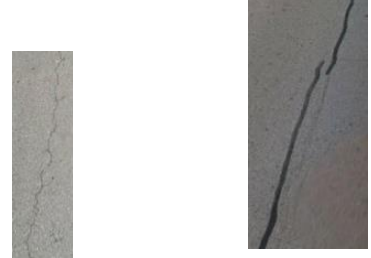

Sealed

Longitudinal Longitudinal

(D3)

(D7)

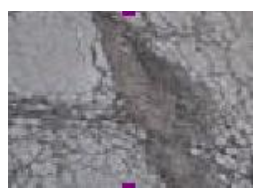

Pothole

(D8)

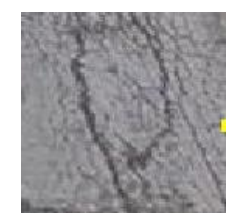

Alligator

(D4)
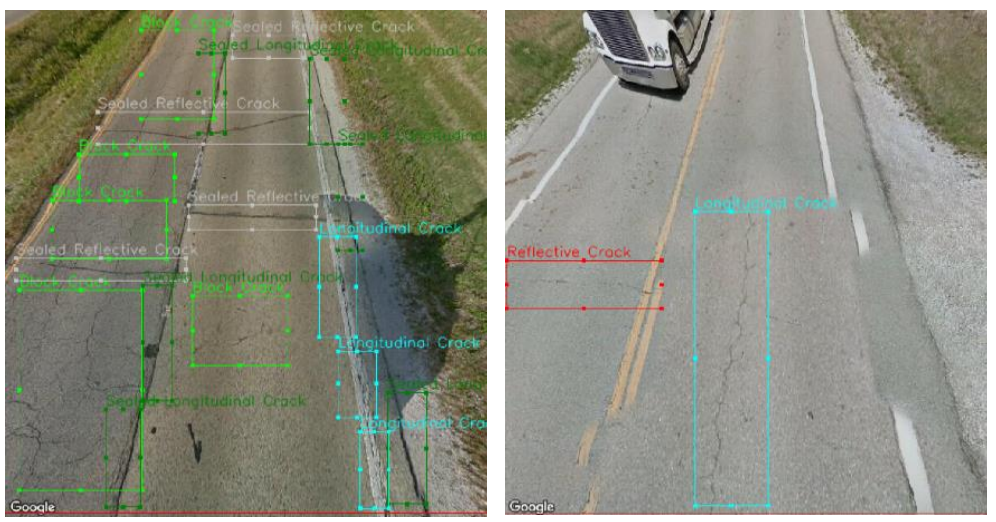

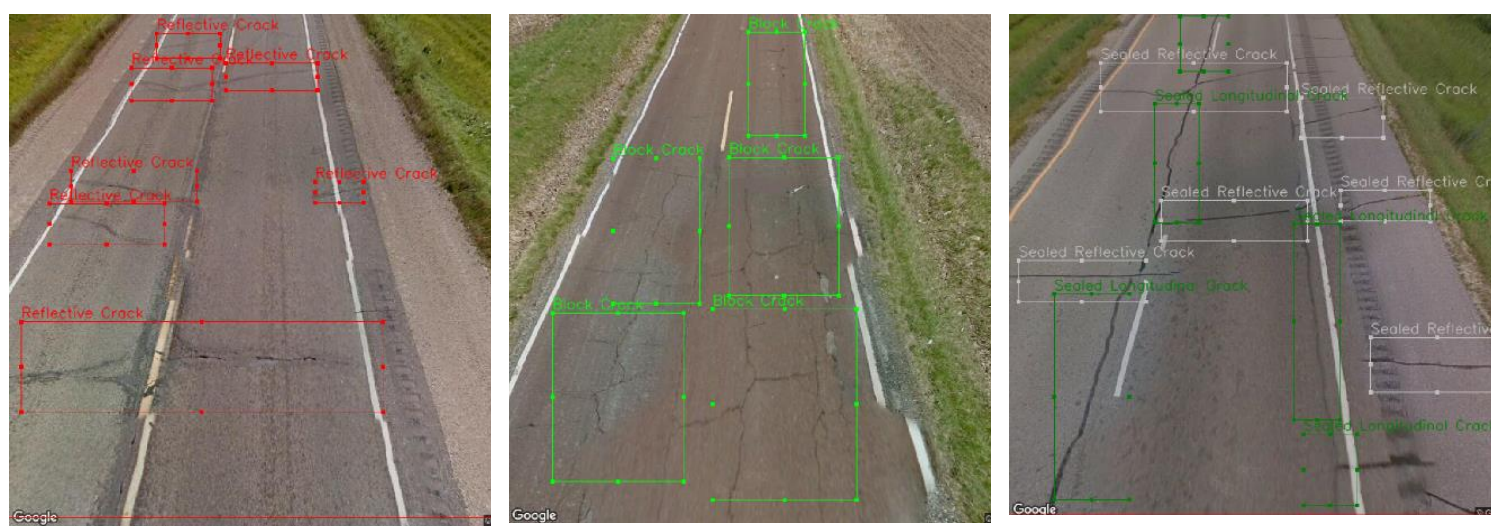

b)

\section{Figure 4-1 a) Nine different distress classes considered in the PID dataset; b) sample of annotated images from wide-view images in the PID dataset.}

The total number of boundary boxes and images for each distress type are shown in

Figure 4-2. Reflective, lane longitudinal, sealed longitudinal, and block cracks are among the highest number of boundary boxes and images found in the selected pavements, mainly concentrated in the Midwest USA. Potholes were the scarcest distress found in our dataset, probably because our dataset focused on 'high-type' interstate and highways roads, where pothole repair is quickly done when needed. 


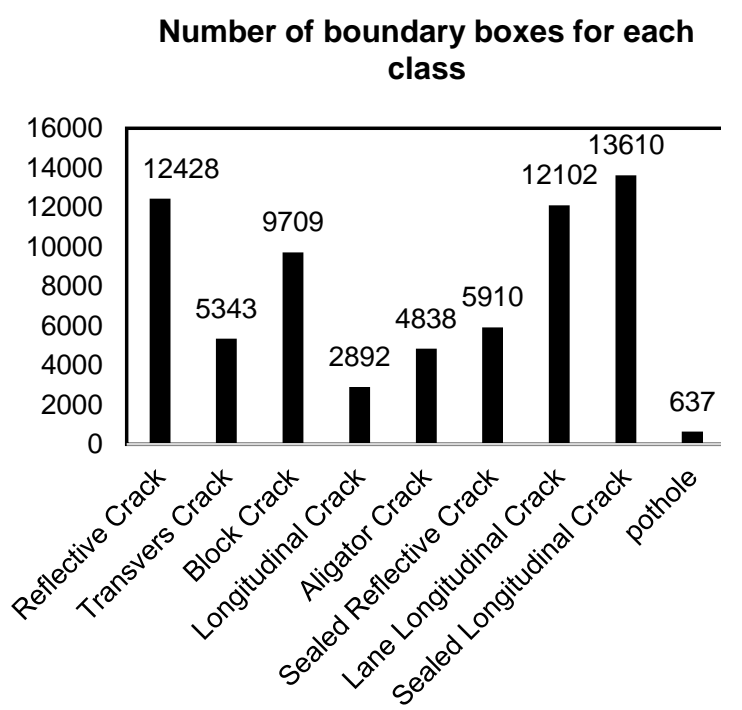

a)
Number of images for each class

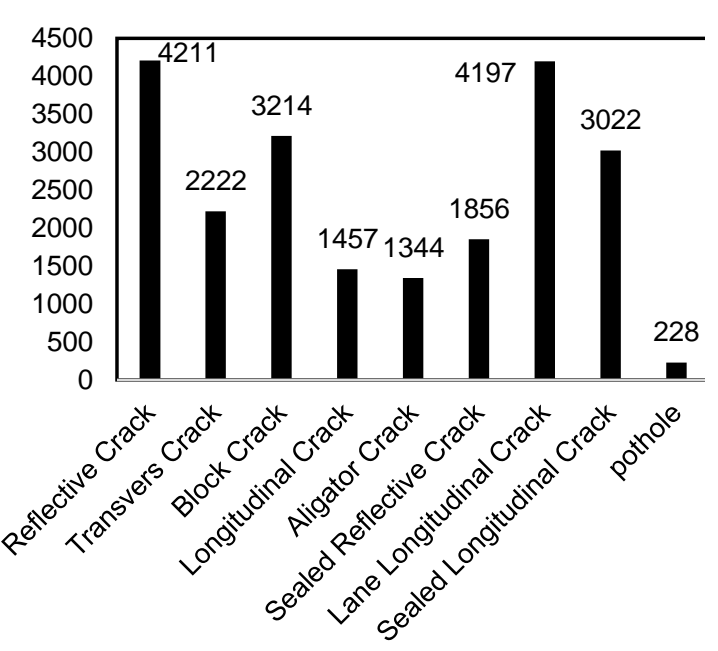

b)

Figure 4-2 a) Number of boundary boxes for each class, b) Number of images for each class.

\subsubsection{Model Training and Testing}

\subsubsection{YOLO v2 Model}

The first framework evaluated was the YOLO v2 deep convolutional neural network. YOLO is a relatively new object detection algorithm, which appears to have the highest accuracy and speed for developing deep learning-based models. YOLO reframes object detection methodology by looking at a particular image only one time to conduct object detections appropriately. Most recently, object detection algorithms use CNN classifiers to facilitate detections. In this manner, the algorithm can do simultaneous prediction of class probabilities. Table 4-2 shows the $\mathrm{CNN}$ architecture implemented for the prediction model developed herein. Standard layer types were used in the model including max pooling with a $2 \times 2$ kernel and convolution with a $3 \times 3$ kernel. The $1 \times 1$ kernel in the last convolutional layer contributes to reshape the data to $13 \times 13 \times 125$. This $13 \times 13$ structure is the size of 
the grid where the image becomes distributed. There are 35 channels of predictions for every grid cell. Through all these grid cells, five bounding boxes are predicted and labeled by seven data factors as follows: $\mathrm{x}$ and $\mathrm{y}$ values; height and width for the rectangle of the bounding box; road crack and non-crack probability distribution, and the confidence score.

Table 4-2 YOLO v2 model architecture.

\begin{tabular}{cccc}
\hline Layer & Kernel & Stride & Output Shape \\
\hline Input & & & {$[416,416,3]$} \\
Convolution & $3 \times 3$ & 1 & {$[416,416,16]$} \\
Max Pooling & $2 \times 2$ & 2 & {$[208,208,16]$} \\
Convolution & $3 \times 3$ & 1 & {$[208,208,32]$} \\
Max Pooling & $2 \times 2$ & 2 & {$[104,104,32]$} \\
Convolution & $3 \times 3$ & 1 & {$[104,104,64]$} \\
Max Pooling & $2 \times 2$ & 2 & {$[52,52,64]$} \\
Convolution & $3 \times 3$ & 1 & {$[52,52,128]$} \\
Max Pooling & $2 \times 2$ & 2 & {$[26,26,128]$} \\
Convolution & $3 \times 3$ & 1 & {$[26,26,256]$} \\
Max Pooling & $2 \times 2$ & 2 & {$[13,13,256]$} \\
Convolution & $3 \times 3$ & 1 & {$[13,13,512]$} \\
Max Pooling & $2 \times 2$ & 1 & {$[13,13,512]$} \\
Convolution & $3 \times 3$ & 1 & {$[13,13,1024]$} \\
Convolution & $3 \times 3$ & 1 & {$[13,13,1024]$} \\
Convolution & $1 \times 1$ & 1 & {$[13,13,35]$} \\
\hline
\end{tabular}

\subsubsection{Faster R-CNN Model}

The Faster R- CNN model involves a two-stage target detection method. The faster R-CNN model is a third generation model in the R-CNN series, which merges four primary steps in target detection. These steps include informative region selection, feature extraction classification, and location refinement into a deep network framework. It improves upon Fast R-CNN [229] by replacing the selective search method with a Region Proposal Network (RPN). First, Faster R- CNN splits an image into multiple, small 
segments. Next, the model passes each segment through a series of convolutional filters to derive the precious feature descriptors, which are subsequently passed through a classifier. The classifier outputs are the probability that an image area includes an object type. An NVIDIA GTX 1080Ti GPU was used to run this algorithm efficiently. The training time for the Faster R- CNN model was approximately four hours.

\subsubsection{Transfer learning}

Transfer learning was utilized to boost the training speed and performance of the YOLO and Faster R-CNN models. Using this method, a new task can benefit from formerly well-trained models. The Microsoft COCO dataset involves over 2 million well-labeled objects (like cars, shadows, etc) in 80 various groups with over 300,000 images. The pretrained weights in the $\mathrm{COCO}$ dataset were used to initiate the detection task in the newly proposed models.

\subsubsection{Results}

\subsubsection{Model Accuracy}

The performance of the proposed model was evaluated on 1,448 test images. The model was trained on 5,789 images for 40,000 iterations with the learning rate set to 0.01 . The accuracy of the proposed model was evaluated by measuring the overlapping percentage between the ground truth and the prediction boxes. If a prediction box captured over 30\% overlap with the ground truth box (Intersection over Union (IoU)), the prediction was considered a successful match, or a true positive (tp). Conversely, if the predicted bounding box had less than $30 \%$ IoU overlap with the ground truth box, it was categorized as a false positive (fp). Also, when there was an overlap of $30 \%$ between the prediction 
and the ground truth, but the predicted classification was incorrect, a false positive was assigned. False negatives (fn) were assigned to the instances where the model was not able to predict any distress.

Precision, Recall, and F1 score were the parameters used to evaluate model accuracy. Precision, shown in equation (4-1), is the ratio of true positives (tp) to all predicted positives $(\mathrm{tp}+\mathrm{fp})$. Correspondingly, Recall is the ratio of true positives to all the actual positives (tp+fn) as represented in equation (4-2). Overall accuracy is measured by the F1 score, which includes the recall values and a measure of statistical precision, as shown in equation (4-1; 4-2; 4-3).

$$
\begin{aligned}
& \text { Precision }=\frac{t p}{t p+f p} \\
& \text { Recall }=\frac{t p}{t p+f n} \\
& F 1=\frac{2 \times \text { Precision } \times \text { Recal }}{\text { Precision }+ \text { Recal }}
\end{aligned}
$$

Figure 4-3 illustrates examples of detection and classification obtained using the YOLO algorithm. A red bounding box corresponds to the ground truth value, whereas a green bounding box represents the predicted value generated by the model. Figure 4-3(a) illustrates distresses that were accurately detected and classified with over $30 \%$ IoU for each crack class and thus, classified as true positive. Figure 4-3(b) illustrates a false positive (boundary box on the left), which has less than $30 \%$ IoU overlap with the ground truth. Distresses that were not detected by the model (False negative) are shown in Figure 
4-3(b) and (c). Although some distresses were inadvertently left unlabeled during the tedious, manual annotation process, the model typically detected and classified them (Figure 4-3(d)). This suggest the high level of performance of the developed model within YOLO v2 algorithm. 


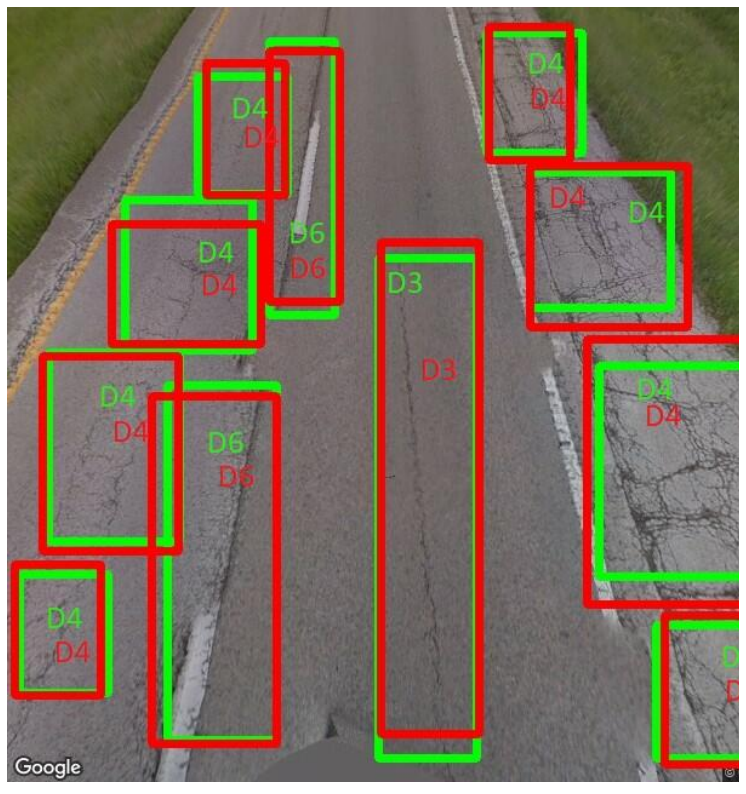

a)

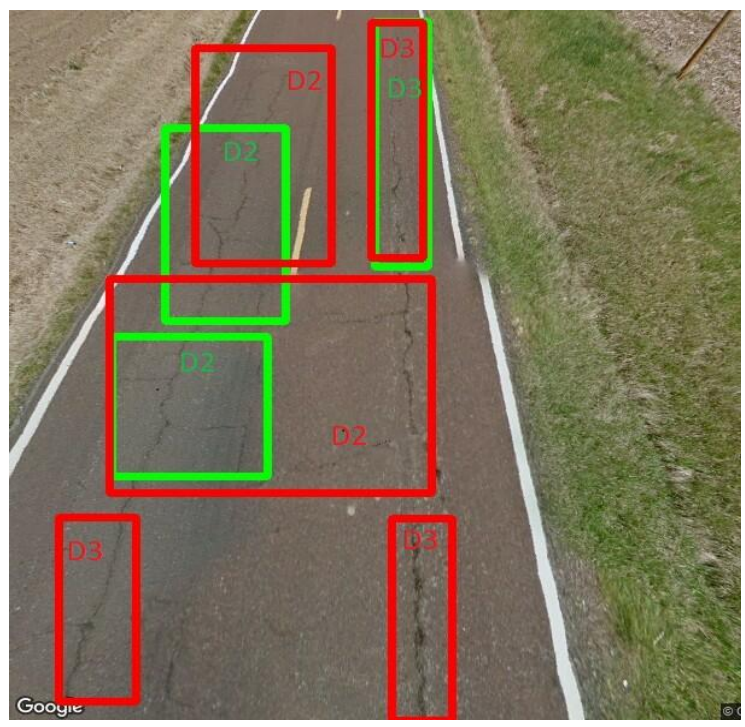

c)

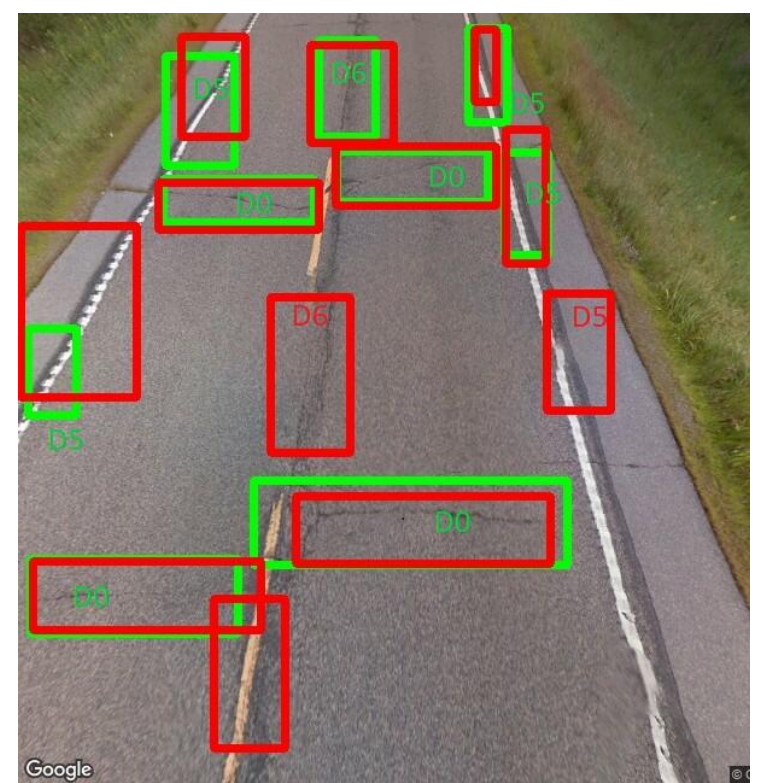

b)

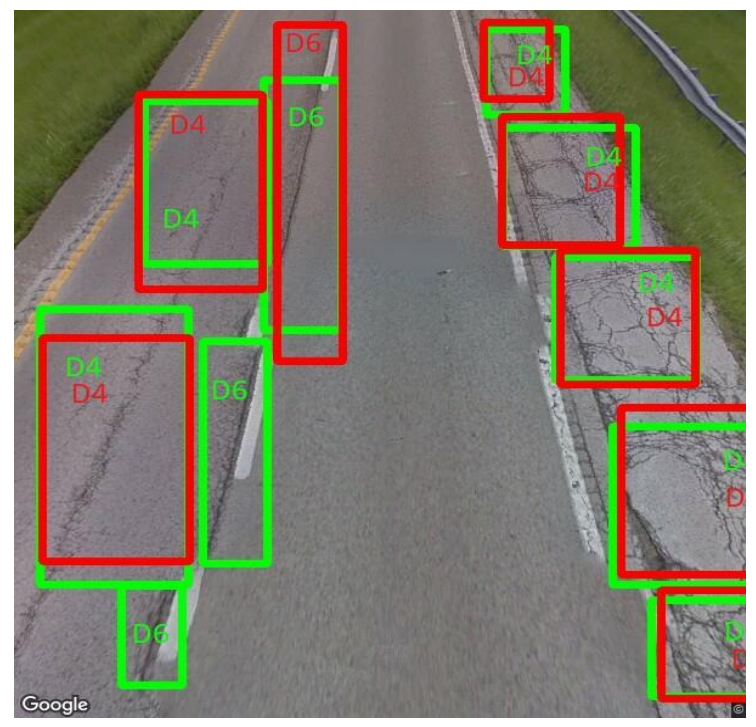

d)

Figure 4-3. Classification of predicted crack from validation dataset: a) true positive, b) false positive and false negative, c) false negative, d) missed annotations identified by YOLO v2.

‘Confusion matrices' from YOLO v2 and Faster R-CNN models are shown in Figure 4-4. Although the accuracy of both models was excellent, the YOLO v2 model achieved higher accuracy as indicated by the values in the confusion matrix as compared to the Faster R-CNN. In both models, confusion results occurred in a small, but significant number of 
cases. Relatively, confusions between classes occurred far more often in the Faster R-CNN model than the YOLO v2 model. Table 4-3 contains the confusions that were detected between classes in both models. Reflective and transverse cracks were the distresses most often confused in both models (Table 4-3). Also, alligator cracking and potholes were confused in several cases in both models. This can be explained by the similarity of these two distresses, as potholes often emerge as a later stage of alligator cracking.

a)

$\begin{array}{cccccccccc}\text { YOLO V2 } & \text { D0 } & \text { D1 } & \text { D2 } & \text { D3 } & \text { D4 } & \text { D5 } & \text { D6 } & \text { D7 } & \text { D8 } \\ \text { D0 } & 0.99 & 0.01 & 0.00 & 0.00 & 0.00 & 0.01 & 0.00 & 0.00 & 0.00 \\ \text { D1 } & 0.02 & 0.97 & 0.01 & 0.00 & 0.00 & 0.00 & 0.00 & 0.00 & 0.00 \\ \text { D2 } & 0.00 & 0.00 & 0.99 & 0.00 & 0.00 & 0.00 & 0.00 & 0.00 & 0.00 \\ \text { D3 } & 0.00 & 0.00 & 0.01 & 0.98 & 0.00 & 0.00 & 0.01 & 0.00 & 0.00 \\ \text { D4 } & 0.00 & 0.00 & 0.00 & 0.00 & 0.99 & 0.00 & 0.00 & 0.00 & 0.00 \\ \text { D5 } & 0.00 & 0.00 & 0.00 & 0.00 & 0.00 & 1.00 & 0.00 & 0.00 & 0.00 \\ \text { D6 } & 0.00 & 0.00 & 0.00 & 0.00 & 0.00 & 0.00 & 0.99 & 0.00 & 0.00 \\ \text { D7 } & 0.00 & 0.00 & 0.00 & 0.00 & 0.00 & 0.00 & 0.00 & 1.00 & 0.00 \\ \text { D8 } & 0.00 & 0.00 & 0.00 & 0.00 & 0.00 & 0.00 & 0.00 & 0.00 & 1.00\end{array}$

Fast R-

$\begin{array}{cccccccccc}\text { CNN } & \text { D0 } & \text { D1 } & \text { D2 } & \text { D3 } & \text { D4 } & \text { D5 } & \text { D6 } & \text { D7 } & \text { D8 } \\ \text { D0 } & 0.96 & 0.02 & 0.01 & 0.00 & 0.00 & 0.01 & 0.00 & 0.00 & 0.00 \\ \text { D1 } & 0.05 & 0.91 & 0.04 & 0.00 & 0.00 & 0.00 & 0.00 & 0.00 & 0.00 \\ \text { D2 } & 0.00 & 0.01 & 0.97 & 0.02 & 0.00 & 0.00 & 0.00 & 0.00 & 0.00 \\ \text { D3 } & 0.00 & 0.00 & 0.07 & 0.92 & 0.00 & 0.00 & 0.01 & 0.00 & 0.00 \\ \text { D4 } & 0.00 & 0.00 & 0.00 & 0.00 & 0.97 & 0.00 & 0.01 & 0.00 & 0.01 \\ \text { D5 } & 0.01 & 0.00 & 0.00 & 0.00 & 0.00 & 0.99 & 0.00 & 0.00 & 0.00 \\ \text { D6 } & 0.00 & 0.00 & 0.00 & 0.01 & 0.00 & 0.00 & 0.99 & 0.00 & 0.00 \\ \text { D7 } & 0.00 & 0.00 & 0.00 & 0.00 & 0.00 & 0.00 & 0.01 & 0.99 & 0.00 \\ \text { D8 } & 0.00 & 0.00 & 0.00 & 0.00 & 0.07 & 0.00 & 0.00 & 0.00 & 0.93\end{array}$

b) 
Figure 4-4. Confusion matrices obtained on the classification dataset using a) YOLO v2 and b) Fast R-CNN models

Table 4-3 Confusion between the classes in the models

\begin{tabular}{|c|c|c|c|}
\hline Model & Distress ID & Distresses & $\begin{array}{c}\text { Number of } \\
\text { Images }\end{array}$ \\
\hline \multirow{7}{*}{ YOLO v2 } & D0 and D1 & Reflective Crack and Transverse Crack & 19 \\
\hline & D0 and D5 & Reflective Crack and Sealed Reflective Crack & 13 \\
\hline & D1 and D2 & Transverse Crack and Block Crack & 6 \\
\hline & D3 and D2 & Longitudinal Crack and & 8 \\
\hline & D3 and D6 & Longitudinal Crack and Lane Longitudinal Crack & 4 \\
\hline & D4 and D6 & Alligator Crack and Lane Longitudinal Crack & 7 \\
\hline & D7 and D6 & $\begin{array}{l}\text { Sealed Longitudinal Crack and Lane Longitudinal } \\
\qquad \text { Crack }\end{array}$ & 7 \\
\hline \multirow{10}{*}{$\begin{array}{l}\text { Faster R- } \\
\text { CNN }\end{array}$} & D0 and D1 & Reflective Crack and Transverse Crack & 34 \\
\hline & D0 and D2 & Reflective Crack and Block Crack & 11 \\
\hline & D0 and D5 & Reflective Crack and Sealed Reflective Crack & 23 \\
\hline & D1 and D2 & Transverse Crack and Block Crack & 27 \\
\hline & D2 and D3 & Block Crack and Longitudinal Crack & 21 \\
\hline & D3 and D6 & Longitudinal Crack and Lane Longitudinal Crack & 12 \\
\hline & D4 and D6 & Alligator Crack and Lane Longitudinal Crack & 8 \\
\hline & D4 and D8 & Alligator Crack and Pothole & 5 \\
\hline & D6 and D3 & Lane Longitudinal Crack and Longitudinal Crack & 12 \\
\hline & D7 and D6 & $\begin{array}{l}\text { Sealed Longitudinal Crack and Lane Longitudinal } \\
\text { Crack }\end{array}$ & 12 \\
\hline
\end{tabular}

Table 4-4 shows detection and classification accuracies of the YOLO v2 and Faster R-CNN models for the nine classes in our dataset. Lower precision, recall, and F1 scores were found in the Faster R-CNN model in the cases of longitudinal, alligator, and longitudinal lane cracks. The F1 scores for the classes in the YOLO v2 model are higher than the scores for the Faster R-CNN model. The range of F1 scores in YOLO v2 models 
were between $0.95-0.98$, while the Faster R-CNN model F1 scores ranged between 0.80.91. Although both are acceptable, the YOLO v2 model achieved better overall accuracy with an F1 score of 0.84 as compared to the Faster R-CNN model, which had an overall F1 score of 0.65 . The precision and recall values for the YOLO v2 model were 0.93 and 0.77 , respectively.

Among previous studies which used the same deep learning frameworks (YOLO v2), Maeda et al. model training resulted in precision and recall values of 0.77 and 0.71 , respectively [215]. Mandal et al. achieved 0.77 and 0.73 for precision and recall values, respectively [218]. The high values of precision, recall and the F1 score of 0.84 in our proposed YOLO v2 model suggest the advantage of using labeled datasets in developing pavement distress detection models.

Table 4-4 Detection and classification results for nine distress types

\begin{tabular}{ccccccc}
\hline Crack class name & \multicolumn{3}{c}{ YOLO v2 } & \multicolumn{3}{c}{ Faster R-CNN } \\
\hline & Precision & Recall & F1 & Precision & Recall & F1 \\
Reflective crack & 0.93 & 0.76 & 0.84 & 0.73 & 0.72 & 0.72 \\
Transverse crack & 0.9 & 0.83 & 0.86 & 0.75 & 0.74 & 0.75 \\
Block crack & 0.93 & 0.79 & 0.85 & 0.82 & 0.59 & 0.68 \\
Longitudinal crack & 0.91 & 0.83 & 0.87 & 0.66 & 0.43 & 0.52 \\
Alligator crack & 0.91 & 0.74 & 0.82 & 0.81 & 0.43 & 0.57 \\
Sealed transverse crack & 0.93 & 0.83 & 0.87 & 0.83 & 0.68 & 0.75 \\
Sealed longitudinal crack & 0.93 & 0.79 & 0.85 & 0.81 & 0.54 & 0.65 \\
Lane longitudinal crack & 0.94 & 0.57 & 0.71 & 0.75 & 0.3 & 0.42 \\
Pothole & 0.96 & 0.78 & 0.86 & 0.83 & 0.78 & 0.8 \\
Average & $\mathbf{0 . 9 3}$ & $\mathbf{0 . 7 7}$ & $\mathbf{0 . 8 4}$ & $\mathbf{0 . 7 8}$ & $\mathbf{0 . 5 8}$ & $\mathbf{0 . 6 5}$ \\
\hline
\end{tabular}




\subsubsection{Model Performance when Using Top-Down Images}

In this section, models developed based solely on wide-view images were tested to allow comparison to results obtained using top-down view images. The motivation for training the models using wide-view images is due to the fact that wide view images are more readily obtained than top-down images. For instance, they can be obtained from smartphones in cars, whereas top-down images are harder to acquire as they emanate from more sophisticated equipment.

Figure 4-5 shows a comparison between the YOLO v2 and faster R-CNN frameworks in detecting pavement distresses from top-down images. Full sunshine images and images containing shadows (for instance, from trees) were selected in an attempt to challenge the robustness of each model. The black boxes in the Figure represents the ground truth, while the red, blue, and green boxes indicated the predicted detections. Both models were able to accurately detect distresses in both the full sunshine and shadow-containing images.

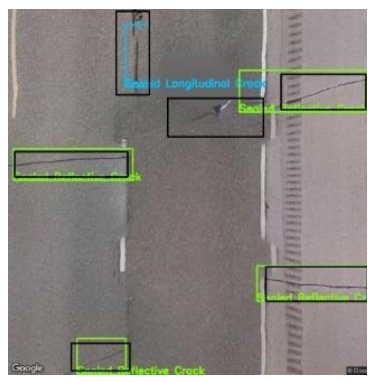

a1)

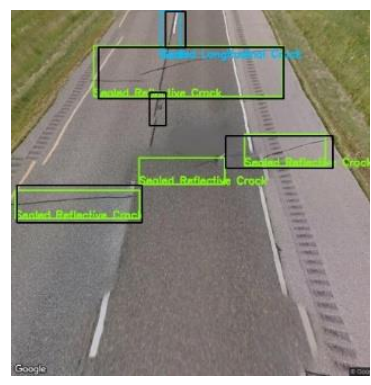

a2)

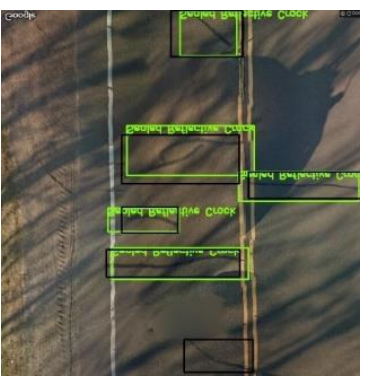

a3)

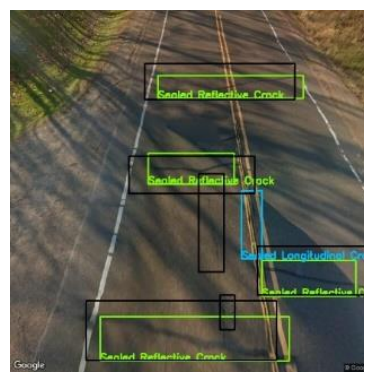

a4) 


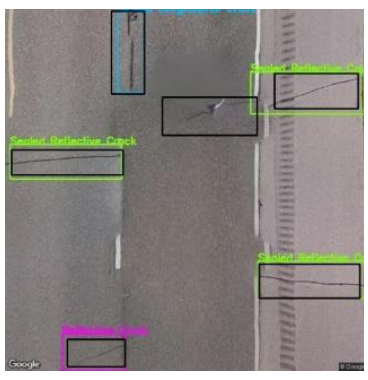

b1)

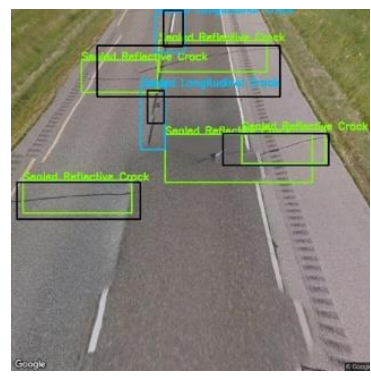

b2)

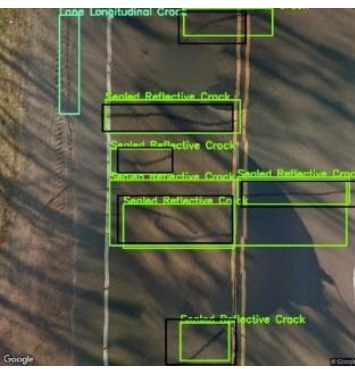

b3)

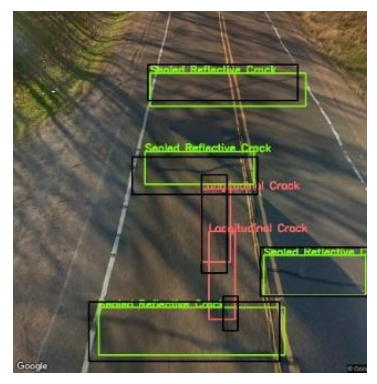

b4)

*The color of the bounding box indicates the type of distress and black is ground truth.

Figure 4-5. a) detection from YOLOv2 model b) detection from Faster R-CNN model, 1, 2, 3 and 4 represent plain top-down, plain wide-view, shadowed top-down and shadowed wide view image.

\subsubsection{Summary}

In this chapter of study, we introduced a comprehensive dataset named Pavement Image Dataset (PID) for training machine learning models for the purpose of automated pavement distress characterization and monitoring. The dataset was created using Google API street-view via a python-based software, which was developed to extract pavement images at desired intervals along roadways. The dataset consists of two image groups: "wide-view images", where 7,237 images with bounding boxes featuring nine different pavement distresses were assembled; and "top-down images", consisting of 7,237 images at identical locations as the wide-view images. The wide-view images were used to classify distresses, while the top-down view images were used for calculating the density of distresses.

The primary focus of this article was to demonstrate how the wide-view images were used along with a deep learning approach to classify distresses. Two state-of-the-art, deep learning frameworks, YOLO v2 and Faster R-CNN, were implemented to automatically detect and classify nine types of pavement distress. The F1 scores, which are often used 
for model accuracy assessment, were obtained as 0.84 for YOLOv2 and 0.65 for the Faster R-CNN models, respectively. According to the F1 scores and confusion matrices for the nine distress classes, the YOLO v2 model results in more accurate distress characterizations than the Faster R-CNN model.

The proposed models offer some advantages over traditional pavement monitoring, and as compared to previous deep learning-based models. First, the models were trained using Google street-view images, which are free and available for virtually all roads in the US and abroad. Second, the models were developed based on a wide variety of common pavement distress types. Finally, the developed models are robust and flexible, able to predict distress from different camera views towards convenient, cost-effective, and accurate pavement evaluation, monitoring, and management.

In the current study, a pre-trained U-Net convolutional network was used, which was originally developed for biomedical image segmentation. Herein, it was used to quantify the density of cracks in roads [230]. The mentioned process was performed on top-down view images. Afterwards, the U-Net output image was reprocessed using a customdeveloped MATLAB code to reduce image noise. Figure 4-6 shows the original, U-Net, and reprocessed image. Future research will be focused on developing improvements in the U-Net analysis. Also, the proposed model in YOLO and U-Net will be integrated with the python-based image extractor software developed in this study to grow the dataset directly from Google maps images. 

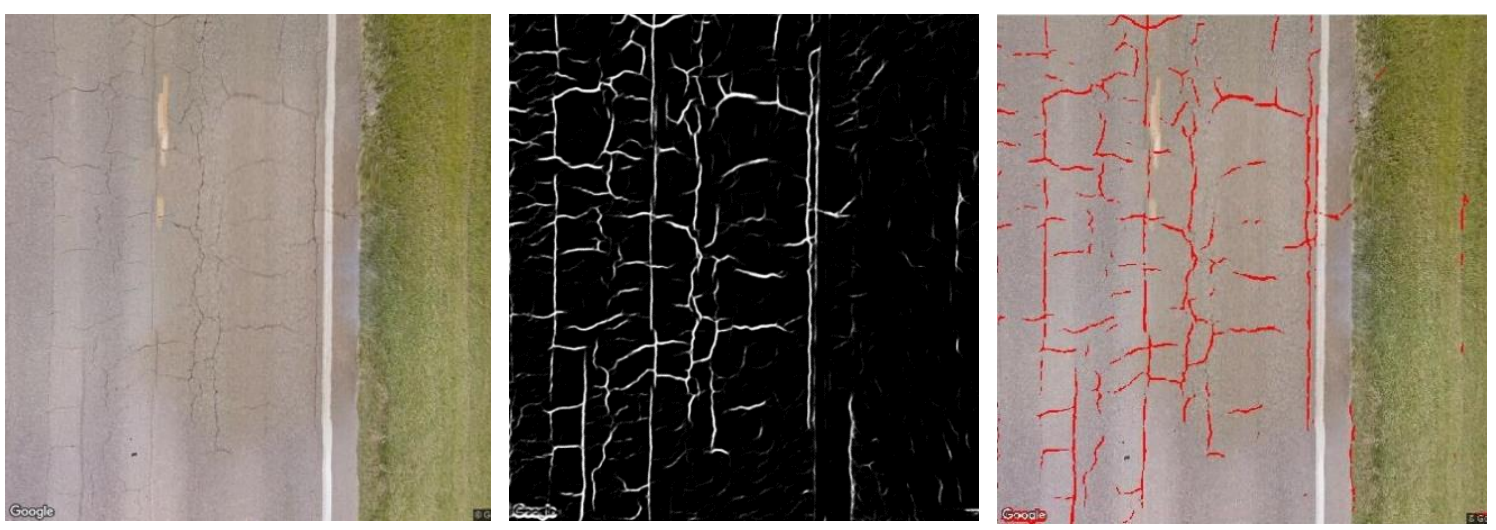

Figure 4-6. Examples of distress densification on top-down images. From left to right: original image, UNET output, overlapped modified UNET on original image 


\subsection{DEEP MACHINE LEARNING APPROACH TO DEVELOP A NEW ASPHALT PAVEMENT CONDITION INDEX}

\subsubsection{Introduction}

Pavement distress detection is a first key step in developing a robust pavement management system. It offers a comprehensive assessment of pavement conditions. Consequently, it generates information needed to make more cost-effective and consistent decisions associated with the pavement network preservation. Generally, pavement distress inspection is performed using sophisticated data collection vehicles and foot-on-ground surveys. In either approach, the current process of distress detection is human-dependent, expensive, inefficient, and unsafe. For example, the total price of an Aran was reported by the Ohio Department of Transportation for US $\$ 1,179,000$, with an annual operating expense of US\$70,000 [194]. Fully automated distress detection systems requiring no specialized data collection equipment, have the potential to lower distress survey costs, reliability and scalability [231]. The primary goal of this study is to leverage recent advances in machine learning to develop a low-cost, robust pavement condition assessment system, capable of detecting, classifying and quantifying the density of pavement cracks in an automated fashion.

The most promising approaches for automated distress analysis, leverage image processing and computer vision algorithms to detect edges of different types and severities of crack in pavement images. The primary advances in automated pavement crack detection techniques are as follow: intensity-thresholding [183], [198]-[200], match filtering [202], edge detection [203], seed-based approach [204], multiscale methods like 
wavelet transforms and empirical mode decomposition [205]-[208], [232]; textureanalysis, and machine learning [209]-[212]. Also, Zou et al developed CrackTree as an automatic procedure for crack detection [213]. There are some challenges related to these approaches. The first challenge is that these techniques rely on image pixel manipulations, which are very slow processes, and then it is not applicable for large scale deployment. Secondly, these techniques only work precisely if the image configuration is static, and the models don't work well if different camera configurations are used. Finally, there are many heuristic rules related to the use of these models, which make it impractical to be implemented extensively.

In order to overcome these challenges, computer vision algorithms which use machine learning models have been proposed as an alternative to traditional image manipulation techniques. In fact, recent progresses in deep learning has directed to substantial improvements in our ability to analyze streams of videos and image at unprecedented accuracies. The models are leading advances in areas like self-driving cars and smart cities [233], nanomaterials [234], healthcare, agriculture, retailing, and finance.

Deployment of machine learning approaches for automated pavement distress detection is not novel anymore, however application of deep learning is still attractive for pavement researchers [214]-[217]. Deep architecture with many hidden layers like deep convolution neural networks (DCNNs) provide frequent abstraction levels [180], [218][220]. CrackNet software was established by Zhang et al as a crack detection model using raw image patches via the CNN [216]. Afterward, Zhang et al applied the Recurrent Neural Network $(\mathrm{RNN})$ to produce CrackNet-R, which is more accurate in detecting small cracks 
and removing noise than CrackNet [183]. It must be noted that none of those mentioned above studies, proposed models based on a comprehensive dataset covering all pavement distress types from sections with different conditions. Also, classification and quantifying the density of the distress did not take into account simultaneously. Furthermore, none of these studies did not provide a pavement condition tool which can be used for evaluating pavement condition based on the proposed detection models.

In the current study, we develop four prediction models to evaluate pavement condition using the proposed pavement distress detection deep learning based models. It must be noted that the variety, quality and quantity of data utilized for training models is the key factor for their robustness, which is not always available. Comprehending the importance of labeled datasets to develop a strong pavement condition detection model, Majidifard et al represented the 'Pavement Image Dataset,' or (PID) [235]. The dataset contains 7,237 images extracted from 22 different pavement sections, including both US highways and interstate routes. A python code was developed to extract images using Google Application Programming Interface (API) in Google street-view [235]. Images were hand annotated by pavement experts carefully considering nine different distress classes. Afterward, the performance of dataset was assessed using a famous deep learning framework named You Look Only Once (YOLO v2) [235]. However, the proposed model by YOLO did not quantify the density of the cracks. Therefore, in our new study we developed a new U-Net based model to quantify the density of the distresses. Finally, various pavement condition indices tried to be developed based on the proposed crack detection models (Yolo-based and U-Net based). The following summarizes the primary contributions of this study (Figure 4-7): 
- First, we introduced a unique dataset annotated for simultaneous classification and densification of pavement distresses. The data is extracted from google street view, which provides us with a variety of camera views needed to improve the system's ability to recognize different types of cracks and estimate their severity.

- Second, we implemented a distress segmentation model capable of delineating the boundaries of different types of cracks in challenging environments characterized by severe shadowing, non-crack-like objects such as cars, trees, etc. We overcome this challenge through intelligent model training techniques and architectural designs.

- Developing a new pavement condition index based on inputs from a crack classification model (YOLO), a density model (U-Net) and a hybrid of machine learning-based models.

- Conduct a comparative analysis of proposed pavement condition index with PASER ratings along pavement profile. 


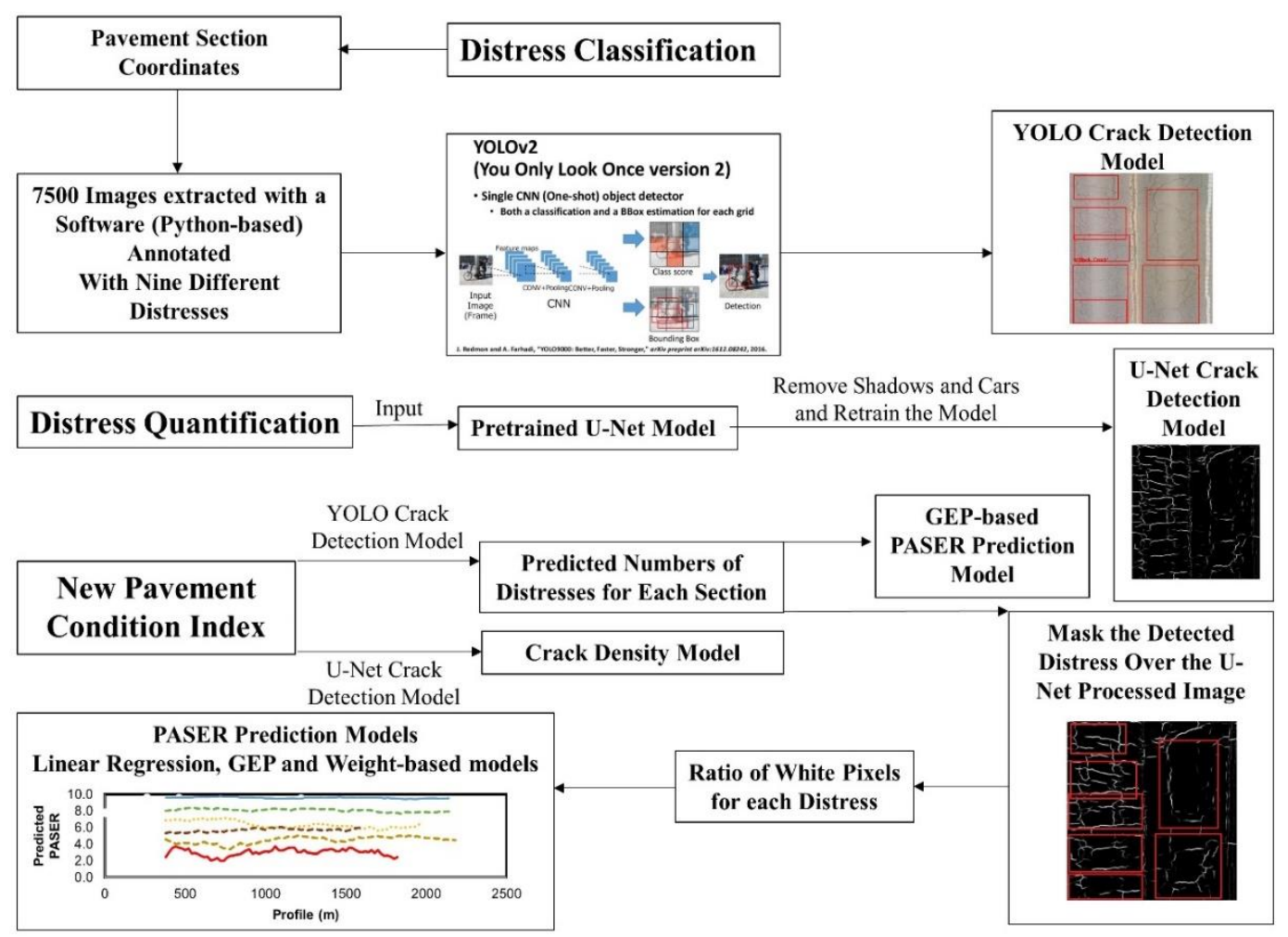

Figure 4-7. Experimental research flowchart

The arrangement of the article is organized as follows. The relate works including pavement condition indices and automated crack detection methods are reviewed in Section 2. The methodology of the research introduces main road segmentation, U-Net based Model for distress quantification, and developing pavement condition indices which discussed in section 3 followed by model validation briefed in Section 4. Section 5 center around the conclusion of the research which summaries the methodology and the implementation of the study. 


\subsubsection{Background}

\subsubsection{Pavement condition index development approaches}

There are various indices that are utilized to characterize pavement conditions, such as the pavement condition index (PCI), present serviceability index (PSI), present serviceability rating (PSR), pavement surface evaluation and rating (PASER) [236]. PCI is the quantitative-based pavement condition index, while the others can be grouped into class of qualitative pavement indices. The PCI, arguably the most prevalent index for pavement condition evaluation, depends on inspection data and visual observation. U.S. Army Corps of Engineers developed PCI for the management of pavement rehabilitation and maintenance system [237]. In the PCI calculation method, various distress types with different severities are incorporated into a univalent PCI value. The length or area and the severity (low, medium, and high) of each distress are taken into account to calculate the deduct values for the surveyed section [238], [239]. The PCI ranges from 100 to 0 , in which 100 is brand new pavement, and 0 is the worst condition possible. The University of Wisconsin-Madison Transportation Information Center developed the PASER system, rates pavement conditions from 1-10. It utilizes visual inspection to assess pavement surface conditions [240]. The type of distress is not required to calculate PASER, as a result, PASER ratings cannot be disaggregated by distress types. The advantage of this method is that pavement sections may be rated promptly, possibly even by "windshield survey." A principal drawback is that the PASER outputs cannot be used in mechanistic-empirical transportation asset management programs because PASER ratings cannot be classified into component distress data [240]. 
Newer approaches for developing pavement condition indices have been proposed by several research teams as a result of the limitations traditional methods such as the PCI, PASER, PSI, etc. Eldin and Senouci used neural-network based algorithm to predict condition of pavement using data provided by the Oregon State Department of Transportation (ODOT) [241]. Fathi et al used a hybrid machine learning (ML) method that combines random forest (RF) and artificial neural network (ANN) to predict the alligator deterioration index (ADI) [90]. Piryonesi and El-Diraby used historical distress data in the LTPP database to develop decition trees-based algorithm to predict PCI of asphalt roads [242]. They developed a Python ${ }^{\mathrm{TM}}$ program to produce the PCI values from distress data according to the ASTM methodology. For this purpose, all deduct value graphs, and correction curves were digitized and mathematically designated [242]. Shahnazari et al., used ANN and GP algorithms to predict the PCI. The models established based on large dataset of PCI values which was collected from road network of Iran. The proposed models were accurate and they can be used instead of Micropaver software to calculate PCI [243].

The limitation of the previous models is that all of them rely on manual inspection of distresses. Inspectors should calculate the area, length, and severity of distresses, and the output can be used as an input in these models to calculate the pavement condition rate. Therefore, a fully automated distress detection model is demanding.

\subsubsection{Automated Crack detection, segmentation and classification approaches}

Image segmentation is the procedure of subdividing an image into several segments (sets of pixels, also known as image objects). Image segmentation is classically performed 
to detect boundaries such as lines and curves in images. In another word, image segmentation is the practice of extracting the interest objects from the background. Object classification is a technology associated with image processing and computer vision that copes with detecting semantic objects of a specific class such as humans, buildings, or cars in videos and images. Object classification and segmentation - both are part of machine learning based image processing to train AI algorithms through computer vision, and both are important for object recognition precisely in machine learning and AI development. The classification process is easier than segmentation; in the classification approaches, all objects in a single image are grouped or categorized into a single class. While in segmentation each object of a single class in an image is highlighted with different shades to make them recognizable to computer vision. In crack segmentation, the severity of the distresses is detectable although there is no ability to classify them into different groups. On the contrary, in the classification approaches the distresses can be categorized into different groups and the severity cannot be measured.

The primary segmentation approaches are thresholding and edge detection. Thresholding-based segmentation is broadly utilized in automated pavement distress systems [244]. Another regular procedure in image processing is edge detection. The significant advantage of edge detection is the fast reduction of image data to beneficial information. There are many useful edge detectors that have been proposed over the past 30 years, such as LOG, Sobel, Roberts, and Prewitt edge detectors [68]. The main problem associated with the most edge detection algorithms is that these algorithms only characterize a spatial scale for edge detection. Pavement images are acknowledged to be challenging to work within the process of pavement distresses because of different details 
at various scales. In the past decade, wavelet-based edge detection at multiple scales became popular in pavement image processing [245]. Shadows and lighting effects in pavement images introduced new challenges in the automatic pavement distress detection field. Region-based image thresholding has been implemented to resolve the difficulties caused by shadow and illumination variations. A neighboring difference histogram procedure was used by Li and Liu to crack image segmentation using a globally optimized threshold [246]. However, histogram-based procedures, do not consider photometric and geometric characteristics of the cracks in road pavement images [247]. Dynamic local thresholding for non-overlapping image blocks, developed by Oliveira and Correia [199]. Although segmentation procedure based on thresholding is beneficial in various image segmentation tasks, it is still problematic for the automatic threshold selection. Image morphological procedure is another primary tool that has often been utilized in the automated pavement distress detection studies [248]. [249] developed a procedure using fundamental top-hat transform and morphological operations to extract structural information from road pavement image, and subsequently cracks detection. Although morphological image processing provides the benefit of extracting prominent geometrical structure related to the cracks in road surface images, the performance is highly parameter dependent [198]. In practice, it is suggested to operate morphological processing along with other image processing techniques.

Object classification generally divided to machine learning-based and deep learning-based methods. In Machine Learning approaches, first the features defined by using Histogram of oriented gradients (HOG), Viola-Jones object detection framework, Scale-invariant feature transform (SIFT) [250], then classification frameworks such as 
support vector machine (SVM) implemented to do the classification. While, deep learning techniques have the capability to do end-to-end object detection without precisely defining features, and are classically based on convolutional neural networks (CNN) such as You Only Look Once (YOLO) [251], Single-Shot Refinement Neural Network for Object Detection (RefineDet) [252], Region Proposals (R-CNN) [229], Fast R-CNN [229], Faster R-CNN [253], Single Shot MultiBox Detector (SSD) [254].

Critical distresses on road surfaces must be detected in order to produce a robust automated pavement distress detection model. Pavement deterioration rate is a function of various features such as climate, structural layering, traffic, and layer age. Strategies are developed by road administrators to repair the road surface based on the type, extent, and severity of the distresses. Former research studies led to improvement in the direction of this target but fell short in one area or another. To clarify, CrackNet (L. Zhang et al., 2016; A. Zhang et al., 2017) focused on detecting the presence of cracks but did not specifically identify individual types. [221] classified the distress types horizontally and vertically, while [222] categorized distresses into three classes - vertical, horizontal, and alligator. Finally, other studies resulted in the blurry road markings detection [223], while others concentrated on cracks classification, including cracks which were sealed [224]. Quality of data used for training machine learning models is the essential key for their robustness. Previous studies introduced several benchmarked datasets (private and public) for training of machine learning models [182]. In spite of this, none of the aforementioned studies employed an inclusive dataset covering all distress types which are annotated cautiously by pavement experts. 
Until recently, very few studies have been performed to address the issue of finegrained classification of pavement distress. The accuracy of distress classification is dependent on factors such as camera view angle, segmentation accuracy, etc. The adoption of recent advances in machine learning has dramatically improved the robustness of distress classification methods. However, despite the great achievement of deep learning models, existence of shadows and poor lighting and low contrast have made it challenging for pavement researchers to develop an intact automated pavement distress detection model. Most of current models only work accurate when the cracks are discernible, generally with salient gray-level and uniform illumination features. Also, the camera angle view parameter is a challenging issue in crack segmentation and classification. Most of the trained crack classification models only work precisely if the same camera angle view images are used for prediction.

Furthermore, the former studies did not try to develop a model to classify and quantify distress density simultaneously. In section 4.2.2.3, the previous research was reviewed, which a dataset introduced with both top-down and wide-view images which are available in GitHub repository [255].

\subsubsection{Developing Crack Detection Model using Deep-Learning Framework (Majidifard et al., 2020)}

The current study is inspired by Majidifard et al' recent work which developed a comprehensive dataset for training deep learning algorithms for classifying different types of pavement distress. This section highlights their work and its relevance to the current study. The study first defined and annotated nine types of the most critical distresses which affect pavement condition selected after reviewing various studies [27], [35], [40], [99], 
[256][91][45], [257][258][258], [259]. Next, a large database of pavement images attained from 22 various pavement sections in the United States via a python-based software which communicate with API of Google street view. All the distresses on the acquired images were then annotated using the pre-defined distress classification rules as shown in Table 4-5. Figure 4-8 offers instances of images annotated with nine different distress types. As shown in the Table 4-5, reflective, lane longitudinal, sealed longitudinal, and block cracks are among the highest number of boundary boxes and images found in our dataset. Two different images were extracted at each coordinate location: Wide-View images with a pitch angle of $-70^{\circ}$ and birds-eye-view at $-90^{\circ}$. The wide view images were found to be beneficial for classification of distresses, whereas the top-down view image resulted in more precise distress severity quantification.

Table 4-5 Distress types versus their corresponding distress ID [31].

\begin{tabular}{|c|c|c|c|}
\hline Distress Type & Distress ID & Image Example & $\begin{array}{c}\text { Number of } \\
\text { boundary boxes for } \\
\text { each class }\end{array}$ \\
\hline Reflective Crack & D0 & 12428 \\
\hline Transvers Crack & D1 & & 5343 \\
\hline Block Crack & D2 & & 9709 \\
\hline
\end{tabular}




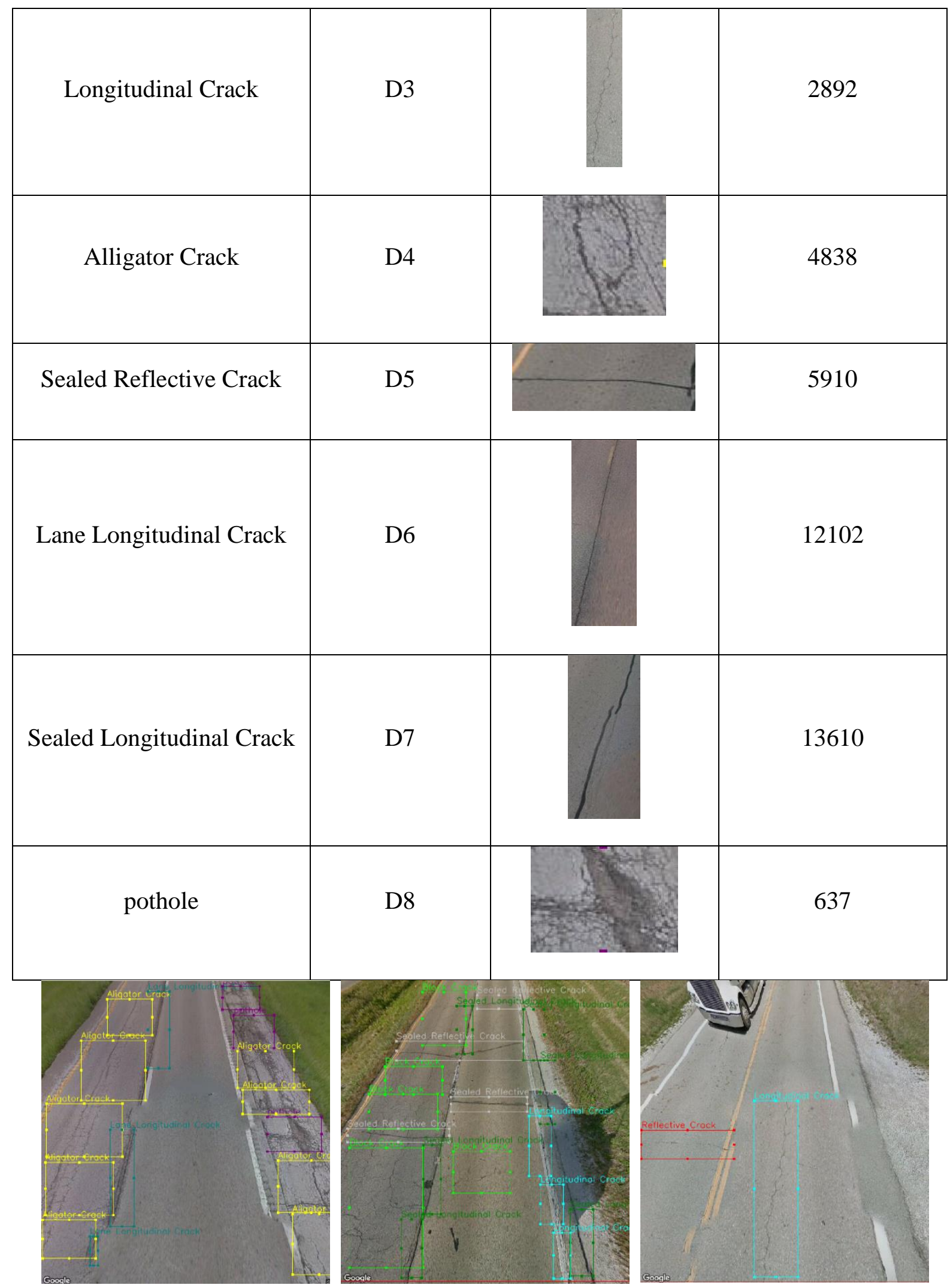



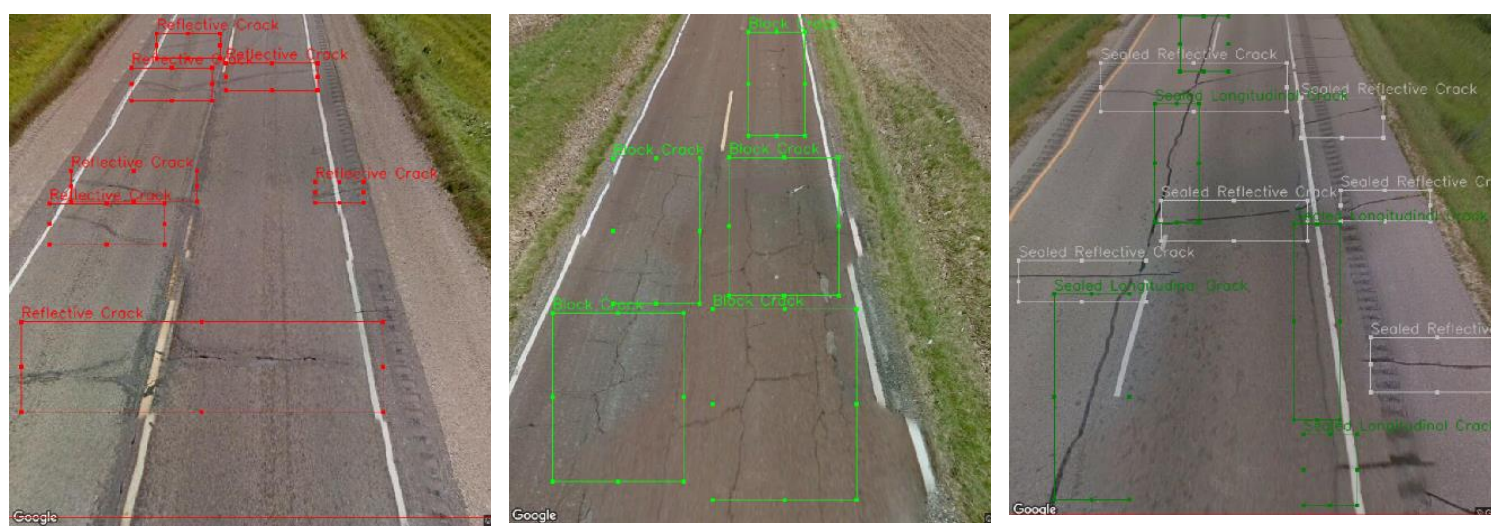

Figure 4-8. Annotated images (wide-view images) in the PID dataset [235].

An advanced deep learning framework named YOLO v2 was employed to detect and classify nine pavement distress types, automatically.

Figure 4-9 shows the architecture of the YOLO model that was applied. The reader is directed to the original paper for model development and testing details (Figure 4-9).

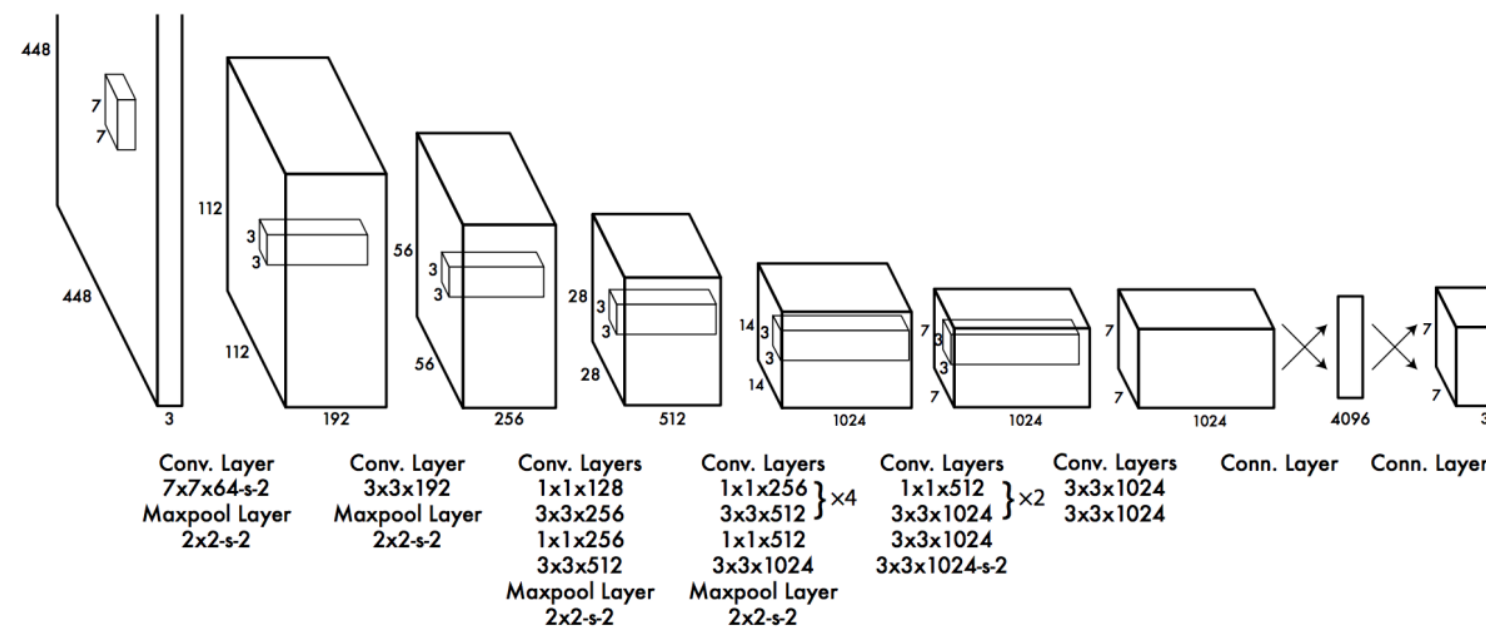

Figure 4-9. YOLO architecture [251]

Figure 4-10 shows some examples of prediction results on top-down images. To challenge the robustness of the model, full sunshine images and images containing shadows (trees and cars) were selected. Although the model was trained on wide-view images, the 
prediction results on top-down images, confirm that the developed model has the capability to detect distresses in both the shadow-containing and full sunshine images, accurately.
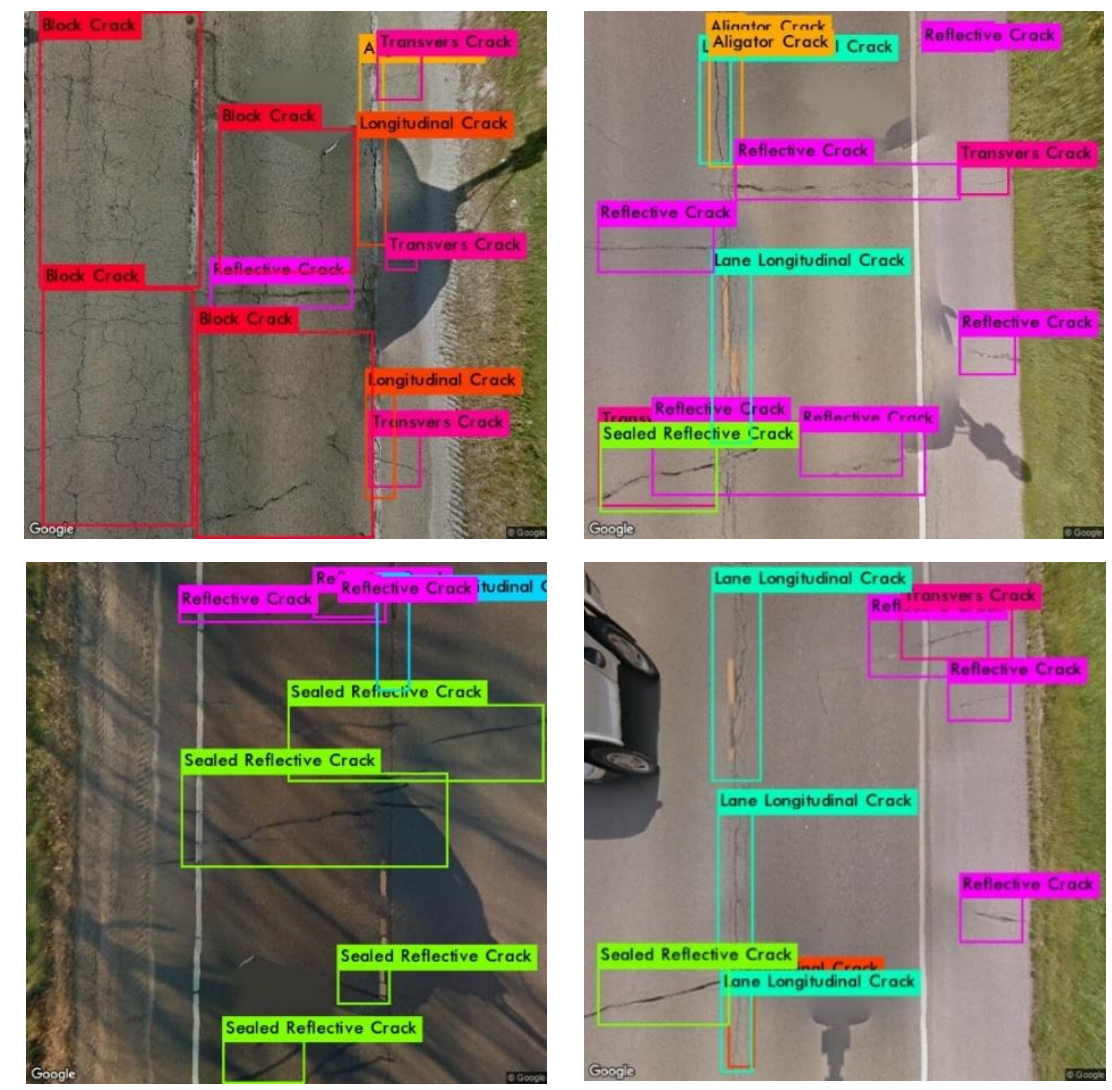

Figure 4-10. Samples of detecting pavement distresses from top-down images [235].

Precision, Recall, F1 scores and confusion matrices were the parameters utilized to assess accuracy of the model.Table 4-6 represents detection and classification accuracies of the proposed model for the nine classes in the dataset. The precision, recall and F1 values for the model were $0.93,0.77$ and 0.84 , respectively [235].

Table 4-6 Detection and classification results for nine distress types [31].

\begin{tabular}{|c|c|c|c|c|c|c|c|c|c|c|}
\hline $\begin{array}{c}\text { Crack } \\
\text { class name }\end{array}$ & D0 & D1 & D2 & D3 & D4 & D5 & D6 & D7 & D8 & Average \\
\hline Precision & 0.93 & 0.9 & 0.93 & 0.91 & 0.91 & 0.93 & 0.93 & 0.94 & 0.96 & $\mathbf{0 . 9 3}$ \\
\hline Recall & 0.76 & 0.83 & 0.79 & 0.83 & 0.74 & 0.83 & 0.79 & 0.57 & 0.78 & $\mathbf{0 . 7 7}$ \\
\hline
\end{tabular}




\begin{tabular}{|l|l|l|l|l|l|l|l|l|l|l|}
\hline F1 & 0.84 & 0.86 & 0.85 & 0.87 & 0.82 & 0.87 & 0.85 & 0.71 & 0.86 & $\mathbf{0 . 8 4}$ \\
\hline
\end{tabular}

In this section, we reviewed approaches to develop pavement condition models and crack detections methods. Also, we explained why we introduced a new pavement image dataset, which was used to develop a deep-learning-based distress detection tool. In the following sections we highlight the main contributions of this study: first, we introduce a robust pavement distress segmentation and densification approach based on deep convolutional neural networks. Second, we develop a pavement condition index based on inputs from distress class types and density information. To the authors' knowledge, none of the previous studies have been able to rate the condition of pavements by taking into account the type and extent of distresses surveyed. Detecting and quantifying of distresses are not enough to evaluate the pavement conditions. As mentioned in the literature review section, PASER is one the pavement condition index which helps to rate roads consistently. However, PASER index is a qualitative and human dependent index, we noticed that there are some mis-ranking and inconsistency between ranking of the sections. Figure 4-11 shows example of inconsistency in PASER rating among the investigated sections.

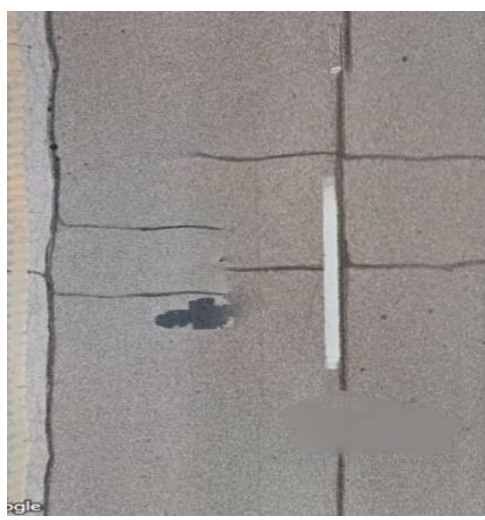

Mo94-PASER= 9

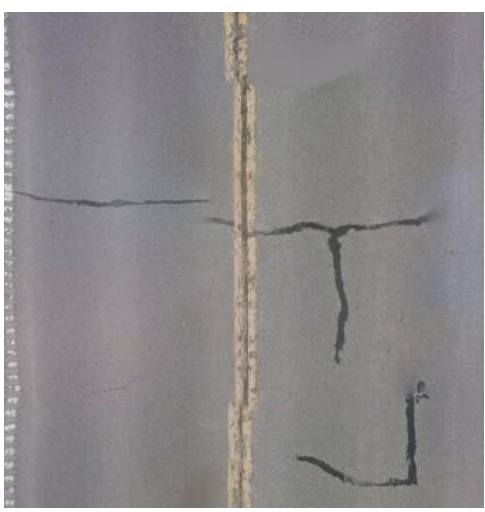

US24-PASER=7.2

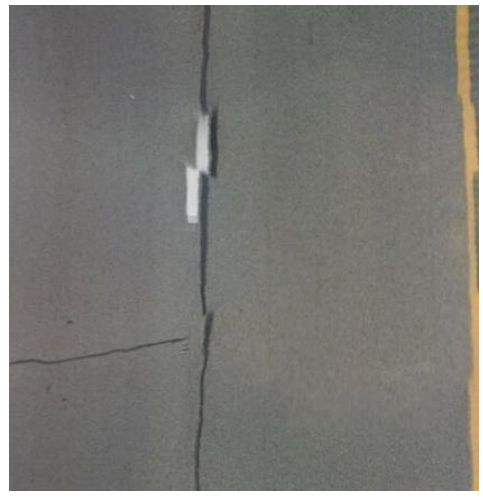

I44-PASER=8 


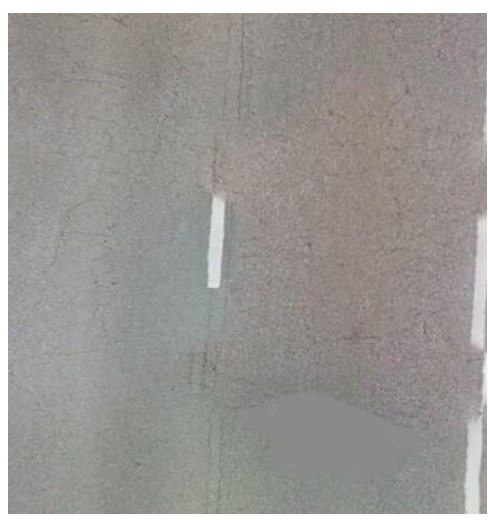

US54-PASER $=5.5$

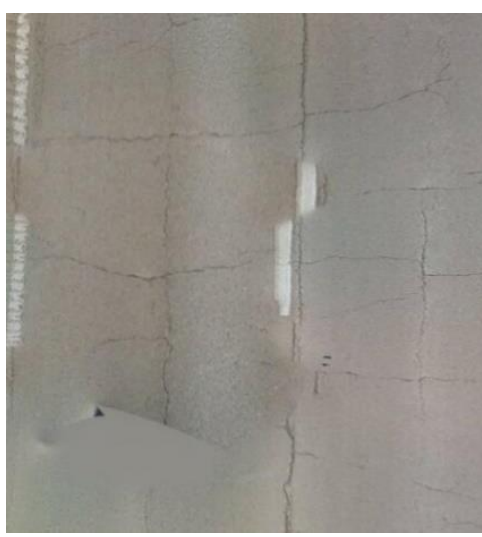

US24-PASER=7

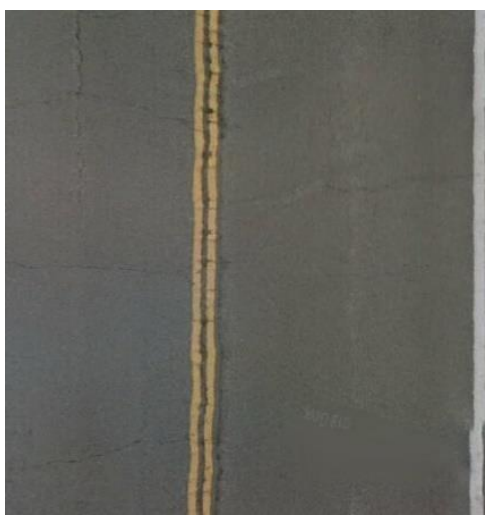

MO13-PASER $=7$

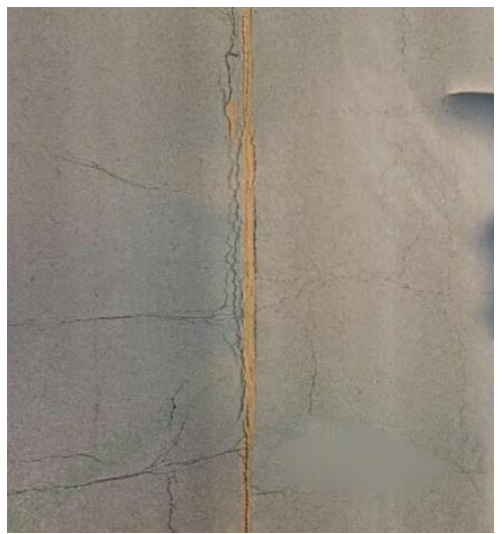

US136-PASER=5

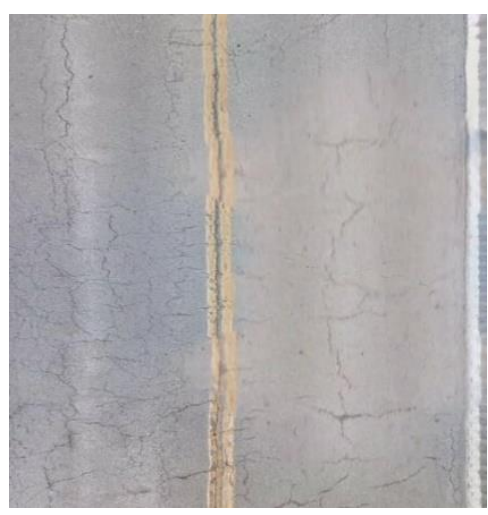

MO7-PASER $=6$

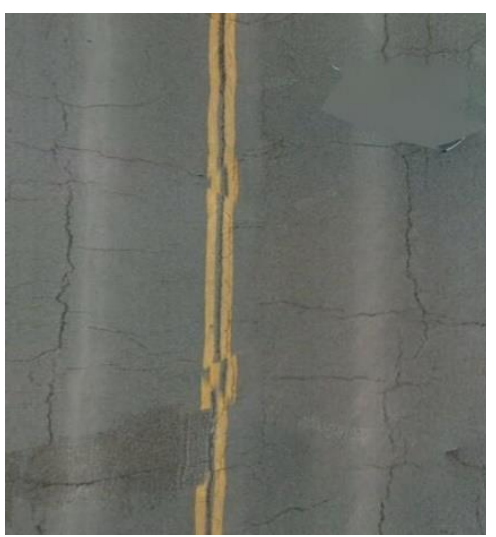

US24(1)-PASER=5

Figure 4-11. Example of inconsistency in PASER rating among the investigated sections

In order to develop a robust prediction tool, we need to have a reliable response. Then, the PASER values were revised by pavement experts and various approaches were implemented to fit a model with the eight features to the response value.

\subsubsection{Methodology of Developing Pavement Condition Prediction Models}

The methodology of developing the pavement condition prediction models consists of four main steps: First, 71 pavement sections were selected in the state of Missouri, and the PASER values were extracted from MoDOT virtual portal. The PASER values were checked and corrected in case of a discrepancy by pavement experts. Afterward, an average 
of 83 images per section was extracted from the Google map at corresponding GPS coordinates using the developed python software. Second, shoulders were removed from images by road segmentation technique, and the revised images were run through the developed YOLO model to classify the distresses. Third, a U-Net based model was developed for distress quantification. Forth the YOLO and U-net models merged together to create the hybrid model, and finally, three prediction PASER models were developed using YOLO and hybrid models by implementing various machine learning techniques.

Figure 4-12 shows the flowchart of the three prediction models.

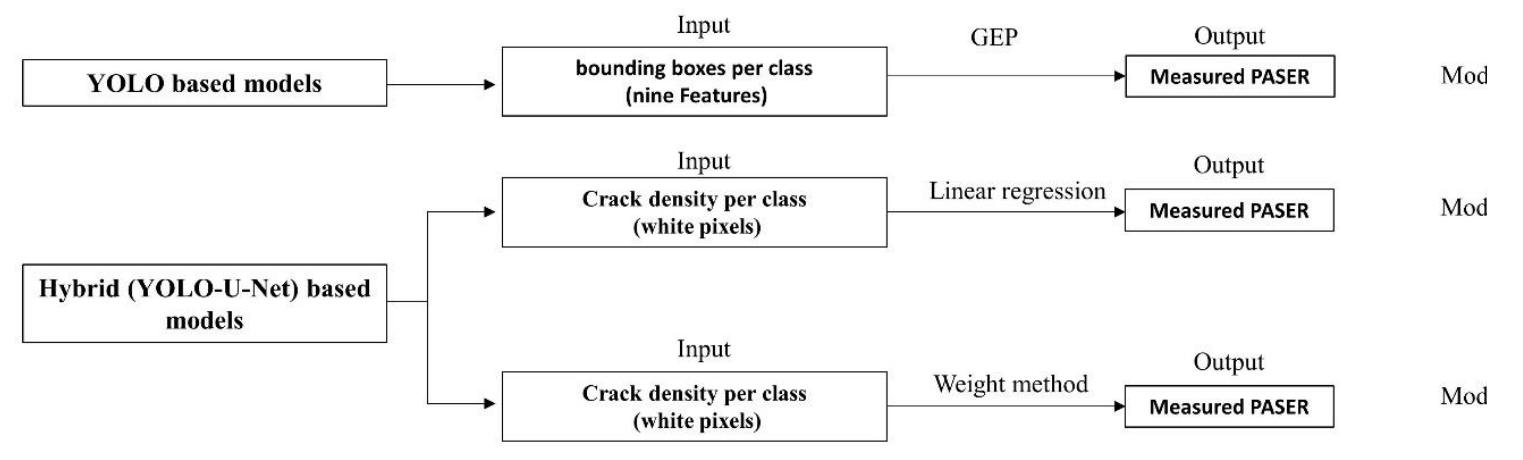

Figure 4-12. The primary PASER prediction models developed in this study.

\subsubsection{Main Road Segmentation}

The types of mixtures used for road shoulder construction usually vary significantly from those used for the main road. As a result, the types of distress and the underlying factors influencing them are also different. In the current study, distress information on road shoulders are not used in calculating the pavement condition index. We annotated and developed a shallow network for cropping out the main road for each pavement image. Distress detection and segmentation and subsequently carried out on the cropped image. Figure 4-13 shows examples of main road cropping. 

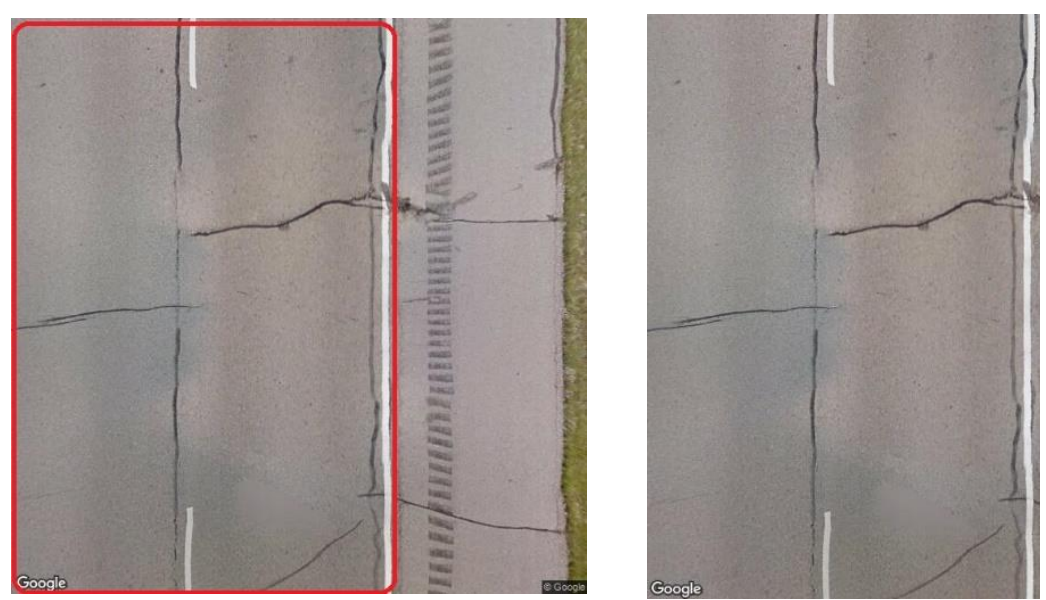

Figure 4-13. Automatic cropping of the main-road from shoulder via CNN

\subsubsection{Developing a U-Net based Model for Distress Quantification}

In the current study, a pre-trained U-Net convolutional network was used, which was originally developed for biomedical image segmentation [260]. Herein, it was used to quantify the density of cracks in roads.

Khanal and Estrada implemented a Neural baseline that does image segmentation to retinal vessel images. The dataset included 40 retinal images (20 for training and 20 for testing) where blood vessels were annotated at the pixel level to mark the presence (1) or absence (0) of a blood vessel at each pixel (i, j) of the image (Figure 4-15). The authors used the U-net architecture to do blood vessel segmentation (Figure 4-14). It is an architecture that is widely used for semantic segmentation tasks, especially in the medical domain. The U-net Architecture is an encoder-decoder with some skip connections between the encoder and the decoder. The ability to consider a wider context when making a prediction for a pixel is the primary benefit of this algorithm. This comes from the large number of channels utilized in the up-sampling process. Figure 4-14 shows the network architecture. The left and right side of the structure represents contracting and an expansive 
path, respectively. The contracting path reflects the typical architecture of a convolutional network. It contains the duplicated application of two $3 \times 3$ convolutions (unpadded convolutions), each accompanied by a rectified linear unit (ReLU) and a $2 \times 2$ max pooling operation with stride 2 for downsampling. At each downsampling step, the number of feature channels is doubled. The expansive path consists of an upsampling of the feature map followed by a $2 \times 2$ convolution that divides feature channels. This feature is connected with the correspondingly cropped feature map from the contracting path, and two $3 \times 3$ convolutions, each followed by a ReLU. At the final layer, a $1 \times 1$ convolution is utilized to map each $64 \times 64$ element feature vector to the desired class numbers. Overall, the network contains 23 convolutional layers [230].

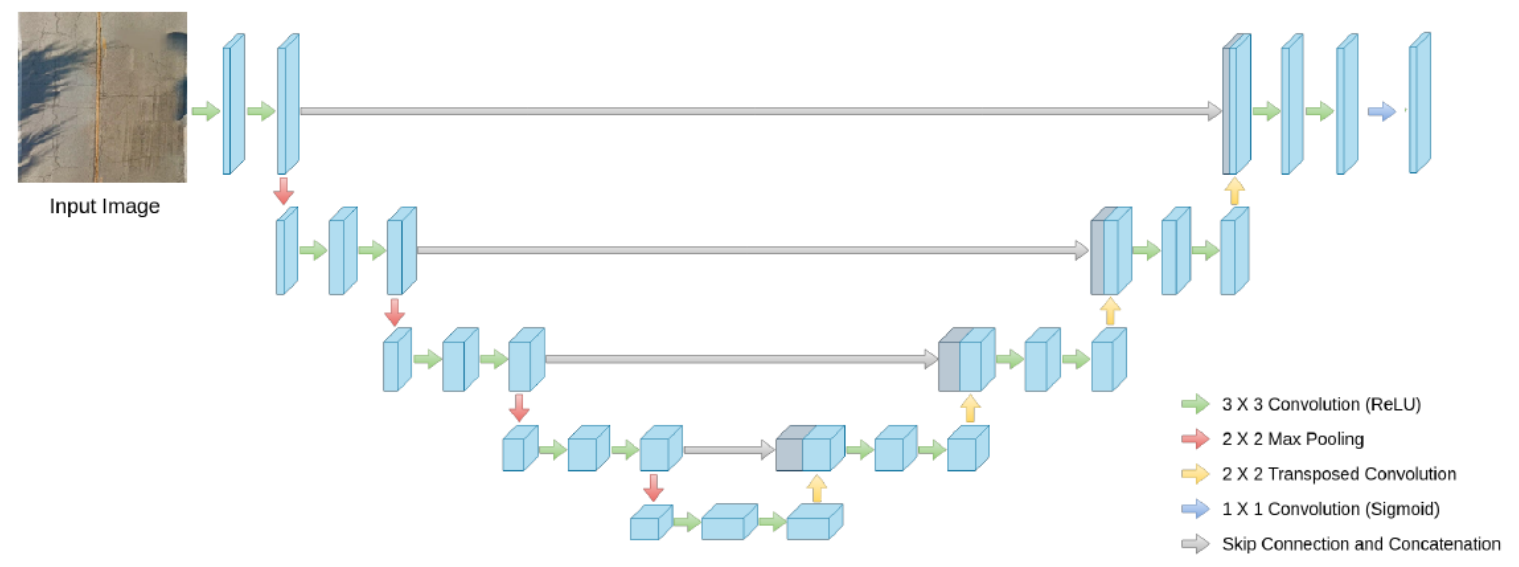

Figure 4-14. U-net Architecture [230].

After processing pavement images with the pretrained model we found it very accurate. However, the problem with this model is that it detects some external items like cars and shadows as distresses. In order to address the mentioned issue, shadows and cars were removed from the U_Net images with hand and the model retrained again. Figure 4-16 shows the images with shadows, cars and cracks. The images in the first, second and 
third columns represent original input, pretrained model and retrained model output, respectively. As seen in the Figure 4-16, cracks were detected accurately in the re-trained model although shadows covered them in some cases. Also, the shadows and cars were removed and the noise decreased significantly in the new model.

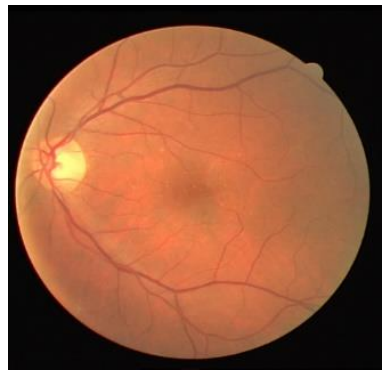

a)

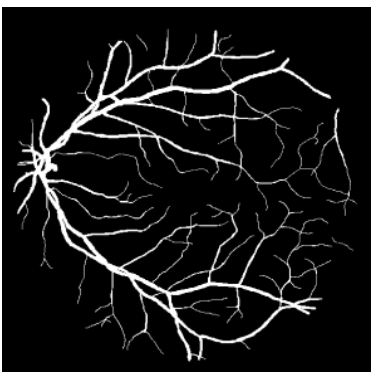

b)

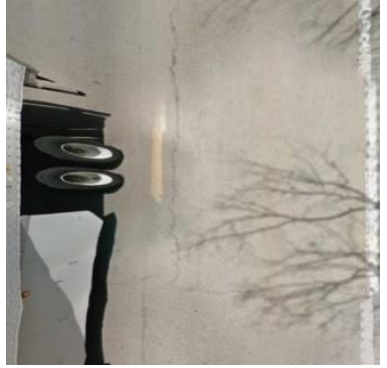

a)

Figure 4-15 a. One example of the training data to developed the U-net model, b. hand-crafted annotations that were used to retrain the model.
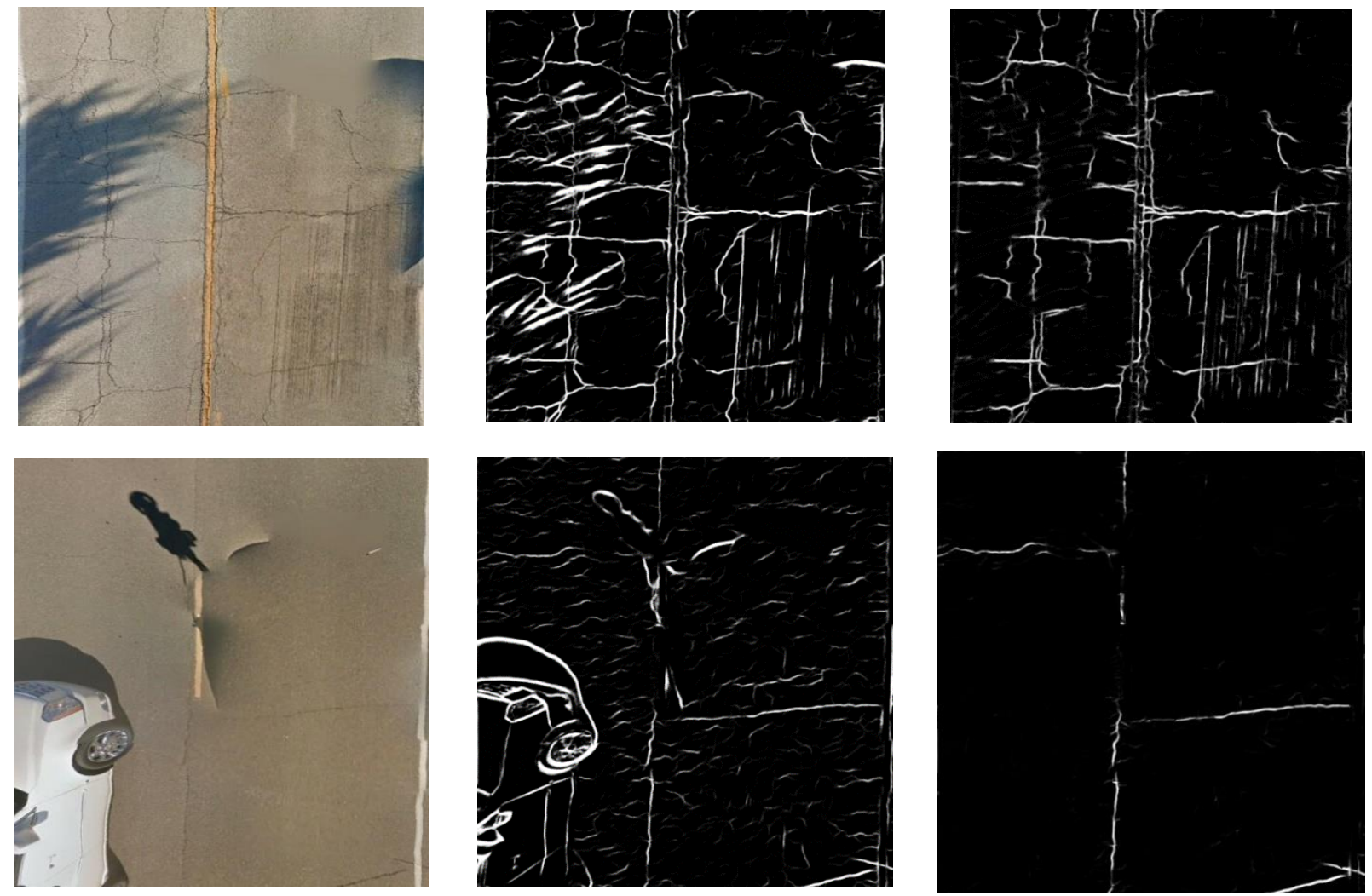

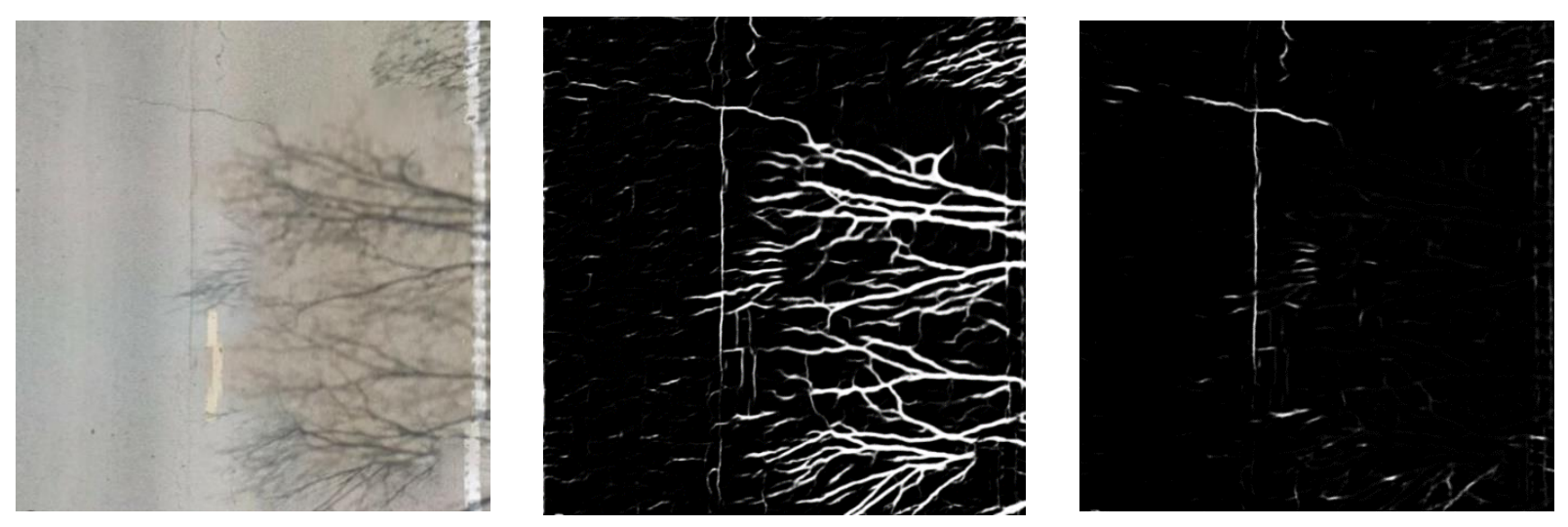

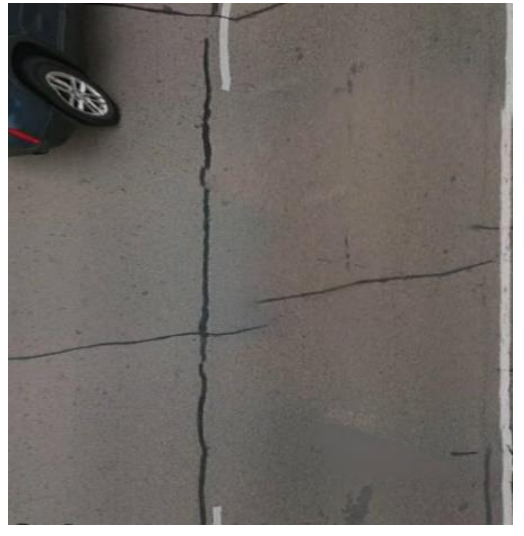

a)

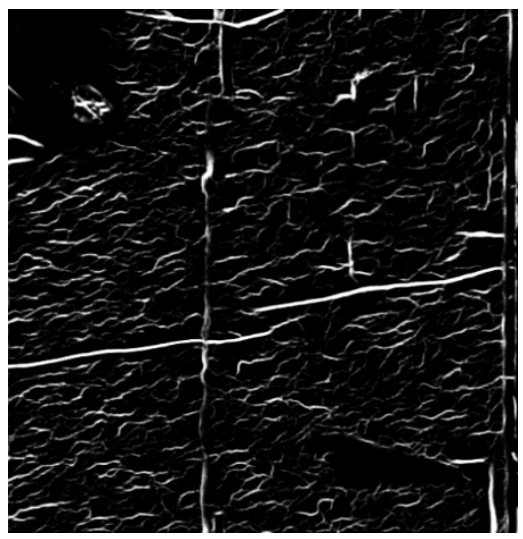

b)

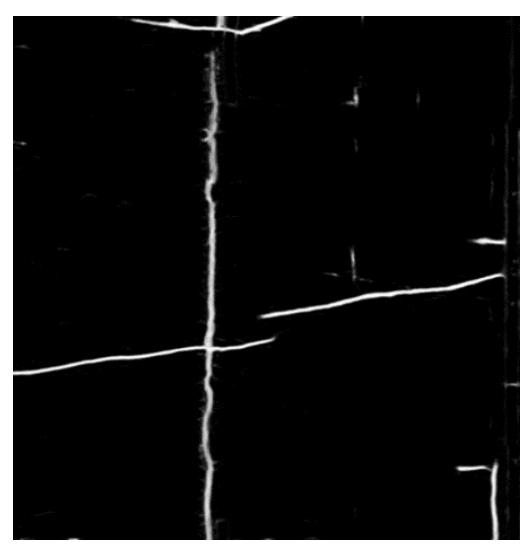

c)

Figure 4-16. Examples of detecting shadows and cars as cracks a) raw image b) pretrained model output, and c) retrained model output.

In order to check the precision of the model, 20 images were selected for testing the model performance. The pixel difference between the ground truth and the predicted image were calculate as MSE (mean square error). Figure 4-17 represents GT, predicted and error for one image in test dataset. The final MSE calculated as 0.25 for the 20 images in the test dataset. 


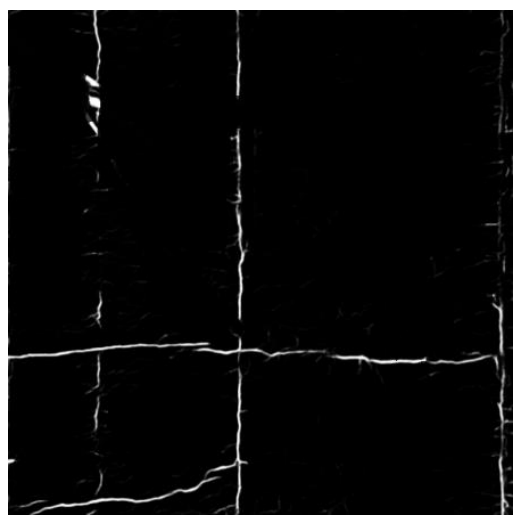

a)

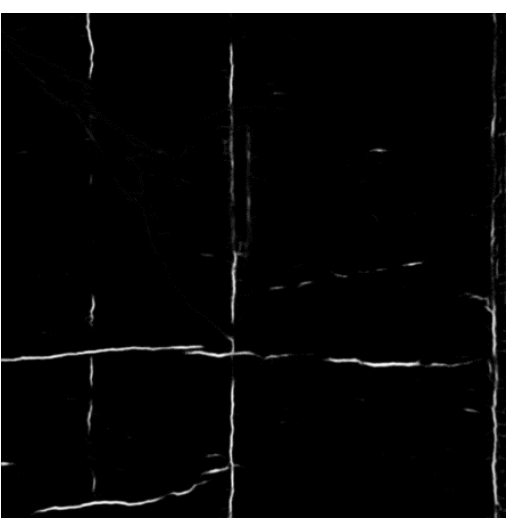

b)

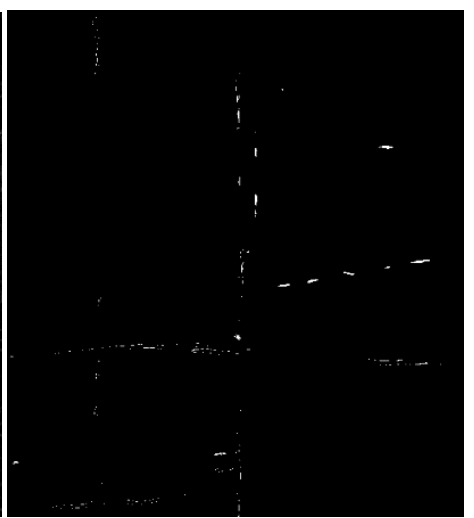

c)

Figure 4-17. Image of a) ground truth, b) predicted and c) error.

\subsubsection{Developing a Hybrid Model of YOLO and U-Net}

In order to develop a robust and comprehensive pavement condition model we needed to consider both type and density of the cracks. As mentioned before, none of the YOLO and U-net models could not provide the most accurate pavement condition model. Therefore, both of these models require to be integrated together. To address this concern, images were processed through the proposed YOLO and U-net models individually and the detected objects in the proposed yolo model image were masked on the corresponding images processed by the U-net model. Afterward the density of cracks were calculated for each type of distresses (Figure 4-18). The ratio of white pixels for each detected distress to the total image size are considered as the new features for the hybrid pavement condition model, which will be explained in detailed in section 3.4.3 and 3.4.4. 


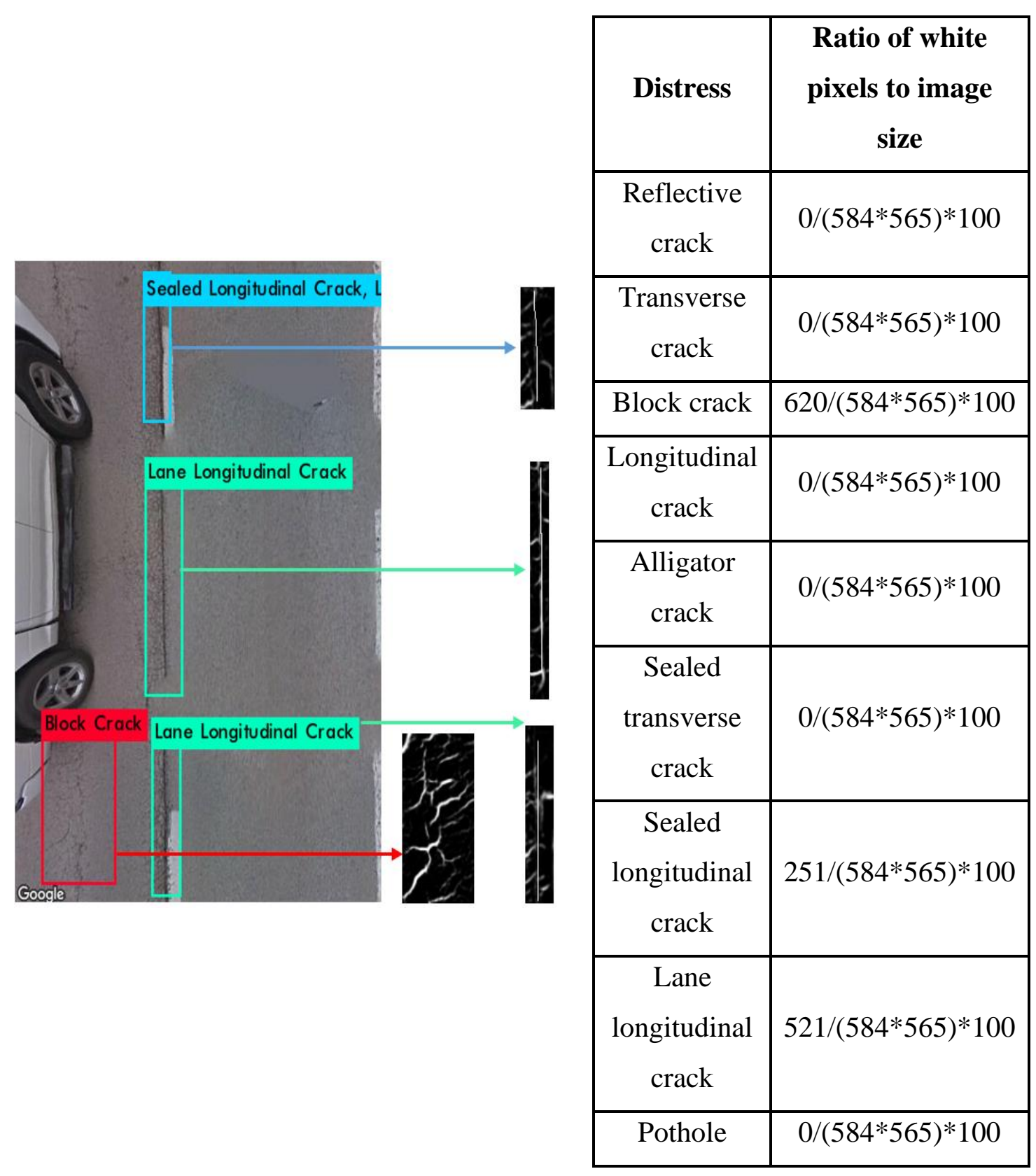

Figure 4-18. Integrated Yolo and U-Net based model and calculation of ratio of white pixels 


\subsubsection{Developing Pavement Condition Prediction Models}

\subsection{Pavement Condition Prediction Model Development based on YOLO Model outputs Using Genetic Expression Programming}

In order to develop pavement condition prediction model, all the extracted images for each section were run through the developed YOLO crack detection model. The average numbers of detected distresses were calculated for each section. Table 4-7 exhibits the detailed variables statistics used in this study. The data (71 pavement sections) are presented by frequency histograms (see Figure 4-19). As seen in the Figure 4-19, the distributions of the predictor variables are not uniform.

Table 4-7 Statistical parameters of the dependent and independent variables.

\begin{tabular}{|c|c|c|c|c|c|c|c|c|c|c|}
\hline & $\mathrm{D}(0)$ & $\mathrm{D}(1)$ & $\mathrm{D}(2)$ & $\mathrm{D}(3)$ & $\mathrm{D}(4)$ & $\mathrm{D}(5)$ & $\mathrm{D}(6)$ & $\mathrm{D}(7)$ & $\mathrm{D}(8)$ & PASER \\
\hline Mean & 0.58 & 0.26 & 0.82 & 0.21 & 0.11 & 0.20 & 0.41 & 0.33 & 0.00 & 7.2 \\
\hline Median & 0.43 & 0.13 & 0.33 & 0.18 & 0.04 & 0.01 & 0.35 & 0.09 & 0.00 & 7.0 \\
\hline Mode & 0.02 & 0.00 & 0.00 & 0.00 & 0.00 & 0.00 & 0.01 & 0.00 & 0.00 & 7.0 \\
\hline Range & 2.09 & 1.07 & 4.32 & 0.94 & 0.92 & 4.07 & 1.37 & 2.65 & 0.08 & 6.0 \\
\hline Maximum & 2.09 & 1.07 & 4.32 & 0.94 & 0.92 & 4.07 & 1.37 & 2.65 & 0.08 & 10.0 \\
\hline Minimum & 0.00 & 0.00 & 0.00 & 0.00 & 0.00 & 0.00 & 0.00 & 0.00 & 0.00 & 4.0 \\
\hline
\end{tabular}



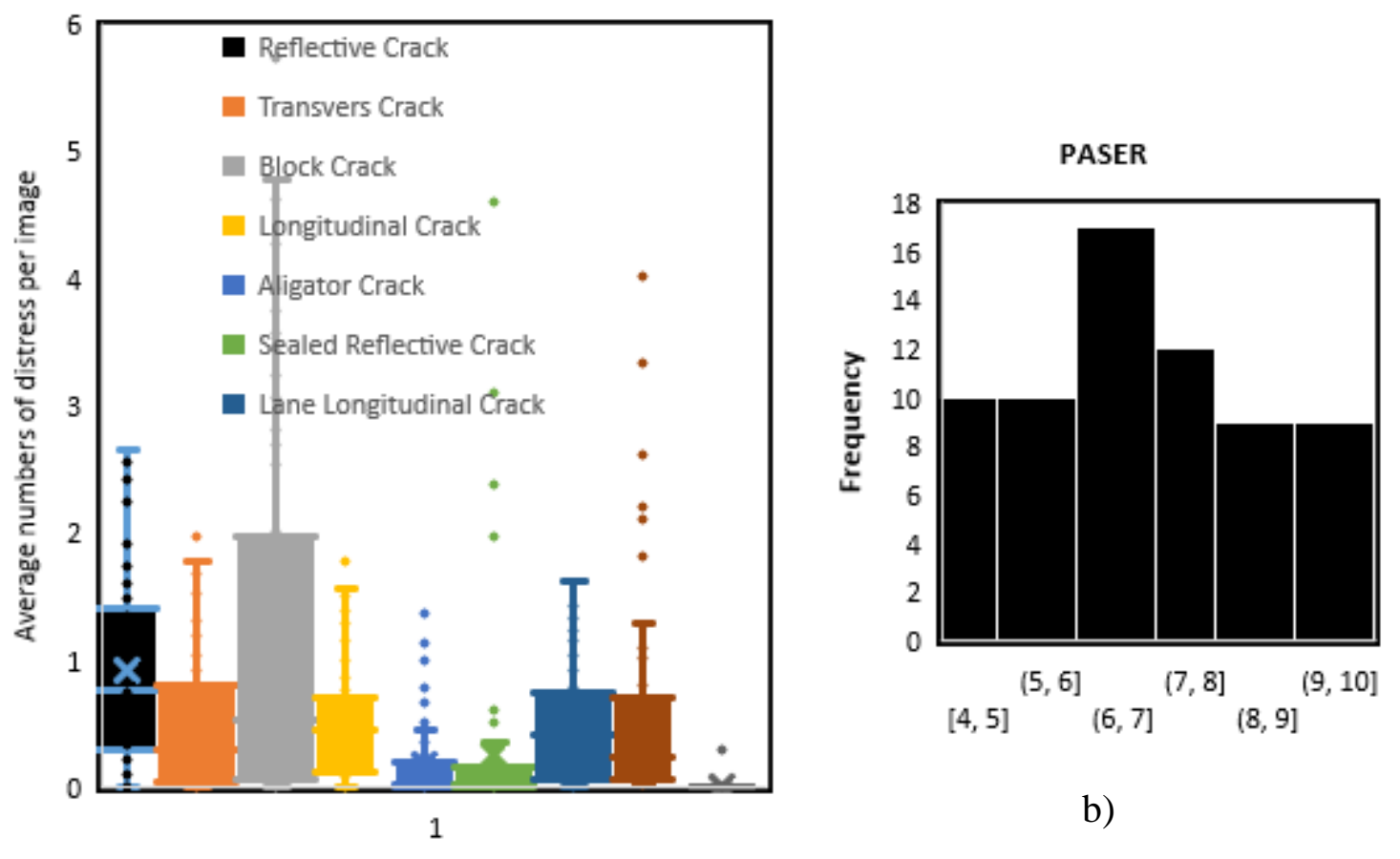

b)

a)

Figure 4-19. Distribution histograms of a) the features and b) output.

GEP is known as the most advanced generation of traditional genetic programming (GP) to generate nonlinear prediction models automatically. These techniques have been extensively deployed for tackling complicated engineering problems [117], [168], [196]. The traditional GP creates computational models by imitating the biological evolution of living organisms. It provides a tree-like form of a solution, which represents the closedform solution of the optimization problem [114], [115]. The primary objective of GP is to generate a population of programs that correlate inputs with outputs for each data point. The initial random functions are then calibrated to seize fitting functions that can accurately estimate the output via an administered trial and error methodology. 
The GP algorithm has some advantages over other machine learning (ML) techniques [105] such as adaptive neuro-fuzzy inference systems and artificial neural networks. The first benefit is that GP is not a black box and outputs are in the form of semi-complex mathematical solutions. The other advantage is that GP-based models lie on their inclination to obtain precise relationships without considering former patterns of the existing relationships. On the other hand, the primary advantage of GEP over the traditional GP method is that it can compile several sub-programs to create a single complex program. Moreover, the GEP algorithm can be implemented significantly faster than GP due to evolving the binary bit patterns. However, more fundamental details about the ML, GP and GEP methods can be found in [103], [114], [115]. In the current study, GEP is deployed for developing the rutting prediction models.

To avoid overfitting, the whole dataset (71 sections) was divided randomly into three categories as training $(70 \%)$, validation (15\%), and testing (15\%). The training and testing subsets were used to calibrate and evaluate the models, respectively, while validation dataset was used as an external output to check the model performance.

This study presents a new ML-based model which predicts the PASER from the following variables:

$$
\text { PASER }=f[d(1), d(2), d(3), d(4), d(5), d(6), d(7), d(8), d(9)]
$$

where,

$\mathrm{d}(1)=$ Reflective Crack, $\mathrm{d}(2)=$ Transverse Crack, $\mathrm{d}(3)=$ Block Crack, $\mathrm{d}(4)=$ Longitudinal Crack, $\mathrm{d}(5)=$ Alligator Crack, $\mathrm{d}(6)=$ Sealed Reflective Crack, $\mathrm{d}(7)=$ Lane Longitudinal Crack, $\mathrm{d}(8)=$ Sealed Longitudinal Crack, $\mathrm{d}(9)=$ Pothole. All the variable including $\mathrm{d}(1)$ to $d(9)$ are the average number of distresses per each section. 
The model was developed using PASER values obtained from 71 pavement sections in the Midwest United States. Various runs were performed to delineate the optimized GEP parameters. There are various principal setting GEP parameters such as general setting, complexity increase, genetic operators, numerical constant, and fitness function. In general part, the number of chromosomes changes the simulation run time. The higher number of chromosomes, the longer running time. The head size represents the complexity of terms in the developed model. Table 4-8 shows a set of parameters used during the GEP simulations.

Table 4-8 The optimal parameter setting for the GEP algorithm.

\begin{tabular}{|c|c|c|}
\hline Parameter & & Settings \\
\hline General & $\begin{array}{c}\text { Chromosomes } \\
\text { Genes } \\
\text { Head size } \\
\text { Linking function }\end{array}$ & $\begin{array}{c}30 \\
6 \\
12 \\
\text { Addition }\end{array}$ \\
\hline Functions & Function set & $\begin{array}{c}+,-, \times, /, \sqrt{ },{ }^{3} \sqrt{ }, \operatorname{Ln}, \text { Log, power, exp } \\
\text {,sin, cos, tan, }\end{array}$ \\
\hline $\begin{array}{l}\text { Complexity } \\
\text { increase }\end{array}$ & $\begin{array}{c}\text { Generations } \\
\text { without change } \\
\text { Number of tries } \\
\text { Max. complexity }\end{array}$ & $\begin{array}{c}2000 \\
3 \\
5\end{array}$ \\
\hline Genetic operators & $\begin{array}{l}\text { Mutation rate } \\
\text { Inversion rate }\end{array}$ & $\begin{array}{c}0.00138,0.044 \\
0.00546\end{array}$ \\
\hline $\begin{array}{l}\text { Numerical } \\
\text { constants }\end{array}$ & $\begin{array}{c}\text { Data type } \\
\text { Lower bound }\end{array}$ & $\begin{array}{c}\text { Floating-point } \\
-10\end{array}$ \\
\hline
\end{tabular}




\begin{tabular}{|l|l|c|}
\hline & Upper bound & +10 \\
\hline
\end{tabular}

The optimal GEP-based prediction model for PASER is as follows:

$$
\begin{aligned}
& Y 1=\arctan \left(\left(d(9)^{\wedge}(1 / 3)-((d(5)\right.\right. \\
& \left.\left.\left.\left.-d(3))^{\wedge} 2\right)\right)\right)\right) \text {; } \\
& Y 2=\sin (\sin (\tanh (((d(9)+(\min ((d(8)-d(1)), \min (G 2 C 4, d(6)))+(G 2 C 7 \\
& +(G 2 C 7)))-d(4))))) \text {; } \\
& Y 3=\left(\operatorname { e x p } \left(\operatorname { m i n } \left(d(6),\left(\left(\left((d(3)+G 3 C 8)^{\wedge}(1 / 3)+d(2)\right) / \exp (d(6))\right)-(G 3 C 6\right.\right.\right.\right. \\
& -(1.0-d(1))))))) \\
& Y 4=\min (d(9), \sin ((\min ((\exp (d(5)) * \min (d(5), d(1))),(G 4 C 2-d(2))) \\
& +d(6)))) \text {; } \\
& Y 5=\left(1.0 /\left(\left((((G 5 C 3+G 5 C 2)+(d(3)+d(1))))^{\wedge}(1 / 3) *((d(7)+G 5 C 9))^{\wedge}(1 / 3)\right.\right.\right. \\
& +\exp (\operatorname{reallog}(\max (d(6), G 5 C 4)))))) \text {; } \\
& Y 6=(1.0-(\min (d(2), G 6 C 6)-(((1.0 /((G 6 C 9-d(4)))) *((d(3)+d(1)) \\
& +d(3)))-d(9)))) \text {; } \\
& Y=Y 1+Y 2+Y 3+Y 4+Y 5+Y 6
\end{aligned}
$$

Where:

$\mathrm{G} 2 \mathrm{C} 7=-0.19, \mathrm{G} 2 \mathrm{C} 4=0.51, \mathrm{G} 3 \mathrm{C} 6=9.60, \mathrm{G} 3 \mathrm{C} 8=10.07, \mathrm{G} 4 \mathrm{C} 2=5.40, \mathrm{G} 5 \mathrm{C} 9=2.57$, $\mathrm{G} 5 \mathrm{C} 4=1.83, \mathrm{G} 5 \mathrm{C} 3=6.68, \mathrm{G} 5 \mathrm{C} 2=-7.18, \mathrm{G} 6 \mathrm{C} 6=-6.74, \mathrm{G} 6 \mathrm{C} 9=-3.36$

$\mathrm{d}(1)=$ Reflective Crack, $\mathrm{d}(2)=$ Transverse Crack, $\mathrm{d}(3)=$ Block Crack, $\mathrm{d}(4)=$ Longitudinal Crack, $\mathrm{d}(5)=$ Alligator Crack, $\mathrm{d}(6)=$ Sealed Reflective Crack, $\mathrm{d}(7)=$ Lane Longitudinal Crack, $d(8)=$ Sealed Longitudinal Crack, $d(9)=$ Pothole . 
Figure 4-20 represents measured versus predicted PASER values for the entire data. Although the dataset size was small, an acceptable performance for the proposed model was achieved (Figure 4-21).

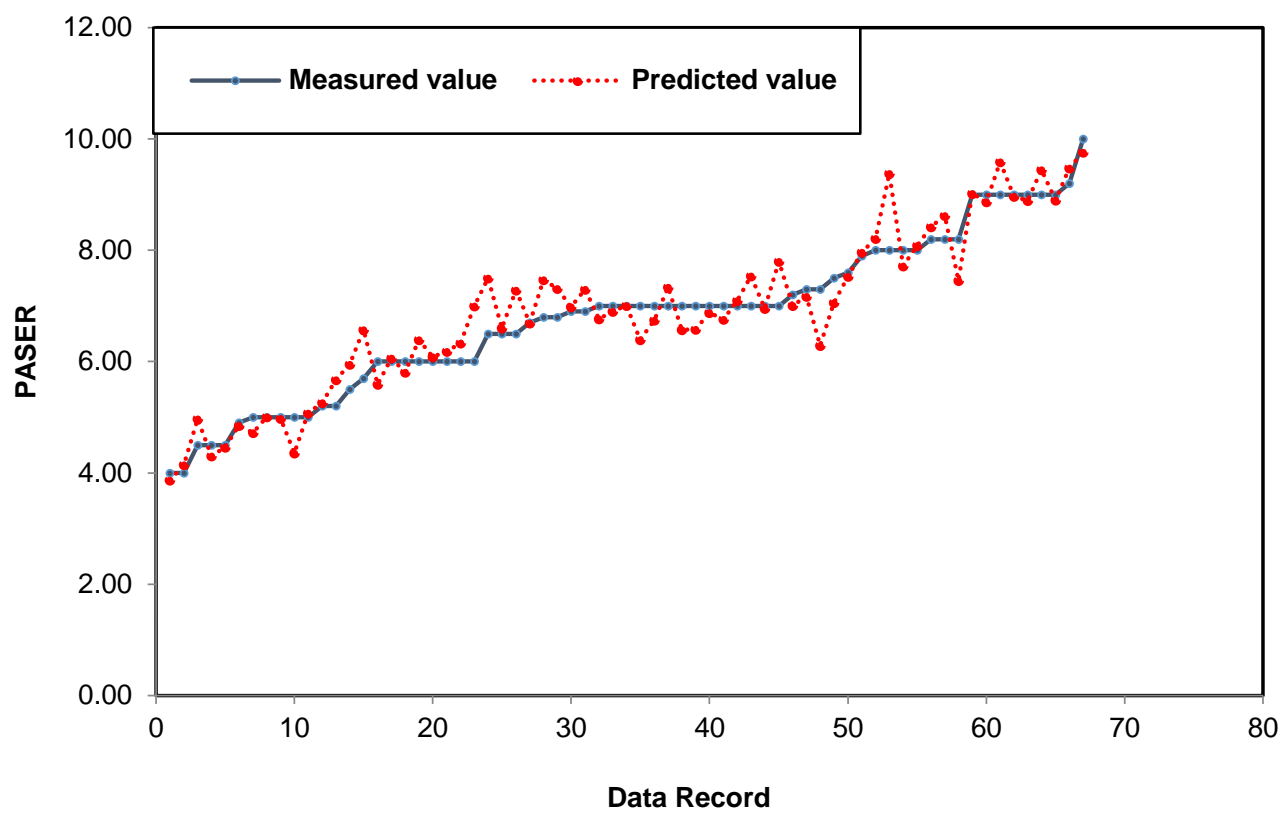

Figure 4-20. Measured versus predicted value for the entire data.

In order to measure the performance of model, coefficient of determination $\left(\mathrm{R}^{2}\right)$, root mean squared error (RMSE) and mean absolute error (MAE) were used.

$$
\begin{aligned}
& R^{2}=\frac{\left(\sum_{i=1}^{n}\left(O_{i}-\overline{O_{l}}\right)\left(t_{i}-\overline{t_{l}}\right)\right)^{2}}{\sum_{1=1}^{n}\left(O_{i}-\overline{O_{l}}\right)^{2} \sum_{i=1}^{n}\left(t_{i}-\overline{t_{l}}\right)^{2}} \\
& R M S E=\sqrt{\frac{\sum_{i=1}^{n}\left(O_{i}-t_{i}\right)^{2}}{n}} \\
& M A E=\frac{\sum_{i=1}^{n}\left|o_{i}-t_{i}\right|}{n}
\end{aligned}
$$


Where,

$O_{i}$ : Measured value

$t_{i}$ : Predicted value

$\overline{O_{i}}$ : Average of measured values

$\overline{t_{i}}$ : Average of predicted values

$n$ : Samples number

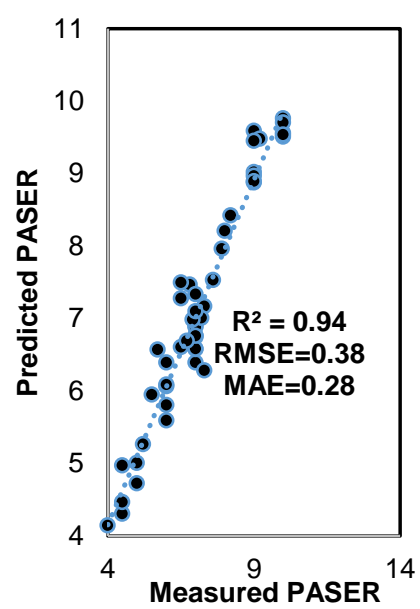

a)

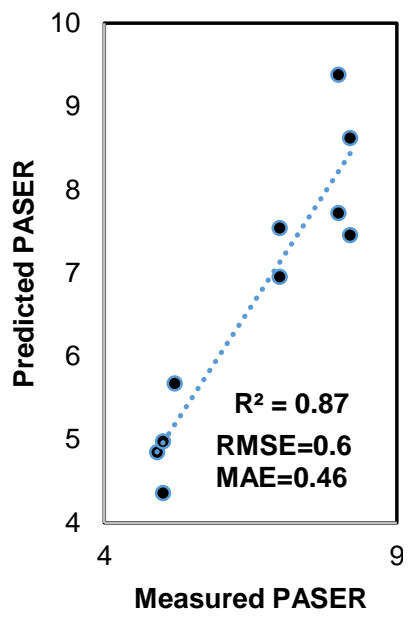

b)

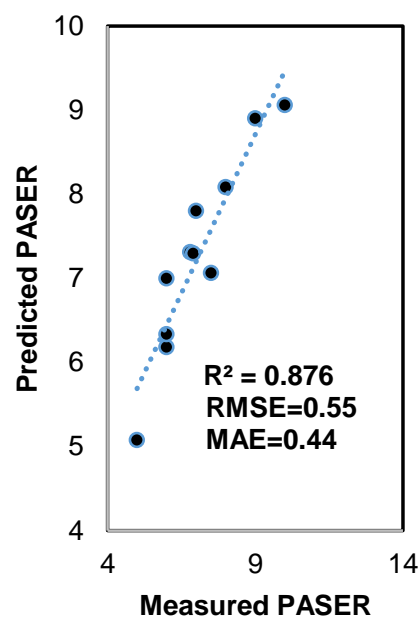

c)

Figure 4-21. Measured against predicted PASER using the GEP model: (a) training data; (b) testing data, and; (c) validation data.

A sensitivity analysis was carried out to explore the relative importance of variables in the GEP model. The results are presented in Figure 4-22. The major observations were:

- Block cracks had the highest influence on the pavement condition (PASER).

- Lane longitudinal, sealed longitudinal cracks and potholes had the lowest influence on PASER. The small effect of potholes on pavement condition may seem nonsense at first glance. However, none of the sections in the dataset have potholes on their surfaces. Therefore, the actual effect of potholes on pavement condition ignored in the prediction model. The same explanation is valid for the alligator and transverse 
cracking.

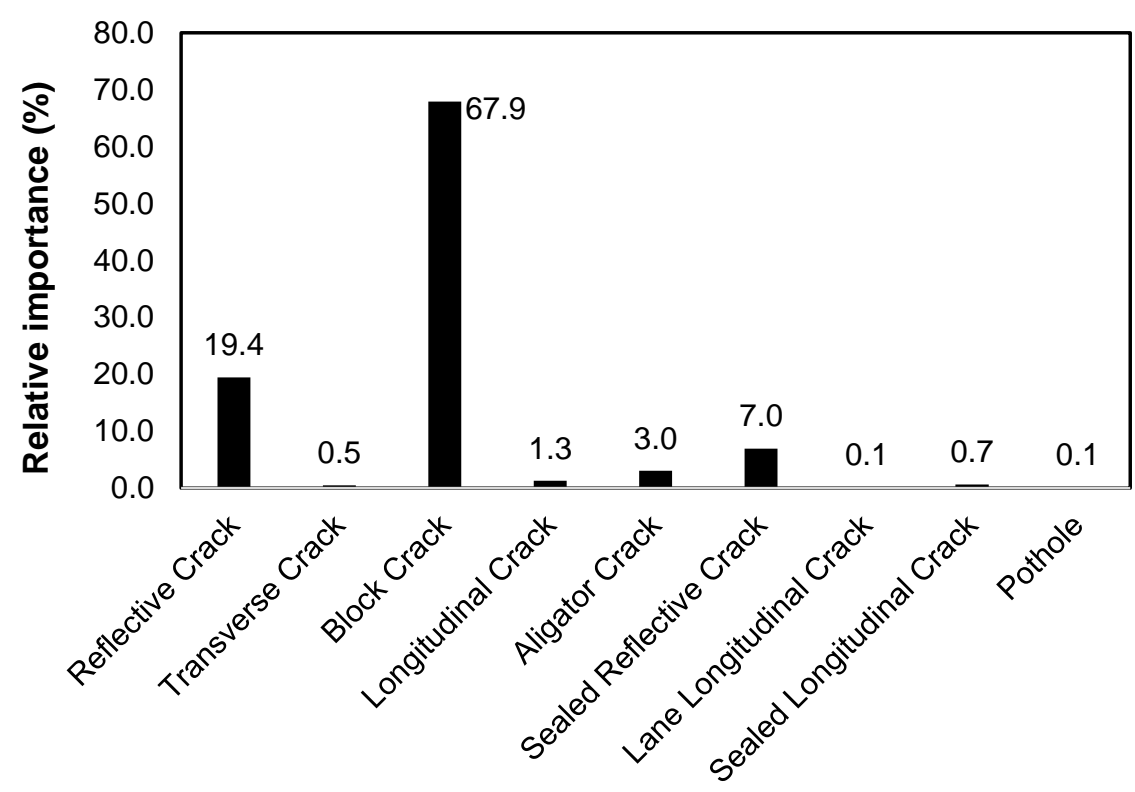

Figure 4-22. Variable importance in the GEP model.

\subsection{Pavement Condition Prediction Model Development based on Unet Model outputs}

In this part of study, a new pavement condition index was developed based solely on the U-net model output. All the 71 sections images were processed through the proposed U-net model. For each processed image, the ratio of white pixels (crack-like features) to the total size of the image was used to measure the crack density. To reduce the effect of spurious, non-crack-like features, the output of the U-Net mode was thresholded. Two thresholds were tested: the first threshold only selects pixels with intensity values ranging from 127 to 255 , and the second one is from 200 to 255 counts as white pixels. The ratio of the white pixels were calculated for all sections and it is reported as the distress index to rank the sections. 
Figure 4-23 shows the correlation of the distress index from U-net model with corresponding PASER values. As shown in the figure, PASER has stronger correlation with the crack density threshold of 127 than the one with 200 threshold.

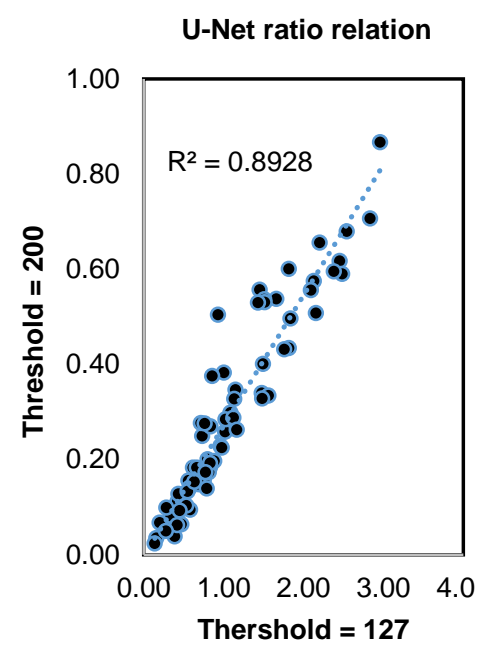

a)

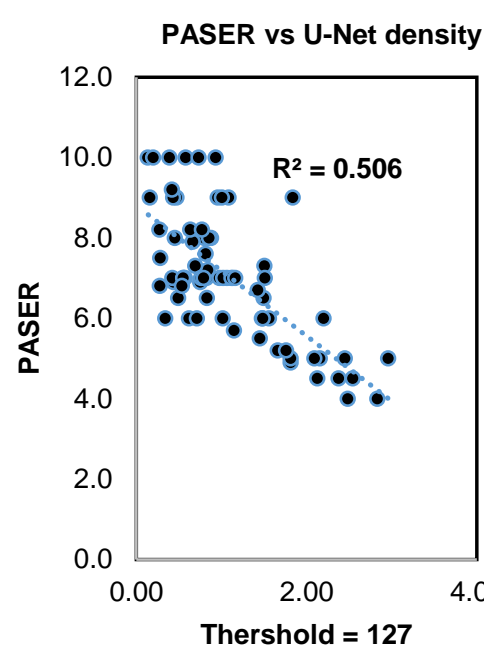

b)

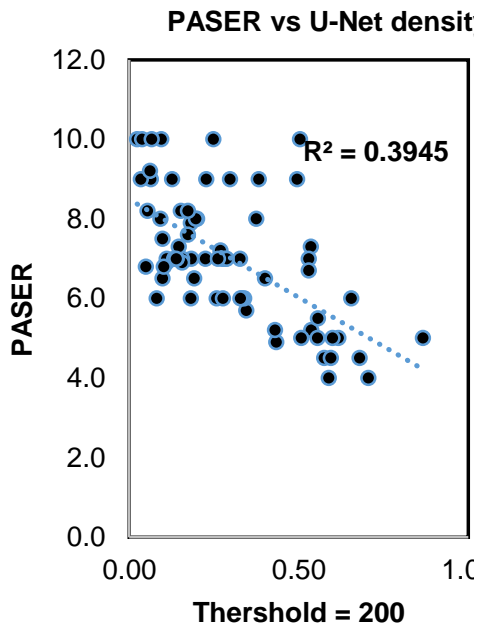

c)

Figure 4-23. Correlation of a) distress density with different threshold, b) PASER versus distress density with threshold $=127$, c) PASER versus distress density with threshold $=127$

This model can rank sections based on the density of distresses. However, it cannot differentiate types of distresses and this may lead to a misleading conclusion. For example, this model cannot discriminate sealed cracks from unsealed cracks; hence it could penalize the condition of a section unfairly. Also sealed cracks are usually thicker than the unsealed ones, the ration of white pixels will therefore be higher than images from unsealed crack sections. Hence, the main problem with U-net model ranking is that this model just rely on detecting the density of distresses not types of them. It has been well known that different distresses affect pavement condition differently (Figure 4-24). 

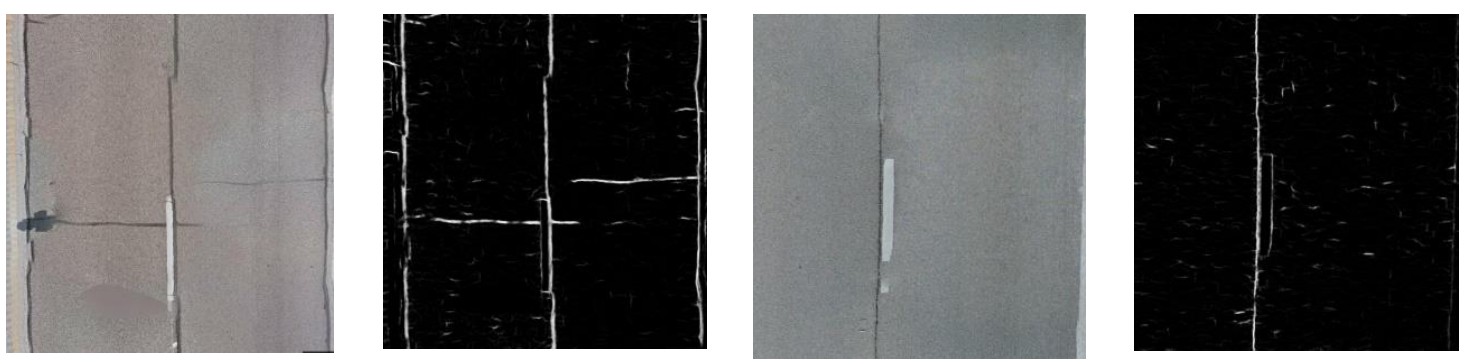

Figure 4-24. Example of problems associated with crack density U-Net based model

According to the limitation of the YOLO and U-net models, we developed a hybrid model including both of these models. Therefore, the type and density of distresses will be considered to develop a new pavement condition index.

\subsection{Linear regression PASER prediction model based on hybrid model}

In this part of study, linear regression method was used to develop a prediction model using the explained variables. In order to make the equation similar to PCI approach, the deducted values introduced as the response instead of the measured PASER values. Table 4-9 shows the estimated coefficient for the predicted model. Also, P-value were calculated for each variable and the variable number three which is block cracking has the lowest pvalue and highest importance in the model. Considering confidence level of $95 \%$, the variable 1, 3, 4, and 8 are significant. Figure 4-25 shows the fitted value versus the measured ones for training, testing and validation dataset.

$$
\text { PASER Predicted }=0.1 *\left(100-\sum_{i=1}^{n}\left(B_{i} d_{i}\right)\right)
$$

where, $d(i)=$ ratio of white pixels to total image size

$\mathrm{d}(1)=$ Reflective Crack, $\mathrm{d}(2)=$ Transverse Crack, $\mathrm{d}(3)=$ Block Crack, $\mathrm{d}(4)=$

Longitudinal Crack, $\mathrm{d}(5)=$ Alligator Crack, $\mathrm{d}(6)=$ Sealed Reflective Crack, $\mathrm{d}(7)=$

Lane Longitudinal Crack, $\mathrm{d}(8)=$ Sealed Longitudinal Crack. 
Table 4-9 Estimated coefficient for the fitted prediction model using linear regression from $R$ software

\begin{tabular}{|c|c|c|c|c|c|}
\hline Variable & Coefficients & Std.Error & $\mathbf{t}$-value & $\operatorname{Pr}(>|\mathbf{t}|)$ & Importance \\
\hline $\mathrm{d}(1)$ & 100.433 & 13.745 & 7.307 & $2.49 \mathrm{E}-09$ & $* * *$ \\
\hline $\mathrm{d}(2)$ & -40.603 & 34.906 & -1.163 & 0.2505 & \\
\hline $\mathrm{d}(3)$ & 47.709 & 3.312 & 14.405 & $2.00 \mathrm{E}-16$ & $* * *$ \\
\hline $\mathrm{d}(4)$ & 70.774 & 16.16 & 4.38 & $6.42 \mathrm{E}-05$ & $* * *$ \\
\hline $\mathrm{d}(5)$ & 40.781 & 27.861 & 1.464 & 0.1498 & \\
\hline $\mathrm{d}(6)$ & 14.959 & 11.834 & 1.264 & 0.2123 & \\
\hline $\mathrm{d}(7)$ & 20.856 & 16.236 & 1.285 & 0.2051 & \\
\hline $\mathrm{d}(8)$ & 7.887 & 3.602 & 2.189 & 0.0335 & $*$ \\
\hline
\end{tabular}

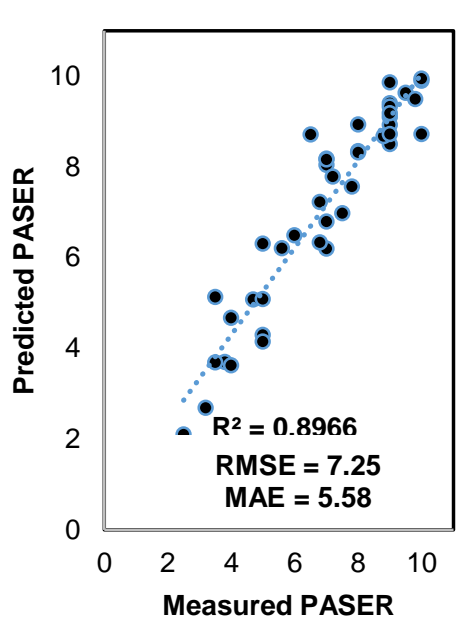

a)

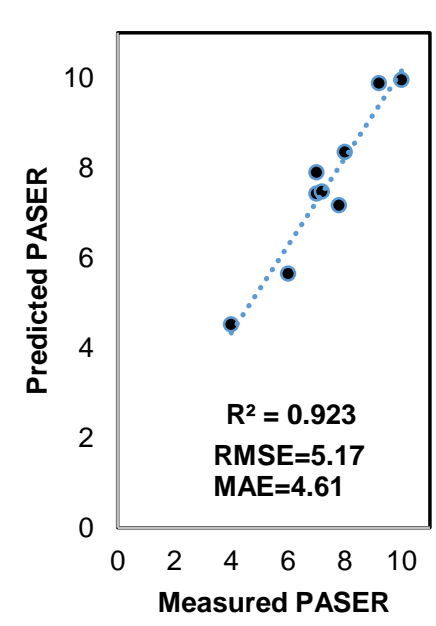

b)

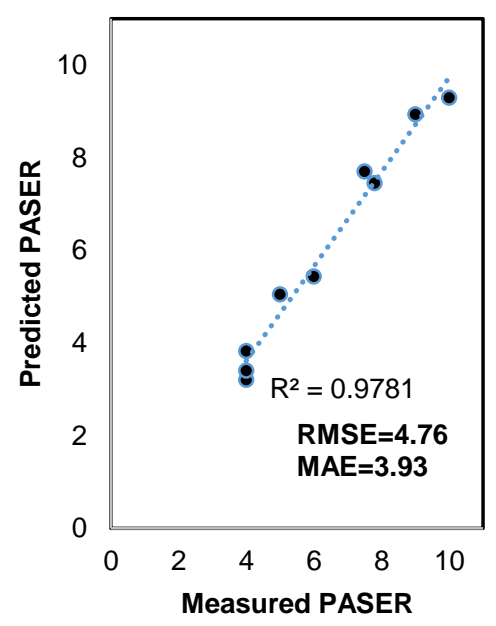

c)

Figure 4-25. Measured PASER versus predicted PASER using linear regression: (a) training data; (b) testing data, and; (c) validation data.

\subsection{Weight based PASER prediction model based on hybrid model}

The PCI decision matrix affords particular guidelines for the repairs required based on road classifications. Using PCI, helps to establish a pinpoint for preventive preservation that diminishes road deteriorating before the point that it needs expensive rehabilitation [33]. 
In this model, different weights were assigned to the distresses, and deduct values were calculated similar to the PCI method (Table 4-10 and equation 4-10). Different weights were considered according to the level of importance of distresses in pavement condition. Afterward, the deduct values were subtracted from 100.

Table 4-10 Distress weights to calculate the predicted PASER using weight method

\begin{tabular}{|c|c|c|}
\hline Variable & Ratio of white pixels to total image size & Weight \\
\hline V1 & Reflective & 0.4 \\
\hline V2 & Transverse & 0.4 \\
\hline V3 & Block & 0.4 \\
\hline V4 & Longitudinal & 0.4 \\
\hline V5 & Alligator & 0.4 \\
\hline V6 & Sealed Reflective & 0.1 \\
\hline V7 & Lane Longitudinal & 0.1 \\
\hline V8 & Sealed Longitudinal & 0.1 \\
\hline
\end{tabular}

$$
\text { PASER }=0.1 \times\left(100-\sum_{i=1}^{n}\left(w_{i} d_{i}\right)\right)
$$

where,

$\mathrm{d}(1)=$ Reflective Crack, $\mathrm{d}(2)=$ Transverse Crack, $\mathrm{d}(3)=$ Block Crack, $\mathrm{d}(4)=$

Longitudinal Crack, $\mathrm{d}(5)=$ Alligator Crack, $\mathrm{d}(6)=$ Sealed Reflective Crack, $\mathrm{d}(7)=$

Lane Longitudinal Crack, $\mathrm{d}(8)=$ Sealed Longitudinal Crack, $\mathrm{d}(9)=$ Pothole

Figure 4-26 shows the measured PASER values versus the predicted PASER using the PCI weighting method. The coefficient of determination $\left(\mathrm{R}^{2}\right)$ for the training, testing and validation dataset are 0.87 .0 .94 , and 0.94 . One of the most interesting benefit about the weight-based method over other approaches is that the weights can be adjusted regarding to the case project. For example, for airport pavement monitoring, where 
reflective cracks are more important than longitudinal cracks. Then desired weights can be assigned to the distresses and a new PASER index will be obtained based on new weights.

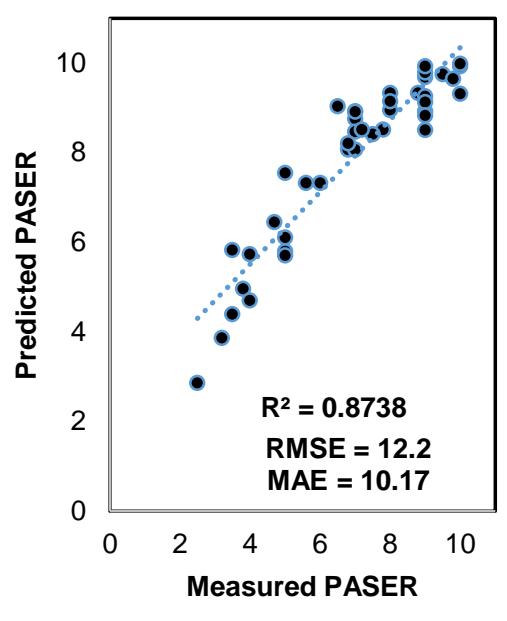

a)

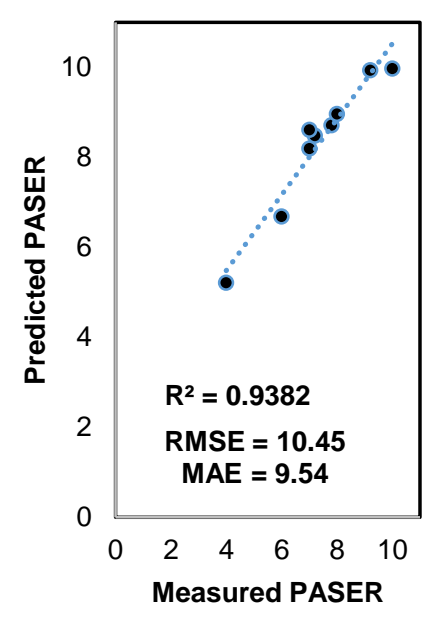

b)

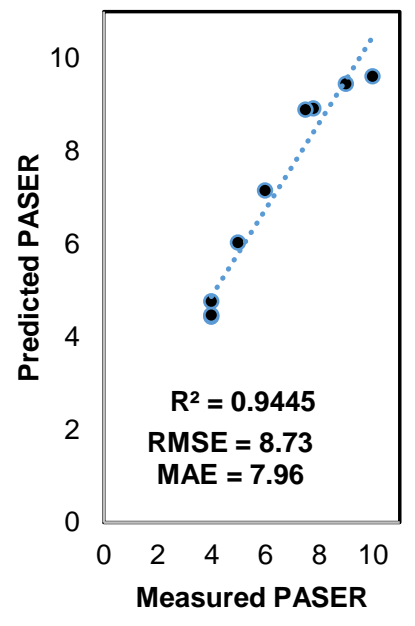

c)

Figure 4-26. Measured PASER versus predicted PASER using Weight Method: (a) training, b) testing and (c) Validation dataset.

\subsubsection{Model Validation}

To further evaluate the generalization capability of the PASER prediction models, the developed models were deployed to predict the condition of six pavement sections with different conditions (Figure 4-27). First, a new set of pavement images was extracted for each section from Google maps using my developed software. Second, the images were analyzed with the developed crack detection and segmentation models (YOLO and U-Net) and the ratio of white pixels for each detected distresses to the total image size were calculated. Finally, the PASER values were predicted using weight based and linear regression models for all the extracted images. The images were extracted by $15 \mathrm{~m}$ and we considered 5 meter as the view of each image. The moving average of PASER values were calculated by a period of 20 images and plotted in Figure 4-28a and b. Figure 4-28c shows 
the predicted PASER which developed based on distress classification alone (YOLO model output). As seen in this figure, the fluctuation of the PASER values from YOLO model is less than the ones from hybrid models. This is also expected because the hybrid models are more susceptible and precise due to its dependency on pixels counting. Also Figure 4-28d shows the corresponding measured PASER values extracted from MoDOT's portal. As seen, the predicted PASER values correlate well with the measured values. The rankings are in order according to the measured PASER except swapping MO11 and US24. Contrary to the developed pavement condition prediction models, PASER rating is very straightforward rating without considering any distress quantification in details. The approximate-based nature and human dependency of the PASER procedure, makes it challenging to correlate the machine learning-based pavement condition model with the measured PASER values in some cases. Also, the PASER values from prediction models are fluctuating more than the corresponding measured PASER values due to variety of detected distresses image by image. While measured PASER values are usually constant along 2000 meter which is unrealistic (Figure 4-28).

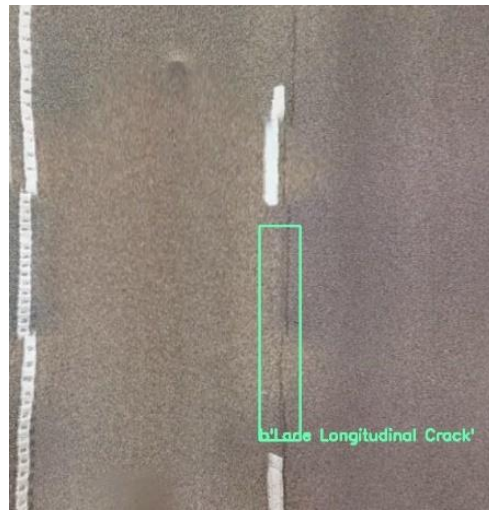

I70

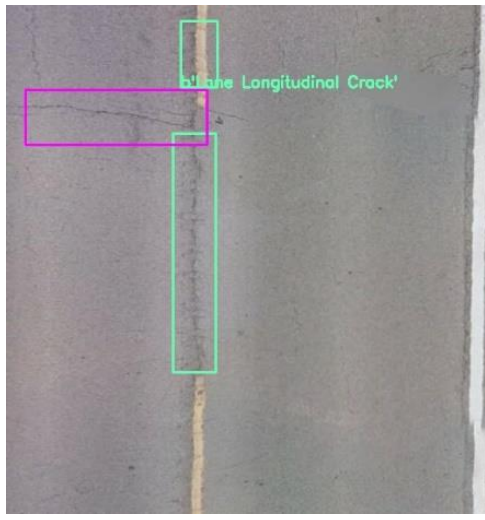

US50_Sedelia

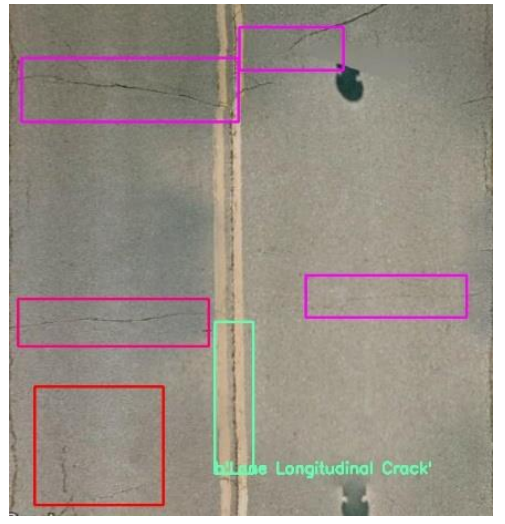

MO11 


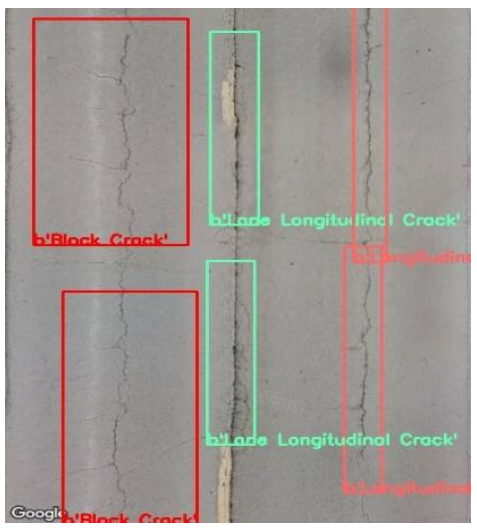

US24_lexington

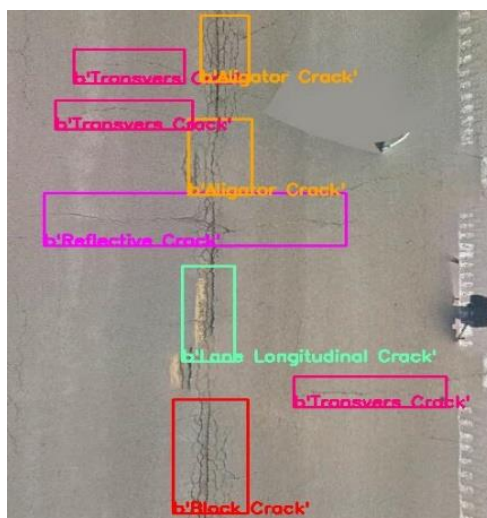

US71

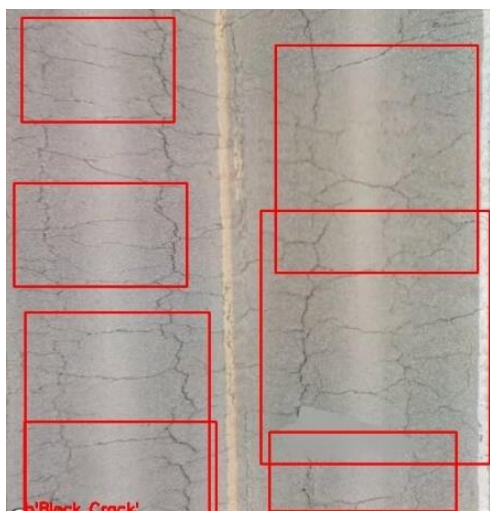

MO52_Stover

Figure 4-27. Pavement condition, a) I70 (good condition), b) US50, c) MO11, d) US24, e)US71 and f) MO52 (dense-block cracks).

Hybrid model-weight based PASER

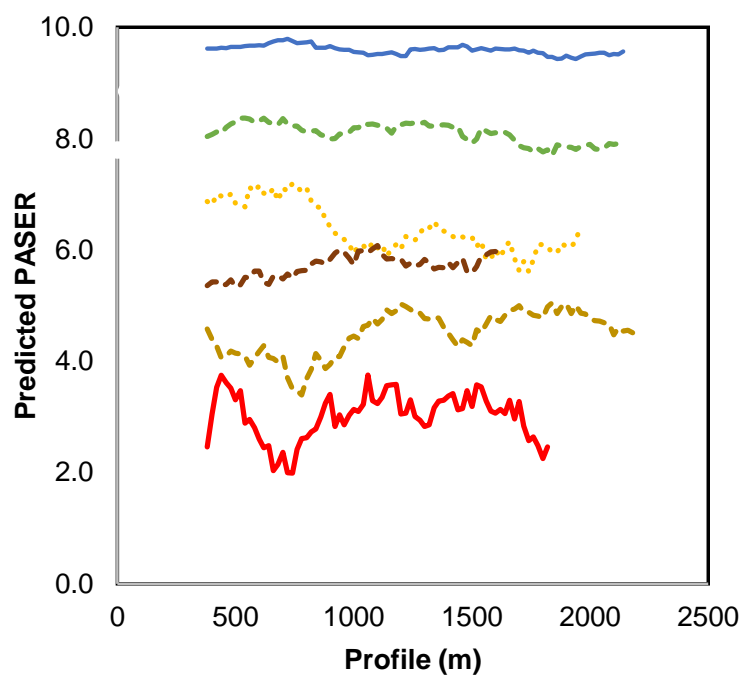

a)
Hybrid model-linear regression PASER

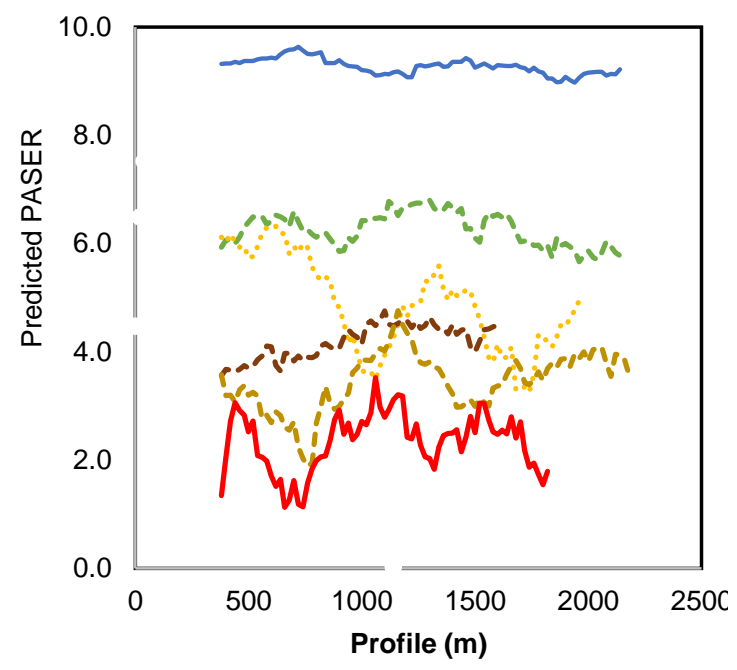

b) 
YOLO model-GEP based PASER

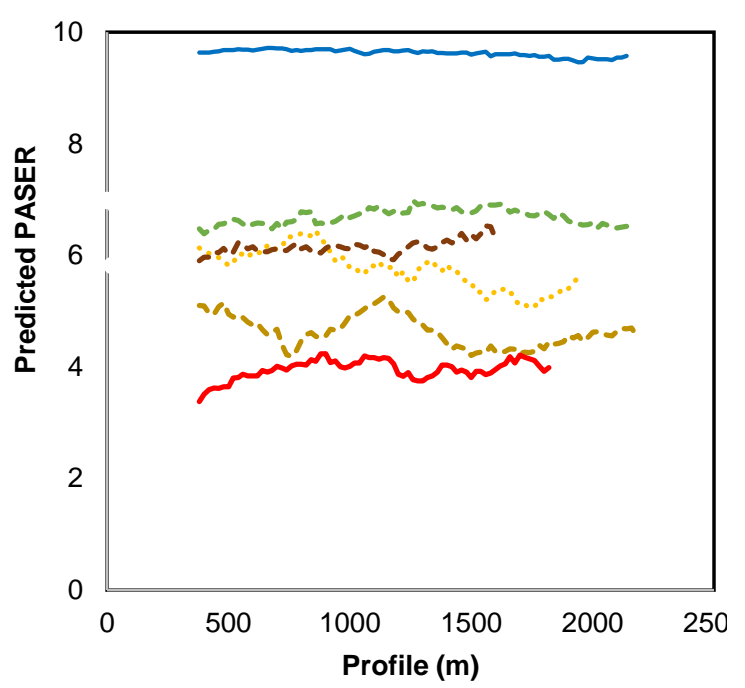

c)

-20 per. Mov. Avg. (I70)
$\ldots \ldots 2$ per. Mov. Avg. (MO11)
$\ldots--20$ per. Mov. Avg. (US71)

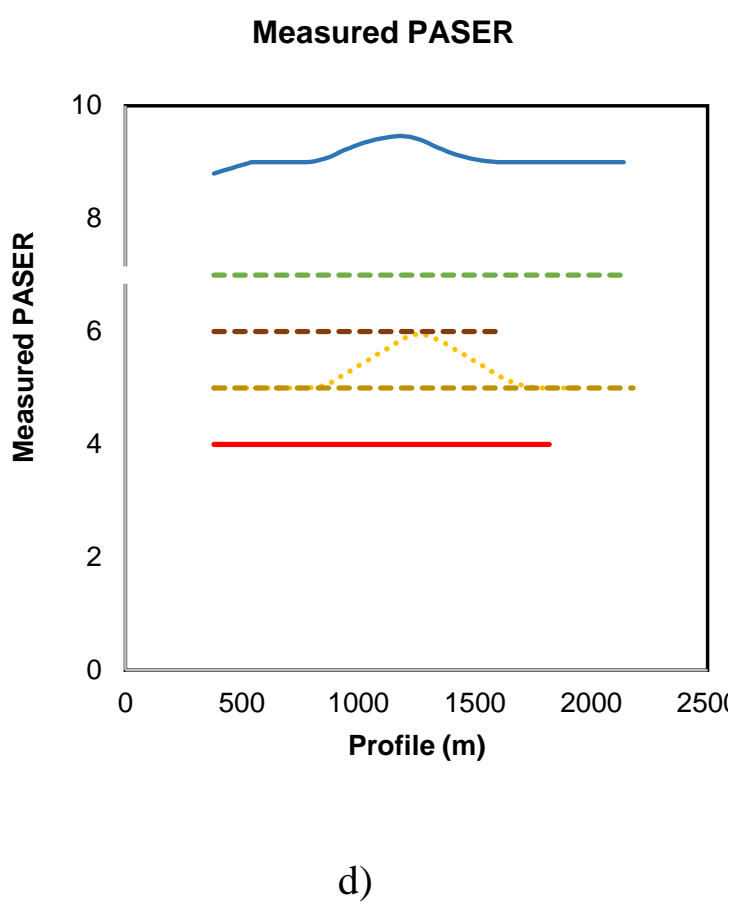

$--\infty$ per. Mov. Avg. (US50_sedelia)

- - 20 per. Mov. Avg. (US24_Lexinton)

20 per. Mov. Avg. (Mo52_stover)

Figure 4-28. Changing of Predicted PASER using different models a) Hybrid modelweight based, b) Hybrid model-linear regression, c) YOLO model-GEP based and d) Measured PASER, for six sections alongside pavement profile.

\subsubsection{Summary}

In this study, a deep machine learning approach was implemented to predict pavement condition of asphalt-surfaced roadways. Models were trained using a comprehensive road condition dataset consisting of 7,237 images extracted from Google Street-view. The images were annotated with bounding boxes featuring nine different pavement distresses. A state-of-the-art deep learning frameworks were employed to detect, classify and segment nine types of pavement distress automatically. A pre-trained U-Net based model was used to calculate the density of the distresses. The pre-trained model was 
fine-tuned by using manually annotated road images acquired in a variety of environmental conditions. The model can accurately differentiate non crack-like features including shadows, cars from pavement distresses. In order to develop a model to classify and quantify severity of the distresses, YOLO and U-net model were integrated together as a hybrid model.

Various pavement condition prediction indices were developed based on the detected distresses by YOLO, and hybrid model. Approximately 82 images per section was extracted from 71 different road sections located in the state of Missouri. The distresses detected for all the images using the developed deep-learning crack detection model, and the average number of distresses per image were calculated for all the 71 sections. Afterward, the measured PASER values were extracted from MoDOT portal for all the corresponding road sections. A GEP-based model was developed to predict PASER using the average number of distresses per image as an input. Also, two more PASER prediction models were developed based on the hybrid model outputs. Linear regression, and weightbased prediction models were developed, and the performance of them were verified by high values of coefficients of determination $\left(\mathrm{R}^{2}\right)$ for all the training, validation, and testing dataset. The results of the sensitivity analysis indicate that block cracking (an extensive distress in the Midway in modern times) is the most effective parameter in explaining the variations in PASER as compared with the other predictor variables. This may be due to the fact that block cracking is a clear indicator of advanced asphalt age and age hardening, both of which are in turn correlated to many other pavement distresses. 
For further model validation, six sections with various surface conditions were selected from Missouri roads. The predicted PASER values correlated well with the measured values. However, there was a swap in ranking between two of the cases. Contrary to the proposed pavement condition prediction models, traditional PASER rating is a qualitative-based rating without considering any distress quantity in detail. The approximate and human-based nature of the PASER procedure makes it challenging to correlate the machine learning-based pavement condition model with the measured PASER values in some cases. Also, the predicted PASER values from the models are fluctuating more than the corresponding measured PASER values due to the variety of detected distresses image by image in each section, while PASER values are usually constant over the whole section which is unrealistic.

Finally, the proposed models offer some advantages over traditional pavement monitoring (expensive cost of ARAN vehicles and laser equipment), and as compared to previous deep learning-based models. First, this tool solved the problem of data inconsistency caused by various human judgment. Also this study is the pioneer in concerning about developing a prediction pavement condition index after developing a model to detect the distresses. Second, the models were trained using Google street-view images, which are free and available for virtually all roads in the US and abroad. Third, the models were developed based on comprehensive pavement image dataset which was annotated considering wide variety of common pavement distress types by pavement experts. Finally, the developed models are robust and flexible, cost-effective, and able to predict distress from different camera views towards convenient. The fact that these firstgeneration models appear to have an acceptable average prediction error suggests that it 
may be very useful for DOTs and road agencies, as a means to evaluate road sections conditions. This tool could be conveniently employed to evaluate the pavement conditions during its service life and help to make valid decisions for rehabilitation or reconstruction of the roads at the right time. 


\section{SUMMARY, CONCLUSIONS AND RECOMMENDATIONS FOR FUTURE WORK}

\subsection{DEVELOPING A MODERN RECYCLED MIX DESIGN}

A comprehensive lab investigation was carried out to evaluate the performance of recycled asphalt mixtures in Missouri. Eighteen field sections were evaluated, including several sections from the recent Long-Term Pavement Performance (LTPP), Special Pavement Sections (SPS-10) project in Osage Beach, Missouri, which was constructed in 2016. Well and poorly performing sections dating back as far as 2003 construction were sampled and tested. Some key observations made in the study are that:

- The DC(T)-Hamburg plot showed that Missouri recycled mixes are generally on the brittle side, although three of 11 newer mixes investigated met recommended Hamburg and DC(T) criteria. Most mixes exhibit sufficient "total" energy to pass Hamburg and $\mathrm{DC}(\mathrm{T})$ recommended criteria without major changes in the aggregate structure, recycling level, or binder cost.

- Adjusting these mixes would simply involve the selection of a softer virgin binder grade, with a similar UTI, and therefore similar cost to the existing binder (which is normally PG 64-22 or PG 64-22H).

- The differences in test range for the DC(T) and IFIT are accompanied by significant differences in test repeatability for these two cracking tests. The DC(T) and I-FIT showed an average COV for all study mixes of $13.2 \%$ and $43.7 \%$, respectively. The DC(T) and I-FIT were both able to identify the best and worst performing section among the eighteen field sections

- Different strategies such as using softer binder, adding rejuvenator, and crumb rubber 
were used to improve the performance of the mixtures. Use of Rubber in conjunction with softer binder was significantly effective to improve the low-temperature cracking performance of asphalt mixtures. With use of this strategy, not only the rutting resistant did not decrease but also it improved. In contrary to fracture energy at low temperature, the intermediate cracking indices including FI and CT index dropped significantly when rubber was incorporated in the mixtures. The reason could be related to the different behavior of asphalt mixtures at low and intermediate temperatures.

- The correlation of deterioration rate of the sections with FI and DC(T) values, confirmed that, $\mathrm{DC}(\mathrm{T})$ test can be a more reliable test than SCB (IFIT) to evaluate the cracking performance. Also DC(T) test results were more repeatable $(\mathrm{COV} \%=19.5)$ than FI $(\mathrm{COV}=52 \%)$ and it can proves the higher reliability of the $\mathrm{DC}(\mathrm{T})$ test compared to SCB IFIT test. Another finding of this part of the research is that the SCB(IFIT) test is highly depend on aging of the section. The recommended values and thresholds should be adjusted based on the section old.

In the last phase of this chapter, a comprehensive evaluation of the existing index parameters derived from the SCB test at intermediate temperature was presented. Several shortcomings of the existing indices motivated the introduction of a new index parameter, called BCI and defined as the fracture energy times the post-peak displacement corresponding to $75 \%$ of the peak load. The usefulness of each index was analyzed and compared using 52 asphalt mixtures and ten field sections. The major findings of this study are enumerated as follows:

- The average index $\mathrm{COV}$ is $34.6 \%, 17.5 \%$, and $11.4 \%$ for the FI, BCI, and CRI, 
respectively, as obtained from the 52 asphalt mixtures tested in this study. The average $\mathrm{COV}$ is smaller than the recommended maximum COV of $20 \%$ only for the $\mathrm{BCI}$ and CRI.

- While the FI and CRI are strongly correlated with the $\mathrm{R}^{2}$ value of 0.97 , the $\mathrm{BCI}$ is only moderately correlated with the other two indices.

- The FI is shown to have a good correlation with post-peak slope but a poor correlation with the fracture energy of the mixtures. The dependency of the FI on the slope parameter leads to misleading conclusions due to the poor repeatability of the slope. In contrast, the correlation of $\mathrm{BCI}$ with its individual parameters, i.e., fracture energy and post-peak displacement at $75 \%$ peak load (L75) is high $\left(\mathrm{R}^{2}=0.85\right)$. The BCI is significantly at odds with both the FI and CRI when a polymer-modified mixture is compared to a soft mixture. While both the FI and CRI sacrifice the fracture energy, the $\mathrm{BCI}$ is equally proportional to both the fracture energy and the relaxation parameter. The SCB indices are not flawless. For one, they almost invariably increase with an elevation of the air voids content. For another, they are sensitive to neither the aggregate type nor the gradation of the mixture. Ten field sections with different conditions were studied to characterize the capability of each SCB index to predict the cracking performance. The FI and CRI did not show a strong correlation with field performance data. Further statistical analysis showed that FI classified the ten field sections into only two groups, and the CRI classified all of them into a single group. Additionally, although the FI identified the most crack resistant mixture, it failed to recognize the most brittle mixture. On the other hand, BCI showed a high correlation with field performance data with an $\mathrm{R}^{2}$ value of 0.6 . Additionally, it categorized the ten 
sections into five different groups, showing its usefulness in distinguishing asphalt mixtures in terms of their cracking potential. Aging significantly lowers the SCB indices from their unaged values. Therefore, the three SCB indices are not a reliable means to evaluate the cracking performance of aged field cores that have variable air voids contents. Furthermore, the air-void-induced change of the SCB indices indicates otherwise (higher value of index implies better performance at higher air voids). It logically follows from this observation that these SCB-based parameters are not wellsuited to evaluate the performance of field cores that are usually compacted between $3-5 \%$ air voids, which is far from the air voids range allowed for performance test specimens $(7.0+0.5 \%)$ by the standard.

Based on the findings of this study, the following recommendations are suggested:

1. More work is needed to further evaluate and fine-tune mix performance tests for use in balanced mix design, which is particularly important for modern, heterogeneous recycled mixes. This should include:

a. Sampling and testing of additional field sections, including a broad array of materials, recycling combinations, and project age;

b. Developing a reliability-based approach for setting performance test thresholds;

c. Considering adjustments to the Hamburg test to make it more performance based and climate based; i.e., making the test temperature related to climate and wheel pass requirements related to traffic level; 
2. More work is needed to evaluate GTR recycling, especially some of the new dry process techniques that are more economical, and more contractor friendly. Ongoing demonstration projects in Missouri should be included in future research.

3. A better physical understanding of recycling physics and chemistry is needed in order to arrive at even higher recycling amounts, in a confident manner. More binder and component characterization is needed, along with micromechanical modeling to better understand the interactions between recycled materials.

\subsection{MACHINE LEARNING-BASED PREDICTION MODELS FOR PERFORMANCE OF ASPHALT MIXTURES}

This chapter involved with developing machine learning-based predictions models for $\mathrm{DC}(\mathrm{T})$ fracture energy test and Hamburg wheel track test.

In the first phase of this chapter, two machine learning methods called GEP and ANN/SA were used to predict the Gf of asphalt mixtures. The proposed models include the simultaneous effect of various test, binder and aggregate-related parameters, as well as modern asphalt ingredients such as recycled materials, rejuvenators, and rubber. The models provide reliable predictions of Gf. The GEP model produces better outcomes than the ANN/SA model for the testing data. The beauty of the GEP analysis is that it can model the Gf behavior without any need to predefine the model's functional structure. Besides, a calculation procedure was proposed to transform the optimal ANN/SA model into a functional representation. The GEP model seems to be more practical than the ANN/SA model because of its better generalization and simpler functional structure. A limitation of the derived models is that they were calibrated using a fairly small set of $\mathrm{DC}(\mathrm{T})$ test results. 
This limitation can be tackled by updating the models with new trained weights when more data become available. The results of the sensitivity analysis indicate that AC, AT, LTPG and $\mathrm{T}$ are more effective to explain the variations of Gf compared with the other predictor variables. Since the proposed models are based on the data alone, they are mostly suitable for pre-design purposes. In any case, the range of the mixture properties should fall within the range considered for the development of the models. It should be noted that performing DC(T) test might be cost-prohibitive for some users. Thus, a trial method to design a mixture with acceptable thermal cracking performance may be expensive. Arguably, using the proposed empirical models for the estimation of the $\mathrm{DC}(\mathrm{T})$ test results will save time and money for such design procedures.

In the second phase of this chapter, a new model was developed to predict the rutting depth of asphalt mixtures using a GEP-based modeling approach. Mix type, ABR \%, PG high temperature grade, and test temperature were found to be more highly related to HWTT rut depth as compared to other variables. The effect of asphalt additives such as ground tire rubber was characterized by the model with respect to both rutting, and by employing a recently developed related model, thermal cracking. By having both high and low temperature prediction models, screening of materials for balanced mix design can be greatly facilitated.

In the last part of this chapter, a new model was developed to predict the rutting depth of asphalt mixtures using a CNN-based modeling approach. The effect of asphalt additives such as ground tire rubber was characterized by the model with respect to both rutting. 
Using both high and low temperature prediction models, screening of materials for balanced mix design can be remarkably facilitated.

The proposed models can and should be updated with more data in the future. Since performing Hamburg wheel track and DC(T) FE tests might be cost-restrictive for some users, using the proposed ML-based model can save time and expense during the material screening phase. Further research will focus on including tests results for mixtures with a much wider range in properties, and will include a larger span of aggregate types and will include more data points conducted across a range of test temperature, to assist agencies in evaluating proper tests temperatures to use for the purpose of balanced mix design in various climates across the US and beyond. Use of the modeling technique to predict the presence and magnitude of the stripping inflection point will also be pursued.

\subsection{DEVELOPING A PREDICTION MODEL FOR PAVEMENT CONDITION OF ASPHALT ROADS USING DEEP- LEARNING APPROACH}

In this chapter, a comprehensive dataset was introduced named Pavement Image Dataset (PID) for training machine learning models for the purpose of automated pavement distress characterization and monitoring. The dataset consists of two image groups: "wideview images", where 7,237 images with bounding boxes featuring nine different pavement distresses were assembled; and "top-down images", consisting of 7,237 images at identical locations as the wide-view images.

The primary focus of this article was to demonstrate how the wide-view images were used along with a deep learning approach to classify distresses. Two state-of-the-art, deep 
learning frameworks, YOLO v2 and Faster R-CNN, were implemented to automatically detect and classify nine types of pavement distress. The F1 scores, which are often used for model accuracy assessment, were obtained as 0.84 for YOLOv2 and 0.65 for the Faster R-CNN models, respectively. According to the F1 scores and confusion matrices for the nine distress classes, the YOLO v2 model results in more accurate distress characterizations than the Faster R-CNN model.

The proposed models offer some advantages over traditional pavement monitoring, and as compared to previous deep learning-based models. First, the models were trained using Google street-view images, which are free and available for virtually all roads in the US and abroad. Second, the models were developed based on a wide variety of common pavement distress types. Finally, the developed models are robust and flexible, able to predict distress from different camera views towards convenient, cost-effective, and accurate pavement evaluation, monitoring, and management.

In the second phase of this chapter, a deep machine learning approach was implemented to predict pavement condition of asphalt-surfaced roadways. A pre-trained U-Net based model was used to calculate the density of the distresses. The pre-trained model was fine-tuned by using manually annotated road images acquired in a variety of environmental conditions. The model can accurately differentiate non crack-like features including shadows, cars from pavement distresses. In order to develop a model to classify and quantify severity of the distresses, YOLO and U-net model were integrated together as a hybrid model. 
Various pavement condition prediction indices were developed based on the detected distresses by YOLO, and hybrid model. Approximately 82 images per section was extracted from 71 different road sections located in the state of Missouri. The distresses detected for all the images using the developed deep-learning crack detection model, and the average number of distresses per image were calculated for all the 71 sections. Afterward, the measured PASER values were extracted from MoDOT portal for all the corresponding road sections. A GEP-based model was developed to predict PASER using the average number of distresses per image as an input. Also, two more PASER prediction models were developed based on the hybrid model outputs. Linear regression, and weightbased prediction models were developed, and the performance of them were verified by high values of coefficients of determination $\left(\mathrm{R}^{2}\right)$ for all the training, validation, and testing dataset. The results of the sensitivity analysis indicate that block cracking (an extensive distress in the Midway in modern times) is the most effective parameter in explaining the variations in PASER as compared with the other predictor variables. This may be due to the fact that block cracking is a clear indicator of advanced asphalt age and age hardening, both of which are in turn correlated to many other pavement distresses.

Finally, the proposed models offer some advantages over traditional pavement monitoring (expensive cost of ARAN vehicles and laser equipment), and as compared to previous deep learning-based models. First, this tool solved the problem of data inconsistency caused by various human judgment. Also this study is the pioneer in concerning about developing a prediction pavement condition index after developing a model to detect the distresses. Second, the models were trained using Google street-view images, which are free and available for virtually all roads in the US and abroad. Third, the 
models were developed based on comprehensive pavement image dataset which was annotated considering wide variety of common pavement distress types by pavement experts. Finally, the developed models are robust and flexible, cost-effective, and able to predict distress from different camera views towards convenient. The fact that these firstgeneration models appear to have an acceptable average prediction error suggests that it may be very useful for DOTs and road agencies, as a means to evaluate road sections conditions. This tool could be conveniently employed to evaluate the pavement conditions during its service life and help to make valid decisions for rehabilitation or reconstruction of the roads at the right time.

A current limitation in the PASER prediction model reported herein is that it was calibrated using a relatively limited set of PASER results (71 sections). The model performance will be enhanced by updating the model, allowing to continue to learn and ultimately culminate into a well-built and broadly predictive tool, as more data becomes available. It is hoped that collaborations with other research groups and owner-agencies will lead to a vastly larger database, and an even more highly predictive model. It is recommended that a major, national study is justified by the current results, and represents a next logical step forward. Also, the same idea can be implemented on 3D images and other distresses like rutting can be taken into account in the final pavement condition prediction model. Furthermore, distresses characteristics can be investigated precisely by introducing 3D images. In order to facilitate the deployment of the proposed model, an integrated software is developed to incorporate all the steps, including extracting images, analyzing images, predicting pavement condition together. The software is available online at www.fardco.net. One of the primary disadvantage of the proposed approach comparing 
to the ARAN machines, is that it could not capture rutting in asphalt pavements. Although there is always trade off between the cost and output of the projects, we are still looking for a way to calculate rutting by developing some correlation models between rutting, cracking and roughness. 


\section{REFERENCES}

[1] D. ASTM, "Standard test method for viscosity determination of asphalt at elevated temperatures using a rotational viscometer," 2015.

[2] ASTM, “ASTM D7313-13: Standard Test Method for Determining Fracture Energy of Asphalt-Aggregate Mixtures Using the Disk-Shaped Compact Tension Geometry,” ASTM Int. Stand., pp. 2-8, 2013, doi: 10.1520/D7313.

[3] M. Marasteanu et al., "Investigation of low temperature cracking in asphalt pavements national pooled fund study-phase II,” 2012.

[4] A. TP124, "Standard Method of Test for Determining the Fracture Potential of Asphalt Mixtures Using Semicircular Bend (SCB) Geometry at Intermediate Temperature," Washingt. DC Am. Assoc. State Highw. Transp. Off. Google Sch., 2016.

[5] F. Zhou, "Development of an IDEAL Cracking Test for Asphalt Mix Design, Quality Control and Quality Assurance," NCHRP-IDEA Progr. Proj. Final Rep., no. $195,2019$.

[6] F. Zhou, S. Im, L. Sun, and T. Scullion, "Development of an IDEAL cracking test for asphalt mix design and QC/QA," J. Assoc. Asph. Paving Technol., vol. 86, pp. 1-23, 2017, doi: 10.1080/14680629.2017.1389082.

[7] P. Paris and F. Erdogan, "A critical analysis of crack propagation laws," 1963.

[8] I. Carol, Z. P. Bažant, and P. C. Prat, "Geometric damage tensor based on microplane model," J. Eng. Mech., vol. 117, no. 10, pp. 2429-2448, 1991.

[9] AASHTO-T-324, "Standard method of test for Hamburg wheel-track testing of compacted hot mix asphalt (HMA)," Standard Specifications for Transportation 
Materials and Methods of Sampling and Testing. AASHTO Washington, DC, 2011.

[10] R. West, C. Rodenzo, F. Leiva, and F. Yin, "Development of a Framework for Balanced Mix Design, NCHRP Project 20-07/Task 406,” p. 168, 2018.

[11] W. G. Buttlar, L. Urra-Contreras, B. Jahangiri, P. Rath, and H. Majidifard, "Support for Balanced Asphalt Mixture Design Specification Development in Missouri,”2020.

[12] R. West, C. Rodezno, F. Leiva, and A. Taylor, "Regressing Air Voids for Balanced HMA Mix Design,” no. 0092, 2018.

[13] S. W. Katicha and G. W. Flintsch, "Improving Mixture Durability Through Design Gyrations , Air Voids , and Binder Content,” 2016.

[14] R. Bonaquist, "Critical Factors Affecting Asphalt Concrete Durability,” 2016.

[15] N. Tran, G. Huber, F. Leiva, B. Pine, and F. Yin, "Mix Design Strategies for Improving Asphalt Mixture Performance,” 2019.

[16] B. Behnia, E. Dave, S. Ahmed, W. Buttlar, and H. Reis, "Effects of Recycled Asphalt Pavement Amounts on Low-Temperature Cracking Performance of Asphalt Mixtures Using Acoustic Emissions,” Transp. Res. Rec. J. Transp. Res. Board, vol. 2208, pp. 64-71, 2011, doi: 10.3141/2208-09.

[17] R. Willis, P. Turner, G. Julian, A. J. Taylor, N. Tran, and F. de G. Padula, "Effects of Changing Virgin Binder Grade and Content on Rap Mixture Properties - NCAT Report 12-03," Natl. Cent. Ashalt Technol., no. 12, pp. 1-47, 2012.

[18] P. Rath, J. Love, W. G. Buttlar, and R. H., "Performance Analysis of Asphalt Mixtures Modified with Ground Tire Rubber and Recycled Materials," Sustain., 
vol. 11 (6), no. 1792, 2019, doi: https://doi.org/10.3390/su11061792.

[19] D. Timm et al., "Phase II NCAT Test Track Results," NCAT Rep. 06-05, no. December, 2006.

[20] R. West et al., "Phase IV NCAT Pavement Test Track Findings," Natl. Cent. Asph. Technol. Auburn Univ., p. 188p, 2012.

[21] W. Buttlar et al., "Develoment of a Performance-Related Asphalt Mix Design Specification for the Illinois Tollway."

[22] D. Newcomb and F. Zhou, "Balanced Design of Asphalt Mixtures," Minnesota. Dept. of Transportation. Research Services \& Library, 2018.

[23] H. Bahia et al., "Analysis and Feasibility of Asphalt Pavement Performance-Based Specifications for WisDOT," WisDOT, no. 0092, 2016.

[24] P. Rath, N. Gettu, S. Chen, and W. G. Buttlar, "Investigation of Cracking Mechanisms in Rubber-Modified Asphalt through Fracture Testing of Mastic Specimen," Road Mater. Pavement Des.

[25] C. Ling, D. Swiertz, T. Mandal, P. Teymourpour, and H. Bahia, "Sensitivity of the Illinois flexibility index test to mixture design factors," Transp. Res. Rec., vol. 2631, no. 1750, 2017, doi: 10.3141/2631-17.

[26] F. Zhou, "IDEAL Cracking Test for QC / QA and Associated Criteria," 2018.

[27] B. Jahangiri, H. Majidifard, J. Meister, and W. G. Buttlar, "Performance Evaluation of Asphalt Mixtures with Reclaimed Asphalt Pavement and Recycled Asphalt Shingles in Missouri," Transp. Res. Rec., 2019, doi: 10.1177/0361198119825638.

[28] L. N. Mohammad, M. A. Elseifi, A. Raghavendra, and Y. Mengqiu, Hamburg 
Wheel-Track Test Equipment Requirements and Improvements to AASHTO T 324. 2016.

[29] W. G. Buttlar et al., "DEVELOPMENT OF A PERFORMANCE-RELATED ASPHALT MIX DESIGN SPECIFICATION FOR THE ILLINOIS TOLLWAY,” Tollw. Rep., 2020.

[30] P. Rath, H. Majidifard, B. Jahangiri, S. Chen, and W. G. Buttlar, "Laboratory and Field Evaluation of Pretreated Dry-Process Rubber Modified Asphalt Binders and Dense-Graded Mixtures," J. Transp. Res. Rec.

[31] H. Majidifard, B. Jahangiri, W. G. Buttlar, and A. H. Alavi, "New machine learning-based prediction models for fracture energy of asphalt mixtures," Measurement, vol. 135, pp. 438-451, 2019.

[32] A. Faheem, A. Hosseini, H. H. Titi, and S. Schwandt, "Evaluation of WisDOT Quality Management Program (QMP) Activities and Impacts on Pavement Performance," Wisconsin. Dept. of Transportation, 2018.

[33] H. Majidifard, Y. Adu-Gyamfi, and W. G. Buttlar, "Deep machine learning approach to develop a new asphalt pavement condition index," Constr. Build. Mater., vol. 247, p. 118513, 2020.

[34] H. Majidifard, P. Jin, Y. Adu-Gyamfi, and W. G. Buttlar, "Pavement Image Datasets: A New Benchmark Dataset to Classify and Densify Pavement Distresses," Transp. Res. Rec., vol. 2674, no. 2, pp. 328-339, 2020.

[35] Y. D. Wang, A. Ghanbari, B. S. Underwood, and Y. R. Kim, "Development of a performance-volumetric relationship for asphalt mixtures," Transp. Res. Rec., vol. 2673, no. 6, pp. 416-430, 2019. 
[36] H. Majidifard, N. Tabatabaee, and W. Buttlar, "Investigating short-term and longterm binder performance of high-RAP mixtures containing waste cooking oil," $J$. Traffic Transp. Eng. (English Ed., vol. 6, no. 4, pp. 396-406, 2019.

[37] H. Majidifard, B. Jahangiri, P. Rath, W. G. Buttlar, and A. H. Alavi, “A Deep Learning Approach to Predict Hamburg Rutting Curve," Road Mater. Pavement Des., 2020.

[38] H. Majidifard, B. Jahangiri, P. Rath, W. G. Buttlar, and A. H. Alavi, "Developing a Prediction Model for Rutting Depth of Asphalt Mixtures Using Gene Expression Programming," Constr. Build. Mater., 2020.

[39] B. Jahangiri, P. Rath, H. Majidifard, L. Urra, and W. G. Buttlar, "OnlyA Comprehensive Performance Investigation of Asphalt Mixtures in Missouri: Laboratory, Field, and ILLI-TC Modeling,” Road Mater. Pavement Des.

[40] W. Buttlar, J. Meister, B. Jahangiri, H. Majidifard, and P. Rath, "Performance Characteristics of Modern Recycled Asphalt Mixes in Missouri, Including Ground Tire Rubber, Recycled Roofing Shingles, and Rejuvenators. Draft Publication (Under Review),” 2018.

[41] W. G. Buttlar, P. Rath, H. Majidifard, E. V. Dave, and H. Wang, "Relating DC(T) Fracture Energy to Field Cracking Observations and Recommended Specification Thresholds for Performance-Engineered Mix Design,” Transp. Res. Circ., vol. EC251, no. September, pp. 51-69, 2019.

[42] R. Rahbar-rastegar, S. M. Asce, E. V Dave, A. M. Asce, J. S. Daniel, and M. Asce, "Fatigue and Thermal Cracking Analysis of Asphalt Mixtures Using ContinuumDamage and Cohesive-Zone Models," vol. 144, no. 4, pp. 1-11, 2018, doi: 


\subsection{1/JPEODX.0000066.}

[43] B. Keshavarzi and Y. R. Kim, "A viscoelastic-based model for predicting the strength of asphalt concrete in direct tension," Constr. Build. Mater., vol. 122, pp. 721-727, 2016, doi: 10.1016/j.conbuildmat.2016.06.089.

[44] Y. R. Kim, C. Castorena, Y. Wang, A. Ghanbari, and J. Jeong, “Comparing Performance of Full-depth Asphalt Pavements and Aggregate Base Pavements in NC," FHWA/NC/2015-02 Report, 2018.

[45] A. Ghanbari, B. S. Underwood, and Y. R. Kim, "Development of a rutting index parameter based on the stress sweep rutting test and permanent deformation shift model," Int. J. Pavement Eng., pp. 1-13, 2020.

[46] Y. Yan, D. Hernando, and R. Roque, "Fracture Tolerance of Asphalt Binder at Intermediate Temperatures," vol. 29, no. 9, pp. 1-10, 2017, doi: 10.1061/(ASCE)MT.1943-5533.0001955.

[47] M. A. Notani, F. Moghadas Nejad, A. Khodaii, and P. Hajikarimi, "Evaluating fatigue resistance of toner-modified asphalt binders using the linear amplitude sweep test," Road Mater. Pavement Des., vol. 20, no. 8, pp. 1927-1940, 2019.

[48] S. Shirzad, M. A. Aguirre, L. Bonilla, M. A. Elseifi, S. Cooper, and L. N. Mohammad, "Mechanistic-empirical pavement performance of asphalt mixtures with recycled asphalt shingles," Constr. Build. Mater., vol. 160, pp. 687-697, 2018, doi: 10.1016/j.conbuildmat.2017.11.114.

[49] A. F. Mirhosseini, A. Kavussi, S. A. Tahami, and S. Dessouky, "Characterizing temperature performance of bio-modified binders containing RAP binder," $J$. Mater. Civ. Eng., vol. 30, no. 8, 2018, doi: 10.1061/(ASCE)MT.1943- 
5533.0002373.

[50] F. Zhou, S. Im, S. Hu, D. Newcomb, and T. Scullion, "Selection and preliminary evaluation of laboratory cracking tests for routine asphalt mix designs," Road Mater. Pavement Des., vol. 0, no. 0, pp. 1-25, 2017, doi:

10.1080/14680629.2016.1266741.

[51] T. L. Anderson, "Fracture Mechanics-Fundamentals and Applications 3rd edn. CRC.” Taylor \& Francis Group, Boca Raton, London, New York, Singapore, 2005.

[52] I. L. Al-Qadi et al., "Testing protocols to ensure performance of high asphalt binder replacement mixes using RAP and RAS," Illinois Center for Transportation/Illinois Department of Transportation, 2015.

[53] Z. Wu, L. N. Mohammad, L. B. Wang, and M. A. Mull, "Fracture resistance characterization of superpave mixtures using the semi-circular bending test," $J$. ASTM Int., vol. 2, no. 3, pp. 1-15, 2005.

[54] K. Majidzadeh, E. M. Kauffmann, and D. V Ramsamooj, "Application of fracture mechanics in the analysis of pavement fatigue," in Association of Asphalt Paving Technologists Proc, 1971, vol. 40.

[55] S. H. Song, G. H. Paulino, and W. G. Buttlar, "A bilinear cohesive zone model tailored for fracture of asphalt concrete considering viscoelastic bulk material," Eng. Fract. Mech., vol. 73, no. 18, pp. 2829-2848, 2006.

[56] M. P. Wagoner, W. G. Buttlar, and G. H. Paulino, "Development of a single-edge notched beam test for asphalt concrete mixtures," J. Test. Eval., vol. 33, no. 6, pp. 452-460, 2005. 
[57] B. S. Underwood, C. Baek, and Y. R. Kim, "Simplified viscoelastic continuum damage model as platform for asphalt concrete fatigue analysis," Transp. Res. Rec., vol. 2296, no. 1, pp. 36-45, 2012.

[58] F. Kaseer, F. Yin, E. Arámbula-Mercado, A. E. Martin, J. S. Daniel, and S. Salari, "Development of an index to evaluate the cracking potential of asphalt mixtures using the semi-circular bending test," Constr. Build. Mater., vol. 167, pp. 286-298, 2018.

[59] A. A. A. Molenaar, A. Scarpas, X. Liu, and S. Erkens, "Semi-circular bending test; simple but useful?," J. Assoc. Asph. Paving Technol., vol. 71, 2002.

[60] R. L. Krans, F. Tolman, and M. F. C. Van de Ven, "Semi-circular bending test: a practical crack growth test using asphalt concrete cores," in RILEM proceedings, 1996, pp. 123-132.

[61] H. Ozer, I. L. Al-Qadi, J. Lambros, A. El-Khatib, P. Singhvi, and B. Doll, "Development of the fracture-based flexibility index for asphalt concrete cracking potential using modified semi-circle bending test parameters," Constr. Build. Mater., vol. 115, pp. 390-401, 2016, doi: 10.1016/j.conbuildmat.2016.03.144.

[62] M. P. Wagoner, W. G. Buttlar, and G. H. Paulino, "Disk-shaped Compact Tension Test for Asphalt Concrete Fracture,” Exp. Mech., vol. 45, no. 3, pp. 270-277, 2005, doi: 10.1177/0014485105053205.

[63] R. Nemati, K. Haslett, E. V Dave, and J. E. Sias, "Development of a ratedependent cumulative work and instantaneous power-based asphalt cracking performance index," Road Mater. Pavement Des., vol. 20, no. sup1, pp. S315S331, 2019. 
[64] P. J. Bickel and K. A. Doksum, Mathematical statistics: basic ideas and selected topics, volume I, vol. 117. CRC Press, 2015.

[65] J.-S. Chen, T. J. Wang, and C.-T. Lee, "Evaluation of a highly-modified asphalt binder for field performance," Constr. Build. Mater., vol. 171, pp. 539-545, 2018.

[66] W. J. Woo et al., "Polymer modified asphalt durability in pavements," Coll. Station. TX Texas Transp. Inst., 2007.

[67] J. R. Willis, D. H. Timm, and R. Kluttz, "Performance of a highly polymermodified asphalt binder test section at the national center for asphalt technology pavement test track,” Transp. Res. Rec., vol. 2575, no. 1, pp. 1-9, 2016.

[68] R. R. Davison et al., "A comprehensive laboratory and field study of high-cure crumb-rubber modified asphalt materials,” Texas Transportation Institute, 2000.

[69] A. W. Ahmed, S. F. Said, X. Lu, and H. Carlsson, "Pavement performance followup and evaluation of polymer-modified test sections," Int. J. Pavement Eng., vol. 20, no. 12, pp. 1474-1487, 2019.

[70] B. Javilla, L. Mo, F. Hao, B. Shu, and S. Wu, "Multi-stress loading effect on rutting performance of asphalt mixtures based on wheel tracking testing," Constr. Build. Mater., vol. 148, pp. 1-9, 2017, doi: 10.1016/j.conbuildmat.2017.04.182.

[71] H. Kim, M. P. Wagoner, and W. G. Buttlar, "Micromechanical fracture modeling of asphalt concrete using a single-edge notched beam test," Mater. Struct., vol. 42, no. 5 , p. $677,2009$.

[72] X. Chen, "Use of Semi-circular Bend Test to Characterize Fracture Properties of Asphalt Concrete with Virgin and Recycled Materials,” 2019.

[73] D. Batioja-Alvarez, J. Lee, and J. E. Haddock, "Understanding the Illinois 
Flexibility Index Test (I-FIT) using Indiana Asphalt Mixtures," Transp. Res. Rec., vol. 2673, no. 6, pp. 337-346, 2019.

[74] J. J. Rivera-Perez, "Effect of specimen geometry and test configuration on the fracture process zone for asphalt materials,” 2017.

[75] M. K. Barry, "An analysis of impact factors on the Illinois flexibility index test." 2016.

[76] X. Chen and M. Solaimanian, "Evaluating fracture properties of crumb rubber modified asphalt mixes," Int. J. Pavement Res. Technol., vol. 12, no. 4, pp. 407415, 2019.

[77] C. A. Bell, R. G. Hicks, and J. E. Wilson, "Effect of percent compaction on asphalt mixture life," in Placement and Compaction of Asphalt Mixtures, ASTM International, 1984.

[78] P. S. Kandhal, Designing and Constructing SMA Mixtures: State-of-the-practice. NAPA, 2002.

[79] Caltrans, "Use of Scrap Tire Rubber: State of the Technology and Best Practices," Caltrans, 2005, [Online]. Available: http://www.dot.ca.gov/hq/esc/Translab/ormt/pdf/T021_ScrapTire.pdf.

[80] S. R. Rebala and C. K. Estakhri, "Laboratory evaluation of crumb rubber modified mixtures designed using TxDOT mixture design method," Transp. Res. Rec., no. 1515, pp. 1-10, 1995.

[81] P. Rath, J. E. Love, W. G. Buttlar, and H. Reis, "Performance analysis of asphalt mixtures modified with ground tire rubber modifiers and recycled materials," Sustainability, vol. 11, no. 6, p. 1792, 2019. 
[82] D. Bergerson, M. Abdelrahman, and M. Ragab, "Environmental study of the release of BTEX from asphalt modified with used motor oil and crumb rubber modifier," 2014.

[83] A. Ghavibazoo and M. Abdelrahman, "Composition analysis of crumb rubber during interaction with asphalt and effect on properties of binder," Int. J. Pavement Eng., vol. 14, no. 5, pp. 517-530, 2013.

[84] S. Eskandarsefat, G. Dondi, and C. Sangiorgi, "Recycled and rubberized SMA modified mixtures: A comparison between polymer modified bitumen and modified fibres," Constr. Build. Mater., vol. 202, pp. 681-691, 2019.

[85] M. Manosalvas-Paredes, J. Gallego, L. Saiz, and J. M. Bermejo, "Rubber modified binders as an alternative to cellulose fiber-SBS polymers in Stone Matrix Asphalt," Constr. Build. Mater., vol. 121, pp. 727-732, 2016.

[86] M. D. Nazzal, M. T. Iqbal, S. S. Kim, A. R. Abbas, M. Akentuna, and T. Quasem, "Evaluation of the long-term performance and life cycle costs of GTR asphalt pavements," Constr. Build. Mater., vol. 114, pp. 261-268, 2016, doi: 10.1016/j.conbuildmat.2016.02.096.

[87] S. Mavridou, “" Integration of end-of-life tires in the life cycle of road construction ,"' Asphalt, vol. 38, no. December, pp. 1-66, 2010.

[88] V. Venudharan, K. P. Biligiri, J. B. Sousa, and G. B. Way, “Asphalt-rubber gapgraded mixture design practices: a state-of-the-art research review and future perspective," Road Mater. Pavement Des., vol. 18, no. 3, pp. 730-752, 2017.

[89] A. Obaid, M. D. Nazzal, S. S. Kim, A. Abbas, M. Arefin, and T. Quasem, “Evaluation of Terminal Blend GTR Mixes with RAP," J. Transp. Eng. Part B 
Pavements, vol. 146, no. 1, p. 4019041, 2020.

[90] A. Fathi, M. Mazari, M. Saghafi, A. Hosseini, and S. Kumar, "Parametric Study of Pavement Deterioration Using Machine Learning Algorithms," Airf. Highw. Pavements, pp. 31-41, 2019.

[91] M. Saghafi, C. Tirado, I. N. Abdallah, and S. Nazarian, "Considering Pavement Structure in Laboratory Fatigue Cracking Assessment," in International Conference on Transportation and Development 2020, 2020, pp. 187-199.

[92] D. Walker, L. Entine, and S. Kummer, "Pavement surface evaluation and rating: PASER manual," 1987.

[93] M. S. Medeiros Jr, J. S. Daniel, and G. R. Chehab, "Framework for lowtemperature cracking analysis of asphalt mixtures using a viscoelastic continuum damage model," J. Mater. Civ. Eng., vol. 27, no. 10, p. 4014265, 2015.

[94] R. Ghabchi, D. Singh, and M. Zaman, "Laboratory evaluation of stiffness, lowtemperature cracking, rutting, moisture damage, and fatigue performance of WMA mixes," Road Mater. Pavement Des., vol. 16, no. 2, pp. 334-357, 2015.

[95] D. J. Mensching, G. M. Rowe, and J. Sias Daniel, “A mixture-based Black Space parameter for low-temperature performance of hot mix asphalt," Road Mater. Pavement Des., vol. 18, no. sup1, pp. 404-425, 2017.

[96] W. G. Buttlar, B. C. Hill, H. Wang, and W. Mogawer, "Performance space diagram for the evaluation of high-and low-temperature asphalt mixture performance," Road Mater. Pavement Des., vol. 18, no. sup1, pp. 336-358, 2017.

[97] M. O. Marasteanu et al., "Investigation of Low Temperature Cracking in Asphalt Pavements, national Pooled Fund Study 776,” 2007. 
[98] E. V Dave and C. Hoplin, "Flexible pavement thermal cracking performance sensitivity to fracture energy variation of asphalt mixtures," Road Mater.

Pavement Des., vol. 16, no. sup1, pp. 423-441, 2015.

[99] W. Buttlar, P. Rath, H. Majidifard, E. V Dave, and H. Wang, "Relating DC (T) Fracture Energy to Field Cracking Observations and Recommended Specification Thresholds for Performance-Engineered Mix Design,” Asph. Mix., vol. 51, 2018.

[100] C. Hoplin, "Cracking Performance Evaluation of Minnesota Asphalt Pavements," 2016.

[101] Y.-R. Kim, "Cohesive zone model to predict fracture in bituminous materials and asphaltic pavements: state-of-the-art review," Int. J. Pavement Eng., vol. 12, no. 4, pp. 343-356, 2011.

[102] Q. Li, H. J. Lee, and T. W. Kim, “A simple fatigue performance model of asphalt mixtures based on fracture energy," Constr. Build. Mater., vol. 27, no. 1, pp. 605$611,2012$.

[103] A. H. Alavi and A. H. Gandomi, "A robust data mining approach for formulation of geotechnical engineering systems," Eng. Comput. Int J Comput. Eng., vol. 28, no. 3, pp. 242-274, 2011.

[104] D. J. Lary, A. H. Alavi, A. H. Gandomi, and A. L. Walker, "Machine learning in geosciences and remote sensing," Geosci. Front., vol. 7, no. 1, pp. 3-10, 2016.

[105] T. M. Mitchell, "Does machine learning really work?," AI Mag., vol. 18, no. 3, p. 11, 1997.

[106] A. Behnood and E. M. Golafshani, "Predicting the compressive strength of silica fume concrete using hybrid artificial neural network with multi-objective grey 
wolves," J. Clean. Prod., vol. 202, pp. 54-64, 2018.

[107] S. M. Mousavi, A. H. Gandomi, A. H. Alavi, and M. Vesalimahmood, "Modeling of compressive strength of HPC mixes using a combined algorithm of genetic programming and orthogonal least squares," Struct. Eng. Mech., vol. 36, no. 2, pp. 225-241, 2010.

[108] P. Ghasemi, M. Aslani, D. K. Rollins, and R. C. Williams, "Principal Component Neural Networks for Modeling, Prediction, and Optimization of Hot Mix Asphalt Dynamics Modulus," Infrastructures, vol. 4, no. 3, p. 53, 2019.

[109] A. H. Alavi and A. H. Gandomi, "Prediction of principal ground-motion parameters using a hybrid method coupling artificial neural networks and simulated annealing," Comput. Struct., vol. 89, no. 23-24, pp. 2176-2194, 2011.

[110] V. W. Porto, D. B. Fogel, and L. J. Fogel, “Alternative Neural Network Training Models," IEEE Expert, pp. 16-22.

[111] R. S. Sexton, B. Alidaee, R. E. Dorsey, and J. D. Johnson, "Global optimization for artificial neural networks: A tabu search application," Eur. J. Oper. Res., vol. 106, no. 2-3, pp. 570-584, 1998.

[112] J. Binner, G. Kendall, and A. Gazely, "Co-evolving neural networks with evolutionary strategies: A new application to divisia money," Appl. Artif. Intell. Financ. Econ., vol. 19, pp. 127-143, 2004.

[113] S. Ledesma, M. Torres, D. Hernández, G. Aviña, and G. García, “Temperature cycling on simulated annealing for neural network learning," in Mexican International Conference on Artificial Intelligence, 2007, pp. 161-171.

[114] J. R. Koza and J. R. Koza, Genetic programming: on the programming of 
computers by means of natural selection, vol. 1. MIT press, 1992.

[115] C. Ferreira, "Gene expression programming: a new adaptive algorithm for solving problems," arXiv Prepr. cs/0102027, 2001.

[116] J. A. Torkestani, “An adaptive learning automata-based ranking function discovery algorithm," J. Intell. Inf. Syst., vol. 39, no. 2, pp. 441-459, 2012.

[117] A. H. Alavi, A. H. Gandomi, H. C. Nejad, A. Mollahasani, and A. Rashed, "Design equations for prediction of pressuremeter soil deformation moduli utilizing expression programming systems," Neural Comput. Appl., vol. 23, no. 6, pp. 1771-1786, 2013.

[118] P. M. Ramos and F. M. Janeiro, "Gene expression programming for automatic circuit model identification in impedance spectroscopy: Performance evaluation,” Measurement, vol. 46, no. 10, pp. 4379-4387, 2013.

[119] D. E. Rumelhart, J. L. McClelland, and P. D. P. R. Group, "Parallel distributed processing, Vol. 1\&2," Cambridge, MA Ml, 1986.

[120] A. Hossein Alavi, A. Hossein Gandomi, A. Mollahassani, A. Akbar Heshmati, and A. Rashed, "Modeling of maximum dry density and optimum moisture content of stabilized soil using artificial neural networks," J. Plant Nutr. Soil Sci., vol. 173, no. 3, pp. 368-379, 2010.

[121] S. Kirkpatrick, C. D. Gelatt, and M. P. Vecchi, "Optimization by simulated annealing,” Science (80-. )., vol. 220, no. 4598, pp. 671-680, 1983.

[122] N. Metropolis, A. W. Rosenbluth, M. N. Rosenbluth, A. H. Teller, and E. Teller, "Equation of state calculations by fast computing mechanics," J Chem Phys. v21 i6, pp. 1087-1092. 
[123] L. M. R. Rere, M. I. Fanany, and A. M. Arymurthy, "Simulated annealing algorithm for deep learning," Procedia Comput. Sci., vol. 72, no. 1, pp. 137-144, 2015.

[124] X. Li, A. F. Braham, M. O. Marasteanu, W. G. Buttlar, and R. C. Williams, "Effect of Factors Affecting Fracture Energy of Asphalt Concrete at Low Temperature," Road Mater. Pavement Des., vol. 9, no. sup1, pp. 397-416, 2008, doi: 10.1080/14680629.2008.9690176.

[125] X.-J. Li and M. O. Marasteanu, "Using semi circular bending test to evaluate low temperature fracture resistance for asphalt concrete," Exp. Mech., vol. 50, no. 7, pp. 867-876, 2010.

[126] E. V. Dave, W. G. Buttlar, S. E. Leon, B. Behnia, and G. H. Paulino, "IlliTC low-temperature cracking model for asphalt pavements," Road Mater. Pavement Des., vol. 14, no. December, pp. 57-78, 2013, doi:

10.1080/14680629.2013.812838.

[127] P. B. Blankenship and A. Zeinali, "Evaluation of the DC (T) test in discerning the variations in cracking properties of asphalt mixtures," Road Mater. Pavement Des., vol. 18, no. sup1, pp. 426-449, 2017.

[128] J. W. Arnold, B. Behnia, M. E. McGovern, B. Hill, W. G. Buttlar, and H. Reis, “Quantitative evaluation of low-temperature performance of sustainable asphalt pavements containing recycled asphalt shingles (RAS)," Constr. Build. Mater., vol. 58, pp. 1-8, 2014.

[129] E. T. Zegeye, K. H. Moon, M. Turos, T. R. Clyne, and M. O. Marasteanu, "Low temperature fracture properties of polyphosphoric acid modified asphalt mixtures," 
J. Mater. Civ. Eng., vol. 24, no. 8, pp. 1089-1096, 2012.

[130] X. Yang et al., "Environmental and mechanical performance of crumb rubber modified warm mix asphalt using Evotherm," J. Clean. Prod., vol. 159, pp. 346358, 2017.

[131] A. F. Braham, W. G. Buttlar, and M. O. Marasteanu, "Effect of binder type, aggregate, and mixture composition on fracture energy of hot-mix asphalt in cold climates," Transp. Res. Rec., vol. 2001, no. 1, pp. 102-109, 2007.

[132] W. G. Buttlar, J. Meister, B. Jahangiri, H. Majidifard, and P. Rath, "Performance characteristics of modern recycled asphalt mixes in Missouri, including ground tire rubber, recycled roofing shingles, and rejuvenators," 2019.

[133] S. Ahmed, "Fracture characterization of thin bonded asphalt concrete overlay systems." University of Illinois at Urbana-Champaign, 2011.

[134] W. Banzhaf, P. Nordin, R. Keller, and F. Francone, "GP-An Introduction; On the Automatic Evolution of Computer Programs and its Applications." Morgan Kaufmann San Francisco, 1998.

[135] S. K. Das, "10 Artificial Neural Networks in Geotechnical Engineering: Modeling and Application Issues," Metaheuristics Water Geotech Transp Eng, vol. 45, pp. 231-267, 2013.

[136] P. K. Muduli, S. K. Das, and S. Bhattacharya, "CPT-based probabilistic evaluation of seismic soil liquefaction potential using multi-gene genetic programming," Georisk Assess. Manag. Risk Eng. Syst. Geohazards, vol. 8, no. 1, pp. 14-28, 2014.

[137] S. K. Babanajad, A. H. Gandomi, and A. H. Alavi, "New prediction models for 
concrete ultimate strength under true-triaxial stress states: An evolutionary approach," Adv. Eng. Softw., vol. 110, pp. 55-68, 2017.

[138] K. Swingler, Applying neural networks: a practical guide. Morgan Kaufmann, 1996.

[139] A. T. C. Goh, F. H. Kulhawy, and C. G. Chua, "Bayesian neural network analysis of undrained side resistance of drilled shafts," J. Geotech. Geoenvironmental Eng., vol. 131, no. 1, pp. 84-93, 2005.

[140] G. N. Smith, "Probability and statistics in civil engineering," Collins Prof. Tech. books, vol. 244, 1986.

[141] G. Alexander and T. Alexander, "Beware of Q2," J Mol Graph Model, vol. 20, no. 4, pp. 269-276, 2002.

[142] P. P. Roy and K. Roy, "On some aspects of variable selection for partial least squares regression models," QSAR Comb. Sci., vol. 27, no. 3, pp. 302-313, 2008.

[143] A. H. Gandomi, A. H. Alavi, and M. G. Sahab, "New formulation for compressive strength of CFRP confined concrete cylinders using linear genetic programming," Mater. Struct., vol. 43, no. 7, pp. 963-983, 2010.

[144] D. G. Garson, "Interpreting neural network connection weights," 1991.

[145] A. K. Naiel, "Flexible pavement rut depth modeling for different climate zones," 2010.

[146] B. Javilla, H. Fang, L. Mo, B. Shu, and S. Wu, "Test evaluation of rutting performance indicators of asphalt mixtures," Constr. Build. Mater., vol. 155, pp. 1215-1223, 2017.

[147] A. Mansourkhaki, M. Ameri, M. Habibpour, and B. Shane Underwood, "Chemical 
Composition and Rheological characteristics of binders containing RAP and rejuvenator," J. Mater. Civ. Eng., vol. 32, no. 4, p. 4020026, 2020.

[148] H. Ziari, A. Amini, A. Goli, and D. Mirzaiyan, "Predicting rutting performance of carbon nano tube (CNT) asphalt binders using regression models and neural networks," Constr. Build. Mater., vol. 160, pp. 415-426, 2018.

[149] M. Ameri, D. Mirzaiyan, and A. Amini, "Rutting resistance and fatigue behavior of gilsonite-modified asphalt binders," J. Mater. Civ. Eng., vol. 30, no. 11, p. 4018292, 2018.

[150] R. F. Bonaquist, Ruggedness testing of the dynamic modulus and flow number tests with the simple performance tester, no. Project 9-29. 2008.

[151] L. F. Walubita, A. N. M. Faruk, J. Zhang, X. Hu, and S. I. Lee, "The Hamburg rutting test-Effects of HMA sample sitting time and test temperature variation," Constr. Build. Mater., vol. 108, pp. 22-28, 2016.

[152] W. Cao and Y. R. Kim, "A viscoplastic model for the confined permanent deformation of asphalt concrete in compression," Mech. Mater., vol. 92, pp. 235247, 2016.

[153] M. K. Darabi, R. K. A. Al-Rub, E. A. Masad, C.-W. Huang, and D. N. Little, “A modified viscoplastic model to predict the permanent deformation of asphaltic materials under cyclic-compression loading at high temperatures," Int. J. Plast., vol. 35, pp. 100-134, 2012.

[154] L. N. Mohammad, M. A. Elseifi, A. Raghavendra, and M. Ye, "Hamburg WheelTrack Test Equipment Requirements and Improvements to AASHTO T 324," 2015. 
[155] S. Schram, R. C. Williams, and A. Buss, "Reporting results from the Hamburg wheel tracking device," Transp. Res. Rec., vol. 2446, no. 1, pp. 89-98, 2014.

[156] F. Yin, E. Arambula, R. Lytton, A. E. Martin, and L. G. Cucalon, "Novel method for moisture susceptibility and rutting evaluation using Hamburg wheel tracking test," Transp. Res. Rec., vol. 2446, pp. 1-7, 2014, doi: 10.3141/2446-01.

[157] I. Sel, Y. Yildirim, and H. B. Ozhan, "Effect of test temperature on Hamburg wheel-tracking device testing," J. Mater. Civ. Eng., vol. 26, no. 8, p. 4014037, 2014.

[158] D. Swiertz, C. Ling, P. Teymourpour, and H. Bahia, "Use of the Hamburg wheeltracking test to characterize asphalt mixtures in cool weather regions," Transp. Res. Rec., vol. 2633, no. 1, pp. 9-15, 2017, doi: 10.3141/2633-03.

[159] F. Xiao, S. Amirkhanian, and C. H. Juang, "Rutting Resistance of Rubberized Asphalt Concrete Pavements Containing Reclaimed Asphalt Pavement Mixtures," J. Mater. Civ. Eng., vol. 19, no. 6, pp. 475-483, 2007, doi: 10.1061/(ASCE)08991561(2007)19:6(475).

[160] S. Vahidi, W. S. Mogawer, and A. Booshehrian, "Effects of GTR and Treated GTR on Asphalt Binder and High-RAP Mixtures," J. Mater. Civ. Eng., vol. 26, no. 4, pp. 721-727, 2013, doi: 10.1061/(asce)mt.1943-5533.0000858.

[161] E. Mahmoud, E. Masad, S. Nazarian, and I. Abdallah, "Modeling and Experimental Evaluation of Influence of Aggregate Blending on Asphalt Mixture Strength,” pp. 48-57, doi: 10.3141/2180-06.

[162] B. Shirini and R. Imaninasab, "Performance evaluation of rubberized and SBS modified porous asphalt mixtures," Constr. Build. Mater., vol. 107, pp. 165-171, 
2016.

[163] S. Grebenschikov and J. A. Prozzi, "Enhancing Mechanistic-Empirical Pavement Design Guide Rutting-Performance Predictions with Hamburg Wheel-Tracking Results," Transp. Res. Rec., vol. 2226, no. 1, pp. 111-118, 2011.

[164] S. Zhao, B. Huang, X. Shu, X. Jia, and M. Woods, "Laboratory performance evaluation of warm-mix asphalt containing high percentages of reclaimed asphalt pavement," Transp. Res. Rec., vol. 2294, no. 1, pp. 98-105, 2012.

[165] J. Zhang, G. S. Simate, X. Hu, M. Souliman, and L. F. Walubita, "Impact of recycled asphalt materials on asphalt binder properties and rutting and cracking performance of plant-produced mixtures," Constr. Build. Mater., vol. 155, pp. 654-663, 2017.

[166] H. Ozer, I. L. Al-Qadi, A. I. Kanaan, and D. L. Lippert, "Performance Characterization of Asphalt Mixtures at High Asphalt Binder Replacement with Recycled Asphalt Shingles," Transp. Res. Rec. J. Transp. Res. Board, vol. 2371, no. 1, pp. 105-112, 2013, doi: 10.3141/2371-12.

[167] M. Faramarzi, K. W. Lee, Y. Kim, and S. Kwon, “A case study on a cement treated RAP containing asphalt emulsion and acryl polymer," Case Stud. Constr. Mater., vol. 9, p. e00211, 2018.

[168] M. Rashidi, M. Saghafi, and H. Takhtfiroozeh, "Genetic programming model for estimation of settlement in earth dams," Int. J. Geotech. Eng., pp. 1-10, 2018.

[169] F. M. Nejad, F. Z. Motekhases, H. Zakeri, and A. Mehrabi, “An image processing approach to asphalt concrete feature extraction," J. Ind. Intell. Inf., vol. 3, 2015.

[170] P. Jiao, M. Roy, K. Barri, R. Zhu, I. Ray, and A. H. Alavi, "High-performance 
fiber reinforced concrete as a repairing material to normal concrete structures: Experiments, numerical simulations and a machine learning-based prediction model," Constr. Build. Mater., vol. 223, pp. 1167-1181, 2019.

[171] F. Zhou, S. Hu, and T. Scullion, "Balanced RAP/RAS mix design and performance evaluation system for project-specific service conditions.," Texas. Dept. of Transportation. Research and Technology Implementation Office, 2012.

[172] F. Zhou, S. Hu, G. Das, and T. Scullion, "High RAP mixes design methodology with balanced performance.," United States. Federal Highway Administration, 2011.

[173] S. B. Cooper III, L. N. Mohammad, S. Kabir, and W. King Jr, "Balanced asphalt mixture design through specification modification: Louisiana's experience," Transp. Res. Rec., vol. 2447, no. 1, pp. 92-100, 2014.

[174] W. G. Buttlar, B. C. Hill, H. Wang, and W. Mogawer, "Performance space diagram for the evaluation of high- and low-temperature asphalt mixture performance," Road Mater. Pavement Des., pp. 1-23, 2016, doi: 10.1080/14680629.2016.1267446.

[175] B. Hill, B. Behnia, W. G. Buttlar, and H. Reis, "Evaluation of Warm Mix Asphalt Mixtures Containing Reclaimed Asphalt Pavement through Mechanical Performance Tests and an Acoustic Emission Approach," J. Mater. Civ. Eng., vol. 25, no. December, pp. 1887-1897, 2013, doi: 10.1061/(ASCE)MT.19435533.0000757.

[176] I. L. Al-Qadi, M. Elseifi, and S. H. Carpenter, "Reclaimed asphalt pavement - A literature review," no. FHWA-ICT-07-001, p. 23, 2007, doi: 
http://hdl.handle.net/2142/46007.

[177] M. V Valueva, N. N. Nagornov, P. A. Lyakhov, G. V Valuev, and N. I.

Chervyakov, "Application of the residue number system to reduce hardware costs of the convolutional neural network implementation," Math. Comput. Simul., 2020.

[178] I. Goodfellow, Y. Bengio, and A. Courville, Deep learning. MIT press, 2016.

[179] B. Rezaeianjouybari and Y. Shang, "Deep learning for prognostics and health management: State of the art, challenges, and opportunities," Measurement, p. 107929, 2020.

[180] W. Liu, Z. Wang, X. Liu, N. Zeng, Y. Liu, and F. E. Alsaadi, “A survey of deep neural network architectures and their applications," Neurocomputing, vol. 234, pp. 11-26, 2017.

[181] Y. Kim, "Convolutional neural networks for sentence classification," arXiv Prepr. $\operatorname{arXiv1408.5882,2014.}$

[182] K. Gopalakrishnan, "Deep learning in data-driven pavement image analysis and automated distress detection: A review," Data, vol. 3, no. 3, p. 28, 2018.

[183] A. Zhang et al., "Automated pixel-level pavement crack detection on 3D asphalt surfaces using a deep-learning network," Comput. Civ. Infrastruct. Eng., vol. 32, no. 10, pp. 805-819, 2017.

[184] J. Jiang, Z. Zhang, Q. Dong, and F. Ni, "Characterization and identification of asphalt mixtures based on Convolutional Neural Network methods using X-ray scanning images," Constr. Build. Mater., vol. 174, pp. 72-80, 2018.

[185] H. Gong, Y. Sun, Z. Mei, and B. Huang, "Improving accuracy of rutting prediction 
for mechanistic-empirical pavement design guide with deep neural networks," Constr. Build. Mater., vol. 190, pp. 710-718, 2018.

[186] G. E. Hinton, N. Srivastava, A. Krizhevsky, I. Sutskever, and R. R. Salakhutdinov, "Improving neural networks by preventing co-adaptation of feature detectors," arXiv Prepr. arXiv1207.0580, 2012.

[187] V. Nair and G. E. Hinton, "Rectified linear units improve restricted boltzmann machines," 2010.

[188] T. Tieleman and G. Hinton, "Lecture 6.5-rmsprop: Divide the gradient by a running average of its recent magnitude," COURSERA Neural networks Mach. Learn., vol. 4, no. 2, pp. 26-31, 2012.

[189] ASCE, “2017 infrastructure report card,” 2017.

[190] N. Kargah-Ostadi, A. Nazef, J. Daleiden, and Y. Zhou, "Evaluation Framework for Automated Pavement Distress Identification and Quantification Applications," Transp. Res. Rec., vol. 2639, no. 1, pp. 46-54, 2017.

[191] W. D. Paterson, "Proposal of universal cracking indicator for pavements (with discussion and closure)," Transp. Res. Rec., no. 1455, 1994.

[192] H. Lee, "Accuracy, precision, repeatability and compatibility of the Pavedex PAS 1 automated distress measuring device," Transp. Res. Rec., vol. 1311, pp. 136143, 1991.

[193] KICT, "Final Report of The National Highway Pavement Management System 2016." Korea Institute of Civil Engineering and Building Technology, Gyeonggido ..., 2017.

[194] W. Vavrik, L. Evans, S. Sargand, and J. Stefanski, "PCR evaluation: considering 
transition from manual to semi-automated pavement distress collection and analysis.," 2013.

[195] P. Fu, J. T. Harvey, J. N. Lee, and P. Vacura, "New method for classifying and quantifying cracking of flexible pavements in automated pavement condition survey," Transp. Res. Rec., vol. 2225, no. 1, pp. 99-108, 2011.

[196] M. Saghafi, S. M. Asgharzadeh, A. Fathi, and A. Hosseini, "Image processing method to estimate the wearing condition of slurry seal mixtures," in Transportation \& Development Institute (T\&DI), International Airfield and Highway Pavements Conference, 2019, vol. 2019.

[197] X. Jia, B. Huang, Q. Dong, D. Zhu, and J. Maxwell, "Influence of pavement condition data variability on network-level maintenance decision," Transp. Res. Rec., vol. 2589, no. 1, pp. 20-31, 2016.

[198] S. Chambon and J.-M. Moliard, “Automatic road pavement assessment with image processing: review and comparison," Int. J. Geophys., vol. 2011, 2011.

[199] H. Oliveira and P. L. Correia, "Automatic road crack segmentation using entropy and image dynamic thresholding," in 2009 17th European Signal Processing Conference, 2009, pp. 622-626.

[200] Y.-C. Tsai, V. Kaul, and R. M. Mersereau, "Critical assessment of pavement distress segmentation methods," J. Transp. Eng., vol. 136, no. 1, pp. 11-19, 2010.

[201] D. Zhang, Q. Li, Y. Chen, M. Cao, L. He, and B. Zhang, “An efficient and reliable coarse-to-fine approach for asphalt pavement crack detection," Image Vis. Comput., vol. 57, pp. 130-146, 2017.

[202] A. Zhang, Q. Li, K. C. P. Wang, and S. Qiu, "Matched filtering algorithm for 
pavement cracking detection," Transp. Res. Rec., vol. 2367, no. 1, pp. 30-42, 2013.

[203] A. Ayenu-Prah and N. Attoh-Okine, "Evaluating pavement cracks with bidimensional empirical mode decomposition," EURASIP J. Adv. Signal Process., vol. 2008, pp. 1-7, 2008.

[204] Y. Zhou, F. Wang, N. Meghanathan, and Y. Huang, "Seed-based approach for automated crack detection from pavement images," Transp. Res. Rec., vol. 2589, no. 1, pp. 162-171, 2016.

[205] J. Zhou, P. Huang, and F.-P. Chiang, "Wavelet-based pavement distress classification," Transp. Res. Rec., vol. 1940, no. 1, pp. 89-98, 2005.

[206] P. Subirats, J. Dumoulin, V. Legeay, and D. Barba, "Automation of pavement surface crack detection using the continuous wavelet transform," in 2006 International Conference on Image Processing, 2006, pp. 3037-3040.

[207] K. C. P. Wang, Q. Li, and W. Gong, "Wavelet-based pavement distress image edge detection with a trous algorithm," Transp. Res. Rec., vol. 2024, no. 1, pp. 7381, 2007.

[208] L. Ying and E. Salari, "Beamlet transform-based technique for pavement crack detection and classification," Comput. Civ. Infrastruct. Eng., vol. 25, no. 8, pp. $572-580,2010$.

[209] C. Koch, K. Georgieva, V. Kasireddy, B. Akinci, and P. Fieguth, "A review on computer vision based defect detection and condition assessment of concrete and asphalt civil infrastructure," Adv. Eng. Informatics, vol. 29, no. 2, pp. 196-210, 2015. 
[210] H. Oliveira and P. L. Correia, "Automatic road crack detection and characterization," IEEE Trans. Intell. Transp. Syst., vol. 14, no. 1, pp. 155-168, 2012.

[211] Y. Fujita, K. Shimada, M. Ichihara, and Y. Hamamoto, "A method based on machine learning using hand-crafted features for crack detection from asphalt pavement surface images," in Thirteenth International Conference on Quality Control by Artificial Vision 2017, 2017, vol. 10338, p. 103380I.

[212] A. Hizukuri and T. Nagata, "Development of a classification method for a crack on a pavement surface images using machine learning," in Thirteenth International Conference on Quality Control by Artificial Vision 2017, 2017, vol. 10338, p. 103380M.

[213] Q. Zou, Y. Cao, Q. Li, Q. Mao, and S. Wang, "CrackTree: Automatic crack detection from pavement images," Pattern Recognit. Lett., vol. 33, no. 3, pp. $227-$ $238,2012$.

[214] Y. Cha, W. Choi, and O. Büyüköztürk, "Deep learning-based crack damage detection using convolutional neural networks," Comput. Civ. Infrastruct. Eng., vol. 32, no. 5, pp. 361-378, 2017.

[215] H. Maeda, Y. Sekimoto, T. Seto, T. Kashiyama, and H. Omata, "Road damage detection using deep neural networks with images captured through a smartphone," arXiv Prepr. arXiv1801.09454, 2018.

[216] L. Zhang, F. Yang, Y. D. Zhang, and Y. J. Zhu, "Road crack detection using deep convolutional neural network," in 2016 IEEE international conference on image processing (ICIP), 2016, pp. 3708-3712. 
[217] Z. Fan, Y. Wu, J. Lu, and W. Li, "Automatic pavement crack detection based on structured prediction with the convolutional neural network," arXiv Prepr. arXiv1802.02208, 2018.

[218] V. Mandal, L. Uong, and Y. Adu-Gyamfi, “Automated road crack detection using deep convolutional neural networks," in 2018 IEEE International Conference on Big Data (Big Data), 2018, pp. 5212-5215.

[219] D. Xie, L. Zhang, and L. Bai, "Deep learning in visual computing and signal processing,” Appl. Comput. Intell. Soft Comput., vol. 2017, 2017.

[220] A. Agrawal and A. Choudhary, "Perspective: Materials informatics and big data: Realization of the 'fourth paradigm' of science in materials science," Apl Mater., vol. 4, no. 5, p. 53208, 2016.

[221] E. Zalama, J. Gómez-García-Bermejo, R. Medina, and J. Llamas, "Road crack detection using visual features extracted by Gabor filters," Comput. Civ. Infrastruct. Eng., vol. 29, no. 5, pp. 342-358, 2014.

[222] B. Akarsu, M. KARAKÖSE, K. PARLAK, A. Erhan, and A. SARIMADEN, “A fast and adaptive road defect detection approach using computer vision with real time implementation," Int. J. Appl. Math. Electron. Comput., no. Special Issue-1, pp. 290-295, 2016.

[223] M. Kawano, K. Mikami, S. Yokoyama, T. Yonezawa, and J. Nakazawa, "Road marking blur detection with drive recorder," in 2017 IEEE International Conference on Big Data (Big Data), 2017, pp. 4092-4097.

[224] K. Zhang, H. D. Cheng, and B. Zhang, "Unified approach to pavement crack and sealed crack detection using preclassification based on transfer learning," $J$. 
Comput. Civ. Eng., vol. 32, no. 2, p. 4018001, 2018.

[225] L. Some, "Automatic image-based road crack detection methods.” 2016.

[226] M. Eisenbach et al., "How to get pavement distress detection ready for deep learning? A systematic approach," in 2017 international joint conference on neural networks (IJCNN), 2017, pp. 2039-2047.

[227] K. Gopalakrishnan, S. K. Khaitan, A. Choudhary, and A. Agrawal, "Deep Convolutional Neural Networks with transfer learning for computer vision-based data-driven pavement distress detection," Constr. Build. Mater., vol. 157, pp. 322330, 2017.

[228] Z. Tong, J. Gao, and H. Zhang, "Recognition, location, measurement, and 3D reconstruction of concealed cracks using convolutional neural networks," Constr. Build. Mater., vol. 146, pp. 775-787, 2017.

[229] R. Girshick, "Fast r-cnn," in Proceedings of the IEEE international conference on computer vision, 2015, pp. 1440-1448.

[230] O. Ronneberger, P. Fischer, and T. Brox, "U-net: Convolutional networks for biomedical image segmentation," in International Conference on Medical image computing and computer-assisted intervention, 2015, pp. 234-241.

[231] H. N. Koutsopoulos and A. B. Downey, "Primitive-based classification of pavement cracking images," J. Transp. Eng., vol. 119, no. 3, pp. 402-418, 1993.

[232] Y. O. Adu-Gyamfi, N. O. A. Okine, G. Garateguy, R. Carrillo, and G. R. Arce, "Multiresolution information mining for pavement crack image analysis," $J$. Comput. Civ. Eng., vol. 26, no. 6, pp. 741-749, 2012.

[233] B. Huval et al., "An empirical evaluation of deep learning on highway driving," 
arXiv Prepr. arXiv1504.01716, 2015.

[234] T. Hajilounezhad et al., "Exploration of Carbon Nanotube Forest SynthesisStructure Relationships Using Physics-Based Simulation and Machine Learning," 2020.

[235] H. Majidifard, P. Jin, Y. Adu-Gyamfi, and W. G. Buttlar, "PID: A New Benchmark Dataset to Classify and Densify Pavement Distresses," arXiv Prepr. arXiv1910.11123, 2019.

[236] Y. H. Huang, "Pavement analysis and design,” 2004.

[237] M. Y. Shahin, Pavement management for airports, roads, and parking lots, vol. 501. Springer New York, 2005.

[238] D. ASTM, "Standard practice for roads and parking lots pavement condition index surveys," 2011.

[239] ASTM, "Standard test method for airport pavement condition index surveys," Designation D5340-12. ASTM International West Conshohocken, PA, US, 2012.

[240] D. Walker, “Asphalt-PASER Manual: Pavement Surface Evaluation and Rating," 1987.

[241] N. N. Eldin and A. B. Senouci, "A pavement condition-rating model using backpropagation neural networks," Comput. Civ. Infrastruct. Eng., vol. 10, no. 6, pp. 433-441, 1995.

[242] S. M. Piryonesi and T. E. El-Diraby, "Data Analytics in Asset Management: CostEffective Prediction of the Pavement Condition Index," J. Infrastruct. Syst., vol. 26, no. 1, p. 4019036, 2020.

[243] H. Shahnazari, M. A. Tutunchian, M. Mashayekhi, and A. A. Amini, "Application 
of soft computing for prediction of pavement condition index," J. Transp. Eng., vol. 138, no. 12, pp. 1495-1506, 2012.

[244] H. N. Koutsopoulos, I. El Sanhouri, and A. B. Downey, “Analysis of segmentation algorithms for pavement distress images," J. Transp. Eng., vol. 119, no. 6, pp. 868-888, 1993.

[245] A. Cuhadar, K. Shalaby, and S. Tasdoken, "Automatic segmentation of pavement condition data using wavelet transform," in IEEE CCECE2002. Canadian Conference on Electrical and Computer Engineering. Conference Proceedings (Cat. No. 02CH37373), 2002, vol. 2, pp. 1009-1014.

[246] Q. Li and X. Liu, "Novel approach to pavement image segmentation based on neighboring difference histogram method," in 2008 Congress on Image and Signal Processing, 2008, vol. 2, pp. 792-796.

[247] S. Jitprasithsiri, H. Lee, R. G. Sorcic, and R. Johnston, "Development of digital image-processing algorithm to compute unified crack index for Salt Lake City," Transp. Res. Rec., vol. 1526, no. 1, pp. 142-148, 1996.

[248] X. Bai, F. Zhou, and B. Xue, "Multiple linear feature detection based on multiplestructuring-element center-surround top-hat transform," Appl. Opt., vol. 51, no. 21, pp. 5201-5211, 2012.

[249] N. Tanaka and K. Uematsu, "A Crack Detection Method in Road Surface Images Using Morphology.," MVA, vol. 98, pp. 17-19, 1998.

[250] N. Dalal and B. Triggs, "Histograms of oriented gradients for human detection," in 2005 IEEE computer society conference on computer vision and pattern recognition (CVPR'05), 2005, vol. 1, pp. 886-893. 
[251] J. Redmon, S. Divvala, R. Girshick, and A. Farhadi, "You only look once: Unified, real-time object detection," in Proceedings of the IEEE conference on computer vision and pattern recognition, 2016, pp. 779-788.

[252] S. Zhang, L. Wen, X. Bian, Z. Lei, and S. Z. Li, "Single-shot refinement neural network for object detection," in Proceedings of the IEEE conference on computer vision and pattern recognition, 2018, pp. 4203-4212.

[253] S. Ren, K. He, R. Girshick, and J. Sun, "Faster r-cnn: Towards real-time object detection with region proposal networks," in Advances in neural information processing systems, 2015, pp. 91-99.

[254] W. Liu et al., "Ssd: Single shot multibox detector," in European conference on computer vision, 2016, pp. 21-37.

[255] H. Majidifard, "PID - Pavement Image Dataset,” 2020.

[256] K. McGhee, A. Habib, and T. Chowdhury, "Development and implementation of pavement condition indices for the Virginia Department of Transportation," Phase I Flex. Pavements, 2002.

[257] M. Saghafi, S. Nazarian, I. Abdallah, C. Tirado, and D. Rodrigues, "Incorporating Wheel Tracking Test Results in Flexible Pavement Rutting Analysis," Int. J. Pavement Eng.

[258] Y. D. Wang, A. Ghanbari, B. S. Underwood, and Y. R. Kim, "Development of preliminary transfer functions for performance predictions in FlexPAVE ${ }^{\mathrm{TM}}$," Constr. Build. Mater., vol. 266, p. 121182, 2021.

[259] A. Ghanbari, "Performance and Reliability Analysis of Asphalt Mixtures in a Performance-Related Specification.,” 2020. 
[260] A. Khanal and R. Estrada, "Dynamic Deep Networks for Retinal Vessel Segmentation," arXiv Prepr. arXiv1903.07803, 2019. 


\section{VITA}

Hamed Majidifard was born in Kerman, Iran. He completed his Bachelors of Science in Civil Engineering at the Shahid Bahonar University of Kerman, graduating in September 2013. In Jan 2016 Hamed Received a Master of Science in Civil Engineering from the Sharif University of Technology, for his thesis work, "Investigating the Effect of Softening Agent on the Performance Characterization of Asphalt Concrete Mixture with High RAP Content ". Finally, he received a Doctoral Degree in Civil Engineering from the University of Missouri for his work, "Developing a Modern Recycled Mix Design and Automatic Pavement Monitoring System". His research primarily focuses on implementing machine learning techniques to predict the performance of asphalt mixtures and pavement conditions, advancement of mixture sustainability, and promoting durability of asphalt mixtures (crack resistance, balanced mix design). Additionally, he has carried out multiple research studies with the focus on devising a programming approach to predict the low and high-temperature performance of asphalt mixtures using machine learning techniques. This tool can be used for pre-design purposes to design an asphalt mixture with balanced performance in rutting and cracking. Finally, his primary research was about using deep learning techniques to devise an automatic pavement monitoring system. This allows for effective assessment of the pavement condition using pictures and videos instead of manual field observation and evaluation (www.fardco.net/softwares). He published several journal and conference papers during the past year. He also peer-reviewed more than 20 articles for Road and Material Pavement Design, Construction Building Material, and Transportation Research Record journals. 